UNIVERSIDAD POLITÉCNICA DE MADRID

E.T.S. DE INGENIEROS DE CAMINOS, CANALES Y PUERTOS

Análisis del efecto de la rugosidad en el contacto rocapilote sobre la resistencia por fuste de pilotes

TESIS DOCTORAL

JOSÉ GREGORIO GUTIÉRREZ CHACON

INGENIERO GEÓLOGO 


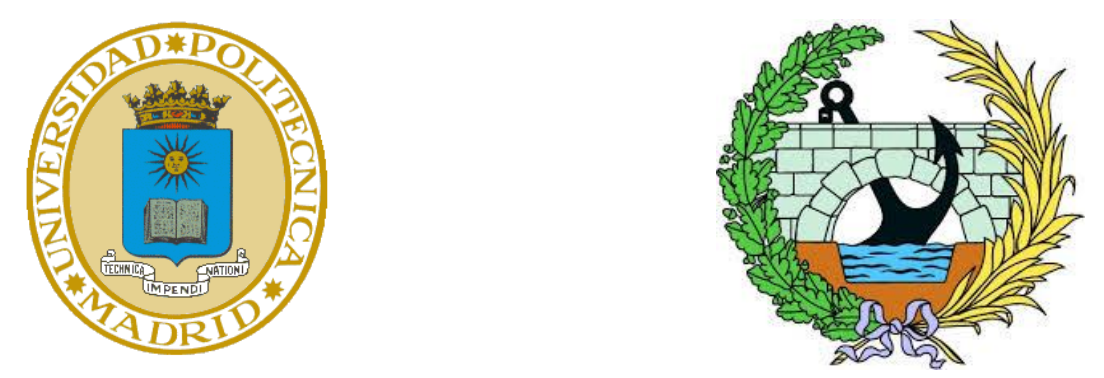

Departamento de Ingeniería y Morfología del Terreno

Escuela Técnica Superior de Ingenieros de Caminos, Canales y Puertos Universidad Politécnica de Madrid

\title{
Análisis del efecto de la rugosidad en el contacto roca- pilote sobre la resistencia por fuste de pilotes
}

\author{
Tesis Doctoral \\ José Gregorio Gutiérrez Chacón \\ Ingeniero Geólogo
}

Directores:

Rafael Jiménez Rodríguez

Doctor Ingeniero de Caminos, Canales y Puertos

Svetlana Melentijevic

Doctora Ingeniero de Caminos, Canales y Puertos

Madrid, 2020 

Título de la tesis:

Análisis del efecto de la rugosidad en el contacto roca-pilote sobre la resistencia por fuste de pilotes

Autor: José Gregorio Gutiérrez Chacón

Directores: Rafael Jiménez Rodríguez y Svetelana Melentijevic

Tribunal nombrado por el Mgfco. Y Excmo. Sr. Rector de la Universidad Politécnica de Madrid, el día de de

TRIBUNAL CALIFICADOR

Presidente:

Vocal 1ㅇ:

Vocal $2^{\circ}$ :

Vocal 3:

Secretario:

Realizado el acto de defensa y lectura de la tesis el día de de en Madrid, los miembros del Tribunal acuerdan otorgar la calificación de: 

Esta Tesis Doctoral ha sido financiada por las siguientes instituciones:

Fundación José Entrecanales Ibarra, mediante una de sus becas "José Entrecanales Ibarra" para el fomento de la investigación entre doctorandos.

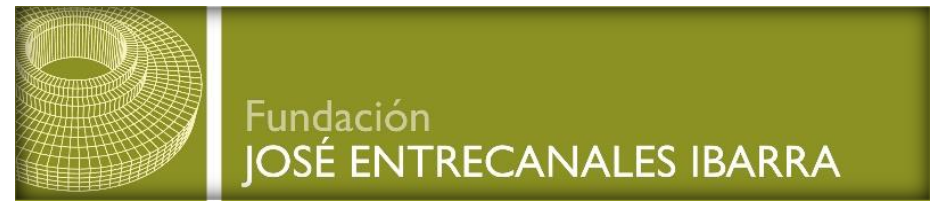

Ministerio de Economía y Competitividad, mediante el Proyecto de Investigación "Predicción y análisis de deformaciones para la seguridad en túneles" (ref. BIA 2015-69152-R).

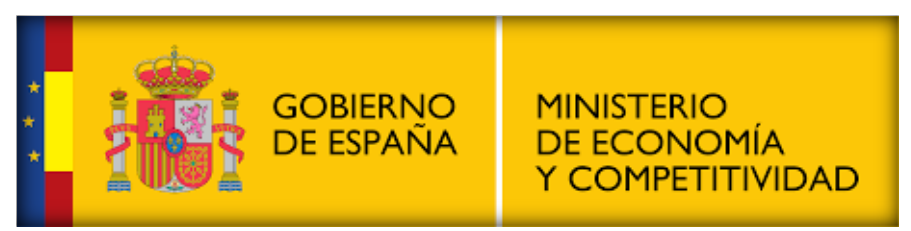



A mi esposa, Eliana

A mis padres Hernan y Maritza 



\section{ÍNDICE}

Listado de Figuras................................................................................................

Listado de Tablas .............................................................................................. xxxix

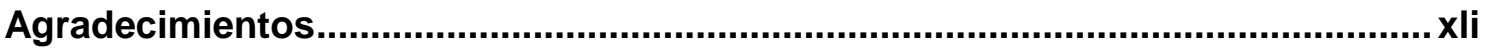

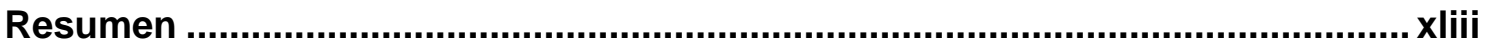

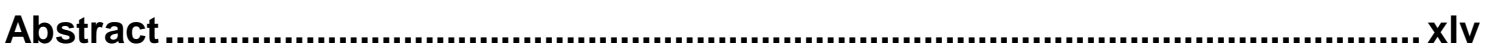

Listado de Figuras ................................................................................................ xvi

Listado de Tablas .............................................................................................. xxxix

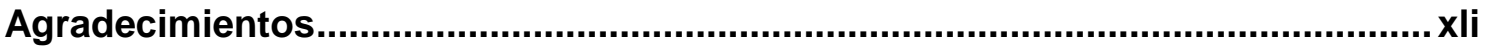

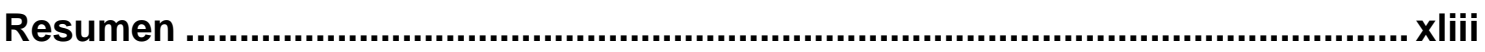

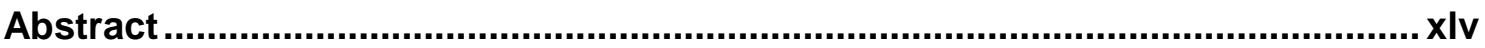

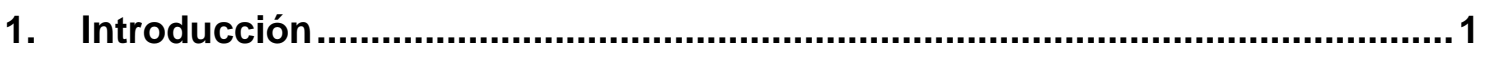

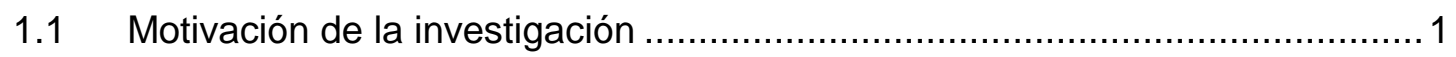

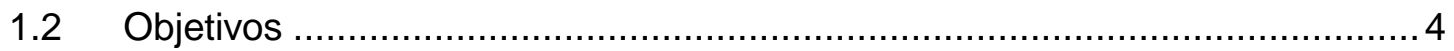

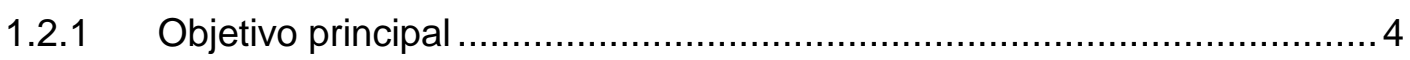

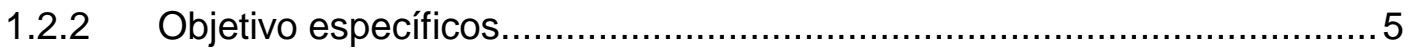

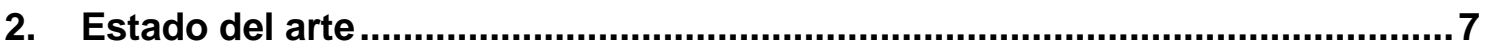

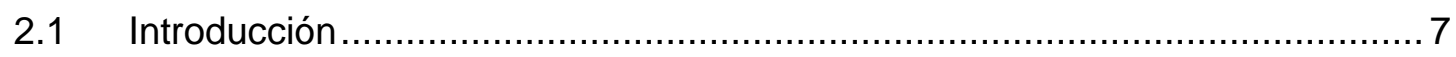

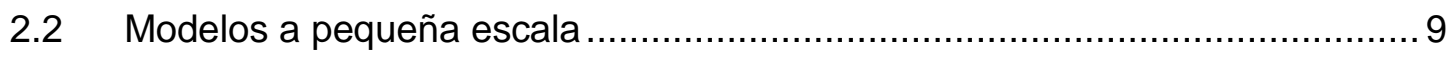

2.2.1. Ensayos de corte directo en laboratorio e in situ................................... 9

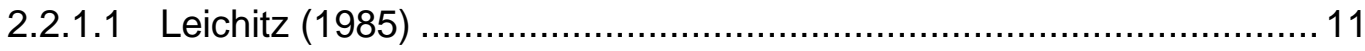

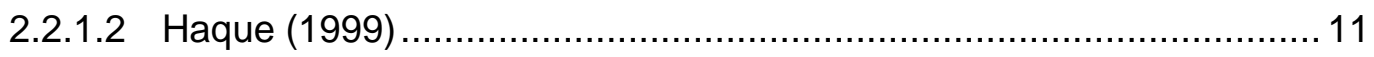


2.2.1.3 Gu et al. (2003)

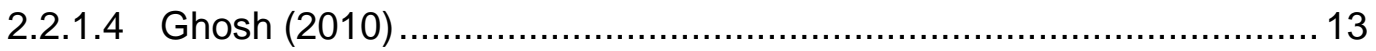

2.2.1.5 Liahagen (2012) y Gutiérrez (2013) .......................................... 14

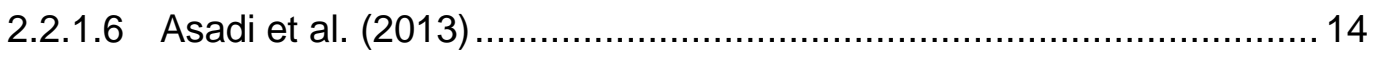

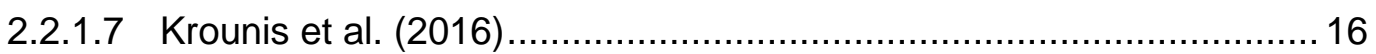

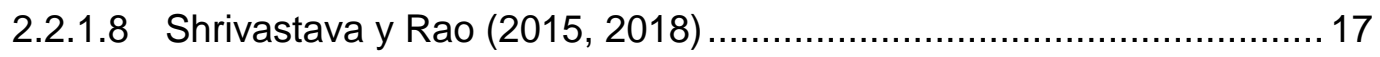

2.2.2. Ensayos de corte directo con modelos numéricos a través de la implementación del Método de Elementos Discretos ....................................... 19

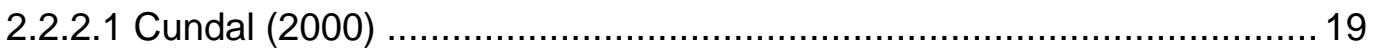

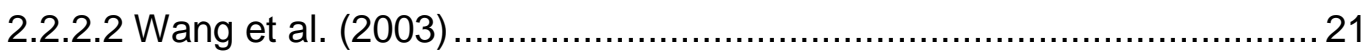

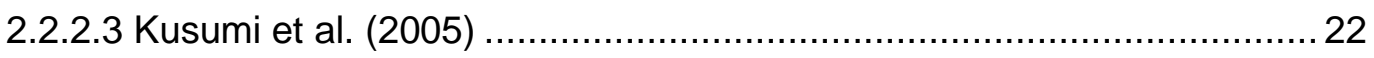

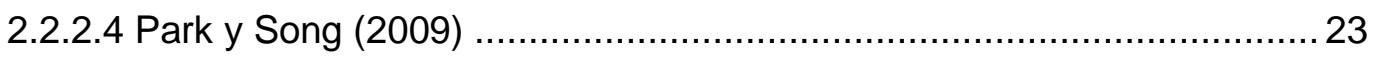

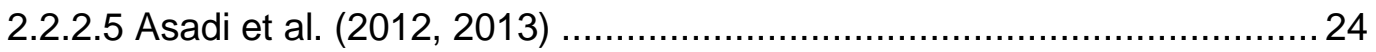

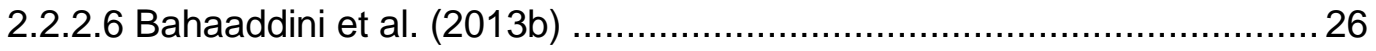

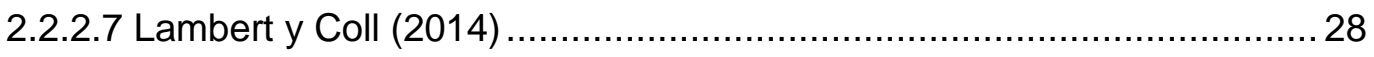

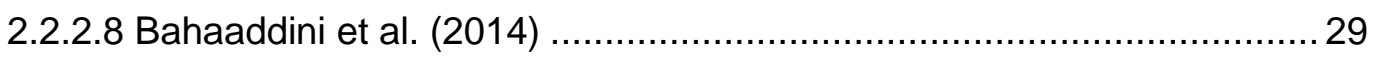

2.2.2.9 Bahaaddini et al. (2016), Bahaaddini (2017)...................................... 30

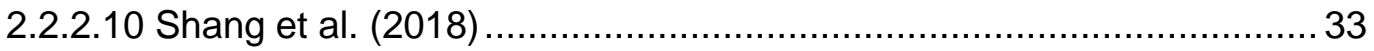

2.2.3. Ensayos de carga sobre pilotes en el laboratorio................................ 34

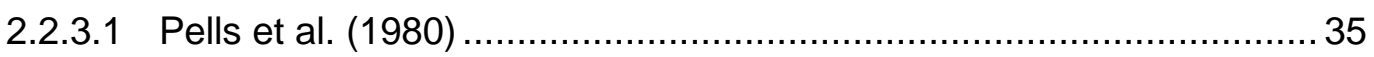

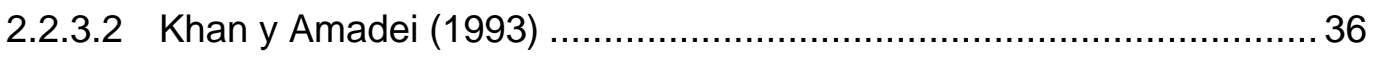

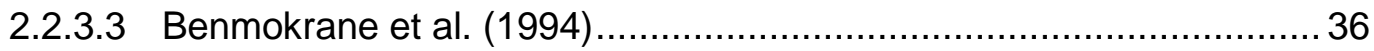

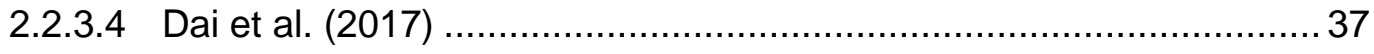


2.3.1. Ensayos de carga sobre pilotes en campo ...................................... 39

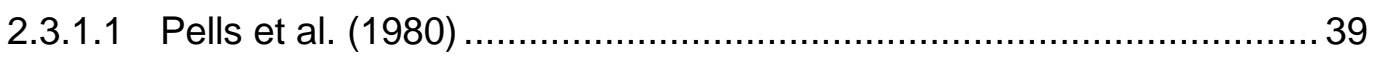

2.3.1.2 Williams (1980), Williams y Pells (1981)...................................... 40

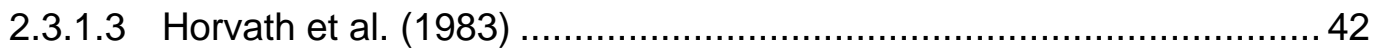

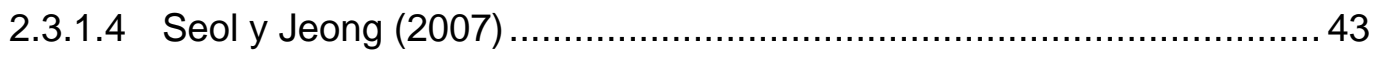

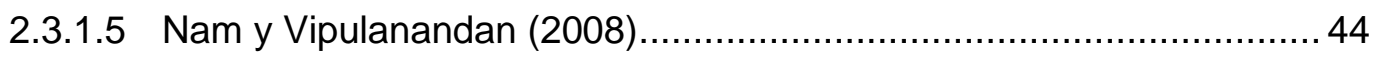

2.3.2. Ensayos de carga sobre pilotes con modelos numéricos a través de la implementación del Método de Elementos Finitos...........................................45

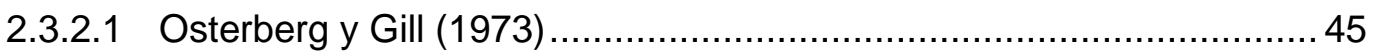

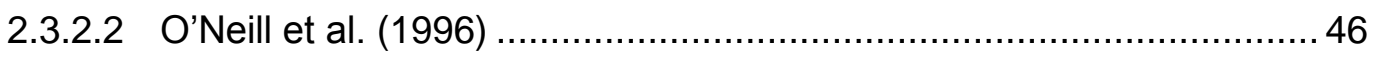

2.3.2.3 Melentijevic et al. (2012), Melentijevic y Olalla (2014) ..................... 47

2.3.2.4 Gutiérrez-Ch (2015), Gutiérrez-Ch y Melentijevic (2016)................... 49

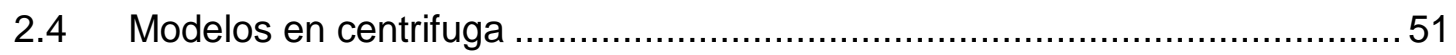

2.4.1 Ensayos de carga sobre pilotes mediante ensayos de centrifuga ..........53

2.4.1.1 Leung y Ko (1993) …...................................................... 53

2.4.1.2 Dykeman y Valsangkar (1996) …........................................... 54

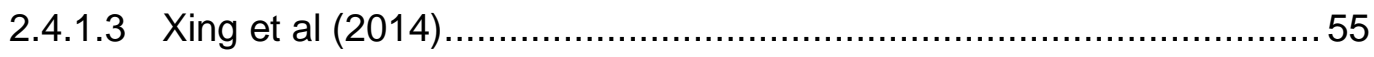

3. Modelos numéricos a pequeña escala considerando la interacción roca-

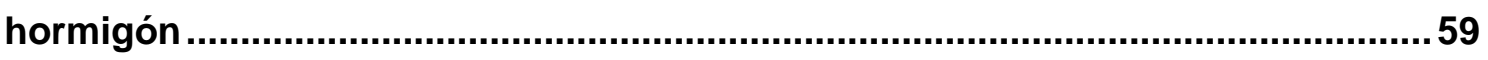

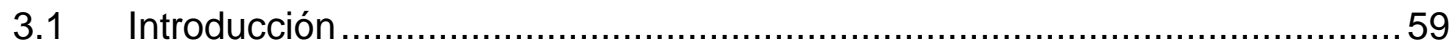

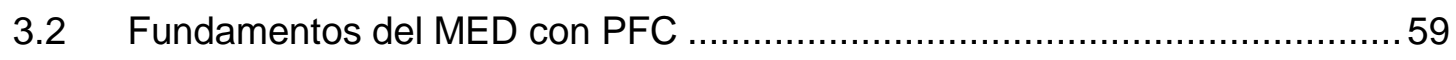

3.2.1 Modelo de contacto flat-joint (FJCM) ......................................... 60 
3.2.2 Modelo de contacto smooth-joint (SJCM)

3.3 Configuración del modelo numérico para el ensayo de corte directo con MED.

64

3.3.1 Descripción de los ensayos experimentales .65

3.3.2 Calibración de los microparámetros del modelo de contacto flat-joint....65

3.3.3 Generación de la probeta para el ensayo de corte directo y aplicación de las condiciones de borde 73

3.3.4 Calibración de los microparámetros del modelo de contacto smooth joint. 81

3.4 Resultados .85

3.4.1 Ensayos de corte directo en condición CNL .86

3.4.2 Ensayos de corte directo en condición CNS 91

3.4.3 Comparación entre la condición CNL y CNS .94

4. Modelos numéricos a gran escala de pilotes empotrados en roca

4.1 Introducción 97

4.2 Configuración del modelo numérico con MED para pilotes cargados axialmente y empotrados en roca. .98

4.2.1 Esquema del modelo MED ${ }^{3 D}$ .98

4.2.2 Rugosidad de la interfaz roca-pilote............................................ 98

4.2.3 Calibración de los microparámetros de los materiales ......................... 100

4.2.4 Ensamblaje inicial del modelo...................................................... 101

4.2.5 Aplicación del esfuerzo isotrópico inicial ........................................ 103

4.2.6 Eliminación de partículas "flotantes" ................................................ 104

4.2.7 Aplicación de los modelos de contacto FJCM y SJCM .........................104 
4.2.8 Aplicación de las cargas actuantes y condiciones de borde. 106

4.3 Resultados de los modelos con MED …................................................ 107

4.3.1 Comportamiento de carga axial-asiento....................................... 107

4.3.2 Comportamiento de resistencia promedio por fuste-asiento ................ 109

4.3.3 Esfuerzo axial y resistencia por fuste movilizada a lo largo del pilote ...114

4.3.4 Mecanismo de transferencia de carga ........................................... 120

4.4 Configuración del modelo numérico con MEF para pilotes cargados axialmente

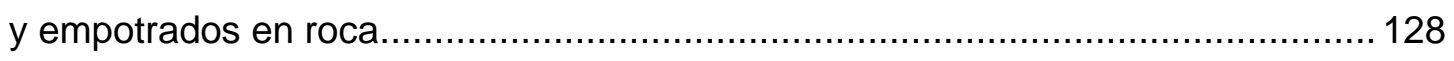

4.4.1 Esquema del modelo MEF...................................................... 129

4.5 Resultados de los modelos con MEF ................................................ 131

4.5.1 Comportamiento de resistencia promedio por fuste-asiento para los modelos de pilotes empotrados en la arenisca-S3

131

4.5.2 Comportamiento para los modelos de pilotes empotrados en la pseudoroca

4.5.2.1 Respuesta carga axial-asiento 134

4.5.2.2 Respuesta de resistencia promedio por fuste y por punta-asiento 135

5. Ensayos de centrífuga de pilotes empotrados en roca 139

5.1 Introducción 139

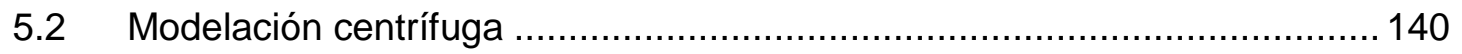

5.2.1 Pseudo-roca 141

5.2.2 Modelo de los Pilotes.................................................................. 143

5.2.2.1 Fabricación

5.2.2.2 Instrumentación 144

5.2.2.3 Calibración de los sensores FBG. 
5.2.3 Preparación del modelo de centrífuga 150

5.2.4 Ensayos de centrífuga 151

5.3 Resultados .153

5.3.1 Respuesta carga-asiento .153

5.3.2 Fuerza axial 156

5.3.3 Resistencia por fuste 159

5.3.4 Comparación con modelos numéricos mediante el MEF 163

6. Formulación para la estimación de la resistencia por fuste de pilotes empotrados en roca considerando la rugosidad de la excavación .167

6.1 Introducción 167

6.2 Formulaciones para el cálculo de la resistencia por fuste de pilotes empotrados en roca 167

6.3 Comparación con formulaciones de diseño 172

6.4 Comparación con el factor empírico $\alpha$ 182

6.5 Factor $\boldsymbol{\alpha R F , 0 . 0 1 D}$ para la estimación de la resistencia por fuste. 186

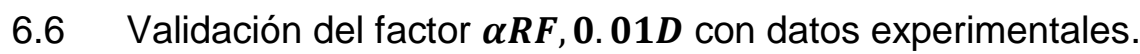
187

6.7 Aplicación del factor $\boldsymbol{\alpha R F , 0 . 0 1 D}$ a nuevos casos de estudio 190

6.7.1 Ensayos de carga realizados en Lexington 191

6.7.1.1 Ensayo de carga TS-1A 192

6.7.1.2 Ensayo de carga TS-2 192

6.7.1.3 Resultados 194

6.7.1.4 Implicación práctica

6.7.2 Ensayo de carga realizado en Kansas 198 
6.7.2.1 Ensayo de carga GT

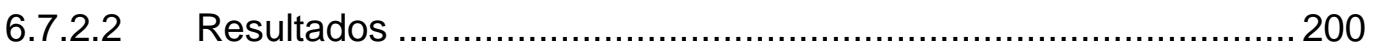

6.7.2.3 Implicación práctica ...............................................................203

7. Conclusiones y líneas de investigación futuras............................................205

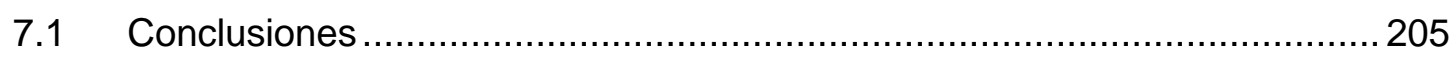

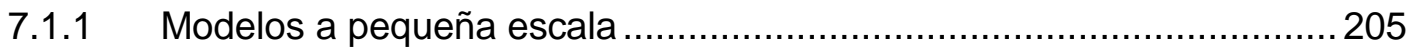

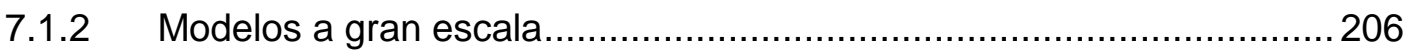

7.1.3 Ensayos de centrífuga ................................................................. 208

7.1.4 Contraste, comparación y propuesta de diseño .................................209

7.2 Líneas de investigación futuras..........................................................210

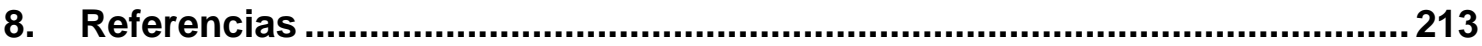

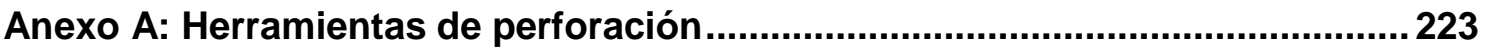

Anexo B: Registro fotográfico de los ensayos de laboratorio .............................227 


\section{Listado de Figuras}

Figura 1.1. Pilotes empotrados en roca: (a) pilote por fuste, (b) pilote por punta y fuste (modificado de Gutiérrez-Ch y Melentijevic 2016). 1

Figura 2.1. (a) envolventes de falla propuestas por Patton (1966) para caracterizar la resistencia al corte de juntas de roca plana y rugosas, (b) detalle de la situación 2, (c) detalle de la situación 3 (modificado de Gutiérrez 2013). 7

Figura 2.2. (a) perfiles de rugosidad típicos y su correspondiente valor JRC (modificado de Barton y Choubey 1977), (b) estimación del perfil de rugosidad de la junta de roca mediante el uso del perfilómetro o "peine" de Barton. 8

Figura 2.3. Talud de roca, condición de borde CNL (modificado de Poturovic et al. 2015).

Figura 2.4. Pilote empotrado en roca, condición de borde CNS (modificado de Johnston et al. 1987) 10

Figura 2.5. Ensayos de corte directo en probetas de arenisca con juntas rugosas: (a) condición CNL, (b) condición CNS (Leichnitz 1985). 11

Figura 2.6. Ensayos de corte directo en probetas de arenisca con juntas rugosas para ángulo base de 9.5: (a) comparación entre condición CNL y CNS, (b) efecto del relleno y su espesor sobre la resistencia al corte pico (modificado de Haque 1999). .12

Figura 2.7. Ensayos de resistencia al corte en juntas triangulares arenisca-hormigón: (a) sin cohesión, condición CNL con $\sigma n=400 \mathrm{kPa}$, (b) sin cohesión, condición CNS con $\sigma n o=400 \mathrm{kPa}$ y $K=800,1600$ y 3200 kPa/mm, (c) con cohesión, condición CNS con $\sigma n o=400 \mathrm{kPa}$ y $K=800 \mathrm{kPa} / \mathrm{mm}$, (d) con relleno de diferente espesor, condición CNS con $\sigma n o=400 \mathrm{kPa}$ y $K=800 \mathrm{kPa} / \mathrm{mm}$ (modificado de Gu et al. 2003). 13 
Figura 2.8. (a) configuración del ensayo de corte directo in situ con interacción rocahormigón, (b) curvas de resistencia al corte vs desplazamiento al corte (modificado de Ghosh 2010) 13

Figura 2.9. Ensayos de corte directo en probetas roca-hormigón con junta rugosa bajo condición CNL: (a) perfiles de rugosidad y probetas con diferente $\alpha$, (b) curvas de carga al corte vs desplazamiento al corte para juntas granito-hormigón con distinta rugosidad, sin cohesión y con $\sigma n=1200 \mathrm{kPa}$, (c) curvas de carga al corte vs desplazamiento al corte para juntas planas granito-hormigón, con y sin cohesión, para $\sigma n=1200 \mathrm{kPa}$, (d) curvas de carga al corte vs desplazamiento al corte para juntas gneis-hormigón con distinta rugosidad, sin cohesión y con $\sigma n=3200 \mathrm{kPa}$, (modificado de Liahagen 2012, Gutiérrez 2013).

Figura 2.10. Ensayos de corte directo en probetas roca-roca con junta rugosa bajo condición CNL: (a) probetas y geometría de los perfiles de rugosidad con $\alpha=15^{\circ}, 30^{\circ}$ y $45^{\circ}$, (b) curvas de resistencia al corte vs desplazamiento al corte, (c) probeta y geometría del perfil de rugosidad sinusoidal, (d) curvas de resistencia al corte vs desplazamiento al corte (modificado de Asadi et al. 2013). 16

Figura 2.11. Resistencia al corte pico vs porcentaje de la junta con cohesión en la junta (datos experimentales tomados de Krounis et al. 2016). 17

Figura 2.12. Ensayos de corte directo en junta triangular roca-roca bajo condición CNL y CNS: (a) probetas con $\alpha=15^{\circ}$ y $30^{\circ}$, (b) probeta con $\alpha=15^{\circ}$, sin relleno, (c) probeta con $\alpha=30^{\circ}$, sin relleno, (b) probeta con $\alpha=30^{\circ}$ y con relleno (modificado de Shrivastava y Rao 2015, 2018). 18

Figura 2.13. Modelo del ensayo de corte directo con MED ${ }^{2 D}$ en junta roca-roca para $c=$ 0 y condición CNL: (a) probeta del ensayo (las partículas de color verde, rojo y negro representan la roca, la junta sin cohesión y la geometría de la probeta, respectivamente),(b) grietas generadas (el color verde y rojo representan las grietas de 
falla por corte y tracción, respectivamente), (c) curva de resistencia al corte normalizada por la resistencia a compresión uniaxial $(\sigma c)$ de la roca vs el desplazamiento al corte (modificado de Cundal 2000).

Figura 2.14. Modelo del ensayo de corte con $\mathrm{MED}^{2 \mathrm{D}}$ en junta roca-roca, con $c=0 \mathrm{y}$ condición CNL: (a) probeta del ensayo después de alcanzar un desplazamiento al corte de $15 \mathrm{~mm}$, (b) curvas de resistencia al corte vs el desplazamiento al corte para diferentes valores de esfuerzo normal (modificado de Wang et al. 2003) 21

Figura 2.15. Ensayos de corte directo en juntas rugosas bajo condición CNL: (a) experimental, (b) MED ${ }^{2 D}$, (c) probeta al inicio y final del ensayo con MED ${ }^{2 D}$ (modificado de Kusumi et al. 2005)

Figura 2.16. Ensayos de corte directo con $\mathrm{MED}^{3 \mathrm{D}}$ en juntas rugosas bajo condición CNL $(\sigma n=15 \mathrm{MPa}):$ (a) probeta del modelo, (b) grietas generadas luego de un desplazamiento al corte de $1.6 \mathrm{~mm}$ (los discos de color negro y gris representan grietas por tracción y corte, respectivamente), (c) curva resistencia al corte-desplazamiento normal-número de grietas vs desplazamiento al corte (modificado de Park y Song 2009) .24

Figura 2.17. Ensayos de corte directo con $\mathrm{MED}^{2 \mathrm{D}}$ en juntas rugosas bajo condición CNL: (a) probetas del modelo con rugosidad triangular simétrica para un desplazamiento al corte de $3 \mathrm{~mm}$, (b) curvas de resistencia al corte vs desplazamiento al corte para $\sigma n=5$ $\mathrm{MPa}$, (c) probetas del modelo con rugosidad triangular y sinusoidal, (d) curvas de resistencia al corte vs desplazamiento al corte para $\sigma n=1 \mathrm{MPa}$ (modificado de Asadi et al. 2002). 25

Figura 2.18. Ensayos de corte directo con $\mathrm{MED}^{2 \mathrm{D}}$ en juntas planas bajo condición CNL: (a) probeta del modelo, (b) curvas de resistencia al corte y desplazamiento normal vs desplazamiento al corte para $\sigma n=1 \mathrm{MPa}$ (modificado de Bahaaddini et al. 2013b)...26 
Figura 2.19. Curvas resistencia al corte vs desplazamiento al corte obtenidas con MED ${ }^{2 D}$ empleando el SJCM y la metodología SBG, en probetas de junta plana roca-roca y con diferentes valores de $\sigma n$ (condición CNL) (modificado de Bahaaddini et al. 2013b)....28

Figura 2.20. Ensayos de corte directo con MED ${ }^{3 D}$ en juntas rugosas bajo condición CNL: (a) probeta del modelo, (b) curvas de resistencia al corte vs desplazamiento al corte (modificado de Lambert y Coll 2014). 28

Figura 2.21. Influencia de la rigidez normal smooth-joint (kn $S J)$ sobre (i) la resistencia al corte pico $(\tau p)$, (ii) el desplazamiento normal normalizado por el desplazamiento al corte y (iii) la rigidez al corte $(K S)$ de la junta: (a) junta triangular con $\alpha=15^{\circ}$,(b) junta con JRC 10-12 (modificado de Bahaaddini et al. 2014). 30

Figura 2.22. Ensayos de corte directo en junta plana y triangular bajo condición CNL (a) esquema del modelo, (b) curvas de resistencia al corte vs desplazamiento al corte para la junta plana, (c) y (d) curvas de resistencia al corte vs desplazamiento al corte para la junta triangular (con $\alpha=30^{\circ}$ ) experimental y numérico con $\mathrm{PFC}^{2 \mathrm{D}}$ (modificado de Bahaaddini et al. 2016)

Figura 2.23. Ensayos de corte directo en juntas de roca-roca, bajo condición CNL: (a) junta con $\alpha=20^{\circ}$, (b) junta con $\alpha=30^{\circ}$, (c) efecto de la condición de borde en el modo de fallo de la probeta con junta con $\alpha=30^{\circ}$ y $\sigma n=7 \mathrm{MPa}$ (modificado de Bahaaddini 2017). 32

Figura 2.24. Ensayos de corte directo en juntas de roca-roca con MED ${ }^{2 D}$, bajo condición CNL: (a) esquema del modelo con junta triangular, (b) curvas de resistencia al corte vs desplazamiento al corte para $\alpha=30^{\circ}$, (c) grietas (líneas rojas) y distribución de esfuerzos (líneas negras) para la probeta con $\sigma n=4 \mathrm{MPa}$ (modificado de Bahaaddini 2017). 33

Figura 2.25. Ensayos de corte directo en juntas de roca: (a) condición CNL, (b) condición CNS, (c) curvas de resistencia al corte vs desplazamiento al corte con PFC $^{3 D}$ y 
experimental para juntas planas con diferentes valores de $\sigma n$ y condición $\mathrm{CNL}$, (d) curvas de resistencia al corte vs deformación al corte con $\mathrm{PFC}^{3 \mathrm{D}}$ de juntas de roca incipientes bajo condición CNL $(\sigma n=20 \mathrm{MPa}$ ) y CNS con $\sigma n o=20 \mathrm{MPa}$ (modificado de Shang et al. 2018).

Figura 2.26 Pruebas de carga en el laboratorio sobre pilotes excavados en roca con diferente rugosidad (modificado de Pells et al. 1980) 35

Figura 2.27 Pruebas de carga a pequeña escala en el laboratorio sobre pilotes excavados en roca: (a) esquema y geometría del ensayo, (b) carga pico en cabeza vs la relación $E c E r$ (modificado de Khan y Amadei 1993). 36

Figura 2.28. Pruebas de carga a pequeña escala en el laboratorio sobre pilotes excavados en roca: (a) geometría de los pilotes, modelo 1 con $D=102 \mathrm{~mm}$ y $L=203$ $\mathrm{mm}$, modelo 2 con $D=51 \mathrm{~mm}$ y $L=102 \mathrm{~mm}$, (b) esquema y geometría del ensayo, (c) resistencia promedio por fuste vs asiento en cabeza del pilote $(1,2$ y 3 representan la resistencia de la roca circundante de 22, 42.9 y $4.7 \mathrm{MPa}$, respectivamente) (modificado de Benmokrane et al. 1994)

Figura 2.29. Pruebas de carga a pequeña escala en el laboratorio sobre pilotes excavados en roca: (a) configuración geométrica del ensayo (unidades en mm), (b) geometría de los pilotes $D=50 \mathrm{~mm}$ y $L=450 \mathrm{~mm}(R F$ es el coeficiente de rugosidad propuesto por Horvath et al. 1983), (c) carga en cabeza vs asiento en cabeza del pilote para los modelos de solo resistencia por fuste (modificado de Dai et al. 2017) .38

Figura 2.30. Pruebas de carga sobre pilotes excavados en arenisca con rugosidad variable en la interfaz roca-pilote: (a) resistencia promedio por fuste vs asiento en cabeza del pilote, (b) resistencia promedio por fuste vs profundidad para dos intervalos de carga aplicada en cabeza del pilote (modificado de Pells et al. 1980). 40

Figura 2.31. Pruebas de carga sobre pilotes excavados en lutitas con rugosidad variable en la interfaz roca-pilote: (a) perfiles de rugosidad medidos dentro de la cavidad 
(unidades en mm), (b) carga en cabeza vs asiento en cabeza del pilote (modificado de Williams 1980) (c) efecto del fluido de perforación y zona de alteración sobre la resistencia de pilotes empotrados en roca (modificado de Williams y Pells 1981)......41

Figura 2.32. Pruebas de carga sobre pilotes excavados en lutitas con rugosidad variable en la interfaz roca-pilote: (a) perfiles de rugosidad medidos dentro de la cavidad (unidades en mm), (b) carga movilizada por fuste vs asiento en cabeza del pilote (modificado de Horvath et al. 1983). 42

Figura 2.33. Pruebas de carga sobre pilotes excavados en gneis (MW) con rugosidad variable en la interfaz roca-pilote: (a) perfiles de rugosidad medidos dentro de la cavidad con un perfilómetro de rugosidad laser, (b) resistencia promedio por fuste vs asiento en cabeza del pilote (modificado de Seol y Jeong 2007). .43

Figura 2.34. Pruebas de carga sobre pilotes excavados en roca: (a) ilustración del tipo de prueba de carga y perfil geológico del sitio HT, (b) perfiles de rugosidad medidos dentro de la cavidad del sitio HT (dimensiones en $\mathrm{mm}$ ) cuyas alturas promedio de rugosidades con Barrena y Core Barrel son 2.5 y $3.7 \mathrm{~mm}$, respectivamente (modificado de Nam y Vipulanandan 2008), (c) resistencia promedio por fuste máxima para todos los ensayos (datos experimentales tomados de Nam y Vipulanandan 2008). 45

Figura 2.35. Modelo numérico de pilotes empotrados en roca con el MEF: (a) geometría del modelo, (b) detalle de la geometría en el contacto pilote-roca, (c) influencia de la relación $L / D$ sobre la carga movilizada a lo largo del pilote (modificado de Osterberg y Gill 1973) 46

Figura 2.36. Modelo numérico de pilotes empotrados en roca con el MEF: (a) geometría del modelo, (b) detalle de la geometría de la rugosidad empleada para el pilote rugoso, (c) resistencia promedio por fuste vs asiento en cabeza del pilote (modificado de O'Neill et al. 1996). 47 
Figura 2.37. Modelo numérico de pilotes empotrados en roca con el MEF: (a) geometría y mallado del modelo y detalle de la rugosidad empleada en la interfaz roca-pilote, (b) resistencia promedio por fuste vs módulo de Young del material presente en la punta del pilote (modificado de Melentijevic y Olalla 2014)

Figura 2.38. Modelo numérico de pilotes empotrados en roca con el MEF: (a) geometría y detalle de la rugosidad empleada en la interfaz roca-pilote, (b) carga en cabeza versus asiento para los modelos con rugosidad sinusoidal (O4 representa el modelo más rugoso y $\mathrm{O} 5$ el menos rugoso en función de la amplitud de la onda), (c) resistencia promedio por fuste obtenida para los modelos con $L=1 \mathrm{~m}$ bajo carga en cabeza de 34.5 MN (modificado de Gutiérrez-Ch y Melentijevic 2016). 50

Figura 2.39. Efecto de la relación $L / D$ sobre la resistencia movilizada de pilotes empotrados en roca: modelo con rugosidad R3 (modificado de Gutiérrez-Ch 2015). . 51

Figura 2.40. Esquema conceptual de los modelos en ensayos de centrifuga $(\mathrm{Ng})$ y su equivalente a escala del prototipo (1g) (modificado de Taylor 1995). 52

Figura 2.41. Pruebas de carga sobre pilotes empotrados en roca mediante ensayos de centrifuga y de escala reducida: (a) esquema y geometría del ensayo, (b) resistencia promedio por fuste vs la relación asiento-diámetro del pilote (modificado de Leung y Ko 1993). 53

Figura 2.42. Pruebas de carga sobre pilotes empotrados en roca mediante ensayos de centrifuga y de escala reducida: (a) esquema y geometría del ensayo para pilotes cargados axialmente, (b) carga en cabeza vs asiento en cabeza del pilote (modificado de Dykeman y Valsangkar 1996). 55

Figura 2.43. Pruebas de carga sobre pilotes empotrados en roca mediante ensayos de centrifuga y de escala reducida: (a) perfil del ensayo para distintos valores de longitud de empotramiento y espesor de suelo, (b) carga en cabeza vs asiento en cabeza del pilote a escala del prototipo (1g) (modificado de Xing et al. 2014).... 56 
Figura 3.1. Comportamiento y componentes del FJCM: (modificado de Itasca Consulting Group Inc. 2014) 61

Figura 3.2. (a) Sección delgada de roca (fuente propia), (b) idealización de material FlatJoint en PFC ${ }^{3 D}$ (modificado de Itasca Consulting Group Inc. 2014). 62

Figura 3.3 Comportamiento y componentes del SJCM (modificado de Itasca Consulting Group Inc. 2014) 62

Figura 3.4 (a) Notación utilizada para definir la junta en el SJCM, nc y tc es el vector normal y perpendicular, respectivamente, de la línea de centros, (b) analogía física, (c) representación del ángulo de dirección $(\theta d)$ y buzamiento $(\theta p)$ de la interfaz SJCM (modificado de Ivars et al. 2011).

Figura 3.5. Esquema idealizado del ensayo de corte directo con condición de borde de: (a) CNL, (b) CNS (Gutiérrez-Ch et al. 2018) 64

Figura 3.6. Procedimiento de generación de la probeta para el ensayo de compresión uniaxial con PFC ${ }^{2 D}$ : (a) generación inicial de las partículas antes del arreglo definitivo (las líneas azules representan las paredes o walls), (b) distribución de fuerzas de contacto después de la aplicación del paso 2 (las líneas negras representan las fuerzas de contacto y los círculos color cian representan los círculos de medición de esfuerzos), (c) identificación de las partículas "flotantes" durante el paso 3, (d) probeta generada después del paso 5 (las líneas negras representan la red de contactos entre partículapartícula y pared-partículas. Notar que para facilitar la ilustración se han empleado partículas de mayor tamaño a las empleadas en los modelos de la investigación (Gutiérrez-Ch et al. 2018).

Figura 3.7. Análisis de sensibilidad del efecto de los microparámetros smooth-joint sobre los parámetros macroscópicos $(E, v$ y $\sigma c)$ de la probeta de arenisca (S1) simulada con DEM ${ }^{2 \mathrm{D}}$ : (a) efecto del módulo efectivo $E *=E *$, (b) efecto de la relación de rigidez 
normal y de corte $k *=k *$, (c) efecto de la cohesión, (d) efecto de la resistencia a tracción (Gutiérrez-Ch et al. 2018) 68

Figura 3.8. Proceso iterativo de calibración de las microparámetros flat-joint (GutiérrezCh et al. 2018). 69

Figura 3.9. Curvas esfuerzo-deformación axial obtenidas en 10 ensayos de compresión uniaxial con diferente valor de la "semilla" en el proceso de generación de la probeta con PFC 2D: (a) arenisca (S1), (b) arenisca (S3), (c) gneis (Gutiérrez-Ch et al. 2018). .......71 Figura 3.10. Comparación de modelos con $\mathrm{PFC}^{2 \mathrm{D}}$ y PFC ${ }^{3 \mathrm{D}}$ : (a) probeta inicial arenisca (S3), (b) vectores de desplazamiento total al final del ensayo (unidades en metros), (c) curva esfuerzo-deformación axial. 72

Figura 3.11. Deficiencia de la técnica BRA: (a) generación de la probeta, (b) instalación del plano hipotético de la junta y aplicación de la técnica BRA, (c) probeta después de la aplicación de la técnica BRA, (d) rugosidad inherente resultante de la técnica BRA. (e) probeta del modelo PFC antes de iniciar el ensayo, (f) concentración de esfuerzos y dilatación de la caja de corte para un desplazamiento al corte alcanzado (las líneas de color negro representan la intensidad de las fuerzas de contacto), (g) detalle de la deficiencia de la técnica BRA

Figura 3.12. Comparación entre ensayo de corte directo experimental y numérico (usando la técnica BRA), en junta plana roca-roca, bajo condición CNL ( $\sigma n=2.67 \mathrm{MPa}$ ): (a) resistencia al corte vs desplazamiento al corte, (b) desplazamiento normal vs desplazamiento al corte 75

Figura 3.13. Deficiencia en el uso del SJCM: (a) generación de la probeta, (b) instalación del plano hipotético de la junta y aplicación del SJCM, (c) probeta del modelo antes de iniciar el ensayo, (d) probeta para un desplazamiento al corte menor a Dmin,.(e) probeta para un desplazamiento al corte mayor a $\operatorname{Dmin}$ (f) concentración de esfuerzos y dilatación de la caja de corte del ensayo después de cierto valor de desplazamiento al 
corte (las líneas de color negro representan la intensidad de las fuerzas de contacto), (g) detalle de la deficiencia en el uso del modelo SJCM. 77

Figura 3.14. Comparación entre ensayo de corte directo experimental y numérico con el modelo SJCM, en junta plana roca-roca, bajo condición CNL $(\sigma n=2.67 \mathrm{MPa})$ : (a) resistencia al corte versus desplazamiento al corte, (b) desplazamiento normal vs desplazamiento al corte. 78

Figura 3.15. Metodología Shear Box Genesis (SBG): (a) generación inicial de las partículas antes de la distribución final (las partículas amarillas y grises representan la roca y el hormigón, respectivamente; las líneas azules representan las paredes de la caja de corte superior e inferior), (b) instalación del esfuerzo isotrópico (las líneas negras representan la intensidad de las fuerzas y las líneas segmentadas azules representan los círculos de medición de esfuerzos), (c) detección de partículas "flotantes", (d) probeta después de la aplicación de los modelos de contacto FJCM y SJCM, (e) probeta con $\mathrm{PFC}^{2 \mathrm{D}}$ antes de comenzar el ensayo de corte directo (Gutiérrez-Ch et al. 2018)........79

Figura 3.16. Probeta para el ensayo de corte directo con PFC $2 \mathrm{D}$ : (a) modelo areniscahormigón (alrededor de 12241 partículas), (b) perfiles con junta o interfaz plana y forma triangular simétrica empleados para los modelos arenisca-hormigón, (c) modelo gneishormigón (alrededor de 14517 partículas), (d) perfiles con junta o interfaz plana y forma triangular simétrica empleados para los modelos gneis-hormigón (las partículas de color gris, amarillo y verde representan al hormigón, arenisca y gneis, respectivamente; las líneas de color azul segmentadas representan el plano de la interfaz roca-hormigón, y la línea de color rojo representa el espaciado entre la caja de corte inferior y superior) (Gutiérrez-Ch et al. 2018). 80

Figura 3.17. Proceso iterativo de calibración de los microparámetros del modelo de contacto smooth-joint: (a) diagrama de flujo, (b) modelo conceptual para calibrar la rigidez normal kn SJ: (i) estado inicial, (ii) desplazamiento normal luego de la aplicación de un esfuerzo normal, (iii) esfuerzo normal versus desplazamiento normal para obtener 
$k n S J$, (c) modelo conceptual para calibrar la rigidez al corte $k s S J$ y $\mu S J$ : (iv) resistencia al corte vs desplazamiento al corte para el cálculo de $k s S J$, (v) resistencia al corte vs esfuerzo normal para el cálculo del coeficiente de fricción $\mu(\tan \emptyset)($ Gutiérrez-Ch et al. 2017). 83

Figura 3.18. Comparación de los resultados de ensayos de corte directo con $\mathrm{PFC}^{2 \mathrm{D}}$ en probetas roca-hormigón de junta plana, sin cohesión, con diferentes valores de esfuerzo normal en condición CNL: (a) arenisca(S1)-hormigón(C1), (b) arenisca(S2)hormigón(C1), (c) arenisca(S3)-hormigón(C1). (datos experimentales tomados de Gu et al. 2003) (modificado de Gutiérrez-Ch et al. 2018, 2019d). 84

Figura 3.19. Comparación de los resultados de ensayo de corte directo con $\mathrm{PFC}^{2 \mathrm{D}}$ en probeta de gneis-hormigón(C2) con junta plana, sin cohesión, en condición CNL ( $\sigma n=$ 3.2 MPa) (datos experimentales tomados de Gutiérrez 2013) (Gutiérrez-Ch et al. 2017).

Figura 3.20. Comparación de resultados de ensayos de corte directo numéricos con $\mathrm{PFC}^{2 \mathrm{D}}$ y experimentales de Gu et al. (2003) realizados en probetas de arenisca(S3)hormigón(C1) con junta sin cohesión y de diferentes perfiles de rugosidad bajo condición CNL con $\sigma n=400 \mathrm{kPa}$ (modificado de Gutiérrez-Ch et al. 2018). 87

Figura 3.21. Comparación de resultados de ensayos de corte directo con $\mathrm{PFC}^{2 \mathrm{D}}$ y experimentales de Gutiérrez. (2013), realizados en probetas gneis-hormigón(C1) con junta sin cohesión y con diferentes perfiles de rugosidad bajo condición CNL con $\sigma n=$ 3.2 MPa (modificado de Gutiérrez-Ch et al. 2018). 89

Figura 3.22. Daño observado en las asperidades de los modelos con $\mathrm{PFC}^{2 \mathrm{D}}$ en juntas de morfología triangular sin cohesión, bajo condición CNL y diferentes valores de ángulo base $\alpha$ : (a) probeta de arenisca(S3)-hormigón(C1) para un desplazamiento al corte de $4 \mathrm{~mm}$ y $\sigma n=400 \mathrm{kPa}$, (b) probeta de gneis-hormigón(C2) para un desplazamiento al corte de $4 \mathrm{~mm}$ y $\sigma n=3.2 \mathrm{MPa}$ (las partículas de color gris, amarillo y verde representan 
al hormigón, arenisca y gneis, respectivamente; las líneas color rojo representan las micro-grietas) (Gutiérrez-Ch et al. 2018). .90

Figura 3.23. Rotura de las asperidades en el ensayo de corte directo de una probeta de gneis-hormigón(C2), con junta triangular simétrica $\left(\alpha=40^{\circ}\right)$ sin cohesión, y condición CNL con $\sigma n=3.2 \mathrm{MPa}$ : (a) resultados experimentales de Gutiérrez (2013), (b) resultado del modelo numérico con PFC ${ }^{2 D}$ (Gutiérrez-Ch et al. 2018). 91

Figura 3.24. Comparación de resultados de ensayos de corte directo numéricos de esta investigación y experimentales de $\mathrm{Gu}$ et al. (2003), realizados en probetas de arenisca(S1)-hormigón(C1), con junta triangular simétrica sin cohesión, bajo condición CNS de $\sigma n o=400 \mathrm{kPa}$ y con $K=800 \mathrm{kPa} / \mathrm{mm}$ ) (Gutiérrez-Ch et al. 2018) 92

Figura 3.25. Comparación de resultados de ensayos de corte directo numéricos con PFC $^{2 D}$ y experimentales de Gu et al. (2003), realizados en probetas de arenisca(S1)hormigón(C1), con junta triangular simétrica de $\alpha=5$ o sin cohesión, bajo condición CNS de $\sigma n o=400 \mathrm{kPa}$, y $K$ variado desde 800 a $3200 \mathrm{kPa} / \mathrm{mm}$ (Gutiérrez-Ch et al. 2018).

Figura 3.26. Comparación de resultados de ensayos de corte directo numéricos con $\mathrm{PFC}^{2 \mathrm{D}}$ y experimentales de Gu et al. (2003), realizados en probetas de arenisca(S3)hormigón(C1), con junta triangular simétrica de $\alpha=20$ o sin cohesión, bajo condición CNS con diferentes valores de esfuerzo normal inicial $(\sigma n o)$, y $K=800 \mathrm{kPa} / \mathrm{mm}$ (Gutiérrez-Ch et al. 2018). 93

Figura 3.27. Comparación de resultados de ensayos de corte directo numéricos con PFC $^{2 D}$ y experimentales de Gu et al. (2003), realizados en probetas de arenisca(S3)hormigón(C1), con junta triangular simétrica de $\alpha=50$ y 20 o sin cohesión, bajo condición CNS con $\sigma n o=400 \mathrm{kPa}$, y $K=800 \mathrm{kPa} / \mathrm{mm}$ (Gutiérrez-Ch et al. 2018)....94 Figura 3.28. Comparación de resultados de ensayos de corte directo numéricos con $\mathrm{PFC}^{2 \mathrm{D}}$ y experimentales de Gu et al. (2003), realizados en probetas de arenisca(S3)- 
hormigón, con junta triangular simétrica de $\alpha=20$ o sin cohesión, bajo condición CNL (línea roja) y CNS (línea azul) (Gutiérrez-Ch et al. 2018).

Figura 4.1. (a) modelo idealizado de un pilote empotrado en roca y su nomenclatura, (b) modelo con PFC $^{3 \mathrm{D}}$ de pilote empotrado en roca (Gutiérrez-Ch et al. 2020b). 99

Figura 4.2. Parámetros para definir el factor de rugosidad $R F$ (modificado de Zhang 2004). 99

Figura 4.3. Esquema idealizado: (a) vista frontal 2D de los perfiles de rugosidad empleados en la interfaz roca-pilote, (b) ejemplo del detalle de los parámetros de la geometría sinusoidal para el modelo con $R F=0.106$ (modificado de Gutiérrez-Ch et al. 2019a). 100

Figura 4.4. Generación del Modelo con PFC ${ }^{3 \mathrm{D}}$ : (a) geometría del ensamblaje inicial del modelo, (b) distribución de las fuerzas después de la instalación del esfuerzo isotrópico (las esferas azules representan las zonas de medición), (c) identificación de las partículas "flotantes" (esferas rojas), (d) asignación de los modelos de contacto FJCM y SJCM 102

Figura 4.5. Selección del radio mínimo (Rmin) de las partículas de los modelos con PFC ${ }^{3 D}$ : análisis de sensibilidad realizado sobre el pilote empotrado en arenisca-S3 con $R F=0.025$ (para facilitar la ilustración solo se representaron el número de partículas correspondientes al cuerpo del pilote). 103

Figura 4.6. Representación de los vectores unitarios normales del modelo de contacto SJCM: (a) vista 3D y vista en planta de los vectores $n j$ (las flechas color purpura representan los vectores $n j$ en cada contacto partícula-partícula en el plano de la interfaz roca-pilote), (b) representación estereográfica de los vectores $n j$ para los modelos con PFC $^{3 \mathrm{D}}$ con $R F=0.025$ y $R F=0.106$ (los puntos rojos representan los polos de los vectores $n j$ ).(Notar que para facilitar la ilustración se ha suprimido las partículas del cuerpo del pilote) (Gutiérrez-Ch et al. 2020b) 105 
Figura 4.7. (a) condiciones de borde y cargas actuantes, (b) vista de los modelos con PFC $^{3 \mathrm{D}}$ de los pilotes empotrados en roca con diferente nivel de rugosidad en función del factor $R F$ 107

Figura 4.8. Comparación de las curvas carga en cabeza vs asiento cabeza obtenidas en los modelos con PFC ${ }^{3 \mathrm{D}}$ para los pilotes (hormigón-C1) empotrados en arenisca-S3 con diferente grado de rugosidad (modificado de Gutiérrez-Ch et al. 2020b).... 108

Figura 4.9. Resistencia promedio por fuste y numero de grietas vs asiento cabeza obtenidos en los modelos con $\mathrm{PFC}^{3 \mathrm{D}}$ en: (a) pilotes (hormigón-C1) empotrados en arenisca-S3 (modificado de Gutiérrez-Ch et al. 2020b), (b) pilotes (hormigón-C1) empotrados en arenisca-S2 (modificado de Gutiérrez-Ch et al. 2019c), (b) pilotes (hormigón-C2) empotrados en gneis, con diferente grado de rugosidad (modificado de Gutiérrez-Ch et al. 2020b). 111

Figura 4.10. Propagación de micro-grietas bajo diferentes pasos de carga axial para los pilotes empotrados en arenisca-S3: (a) modelo con $R F=0.050$, (b) modelo con $R F=$ 0.010. (los colores azul y rojo representan las grietas por cortante y por tracción, respectivamente) (modificado de Gutiérrez-Ch et al. 2019b). 112

Figura 4.11. Resistencia promedio por fuste vs factor de rugosidad para valores de asiento en cabeza de $1 \%$ al $3 \%$, obtenidos con modelos $\mathrm{PFC}^{3 \mathrm{D}}$ en pilotes (hormigónC1) empotrados en arenisca-S3 y con diferente grado de rugosidad (modificado de Gutiérrez-Ch et al. 2020b)

Figura 4.12. Esfuerzo axial movilizado para los modelos con PFC ${ }^{3 D}$ de pilotes empotrados en la arenisca-S3 con diferente nivel de rugosidad: (a) ubicación de la sección axial trazada, (b) distribución de esfuerzo axial vs profundidad.....

Figura 4.13. Resistencia por fuste movilizada para los modelos con PFC ${ }^{3 D}$ de pilotes empotrados en la arenisca-S3 con diferente nivel de rugosidad: (a) ubicación de la 
sección en la interfaz roca-pilote, (b) distribución de resistencia por fuste vs profundidad.

Figura 4.14. Resultados registrados a varios niveles en profundidad para los modelos con PFC ${ }^{3 D}$ de pilotes empotrados en la arenisca-S3 con diferente nivel de rugosidad: (a) ubicación en profundidad de los puntos de medición en la interfaz roca-pilote, (b) resistencia por fuste movilizada vs asiento en cabeza.

Figura 4.15. Mecanismo de transferencia de carga por fuste para pilotes rugosos empotrados en roca: (a) estado inicial y nomenclatura de la asperidad, (b) estado para deformación elástica, (c) estado para deformación inelástica (modificado de Hassan y O’Neill 1997). 120

Figura 4.16. Curvas de las componentes de esfuerzos vs asiento en cabeza para el modelo con $\mathrm{PFC}^{3 \mathrm{D}}$ del pilote con $R F=0.050$ empotrado en la arenisca-S3: (a) localización de los puntos de medición, (b) frente de la asperidad, (c) parte posterior de la asperidad, (d) base de la asperidad.

Figura 4.18. Curvas de las componentes de esfuerzos vs asiento en cabeza para el modelo con $\mathrm{PFC}^{3 \mathrm{D}}$ del pilote con $R F=0.010$ empotrado en la arenisca-S3: (a) localización de los puntos de medición, (b) frente de la asperidad, (c) parte posterior de la asperidad, (d) base de la asperidad.

Figura 4.19. Distribución de esfuerzos alrededor de la roca circundante (arenisca-S3) del modelo con PFC $^{3 \mathrm{D}}$ y $R F=0.050$ : (a) ubicación de las secciones, (b) esfuerzos en el frente de la asperidad $(-10 \mathrm{~cm}),(\mathrm{c})$ esfuerzos en la parte posterior de la asperidad $(-27$ $\mathrm{cm})$, (d) esfuerzos en la base de la asperidad $(-37 \mathrm{~cm})$. 126

Figura 4.20. Distribución de esfuerzos alrededor de la roca circundante (arenisca-S3) del modelo con $\mathrm{PFC}^{3 \mathrm{D}}$ y $R F=0.010$ : (a) ubicación de las secciones, (b) esfuerzos en el frente de la asperidad $(-10 \mathrm{~cm}),(\mathrm{c})$ esfuerzos en la parte posterior de la asperidad $(-27$ $\mathrm{cm})$, (d) esfuerzos en la base de la asperidad $(-37 \mathrm{~cm})$. 127 
Figura 4.21. Esquema del proceso de simulación numérica con MEF en Abaqus (modificado de Gutiérrez-Ch y Melentijevic, 2016) 130

Figura 4.22. Geometría de los modelos con MEF considerando la resistencia por punta y fuste, Grupo 2.

Figura 4.23. Comparación entre modelos con MED y con MEF: (a) resistencia promedio por fuste vs asiento en cabeza (b) resistencia promedio por fuste para un asiento de 1\%D (fave, 0.01D) (modificado de Gutiérrez-Ch et al. 2019a).

Figura 4.24. Ejemplo de los esfuerzos tangenciales movilizados por fuste para los modelos con Abaqus de pilotes empotrados en la arenisca-S3 para un asiento en cabeza de $1 \% D:$ (a) $R F=0.025$, (b) $R F=0.050$, (c) $R F=0.106$ 133

Figura 4.25. Comparación de las curvas carga en cabeza vs asiento cabeza obtenidas en los modelos con Abaqus para los pilotes empotrados en la pseudo-roca con diferente grado de rugosidad.

Figura 4.26. Resistencia promedio por fuste y por punta vs asiento cabeza obtenidos en los modelos con Abaqus para los pilotes empotrados en la pseudo-roca con diferente grado de rugosidad. 136

Figura 4.27. Contribución del porcentaje de resistencia promedio movilizada por fuste y por punta vs asiento cabeza obtenidos en los modelos con Abaqus para los pilotes empotrados en la pseudo-roca con diferente grado de rugosidad. 137

Figura 5.1. Esquema idealizado a escala del prototipo $(1 g)$ y del modelo $(N g)$. 140 Figura 5.2. Máquina de centrífuga del Centro de Geomecánica de la Universidad de Nottingham y sus componentes: (a) vista panorámica, (b) vista frontal. 141

Figura 5.3. Proceso de preparación de la pseudo-roca: (a) arena, cemento y bentonita antes de mezclarlos, (b) arena, cemento y bentonita después de mezclarlos, (c) mezcla de arena, cemento y bentonita después de agregar la proporción de agua, (d) probetas 
cúbicas tras verter y vibrar la mezcla de la pseudo-roca en 3 capas iguales, (e) probeta de la pseudo-roca desmoldada a los 44 días para ensayo de compresión uniaxial. . 142 Figura 5.4. Detalle de la geometría idealizada del modelo de pilote.

Figura 5.5. Pilotes de aluminio con diferente factor de rugosidad $(R F)$ a escala del modelo.

Figura 5.6. Esquema de la estructura y repuesta espectral de un sensor FBG (modificado de Kreuzer 2006).

Figura 5.7. (a) ejemplo de la estructura de una fibra óptica con varios sensores FBG, (b) detalle de la fibra óptica y sensores FBG, (c) esquema del proceso de instalación de la instrumentación del modelo del pilote. 146

Figura 5.8. Instrumentación de cada modelo de pilote: (a) esquema ilustrativo de la localización de líneas de fibra óptica y localización de los sensores FBG $\left(F B G_{n}\right)$, (modificado de Gutiérrez-Ch et al. 2020a), (b) modelo de pilote con $R F=0.000$ después de instalar su instrumentación.

Figura 5.9. Esquema ilustrativo de la configuración adoptada durante el proceso de calibración de los sensores FBG.

Figura 5.10. Ejemplo de los resultados obtenidos durante la calibración de los sensores para el modelo de pilote con $R F=0.025$ (para el sensor $F B G 5$ ): (a) ciclos de carga y descarga vs tiempo, (b) cambio en la longitud de onda vs tiempo, (c) relación fuerzacambio de longitud de onda (modificado de Gutiérrez-Ch et al. 2020a); (d) relación fuerza-cambio de longitud de onda para todos los sensores.

Figura 5.11. Preparación del modelo de centrífuga: (a) modelo del pilote de aluminio con $R F=0.106$ tras adherirle la pieza de poliestireno en la punta, (b) molde cilíndrico metálico tras verter la mezcla húmeda de la pseudo-roca, (c) ensamblaje pseudo-rocapilote del modelo de centrífuga, (d) modelo idealizado de un ensamblaje típico pseudoroca-pilote. 
Figura 5.12. Configuración del ensayo de centrífuga: (a) sistema de carga, (b) localización del interrogador FBG, (c) detalle de la instrumentación de medición ...... 152

Figura 5.13. (a) curvas carga axial vs asiento normalizado por el diámetro, (b) curvas rigidez global vs asiento normalizado por el diámetro. 153

Figura 5.14. Distribución de la carga axial movilizada (en profundidad) para un asiento dado para los ensayos de centrifuga realizados sobre pilotes con diferente nivel de rugosidad: (a) $R F=0.000$, (b) $R F=0.025$, (c) $R F=0.050$, (d) $R F=0.106$; (d) carga axial movilizada vs tiempo, registrada por la célula de carga y por los sensores FBG para modelo de pilote con $R F=0.025$ 157

Figura 5.15. Variación del factor $K_{F B G_{i}}$ para el modelo de pilote con $R F=0.025 \ldots \ldots 158$

Figura 5.16. Resistencia promedio por fuste (vs profundidad) para todos los ensayos de centrífuga realizados sobre modelos de pilotes con diferente nivel de rugosidad en la interfaz roca-pilote: (a) $R F=0.000$, (b) $R F=0.025$, (c) $R F=0.050$, (d) $R F=0.106 .160$ Figura 5.17. Resistencia promedio por fuste (vs asiento en cabeza del pilote) registrada durante todos los ensayos de centrífuga a diferentes profundidades: (a) $R F=0.000$, (b) $R F=0.025$, (c) $R F=0.050$, (d) $R F=0.106$. 162

Figura 5.18. Resistencia promedio por fuste ( fave) versus el asiento en cabeza $(\delta)$ para los ensayos de centrífuga realizados sobre pilotes con diferente nivel de rugosidad.163 Figura 5.19. Comparación entre la distribución en profundidad de la resistencia promedio por fuste obtenida mediante el ensayo de centrífuga y el MEF para el modelo de pilote con $R F=0.106: \delta=0.01 D$, (b) $\delta=0.02 D$, (c) $\delta=0.05 D$, (d) $\delta p i c o=0.067 D$. 165

Figura 6.1. Curvas para la estimación de la resistencia media por fuste de pilotes empotrados en roca, considerando la rugosidad de la excavación: (a) modificado de Pells et al. (1980), (b) modificado de Horvath et al (1983). 171

Figura 6.2. Curvas para la estimación de la resistencia por fuste de pilotes empotrados en roca propuesta por Kodikara et al. (1992). 
Figura 6.3. Curvas para la estimación de la resistencia por fuste de pilotes empotrados en roca propuesta por Seidel y Collingwood (2001)

Figura 6.4. Estimación de la resistencia por fuste para los pilotes empotrados en la arenisca-S3: comparación entre los resultados numéricos con $\mathrm{PFC}^{3 \mathrm{D}}$ vs las formulaciones analíticas y empíricas que no consideran la rugosidad de la interfaz rocapilote. 176

Figura 6.5. Estimación de la resistencia por fuste para los pilotes empotrados en la arenisca-S3: comparación entre los resultados numéricos con $\mathrm{PFC}^{3 \mathrm{D}}$ y las formulaciones empíricas que consideran la rugosidad de la interfaz roca-pilote.

Figura 6.6. Estimación de la resistencia por fuste para los pilotes empotrados en el gneis: comparación entre los resultados numéricos con $\mathrm{PFC}^{3 \mathrm{D}}$ y las formulaciones empíricas y analíticas que consideran o no la rugosidad de la interfaz roca-pilote.

Figura 6.7. Estimación de la resistencia por fuste para los pilotes empotrados en la arenisca-S2: comparación entre los resultados numéricos con $\mathrm{MED}^{3 \mathrm{D}}$ y las formulaciones empíricas y analíticas que consideran o no la rugosidad de la interfaz roca-pilote. 180

Figura 6.8. Estimación de la resistencia por fuste para los pilotes empotrados en la pseudo-roca: comparación entre los resultados de los ensayos de centrífuga y las formulaciones analíticas y empíricas que no consideran la rugosidad de la interfaz rocapilote.

Figura 6.9. Estimación de la resistencia por fuste para los pilotes empotrados en la pseudo-roca: comparación entre los resultados de los ensayos de centrífuga y las formulaciones empíricas que consideran la rugosidad de la interfaz roca-pilote 182

Figura 6.10. Comparación entre los resultados numéricos con PFC ${ }^{3 \mathrm{D}}$ vs las curvas de diseño propuestas por Pells et al. (1980). 183 
Figura 6.11. Comparación entre los resultados numéricos con $\mathrm{PFC}^{3 \mathrm{D}}$ y de centrífuga vs las curvas de diseño propuestas por Williams y Pells (1981) 184

Figura 6.12. Comparación entre los resultados numéricos con $\mathrm{PFC}^{3 \mathrm{D}}$ y de centrífuga vs las curvas de diseño propuestas por Seidel y Collingwood (2001) después de incorporar los resultados de ensayos de carga de Kulhawy and Phoon (1993). 185

Figura 6.13. Factor $\alpha R F, 0.01 D$ propuesto para la estimación de la resistencia por fuste de pilotes empotrados en roca considerando un asiento en cabeza de $1 \% D$, fave, $0.01 D$ (los puntos de color azul y rojo representan los casos en que el material el más débil roca o pilote- con $\sigma c \geq 10 \mathrm{MPa}$ y $\sigma c<10 \mathrm{MPa}$, respectivamente) 186

Figura 6.14. Validación del factor $\alpha R F, 0.01 D$ a partir de ensayos sobre pilotes empotrados en roca publicados en la literatura (los puntos de color azul y rojo representan los casos en que el material el más débil -roca o pilote-presenta $\sigma c \geq 10$ MPa o $\sigma c<10 \mathrm{MPa}$, respectivamente) 188

Figura 6.15. Vista longitudinal del viaducto: perfil geológico, ubicación de las pilas (1924) de fundación y localización de las pruebas de carga (TS-1A y TS-2) (modificado de Miller 2003). 191

Figura 6.16. Esquema del ensayo de carga TS-1A: (a) perfil geológico y geometría del ensayo (modificado de Miller 2003), (b) perfil de rugosidad y factor de rugosidad calculado a partir de los datos de Miller (2003) 193

Figura 6.17. Esquema del ensayo de carga TS-2: (a) perfil geológico y geometría del ensayo (modificado de Miller 2003), (b) perfil de rugosidad y factor de rugosidad calculado a partir de los datos de Miller (2003). 193

Figura 6.18. Resistencia promedio por fuste vs desplazamiento de la célula de carga obtenido en los ensayos de carga: (a) ensayo TS-1A, (b) ensayo TS-2 (datos experimentales tomados de Miller 2003) 195 
Figura 6.19. Resultados de la aplicación del factor $\alpha R F, 0.01 D$ en los ensayos de carga TS-1A y TS-2 (los puntos de color rojo representan el material el más débil -roca o pilote- presenta $\sigma c<10 \mathrm{MPa}$ ). 197

Figura 6.20. Longitud de perforación en roca por cada pilote de soporte de las pilas del viaducto (datos del proyecto tomados de Miller 2003). 198

Figura 6.21. Vista longitudinal del viaducto A6252: perfil geológico, ubicación de las pilas (3-7) de dimentación y localización de la prueba de carga GT (modificado de Miller 2003). 199

Figura 6.22. Esquema del ensayo de carga GT: (a) perfil geológico y geometría del ensayo (modificado de Miller 2003), (b) detalle de rugosidad del hoyo y factor de rugosidad calculado a partir de los datos de Miller (2003). 200

Figura 6.23. Resistencia promedio por fuste vs desplazamiento de la célula de carga obtenido en el ensayo de carga GT (datos experimentales tomados de Miller 2003).

201

Figura 6.24. Resultados de la aplicación del factor $\alpha R F, 0.01 D$ en el ensayo de carga GT (los puntos de color azul y rojo representan los casos en que el material el más débil roca o pilote- presenta $\sigma c \geq 10 \mathrm{MPa}$ o $\sigma c<10 \mathrm{MPa}$, respectivamente). 202

Figura A.1. Barrena (Auger) (tomado de Nam y Vipulanandan 2008). 223

Figura A.2. Core Barrel (tomado de Collingwood 2000). .223

Figura A.3. Barrena Bullet Tooth Rock (tomado de TEREX 2015). 224

Figura A.4. Barrena Bucket. (tomado de Collingwood 2000). .224

Figura A.5. Herramienta de corte circunferencial (WRT1) para incrementar la rugosidad de la excavación (modificado de Collingwood 2000) 224

Figura A.6. Core Bit (tomado de Karandikar 2018) 225

Figura A.7. Rock roller (tomado de Karandikar 2018). .225 
Figura A.8. Barrena con "dientes" de corte para incrementar la rugosidad de la excavación (modificado de Collingwood 2000). .225

Figura B.1. Ejemplo del proceso de preparación de la pseudo-roca: (a) arena, cemento y bentonita antes de mezclarlos, (b) arena, cemento y bentonita después de mezclarlos, (c) mezcla de arena, cemento y bentonita después de agregar la proporción de agua, (d) molde de madera de $36 \times 21 \times 15 \mathrm{~cm}^{3}$ antes de verter la mezcla, (e) molde de madera después de verter y vibrar la mezcla de la pseudo-roca en 3 capas iguales, (f) probeta de la pseudo-roca desmoldada después del proceso de curado, (g) extracción de probetas cilíndricas de la pseudo-roca para realización de ensayos. 227

Figura B.2. Ejemplo de ensayo de compresión uniaxial: (a) probeta de la pseudo-roca antes de comenzar el ensayo, (b) modo de fallo de la probeta. 228

Figura B.3. Ejemplo de ensayo triaxial consolidado no drenado: (a) preparación de la probeta de la pseudo-roca, (b) probeta durante el ensayo, (c) modo de fallo de la probeta. 228

Figura B.4. Ejemplo de ensayo brasileño (tracción indirecta): probeta de la pseudo-roca antes de comenzar el ensayo, (b) modo de fallo de la probeta. .229

Figura B.5. Ejemplo de prueba realizada para evaluar la rugosidad resultante en la interfaz roca-pilote: (a) modelos de pilotes de plástico con diferente nivel de rugosidad, (b) colocación de los modelos de pilotes dentro del molde de madera, (c) probeta del ensayo después de verter y vibrar la mezcla de pseudo-roca, (d) probeta del ensayo después de ser desmoldada a las 24 horas. 229

Figura B.6. Ejemplo de la rugosidad en la interfaz roca-pilote: (a) remoción de los modelos de pilotes a las 24 horas, (b) rugosidad resultante en la mezcla de la pseudoroca luego de remover el modelo de pilote con $R F=0.050$, (C) rugosidad resultante en la mezcla de la pseudo-roca luego de remover el modelo de pilote con $R F=0.085$, (d) detalle de la rugosidad resultante en la pseudo-roca del ensayo. 230 


\section{Listado de Tablas}

Tabla 2.1. Sistema de clasificación de rugosidades (Pells et al. 1980) 40

Tabla 2.2. Leyes de escala en ensayos de centrifuga (Taylor 1995; Xing et al. 2014) 52 Tabla 3.1. Micropropiedades para el modelo de contacto flat-joint calibradas a partir de los datos experimentales de Gu et al. (2003) y Gutiérrez (2013). 70

Tabla 3.2. Macropropiedades obtenidas con el ensayo de compresión uniaxial experimental y numérico con PFC $2 \mathrm{D}$. .70

Tabla 3.3. Macropropiedades obtenidas con el ensayo de compresión uniaxial experimental y numérico con $\mathrm{PFC}^{2 \mathrm{D}}$ y $\mathrm{PFC}^{3 \mathrm{D}}$ 72

Tabla 3.4. (a) Micropropiedades del modelo de contacto smooth-joint calibradas con $\mathrm{PFC}^{2 \mathrm{D}}$ para las diferentes juntas roca-hormigón empleadas en la validación; (b) comparación de los macroparámetros de la junta roca-hormigón obtenidos en los ensayos de corte directo experimentales y con $\mathrm{PFC} 2 \mathrm{D}$ .85

Tabla 4.1. Distribución del tamaño de partícula empleado en la modelización con PFC ${ }^{3 D}$ 103

Tabla 4.2 Carga axial soportada en cabeza de pilotes excavados en roca con diferente nivel de rugosidad y para un asiento en cabeza equivalente a $2 \%$ el diámetro del pilote.

Tabla 4.3. Propiedades de los materiales empleados en los modelos con MEF.

Tabla 5.1. Proporciones de la mezcla expresadas como porcentaje en masa y resultados de ensayos realizados en probetas a los 44 días. 142

Tabla 5.2. Propiedades del pilote a escala del prototipo y del modelo 143

Tabla 6.1. Coeficientes empíricos $\alpha$ y $\beta$ para la estimación de la resistencia por fuste promedio pico por fuste (ver Ecuación 6.1) (tomado principalmente de O’Neil et al. 1996 
y Serrano et al. 2008, más algunos casos adicionales incorporados en esta investigación) 169

Tabla 6.2. Altura de rugosidades obtenidas en las excavaciones de pilotes empotrados en roca con diferentes herramientas de perforación (ver el Anexo A) (Gutiérrez-Ch et al. 2020b) 174

Tabla 6.3. Datos empleados para la validación del factor $\alpha R F, 0.01 D$. 188

Tabla 6.4. Datos empleados para la aplicación del factor $\alpha R F, 0.01 D$ a los ensayos de carga TS-1A y TS-2 (datos experimentales tomados de Miller 2003) 196

Tabla 6.5. Costo total ahorrado en la perforación de los pilotes de soporte de las pilas del viaducto realizado en Lexington (datos del proyecto tomados de Miller 2003).... 198 Tabla 6.6. Datos empleados para la aplicación del factor $\alpha R F, 0.01 D$ al ensayo de carga GT (datos experimentales tomados de Miller 2003) 201 


\section{Agradecimientos}

El desarrollo de una tesis doctoral conlleva varios años de continua dedicación, investigación y desarrollo de diversas actividades, no solo por parte del doctorando, sino también de los directores de tesis, instituciones, entre otros, que contribuyen al feliz término de la investigación. Por tal razón, aprovecho estás líneas para agradecer a todos los que me acompañaron a lo largo de estos años.

A mis directores de tesis, Dr. Rafael Jiménez y Dra. Svetlana Melentijevic, gracias por su dedicación, implicación e ideas, las cuales en su conjunto permitieron el buen desarrollo y culminación de esta etapa de mi formación profesional. Ha sido un inmenso placer trabajar bajo la dirección de ambos durante estos años.

Al Centro de Geomecánica de la Universidad de Nottingham (Reino Unido), en especial a los Doctores Alec Marshall, Charles Heron y Geyang Song por su contribución en los ensayos centrífuga realizados en esta tesis doctoral.

Al personal del Laboratorio de Geotecnia tanto de la Escuela Técnica Superior de Ingenieros de Caminos, Canales y Puertos (Universidad Politécnica de Madrid) como del Centro de Estudios y Experimentación de Obras Públicas (CEDEX) por su colaboración durante parte de la fase experimental de esta tesis doctoral.

A mi esposa, Eliana Graterol, gracias por tu confianza y compresión, por cada palabra de aliento brindada durante las fases complejas de esta investigación, pero sobre todo gracias por tu amor, tiempo y entrega a lo largo de estos años.

A mis padres, Hernan y Maritza, quienes desde la distancian me brindaban su amor y orientación en cada paso dado durante estos años lejos de casa.

Al Dr. Salvador Senent, gracias por el apoyo e implicación no solo profesional, sino también humana, haciendo más llevaderos estos años de investigación. 
A mis compañeros del Laboratorio de Geotecnia-UPM: Daniel Panique, Ramiro Luna, Andrea Franza, Natalia Montero, Cong Yi y Ana Santos, gracias por todos momentos compartidos durante estos años. 


\section{Resumen}

El diseño de pilotes empotrados en roca tiene como objetivo principal transferir cargas estructurales a geomateriales más profundos y más resistentes. La estimación de la resistencia por fuste de este tipo de cimentación se realiza usualmente a través de las normativas o experiencias de cada país, o mediante el uso de correlaciones empíricas expresadas en función de la resistencia a compresión simple del material más débil (roca o pilote). No obstante, estas recomendaciones no consideran la influencia que otros aspectos importantes tienen sobre la resistencia por fuste de pilotes como, por ejemplo, la rugosidad en la interfaz roca-pilote. Trabajos y experiencias previas en campo sugieren que la rugosidad resultante durante la perforación de pilotes empotrados en roca es un factor que depende principalmente de la herramienta de perforación empleada, así como del tipo y resistencia de la roca. Esta tesis doctoral está enfocada en evaluar la influencia de la rugosidad en el contacto roca-pilote sobre la resistencia por fuste de pilotes empotrados en roca.

Para ello, en primer lugar, se ha analizado el efecto de la rugosidad en juntas de rocahormigón a través de ensayos numéricos de corte directo en $\mathrm{PFC}{ }^{2 \mathrm{D}}$ bajo condición de borde de Carga Normal Constante (CNL) y Rigidez Normal Constante (CNS) y su validación con resultados experimentales de ensayos de corte directo en juntas de arenisca-hormigón y gneis-hormigón realizados por Gu et al. (2003) y Gutiérrez (2013), respectivamente. Los resultados demuestran que cuando los modelos numéricos realizados en $\mathrm{PFC}^{2 \mathrm{D}}$ son calibrados apropiadamente a través de ensayos de compresión simple y ensayos de corte directo en juntas planas, éstos permiten modelar de manera adecuada el comportamiento al corte de juntas roca-hormigón, (i) bajo condición de borde CNL y CNS, (ii) en rocas con diferente resistencia, y (iii) con diferente nivel de rugosidad en la junta, y además, con buena correspondencia al comportamiento experimental obtenido por Gu et al. (2003) y Gutiérrez (2013). En segundo lugar, se ha evaluado el efecto de la rugosidad sobre la resistencia por fuste de pilotes empotrados 
en roca considerando diferente nivel de rugosidad en la interfaz roca-pilote. Para ello, se han elaborado (i) modelos numéricos empleando el Método de Elementos Discretos (MED) en PFC ${ }^{3 \mathrm{D}}$, (ii) modelos numéricos utilizando el Método de Elementos Finitos (MEF) en Abaqus, y (iii) ensayos de centrífuga con pilotes fabricados de aluminio e instrumentados empleando una de las últimas tecnologías como lo es la fibra óptica. Los resultados han sido comparados con las predicciones obtenidas con correlaciones empíricas propuestas por otros autores basadas en resultados de ensayos de carga. Los resultados obtenidos sugieren que los modelos numéricos y ensayos de centrífuga son adecuados para reproducir el comportamiento de pilotes empotrados en roca considerando la rugosidad de la interfaz roca-pilote. Además, los resultados sugieren que cuando se perforan rocas de baja a mediana resistencia con herramientas de perforación estándar, las paredes de la cavidad resultan relativamente lisas a menos que se incremente su rugosidad de forma artificial con herramientas especiales de perforación; y que el daño en las asperidades de la interfaz roca-pilote comienza a ser excesivo cuando se producen asientos en cabeza del pilote superiores a $1 \%$ del diámetro del pilote, especialmente para pilotes rugosos. Finalmente, los resultados obtenidos con los modelos numéricos y ensayos de centrífuga realizados en esta tesis doctoral, junto con una base de datos de ensayos de carga realizados por otros autores y compilados en esta investigación, han sido empleados para proponer un nuevo factor $\alpha_{R F, 0.01 D}$ para la estimación de la resistencia promedio por fuste de pilotes empotrados en roca considerando (i) un asiento en cabeza equivalente a $1 \%$ del diámetro del pilote, (ii) la rugosidad de la interfaz roca-pilote, y (iii) la resistencia a compresión simple del material intacto menos resistente (roca o pilote). 


\section{Abstract}

The design of axially loaded rock-socketed piles aims to transfer the structural load to a stronger and deeper foundation material. The side shear resistance of rock-socketed piles is usually estimated using recommendations from codes and standards, or using local knowledge obtained from full-scale static load tests, or employing empirical correlations as a function of the uniaxial compressive strength (UCS) of the weaker materials (rock or pile). However, this approach neglects the influence of other important aspects, such as the roughness of the pile-socket interface. Previous research and field experiences suggest that socket roughness is mainly a factor of the drilling tools employed, and on the type and strength of the rock. This work focuses on the influence of roughness at the socket's pile-rock interface on the side shear resistance of rocksocketed piles.

To do that, first, the effect of roughness at rock-pile interface has been analyzed using $\mathrm{PFC}^{2 \mathrm{D}}$ to model direct shear tests of rock-concrete interfaces under Constant Normal Load (CNL) and Constant Normal Stiffness (CNS) boundary conditions with the corresponding validation of results with experimental data previously published in the literature. Results corresponding to direct shear test conducted on gneiss-concrete (Gutiérrez, 2013) and sandstone-concrete (Gu et al., 2003) interfaces are employed. Numerical results show that PFC ${ }^{2 D}$ numerical models, when adequately calibrated using information from uniaxial compression tests and from direct shear tests conducted on smooth interfaces, can satisfactorily reproduce the shear behaviour of rock-concrete interfaces, (i) under CNL and CNS boundary conditions, (ii) with rocks of different strengths, and (iii) with different interfaces roughness profiles, resulting in a good agreement with laboratory results obtained by Gutiérrez (2013) and Gu et al. (2003). Next, the socket roughness effect on side shear resistance of rock-socketed piles is examined. To do that, (i) numerical discrete element models -DEM, using PFC ${ }^{3 \mathrm{D}}$-, (ii) numerical finite element models -FEM, using Abaqus-, and (iii) centrifuge tests -using 
aluminum piles and Fiber Bragg gratings instrumentation- of rock-socketed piles with different degrees of socket roughness, are employed. Results are compared with predictions obtained using empirical correlations based on load test results and proposed by other authors. Results suggest that numerical models and centrifuge tests are suitable to reproduce rock-socket pile behaviour considering socket roughness, they also suggest that sockets drilled with standard tools in soft to medium rock tend to be relatively smooth, unless artificially roughened with special tools; and that damage to the interface asperities becomes more relevant after socket settlement of about $1 \%$ of the socket diameter, especially for rougher piles. Finally, numerical and centrifuge tests results conducted herein together with a load database compiled by the author, are employed to proposed the factor $\alpha_{R F, 0.01 D}$ to predict the average side shear resistance of rocksocketed piles considering (i) a pile head settlement of $1 \%$ of the pile diameter, (ii) the socket roughness, and (iii) the UCS of the weaker materials (rock or pile). 


\section{Introducción}

\subsection{Motivación de la investigación}

Los pilotes cargados axialmente son un tipo de cimentación profunda que se emplea para recibir y transferir cargas estructurales elevadas a geomateriales infrayacentes más resistentes (e.g., rocas), así como también para limitar o reducir los posibles asientos que puedan producirse en las estructuras, en donde han sido empleados. Tradicionalmente, el diseño de pilotes empotrados en roca se realiza considerando que el mecanismo de transferencia de carga se realiza mediante una de siguientes formas: solo por fuste $(f)$, solo por punta $\left(q_{p}\right)$, o por ambas simultáneamente (ver la Figura 1.1).

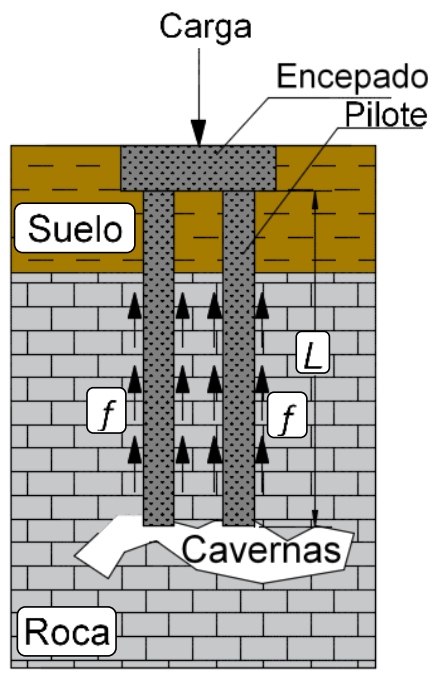

(a)

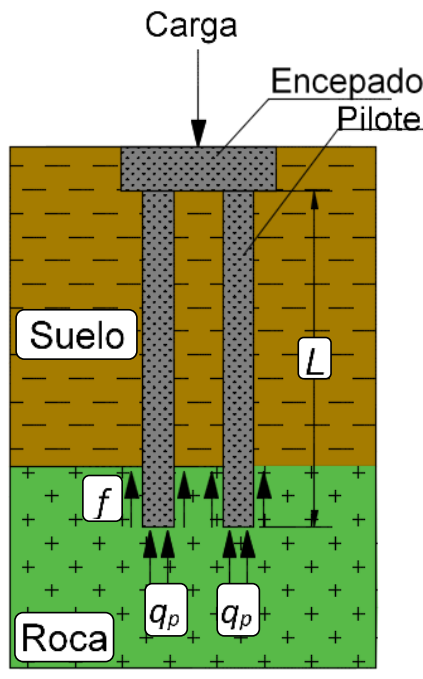

(b)

Figura 1.1. Pilotes empotrados en roca: (a) pilote por fuste, (b) pilote por punta y fuste (modificado de Gutiérrez-Ch y Melentijevic 2016).

Las situaciones en las que un pilote puede desarrollar su resistencia solo por fuste pueden ser diversas, por ejemplo, residuos acumulados en el fondo de la excavación, o roca con cavidades (ver la Figura 1.1(a)), mientras que cuando la totalidad del pilote se encuentra en un estrato de baja resistencia con la punta apoyada sobre roca, se dice que el pilote trabaja sólo por punta (Seidel y Collingwood 2001). No obstante, el comportamiento habitual es que el mecanismo de transferencia de carga ocurra a través 
de la combinación de ambas componentes (ver la Figura 1.1(b)); bajo estas circunstancias, la contribución de la resistencia por fuste es de gran importancia, debido a que su movilización total ocurre para pequeñas deformaciones -o para asientos en cabeza del pilote equivalente a $1 \%$ del diámetro del pilote (Whitaker y Cooke 1966; Horvath y Kenney 1979)- en comparación a la resistencia por punta cuya completa movilización ocurre para asientos cercanos a $10 \%$ del diámetro del pilote (ISSMFE 1985).

La estimación de la resistencia por fuste de pilotes empotrados en roca usualmente se basa en normativas o experiencias adquiridas en cada país, así como también, en correlaciones empíricas en función de la resistencia a compresión simple del material más débil (roca o pilote) (Seidel y Collingwood 2001). Sin embargo, el principal problema que surge al usar estas formulaciones es la gran dispersión de resultados que se obtiene al emplear una u otra. La consecuencia es que los diseños de pilotes empotrados en roca resultan a menudo muy conservadores, de grandes longitudes, con mayores costos y mayor tiempo en obra, etc. (Miller 2003).

De acuerdo con O'Neill et al. (1996), otros factores adicionales a la resistencia a compresión simple deben ser considerados para realizar una adecuada estimación de la resistencia por fuste de pilotes empotrados en roca. Tal es el caso del método constructivo y la herramienta de perforación empleada (Nam y Vipulanandan 2008; O’Neill et al.1996; $\mathrm{Ng}$ et al. 2001), el tipo y la calidad del macizo rocoso (Haberfield 2013; Melentijevic y Olalla 2014; Haberfield y Lochaden 2018), la longitud y diámetro del pilote (Seidel y Haberfield 1995; Seol y Jeong 2007), el fluido empleado y el espesor de la zona de alteración durante el proceso de perforación de la roca (Williams y Pells 1981; Haberfield 2013), y el esfuerzo normal actuante y la rugosidad en la interfaz roca-pilote (Horvath y Kenney 1979; Pells et al. 1980; Horvath et al. 1983; Seidel y Collingwood 2001; Seol et al. 2009). En particular, la rugosidad es un factor que ha demostrado tener gran influencia en el aumento de la resistencia por fuste de pilotes empotrados en roca 
(e.g., ver Pells et al. 1980; Horvath et al. 1983); sin embargo, es poco usual medirla durante la fase de construcción o considerarla durante el proceso de diseño de este tipo de cimentación.

En relación con esto último, la constante evolución de la tecnología ha permitido que, hoy en día, la medición de la rugosidad (resultante en la excavación de pilotes empotrados en roca) pueda ser realizada a través de diversas técnicas -e.g., SoniCaliper (Loadtest 2014), Fotogrametría (García-Luna et al. 2019), LiDAR (Riquelme et al. 2016), etc.- y, por tanto, se abre la posibilidad de que la misma pueda ser incorporada en la estimación de la resistencia por fuste de pilotes. Sin embargo, a pesar de que se han realizado algunos esfuerzos -e.g., ver Horvath et al. 1983; Seidel y Collingwood 2001- aún no existe en la literatura una formulación que permita realizar una estimación más "real" y no tan conservadora de la resistencia por fuste de pilotes empotrados en roca considerando la rugosidad en la interfaz roca-pilote. Ese es precisamente el objetivo de esta tesis doctoral que, sobre la base de las consideraciones anteriores y siguiendo con la introducción y objetivos de la investigación, se estructura en los siguientes capítulos:

Capítulo 2: Estado del arte, en el que se presenta un compendio de las diversas investigaciones realizadas para estudiar el efecto de la rugosidad sobre el comportamiento resistente de pilotes empotrados en roca a partir de (i) modelos a pequeña escala, (ii) modelos a gran escala y (iii) ensayos de centrifuga.

Capítulo 3: Modelos numéricos a pequeña escala considerando la interacción rocahormigón, en el que se evalúa el comportamiento de resistencia al corte de juntas rocahormigón, con diferentes niveles de rugosidad en las juntas, mediante la simulación numérica de ensayos de corte directo y su validación con ensayos experimentales publicados en la literatura. 
Capítulo 4: Modelos numéricos a gran escala de pilotes empotrados en roca, en el cual, se evalúa el efecto de la rugosidad sobre la resistencia por fuste de pilotes empotrados en roca mediante la simulación numérica (mediante el Método de los Elementos Discretos y el Método de los Elementos Finitos) de pilotes cargados axialmente, considerando diferentes niveles de rugosidad en la interfaz roca-pilote. Además, se presenta y analiza el mecanismo de transferencia de carga que se produce debido a la rugosidad en la interfaz roca-pilote.

Capítulo 5: Ensayos de centrifuga de pilotes empotrados en roca, en el cual se emplean ensayos de centrifuga para evaluar el efecto de la rugosidad sobre la resistencia por fuste de pilotes empotrados en roca, considerando diferentes niveles de rugosidad en la interfaz roca-pilote, así como una comparación de resultados a través de modelos numéricos realizados con el MEF.

Capítulo 6: Formulación para la estimación de la resistencia por fuste de pilotes empotrados en roca considerando la rugosidad de la excavación, donde se compara y contrastan los resultados obtenidos con los modelos numéricos y ensayos de centrifuga con aquellos resultados obtenidos mediante las diversas formulaciones publicadas en la literatura. Adicionalmente, en este capítulo se propone y valida una formulación derivada de esta investigación para estimar la resistencia por fuste de pilotes empotrados en roca, considerando la rugosidad de la interfaz roca-pilote.

Capítulo 7: Conclusiones y líneas de investigación futuras derivadas de este trabajo de investigación.

\subsection{Objetivos}

\subsubsection{Objetivo principal}

Evaluar el efecto de la rugosidad en el contacto roca-pilote sobre la resistencia por fuste de pilotes empotrados en roca. 


\subsubsection{Objetivo específicos}

- Describir el "Estado del Arte" de las diversas investigaciones realizadas para estudiar el comportamiento de la rugosidad en la interfaz roca-pilote y su efecto sobre el comportamiento por fuste.

- Analizar del comportamiento al corte de juntas roca-hormigón, con diferente nivel de rugosidad en su interfaz, mediante modelos numéricos aplicando el Método de Elementos Discretos (MED), los cuales se validan con ensayos de laboratorio publicados en la literatura.

- Analizar el efecto de la rugosidad sobre la resistencia por fuste de pilotes empotrados en roca, a través de modelos numéricos utilizando el Método de Elementos Discretos (MED) y su comparación con el Método de Elementos Finitos (MEF).

- Analizar el efecto de la rugosidad sobre la resistencia por fuste de pilotes empotrados en roca a través de ensayos de centrifuga.

- Contrastar y comparar la resistencia por fuste de pilotes empotrados en roca obtenida mediante el uso de modelos numéricos y ensayos de centrifuga con aquella obtenida a través de las diversas formulaciones existente en la literatura.

- Proponer una formulación de diseño para la estimación de la resistencia por fuste de pilotes empotrados en roca, considerando la rugosidad en la interfaz rocapilote. 


\section{Estado del arte}

\subsection{Introducción}

La resistencia al corte en juntas roca-roca depende, en gran medida, de las propiedades resistentes de la misma roca y de la geometría de la discontinuidad. Patton (1966) fue uno de los primeros investigadores en abordar este tema, a través de una serie de ensayos de corte directo sobre juntas de roca bajo la acción de pequeños esfuerzos normales constantes y así analizar los múltiples modos de falla por corte que podrían presentarse en juntas de roca. En la Figura 2.1 (situación 2 y 3), se presenta la envolvente de falla bilineal propuesta en su investigación y, en el que además, valida el criterio de Mohr-Coulomb sugerido para juntas planas (ver la Figura 2.1, situación 1).
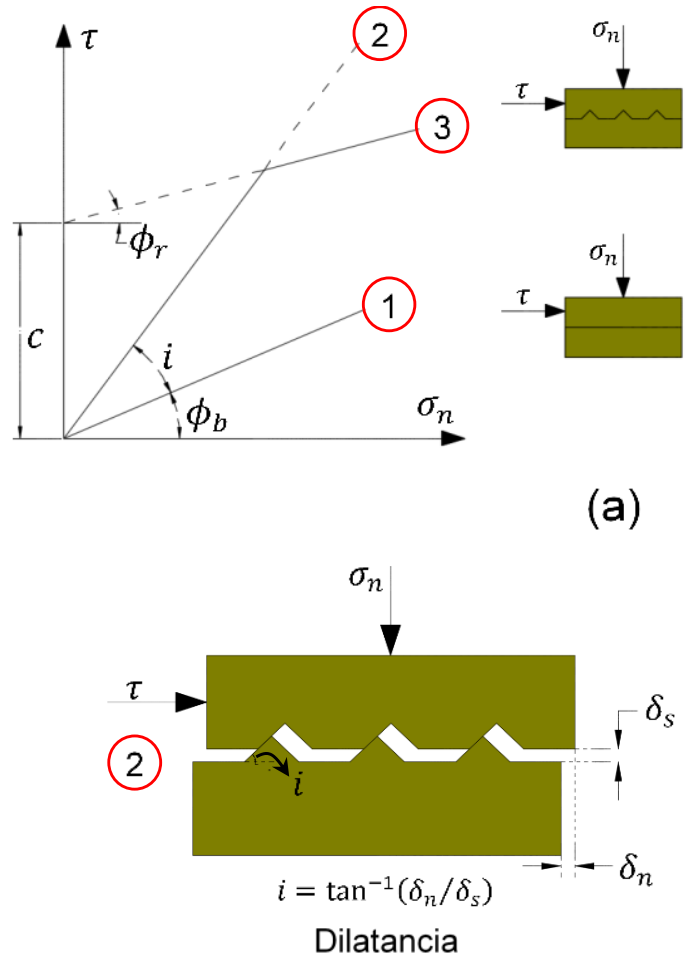

(b)

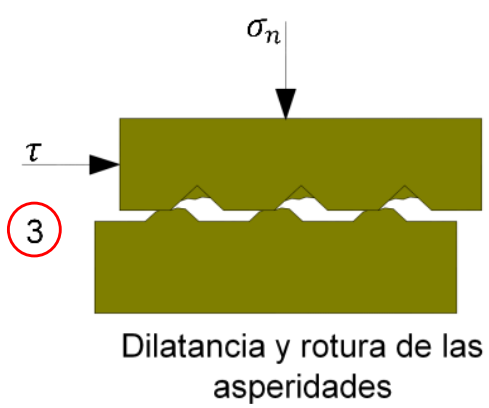

(c)

Figura 2.1. (a) envolventes de falla propuestas por Patton (1966) para caracterizar la resistencia al corte de juntas de roca plana y rugosas, (b) detalle de la situación 2 , (c) detalle de la situación 3 (modificado de Gutiérrez 2013).

En la Figura 2.1, $c$ es la cohesión, $\phi_{b}$ es el ángulo de fricción básico o de resistencia al corte (o deslizamiento), $i$ es el ángulo de inclinación de asperidad con respecto a la 
dirección de aplicación de la fuerza de corte, $\phi_{r}$ es el ángulo de fricción residual, $\tau$ es la resistencia al corte, $\sigma_{n}$ es el esfuerzo normal actuante, $\delta_{n}$ y $\delta_{s}$ son los desplazamientos en la dirección normal y de corte, respectivamente.

Posteriormente, Barton (1976), Barton y Choubey (1977) propusieron un modelo empírico para estimar la resistencia al corte de juntas de roca rugosas de la forma:

$$
\tau=\sigma_{n} \tan \left(\phi_{b}+J R C \log _{10}\left(\frac{J C S}{\sigma_{n}}\right)\right)
$$

Donde $J R C$ es el coeficiente de rugosidad de la junta (Joint Roughness Coefficient) obtenido a partir de un perfil de rugosidad trazado sobre la junta (ver la Figura 2.2) y JCS es la resistencia a compresión simple determinada sobre el plano de la junta (e.g., mediante el uso del esclerómetro de Schmidt o a través de un ensayo de carga puntual).
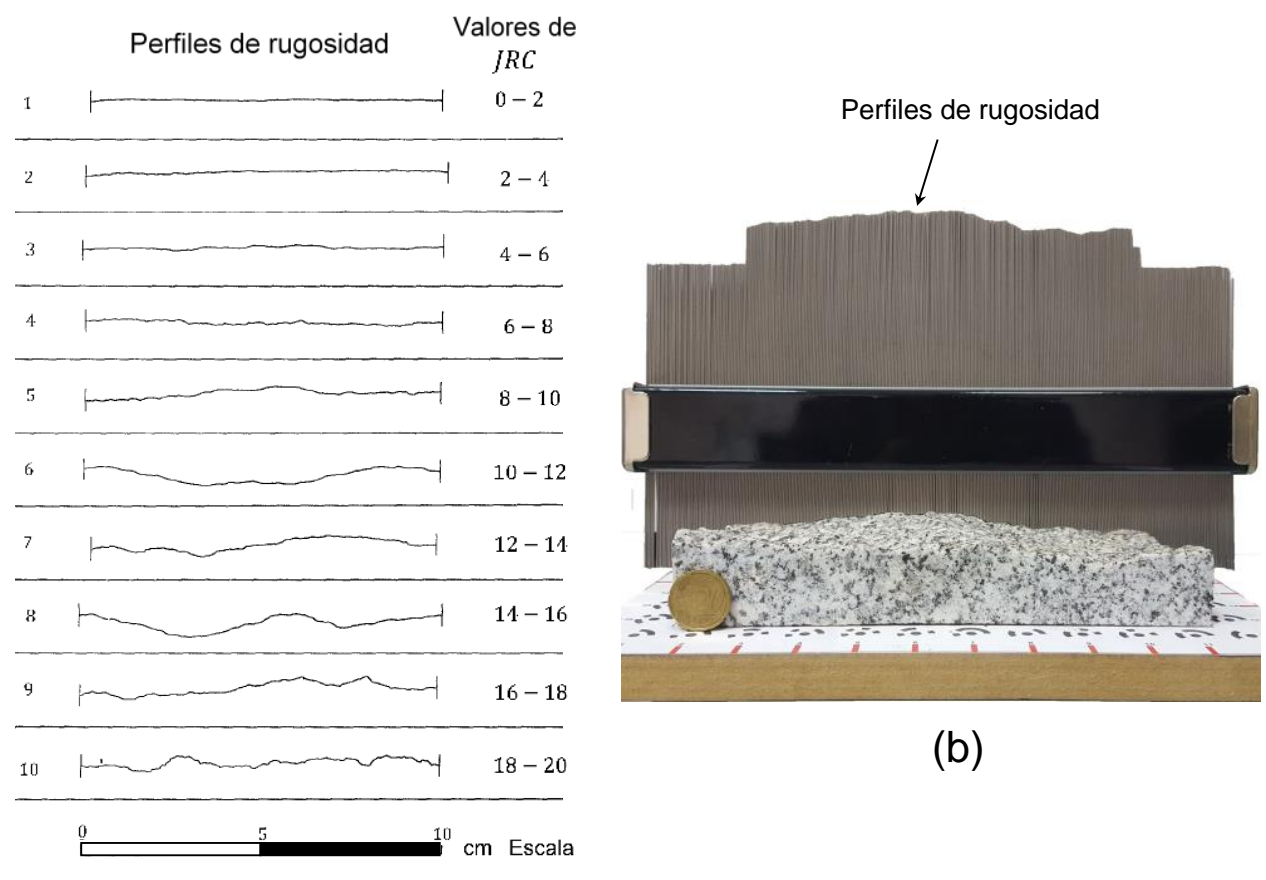

(a)

Figura 2.2. (a) perfiles de rugosidad típicos y su correspondiente valor JRC (modificado de Barton y Choubey 1977), (b) estimación del perfil de rugosidad de la junta de roca mediante el uso del perfilómetro o "peine" de Barton.

Otro tópico que también ha sido objeto de investigación en la ingeniería geotécnica, en adición al estudio del comportamiento al corte de juntas roca-roca, es el efecto de la 
rugosidad sobre el comportamiento al corte en la interfaz roca-hormigón; principalmente por la común interacción de estos dos materiales en diversos proyectos de ingeniería (e.g., presas, anclajes en roca, pilotes, etc.). En particular, en esta tesis se analiza el efecto que tiene la rugosidad sobre la resistencia por fuste de pilotes empotrados en roca, en la cual, la interacción roca-hormigón está presente. Por tal razón, en las siguientes secciones se presentan algunos trabajos desarrollados en diversas investigaciones, para estudiar y analizar este comportamiento a partir de modelos a pequeña escala (ver Sección 2.2), así como mediante el uso de modelos a gran escala (ver Sección 2.3) y ensayos de centrifuga (ver Sección 2.4).

\subsection{Modelos a pequeña escala}

El estudio y comprensión del comportamiento al corte de juntas con interfaz roca-roca y juntas con interfaz roca-hormigón es un aspecto de gran relevancia en muchos proyectos tales como: presas de hormigón (Krounis et al. 2016), cimentaciones de puentes y viaductos (Tian et al. 2015), estabilidad de macizos rocosos (Leichnitz 1985; Poturovic et al. 2015), anclajes (Tian et al. 2015) y pilotes empotrados en roca (Johnston et al. 1987; Melentijevic et al. 2012; Melentijevic y Olalla 2014; Gutiérrez-Ch y Melentijevic 2016). En los apartados siguientes, se describen brevemente algunos de los trabajos publicados en la literatura sobre este tema.

\subsubsection{Ensayos de corte directo en laboratorio e in situ}

Tradicionalmente, la resistencia al corte de juntas roca-roca y juntas roca-hormigón ha sido investigada mediante ensayos in situ o de laboratorio. En ambos casos, el ensayo más comúnmente empleado es el ensayo de corte directo bien bajo carga normal constante (constant normal load, CNL, ver la Figura 2.3), o bajo rigidez normal constante (constant normal stiffness, CNS, ver la Figura 2.4). 


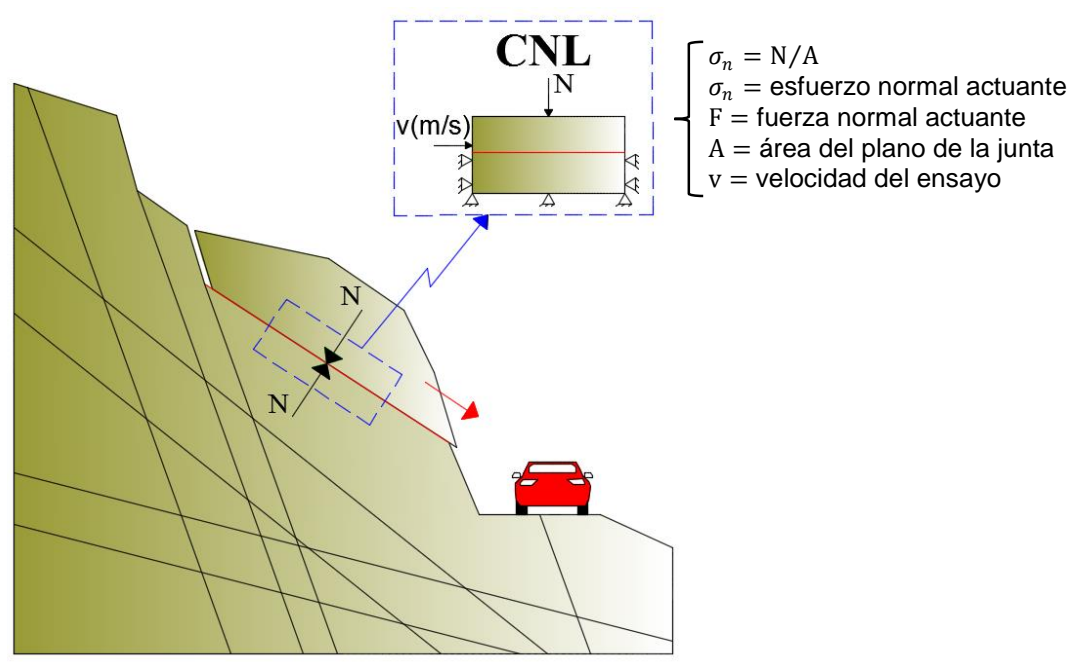

Figura 2.3. Talud de roca, condición de borde CNL (modificado de Poturovic et al. 2015).

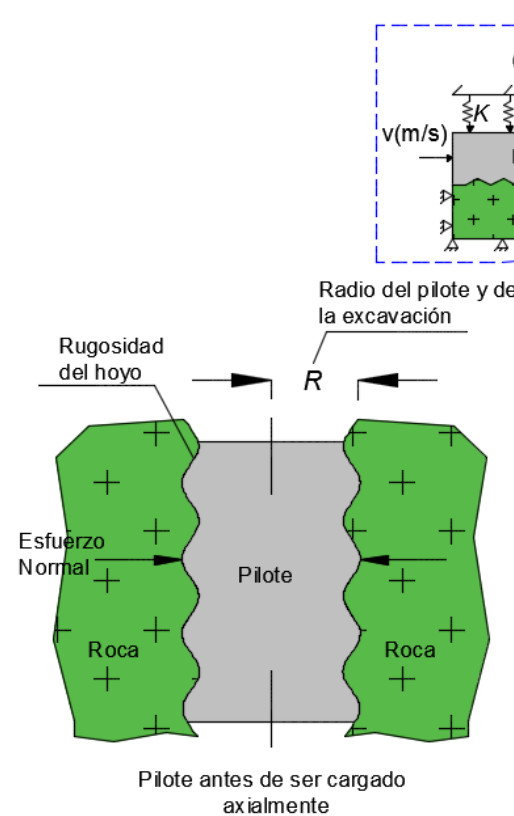

(a)

$$
\begin{aligned}
& \sigma_{n}=\sigma_{n o}+K y \\
& \sigma_{n}=\text { esfuerzo normal actuante } \\
& \sigma_{n o}=\text { esfuerzo normal inicial } \\
& K=\text { rigidez externa } \\
& \mathrm{v}=\text { velocidad del ensayo } \\
& \mathrm{y}=\text { desplazamiento vertical } \\
& \text { каaı de ı }
\end{aligned}
$$
excavación

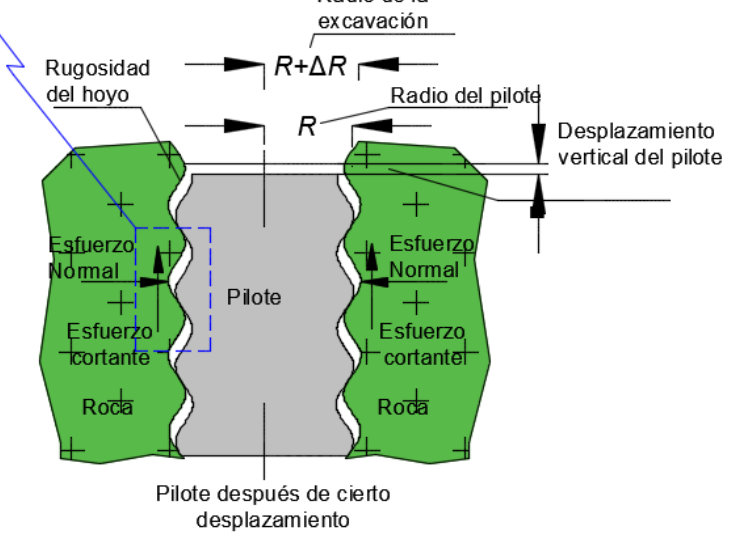

(b)

Figura 2.4. Pilote empotrado en roca, condición de borde CNS (modificado de Johnston et al. 1987).

En los ensayos de corte directo bajo condición $\mathrm{CNL}$, la carga normal permanece (aproximadamente) constante, y la interfaz puede dilatar o contraer libremente durante la realización del ensayo; esta condición es representativa, por ejemplo, para juntas presentes en taludes de roca (ver la Figura 2.3). Por otra parte, en los ensayos de corte bajo condición CNS, la carga actuante depende de la rigidez normal y de corte de la junta, así como del desplazamiento que la interfaz pueda permitir. La condición CNS es válida para situaciones en los que la interfaz está sometida a grandes esfuerzos 
normales y reducidas posibilidades de poder dilatar, por ejemplo, para pilotes empotrados en roca (ver la Figura 2.4) (Asadi et al. 2013; Shrivastava y Rao 2015; Thirukumaran y Indraratna 2016). A continuación, se presentan algunos ensayos de corte directo publicados en la literatura.

\subsubsection{Leichitz (1985)}

Con el fin de analizar la interacción en juntas roca-roca, Leichitz (1985) realizó 31 ensayos de corte directo en areniscas bajo condición de (i) carga normal constante (CNL), y de (ii) rigidez normal constante (CNS). Los resultados experimentales muestran que la condición de borde influye sobre el comportamiento al corte para las probetas ensayadas en esta investigación, principalmente para pequeños valores de esfuerzo normal (ver la Figura 2.5).

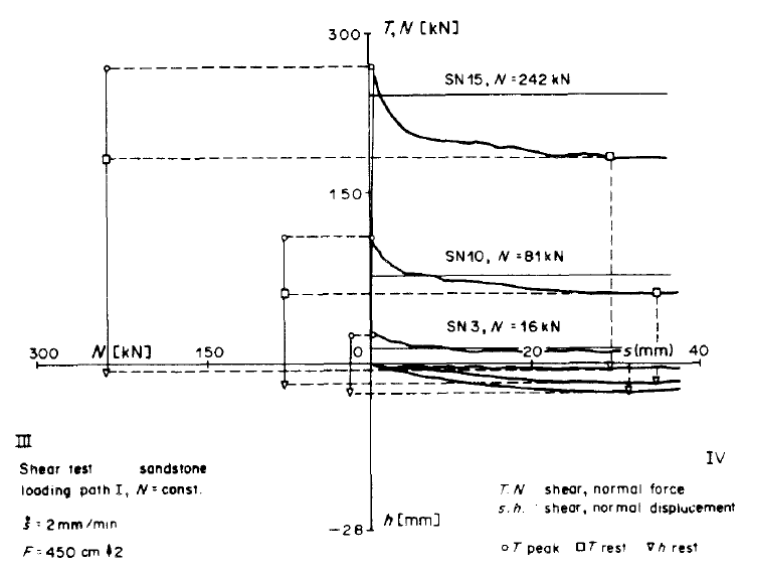

(a)

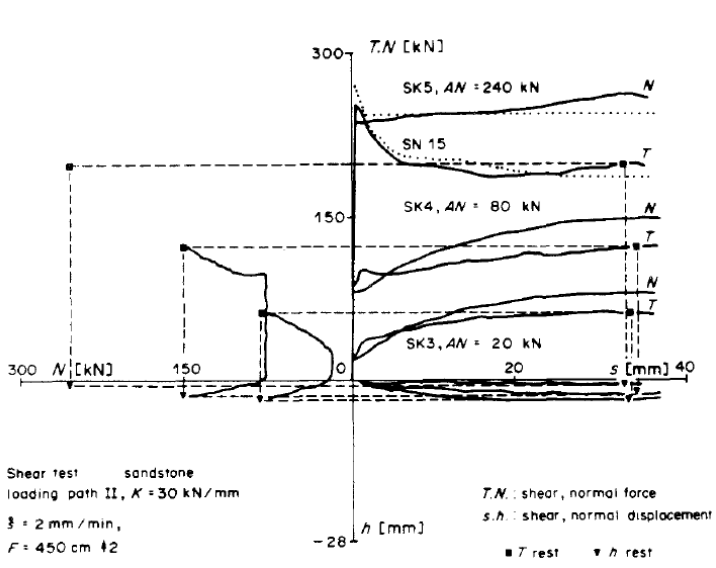

(b)

Figura 2.5. Ensayos de corte directo en probetas de arenisca con juntas rugosas: (a) condición CNL, (b) condición CNS (Leichnitz 1985).

\subsubsection{Haque (1999)}

Para analizar el comportamiento al corte en las areniscas de New South-Australia, Haque (1999) realizó una serie de ensayos de corte directo en probetas roca-roca, sin cohesión, con relleno y sin relleno, con junta de morfología natural y triangular simétrica (con ángulo respecto al plano horizontal de $9.5^{\circ}, 18.5^{\circ}$ y $26.5^{\circ}$ ), empleando condición de borde CNL y CNS. Los resultados sugieren que (i) la condición de borde CNS respecto 
a la CNL arroja mayor resistencia al corte pico con su correspondiente desplazamiento al corte, y con menor dilatación; (ii) a mayor rugosidad en la interfaz se alcanza mayor resistencia al corte y, (iii) la presencia y espesor del relleno en el plano de contacto disminuye la resistencia al corte pico alcanzada en el ensayo (ver la Figura 2.6).

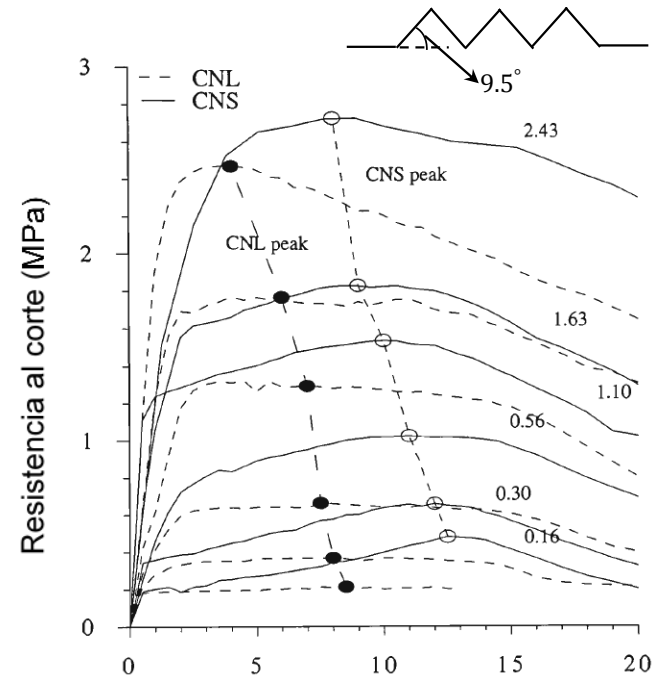

(a)

Desplazamiento al corte $(\mathrm{mm})$

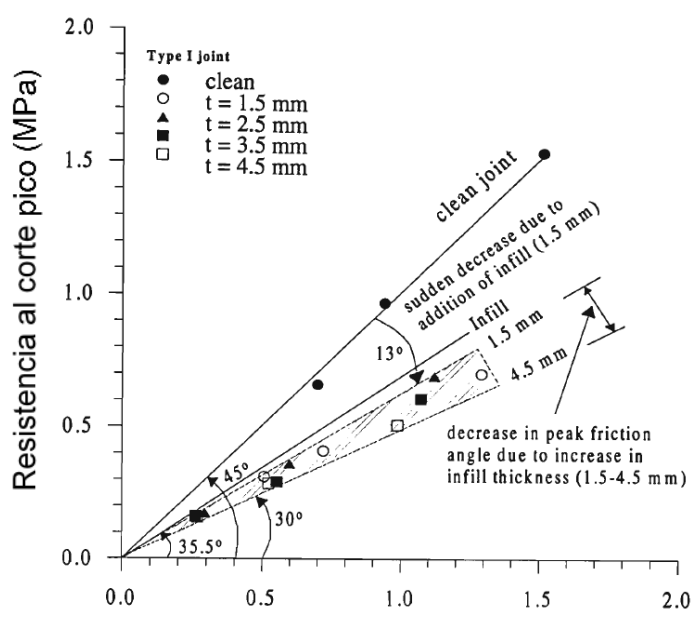

(b) Esfuerzo normal (MPa)

Figura 2.6. Ensayos de corte directo en probetas de arenisca con juntas rugosas para ángulo base de $9.5^{\circ}$ : (a) comparación entre condición CNL y CNS, (b) efecto del relleno y su espesor sobre la resistencia al corte pico (modificado de Haque 1999).

\subsubsection{Gu et al. (2003)}

Para entender el comportamiento de pilotes empotrados en roca, Gu et al. (2013) realizaron 112 ensayos de corte directo en probetas con interacción arenisca-hormigón, considerando la interfaz sin cohesión, con cohesión y con relleno, así como juntas de morfología fractal y triangular simétrica de diferente ángulo base $\left(\alpha=5^{\circ}, 10^{\circ}\right.$, $20^{\circ}, 30^{\circ}, 45^{\circ}$ ) y, bajo condición de borde CNL y CNS. Los resultados presentados en la Figura 2.7 son similares a los obtenidos por Haque (1999), en los cuales, se observa que: (i) a mayor rugosidad en la interfaz se obtiene mayor resistencia al corte pico movilizada, (ii) la resistencia al corte pico en condición CNS es mayor que en condición CNL, (iii) las juntas con cohesión tiene mayor resistencia pico que aquellas sin cohesión, y (iv) el relleno y su espesor disminuyen la resistencia al corte pico (ver la Figura 2.7). 

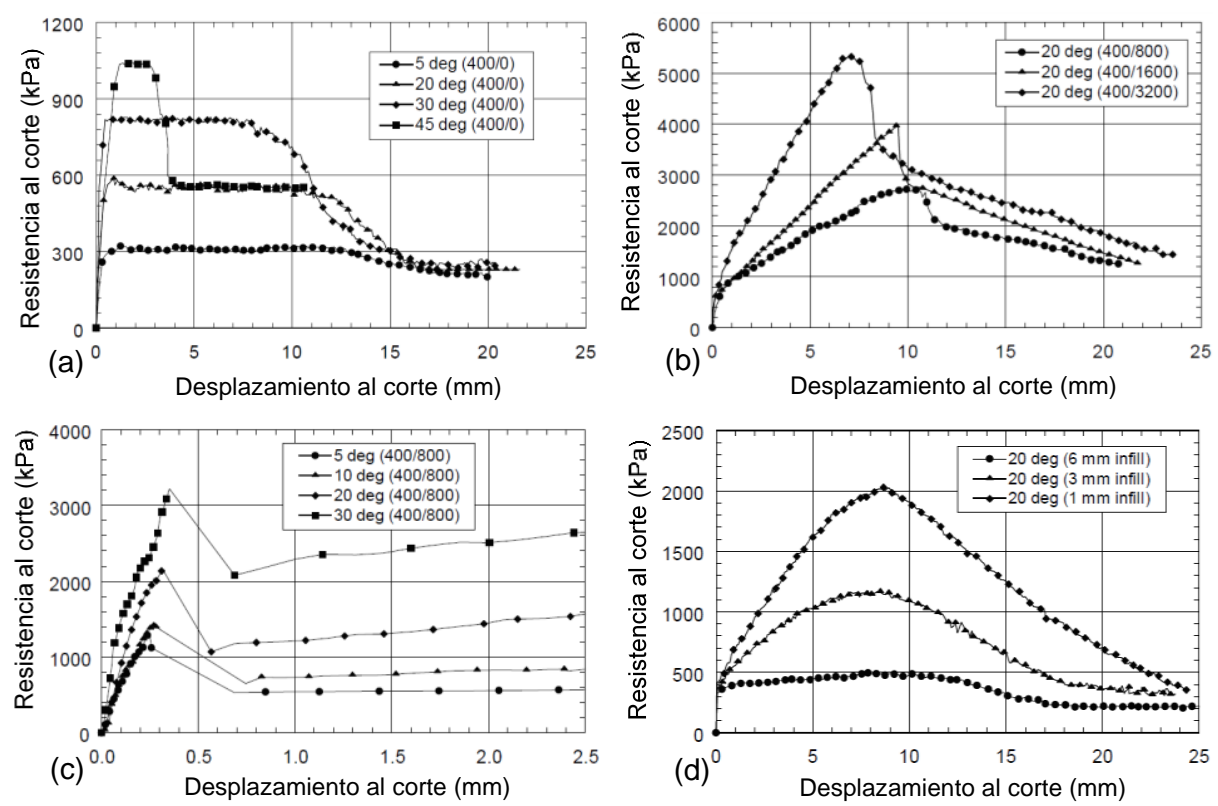

Figura 2.7. Ensayos de resistencia al corte en juntas triangulares arenisca-hormigón: (a) sin cohesión, condición CNL con $\sigma_{n}=400 \mathrm{kPa}$, (b) sin cohesión, condición CNS con $\sigma_{n o}=400 \mathrm{kPa}$ y $K=800,1600$ y $3200 \mathrm{kPa} / \mathrm{mm}$, (c) con cohesión, condición CNS con $\sigma_{n o}=400 \mathrm{kPa}$ y $K=$ $800 \mathrm{kPa} / \mathrm{mm}$, (d) con relleno de diferente espesor, condición CNS con $\sigma_{n o}=400 \mathrm{kPa}$ y $K=800$ $\mathrm{kPa} / \mathrm{mm}$ (modificado de Gu et al. 2003).

\subsubsection{Ghosh (2010)}

Para estudiar el comportamiento al corte de una presa de hormigón (de $26.2 \mathrm{~m}$ de alto y 700 m de largo) cimentada sobre roca (granito), Ghosh (2010) realizó 6 ensayos de corte directo in situ bajo condición CNL (ver la Figura 2.8).

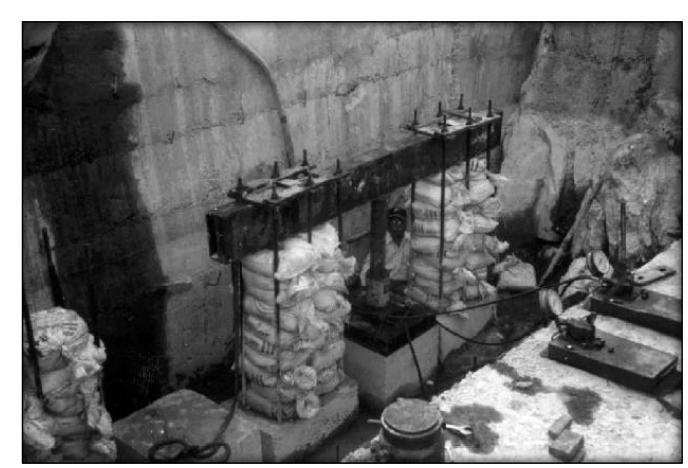

(a)

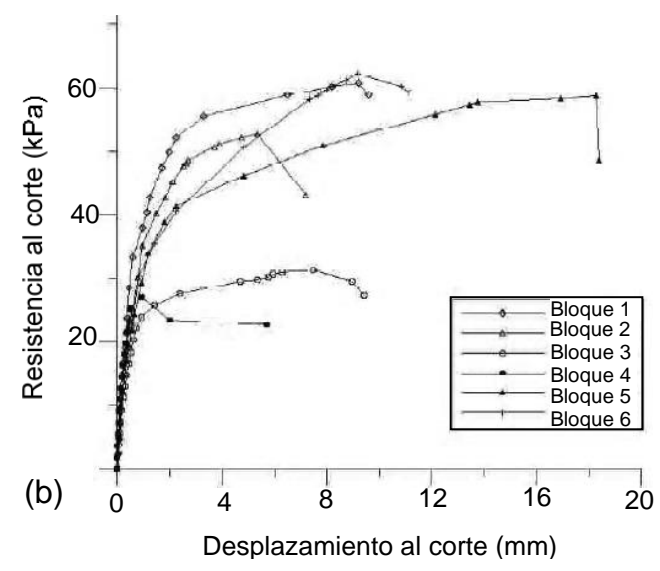

Figura 2.8. (a) configuración del ensayo de corte directo in situ con interacción roca-hormigón,

(b) curvas de resistencia al corte vs desplazamiento al corte (modificado de Ghosh 2010).

Para ello, fabricaron 6 bloques de hormigón con dimensiones de $700 \mathrm{~mm} \times 600 \mathrm{~mm} \times$ $600 \mathrm{~mm}$, con período de curado de tres semanas, los cuales posteriormente fueron 
colocadas sobre la roca del cimiento -en diferentes lugares con distinta rugosidad-para la realización del ensayo. Los resultados sugieren que los parámetros de resistencia en la interfaz roca-hormigón dependen de la rugosidad en la junta.

\subsubsection{Liahagen (2012) y Gutiérrez (2013)}

Para estudiar el comportamiento en la interfaz roca-hormigón de una presa cimentada sobre roca (granito o gneis), Liahagen (2012) y Gutiérrez (2013) realizaron ensayos de corte directo en probetas hormigón-granito y hormigón-gneis, respectivamente, bajo condición CNL, en juntas rugosas de geometría triangular simétrica, con $\alpha=0^{\circ}, 10^{\circ}$, $20^{\circ}$, y $40^{\circ}$ (ver la Figura 2.9(a)), con y sin cohesión en la interfaz de ambos materiales. Los resultados obtenidos en estos ensayos (ver la Figura 2.9(b)-(d)) son similares al comportamiento reflejado por Gu et al. (2003), de modo que la rugosidad de la junta, la cohesión y el esfuerzo normal aplicado influyen sobre el comportamiento al corte de juntas roca-hormigón.

\subsubsection{Asadi et al. (2013)}

El mecanismo de fallo y la degradación de las asperidades ha sido otro aspecto de gran interés en la interacción de juntas roca-roca. Por ello, Asadi et al. (2013) elaboraron probetas de roca artificial con resistencia a compresión simple $\left(\sigma_{c}\right)$ de $29.2 \mathrm{MPa}$ y realizaron ensayos de corte directo sobre la interfaz roca-roca, empleando perfiles de rugosidad de morfología triangular simétrica de ángulo base variable $\left(15^{\circ}, 30^{\circ}\right.$ y $\left.45^{\circ}\right)$, y con forma sinusoidal, empleando la condición CNL en todos los ensayos (ver la Figura 2.10).

Los resultados presentados en la Figura 2.10 confirman lo obtenido en investigaciones previas, en las que el aumento de la rugosidad aumenta la resistencia al corte pico alcanzada en juntas roca-roca. Adicionalmente, se nota que la morfología de la junta influye en el comportamiento post-pico y, en el mecanismo de fallo, es decir, a medida 
que se incrementa el ángulo de la rugosidad, el mecanismo de fallo pasa de ser deslizante a fallo por rotura de las asperidades - notar los cambios bruscos en las curvas esfuerzo al corte versus desplazamiento al corte de la Figura 2.10(b)-; este comportamiento también fue observado por Gu et al. (2003). Finalmente, en la Figura 2.10(d) se aprecia que la dirección de realización del ensayo es otro factor que influye sobre el comportamiento al corte, movilizándose en este caso mayor resistencia de derecha a izquierda -denotado como $R L$ shearing, ver la Figura 2.10(d)-.
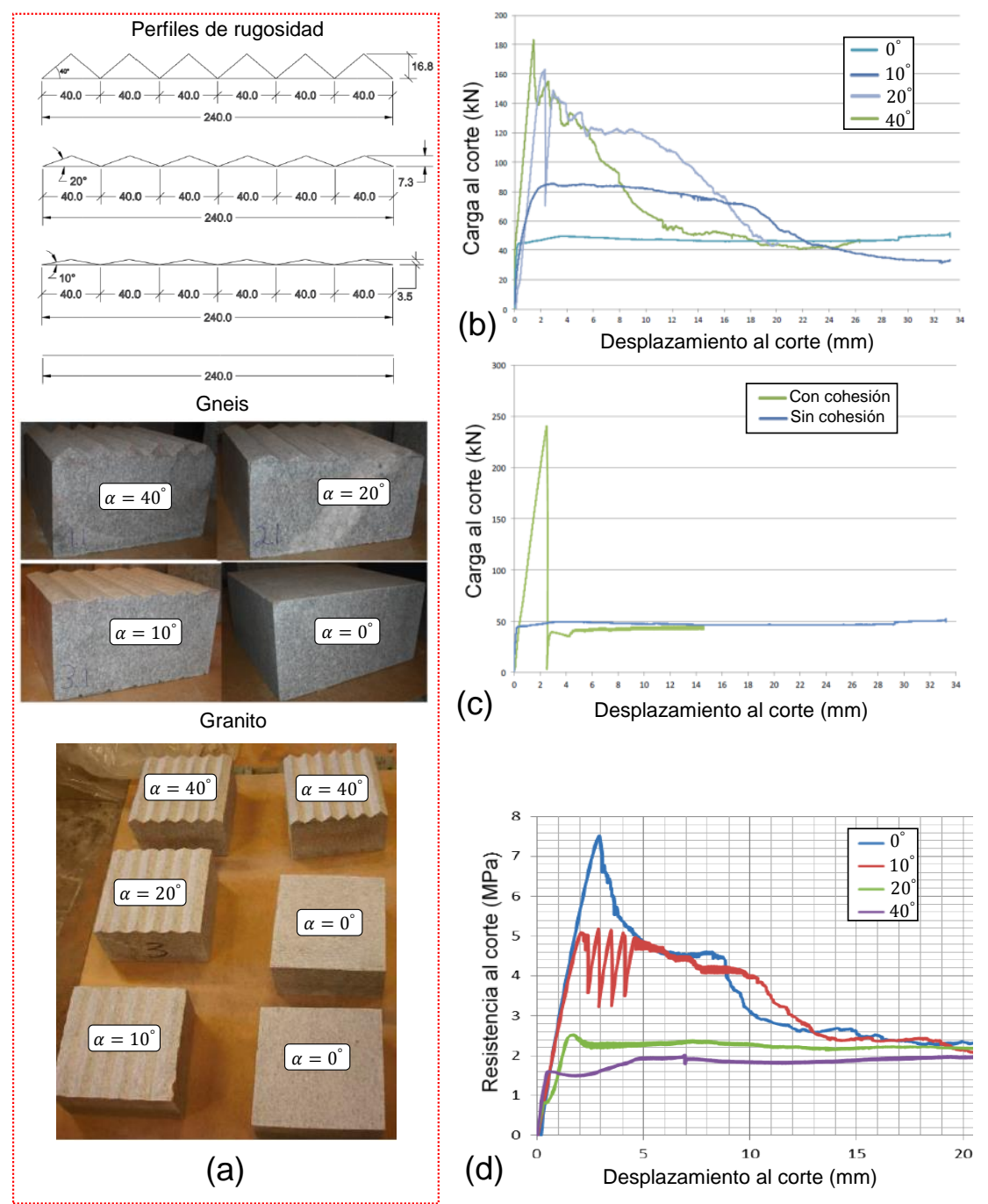

Figura 2.9. Ensayos de corte directo en probetas roca-hormigón con junta rugosa bajo condición CNL: (a) perfiles de rugosidad y probetas con diferente $\alpha$, (b) curvas de carga al corte vs desplazamiento al corte para juntas granito-hormigón con distinta rugosidad, sin cohesión y con $\sigma_{n}=1200 \mathrm{kPa}$, (c) curvas de carga al corte vs desplazamiento al corte para juntas planas granito-hormigón, con y sin cohesión, para $\sigma_{n}=1200 \mathrm{kPa}$, (d) curvas de carga al corte vs desplazamiento al corte para juntas gneis-hormigón con distinta rugosidad, sin cohesión y con $\sigma_{n}=3200 \mathrm{kPa}$, (modificado de Liahagen 2012, Gutiérrez 2013). 

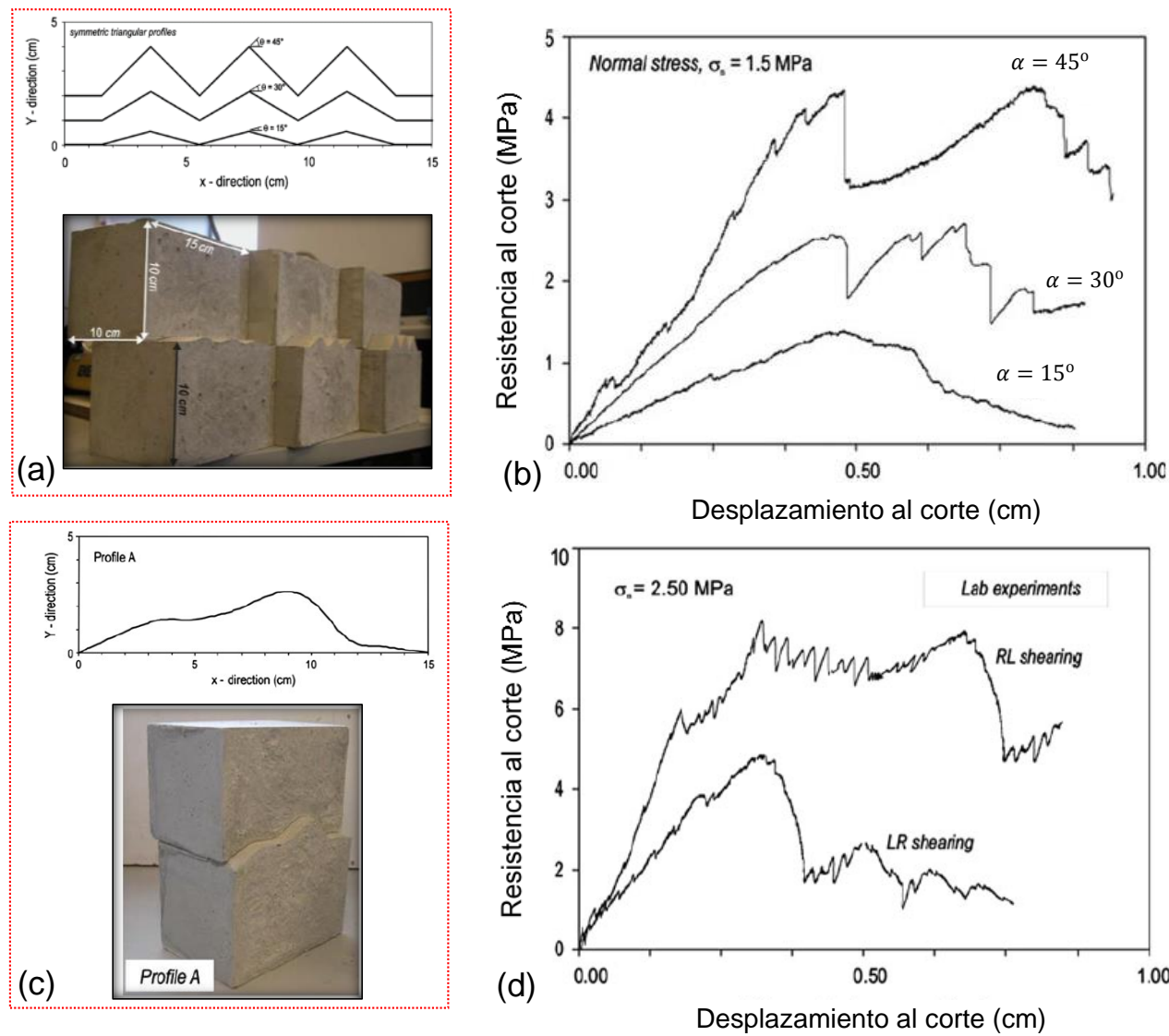

Figura 2.10. Ensayos de corte directo en probetas roca-roca con junta rugosa bajo condición CNL: (a) probetas y geometría de los perfiles de rugosidad con $\alpha=15^{\circ}, 30^{\circ}$ y $45^{\circ}$, (b) curvas de resistencia al corte vs desplazamiento al corte, (c) probeta y geometría del perfil de rugosidad sinusoidal, (d) curvas de resistencia al corte vs desplazamiento al corte (modificado de Asadi et al. 2013).

\subsubsection{Krounis et al. (2016)}

Otro aspecto de interés en la estabilidad al deslizamiento de presas de hormigón cimentadas sobre roca, es el porcentaje de cohesión resultante en la interfaz rocahormigón y su efecto sobre la resistencia al corte entre ambos materiales. Esto llevó a Krounis et al. (2016) a realizar ensayos de corte directo bajo condición CNL, en probetas con interfaz roca-hormigón, de rugosidad natural variable y con diferente porcentaje $(0$, $25,50,75$ y $100 \%$ ) de cohesión en la junta. En la Figura 2.11, se presentan los valores de resistencia al corte pico alcanzados para diferentes porcentajes de cohesión en la junta. Como puede observarse, hay un claro efecto de que las juntas con $100 \%$ de cohesión alcanzan mayor resistencia al corte pico que las juntas sin cohesión ( $0 \%)$; no obstante, el comportamiento observado en juntas con 50 y 75\% de cohesión arrojaron 
valores menores de resistencia al corte pico que aquellos obtenidos para las probetas con $25 \%$ de cohesión, este comportamiento pareciera no ser lógico y Krounis et al. (2016) sugieren que quizás puede deberse a que se haya producido una rotura de las asperidades en la interfaz roca-hormigón antes de haberse movilizado totalmente la resistencia debida a la cohesión existente entre ambos materiales.

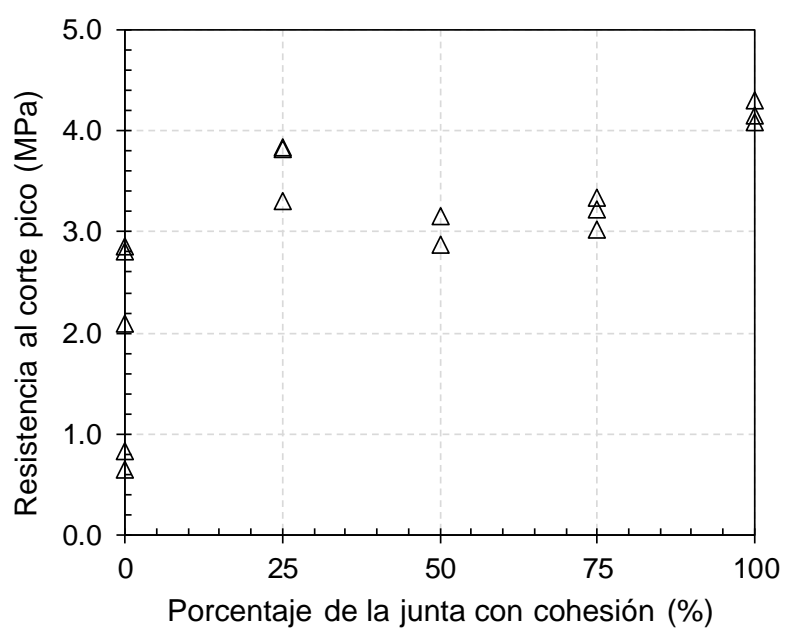

Figura 2.11. Resistencia al corte pico vs porcentaje de la junta con cohesión en la junta (datos experimentales tomados de Krounis et al. 2016).

\subsubsection{Shrivastava y Rao $(2015,2018)$}

El efecto del relleno entre juntas roca-roca sobre el comportamiento al corte ha sido un aspecto de interés en diversos proyectos geotécnicos. Por tal razón, Shrivastava y Rao $(2015,2018)$ realizaron ensayos de corte directo en juntas de roca-roca -la roca fue preparada artificialmente con una resistencia a la compresión uniaxial $\left(\sigma_{c}\right)$ de 11.75 $\mathrm{MPa}-$, bajo condición de borde CNL y CNS, con interfaz de rugosidad triangular simétrica $\left(\alpha=0^{\circ}, 15^{\circ}\right.$ y $\left.30^{\circ}\right)$ y agregando material de relleno (preparado artificialmente con $\sigma_{c}=3.47 \mathrm{MPa}$ ) al plano de contacto roca-roca. Los resultados obtenidos están cualitativamente en concordancia con aquellos reportados por Haque (1999) y Gu et al. (2003), en los cuales se nota que la condición de borde, el relleno en la junta y su espesor, así como la morfología en la interfaz, tienen efecto sobre la resistencia al corte en probetas de junta roca-roca (ver la Figura 2.12). 
Por otra parte, el comportamiento al corte en juntas roca-roca o roca-hormigón también ha sido estudiado mediante formulaciones analíticas (Patton 1966; Ladanyi y Archambault 1980) y empíricas (Barton 1976, Barton y Chouby 1977), que han permitido el desarrollo de modelos para estimar (i) la resistencia al corte de juntas roca-roca (Gens et al. 1990; Grasselli y Egger 2003; Haque y Kodikara 2012; Serrano et al. 2014; Indraratna et al. 2015; Tian et al. 2018), o de juntas roca-hormigón (Lo et al. 1991; Kodikara y Johnston 1994); y (ii) reproducir su comportamiento al corte (Barton y Chouby 1977; Seidel y Haberfiel 2002). No obstante, al no ser un objetivo de estudio la comparación entre resultados experimentales de ensayos de corte directo versus aquellos valores obtenidos con formulaciones, no se presentan en detalle las formulaciones antes mencionadas.

(a)
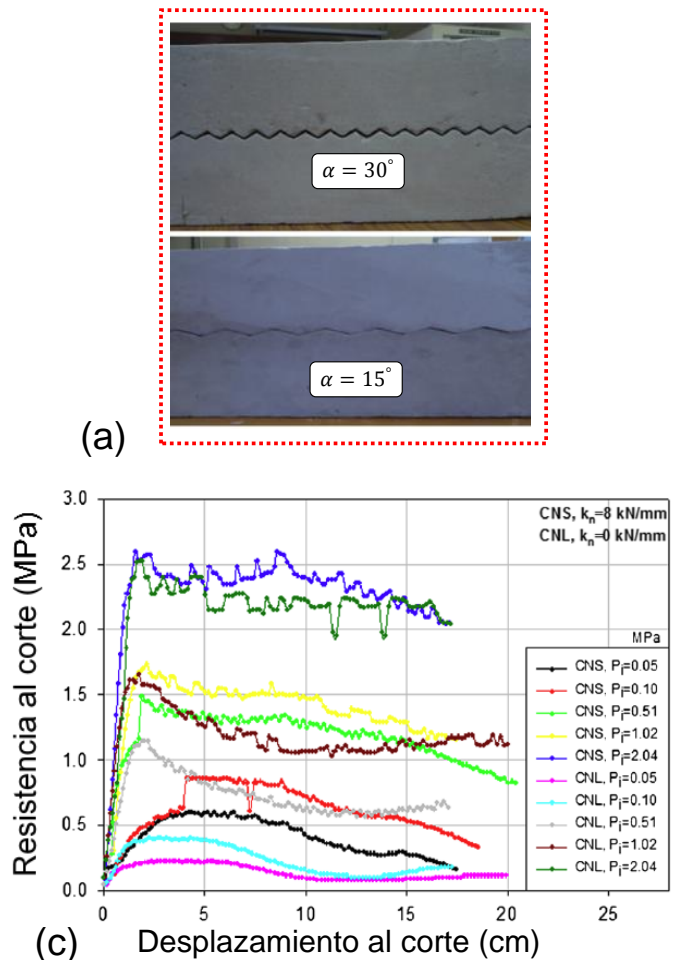
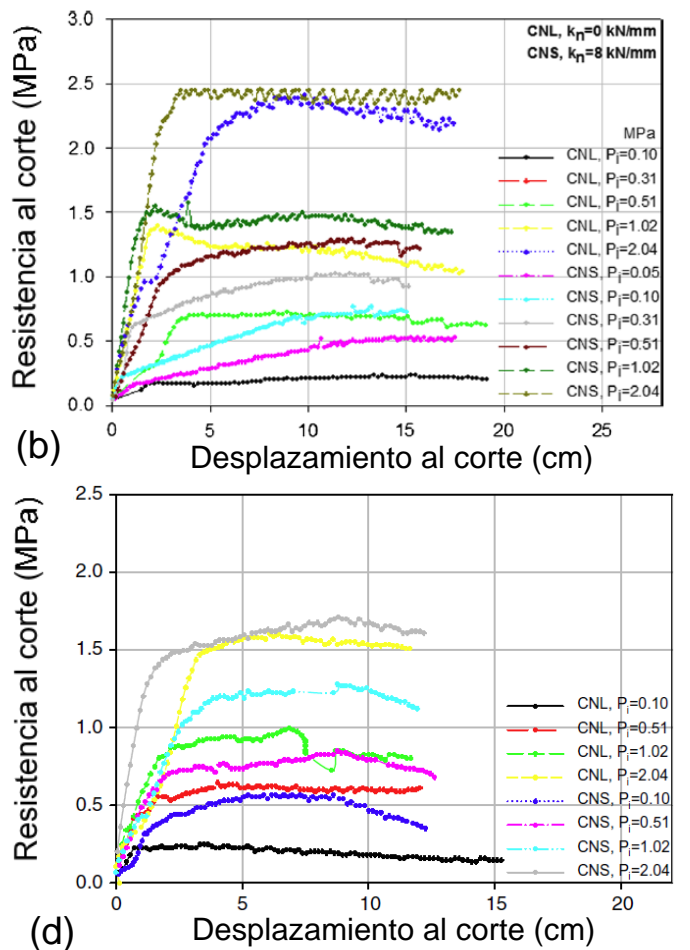

Figura 2.12. Ensayos de corte directo en junta triangular roca-roca bajo condición CNL y CNS: (a) probetas con $\alpha=15^{\circ}$ y $30^{\circ}$, (b) probeta con $\alpha=15^{\circ}$, sin relleno, (c) probeta con $\alpha=30^{\circ}$, sin relleno, (b) probeta con $\alpha=30^{\circ}$ y con relleno (modificado de Shrivastava y Rao 2015, 2018). 


\subsubsection{Ensayos de corte directo con modelos numéricos a través de la implementación del Método de Elementos Discretos}

Los enfoques analíticos y experimentales para estudiar el comportamiento al corte en juntas roca-roca y roca-hormigón mencionados en la Sección 2.2.1 pueden presentar problemas debido a su complejidad matemática, o a las dificultades y costos asociados que conlleva la realización de ensayos de laboratorio. Por esta razón, el uso de los métodos numéricos -como el Método de Elementos Finitos (MEF) o el Método de Elementos Discretos (MED)- ha tomado auge en los últimos años. Entre ellos, el enfoque del MED con su implementación en el programa comercial Particle Flow Code (PFC) (Park y Song, 2009; Asadi et al., 2012; Bahaaddini et al., 2013a), ha demostrado ser muy versátil para estudiar el comportamiento al corte en juntas roca-roca y rocahormigón, debido a que éste permite reproducir de forma más "real", aspectos importantes del comportamiento de la junta como la dilatancia, la rugosidad en los contactos y la rotura de las asperidades que conforman la interfaz, así como también la degradación que conduce al desarrollo de "terceros cuerpos" -i.e., aquel material generado por la degradación y rotura de las asperidades de la junta- (Royo y Melentijevic, 2014; Mollon, 2015; Bahaaddini, 2017). No obstante, a pesar de las capacidades que tiene el MED para caracterizar el comportamiento al corte de juntas roca-roca o roca-hormigón, los modelos con el MED aún tiene algunos aspectos por mejorar (e.g., tiempo de cálculo, rotura de las propias partículas, etc.). A continuación, se presentan algunas investigaciones realizadas para estudiar el comportamiento al corte en juntas roca-roca y roca-hormigón con el MED.

\subsubsection{Cundal (2000)}

Cundal (2000) modelizó el comportamiento al corte en juntas rugosas roca-roca, sin cohesión, a través de ensayos de corte directo bajo condición de borde CNL con el MED (en PFC ${ }^{2 \mathrm{D}}$ ). Para ello, empleó el método Bonded Particle Model (BPM), el cual permite 
generar materiales sintéticos (e.g., roca o hormigón), a partir de partículas circulares que representen el material en estudio. Así, en este trabajo se generó tanto la probeta como la geometría de la caja de corte mediante el uso de partículas circulares (ver la Figura 2.13(a)); a las partículas que representan la caja de corte les fue asignada propiedades resistentes 10 veces superiores a las de la roca, evitando así una posible rotura de estas partículas durante la realización del ensayo. Adicionalmente, para la asignación de propiedades a los contactos partícula-partícula en la junta, Cundal (2000) propuso una metodología llamada Bond Removal Approach (BRA), que consiste en eliminar la cohesión $(c=0)$ entre los contactos partícula-partícula que son atravesados por la línea imaginaria del plano de la junta roca-roca.

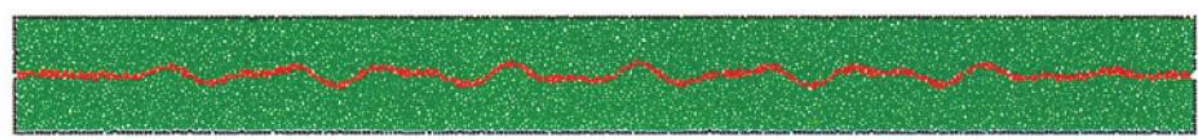

(a)

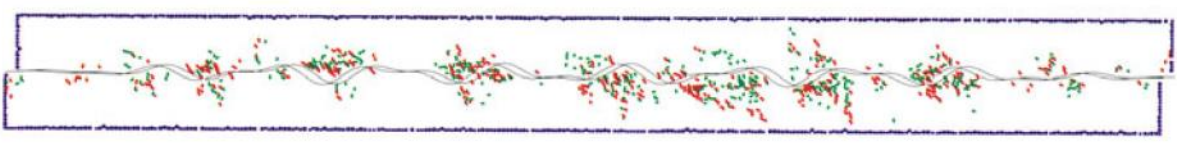

(b)

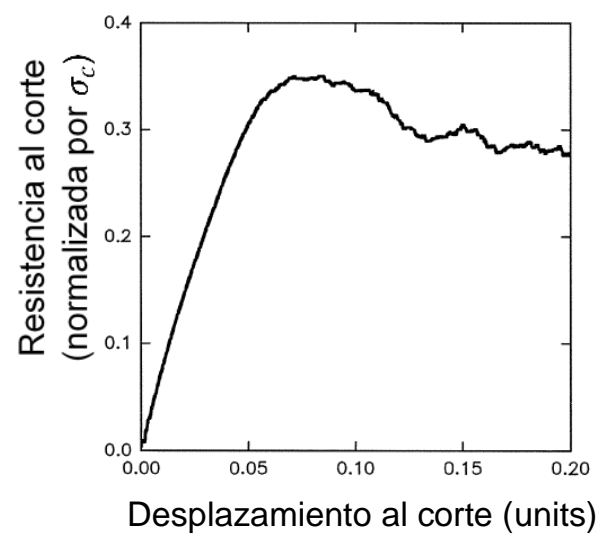

(c)

Figura 2.13. Modelo del ensayo de corte directo con MED ${ }^{2 D}$ en junta roca-roca para $c=0 \mathrm{y}$ condición CNL: (a) probeta del ensayo (las partículas de color verde, rojo y negro representan

la roca, la junta sin cohesión y la geometría de la probeta, respectivamente),(b) grietas generadas (el color verde y rojo representan las grietas de falla por corte y tracción, respectivamente), (c) curva de resistencia al corte normalizada por la resistencia a compresión uniaxial $\left(\sigma_{c}\right)$ de la roca vs el desplazamiento al corte (modificado de Cundal 2000).

Los resultados obtenidos por Cundal (2000) sugieren que los ensayos numéricos de corte directo realizados con el $\mathrm{MED}^{2 \mathrm{D}}$ son capaces de reproducir el comportamiento 
observado en juntas rugosas "reales", así como también identificar que el daño producido en las asperidades de la junta se incrementa con el aumento del esfuerzo normal aplicado durante el ensayo (ver la Figura 2.13(b)-(c)). Adicionalmente, Cundall (2000) sugiere que, si el modelo numérico es calibrado adecuadamente, su aplicación permite estudiar varios aspectos como el efecto de la rugosidad de la junta sobre su comportamiento resistente, o analizar el efecto escala del tamaño de la junta, entre otros.

\subsubsection{Wang et al. (2003)}

Para realizar un análisis de estabilidad de un talud en un macizo rocoso altamente fracturado en $\mathrm{PFC}^{2 \mathrm{D}}$, Wang et al. (2003) realizaron ensayos de corte directo con el $\operatorname{MED}^{2 D}$ (ver la Figura 2.14(a)), para calibrar de esta manera los microparámetros que han de asignarse a las juntas del macizo rocoso para el modelo numérico del talud. En el ensayo numérico con el $\mathrm{MED}^{2 \mathrm{D}}$, la roca y la junta fueron simuladas con el modelo constitutivo Linear Parallel Bond (LPB) de PFC ${ }^{2 \mathrm{D}}$, pero a los contactos partícula-partícula de la junta les fue asignado (usando la metodología BRA) (i) una rigidez de dos órdenes de magnitud menor a la empleada para la roca, (ii) sin cohesión $(c=0)$ y (iii) $\sin$ coeficiente de fricción $(\mu=0)$.

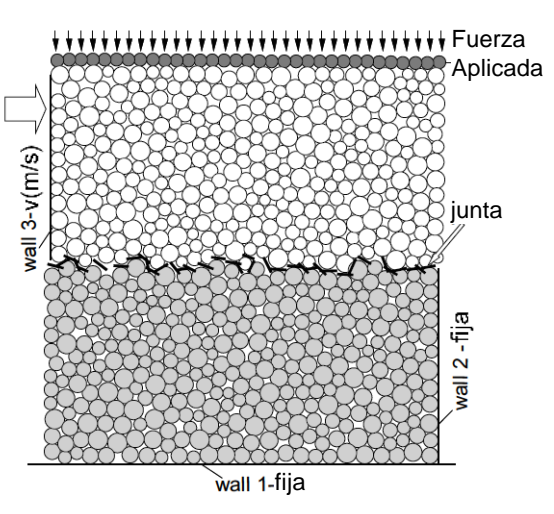

(a)

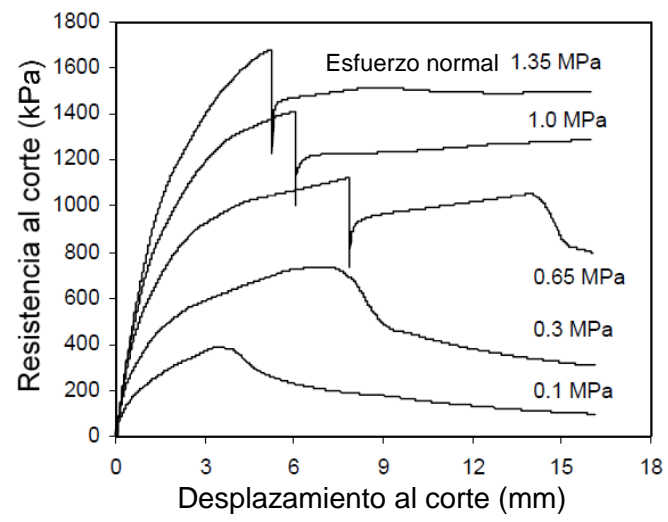

(b)

Figura 2.14. Modelo del ensayo de corte con $M E D^{2 D}$ en junta roca-roca, con $c=0$ y condición CNL: (a) probeta del ensayo después de alcanzar un desplazamiento al corte de $15 \mathrm{~mm}$, (b) curvas de resistencia al corte vs el desplazamiento al corte para diferentes valores de esfuerzo normal (modificado de Wang et al. 2003). 
Los resultados obtenidos con los modelos numéricos se presentan en la Figura 2.14(b).

Como puede observarse, una vez alcanzada la resistencia al corte pico, se producen caídas repentinas de la resistencia al corte; Wang et al. (2003) sugieren que dichas caídas pueden ser producto de la rotura de los contactos existentes entre las partículas de la junta y las partículas circundantes. Por otra parte, al no existir fricción ni cohesión ( $\mu=0$ y $c=0)$ en los contactos partícula-partícula de la junta, Wang et al. (2003) indicaron que la resistencia al corte movilizada durante el ensayo se debe a la rugosidad inherente de la propia junta -i.e., a la morfología no plana de la junta, en la cual las partículas se ven obligadas a pasar unas por encima las otras a lo largo de su geometría circunferencial-.

\subsubsection{Kusumi et al. (2005)}

Con el propósito de analizar el comportamiento al corte en juntas roca-roca, bajo condición de borde CNL y de rugosidad variable -según el sistema de clasificación Joint Roughness Coefficient (JRC) de 18-20-, Kusami et al. (2015) realizaron ensayos de corte directo experimentales y numéricos con PFC $2 \mathrm{D}$ (ver la Figura 2.15).
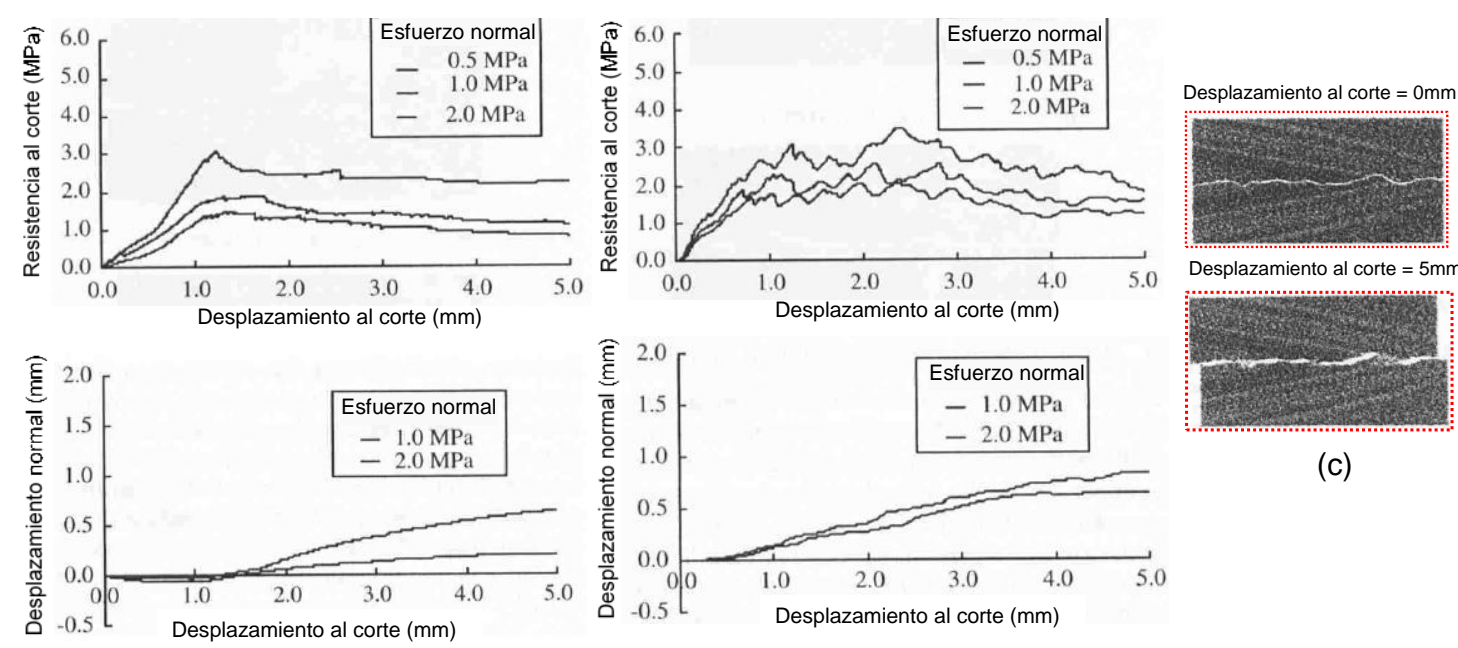

(c)

(a)

(b)

Figura 2.15. Ensayos de corte directo en juntas rugosas bajo condición CNL: (a) experimental, (b) MED ${ }^{2 D}$, (c) probeta al inicio y final del ensayo con MED2D (modificado de Kusumi et al. 2005). 
Los resultados muestran un comportamiento similar de incremento de resistencia al corte pico (obtenido para un desplazamiento al corte menor a $1.5 \mathrm{~mm}$ en los ensayos experimentales) con el aumento del esfuerzo normal aplicado para ambos modelos (experimental y numérico); no obstante, se observa un comportamiento fluctuante en las curvas obtenidas con el $\mathrm{MED}^{2 \mathrm{D}}$, alcanzando valores de resistencia al corte y de desplazamiento normal superiores a aquellos obtenidos experimentalmente para desplazamientos al corte superiores a 2 mm (ver la Figura 2.15); Kusumi et al. (2005) indican que esto puede deberse al coeficiente de amortiguamiento empleado en sus modelos DEM.

\subsubsection{Park y Song (2009)}

Park y Song (2009) estudiaron la capacidad que tienen los modelos numéricos elaborados en $\mathrm{PFC}^{3 \mathrm{D}}$ para reproducir el comportamiento al corte en juntas roca-roca, de rugosidad variable. Adicionalmente, realizaron un estudio paramétrico para determinar la influencia que tienen los microparámetros empleados en los modelos con el MED ${ }^{3 D}$ sobre el comportamiento resistente de juntas roca-roca. Para ello, asignaron el modelo LPB a todas las partículas que conforman la probeta del ensayo (utilizando la metodología BPM), y emplearon la metodología BRA para eliminar la cohesión $(c=0)$ y asignar un coeficiente de fricción nulo $(\mu=0)$ a todos los contactos partícula-partícula en el plano de la junta -i.e., a los contactos existentes entre la caja de corte superior e inferior del modelo numérico (ver la Figura 2.16(a))-.

Park y Song (2009) indican que los resultados obtenidos con los modelos numéricos son capaces de reproducir el proceso de rotura (ver la Figura 2.16(b)) y el comportamiento al corte (ver la Figura 2.16(c)) observado experimentalmente en juntas de este tipo. Por otra parte, con base al estudio paramétrico realizado, Park y Song (2009) sugieren que los microparámetros del modelo con el MED ${ }^{3 D}$ que más influyen sobre el comportamiento al corte de juntas roca-roca son el coeficiente de fricción, la 
rugosidad y, para el caso de juntas con cohesión, la relación entre la resistencia a tracción y la cohesión del contacto partícula-partícula en el plano de la junta.

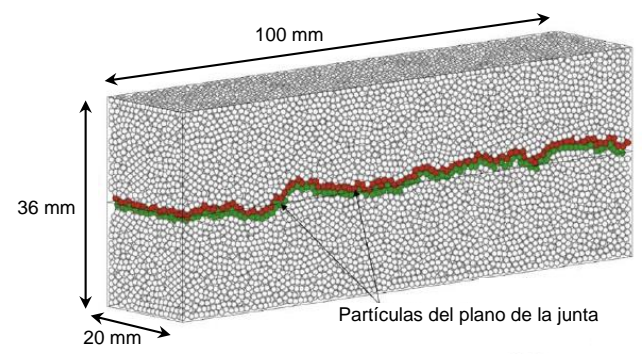

(a)

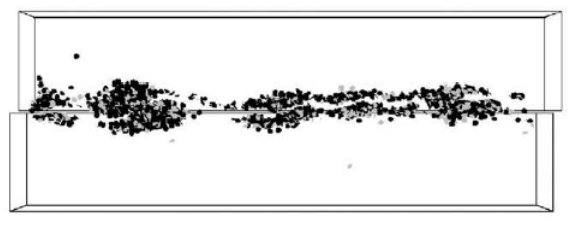

(b)

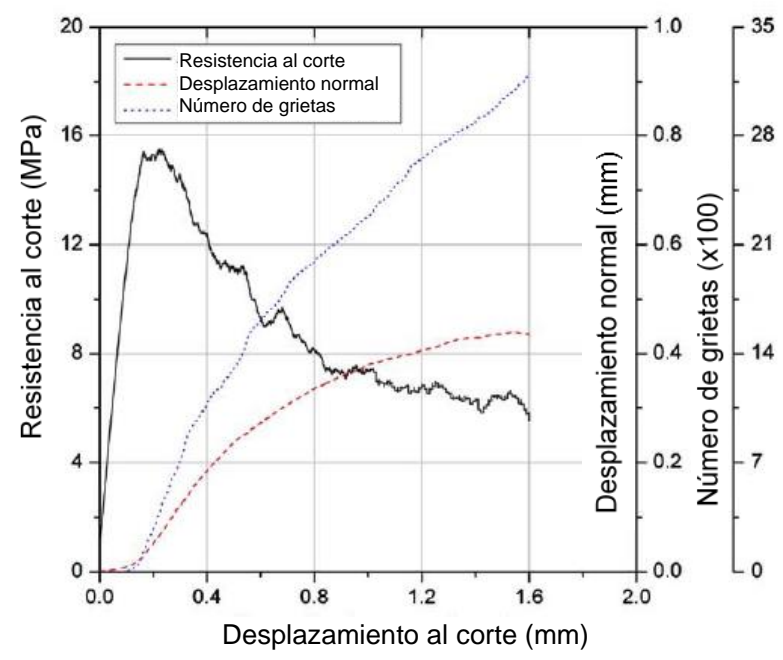

(c)

Figura 2.16. Ensayos de corte directo con MED ${ }^{3 D}$ en juntas rugosas bajo condición CNL $\left(\sigma_{n}=\right.$ $15 \mathrm{MPa}$ ): (a) probeta del modelo, (b) grietas generadas luego de un desplazamiento al corte de $1.6 \mathrm{~mm}$ (los discos de color negro y gris representan grietas por tracción y corte, respectivamente), (c) curva resistencia al corte-desplazamiento normal-número de grietas vs desplazamiento al corte (modificado de Park y Song 2009).

2.2.2.5 Asadi et al. $(2012,2013)$

Asadi et al. $(2012,2013)$ realizaron ensayos de corte directo en PFC ${ }^{2 D}$ con el objetivo de estudiar (numérica y experimentalmente), el comportamiento al corte y la degradación que ocurre en juntas roca-roca, sin cohesión, con perfiles de rugosidad triangular simétrica, sinusoidal y fractal, bajo condición de borde CNL (ver la Figura 2.17). La generación de la roca sintética fue realizada a través de la metodología BPM, asignando el modelo LPB al contacto partícula-partícula de la roca sintética, mientras que para los contactos partícula-partícula del plano de la junta, emplearon la metodología BRA con $c=0$ y $\mu=0$.

Los resultados numéricos indican que el mecanismo de rotura y el comportamiento al corte de juntas roca-roca dependen de la rugosidad de la junta y del esfuerzo normal aplicado durante el ensayo. Finalmente, Asadi et al. (2013) reportaron que los resultados 
de resistencia al corte pico numéricos resultaron superiores a los obtenidos experimentalmente.

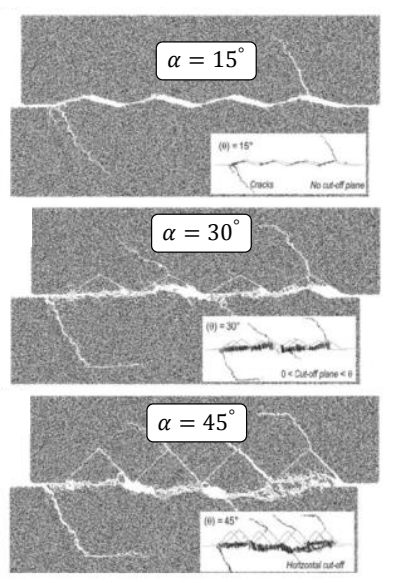

(a)
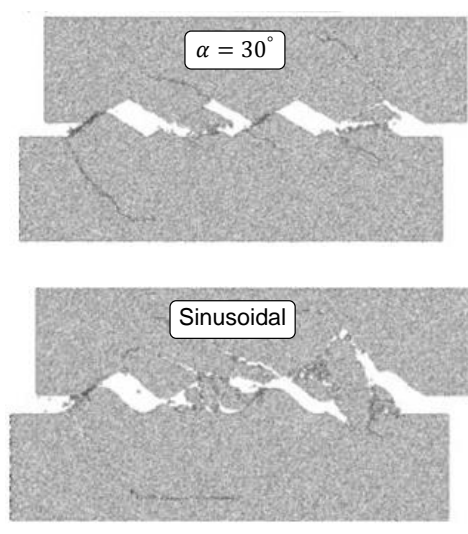

(c)

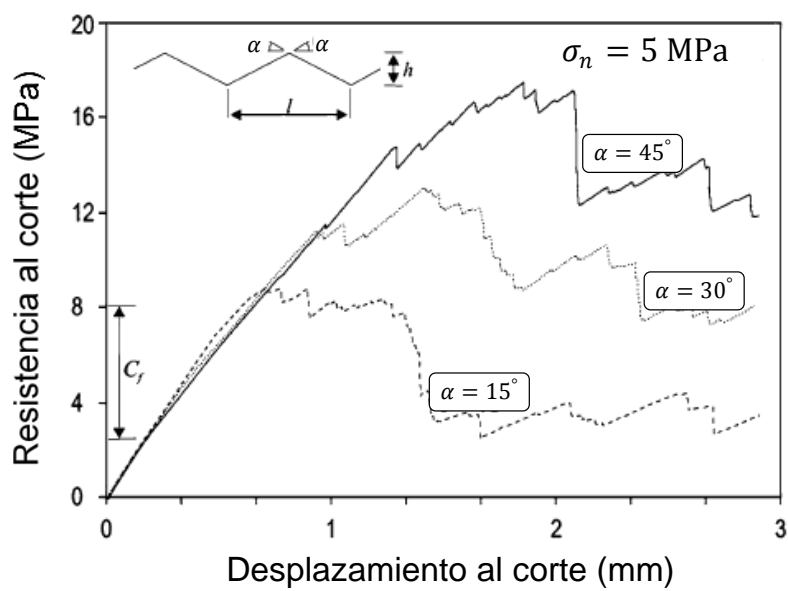

(b)

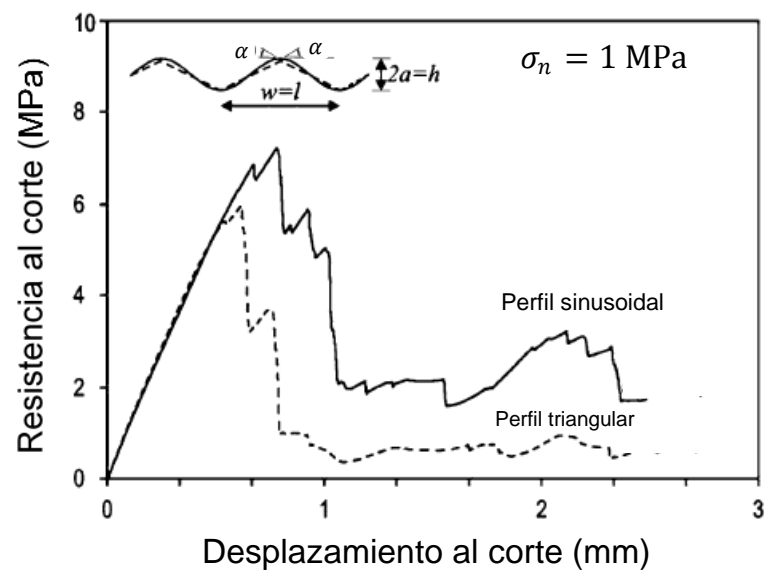

(d)

Figura 2.17. Ensayos de corte directo con MED ${ }^{2 D}$ en juntas rugosas bajo condición CNL: (a) probetas del modelo con rugosidad triangular simétrica para un desplazamiento al corte de 3 $\mathrm{mm}$, (b) curvas de resistencia al corte vs desplazamiento al corte para $\sigma_{n}=5 \mathrm{MPa}$, (c) probetas del modelo con rugosidad triangular y sinusoidal, (d) curvas de resistencia al corte vs desplazamiento al corte para $\sigma_{n}=1 \mathrm{MPa}$ (modificado de Asadi et al. 2002).

Los trabajos comentados anteriormente, estudiaron el comportamiento al corte con ensayos de corte directo en juntas roca-roca, a través de la metodología BRA mediante el uso del MED ${ }^{2 D \& 3 D}$. No obstante, dichas investigaciones reportaron que uno de los inconvenientes más importantes de esta metodología es la concentración de fuerzas "no reales", producto de la rugosidad inherente generada por las partículas del plano de la junta (y no por los parámetros resistentes en los contactos cuyas propiedades son $c=0$ y $\mu=0$ ). Esto trae como consecuencia la obtención de valores de resistencia al corte 
superiores (con el MED ${ }^{2 \mathrm{D} \& 3 \mathrm{D}}$ ) a los obtenidos mediante ensayos de laboratorio para las mismas condiciones del problema. En este sentido, a continuación, se presentan algunos estudios realizados para identificar y mejorar esta deficiencia.

\subsubsection{Bahaaddini et al. (2013b)}

Para estudiar la deficiencia de la metodología BRA propuesta por Cundall (2000), Bahaaddini et al. (2013b) realizaron modelos numéricos (en PFC ${ }^{2 \mathrm{D}}$ ) de ensayos de corte directo en juntas roca-roca, planas (ver la Figura 2.18(a)) y de rugosidad triangular simétrica (con $\alpha=15^{\circ}$ ), sin cohesión y bajo condición de borde CNL. La generación del material sintético (roca) de las cajas de corte superior e inferior se realizó de forma simultánea a través de la metodología BPM, con el modelo de contacto LPB.

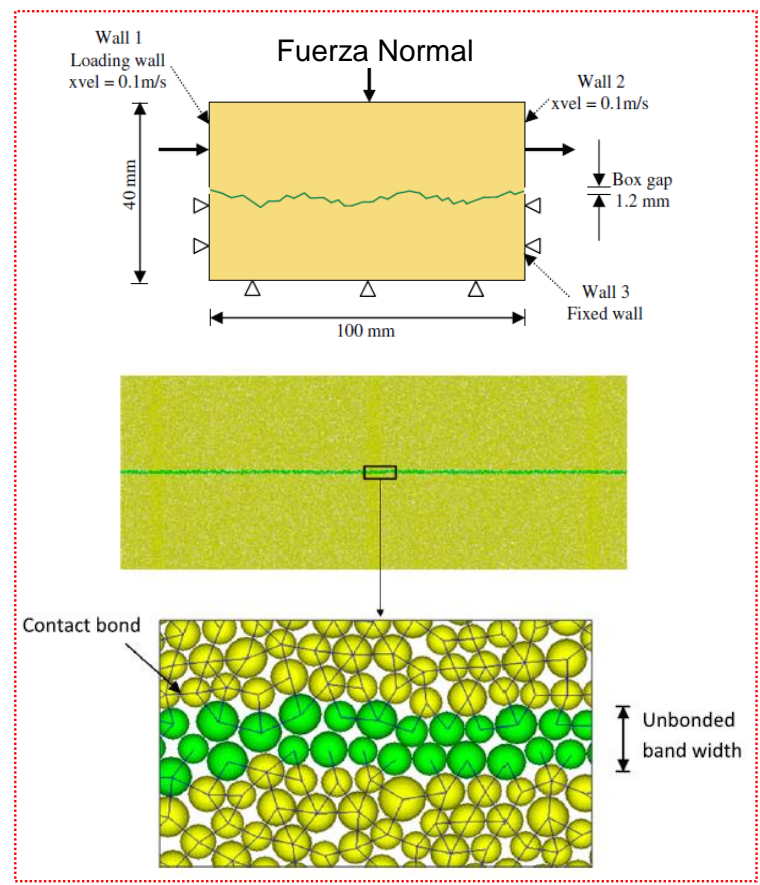

(a)
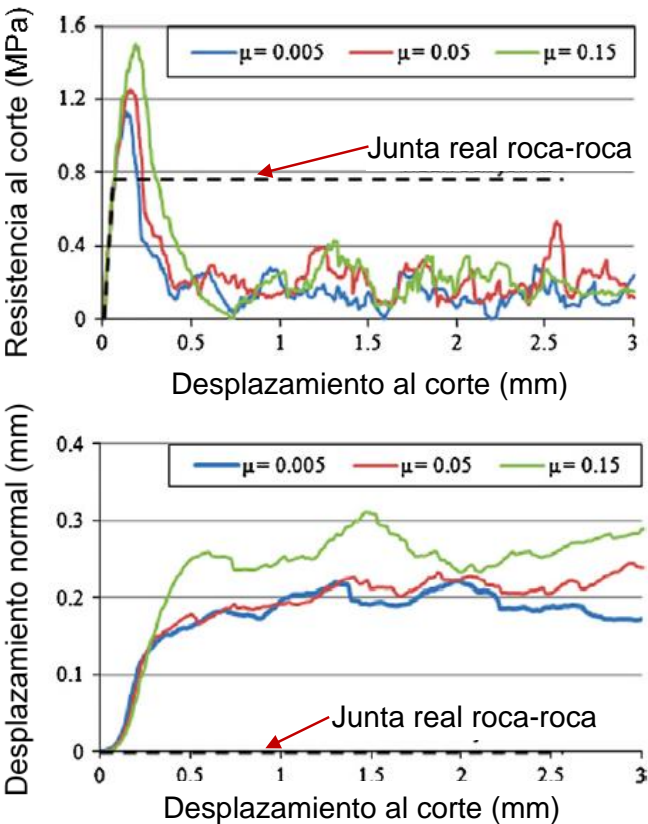

(b)

Figura 2.18. Ensayos de corte directo con MED ${ }^{2 \mathrm{D}}$ en juntas planas bajo condición CNL: (a) probeta del modelo, (b) curvas de resistencia al corte y desplazamiento normal vs desplazamiento al corte para $\sigma_{n}=1 \mathrm{MPa}$ (modificado de Bahaaddini et al. 2013b).

Los resultados obtenidos por Bahaaddini et al. (2013b) (ver la Figura 2.18(b)) reflejan un claro inconveniente de la técnica BRA para el estudio de resistencia al corte, debido a que la misma arroja valores de resistencia al corte muy superiores al valor esperado 
en juntas "reales". Adicionalmente, se observa que, una vez alcanzado el valor de resistencia al corte pico, se produce una caída abrupta de la resistencia al corte, incluso por debajo del valor de resistencia al corte residual de la junta "real". Por otra parte, se observa que con la técnica BRA se obtienen valores de desplazamientos en la dirección normal (dilatancia) mayores a aquellos obtenidos en el ensayo experimental, siendo esto un comportamiento no esperado en este tipo de juntas. Esta limitación de la técnica BRA fue llamado "interlocking" particles por Bahaaddini et al. (2013b).

Ante las deficiencias observadas en la técnica BRA, Ivars et al. (2008) propusieron un nuevo modelo de interacción entre partículas Ilamado Smooth-Joint Contact Model (SJCM, explicado en detalle en el Capítulo 3, Sección 3.2.2). A fin de analizar las potencialidades del SJCM, Bahaaddini et al. (2013b) realizaron ensayos de corte directo en $\mathrm{PFC}^{2 \mathrm{D}}$, en juntas (plana y triangular simétrica) roca-roca, bajo condición CNL. Los resultados obtenidos por Bahaaddini et al. (2013b) sugieren que este nuevo modelo de contacto reproduce en forma adecuada el comportamiento al corte para valores de desplazamiento al corte menores al diámetro mínimo $\left(D_{\min }\right)$ del tamaño de partículas empleados en el modelo con el MED. No obstante, para desplazamientos al corte superiores a $D_{\min }$, Bahaaddini et al. (2013b) observaron que se generaba nuevamente el problema debido a "interlocking" particles.

Para superar esta deficiencia del modelo SJCM, Bahaaddini et al. (2013b) propusieron la metología Shear Box Genesis (SBG) para simular ensayos de corte directo en PFC ${ }^{2 D}$ usando el modelo de contacto SJCM (esta metodología se presenta en detalle en el Capítulo 3 Sección 3.3.3). Los resultados obtenidos por Bahaaddini et al. (2013b) demuestran que la metodología SBG, junto con el modelo SCJM, puede reproducir en forma adecuada el comportamiento al corte en ensayos de corte directo en $\mathrm{PFC}{ }^{2 \mathrm{D}}$, en juntas roca-roca, sin cohesión bajo condición de borde CNL (ver la Figura 2.19). 


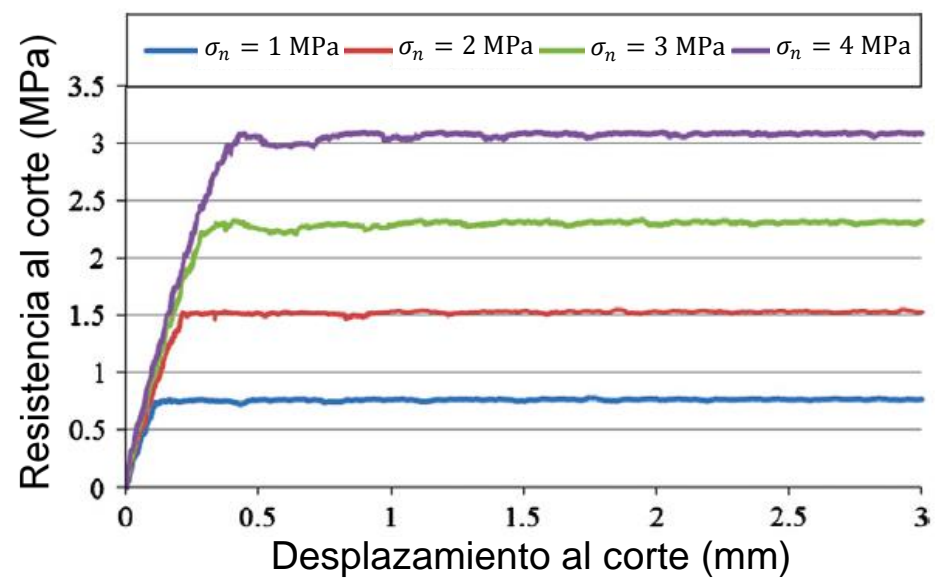

Figura 2.19. Curvas resistencia al corte vs desplazamiento al corte obtenidas con MED $2 \mathrm{D}$ empleando el SJCM y la metodología SBG, en probetas de junta plana roca-roca y con diferentes valores de $\sigma_{n}$ (condición CNL) (modificado de Bahaaddini et al. 2013b).

\subsubsection{Lambert y Coll (2014)}

Con el objetivo de estudiar la eficacia el modelo SJCM, Lambert y Coll (2014) realizaron ensayos de corte directo en $\mathrm{PFC}^{3 \mathrm{D}}$ para juntas de roca-roca, sin cohesión, de rugosidad variable (creada sintéticamente) y bajo condición CNL. Las dimensiones de la probeta y las curvas resistencia al corte - desplazamiento al corte obtenidas con el MED ${ }^{3 D}$ se presentan en la Figura 2.20. Lambert y Coll (2014) indican que los resultados numéricos con el modelo SJCM reproducen en forma adecuadamente el comportamiento elastoplástico esperado en este tipo de juntas.

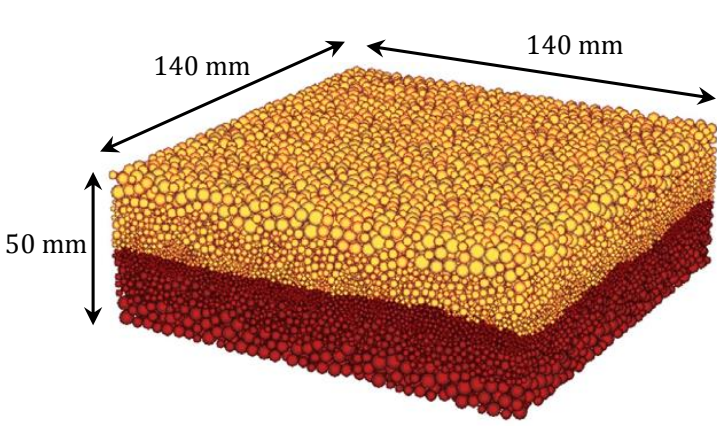

(a)

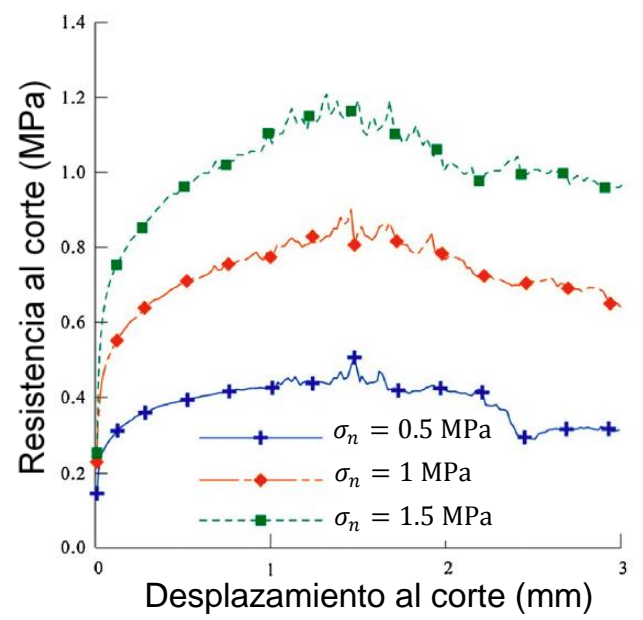

(b)

Figura 2.20. Ensayos de corte directo con MED ${ }^{3 D}$ en juntas rugosas bajo condición CNL: (a) probeta del modelo, (b) curvas de resistencia al corte vs desplazamiento al corte (modificado de Lambert y Coll 2014). 


\subsubsection{Bahaaddini et al. (2014)}

Bahaaddini et al. (2014) realizaron un estudio paramétrico a partir de ensayos de corte directo en $\mathrm{PFC}{ }^{2 \mathrm{D}}$, para analizar la influencia de los microparámetros del SJCM -i.e., la rigidez normal y de corte de los contactos smooth-joint $\left(k_{n S J}\right.$ y $\left.k_{s S J}\right)$; y su coeficiente de fricción $\left(\mu_{S J}\right)$ - sobre el comportamiento resistente de juntas roca-roca, de rugosidad variable (clasificada como JRC 10-12) y triangular simétrica $\alpha=15^{\circ}$. Los resultados de Bahaaddini et al. (2014) indican que el incremento de $k_{n s J}$ : (i) no tiene influencia sobre la resistencia al corte pico $\left(\tau_{p}\right)$ en juntas cuyo mecanismo de fallo es por deslizamiento (junta triangular con $\alpha=15^{\circ}$, ver la Figura 2.21(a)); no obstante, para juntas con mecanismo de fallo por cizallamiento de las asperidades (clasificadas como JRC 1012) se produce una disminución en la $\tau_{p}$ obtenida (ver la Figura 2.21(b)); (ii) produce un incremento en el valor del desplazamiento normal normalizado (i.e., desplazamiento normal/desplazamiento al corte, $\delta_{n} / \delta_{s}$ ) al alcanzar $\tau_{p}$ para ambas juntas (ver la Figura 2.21(a)-(b)), y (iii) se disminuye la rigidez al corte $\left(K_{S}\right)$ del ensayo (ver la Figura 2.21(a)(b)) con su correspondiente desplazamiento al corte pico (la rigidez al corte de ensayos de corte directo es representada por la pendiente de la curva antes de alcanzar la resistencia al corte pico).

En cuanto a la influencia de la rigidez de corte del smooth-joint $\left(k_{s} s J\right.$, Bahaaddini et al. (2014) indican que este parámetro no tiene un efecto considerable sobre la $\tau_{p}$ y sobre el desplazamiento normal normalizado de la junta triangular (con $\alpha=15^{\circ}$ ) al alcanzar la $\tau_{p}$, mientras que para la junta de JRC $10-12$, el incremento de $k_{s} s J$ aumenta la $\tau_{p} \mathrm{y}$ el desplazamiento normal normalizado. Adicionalmente, estos autores indican que un incremento en $k_{s S J}$ produce un incremento de la rigidez al corte $K_{S}$ para ambas tipologías de juntas. 
Por otra parte, Bahaaddini et al. (2014) reportan que un incremento en el coeficiente de fricción del smooth-joint $\left(\mu_{S J}\right)$ (i) aumenta el valor de resistencia al corte pico $\left(\tau_{p}\right)$ de ambas juntas, (ii) no produce efectos considerables sobre el desplazamiento normal normalizado de la junta triangular (con $\alpha=15^{\circ}$ ), e (iii) incrementa el desplazamiento normal normalizado para la junta con JRC 10-12.
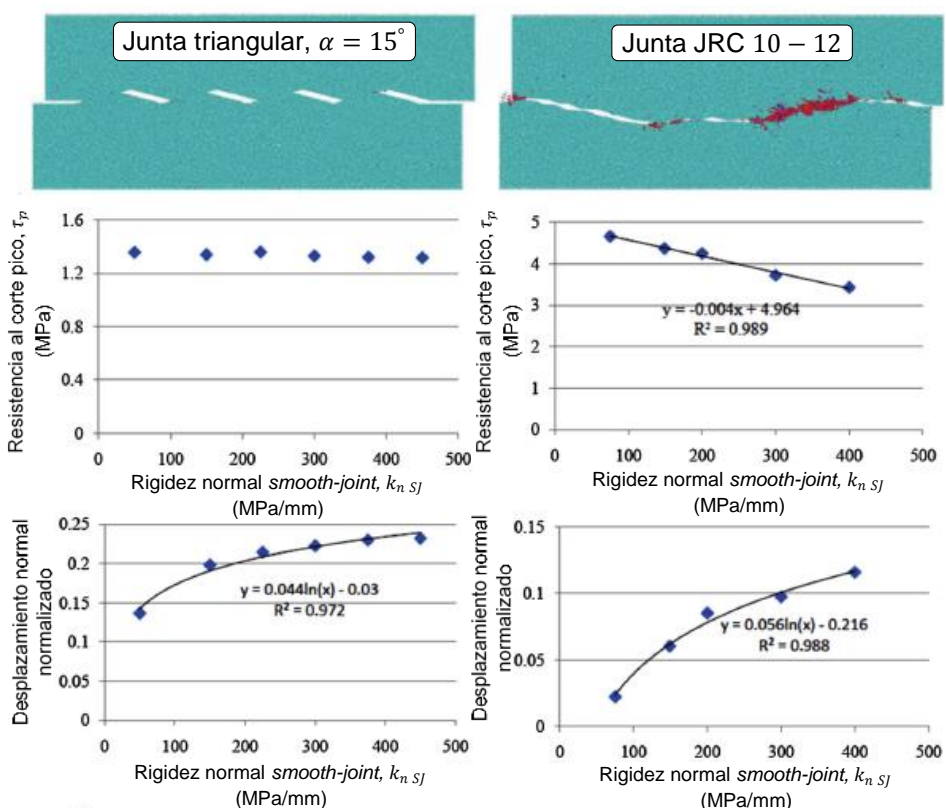

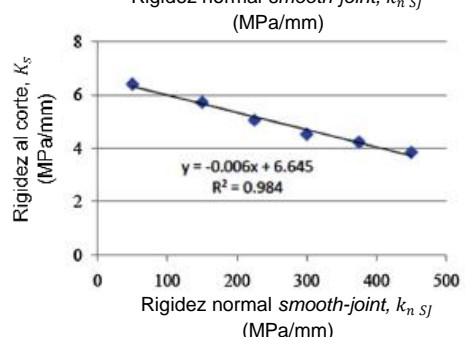

(a)

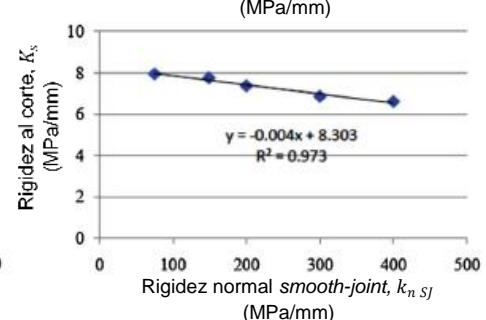

(b)

Figura 2.21. Influencia de la rigidez normal smooth-joint $\left(k_{n s J}\right)$ sobre (i) la resistencia al corte pico $\left(\tau_{p}\right)$, (ii) el desplazamiento normal normalizado por el desplazamiento al corte y (iii) la rigidez al corte $\left(K_{S}\right)$ de la junta: (a) junta triangular con $\alpha=15^{\circ}$,(b) junta con JRC 10-12 (modificado de Bahaaddini et al. 2014).

\subsubsection{Bahaaddini et al. (2016), Bahaaddini (2017)}

Con el fin de validar la metodología del Shear Box Genesis (SBG), Bahaaddini et al. (2016) realizaron ensayos de corte directo experimentales y numéricos (en PFC ${ }^{2 D}$ usando como modelo de contacto entre las partículas de la junta el SJCM), en juntas roca-roca, de morfología plana y triangular simétrica (con $\alpha=30^{\circ}$ ), bajo condición CNL. 
Los resultados obtenidos por estos investigadores indican que la metodología SBG, con una adecuada calibración de los microparámetros que representen el comportamiento macroscópico de la probeta y de la junta de roca, permiten reproducir en forma adecuada el comportamiento al corte en este tipo de ensayos (ver la Figura 2.22).

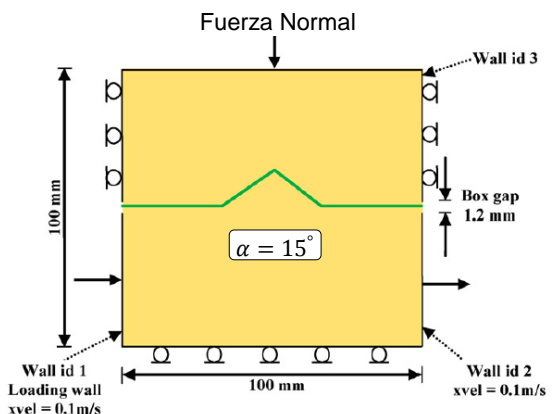

(a)

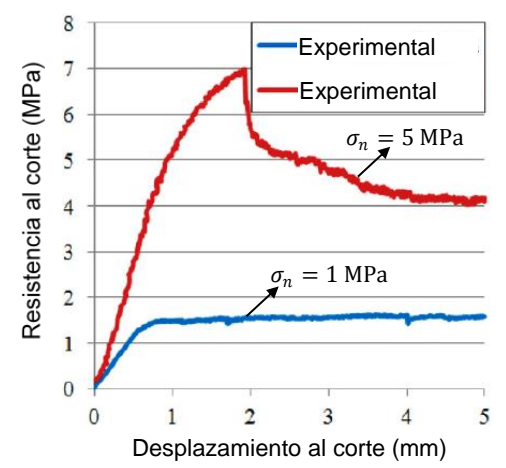

(c)

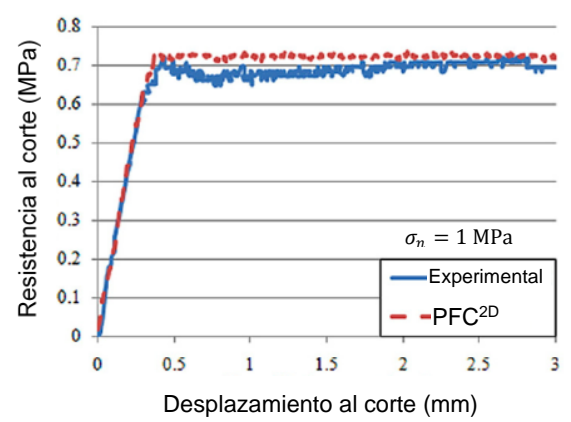

(b)

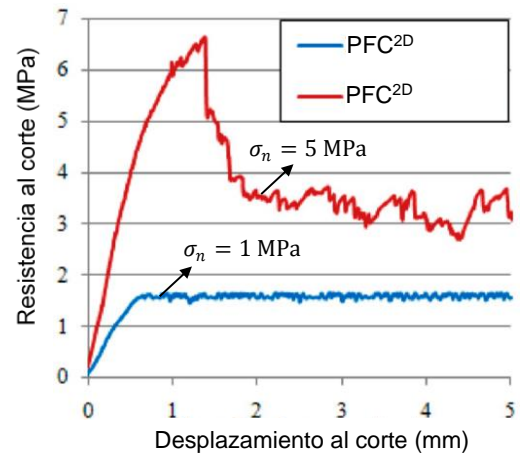

(d)

Figura 2.22. Ensayos de corte directo en junta plana y triangular bajo condición CNL (a) esquema del modelo, (b) curvas de resistencia al corte vs desplazamiento al corte para la junta plana, (c) y (d) curvas de resistencia al corte vs desplazamiento al corte para la junta triangular

(con $\alpha=30^{\circ}$ ) experimental y numérico con PFC ${ }^{2 \mathrm{D}}$ (modificado de Bahaaddini et al. 2016).

Más recientemente, Bahaaddini (2017) analizó el efecto de la condición de borde sobre la resistencia al corte obtenido en ensayos de corte directo experimentales y numéricos. En particular, Bahaaddini (2017) estudió el efecto que tiene la separación existente entre la caja de corte superior e inferior de la probeta, sobre la resistencia al corte de la junta. Para ello, realizó ensayos de corte experimentales en juntas de roca sintéticas, con morfología triangular simétrica ( $\operatorname{con} \alpha=20^{\circ}$ y $30^{\circ}$ ) y bajo condición CNL. En los ensayos experimentales, Bahaaddini (2017) notó que el proceso de rotura en el ensayo de la junta roca-roca con $\alpha=30^{\circ}$ y $\sigma_{n}=7 \mathrm{MPa}$ (ver la Figura 2.23(b)-(c)), se producía por el inicio de grietas desde el borde de la caja de corte de la probeta hacia el interior, en 
lugar de producirse por concentración de esfuerzo y rotura de las asperidades de la junta. No obstante, para la probeta con $\alpha=30^{\circ}$, este comportamiento no se observó porque su modo de fallo ocurrió por deslizamiento (ver la Figura 2.23(a)).

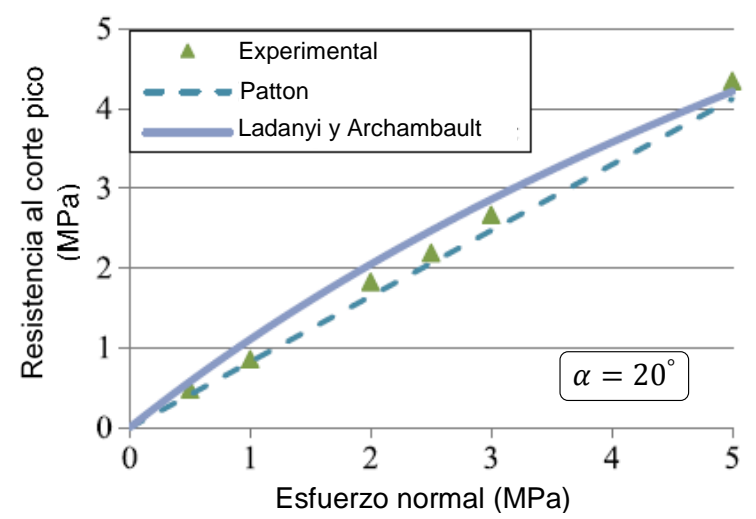

(a)

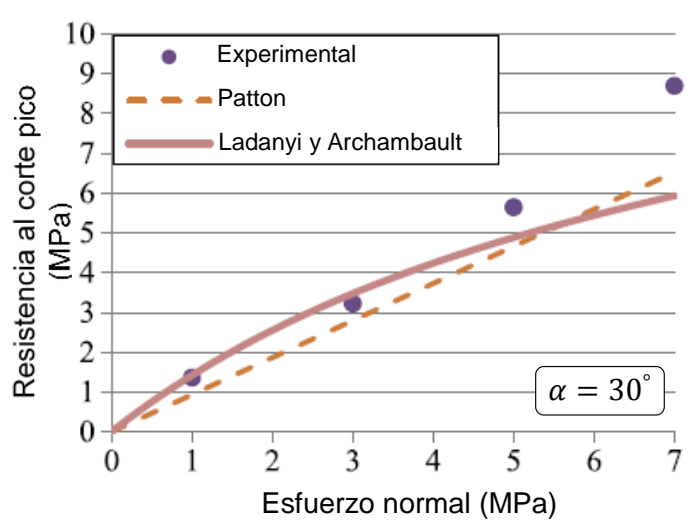

(b)

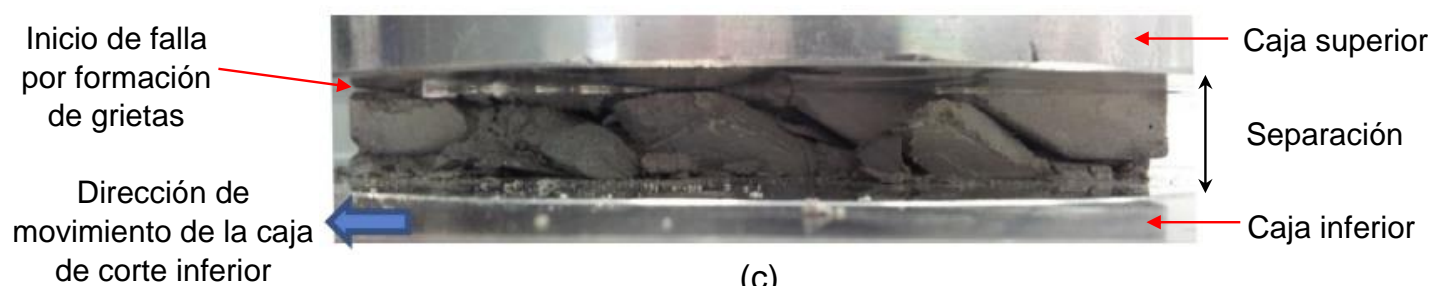

(c)

Figura 2.23. Ensayos de corte directo en juntas de roca-roca, bajo condición CNL: (a) junta con $\alpha=20^{\circ}$, (b) junta con $\alpha=30^{\circ}$, (c) efecto de la condición de borde en el modo de fallo de la probeta con junta con $\alpha=30^{\circ}$ y $\sigma_{n}=7 \mathrm{MPa}$ (modificado de Bahaaddini 2017).

Con base en el comportamiento observado en los ensayos experimentales, Bahaaddini (2017) realizó ensayos de corte directo en $\mathrm{PFC}^{2 \mathrm{D}}$ para la junta roca-roca con $\alpha=30^{\circ}$, para evaluar así la influencia que tiene la separación entre la caja de corte superior e inferior sobre el comportamiento al corte de este tipo de ensayos. Los resultados del modelo $\mathrm{DEM}^{2 \mathrm{D}}$ (ver la Figura 2.24(b)-(c)) demuestran que la separación existente entre la caja de corte superior e inferior del ensayo tienen influencia sobre la resistencia al corte del modelo y su mecanismo de fallo -i.e., se observa que para valores de separación pequeños, se obtiene una distribución de esfuerzos y propagación de grietas más "real" a lo largo de las asperidades de la junta. 


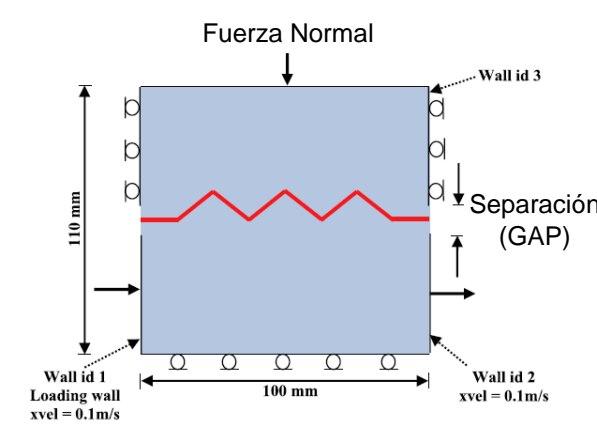

(a)

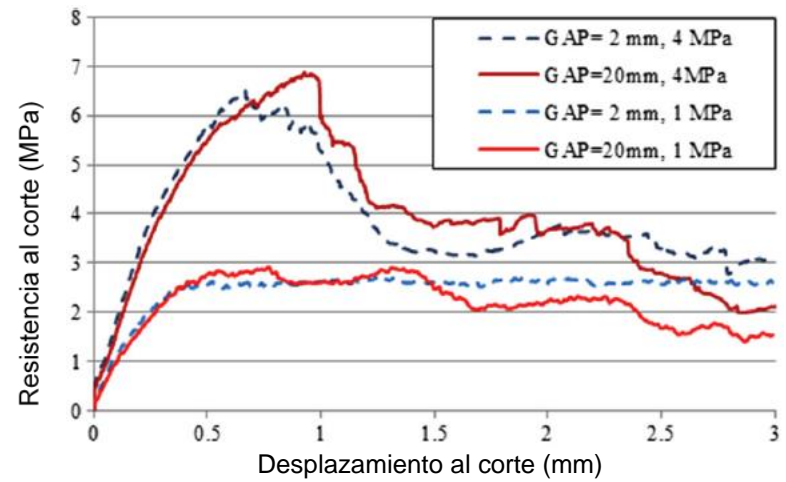

(b)

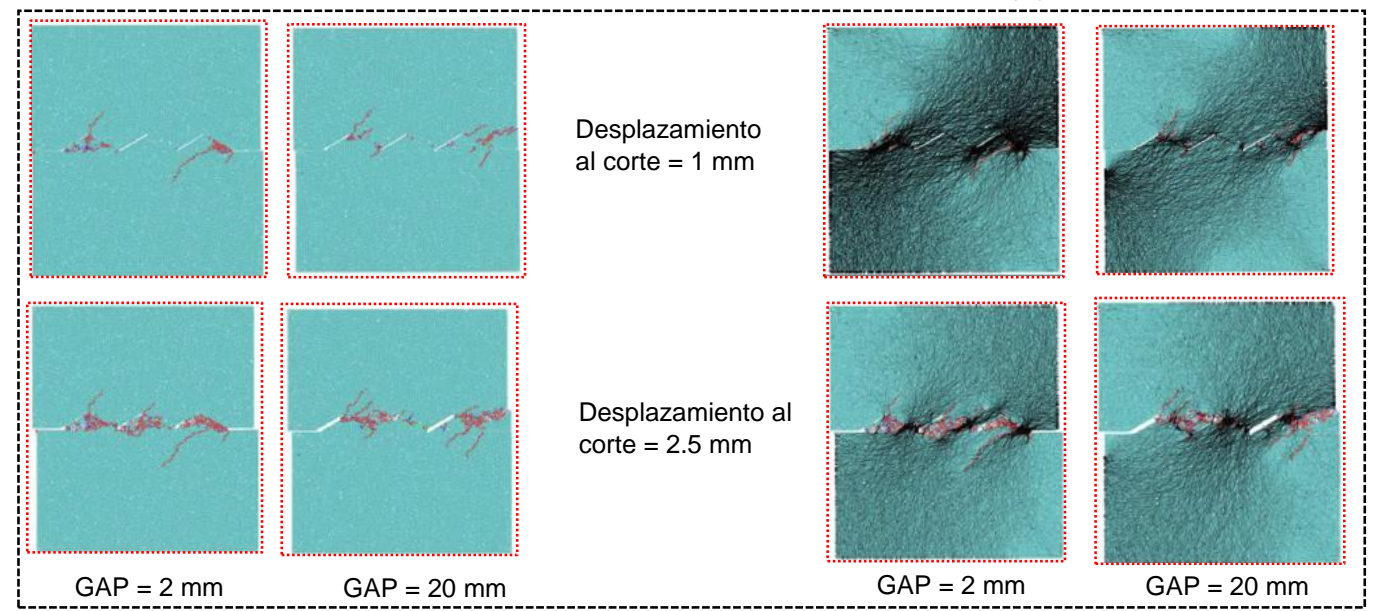

(c)

Figura 2.24. Ensayos de corte directo en juntas de roca-roca con MED ${ }^{2 D}$, bajo condición CNL:

(a) esquema del modelo con junta triangular, (b) curvas de resistencia al corte vs desplazamiento al corte para $\alpha=30^{\circ}$, (c) grietas (líneas rojas) y distribución de esfuerzos (líneas negras) para la probeta con $\sigma_{n}=4 \mathrm{MPa}$ (modificado de Bahaaddini 2017).

\subsubsection{Shang et al. (2018)}

Para analizar la influencia de la condición de borde (CNL y CNS) sobre la resistencia al corte en juntas incipientes -i.e., no persistentes- en rocas, Shang et al. (2018) realizaron ensayos de corte directo en $\mathrm{PFC}^{3 \mathrm{D}}$, usando como modelo de contacto entre las partículas de la junta el modelo SJCM, sin cohesión y con condición de borde CNL y CNS (ver la Figura 2.25(a)-(b)). Sus resultados demuestran que la calibración adecuada de los parámetros del modelo SJCM permite reproducir adecuadamente el comportamiento al corte en juntas roca-roca planas (ver la Figura 2.25(c)); también demostraron que la condición de borde influye notablemente sobre la resistencia al corte de juntas no persistentes en roca, siendo mayor la resistencia al corte pico en ensayos bajo condición CNS que en CNL (ver la Figura 2.25(d)). 


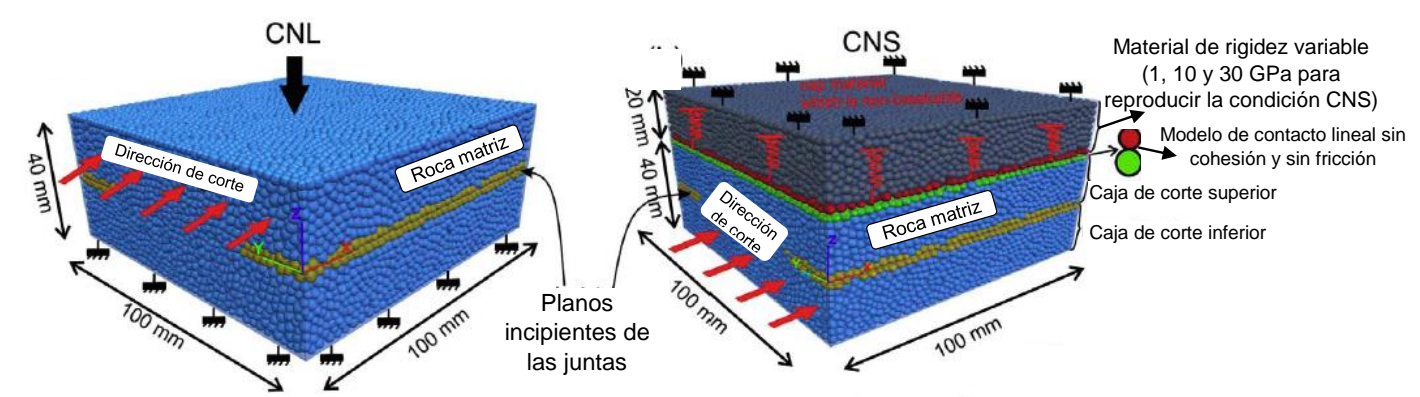

(a)

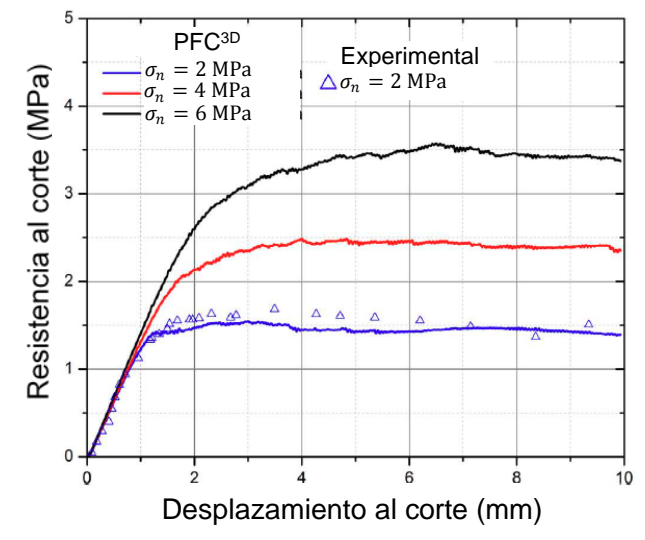

(c) (b)

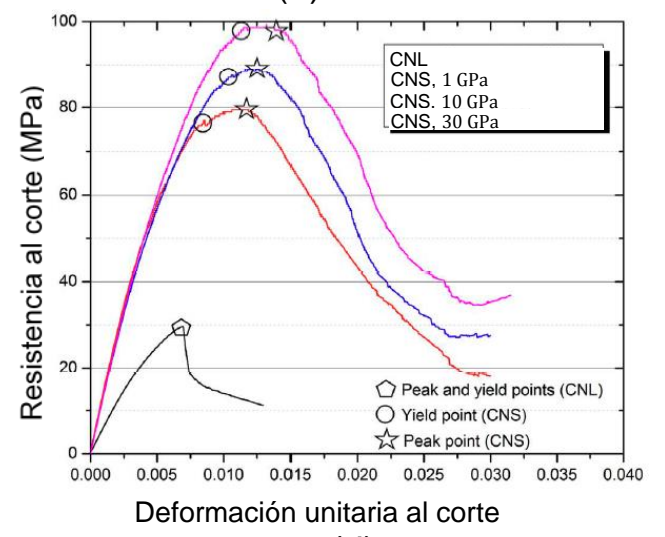

(d)

Figura 2.25. Ensayos de corte directo en juntas de roca: (a) condición CNL, (b) condición CNS, (c) curvas de resistencia al corte vs desplazamiento al corte con $\mathrm{PFC}^{3 \mathrm{D}}$ y experimental para juntas planas con diferentes valores de $\sigma_{n}$ y condición CNL, (d) curvas de resistencia al corte vs deformación al corte con PFC ${ }^{3 \mathrm{D}}$ de juntas de roca incipientes bajo condición CNL $\left(\sigma_{n}=20\right.$ $\mathrm{MPa}$ ) y CNS con $\sigma_{n o}=20 \mathrm{MPa}$ (modificado de Shang et al. 2018).

Con base a los resultados de ensayos de laboratorio e in-situ descritos previamente, se evidencia que los principales factores que afectan al comportamiento al corte de juntas roca-roca y roca-hormigón son: (i) las propiedades mecánicas de los materiales en contacto, (ii) la rugosidad en el plano de la junta, (iii) el esfuerzo normal actuante sobre la interfaz, (iv) la condición de borde aplicada (CNL o CNS), (v) la existencia y porcentaje de cohesión en la interfaz, y (vi) la presencia de material de relleno en el plano de la junta y su espesor.

\subsubsection{Ensayos de carga sobre pilotes en el laboratorio}

El estudio del efecto de la rugosidad sobre el comportamiento de pilotes empotrados en roca también se ha realizado empleando pruebas de carga en el laboratorio sobre pilotes a escala reducida. A continuación, se presentan los resultados de algunas de estas investigaciones. 


\subsubsection{Pells et al. (1980)}

Para analizar el efecto de la rugosidad y de las condiciones de confinamiento (aplicado mediante anillos de acero) en pilotes, Pells et al. (1980) realizaron 39 pruebas de carga sobre pilotes excavados en cubos roca (arenisca, $\sigma_{c}=17.7 \mathrm{MPa}$ ) en el laboratorio. Los pilotes tenían diámetros de hasta $250 \mathrm{~mm}$, fabricados con hormigón de $\sigma_{c}=38 \mathrm{MPa}$ (a los 28 días) y de diferente nivel de rugosidad. En la Figura 2.26 se presentan los resultados obtenidos en algunos de los pilotes.

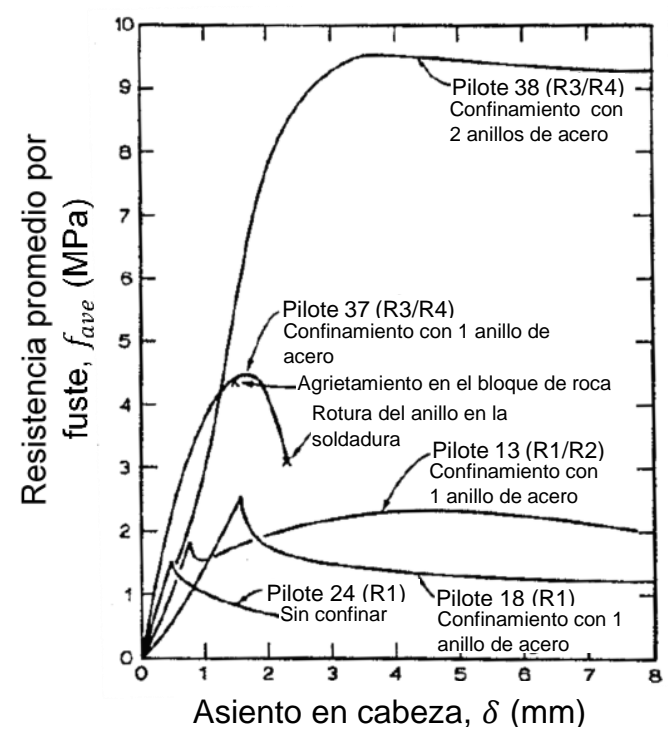

Figura 2.26 Pruebas de carga en el laboratorio sobre pilotes excavados en roca con diferente rugosidad (modificado de Pells et al. 1980).

Las denominaciones R1 (liso), R2, R3 y R4 (muy rugoso), representan el nivel rugosidad según el sistema de clasificación de rugosidades propuesto por estos mismos autores. Como puede observarse en la Figura 2.26, hay una clara influencia del nivel de rugosidad en el incremento de la resistencia promedio por fuste, tal y como se demuestra en los resultados para los ensayos con las mismas condiciones de confinamiento pero con diferente rugosidad (e.g., pilotes 13 y 37 de la Figura 2.26). Por otra parte, se observa como para dos ensayos con el mismo grado de rugosidad (e.g., pilotes 37 y 38 de la Figura 2.26), la resistencia por fuste se incrementa con el aumento 
del confinamiento, siendo éste otro factor importante en la respuesta de pilotes excavados en roca.

\subsubsection{Khan y Amadei (1993)}

Con el propósito de estudiar el efecto de la rugosidad y de la relación entre el módulo de Young del hormigón $\left(E_{c}\right)$ y de la roca $\left(E_{r}\right)$, Khan y Amadei (1993) realizaron 12 ensayos de carga en el laboratorio sobre pilotes de diámetro $(D)$ y longitud $(L)$ de 6.34 cm y $25.4 \mathrm{~cm}$, respectivamente (ver la Figura 2.27(a)). Los pilotes fueron elaborados con hormigón ( $\sigma_{c}=27.5 \mathrm{MPa}$ ) y se fabricaron con rugosidad triangular simétrica de diferente ángulo base $\left(0^{\circ}, 18^{\circ}\right.$ y $36^{\circ}$, ver la Figura $\left.2.27(\mathrm{~b})\right)$. La roca artificial fue preparada teniendo relaciones $E_{c} / E_{r}$ con valores de $4,7,12$ y 24 . Los resultados, presentados en la Figura 2.27(b), reflejan una clara influencia tanto de la rugosidad como de la relación $E_{c} / E_{r}$, sobre el comportamiento de pilotes empotrados en roca, cuyo efecto parece ser más notorio con la disminución de $E_{c} / E_{r}$.

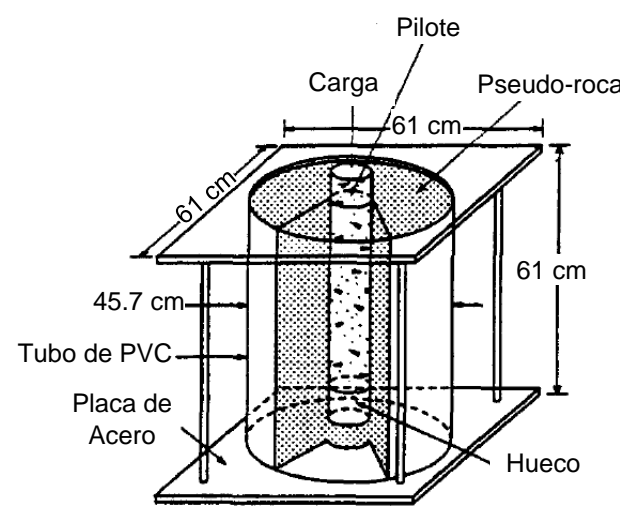

(a)

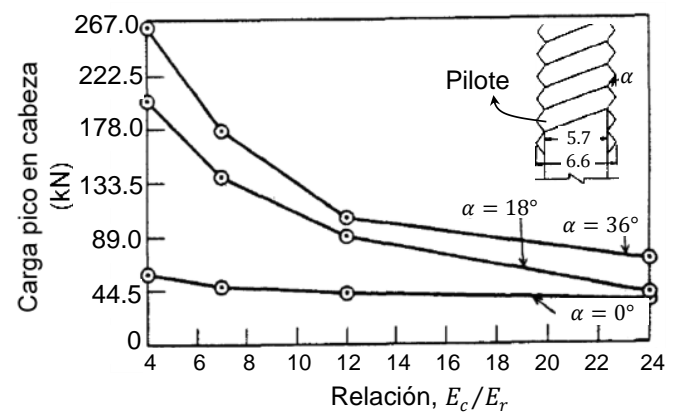

(b)

Figura 2.27 Pruebas de carga a pequeña escala en el laboratorio sobre pilotes excavados en roca: (a) esquema y geometría del ensayo, (b) carga pico en cabeza vs la relación $E_{c} / E_{r}$ (modificado de Khan y Amadei 1993).

\subsubsection{Benmokrane et al. (1994)}

Benmokrane et al. (1994) realizaron 6 ensayos sobre pilotes rugosos excavados en roca con el objetivo de evaluar su comportamiento ante la variación del material al alrededor del pilote. Para ello, prepararon tres categorías de pseudo-roca o roca artificial con 
resistencia a compresión simple de 4.7, 22 y $42.9 \mathrm{MPa}$, mientras que, para el pilote, emplearon el mismo material de la pseudo-roca con $\sigma_{c}=22 \mathrm{MPa}$. De esta manera, se consideran tres opciones en las cuales el pilote es más, igual o menos resistente que la roca circundante. Todos los ensayos se realizaron con el mismo nivel de rugosidad. La geometría del ensayo y los resultados obtenidos se presentan en la Figura 2.28. Como se observa, esta investigación también refleja la influencia de la roca circundante sobre la resistencia movilizada de los pilotes, alcanzándose los valores máximos y mínimos para los modelos en los que la roca circundante tiene igual y menor resistencia, respectivamente, a la resistencia del pilote (ver la Figura 2.28(c)). Adicionalmente, se evidencia la influencia que tiene el diámetro en pilotes rugosos sobre los valores obtenidos de resistencia promedio por fuste, siendo mayores en los modelos de pilotes con menor diámetro (ver la Figura 2.28(c)).

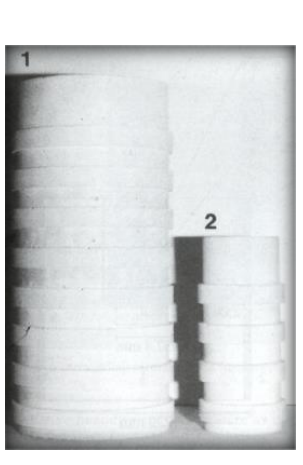

(a)

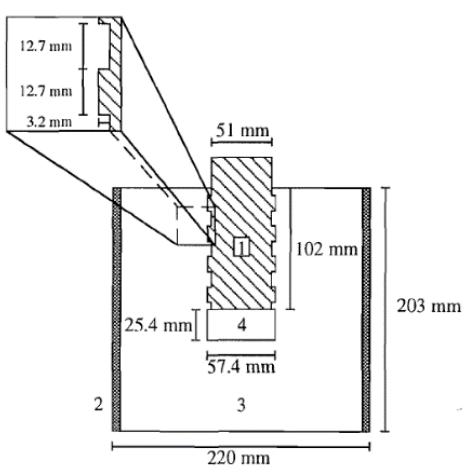

(b)

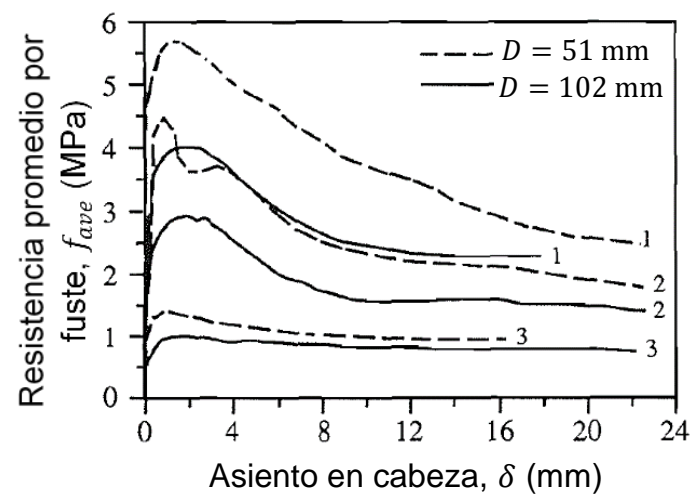

(c)

Figura 2.28. Pruebas de carga a pequeña escala en el laboratorio sobre pilotes excavados en roca: (a) geometría de los pilotes, modelo 1 con $D=102 \mathrm{~mm}$ y $L=203 \mathrm{~mm}$, modelo 2 con $D=$ $51 \mathrm{~mm}$ y $L=102 \mathrm{~mm}$, (b) esquema y geometría del ensayo, (c) resistencia promedio por fuste vs asiento en cabeza del pilote (1, 2 y 3 representan la resistencia de la roca circundante de 22 , 42.9 y $4.7 \mathrm{MPa}$, respectivamente) (modificado de Benmokrane et al. 1994)

\subsubsection{Dai et al. (2017)}

Recientemente, Dai et al. (2017) realizaron una batería de 10 ensayos a pequeña escala sobre pilotes, para analizar el efecto de la rugosidad sobre la resistencia por fuste y punta (ver la Figura 2.29(a)). A efectos de este trabajo, solo se muestran los resultados de los modelos en los que se considera solo la resistencia por fuste -i.e., con un hueco 
o un material muy débil en la punta del pilote-. La roca fue preparada con una mezcla de arena, cemento, yeso y agua cuyo valor de $\sigma_{c}$ a los 20 días fue de $6 \mathrm{MPa}$. Los pilotes fueron construidos con plexiglás sólido $\left(\sigma_{c}=100 \mathrm{MPa}\right)$ y con diferentes grados de rugosidad (ver la Figura 2.29(b)). Para los modelos que solo consideran la resistencia por fuste, se pegó un trozo de espuma de poliestireno a la punta del pilote. Los resultados que se presentan en la Figura 2.29(c) muestran que hay un incremento en la capacidad de carga en los pilotes rugosos respecto a los lisos; sin embargo, se observa que pareciera existir un límite en el cual, a pesar de incrementar la rugosidad en la interfaz roca-pilote, no se evidencia gran efecto sobre la capacidad de carga de los pilotes.

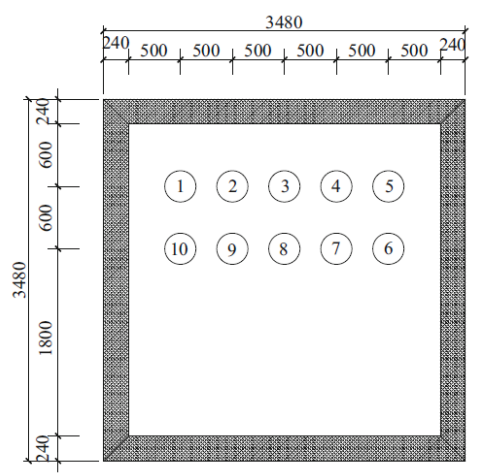

Vista en Planta

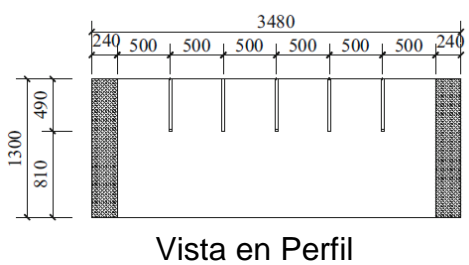

(a)

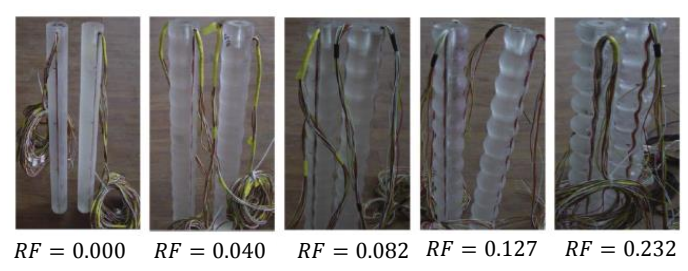

(b)

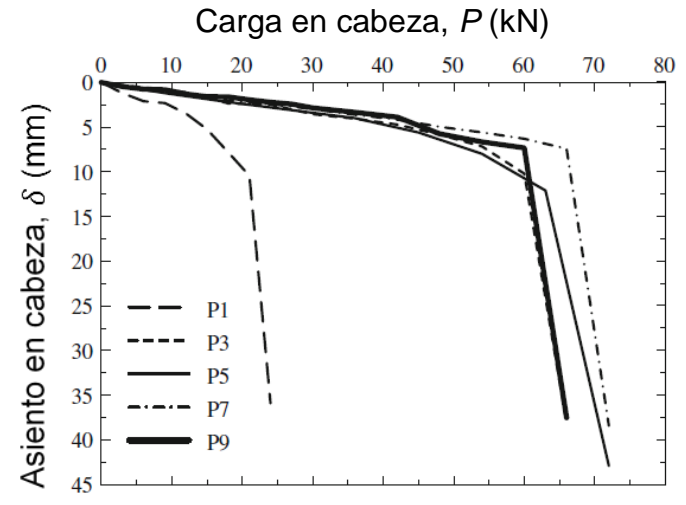

(c)

Figura 2.29. Pruebas de carga a pequeña escala en el laboratorio sobre pilotes excavados en roca: (a) configuración geométrica del ensayo (unidades en $\mathrm{mm}$ ), (b) geometría de los pilotes

$D=50 \mathrm{~mm}$ y $L=450 \mathrm{~mm}(R F$ es el coeficiente de rugosidad propuesto por Horvath et al. 1983), (c) carga en cabeza vs asiento en cabeza del pilote para los modelos de solo resistencia por fuste (modificado de Dai et al. 2017).

\subsection{Modelos a gran escala}

Como ya se describió en Capítulo 1, el objetivo principal de esta investigación es analizar el efecto que tiene la rugosidad sobre la resistencia por fuste de pilotes 
empotrados en roca. Por tal razón, el estado del arte que se describirá en esta sección, está enmarcado en aquellas investigaciones en las que se estudió el comportamiento de pilotes empotrados en roca, con principal énfasis en el comportamiento de la resistencia movilizada por fuste.

Los resultados experimentales y numéricos presentados en la Sección 2.2, evidencian la gran influencia que tiene la rugosidad, en juntas roca-roca o roca-hormigón, sobre el comportamiento al corte en la interfaz de estos materiales. Con base a este comportamiento, se han realizado grandes esfuerzos por diversos investigadores (e.g., Williams 1980; Horvath et al. 1983), para estudiar el efecto de la rugosidad sobre el comportamiento de pilotes empotrados en roca. En las secciones siguientes, se presentan algunos resultados de ensayos de carga realizados sobre pilotes empotrados en roca, en los cuales se considera la rugosidad en la interfaz roca-pilote.

\subsubsection{Ensayos de carga sobre pilotes en campo}

\subsubsection{Pells et al. (1980)}

Pells et al. (1980), realizaron una serie de pruebas de carga con el fin de estudiar el efecto de la rugosidad, de la longitud de empotramiento, etc., sobre el comportamiento resistente de pilotes empotrados en roca. Para ello, realizaron 9 ensayos en pilotes de pequeño diámetro (hasta $160 \mathrm{~mm}$ ) con longitudes de hasta $255 \mathrm{~mm}$; y 18 ensayos en pilotes con un mayor diámetro (hasta $710 \mathrm{~mm}$ ) con longitudes de hasta $1370 \mathrm{~mm}$. Los ensayos se llevaron a cabo en las areniscas en Sídney (Australia), cuya resistencia a la compresión simple $\left(\sigma_{c}\right)$ varía en un rango de 5-40 $\mathrm{MPa}$. Se emplearon pilotes fabricados de hormigón ( $\sigma_{c}=28-42 \mathrm{MPa}$ ). En la Figura 2.30(a) se presentan algunos resultados de las pruebas de carga realizadas por Pells et al. (1980) para pilotes con distinto nivel de rugosidad (R1, R2 y R3, son las denominaciones en función del sistema de rugosidades propuesto por Pells et al. 1980, ver la Tabla 2.1). 


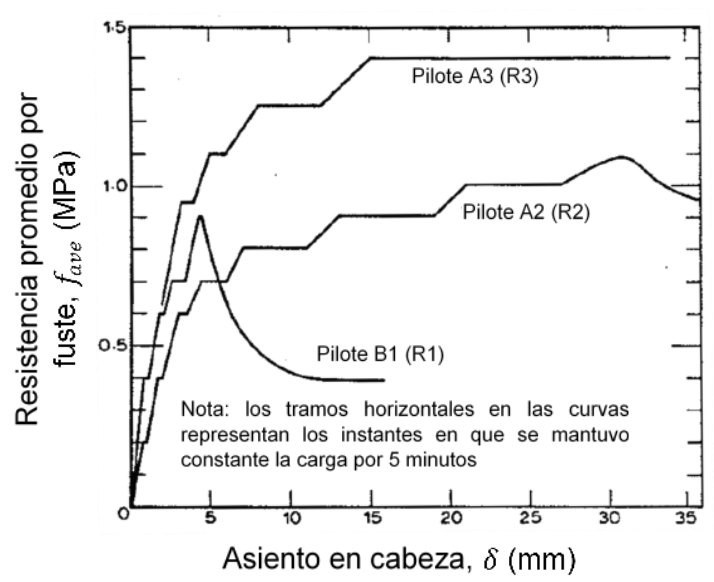

(a)

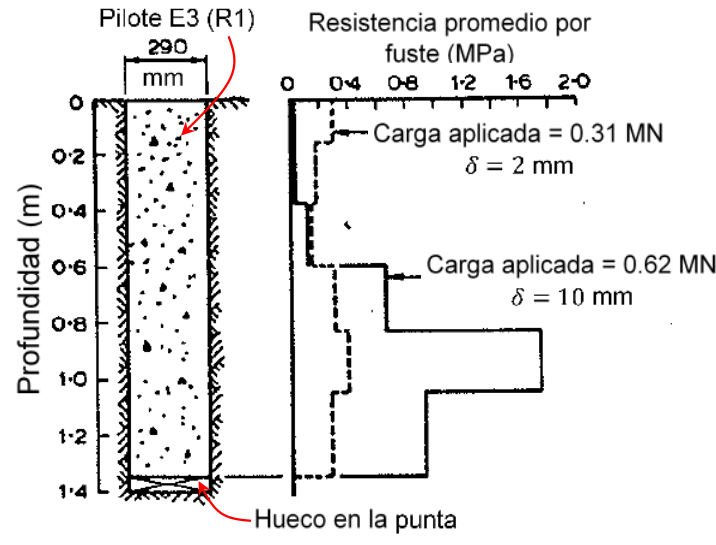

(b)

Figura 2.30. Pruebas de carga sobre pilotes excavados en arenisca con rugosidad variable en la interfaz roca-pilote: (a) resistencia promedio por fuste vs asiento en cabeza del pilote, (b) resistencia promedio por fuste vs profundidad para dos intervalos de carga aplicada en cabeza del pilote (modificado de Pells et al. 1980).

Tabla 2.1. Sistema de clasificación de rugosidades (Pells et al. 1980)

\begin{tabular}{cr}
\hline Clase de rugosidad & Descripción \\
\hline R1 & $\begin{array}{r}\text { Pared lisa, con surcos de profundidad menor a } 1 \mathrm{~mm} \\
\text { R2 }\end{array}$ \\
R3 & $\begin{array}{r}\text { Pared rugosa con surcos de profundidad comprendida entre } 1-4 \mathrm{~mm}, \\
\text { con altura mayor a } 2 \mathrm{~mm} \text {, espaciados en un rango de } 50-200 \mathrm{~mm}\end{array}$ \\
R4 & $\begin{array}{r}\text { Pared rugosa con surcos de profundidad comprendida entre } 4-10 \mathrm{~mm}, \\
\text { con altura mayor a } 5 \mathrm{~mm} \text {, espaciados en un rango de } 50-200 \mathrm{~mm}\end{array}$ \\
& $\begin{array}{r}\text { Pared muy rugosa con surcos de profundidad mayor a } 10 \mathrm{~mm}, \text { con altura } \\
\text { mayor a } 10 \mathrm{~mm} \text {, espaciados en un rango de } 50-200 \mathrm{~mm}\end{array}$ \\
\hline
\end{tabular}

Como puede observarse en la Figura 2.30(a), la rugosidad incrementa la resistencia promedio por fuste $\left(f_{\text {ave }}\right)$ de pilotes empotrados en roca. Por otra parte, en la Figura 2.30(b) se ilustra el comportamiento de $f_{\text {ave }}$ con la profundidad para dos valores de carga aplicada en cabeza; notar que al incrementar la carga -i.e., de $0.31 \mathrm{MN}$ a 0.62 MN-, la distribución de $f_{\text {ave }}$ cambia, de modo que la resistencia por fuste es totalmente movilizada en la zona cercana a la cabeza del pilote y, los valores máximos se producen hacia la parte inferior del mismo.

\subsubsection{Williams (1980), Williams y Pells (1981)}

Williams (1980) realizaron 14 pruebas de carga sobre pilotes excavados en las lutitas $\left(\sigma_{c}=0.5-2.8 \mathrm{MPa}\right)$ de Melbourne (Australia). El objetivo de estos ensayos fue estudiar 
la influencia sobre la resistencia por fuste de las dimensiones del pilote, del método constructivo, de la relación longitud de empotramiento del pilote-diámetro del pilote $(L / D)$, y de la rugosidad en la interfaz roca-pilote, entre otros. En la gran mayoría de estos ensayos se realizó un registro de la rugosidad de la perforación previo a la colocación del hormigón del pilote (e.g., ver perfiles medidos en los pilotes M1 y M3 de Figura 2.31(a)).
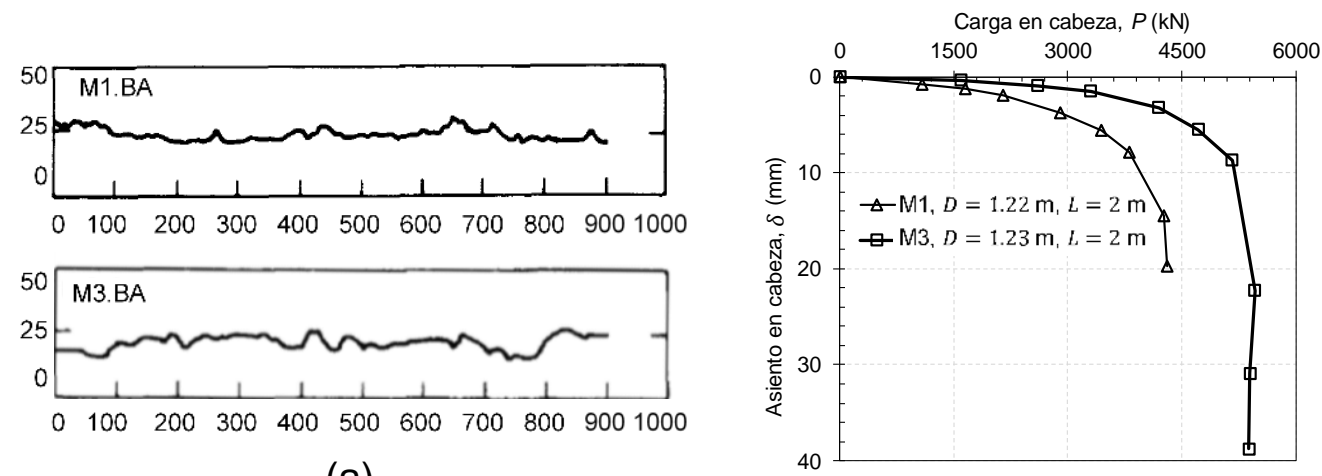

(a)

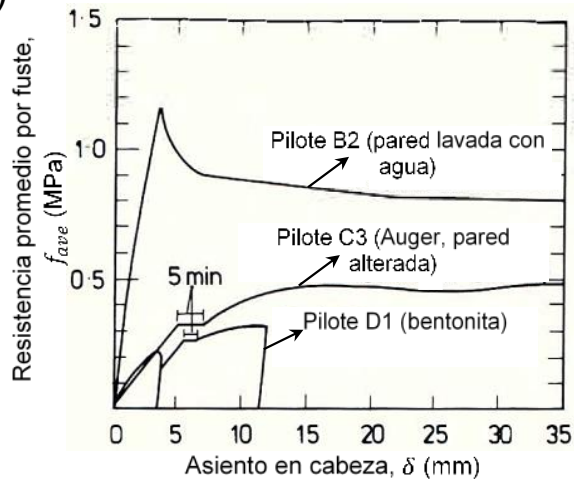

(b)

(c)

Figura 2.31. Pruebas de carga sobre pilotes excavados en lutitas con rugosidad variable en la interfaz roca-pilote: (a) perfiles de rugosidad medidos dentro de la cavidad (unidades en $\mathrm{mm}$ ), (b) carga en cabeza vs asiento en cabeza del pilote (modificado de Williams 1980) (c) efecto del fluido de perforación y zona de alteración sobre la resistencia de pilotes empotrados en roca (modificado de Williams y Pells 1981).

Los pilotes se realizaron con diámetros en un rango de $300-1500 \mathrm{~mm}$ y longitudes de empotramiento en un rango de $1.5-2.6 \mathrm{~m}$. En la Figura 2.31(b) se presentan los resultados obtenidos en los pilotes $\mathrm{M} 1\left(\sigma_{c}=2.46 \mathrm{MPa}\right)$ y $\mathrm{M} 3\left(\sigma_{c}=2.3 \mathrm{MPa}\right)$ cuyas geometrías son similares pero de rugosidades diferentes (ver la Figura 2.31(a)). Como puede observarse, el pilote M3 (que es más rugoso) resiste mayor carga en cabeza que el pilote M1 (menos rugoso, ver la Figura 2.31(b)). Adicionalmente, con base a 
resultados obtenidos sobre pruebas de carga de pilotes empotrados en arenisca $\left(\sigma_{c}=6\right.$ MPa), Williams y Pells (1981) indicaron que no solo la rugosidad influye sobre la resistencia por fuste, sino que también influyen el fluido de perforación empleado y la alteración de la pared de la cavidad durante la construcción del pilote (ver la Figura 2.31(c)), así como la relación longitud de empotramiento-diámetro del pilote $(L / D)$; esta última principalmente sobre pilotes lisos.

\subsubsection{Horvath et al. (1983)}

Con el objetivo de estudiar el efecto de la rugosidad sobre la resistencia de pilotes de hormigón $\left(\sigma_{c}=49 \mathrm{MPa}\right)$ excavados en las lutitas $\left(\sigma_{c}=5.4-5.6 \mathrm{MPa}\right)$ de Queenston (Canadá), Horvath et al. (1983) realizaron tres ensayos de carga considerando solo la resistencia por fuste y tres ensayos tomando en cuenta las resistencias por punta y fuste en forma simultánea. En esta tesis solo se presentan los resultados de los ensayos en los que se considera sólo la resistencia por fuste (ver la Figura 2.32).

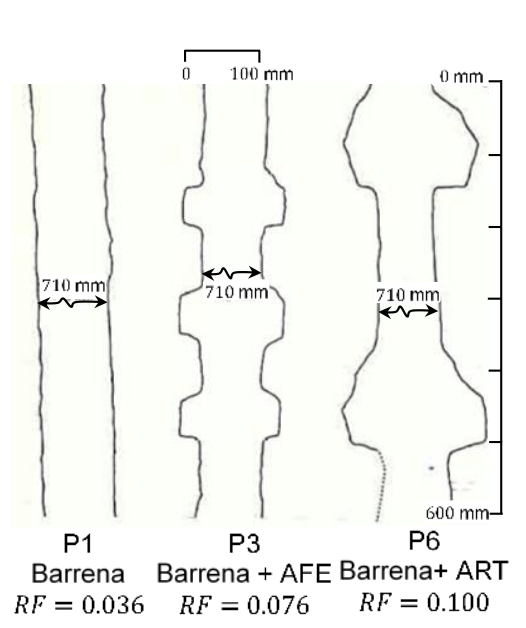

(a)

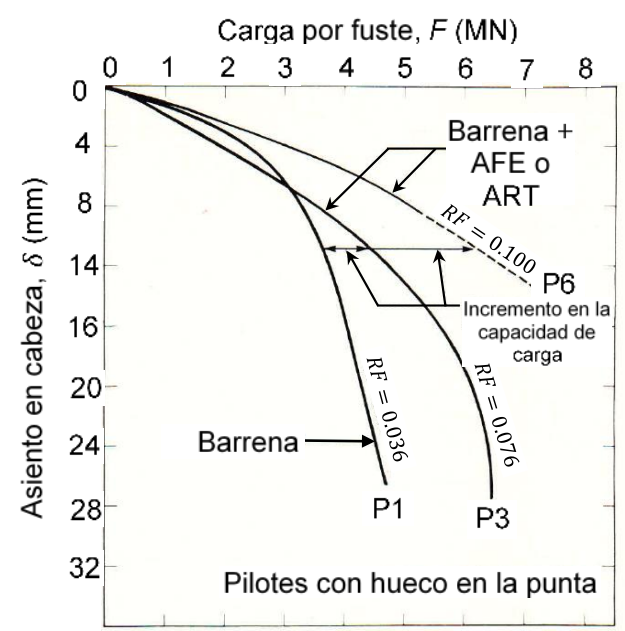

(b)

Figura 2.32. Pruebas de carga sobre pilotes excavados en lutitas con rugosidad variable en la interfaz roca-pilote: (a) perfiles de rugosidad medidos dentro de la cavidad (unidades en $\mathrm{mm}$ ),

(b) carga movilizada por fuste vs asiento en cabeza del pilote (modificado de Horvath et al. 1983).

El pilote P1 se perforó mediante el método convencional con Barrena, mientras que los pilotes P3 y P6, fueron inicialmente perforados con Barrena y, posteriormente emplearon herramientas especiales para hacer más rugosa la pared de la cavidad (ver la Figura 
2.32(a)). El diámetro y la longitud de todos los pilotes son de $710 \mathrm{~mm}$ y $1370 \mathrm{~mm}$, respectivamente. Los resultados, presentados en la Figura 2.32(b), reflejan un incremento en la resistencia por fuste de pilotes excavados en roca con el incremento de la rugosidad en la interfaz roca-pilote, siendo este comportamiento cuantitativamente similar al reportado en los trabajos de Pells et al. (1980) y Williams (1980).

\subsubsection{Seol y Jeong (2007)}

Para analizar la influencia de la rugosidad, del diámetro y de la longitud de pilote empotrados en roca, Seol y Jeong (2007) Ilevaron a cabo una campaña de 9 pruebas de carga sobre pilotes de hormigón en Dongtan (China). Cinco de estos ensayos se realizaron en gneis completamente fracturado (denotado como $\mathrm{CW}, \sigma_{c}=5 \mathrm{MPa}$ ), mientras que los cuatro restantes en gneis moderadamente fracturado (denotado como $\left.\mathrm{MW}, \sigma_{c}=50.3 \mathrm{MPa}\right)$. El diámetro $(D)$ y longitud $(L)$ de todos los pilotes están en un rango de $200-400 \mathrm{~mm}$ y $1000-2000 \mathrm{~mm}$, respectivamente, y con diferente nivel de rugosidad en la interfaz roca-pilote (ver la Figura 2.33(a)). La resistencia a compresión simple (a los 28 días) del hormigón empleado fue de 40 y $80 \mathrm{MPa}$ para CW y MW, respectivamente.

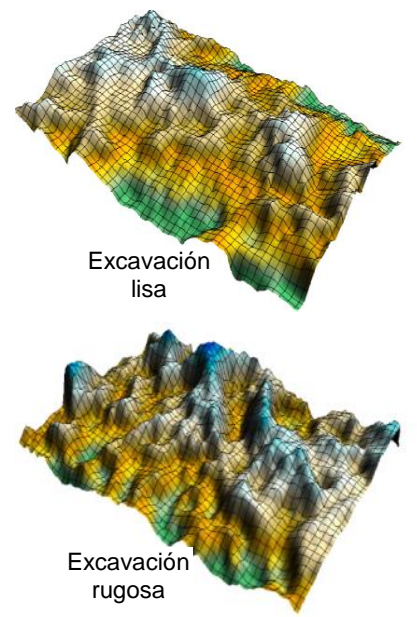

(a)

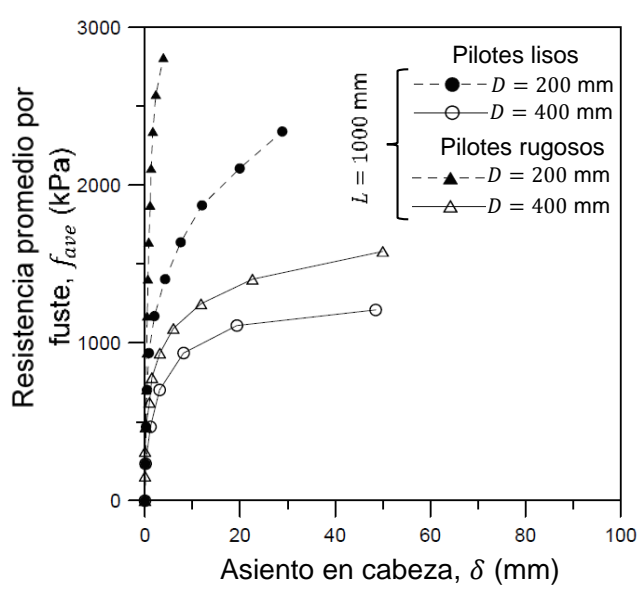

(b)

Figura 2.33. Pruebas de carga sobre pilotes excavados en gneis (MW) con rugosidad variable en la interfaz roca-pilote: (a) perfiles de rugosidad medidos dentro de la cavidad con un perfilómetro de rugosidad laser, (b) resistencia promedio por fuste vs asiento en cabeza del pilote (modificado de Seol y Jeong 2007). 
Adicionalmente, con el fin de estudiar solo la resistencia por fuste, una pieza de poliestireno fue añadida en la punta de todos los pilotes antes del hormigonado. Los resultados, presentados en la Figura 2.33(b), reflejan un aumento de la resistencia promedio por fuste de pilotes excavados en roca con el incremento de la rugosidad y, una disminución de $f_{\text {ave }}$ con el aumento del diámetro del pilote.

\subsubsection{Nam y Vipulanandan (2008)}

La herramienta empleada para la perforación de pilotes empotrados en roca es uno de los factores que también influye sobre la rugosidad, como ya se ha visto en los apartados anteriores. Nam y Vipulanandan (2008) realizaron una investigación para evaluar el efecto de las herramientas de perforación (Barrena y Core Barrel) sobre la rugosidad resultante en la pared de la perforación de pilotes perforados en pizarras $\left(\sigma_{c}=1.2 \mathrm{MPa}\right.$ y $\sigma_{c}=3.1 \mathrm{MPa}$ para las pruebas denotadas como HT y DT, respectivamente) y calizas $\left(\sigma_{c}=10.2 \mathrm{MPa}\right.$ para la prueba denotada como $\mathrm{RC}$ ) al norte de Texas (EEUU). El diámetro de todos los pilotes fue de $760 \mathrm{~mm}$ y su longitud de empotramiento en roca fue variable en un rango de $4.5-5.2 \mathrm{~m}$; no obstante, debido a que en el ensayo de carga se empleó célula Osterberg (ver la Figura 2.34(a)), el cálculo de la resistencia por fuste se realizó considerando solo la longitud de pilote de la parte superior de la célula Osterberg -i.e., sólo resistencia por fuste-.

En la Figura 2.34(b) se presenta un ejemplo de los perfiles de rugosidad resultantes en una de las excavaciones. En la Figura 2.34(c) se presenta la resistencia promedio por fuste movilizada en cada uno de los ensayos, en función de la herramienta de perforación usada. Como puede observarse, la rugosidad y la herramienta de perforación empleada afectan los valores de resistencia por fuste de pilotes empotrados en roca, obteniéndose mayores valores en las perforaciones realizadas con Core Barrel cuyas rugosidades resultantes son mayores a las producidas en perforaciones con Barrena. 


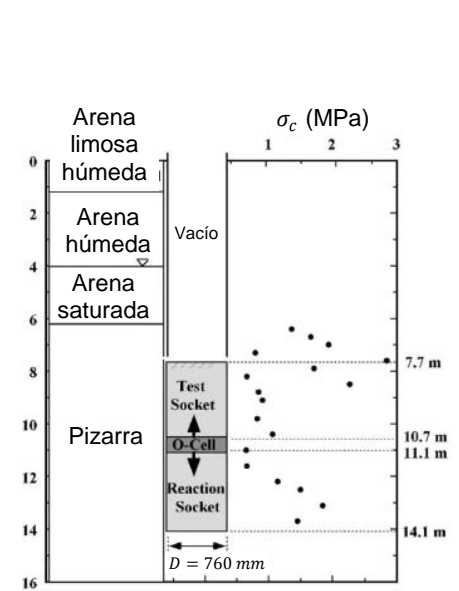

(a)

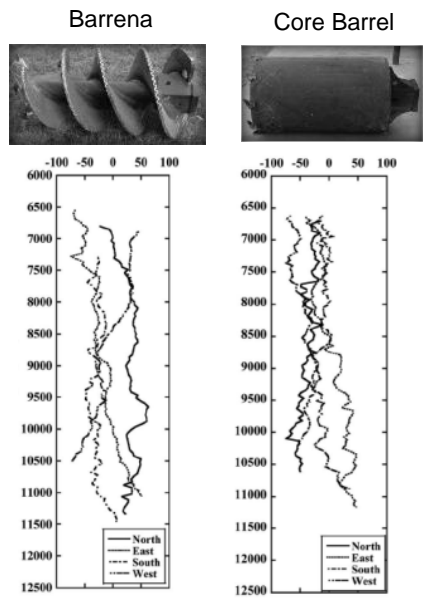

(b)

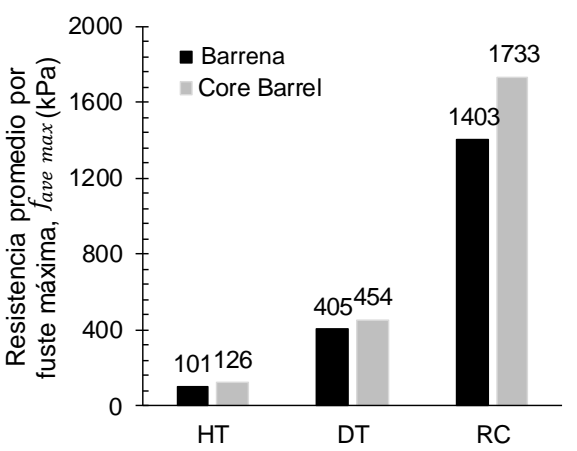

(c)

Figura 2.34. Pruebas de carga sobre pilotes excavados en roca: (a) ilustración del tipo de prueba de carga y perfil geológico del sitio HT, (b) perfiles de rugosidad medidos dentro de la cavidad del sitio HT (dimensiones en $\mathrm{mm}$ ) cuyas alturas promedio de rugosidades con Barrena y Core Barrel son 2.5 y $3.7 \mathrm{~mm}$, respectivamente (modificado de Nam y Vipulanandan 2008),

(c) resistencia promedio por fuste máxima para todos los ensayos (datos experimentales tomados de Nam y Vipulanandan 2008).

\subsubsection{Ensayos de carga sobre pilotes con modelos numéricos a través de la implementación del Método de Elementos Finitos}

\subsubsection{Osterberg y Gill (1973)}

A fin de analizar el mecanismo de transferencia de carga sobre pilotes empotrados roca en función (i) de la relación entre la longitud de empotramiento del pilote-diámetro del pilote $(L / D)$, (ii) de la relación entre el módulo de Young de la roca y del hormigón $\left(E_{r} / E_{c}\right)$ y (iii) de la relación entre el coeficiente de Poisson de la roca y del hormigón $\left(v_{r} / v_{c}\right)$, Osterberg y Gill (1973) modelizaron ensayos de carga sobre pilotes mediante el Método de Elementos Finitos (MEF) (ver la Figura 2.35(a)-(b)). Los resultados obtenidos por estos autores sugieren que gran parte de la carga aplicada sobre el pilote es transferida por fuste en la parte superior del pilote (ver la Figura 2.35(c)) y que, dependiendo de la relación de $E_{r} / E_{c}$ y de $L / D$, solo un pequeño porcentaje de esa carga llega a la punta del pilote. En este sentido, Osterberg y Gill (1973) indican que para $L / D>3$, ya se ha producido una completa transferencia de la carga aplicada en cabeza hacia la resistencia por fuste de la roca circundante para modelos elásticos, y que por tanto, la 
relación máxima de la longitud de empotramiento del pilote-diámetro del pilote debería $\operatorname{ser} L / D=3$.

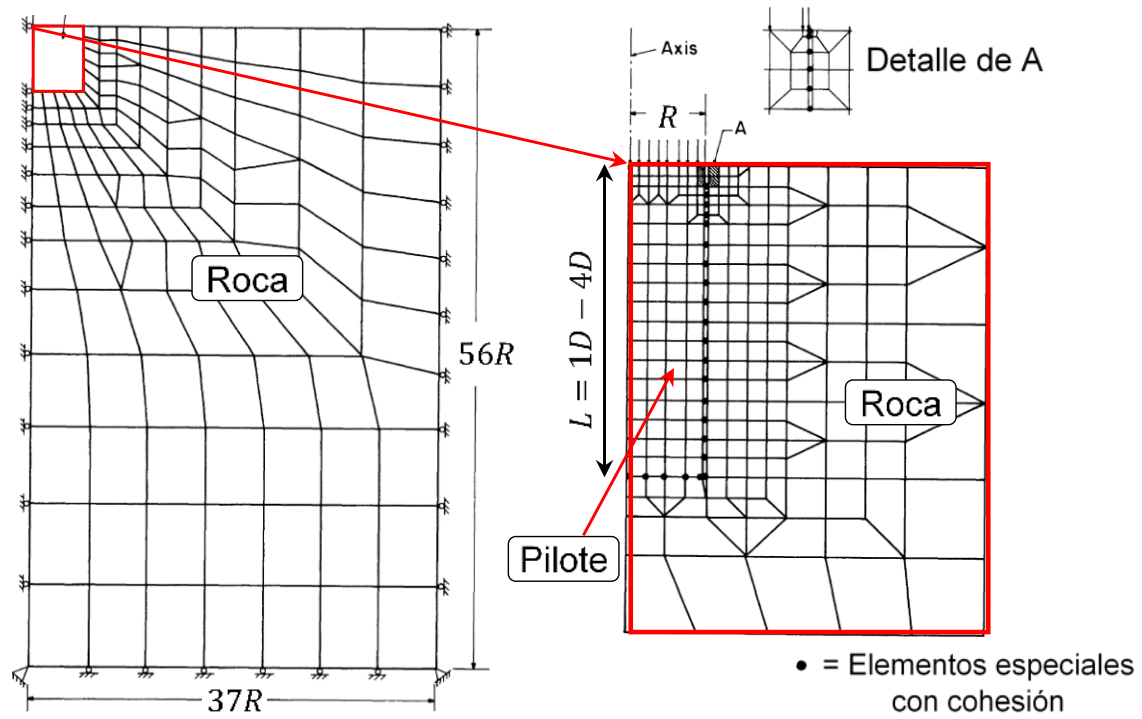

(a)

(b)

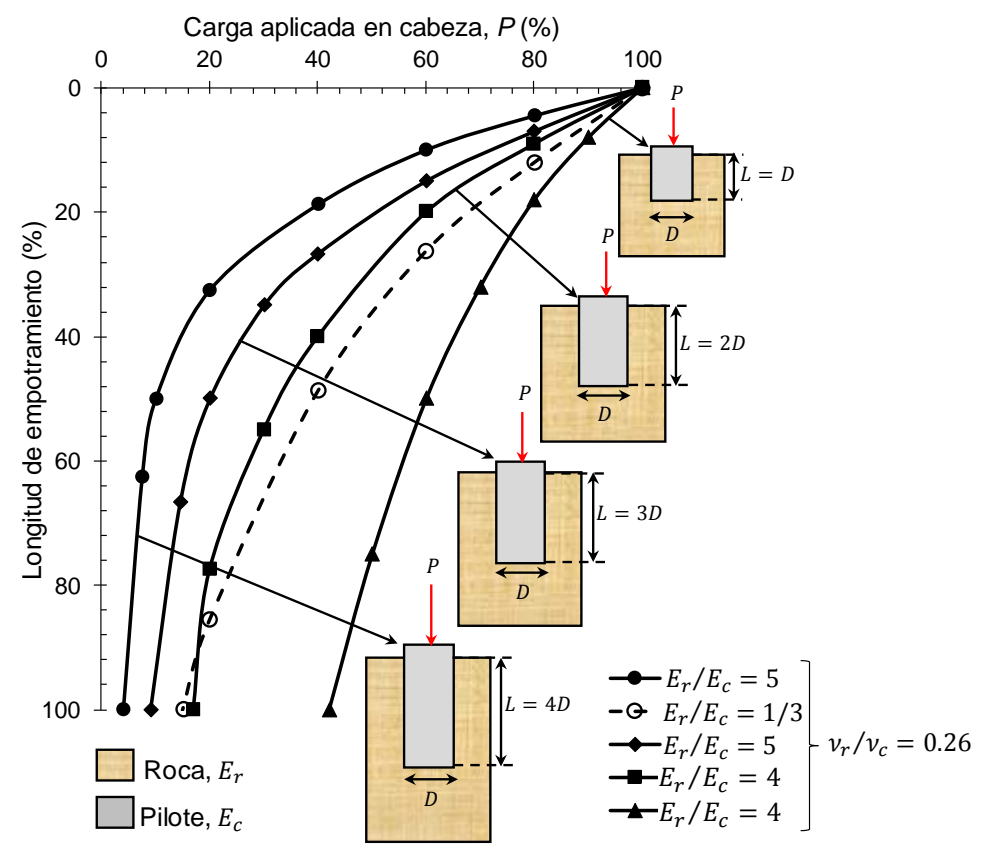

(c)

Figura 2.35. Modelo numérico de pilotes empotrados en roca con el MEF: (a) geometría del modelo, (b) detalle de la geometría en el contacto pilote-roca, (c) influencia de la relación $L / D$ sobre la carga movilizada a lo largo del pilote (modificado de Osterberg y Gill 1973).

2.3.2.2 O'Neill et al. (1996)

Para estudiar el efecto de la rugosidad sobre pilotes empotrados en roca, O'Neill et al. (1996) realizaron dos modelos numéricos axisimetricos de pilotes sometidos a carga 
axial (ver la Figura 2.36(a)), a través del MEF con el código comercial Abaqus. El diámetro y la longitud del pilote del modelo es de $0.61 \mathrm{~m}$ y $6.1 \mathrm{~m}$, respectivamente. La roca del modelo representa un material de resistencia intermedia -e.g., arenisca, caliza con $\sigma_{c}=0.5-5 \mathrm{MPa}-$. Para el modelo rugoso, la interfaz roca-pilote fue modelada como una superficie sinusoidal idealizada (ver la Figura 2.36(b)) cuya morfología, según O’Neill et al. (1996), es típica en excavaciones realizadas con Barrena en este tipo de materiales. En sus modelos numéricos, O’Neill et al. (1996) consideraron la movilización simultánea de resistencia por fuste y punta del pilote. En los resultados, presentados en la Figura 2.36(c), se observa el incremento de la resistencia promedio por fuste del pilote como consecuencia de incorporar la rugosidad en la interacción roca-pilote.

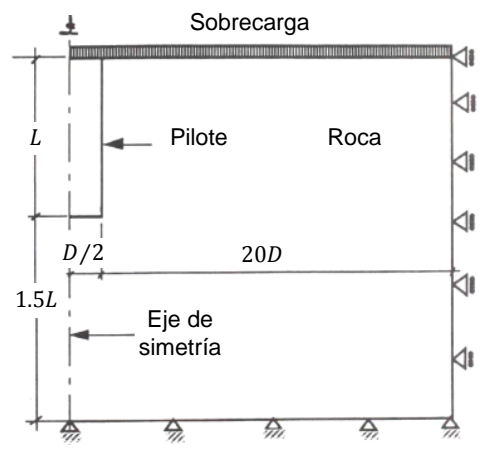

(a)

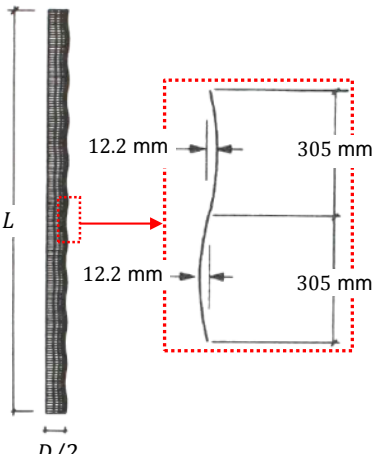

(b)

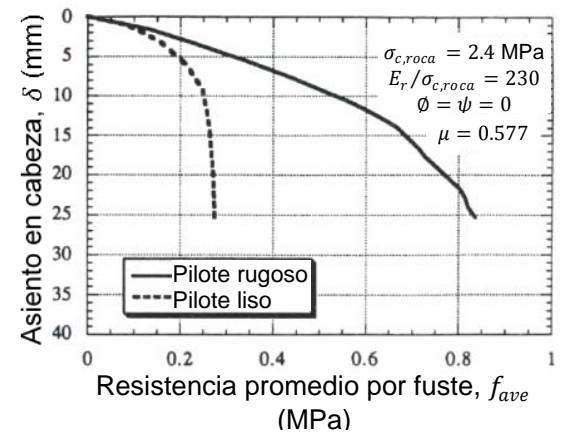

(c)

Figura 2.36. Modelo numérico de pilotes empotrados en roca con el MEF: (a) geometría del modelo, (b) detalle de la geometría de la rugosidad empleada para el pilote rugoso, (c) resistencia promedio por fuste vs asiento en cabeza del pilote (modificado de O'Neill et al. 1996).

\subsubsection{Melentijevic et al. (2012), Melentijevic y Olalla (2014)}

Con el objetivo de analizar el comportamiento resistente de pilotes en roca, estos investigadores realizaron un estudio paramétrico mediante modelos numéricos axisimetricos con diferente grado de rugosidad idealizada $-R 1, R 2$, R3 y $R 4$ según el sistema de clasificación de Pells et al. (1980)- en la interfaz roca-pilote. La simulación se realizó mediante el programa comercial Plaxis, cuya geometría y detalle de la rugosidad se presentan en la Figura 2.37(a). El modelo conceptual, presentado en la Figura 2.37(a), es representativo de ensayos de carga en campo mediante célula 
Osterberg realizado por estos mismos autores. El diámetro $(D)$ y la longitud del pilote $(L)$ de hormigón ( $\sigma_{c}=44 \mathrm{MPa}$ ) de todos los modelos es de $1 \mathrm{~m}$ y $5 \mathrm{~m}$, respectivamente. La roca circundante está formada por una secuencia sedimentaria tipo flysch $\left(\sigma_{c}=48\right.$ $\mathrm{MPa})$.

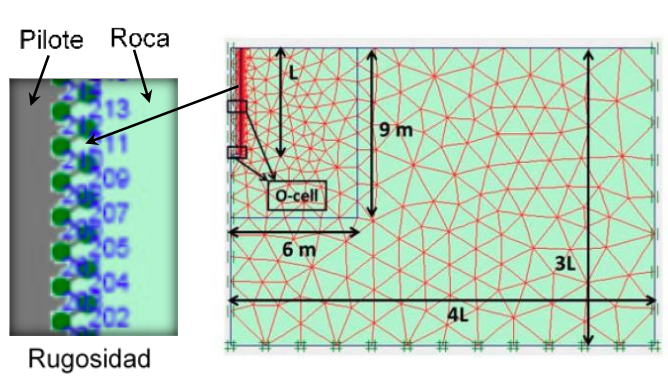

(a)

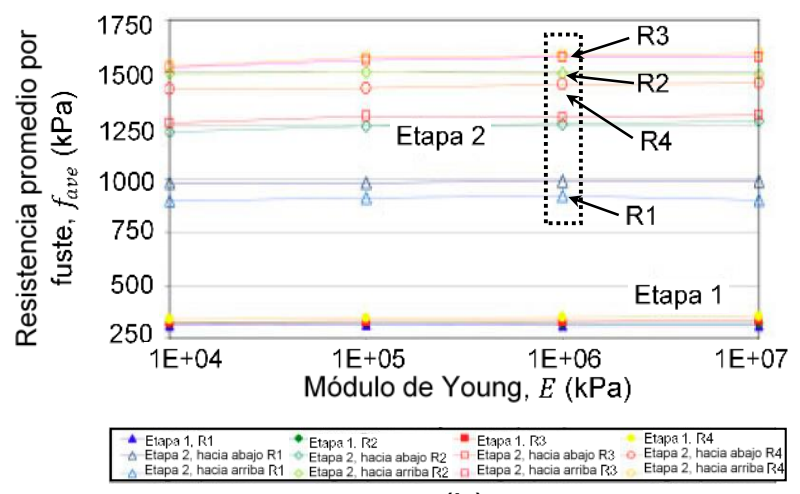

(b)

Figura 2.37. Modelo numérico de pilotes empotrados en roca con el MEF: (a) geometría y mallado del modelo y detalle de la rugosidad empleada en la interfaz roca-pilote, (b) resistencia promedio por fuste vs módulo de Young del material presente en la punta del pilote (modificado de Melentijevic y Olalla 2014).

Adicionalmente, con el fin de simular la calidad del acabado en la punta del pilote, o el equivalente a un hueco en la punta, el material rocoso bajo la punta del pilote fue simulado con valores de módulo de Young $(E)$ en un rango de $1.10^{4}-1.10^{7} \mathrm{kPa}$. Los resultados de resistencia promedio por fuste $\left(f_{\text {ave }}\right)$ obtenida para todos los modelos se presentan en la Figura 2.37(b). La etapa 1 y 2 representan la aplicación de la carga del ensayo en cada nivel de la célula Osterberg (ver la Figura 2.37(a)-(b)). Los resultados mostrados en la Figura 2.37(b) muestran un incremento de $f_{\text {ave }}$ con el aumento de la rugosidad (e.g., ver la Figura 2.37 (b) para $E=1.10^{6} \mathrm{kPa}$ y etapa 2). No obstante, se aprecia que, a pesar de incrementar la rugosidad en la interfaz desde $R 2$ hasta $R 4$, no hay un incremento sustancial de $f_{a v e}$ en la etapa 2 del ensayo; mientras que, para la etapa 1, no se observa una gran influencia de la rugosidad sobre la resistencia por fuste de todos los modelos para diferentes valores de $E$. 


\subsubsection{Gutiérrez-Ch (2015), Gutiérrez-Ch y Melentijevic (2016)}

Para estudiar el efecto de la rugosidad en la interfaz roca-pilote sobre el comportamiento de resistencia por punta y fuste de pilotes empotrados en roca, Gutiérrez-Ch y Melentijevic (2016) realizaron modelos numéricos axisimetricos con el MEF de los códigos comerciales Plaxis y Abaqus. La interfaz roca-pilote fue modelizada con diferente grado de rugosidad según el sistema de clasificación de rugosidades de Pells et al. (1980) para los modelos elaborados en Plaxis, mientras que para los modelos realizados en Abaqus, incorporaron la morfología de rugosidades sinusoidales sugeridas por O’Neil et al. (1996) (ver Sección 2.3.2.2). Las propiedades de la roca y del hormigón fueron las mismas que se emplearon en el trabajo de Melentijevic y Olalla (2014). La geometría y condiciones de borde de los modelos realizados se presentan en la Figura 2.38(a). Adicionalmente, para evaluar la influencia de la longitud de empotramiento sobre la resistencia de pilotes empotrados en roca, la longitud del pilote se modeló en un rango de $L=1-5 \mathrm{~m}$, con $D=1 \mathrm{~m}$. En todos los modelos se consideró la resistencia movilizada por fuste y punta de forma simultánea.

En la Figura 2.38(b) se presenta la carga en el cabezal en función del asiento para los modelos con rugosidad sinusoidal en la interfaz roca-pilote y $L=1$ y $4 \mathrm{~m}$. Los resultados muestran una ligera influencia tanto de la rugosidad como de la longitud de empotramiento sobre el comportamiento carga-asiento de pilotes empotrados en roca. En la Figura 2.38 (c) se muestra la resistencia promedio por fuste $\left(f_{\text {ave }}\right)$ de todos los modelos para $L=1 \mathrm{~m}$ movilizada para un mismo valor de carga en cabeza de 34.5 MN. Nuevamente, se observa un ligero incremento de $f_{\text {ave }}$ con el incremento de la rugosidad en la interfaz (e.g., el modelo con R1 $f_{\text {ave }}=8.3 \mathrm{MPa}$ y el modelo con R3 $f_{\text {ave }}=8.5 \mathrm{MPa}$ ); quizás el que no se produzca un efecto más acentuado de la rugosidad sobre $f_{\text {ave }}$ de manera pueda atribuirse al hecho de que el pilote está trabajando, de forma simultánea, tanto por punta como por fuste. 


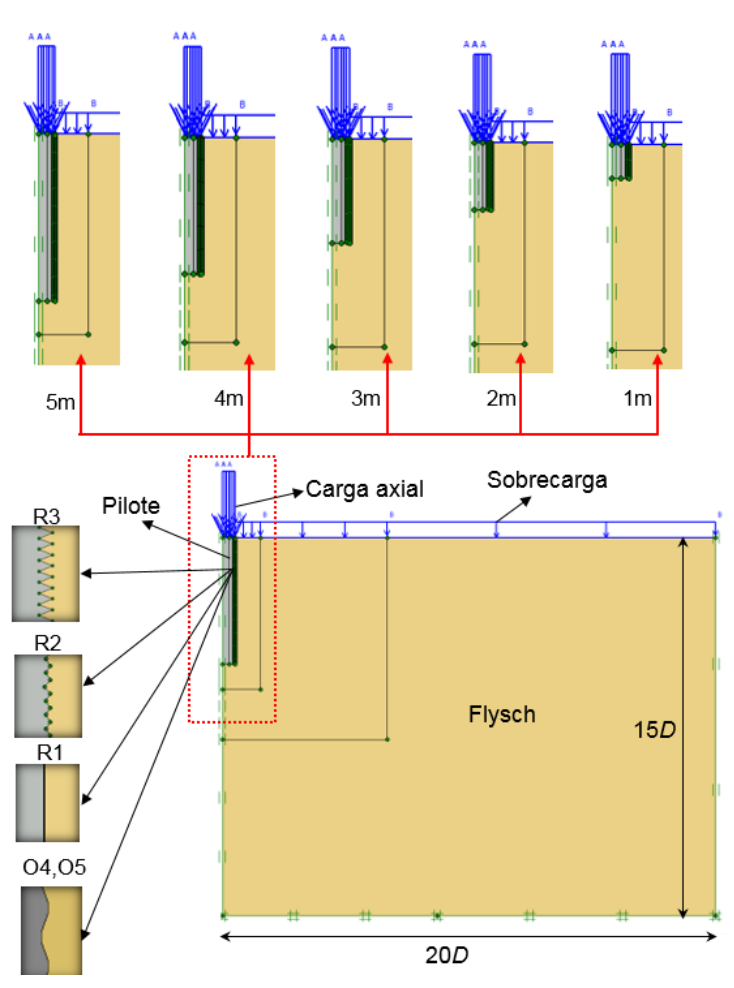

(a)

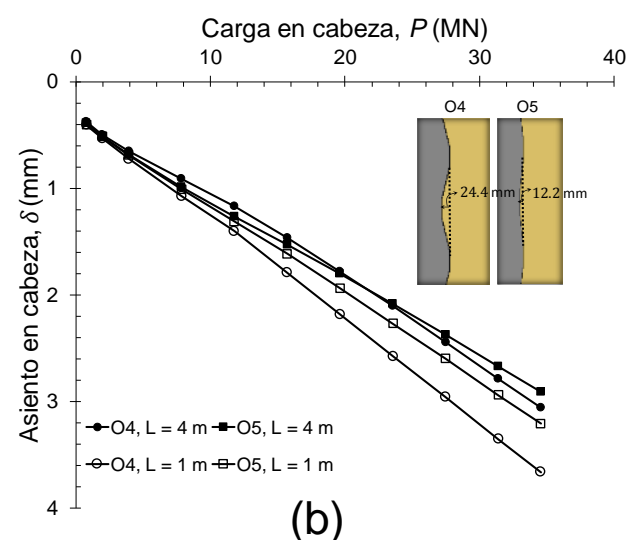

(b)

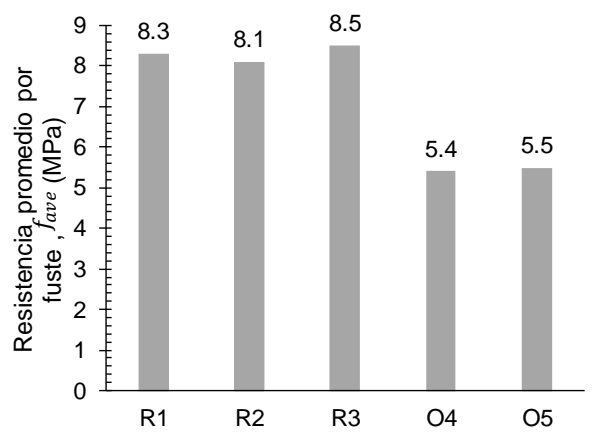

(c)

Figura 2.38. Modelo numérico de pilotes empotrados en roca con el MEF: (a) geometría y detalle de la rugosidad empleada en la interfaz roca-pilote, (b) carga en cabeza versus asiento para los modelos con rugosidad sinusoidal (O4 representa el modelo más rugoso y O5 el menos rugoso en función de la amplitud de la onda), (c) resistencia promedio por fuste obtenida para los modelos con $L=1 \mathrm{~m}$ bajo carga en cabeza de 34.5 MN (modificado de Gutiérrez-Ch y Melentijevic 2016).

Adicionalmente, los resultados reportados por Gutiérrez-Ch (2015) reflejan un comportamiento similar al observado en los modelos de Osterberg y Gill (1973), en el cual se aprecia la influencia de la relación $L / D$ sobre la resistencia movilizada en pilotes empotrados en roca. Por ejemplo, en la Figura 2.39 se muestra que, para el modelo con rugosidad tipo R3 y para los modelos con $L / D>3$, más del $90 \%$ de la resistencia es movilizada por la componente de resistencia por fuste. En este sentido, los resultados numéricos están de acuerdo con la relación máxima de $L / D=3$ sugerida por Osterberg y Gill (1973). 


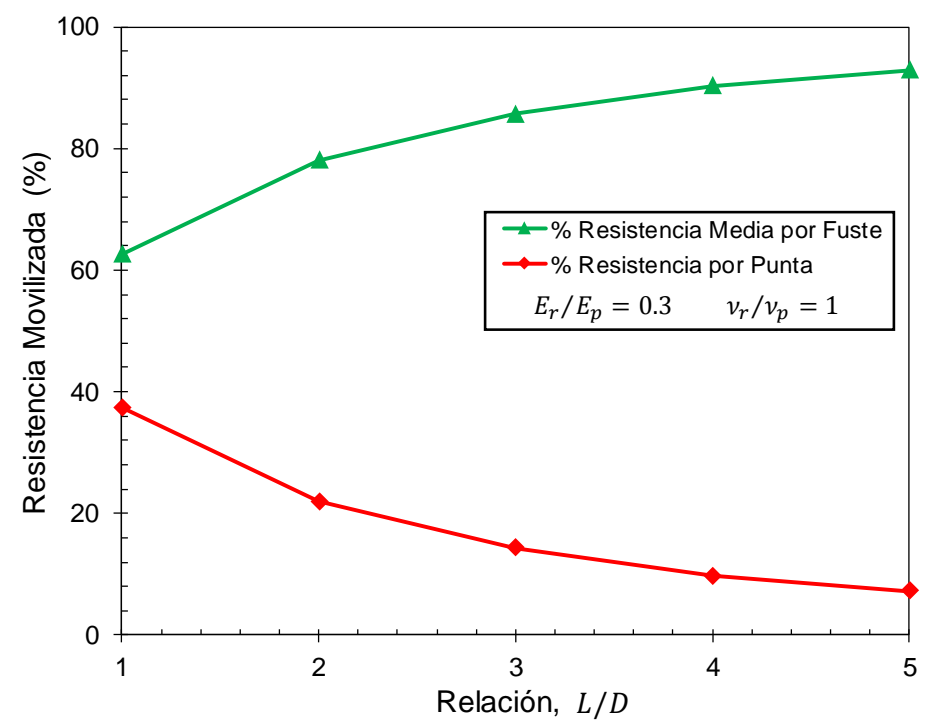

Figura 2.39. Efecto de la relación $L / D$ sobre la resistencia movilizada de pilotes empotrados en roca: modelo con rugosidad R3 (modificado de Gutiérrez-Ch 2015).

Con base a los diversos ensayos de carga realizados sobre pilotes empotrados en roca (tanto a pequeña escala (en laboratorio) como a gran escala (en campo)) expuestos en las secciones previas, se observa que los principales factores que afectan al comportamiento de resistencia por fuste de pilotes empotrados son: (i) la rugosidad y el esfuerzo normal en la interfaz roca-pilote, (ii) el diámetro y la longitud de empotramiento del pilote, (iii) las propiedades resistentes de la roca y del pilote, y (iv) la herramienta empleada en el proceso constructivo.

\subsection{Modelos en centrifuga}

Los ensayos de centrifuga son una alternativa bastante atractiva, por su bajo costo y por su facilidad, para simular fenómenos de interés geotécnico (Leung y Ko 1993). En este tipo de ensayo, los modelos se elaboran a escala reducida (siguiendo las leyes de escala, ver la Figura 2.40 y la Tabla 2.2), de modo que al ser sometidos a un campo de aceleración de magnitud $N$ veces la gravedad $(g)$ de la tierra -i.e., $N g$, donde $N$ representa el factor de escala-, se obtiene como resultado que el estado tensional en el modelo es equivalente al estado tensional del prototipo a gran escala (Taylor 1995). 


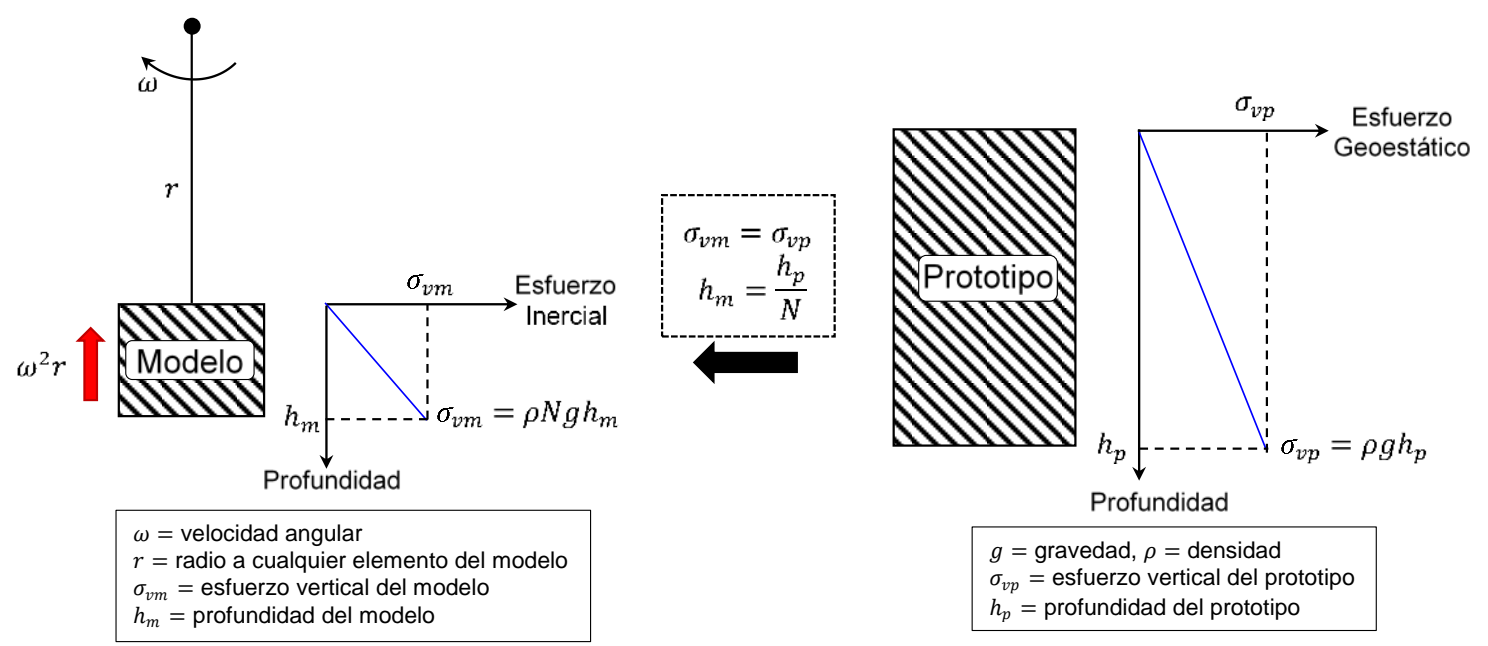

Figura 2.40. Esquema conceptual de los modelos en ensayos de centrifuga $(\mathrm{Ng})$ y su equivalente a escala del prototipo $(1 \mathrm{~g})$ (modificado de Taylor 1995).

Tabla 2.2. Leyes de escala en ensayos de centrifuga (Taylor 1995; Xing et al. 2014)

\begin{tabular}{|c|c|c|}
\hline Cantidad física & Prototipo $(1 g)$ & Modelo $(\mathrm{Ng})$ \\
\hline Longitud, $l$ & 1 & $1 / N$ \\
\hline Desplazamiento, $u$ & 1 & $1 / N$ \\
\hline Aceleración de la gravedad, $g$ & 1 & $N$ \\
\hline Temperatura, $T$ & 1 & 1 \\
\hline Esfuerzo, $\sigma$ & 1 & 1 \\
\hline Deformación, $\varepsilon$ & 1 & 1 \\
\hline Fuerza, $F$ & 1 & $1 / N^{2}$ \\
\hline Rigidez, $E A$ & 1 & $1 / N^{2}$ \\
\hline Área, $A$ & 1 & $1 / N^{2}$ \\
\hline Densidad, $\rho$ & 1 & $1 / N^{3}$ \\
\hline Peso unitario, $\gamma$ & 1 & $N$ \\
\hline \multicolumn{3}{|l|}{ Tiempo, $t$} \\
\hline Procesos de difusión & 1 & $1 / N^{2}$ \\
\hline Eventos inerciales & 1 & $1 / N$ \\
\hline Procesos viscosos & 1 & 1 \\
\hline Energía potencial total del agua, $\psi$ & 1 & 1 \\
\hline Velocidad intersticial del agua, $v_{w}$ & 1 & $N$ \\
\hline Flujo de calor, $\phi_{q}$ & 1 & $N$ \\
\hline
\end{tabular}

A continuación, se presentan algunas investigaciones en las que se realizaron ensayos de centrifuga para evaluar el efecto de algunos de los factores mencionados al final de la Sección 2.3 sobre la resistencia por fuste de pilote empotrados. A efectos de 
nomenclatura, cualquier cantidad física con subíndice " $m$ " está referida a la escala del modelo (i.e., a la escala del ensayo de centrifuga).

\subsubsection{Ensayos de carga sobre pilotes mediante ensayos de centrifuga}

\subsubsection{Leung y Ko (1993)}

Con el objetivo de evaluar el comportamiento por punta y fuste de pilotes empotrados en rocas de distinta resistencia, Leung y Ko (1993) realizaron una serie de ensayos en centrifuga a $40 \mathrm{~g}$ y $1 \mathrm{~g}$ (i.e., a pequeña escala) sobre pilotes cargados axialmente y empotrados en rocas sintéticas (mezclas de cemento y agua) con resistencias a compresión simple $\left(\sigma_{c}\right)$ menores a $12 \mathrm{MPa}$. Los pilotes se fabricaron de aluminio, con $\sigma_{c}=294 \mathrm{MPa}$ y $E=70 \mathrm{GPa}$. El esquema y las dimensiones del ensayo se presentan en la Figura 2.41(a).

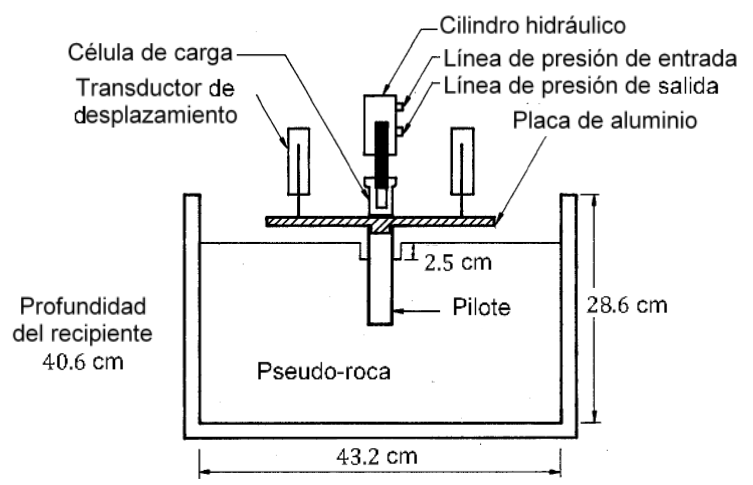

(a)

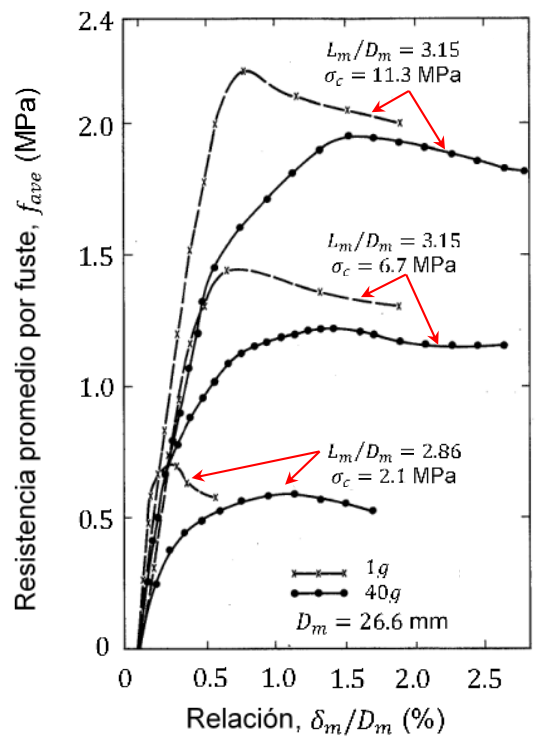

(b)

Figura 2.41. Pruebas de carga sobre pilotes empotrados en roca mediante ensayos de centrifuga y de escala reducida: (a) esquema y geometría del ensayo, (b) resistencia promedio por fuste vs la relación asiento-diámetro del pilote (modificado de Leung y Ko 1993).

En la Figura 2.41(b) se presentan los resultados de los ensayos en los que solo se considera la resistencia promedio por fuste $\left(f_{\text {ave }}\right)$, debido a que la punta se aisló mediante la colocación de una pieza de espuma de poliestireno en la punta del pilote. 
Los resultados presentados en la Figura 2.41(b) sugieren que los siguientes factores influyen sobre el comportamiento por fuste de pilotes empotrados en roca: (i) resistencia de la roca circundante -a mayor $\sigma_{c}$ mayor $f_{\text {ave }}$ para las mismas condiciones $L_{m} / D_{m}-\mathrm{y}$, (ii) el tipo de ensayo realizado. En particular, se evidencia que estos ensayos a pequeña escala $(1 g)$ se alcanzan mayores valores de resistencia pico que los ensayos de centrifuga para menor relación de $\delta_{m} / D_{m}$, así como que el comportamiento post-pico en los ensayos $1 g$ tienden a presentar una caída más brusca hacia el valor residual en comparación al comportamiento observado en los ensayos a $40 \mathrm{~g}$, en el cual, el comportamiento post-pico es más dúctil.

\subsubsection{Dykeman y Valsangkar (1996)}

Dykeman y Valsangkar (1996) realizaron pruebas de carga axial y lateral en pilotes excavados en roca blanda, mediante ensayos de centrifuga a $40 \mathrm{~g}$ (ver la Figura 2.42(a)). La pseudo-roca fue elaborada con la mezcla de arena, cemento, bentonita y agua, resultando una $\sigma_{c}=1.51 \mathrm{MPa}$ (a los 14 días). Los pilotes del modelo se elaboraron de aluminio, de sección hueca (espesor de $1.59 \mathrm{~mm}$ ), y con $D_{m}=25 \mathrm{~mm}, L_{m}=63.5 \mathrm{~mm}-$ i.e., $D=1 \mathrm{~m}$ y $L=2.54 \mathrm{~m}$ a escala del prototipo-. Para los pilotes rugosos, se elaboraron surcos de $0.5 \mathrm{~mm}$ de altura y de profundidad, espaciados cada $5 \mathrm{~mm}$ a lo largo de toda la longitud del pilote del modelo.

En la Figura 2.42(b) se presentan los resultados de los ensayos, en los cuales se considera toda la resistencia movilizada se debe a la resistencia por fuste del pilote (se pegó un trozo de espuma de poliestireno en la punta). Los pilotes P1 y P4 son lisos y los pilotes PR1 y PR2 son rugosos. Los resultados de la Figura 2.42(b) reflejan que la rugosidad no sólo influye sobre el comportamiento resistente de los pilotes empotrados en roca, sino también sobre la forma en que se alcanza los valores máximos de carga en cabeza. Nótese, como para los pilotes lisos se produce un comportamiento lineal hasta alcanzar el valor pico, mientras que para los pilotes rugosos este comportamiento 
lineal es menos prolongado y se transforma en no lineal de forma gradual hasta alcanzar la carga máxima en cabeza del pilote.

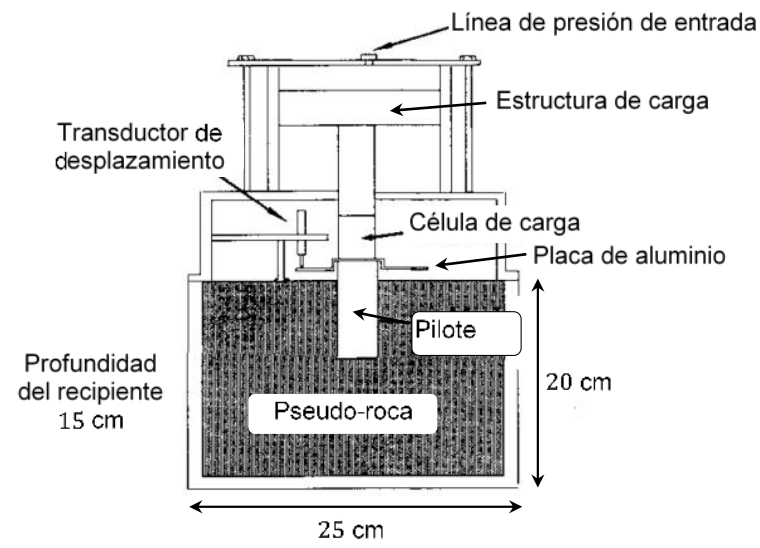

(a)

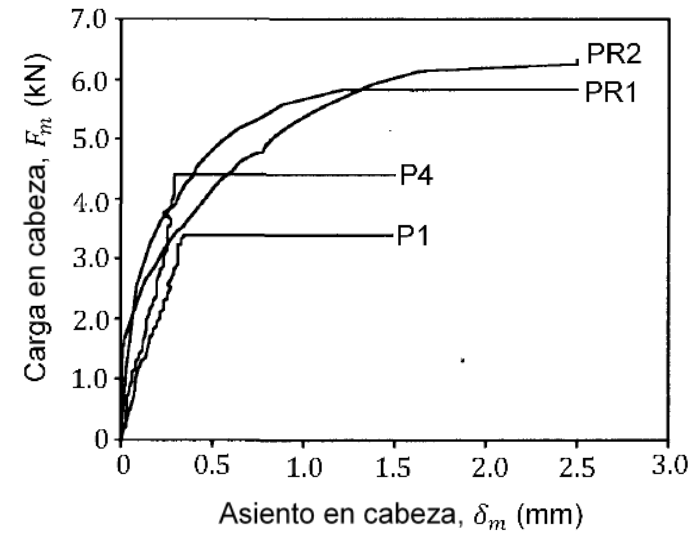

(b)

Figura 2.42. Pruebas de carga sobre pilotes empotrados en roca mediante ensayos de centrifuga y de escala reducida: (a) esquema y geometría del ensayo para pilotes cargados axialmente, (b) carga en cabeza vs asiento en cabeza del pilote (modificado de Dykeman y Valsangkar 1996).

\subsubsection{Xing et al (2014)}

Xing et al (2014) realizaron ensayos de centrifuga a $100 \mathrm{~g}$, en pilotes excavados en roca y cargados axialmente. El propósito de estos ensayos fue evaluar su comportamiento resistente (punta y fuste de manera simultánea) ante (i) distintos valores de longitud de empotramiento y (ii) distinto espesor de suelo suprayacente variable. La pseudo-roca fue preparada con la mezcla de arena, cemento y agua que alcanzaba $\sigma_{c}=38.4 \mathrm{MPa}$ (a los 28 días). El estrato de suelo suprayacente se modeló con partículas de arena con tamaño de partícula comprendido entre $0.25-1 \mathrm{~mm}$. Los pilotes se fabricaron de aluminio de sección hueca (espesor de pared de $8 \mathrm{~mm}$ y diámetro exterior de $D_{m}=22$ $\mathrm{mm}$ ) con longitud variable (ver la Figura 2.43(a)).

En la Figura $2.43(b)$ se presenta (a escala del prototipo, $1 g$ ) la respuesta carga en cabeza - asiento obtenido para todos los ensayos. Los resultados muestran que la longitud de empotramiento en roca y el espesor de suelo suprayacente influyen notablemente sobre la resistencia de pilotes empotrados en roca. (Nótese que, como 
para los modelos con denotación OST(125 - 425) -en los que la porción de pilote empotrado en roca se mantiene constante y se varia el espesor de suelo-, el incremento del estrato de suelo contribuye a un aumento de resistencia, y por tanto, su contribución no debería omitirse durante el proceso de diseño de este tipo de cimentación).

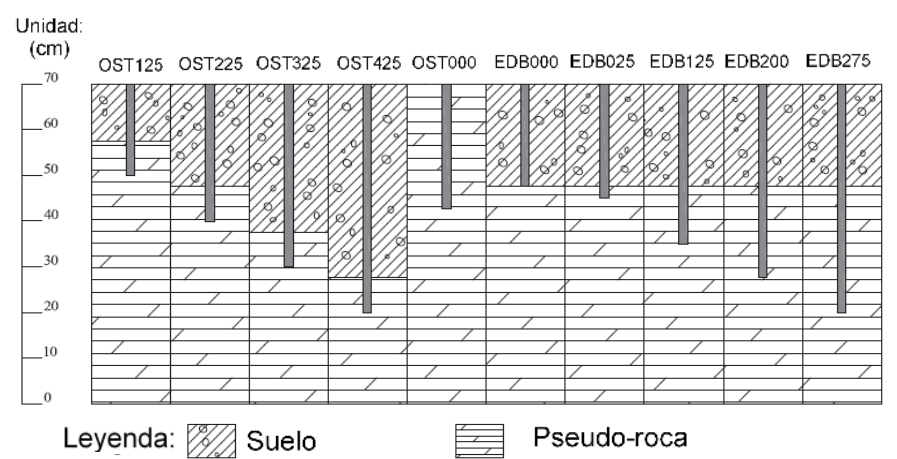

(a)

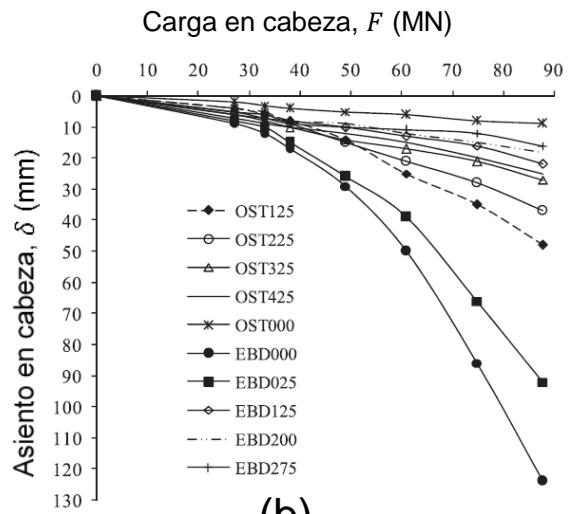

(b)

Figura 2.43. Pruebas de carga sobre pilotes empotrados en roca mediante ensayos de centrifuga y de escala reducida: (a) perfil del ensayo para distintos valores de longitud de empotramiento y espesor de suelo, (b) carga en cabeza vs asiento en cabeza del pilote a escala del prototipo ( $1 \mathrm{~g})$ (modificado de Xing et al. 2014).

Por otra parte, de la Figura $2.43(\mathrm{~b})$ se puede inferir que, para los modelos con espesor de suelo constante pero con aumento de la longitud de pilote empotrada en roca (denotación EDB025 - 275), se produce un aumento de la resistencia de los pilotes. Adicionalmente, se observa que, para los dos casos extremos -pilote solo empotrado en roca (OST000) y pilote solo empotrado en suelo (EDB000)- se presenta la situación de mayor y menor resistencia de todos los modelos. Por tanto, se observa que la longitud de los pilotes es un factor importante en el comportamiento resistente de este tipo de cimentación profunda; no obstante, es importante tener presente que grandes longitudes de pilotes empotrados en roca representan altos costos e inviabilidad económica.

Después de los anteriormente expuesto, los resultados de los ensayos de centrifuga confirman que los principales factores que afectan el comportamiento de resistencia por fuste de pilotes empotrados son: (i) la rugosidad y el esfuerzo normal en la interfaz roca- 
pilote, (ii) la longitud de empotramiento del pilote, y (iii) las propiedades resistentes de la roca.

Finalmente, basado en los resultados obtenidos a través de los ensayos de campo, de laboratorio y modelos numéricos mencionados a lo largo de las secciones previas, se han desarrollado diversas formulaciones para estimar la resistencia por fuste de pilotes empotrados en roca. El compendio de estas formulaciones se desarrolla más adelante, en el Capítulo 6. 


\section{Modelos numéricos a pequeña escala considerando la interacción roca-hormigón}

\subsection{Introducción}

En el Capítulo 2, se presentaron algunas investigaciones publicadas en la literatura, sobre la resistencia al corte de juntas roca-roca o roca-hormigón. Sin embargo, un análisis más exhaustivo del comportamiento al corte de juntas roca-hormigón mediante el Método de Elementos Discretos (MED), y su validación con ensayos de laboratorio, está aún ausente en la literatura. Este capítulo proporciona una contribución en esa dirección, mediante: (i) la aplicación del método Shear Box Genesis (SBG, ver la Sección 3.3.3) propuesto por Bahaaddini et al. (2013b) al estudio del comportamiento al corte en juntas de roca-hormigón, bajo condición de borde CNL y CNS, (ii) el efecto de la rugosidad en la interfaz sobre el comportamiento al corte en juntas de roca-hormigón, y (iii) la validación de resultados numéricos con resultados experimentales de ensayos de corte directo realizados en juntas de arenisca-hormigón y gneis-hormigón publicados en la literatura por Gu et al. (2003) y Gutiérrez (2013), respectivamente.

\subsection{Fundamentos del MED con PFC}

PFC (Particle Flow Code) es un código basado en el MED, el cual puede simular el comportamiento -interacciones, movimientos, etc.-, de un sistema compuesto de muchas partículas de tamaño finito, consideradas como cuerpos rígidos, con una determinada masa y que se mueven independientemente unas respecto a las otras mediante traslación y rotación. La interacción entre partículas se produce a través de fuerzas internas y momentos, cuyas leyes de contacto son actualizadas en cada instante a través del MED, proporcionando una solución dinámica y explicita de las leyes de movimiento de Newton (Itasca Consulting Group Inc. 2014). 
Para simular el comportamiento de materiales cohesivos (como el hormigón o la roca) en PFC, se dispone del modelo denominado Bonded-Particle Model (BPM) (Potyondy 2015), con sus modelos de contacto asociados. Estos modelos han mostrado ser muy útiles para reproducir el comportamiento macroscópico de diversos materiales, así como para estudiar el efecto que los aspectos micromecánicos tienen sobre el comportamiento macroscópico de los materiales (Potyondy y Cundall 2004). Adicionalmente, PFC cuenta con modelos para simular "interfaces" existentes entre los materiales, lo que permite caracterizar y reproducir su comportamiento cuando están en contacto.

En esta tesis doctoral, la simulación numérica de ensayos de corte directo con interfaz roca-hormigón se realizó asignando el modelo de contacto flat-joint (FJCM) para la roca y hormigón, mientras que en la interfaz entre ambos materiales se empleó el modelo de contacto smooth-joint (SJCM). A continuación, se describen brevemente ambos modelos de contacto; para mayor detalle sobre ellos ver Potyondy (2015); Ivars et al. (2008) e Itasca Consulting Group Inc. (2014).

\subsubsection{Modelo de contacto flat-joint (FJCM)}

EI FJCM simula el contacto entre dos partículas en el BPM usando superficies planas hipotéticas (Potyondy 2012). Estas superficies son discretizadas en elementos, los cuales pueden ser bonded o unbonded (ver la Figura 3.1; $g_{s}$ es el gap o separación entre elementos unbonded, $F^{e}$ y $M^{e}$ son la fuerza y el momento actuante sobre cada elemento, respectivamente; $x_{c}$ es el centro del volumen de interpenetración de las dos

partículas, $\bar{F}$ y $\bar{M}$ son la fuerza y momento total actuante en todo el contacto flat-joint, $r_{e}$ es la distancia relativa entre $x_{c}$ y el centro de cada elemento, $x_{c}$ es el centroide del contacto y $\mu$ es el coeficiente de fricción entre elementos unbonded). Los elementos bonded tienen comportamiento lineal elástico hasta que se excede su resistencia límite y se rompe el enlace, convirtiéndose este elemento en tipo unbonded, en consecuencia, 
su comportamiento pasa a ser lineal elástico y friccional, con deslizamiento de acuerdo a la fuerza límite al corte impuesto por el criterio de Coulomb (Itasca Consulting Group Inc. 2014). En la Figura 3.2 se muestra la idealización esquemática realizada al emplear el FJCM en un material tipo roca.

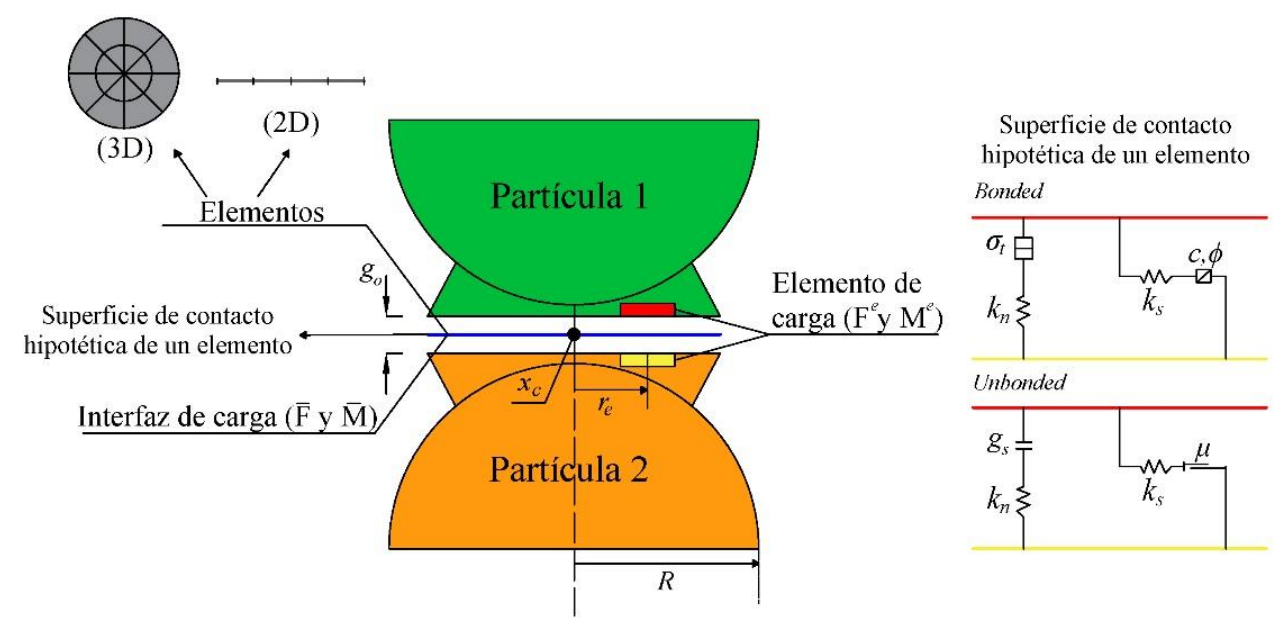

Figura 3.1. Comportamiento y componentes del FJCM: (modificado de Itasca Consulting Group Inc. 2014).

Los principales parámetros a calibrar en este modelo son: el módulo efectivo de la partícula y del contacto flat-joint $\left(E^{*}, \overline{E^{*}}\right)$, la relación de rigidez normal $\left(k_{n}\right)$ y cortante $\left(k_{s}\right)$ de la partícula y del contacto flat-joint $\left(k^{*}, \overline{k^{*}}\right)$; la cohesión $(c)$, y la resistencia a tracción $\left(\sigma_{t}\right)$ en el contacto flat-joint. Existen otros parámetros del FJCM -como el ángulo de fricción del material $(\phi)$, el número de elementos en la dirección radial para modelos 2D $\left(N_{R}\right)$, el número de elementos en la dirección circunferencial para modelos 3D $\left(N_{\alpha}\right)$, la fracción bonded $\left(\phi_{B}=\eta_{B} / \eta_{F J}, \eta_{B}\right.$ y $\eta_{F J}$ son el número de contactos bonded y total del FJCM, respectivamente), la fracción gapped $\left(\phi_{G}=\eta_{G} / \eta_{F J}, \eta_{G}\right.$ es el número de contactos gapped del FJCM) y el gap o separación inicial $\left(g_{o}\right)$ - que podrían tener influencia en el comportamiento del material, pero éstos no son considerados en el proceso de calibración llevado a cabo en esta tesis. 


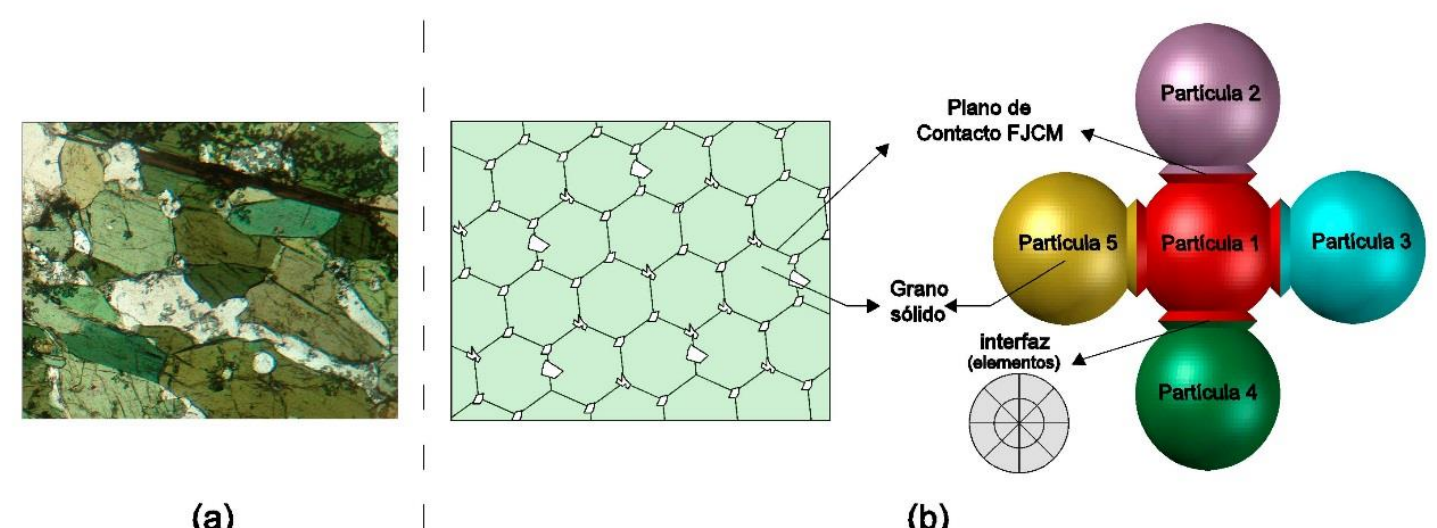

Figura 3.2. (a) Sección delgada de roca (fuente propia), (b) idealización de material Flat-Joint en PFC ${ }^{3 D}$ (modificado de Itasca Consulting Group Inc. 2014).

\subsubsection{Modelo de contacto smooth-joint (SJCM)}

EI SJCM simula el comportamiento de una interfaz plana existente entre varias partículas. La interfaz puede ser de tipo bonded o unbonded: la interfaz bonded tiene comportamiento lineal elástico hasta que se supera la resistencia límite y se rompe la unión, convirtiéndose entonces la interfaz a una de tipo unbonded; ésta última es de comportamiento lineal elástico y friccional (con dilatancia), estando su deslizamiento controlado por la fuerza límite al corte impuesta por el criterio de Coulomb (ver la Figura 3.3). La interfaz con SJCM no permite rotación relativa y, por tanto, los pares de partículas pueden superponerse o deslizarse, en lugar de ser forzadas a moverse unas sobre otras. En la Figura 3.4, se ilustra el patrón de desplazamientos a seguir por las partículas que interaccionan en el plano de la junta.
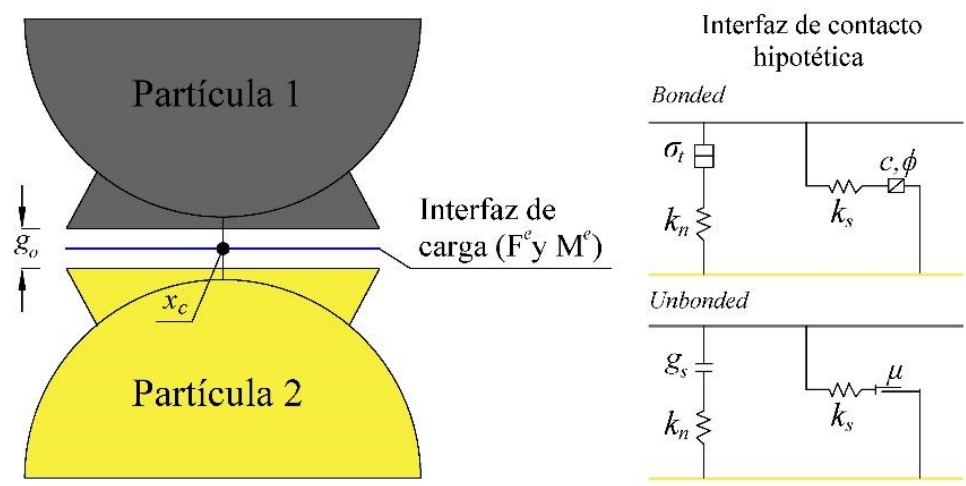

Figura 3.3 Comportamiento y componentes del SJCM (modificado de Itasca Consulting Group Inc. 2014). 
Los contactos smooth-joint se modelizan como dos superficies planas coincidentes (una por cada lado de la junta, ver la Figura 3.4(a)) y las partículas que están a cada lado de la interfaz son asociadas con su superficie correspondiente. Luego, durante cada paso de cálculo, los incrementos de desplazamientos de traslación relativos se descomponen en sus componentes normal $\left(\hat{n}_{j}\right)$ y tangencial $\left(\hat{n}_{t}\right)$ a la junta, y dichos componentes de desplazamientos son multiplicados por sus rigideces de la smooth-joint normal $\left(k_{n S J}\right)$ y de corte $\left(k_{s} s_{J}\right)$ para obtener los incrementos de fuerzas resultantes en la interfaz (Ivars et al. 2011).

Los parámetros principales que deben ser calibrados para el SJCM son: la rigidez normal de la smooth-joint $\left(k_{n S J}\right)$ y de corte $\left(k_{s S J}\right)$. Adicionalmente, si la interfaz es de tipo bonded, se debe calibrar la cohesión $\left(c_{S J}\right)$, la resistencia a tracción $\left(\sigma_{t S J}\right)$ y el ángulo de fricción $\left(\emptyset_{S J}\right)$, mientras que para interfaz unbonded, debe calibrarse el coeficiente de fricción en la interfaz $\left(\mu_{S J}\right)$.

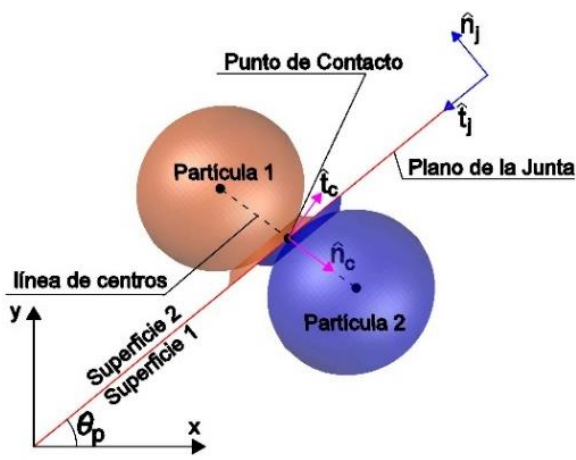

(a)

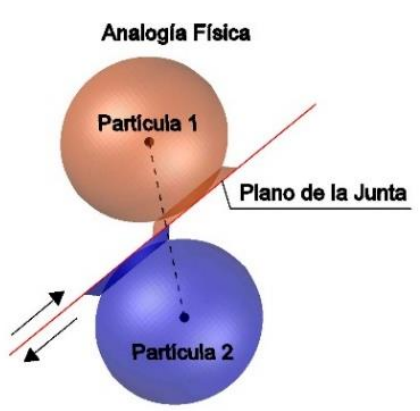

(b)

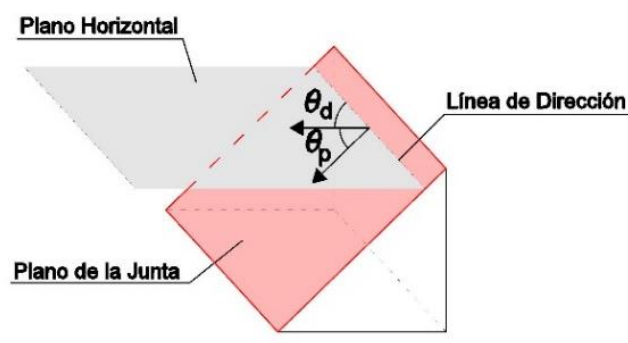

(c)

Figura 3.4 (a) Notación utilizada para definir la junta en el SJCM, $\hat{n}_{c}$ y $\hat{t}_{c}$ es el vector normal y perpendicular, respectivamente, de la línea de centros, (b) analogía física, (c) representación del ángulo de dirección $\left(\theta_{d}\right)$ y buzamiento $\left(\theta_{p}\right)$ de la interfaz SJCM (modificado de Ivars et al. 2011). 


\subsection{Configuración del modelo numérico para el ensayo de corte directo con MED}

El objetivo de esta fase es simular numéricamente el comportamiento de juntas de rocahormigón a través de ensayos de corte directo con condiciones de borde CNL y CNS, se sigue el esquema conceptual presentado en la Figura 3.5. Para ello se ha empleado el código PFC ${ }^{2 D}$ asignando, (i) el FJCM (ver la Sección 3.2.1) a las partículas que representan el comportamiento macroscópico del hormigón y la roca del ensayo, y (ii) el SJCM a las partículas ubicadas en la interfaz roca-hormigón (ver la Sección 3.2.2).

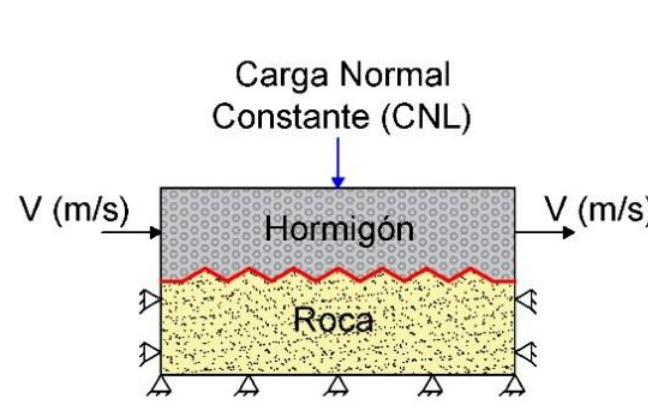

(a)

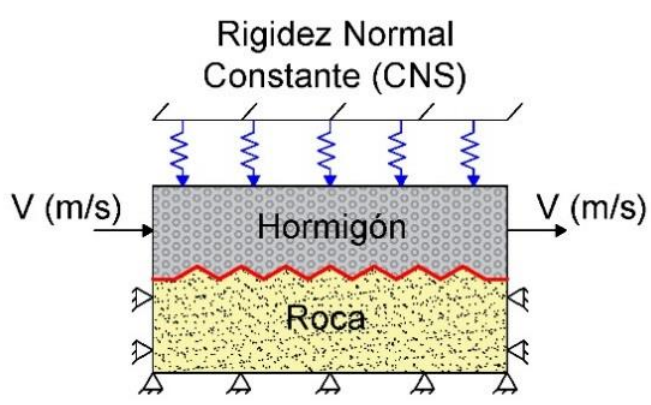

(b)

Figura 3.5. Esquema idealizado del ensayo de corte directo con condición de borde de: (a) CNL, (b) CNS (Gutiérrez-Ch et al. 2018).

La asignación de los microparámetros para reproducir el comportamiento macroscópico de los materiales presentes en el ensayo requiere de un proceso de calibración previo (ver la Sección 3.3.2), el cual se ha validado con datos experimentales de ensayos de compresión uniaxial realizados por Gu et al. (2003) y Gutiérrez (2013) (ver la Sección 3.3.1). Luego, se ha aplicado la metodología SBG propuesta por Bahaaddini et al. (2013b) para generar las probetas del ensayo corte directo con interfaz roca-hormigón con PFC $^{2 D}$ (ver la Sección 3.3.3). Finalmente, se ha realizado la calibración de los microparámetros del SJCM empleando los ensayos de corte experimentales de Gu et al. (2003) y Gutiérrez (2013). 


\subsubsection{Descripción de los ensayos experimentales}

Los datos experimentales provienen de ensayos de corte directo con interfaz rocahormigón sin cohesión publicados en la literatura. El primer grupo de ensayos fue publicado por Gutiérrez (2013), el cual realizó ensayos de corte directo para analizar la influencia de la rugosidad sobre el comportamiento al corte en la interfaz gneis-hormigón en un proyecto de presas de hormigón. Gutiérrez (2013) presenta información de 3 (gneis) + 4 (hormigón) ensayos de compresión uniaxial, así como resultados de 12 ensayos de corte directo con interfaz gneis-hormigón, considerando diferentes perfiles de rugosidad en el plano de la interfaz y condición de borde CNL. El segundo grupo de ensayos fue publicado por Gu et al. (2003) con el fin de estudiar el comportamiento al corte en la interfaz arenisca-hormigón de pilotes empotrados en roca; en particular, esta investigación presenta resultados de 37 (arenisca) + 6 (hormigón) ensayos de compresión uniaxial, y 112 ensayos de corte directo con interfaz arenisca-hormigón bajo condición de borde CNL y CNS.

\subsubsection{Calibración de los microparámetros del modelo de contacto flat-joint}

La elaboración y ejecución del ensayo de corte directo con MED, requiere la calibración previa de los microparámetros del FJCM asignado a los materiales (roca o hormigón) presentes en la simulación. Para ello, usualmente se realiza la simulación numérica con MED de ensayos de compresión uniaxial (UCT) y se validan los resultados con los datos experimentales obtenidos en este mismo tipo de ensayo para los materiales en análisis. En este caso, se han empleado para la validación los resultados experimentales de ensayos UCT publicados por Gutiérrez (2013) -gneis y hormigón (C2)- y por Gu et al. (2003) -arenisca (S1, S2, S3) y hormigón (C1)-.

Para comenzar la calibración se requiere la generación de la probeta sobre la cual se realizará el UCT con PFC ${ }^{2 D}$. Las probetas han sido generadas siguiendo la metodología propuesta por Potyondy y Cundall (2004), que consiste en los siguientes pasos: 
1) Ensamblaje inicial de la probeta: consiste en la distribución aleatoria de partículas dentro de una geometría acotada por paredes o walls, las cuales representan las dimensiones de la probeta a ensayar (ver la Figura 3.6(a)). En este paso, se asigna a las paredes del modelo una rigidez normal superior a la rigidez normal promedio de las partículas, para asegurar que la superposición entre los contactos pared-partícula sea pequeña. Los diámetros de las partículas que conforman la muestran cumplen una distribución uniforme definida por el diámetro mínimo $\left(D_{\min }\right)$ y máximo $\left(D_{\max }\right)$ de las mismas; en el cual, $D_{\min }$ y $D_{\max }$ se han establecido a partir de un análisis de sensibilidad en el cual se observó que para un diámetro de partículas promedio menor a $1.2 \mathrm{~mm}$ la resistencia al cortante pico no es afectada por el tamaño de las partículas.

2) Aplicación del esfuerzo isotrópico inicial: para reducir la magnitud de las fuerzas localizadas y obtener una mejor distribución de estas fuerzas a lo largo de todos los contactos, el radio de todas las partículas es cambiado de forma iterativa hasta alcanzar un valor de esfuerzo isotrópico definido por el usuario $\left(\sigma_{o}^{c} \cong 1 \%\right.$ de la resistencia a compresión uniaxial). Para ello, el código $\mathrm{PFC}^{2 \mathrm{D}}$ permite el uso de círculos de medición de esfuerzos (e.g., 3 círculos, ver la Figura 3.6(b)), los cuales son instalados dentro de la geometría de la probeta y, para cada paso de cálculo, el esfuerzo isotrópico dentro de cada círculo es calculado $\left(\sigma_{o}=\left(\sigma_{11}+\sigma_{22}\right) / 2\right)$. El proceso iterativo culmina cuando la diferencia normalizada entre el valor del esfuerzo isotrópico $\left(\sigma_{o}\right)$ medido dentro de todos los círculos y el valor de esfuerzo definido por el usuario $\left(\sigma_{o}^{c}\right)$ es menor que la tolerancia de esfuerzos establecida previamente por el usuario (de acuerdo con Bahaaddini et al. 2013b, se ha empleado la siguiente relación de tolerancia $\left.\frac{\sigma_{o}^{c}-\sigma_{o}}{\sigma_{o}^{c}} \leq 0.5\right)$.

3) Eliminación de partículas "flotantes": durante los pasos previos, es posible que resulten partículas con menos de tres (3) contactos dentro de la probeta (ver la Figura 3.6(c)). Como este comportamiento es "irreal", en este paso el radio de todas las 
partículas "flotantes" es incrementado hasta que éstas tengan al menos 3 contactos cada una.

4) Aplicación del FJCM: consiste en asignar e instalar el modelo de contacto flat-joint (con sus microparámetros correspondientes) a cada uno de los contactos entre partículas que cumplen con un gap inicial $\left(g_{o}\right)$ definido por el usuario.

5) Eliminación de las paredes laterales: para culminar la generación de la probeta, las paredes laterales son eliminadas y, mediante pasos de cálculo adicionales se le permite a la probeta alcanzar el equilibrio estático (ver la Figura 3.6(d)).
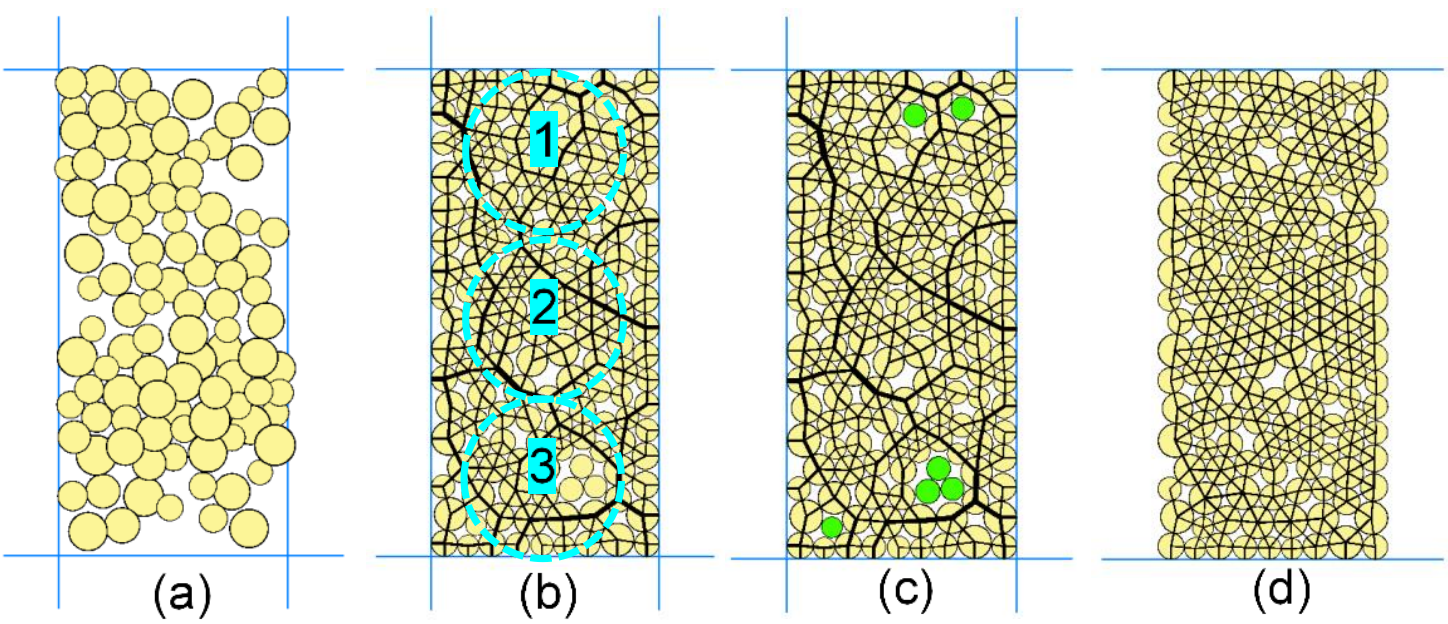

(d)

Figura 3.6. Procedimiento de generación de la probeta para el ensayo de compresión uniaxial con PFC2D: (a) generación inicial de las partículas antes del arreglo definitivo (las líneas azules representan las paredes o walls), (b) distribución de fuerzas de contacto después de la aplicación del paso 2 (las líneas negras representan las fuerzas de contacto y los círculos color cian representan los círculos de medición de esfuerzos), (c) identificación de las partículas "flotantes" durante el paso 3, (d) probeta generada después del paso 5 (las líneas negras representan la red de contactos entre partícula-partícula y pared-partículas. Notar que para facilitar la ilustración se han empleado partículas de mayor tamaño a las empleadas en los modelos de la investigación (Gutiérrez-Ch et al. 2018).

Una vez la probeta ha sido generada para el UCT con el MED, el proceso de calibración de los microparámetros se realiza de manera iterativa. En la literatura se han propuesto diferentes procedimientos para realizar la calibración (Lambert y Coll 2014; Potyondy y Cundall, 2004; Bahaaddini et al. 2014); sin embargo, en esta tesis se propone un procedimiento de calibración distinto, en base a los resultados de un análisis de sensibilidad de los microparámetros del FJCM (ver la Figura 3.7, tendencias similares 
fueron obtenidas en un análisis de sensibilidad realizado por Castro-Filgueira et al. 2017).

(a)
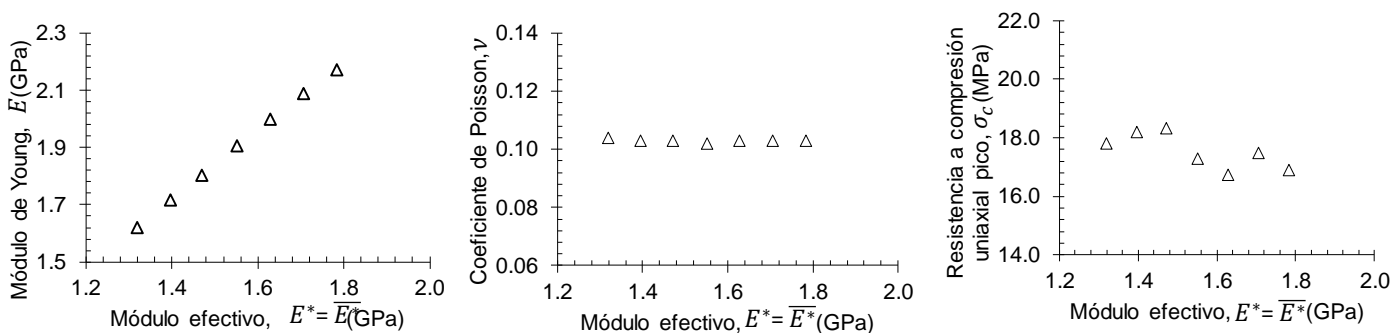

(b)
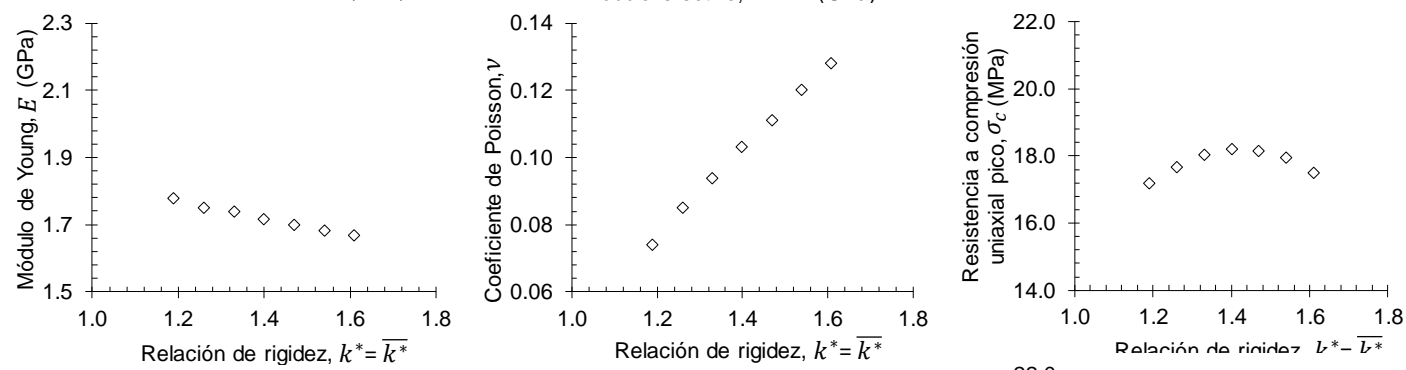

(c)
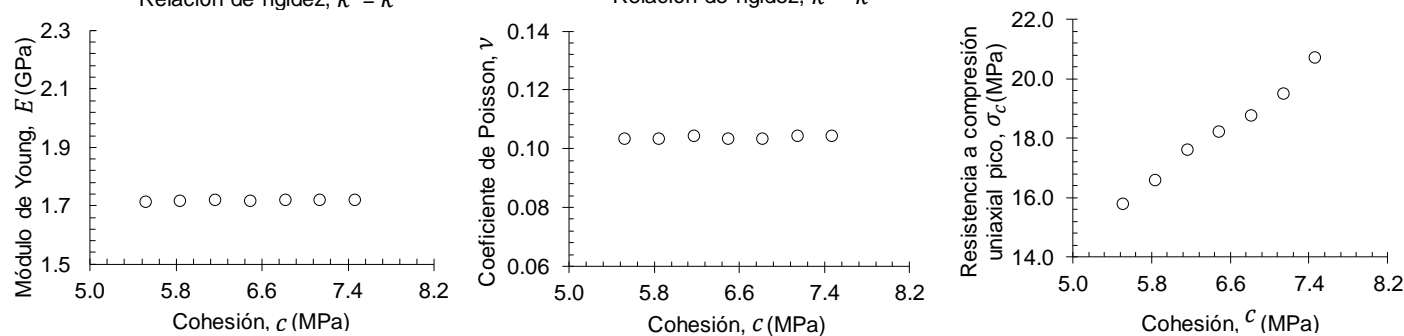

(d)
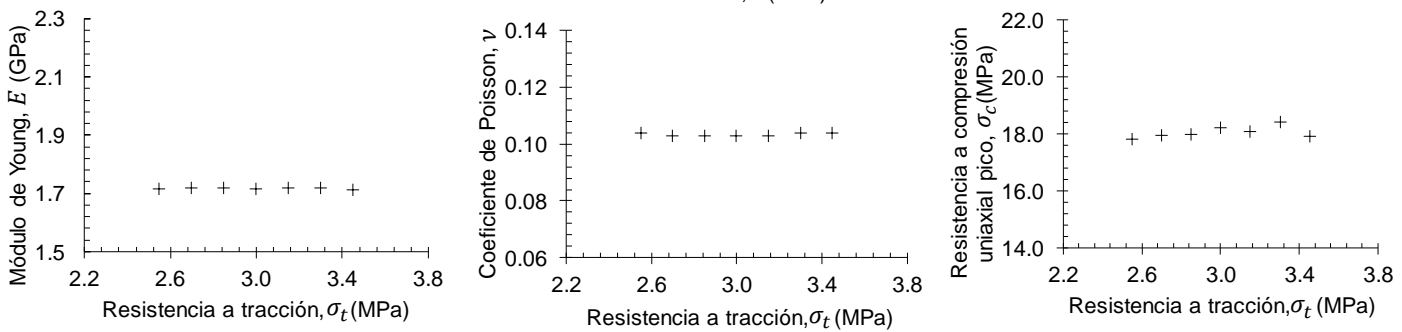

Figura 3.7. Análisis de sensibilidad del efecto de los microparámetros smooth-joint sobre los parámetros macroscópicos $\left(E, v\right.$ y $\left.\sigma_{c}\right)$ de la probeta de arenisca (S1) simulada con DEM ${ }^{2 \mathrm{D}}$ : (a) efecto del módulo efectivo $E^{*}=\overline{E^{*}}$, (b) efecto de la relación de rigidez normal y de corte $k^{*}=$ $\bar{k}^{*}$, (c) efecto de la cohesión, (d) efecto de la resistencia a tracción (Gutiérrez-Ch et al. 2018).

El procedimiento de calibración se presenta en el diagrama de flujo de la Figura 3.8, y se inicia con el ajuste de la relación entre la rigidez normal $\left(k_{n}\right)$ y cortante $\left(k_{s}\right)$ de la partícula y del contacto flat-joint $\left(k^{*}, \overline{k^{*}}\right)$, las cuales tienen influencia principalmente sobre la calibración del coeficiente de Poisson $(v)$ obtenido en la probeta (i.e., a escala real). Luego, se calibra el módulo de Young macroscópico $(E)$ modificando el módulo efectivo de la partícula y del contacto flat-joint $\left(E^{*}, \overline{E^{*}}\right)$. Finalmente, la resistencia a 
compresión uniaxial pico $\left(\sigma_{c}\right)$ obtenida experimentalmente se calibra mediante el ajuste de la cohesión $(c)$ y de la resistencia a tracción $\left(\sigma_{t}\right)$ en el contacto flat-joint.

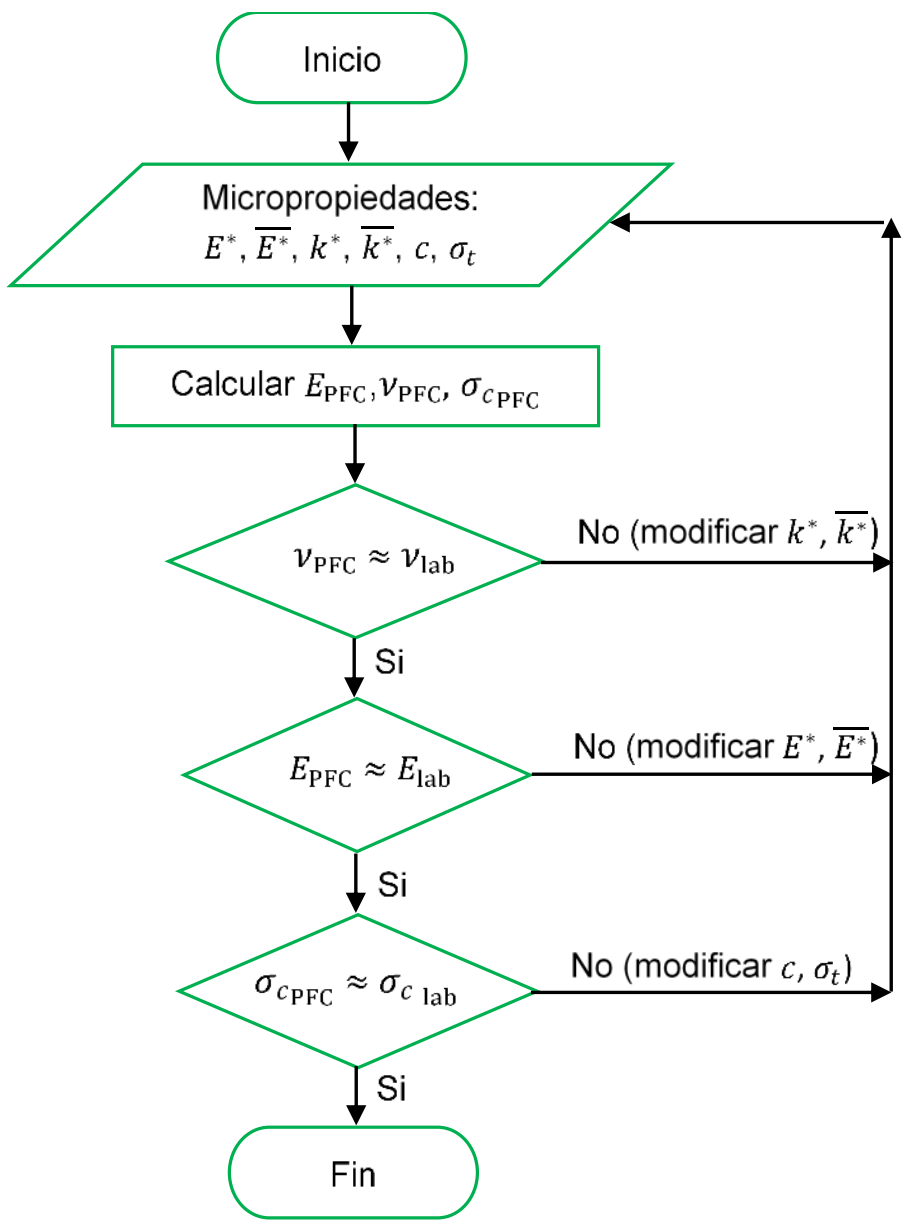

Figura 3.8. Proceso iterativo de calibración de las microparámetros flat-joint (Gutiérrez-Ch et al. 2018).

En la Tabla 3.1 se presentan las propiedades micromecánicas obtenidas para el FJCM después de que el proceso de calibración se ha completado, y la Tabla 3.2 compara las propiedades macroscópicas $-\sigma_{c}, E, v-$ calculadas en los ensayos de compresión uniaxial experimentales con aquellas obtenidas mediante los modelos numéricos con PFC ${ }^{2 D}$. En la Tabla 3.2 se presentan los promedios y desviaciones estándar de cada parámetro; la variación se debe a que los resultados de los modelos numéricos con MED dependen de la disposición inicial de las partículas, que a su vez depende de la "semilla" empleada en la generación aleatoria de las partículas que conforman la probeta. En la Figura 3.9 
se presentan diversas curvas obtenidas en los ensayos de compresión uniaxial con PFC ${ }^{2 D}$.

Tabla 3.1. Micropropiedades para el modelo de contacto flat-joint calibradas a partir de los datos experimentales de Gu et al. (2003) y Gutiérrez (2013).

\begin{tabular}{|c|c|c|c|c|c|c|}
\hline & \multicolumn{6}{|c|}{ Probeta } \\
\hline & (a) & (b) & (c) & (d) & (e) & $(\mathrm{f})$ \\
\hline \multicolumn{7}{|c|}{ Propiedades de las partículas } \\
\hline$E^{*}(\mathrm{GPa})$ & 1.55 & 1.70 & 1.90 & 27.00 & 43.00 & 26.20 \\
\hline$k^{*}=k_{n} / k_{s}$ & 1.40 & 1.35 & 1.45 & 2.75 & 3.25 & 3.00 \\
\hline Ángulo de fricción $\emptyset(\stackrel{\circ}{)})$ & 35 & 32 & 35 & 30 & 35 & 30 \\
\hline Densidad, $\rho\left(\mathrm{kg} / \mathrm{m}^{3}\right)$ & 2550 & 2500 & 2550 & 2500 & 2690 & 2500 \\
\hline Radio mínimo, $R_{\min }(\mathrm{mm})$ & 1.0 & 1.0 & 1.0 & 0.8 & 0.8 & 1.0 \\
\hline$R_{\max } / R_{\min }$ & 1.4 & 1.4 & 1.4 & 1.5 & 1.5 & 1.4 \\
\hline \multicolumn{7}{|c|}{ Propiedades de los contactos flat-joint } \\
\hline$\overline{E^{*}}(\mathrm{GPa})$ & 1.55 & 1.70 & 1.90 & 27.00 & 43.00 & 26.20 \\
\hline$\overline{k^{*}}$ & 1.40 & 1.35 & 1.45 & 2.75 & 3.25 & 3.00 \\
\hline$c(\mathrm{MPa})$ & 6.50 & 4.85 & 7.90 & 13.55 & 40.8 & 18.8 \\
\hline$\sigma_{t}(\mathrm{MPa})$ & 3.0 & 2.8 & 3.5 & 6.0 & 20.0 & 9.0 \\
\hline Relación bonding, $c / \sigma_{t}$ & 2.17 & 1.73 & 2.26 & 2.26 & 2.04 & 2.08 \\
\hline Fracción bonded, $\phi_{B}$ & 0.90 & 0.88 & 0.90 & 0.93 & 0.96 & 0.94 \\
\hline Fracción gapped, $\phi_{G}$ & 0.10 & 0.12 & 0.10 & 0.07 & 0.04 & 0.06 \\
\hline Gap inicial, $g_{o}(\mathrm{~mm})$ & 0.05 & 0.05 & 0.05 & 0.05 & 0.05 & 0.05 \\
\hline
\end{tabular}

Tabla 3.2. Macropropiedades obtenidas con el ensayo de compresión uniaxial experimental y numérico con $\mathrm{PFC}^{2 \mathrm{D}}$.

\begin{tabular}{cccc}
\hline \multirow{2}{*}{ Probeta } & Macropropiedades & Experimental & PFC $^{2 \mathrm{D}}$ \\
\cline { 2 - 4 } & \multicolumn{2}{c}{ Promedio } & Promedio \\
\hline a) Gneis y hormigón experimental publicados por Gutiérrez $(2013)$ \\
\hline \multirow{2}{*}{ Gneis } & $\sigma_{c}(\mathrm{MPa}) \pm \mathrm{DE}$ & $158.87 \pm 7.11(n=3)$ & $158.84 \pm 7.14(n=10)$ \\
& $E(\mathrm{GPa}) \pm \mathrm{DE}$ & 50 & $50.23 \pm 0.40(n=10)$ \\
& $v \pm \mathrm{DE}$ & 0.25 & $0.24 \pm 0.03(n=10)$ \\
\hline \multirow{2}{*}{ Hormigón } & $\sigma_{c}(\mathrm{MPa}) \pm \mathrm{DE}$ & $62.20 \pm 0.74(n=4)$ & $62.12 \pm 3.01(n=10)$ \\
$(\mathrm{C} 2)$ & $E(\mathrm{GPa}) \pm \mathrm{DE}$ & 30 & $29.98 \pm 0.26(n=10)$ \\
& $v \pm \mathrm{DE}$ & 0.20 & $0.20 \pm 0.02(n=10)$ \\
\hline b) Arenisca y hormigón experimental publicados por Gu et al. $(2003)$ \\
\hline \multirow{2}{*}{ Arenisca } & $\sigma_{c}(\mathrm{MPa}) \pm \mathrm{DE}$ & $17.44 \pm 0.71(n=9)$ & $17.39 \pm 0.83(n=10)$ \\
$(\mathrm{S} 1)$ & $E(\mathrm{GPa}) \pm \mathrm{DE}$ & $2.75 \pm 0.25(n=5)$ & $1.89 \pm 0.02(n=10)$ \\
& $v \pm \mathrm{DE}$ & $0.09 \pm 0.00(n=9)$ & $0.10 \pm 0.02(n=10)$ \\
\hline \multirow{2}{*}{ Arenisca } & $\sigma_{c}(\mathrm{MPa}) \pm \mathrm{DE}$ & $11.49 \pm 3.19(n=8)$ & $11.59 \pm 0.97(n=10)$ \\
$(\mathrm{S} 2)$ & $E(\mathrm{GPa}) \pm \mathrm{DE}$ & $1.98 \pm 0.68(n=5)$ & $2.01 \pm 0.02(n=10)$ \\
& $v \pm \mathrm{DE}$ & $0.10 \pm 0.00(n=3)$ & $0.10 \pm 0.01(n=10)$ \\
\hline \multirow{2}{*}{ Arenisca } & $\sigma_{c}(\mathrm{MPa}) \pm \mathrm{DE}$ & $21.77 \pm 1.19(n=8)$ & $21.65 \pm 1.43(n=10)$ \\
$(\mathrm{S} 3)$ & $E(\mathrm{GPa}) \pm \mathrm{DE}$ & $3.25 \pm 0.39(n=5)$ & $2.32 \pm 0.02(n=10)$ \\
& $v \pm \mathrm{DE}$ & $0.10 \pm 0.00(n=3)$ & $0.11 \pm 0.01(n=10)$ \\
\hline \multirow{2}{*}{ Hormigón } & $\sigma_{c}(\mathrm{MPa}) \pm \mathrm{DE}$ & $40 \pm 0.33(n=3)$ & $39.87 \pm 2.73(n=10)$ \\
$(\mathrm{C} 1)$ & $E(\mathrm{GPa}) \pm \mathrm{DE}$ & $29.95 \pm 0.08(n=3)$ & $30.08 \pm 0.23(n=10)$ \\
& $v \pm \mathrm{DE}$ & 0.20 & $0.20 \pm 0.02(n=10)$ \\
\hline
\end{tabular}

DE: Desviación estándar; $n$ : número de ensayos 
Adicionalmente, en la Tabla 3.2 se observa que (i) los valores promedios de las macropropiedades $-\sigma_{c}, E, v-$ calculados a partir de los modelos numéricos reproducen bien los valores calculados en los ensayos experimentales, y (ii) que la desviación estándar obtenida en los ensayos experimentales es mayor en las probetas de roca que en las de hormigón. Esto probablemente puede atribuirse a la presencia de defectos de la propia génesis de la roca -e.g., planos de debilidad, microgrietas, etc.- que quizás no son comunes en materiales con fabricación controlada como ocurre con el hormigón. Notar, además, que los resultados de los modelos numéricos con PFC presentan variaciones similares para la muestra de roca y hormigón debido a que, en el proceso de generación de la probeta de hormigón o roca, el algoritmo con MED no toma en cuentas estas diferencias en su génesis.

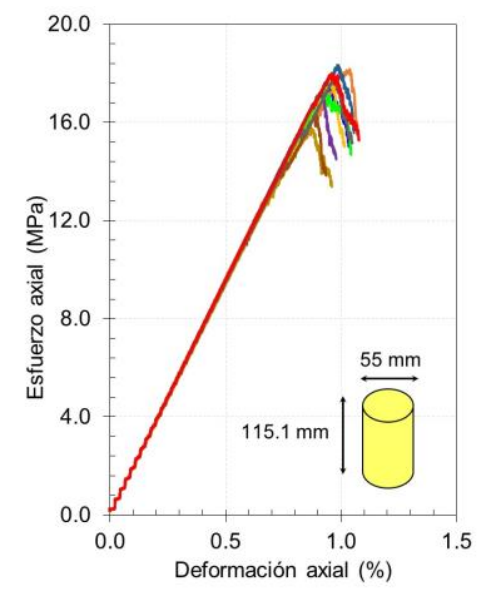

(a)

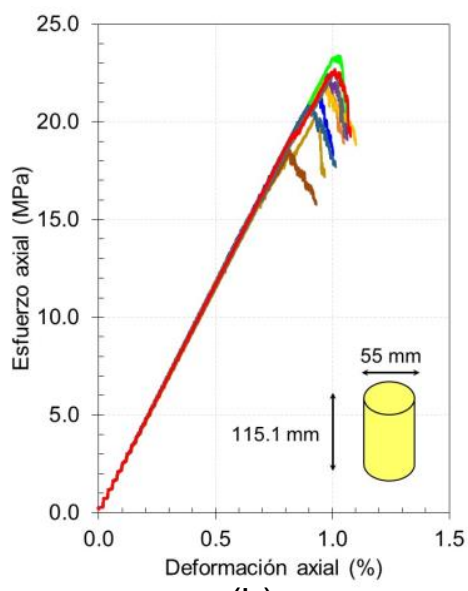

(b)

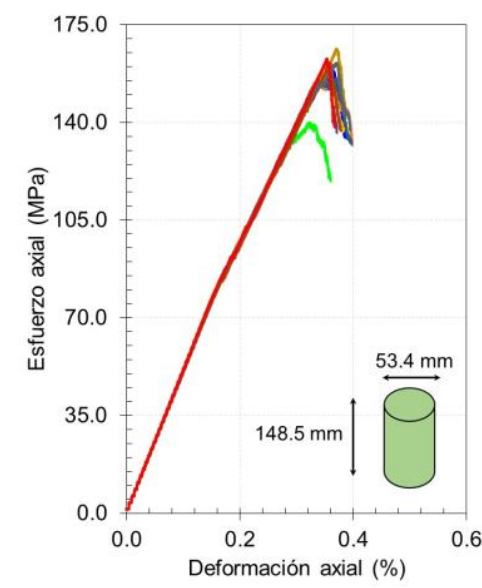

(c)

Figura 3.9. Curvas esfuerzo-deformación axial obtenidas en 10 ensayos de compresión uniaxial con diferente valor de la "semilla" en el proceso de generación de la probeta con PFC2D: (a) arenisca (S1), (b) arenisca (S3), (c) gneis (Gutiérrez-Ch et al. 2018).

Adicionalmente, con el fin de analizar el posible efecto escala al considerar modelos 2D ó 3D en el proceso de calibración, se realizaron ensayos de compresión uniaxial con $\mathrm{PFC}^{3 \mathrm{D}}$ en probetas de arenisca (S3), siguiendo el proceso iterativo descrito en la Figura 3.8 y con los microparámetros presentados en la Tabla 3.1. Los resultados se presentan en la Tabla 3.3 y Figura 3.10. Como puede observarse, ambas probetas presentan un mecanismo de rotura similar (ver la Figura $3.10(b)$ ), y no se aprecia una diferencia significativa de los resultados macroparamétricos obtenidos $-\sigma_{c}, E, v-$ al emplear 
modelos $P F C^{2 D}$ ó $P F C^{3 D}$, excepto por una disminución en el coeficiente de variación (COV) y una ligera sobreestimación de la resistencia a compresión uniaxial pico obtenida cuando se emplean modelos PFC ${ }^{3 D}$ (ver la Tabla 3.3 y la Figura 3.10(c)). Estos resultados están en concordancia con los obtenidos por Ding et al. (2014) en ensayos similares.

Tabla 3.3. Macropropiedades obtenidas con el ensayo de compresión uniaxial experimental y numérico con $\mathrm{PFC}^{2 \mathrm{D}}$ y $\mathrm{PFC}^{3 \mathrm{D}}$

\begin{tabular}{|c|c|c|c|c|c|c|}
\hline \multirow{2}{*}{ Macropropiedades } & \multicolumn{2}{|c|}{$\begin{array}{c}\text { Experimental } \\
\text { de Gu et al. (2003) }\end{array}$} & \multicolumn{2}{|c|}{$\mathrm{PFC}^{2 \mathrm{D}}$} & \multicolumn{2}{|c|}{$\mathrm{PFC}^{3 \mathrm{D}}$} \\
\hline & Promedio & $\begin{array}{l}\text { COV } \\
(\%)\end{array}$ & Promedio & $\begin{array}{l}\text { COV } \\
(\%)\end{array}$ & Promedio & $\begin{array}{l}\text { COV } \\
(\%)\end{array}$ \\
\hline$\sigma_{c}(\mathrm{MPa}) \pm \mathrm{DE}$ & $\begin{array}{c}21.77 \pm 1.19 \\
(n=8)\end{array}$ & 5.5 & $\begin{array}{c}21.65 \pm 1.43 \\
(n=10)\end{array}$ & 6.6 & $\begin{array}{c}24.66 \pm 0.28 \\
(n=10)\end{array}$ & 1.1 \\
\hline$E(\mathrm{GPa}) \pm \mathrm{DE}$ & $\begin{array}{c}3.25 \pm 0.39 \\
(n=5)\end{array}$ & 11.9 & $\begin{array}{c}2.32 \pm 0.02 \\
(n=10)\end{array}$ & 0.9 & $\begin{array}{c}2.35 \pm 0.01 \\
(n=10)\end{array}$ & 0.4 \\
\hline$v \pm \mathrm{DE}$ & $\begin{array}{c}0.1 \pm 0.00 \\
(n=3)\end{array}$ & 0.0 & $\begin{array}{c}0.11 \pm 0.01 \\
(n=10)\end{array}$ & 13.3 & $\begin{array}{c}0.10 \pm 0.0 \\
(n=10)\end{array}$ & 5.0 \\
\hline
\end{tabular}

DE: Desviación estándar; COV: Coeficiente de variación; $n$ : número de ensayos

(a)

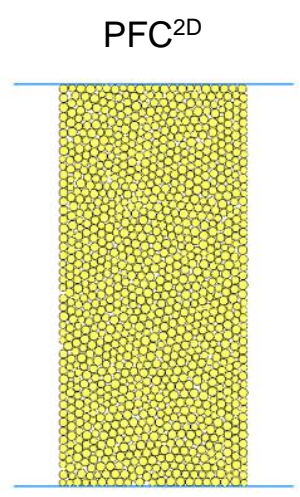

(b)

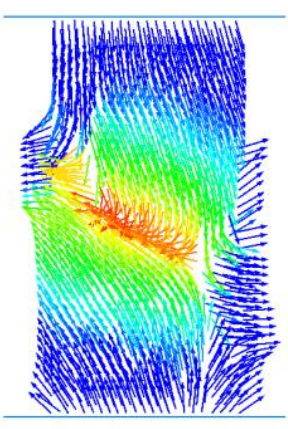

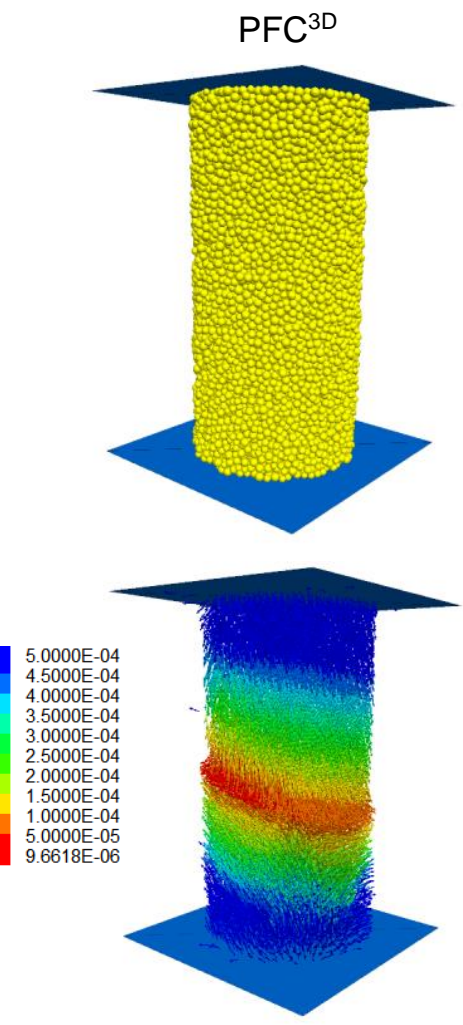

(c)

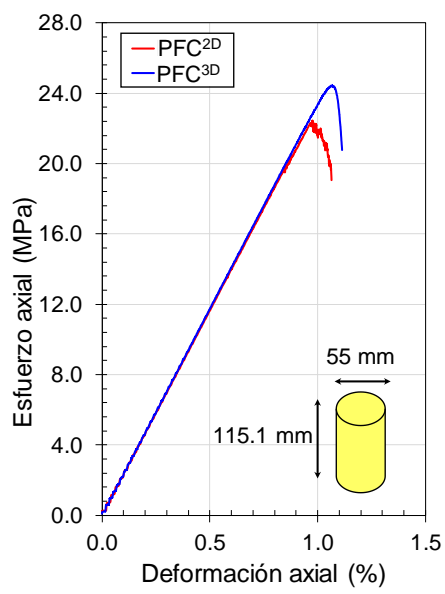

Figura 3.10. Comparación de modelos con PFC 2D y PFC 3D: (a) probeta inicial arenisca (S3), (b) vectores de desplazamiento total al final del ensayo (unidades en metros), (c) curva esfuerzodeformación axial. 


\subsubsection{Generación de la probeta para el ensayo de corte directo y aplicación de las condiciones de borde}

En el Capítulo 2 (Sección 2.2.2.6) se presentaron los diferentes métodos existentes en la literatura -como el Bond-Removal Approach (BRA) o el Smooth Joint Contact Model (SJCM) - para simular ensayos de corte directo con interfaz roca-roca o roca-hormigón, así como las dificultades asociadas con cada una de estas metodologías.

Para analizar en detalle la deficiencia de la técnica BRA debida al "interlocking" particles, en este trabajo de tesis doctoral se realizó un modelo simplificado de un ensayo de corte directo en $\mathrm{PFC}^{2 \mathrm{D}}$, en junta roca-roca, sin cohesión y con condición CNL. (Notar que para facilitar su ilustración se emplearon tamaños de partículas superiores a los empleados en un modelo con MED definitivo, ver la Figura 3.11). Para ello:

(a) Se genera en forma simultánea y aleatoria, la distribución de las partículas dentro la caja de corte delimitadas por las paredes del modelo. Para esto, se emplea la metodología BPM con el modelo de contacto Linear Parallel Bond (LPB) (las líneas negras de representan la red de contactos entre partículas con el modelo LPB, ver la Figura 3.11(a)).

(b) Posteriormente, se instala el plano hipotético de la junta "real" (línea azul segmentada en la Figura 3.11(b)) y, mediante la técnica BRA, se remueve la cohesión de todos aquellos contactos partícula-partícula (puntos rojos y partículas de color verde en la Figura 3.11(c)) que son atravesadas por dicho plano -i.e., a todos aquellos contactos cuyas línea de "centros" son cortadas por la línea de la junta "real", ver la Figura 3.11(g)-. (Notar como las líneas negras de la red de contactos de la probeta inicial han sido removidas de los contactos entre partículas de color verde de la caja de corte superior hacia la inferior). Como consecuencia, se produce una rugosidad inherente a lo largo de toda la junta. La rugosidad inherente es aquella generada producto de la eliminación de 
la cohesión entre contactos partícula-partícula en el plano de la junta, en la cual, la junta resultante del ensayo de corte directo con MED queda conformada por la unión de los segmentos de cada plano hipotético del contacto cuya cohesión ha sido removida (línea roja en la Figura 3.11(d)). El plano hipotético en cada contacto partícula-partícula es la línea perpendicular a la línea de "centros", ver la Figura 3.11(g). Adicionalmente, para representar el problema "interlocking" particles se emplean 4 partículas de control (2 partículas rojas y 2 partículas azules, ver la Figura 3.11(d)).

(c) Finalmente, el ensayo inicia con la aplicación de una velocidad $\left(V_{x}\right)$ constante sobre la caja de corte superior mientras la caja inferior permanece fija (ver Figura 3.11(e)). (Notar que a fin de generar la separación que usualmente hay entre la caja de corte superior e inferior de estos ensayos, se eliminan segmentos de las paredes laterales medidos hacia arriba y hacia abajo del plano de la junta).

Como puede observarse en la Figura 3.11(f), después de cierto desplazamiento al corte alcanzado en el ensayo, se genera una concentración de esfuerzos en algunas partículas del plano de contacto; esto es debido a que algunas partículas (e.g., la partícula azul en la Figura 3.11(e)-(g)) se ven forzadas a moverse sobre la superficie perimetral de su partícula vecina, con dilatación (e.g., la partícula roja en la Figura 3.11(e)-(g)), y en consecuencia, hay una concentración momentánea de energía con su posterior disipación (después de haberse superado cierto desplazamiento). Este comportamiento es el causante de que resistencia al corte pico obtenida con el MED sea superior al valor hallado experimentalmente, así como también, que la resistencia al corte residual (con el MED) sea menor a la hallada en el ensayo de laboratorio, siendo esto un comportamiento al corte poco realista en comparación con lo observado ensayos de laboratorio (ver la Figura 3.12). 


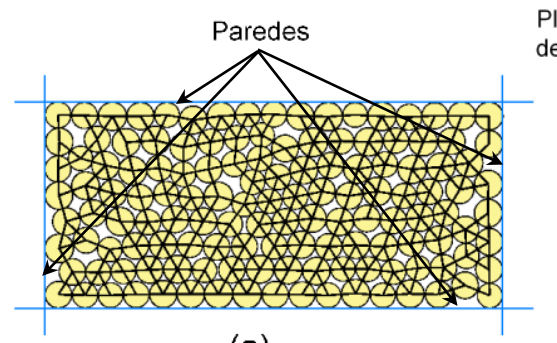

(a)

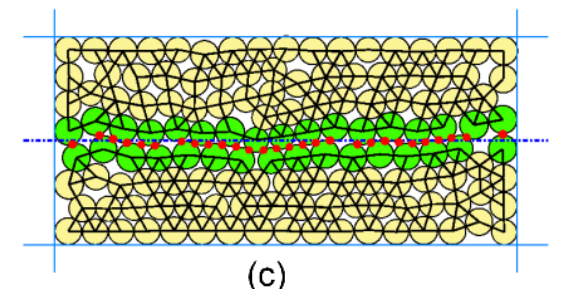

(c)

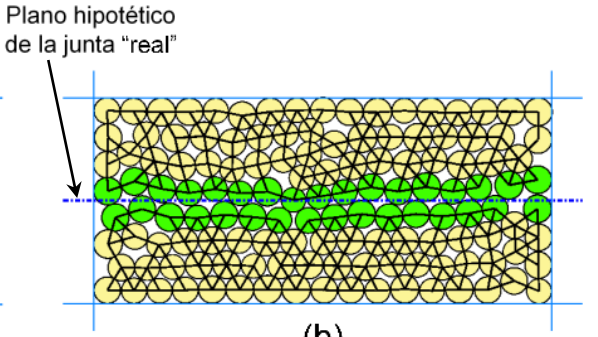

(b)

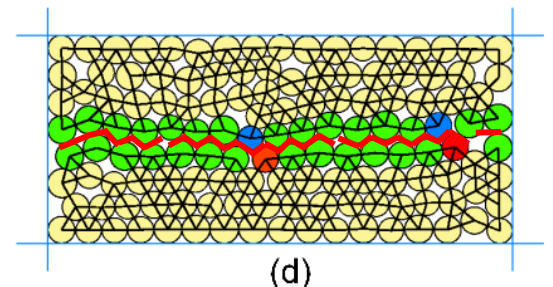

(d)

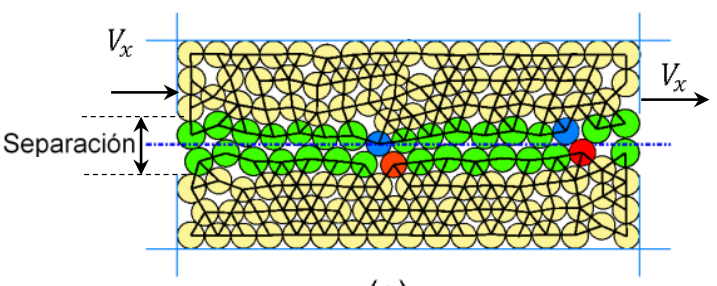

(e)

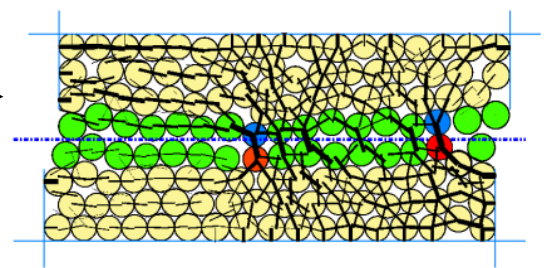

(f)

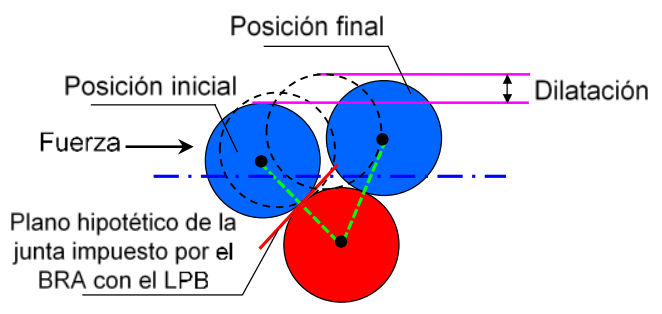

(g)

Figura 3.11. Deficiencia de la técnica BRA: (a) generación de la probeta, (b) instalación del plano hipotético de la junta y aplicación de la técnica BRA, (c) probeta después de la aplicación de la técnica BRA, (d) rugosidad inherente resultante de la técnica BRA. (e) probeta del modelo PFC antes de iniciar el ensayo, (f) concentración de esfuerzos y dilatación de la caja de corte para un desplazamiento al corte alcanzado (las líneas de color negro representan la intensidad de las fuerzas de contacto), (g) detalle de la deficiencia de la técnica BRA.
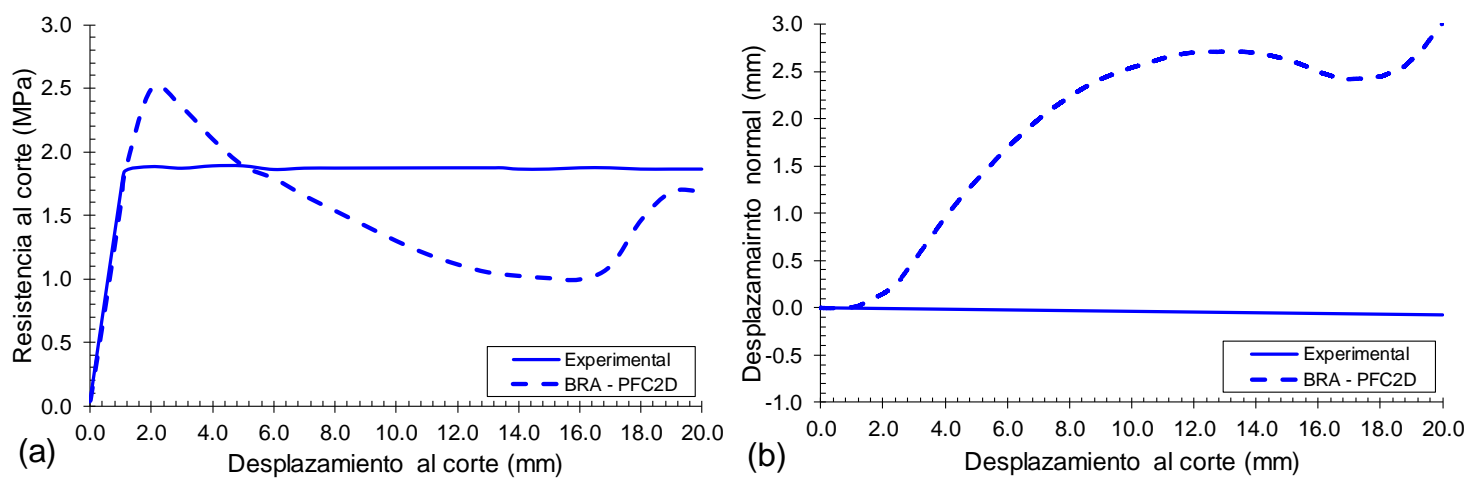

Figura 3.12. Comparación entre ensayo de corte directo experimental y numérico (usando la técnica BRA), en junta plana roca-roca, bajo condición CNL $\left(\sigma_{n}=2.67 \mathrm{MPa}\right)$ : (a) resistencia al corte vs desplazamiento al corte, (b) desplazamiento normal vs desplazamiento al corte. 
La deficiencia de la técnica BRA descrita anteriormente, conllevo a la nueva propuesta del modelo de contacto SJCM (ver la Sección 3.2.2). Sin embargo, Bahaaddini et al. (2013b) reportaron que éste nuevo modelo de contacto también presentaba inconvenientes en reproducir el comportamiento de resistencia al corte. A continuación, se presenta un modelo simplificado (similar al empleado en la explicación de la deficiencia de la técnica BRA) con el objetivo de ilustrar en detalle esta limitación del modelo SJCM para reproducir el comportamiento al corte. Este modelo consiste en:

(a) Se genera, en forma simultánea y aleatoria, la distribución de las partículas dentro la caja de corte delimitadas por las paredes del modelo. Para esto, se emplea la metodología BPM con el modelo de contacto Linear Parallel Bond (LPB) (las líneas negras de representan la red de contactos entre partículas con el modelo LPB, ver la Figura 3.13(a))

(b) Posteriormente, se instala el plano hipotético de la junta "real" (línea azul segmentada en la Figura 3.13(b)) y se asigna el modelo SJCM a los contactos partícula-partícula cuyas línea de "centros" son cortadas por dicho plano (partículas de color verde en la Figura 3.13(b)). Cada contacto con este modelo lleva asociado un vector unitario normal al plano de la junta (líneas color morado en la Figura 3.13(b)), con su correspondiente red de contactos (líneas amarillas en la Figura 3.13(c)). Adicionalmente, para representar el problema "interlocking" particles con el modelo SJCM, se emplean 3 partículas de control (1 roja, 1 azul, y 1 fucsia, ver la Figura 3.13(c)).

(c) Finalmente, el ensayo se inicia con la aplicación de una velocidad $\left(V_{x}\right)$ constante sobre la caja de corte superior mientras que la caja inferior permanece fija (ver la Figura 3.13(c)). 


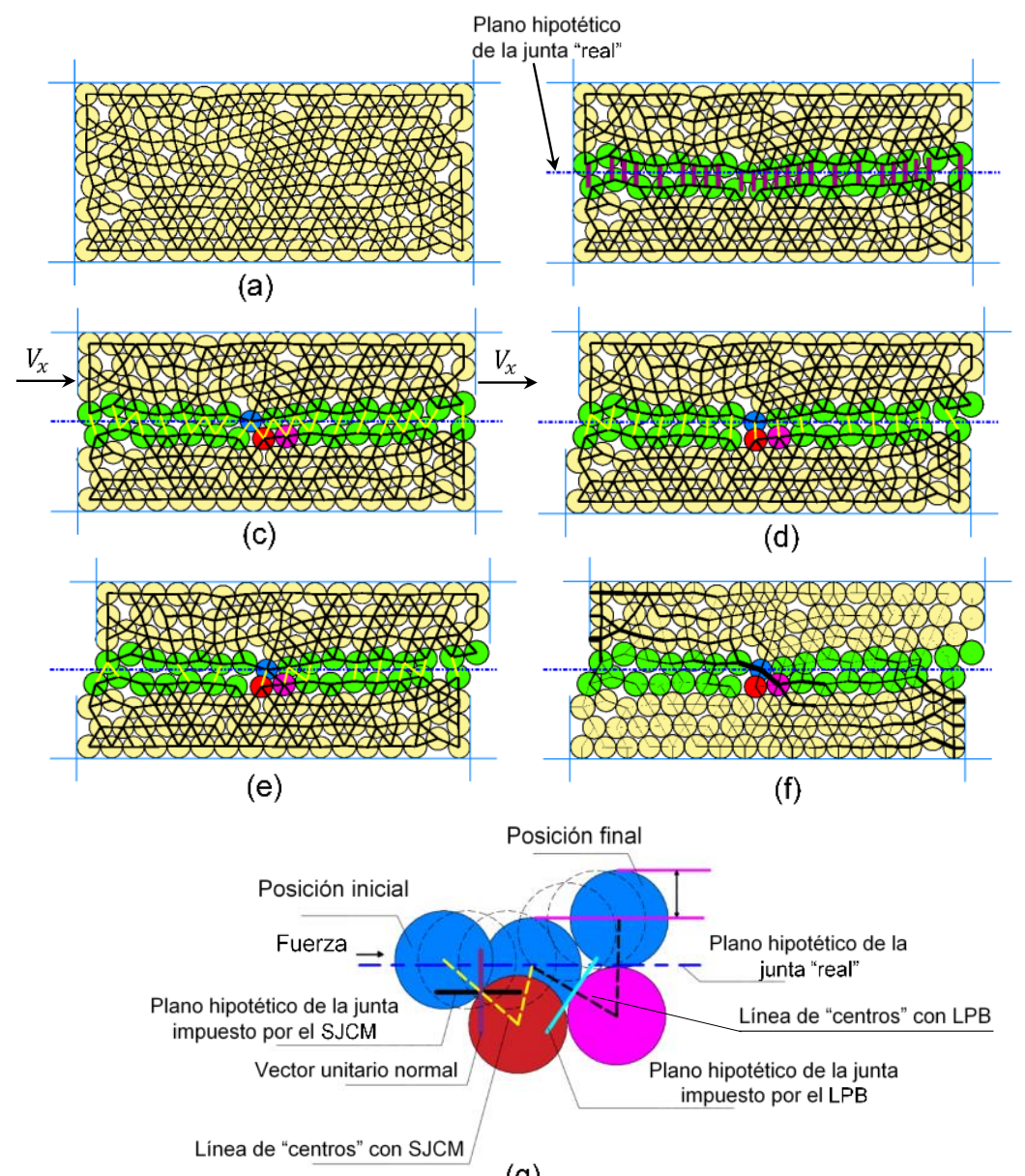

(g)

Figura 3.13. Deficiencia en el uso del SJCM: (a) generación de la probeta, (b) instalación del plano hipotético de la junta y aplicación del SJCM, (c) probeta del modelo antes de iniciar el

ensayo, (d) probeta para un desplazamiento al corte menor a $D_{\min }$..(e) probeta para un desplazamiento al corte mayor a $D_{\min }$ (f) concentración de esfuerzos y dilatación de la caja de corte del ensayo después de cierto valor de desplazamiento al corte (las líneas de color negro representan la intensidad de las fuerzas de contacto), (g) detalle de la deficiencia en el uso del modelo SJCM.

Una vez comenzado el ensayo de corte directo en el modelo $\mathrm{DEM}^{2 \mathrm{D}}$, las partículas (e.g., las partículas azul y roja en la Figura 3.13(c)) que tienen el modelo SJCM, deslizan unas respecto a las otras a través de plano hipotético de la junta impuesto por el modelo SJCM (ver detalle en la Figura 3.13(g)). Por tanto, para desplazamientos al corte menores a $D_{\text {min }}$, el comportamiento al corte es muy similar al comportamiento experimental de la probeta del ensayo (ver la Figura 3.13). Sin embargo, para desplazamientos al corte mayores a $D_{\min }$, se producen nuevos contactos entre las partículas. Si la línea de "centros" (e.g., la línea negra de la red de contactos entre las partícula azul y fucsia de la Figura 3.13(e), o la línea negra segmentada para estas mismas partículas en la Figura 3.13(g)) de los nuevos contactos que se generan entre 
partículas se encuentran por debajo del plano hipotético de la junta, se produce una inadecuada asignación del modelo a estos nuevos contactos. (Notar además que, debido a la acción de la carga normal actuante sobre la caja de corte, la partícula azul se movió hacia abajo, ver detalle en la Figura 3.13(g)). Luego, estos nuevos contactos son asignados de forma errónea como LPB en lugar de SJCM, produciéndose el problema "interlocking" particles (descrito previamente en el uso de la técnica BRA). Este comportamiento genera una inadecuada concentración de esfuerzos y la dilatancia entre las partículas azul y fucsia (ver la Figura 3.13(f)-(g)), y en consecuencia, no se obtiene un resultado adecuado de resistencia al corte en el ensayo numérico para desplazamientos al corte mayores que $D_{\min }$ (ver la Figura 3.14).
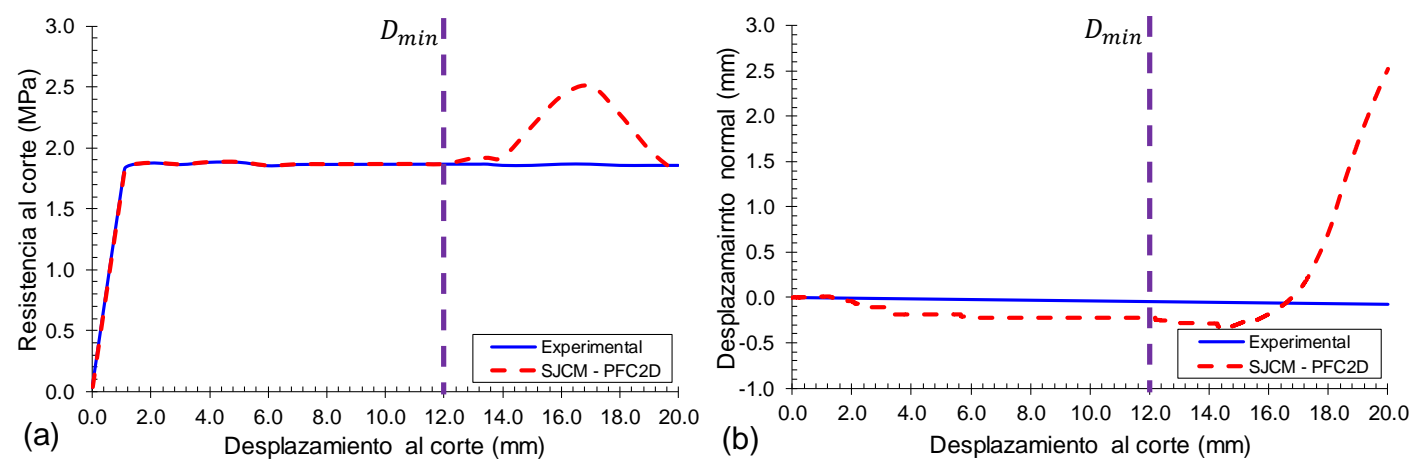

Figura 3.14. Comparación entre ensayo de corte directo experimental y numérico con el modelo SJCM, en junta plana roca-roca, bajo condición CNL $\left(\sigma_{n}=2.67 \mathrm{MPa}\right)$ : (a) resistencia al corte versus desplazamiento al corte, (b) desplazamiento normal vs desplazamiento al corte.

Para superar estas deficiencias, Bahaaddini et al. (2013b) propusieron el método Shear Box Genesis (SBG) para simular ensayos de corte directo con PFC ${ }^{2 D}$. El procedimiento está basado en la metodología para la génesis de probetas descrita en la Sección 3.3.2, utilizando el SJCM propuesto por Potyondy y Cundall (2004). Consiste en:

1) Ensamblaje inicial de la caja de corte directo: consiste en la distribución aleatoria de partículas dentro de las paredes que conforman caja de corte superior (hormigón) e inferior (roca) del ensayo. Esta generación se realiza de forma simultánea e independiente para cada material, siguiendo los pasos 1 al 3 descritos en la Sección 3.3.2 (ver la Figura 3.15(a)-(c)). 
2) Aplicación del FJCM y SJCM: en primer lugar, es asignado el modelo de contacto flatjoint a cada uno de los contactos entre partículas de la roca y del hormigón (líneas negras de la Figura 3.15(d)), y se retiran las paredes que representan el plano de contacto de la interfaz roca-hormigón; así, las partículas que están en contacto con el plano de la junta son identificadas y el modelo de contacto smooth-joint es asignado a los contactos (líneas rojas y moradas de la Figura 3.15(d)-(e)). De esta manera, se resuelve el problema de la detección inadecuada de las partículas presentes en la interfaz de ambos materiales y se permite su fácil aplicación en ensayos de corte directo con juntas de cualquier morfología (Bahaaddini et al. 2013b). Para más detalles de la metodología SBG se sugiere consultar a Bahaaddini et al. (2013b).

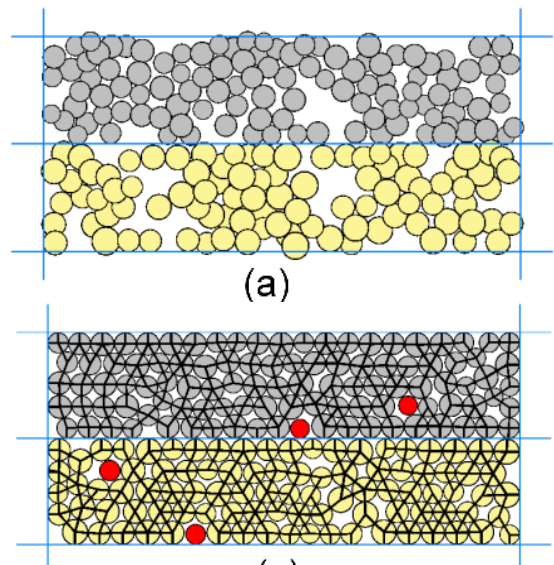

(c)

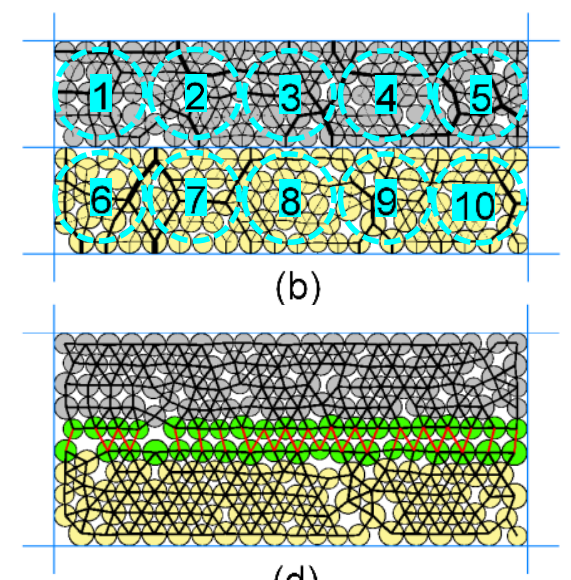

(d)

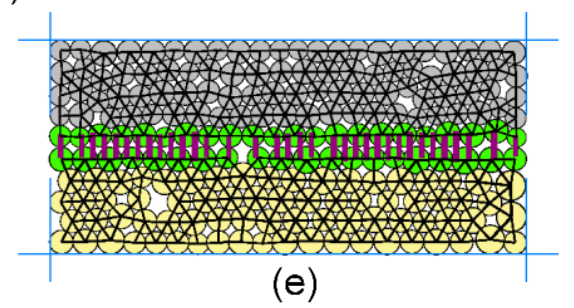

Figura 3.15. Metodología Shear Box Genesis (SBG): (a) generación inicial de las partículas antes de la distribución final (las partículas amarillas y grises representan la roca y el hormigón, respectivamente; las líneas azules representan las paredes de la caja de corte superior e inferior), (b) instalación del esfuerzo isotrópico (las líneas negras representan la intensidad de las fuerzas y las líneas segmentadas azules representan los círculos de medición de esfuerzos), (c) detección de partículas "flotantes", (d) probeta después de la aplicación de los modelos de contacto FJCM y SJCM, (e) probeta con PFC ${ }^{2 D}$ antes de comenzar el ensayo de corte directo (Gutiérrez-Ch et al. 2018).

Utilizando el procedimiento anteriormente descrito, se han generado las probetas de los modelos numéricos con $\mathrm{PFC}^{2 \mathrm{D}}$ que representa los ensayos experimentales de corte 
directo con interfaz roca-hormigón empleados para comparar y validar los parámetros del SJCM. En particular, para los modelos numéricos que simulan las probetas de $135 \times$ 140 mm² con interfaz arenisca-hormigón ensayadas por Gu et al. (2003); así como las probetas de $240 \times 260 \mathrm{~mm}^{2}$ con interfaz gneis-hormigón ensayados por Gutiérrez (2013). La Figura 3.16 ilustra las probetas generadas con $P F C^{2 D}$, así como los perfiles de rugosidad empleados en cada caso.

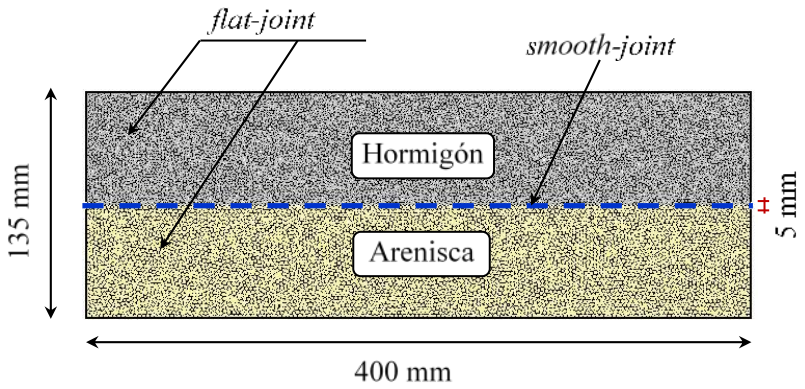

(a)

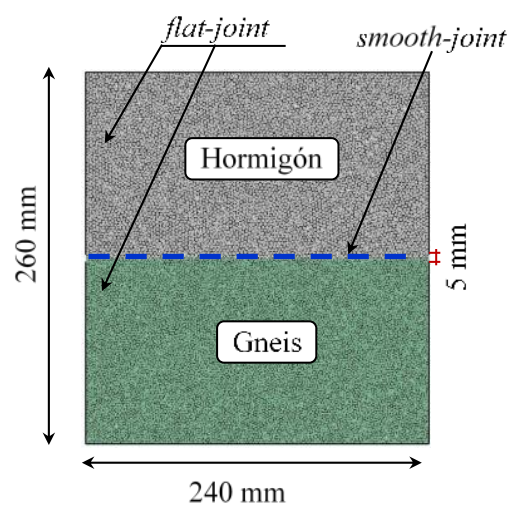

(c)

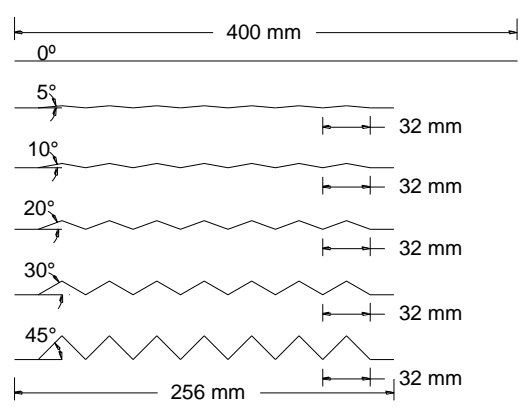

(b)

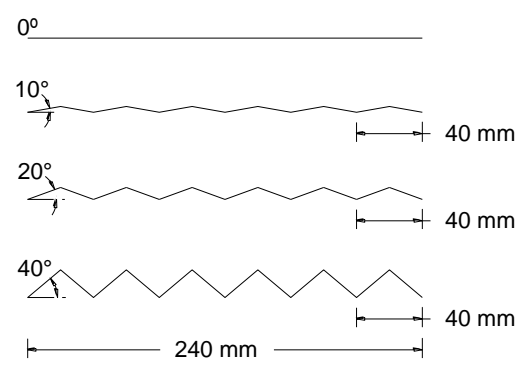

(d)

Figura 3.16. Probeta para el ensayo de corte directo con PFC2D: (a) modelo arenisca-hormigón (alrededor de 12241 partículas), (b) perfiles con junta o interfaz plana y forma triangular simétrica empleados para los modelos arenisca-hormigón, (c) modelo gneis-hormigón (alrededor de 14517 partículas), (d) perfiles con junta o interfaz plana y forma triangular simétrica empleados para los modelos gneis-hormigón (las partículas de color gris, amarillo y verde representan al hormigón, arenisca y gneis, respectivamente; las líneas de color azul segmentadas representan el plano de la interfaz roca-hormigón, y la línea de color rojo representa el espaciado entre la caja de corte inferior y superior) (Gutiérrez-Ch et al. 2018).

Posteriormente, la generación de la probeta para el modelo numérico culmina con la aplicación de las condiciones de borde que representen las condiciones del problema analizado, es decir, condición de borde $\operatorname{CNL}\left(\sigma_{n}=\sigma_{n o}\right) \circ \operatorname{CNS}\left(\sigma_{n}=\sigma_{n o}+K y\right)$, donde $\sigma_{n o}$ es el esfuerzo normal inicial, $K$ es la constante de rigidez normal e $y$ es el desplazamiento vertical de la caja de corte superior (ver la Figura 3.5). La condición de 
borde se aplica sobre la caja de corte superior del modelo numérico a través del mecanismo "servo-control" de PFC, el cual permite controlar la velocidad de traslación de las paredes seleccionadas y, de esta manera, aplicar o mantener sobre ellas una fuerza determinada (Itasca Consulting Group Inc. 2014).

\subsubsection{Calibración de los microparámetros del modelo de contacto smooth joint}

Una vez que se ha generado la probeta del modelo numérico y se han aplicado las condiciones de borde sobre ella, es necesario calibrar los microparámetros del modelo SJCM para las partículas de la interfaz roca-hormigón. La calibración de la rigidez normal y de corte de una junta impone un gran desafío, debido a que estos parámetros no se obtienen fácilmente a partir de ensayos experimentales y no hay pautas específicas para su estimación (Kulatilake et al. 2016). En el laboratorio, el método más utilizado para estimar la rigidez de las juntas es el propuesto por Bandis et al. (1983), el cual se basa en una modificación del modelo hiperbólico de Goodman que utiliza información de los ciclos de carga y descarga en ensayos de compresión uniaxial. Más recientemente, Kulalitake et al. (2016) formularon métodos para (i) estimar la rigidez normal de la junta $\left(K_{n}\right.$ lab $)$ en el laboratorio utilizando una prueba de compresión uniaxial modificada que no requiere el ciclo de descarga; y (ii) estimar la rigidez al corte de la junta $\left(K_{\text {s lab }}\right)$ a través de ensayos de corte directo a pequeña escala. Adicionalmente, en caso de no disponer del dato experimental de la rigidez normal de la junta, Malkowski (2015) sugiere que la rigidez normal $\left(K_{n}\right)$ será entre 1 y 10 veces mayor que la rigidez al corte $\left(K_{S}\right)$ de la junta.

La calibración de los microparámetros del SJCM -i.e., la rigidez normal y de corte de los contactos smooth-joint $\left(k_{n S J}\right.$ y $\left.k_{S S J}\right)$; y su coeficiente de fricción $\left(\mu_{S J}\right)$ - para los ensayos de corte directo con MED, se ha realizado en dos etapas mediante el siguiente proceso iterativo (ver la Figura 3.17(a)) ${ }^{1}$ :

${ }^{1}$ Es importante recordar que los modelos aquí considerados no tienen cohesión en la interfaz (i.e., $c=0$ ) 
1) En primer lugar, se ha realizado la calibración de la rigidez normal smoot-joint $\left(k_{n S J}\right)$. Para esto, se ha empleado el mecanismo "servo-control" de PFC para aplicar sobre la probeta del ensayo un valor de esfuerzo normal 1.3 veces el valor de esfuerzo aplicado en los ensayos experimentales. Durante esta fase del modelo numérico, se registran los valores de esfuerzo normal $\left(\sigma_{n}\right)$ y de desplazamiento normal $\left(\delta_{n}\right)$ generados sobre la pared superior de la caja de corte, lo que permite el ajuste de $k_{n S J}$ hasta reproducir la rigidez normal obtenida en el ensayo experimental $\left(K_{n \text { lab }}\right)$ (ver la Figura 3.17(b)). (No obstante, debido a $K_{n \text { lab }}$ no se reportaba en los datos experimentales de Gu et al. 2003 y Gutiérrez 2013, en esta investigación el valor de $K_{n \text { lab }}$ fue estimado a partir de la formulación sugerida por Malkowski 2015).

2) En segundo lugar, se ha calibrado la rigidez al corte smoot-joint $\left(k_{s} s J\right)$ y su coeficiente de fricción $\left(\mu_{S J}\right)$, a través del ensayo de corte directo con PFC ${ }^{2 \mathrm{D}}$, empleando como referencia los resultados experimentales de ensayos de corte directo en juntas planas. En particular, se varía $\mu$ hasta que el valor de la resistencia al corte pico $\left(\tau_{p}\right)$ del modelo numérico es similar al obtenido en el ensayo experimental, y $k_{s S J}$ se ajusta hasta reproducir el comportamiento al corte $(\tau)$ obtenido en el ensayo experimental, $K_{s}$ lab (o, en otras palabras, hasta que el valor de desplazamiento al corte pico es igual al obtenido en el ensayo experimental). Ver la Figura 3.17(c).

Durante la simulación del ensayo corte directo con $\mathrm{PFC}{ }^{2 \mathrm{D}}$, se han restringido los desplazamientos de la caja de corte inferior y se ha aplicado a la caja de corte superior (de izquierda a derecha) una velocidad horizontal $\left(V_{x}\right)$ de $0.01 \mathrm{~m} / \mathrm{s}$, -o lo suficientemente baja para garantizar que la probeta permanezca en equilibrio cuasiestático-. Durante el ensayo los desplazamientos en la dirección normal y de corte se han obtenido a partir de los desplazamientos horizontales y verticales registrados en la caja de corte superior, respectivamente, mientras que la resistencia al corte se ha calculado dividiendo la 
reacción de la fuerza horizontal promedio registrada en las paredes superior (izquierda) e inferior (derecha) por la longitud total de la junta.

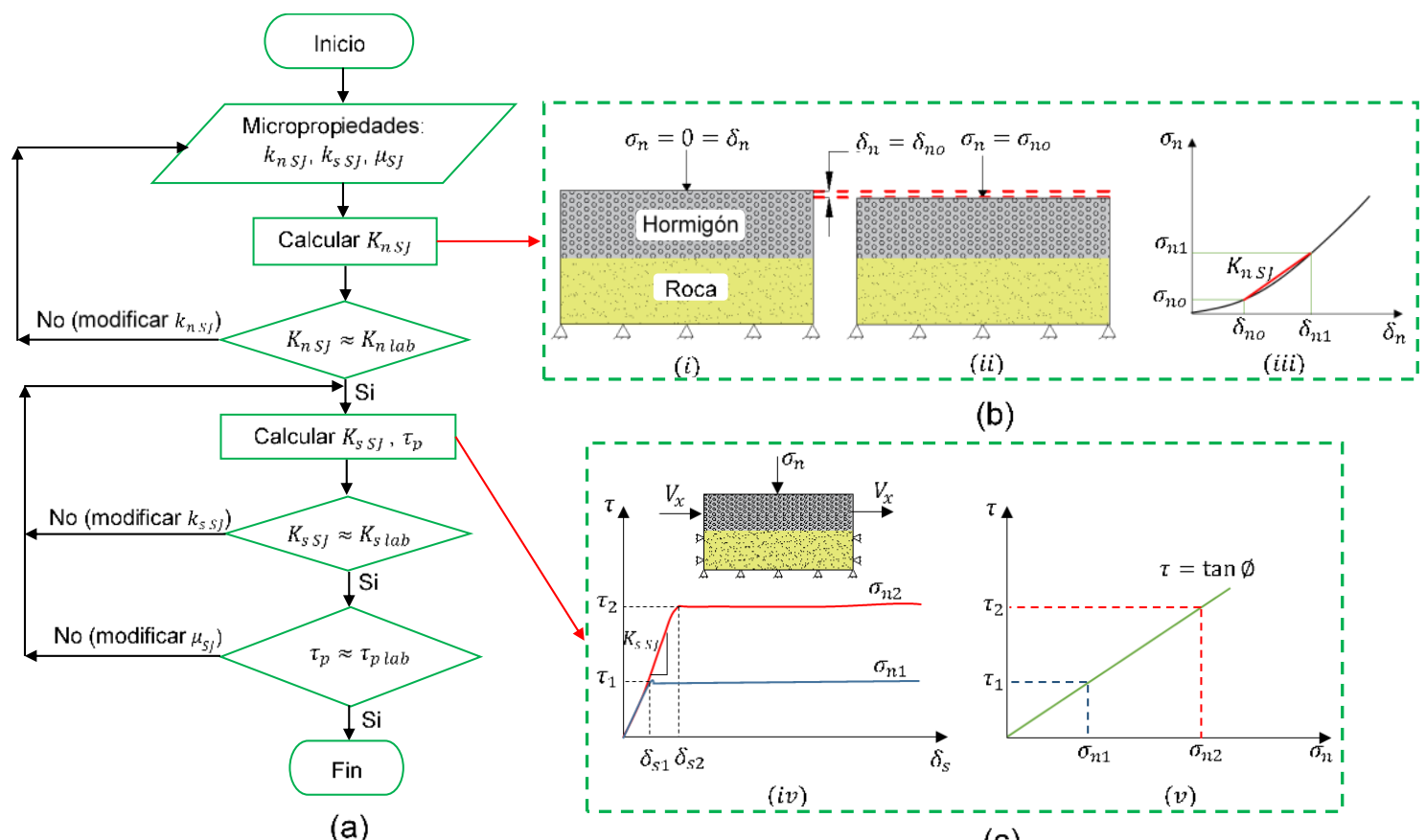

Figura 3.17. Proceso iterativo de calibración de los microparámetros del modelo de contacto smooth-joint: (a) diagrama de flujo, (b) modelo conceptual para calibrar la rigidez normal $k_{n s j}$ : (i) estado inicial, (ii) desplazamiento normal luego de la aplicación de un esfuerzo normal, (iii) esfuerzo normal versus desplazamiento normal para obtener $k_{n S J}$, (c) modelo conceptual para calibrar la rigidez al corte $k_{S S J}$ y $\mu_{S J}$ : (iv) resistencia al corte vs desplazamiento al corte para el cálculo de $k_{s S}$, (v) resistencia al corte vs esfuerzo normal para el cálculo del coeficiente de fricción $\mu$ (tan $\emptyset$ ) (Gutiérrez-Ch et al. 2017).

En las Figuras 3.18 y 3.19 se presentan los resultados obtenidos tras aplicar el procedimiento de calibración descrito anteriormente en los modelos con PFC $2 \mathrm{D}$ desarrollados en esta investigación (i) bajo condición de borde CNL, con la acción de diferentes valores de esfuerzo normal y (ii) considerando junta plana sin cohesión (i.e., $c=0$ ) en la interfaz roca-hormigón. Como puede observarse, los modelos numéricos con $\mathrm{MED}^{2 \mathrm{D}}$ reproducen adecuadamente el comportamiento obtenido en los ensayos experimentales en probetas con juntas roca-hormigón sin cohesión; igualmente, los parámetros de resistencia al corte calculados a partir de los modelos numéricos con $\mathrm{PFC}^{2 \mathrm{D}}$ tienen buena correspondencia con los obtenidos experimentalmente por Gu et al. (2003) y Gutiérrez (2013) (ver la Tabla 3.4). 


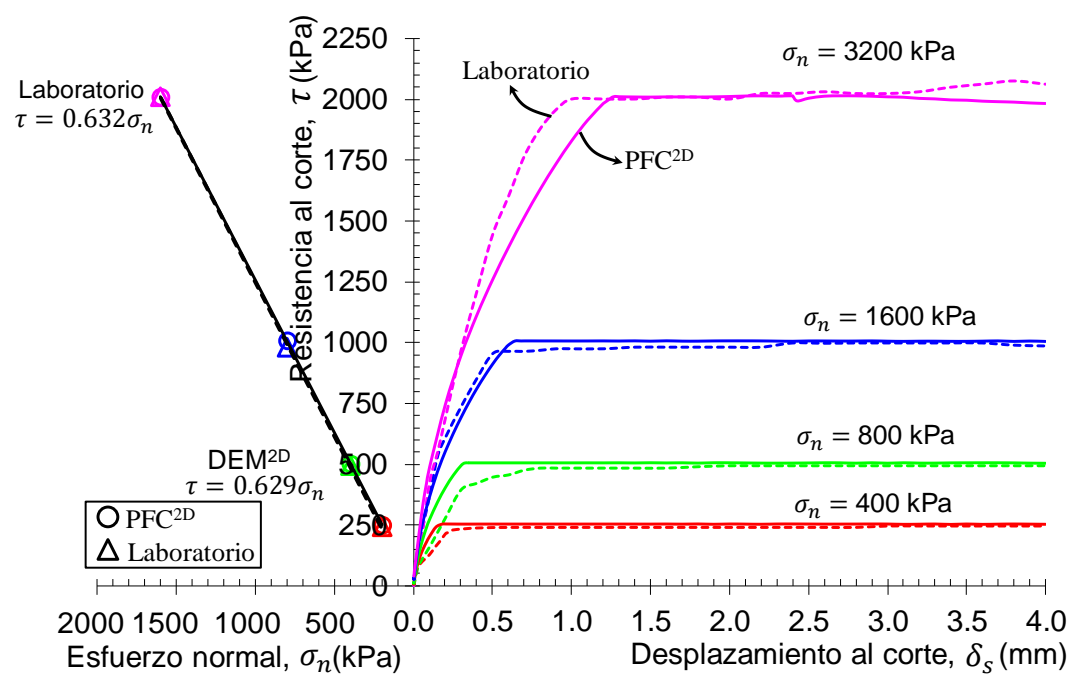

(a)

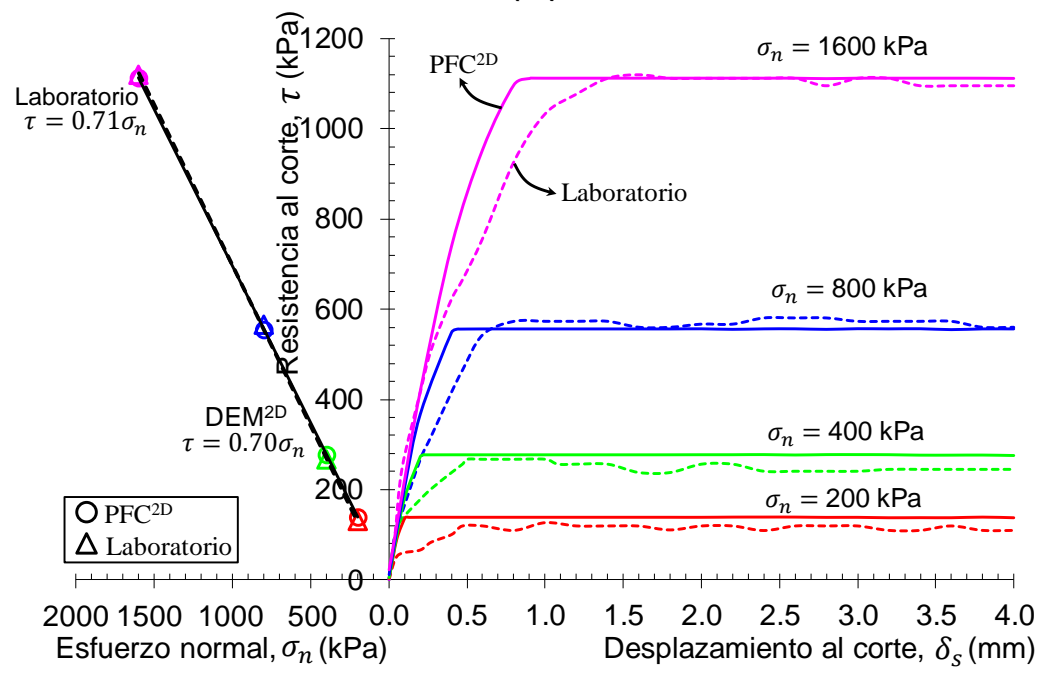

(b)

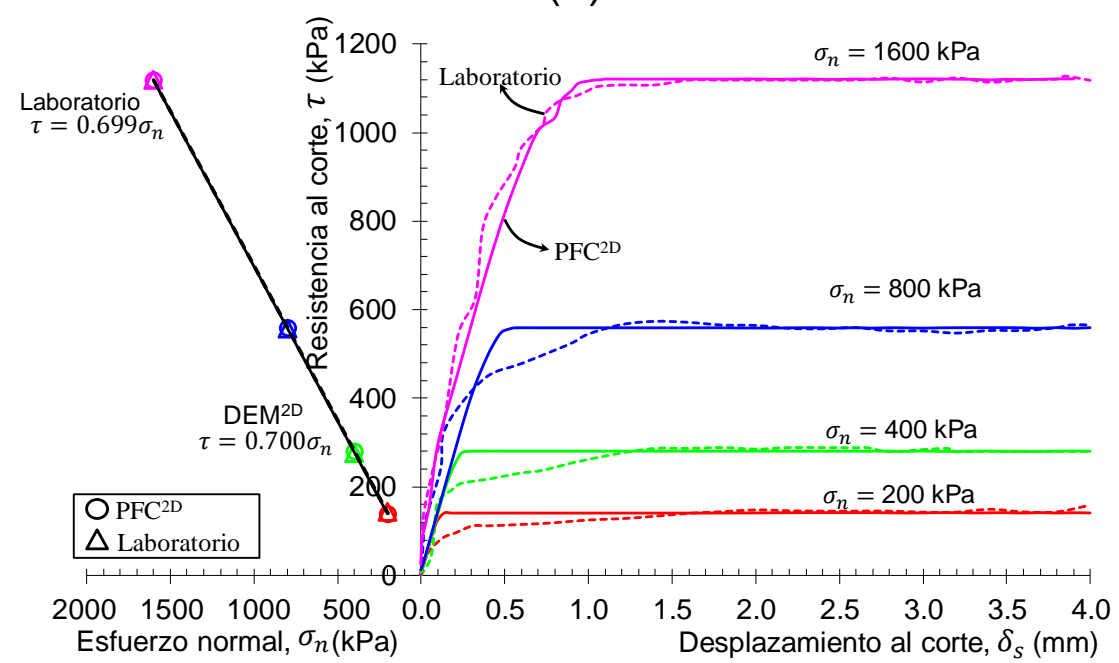

(c)

Figura 3.18. Comparación de los resultados de ensayos de corte directo con $\mathrm{PFC}^{2 \mathrm{D}}$ en probetas roca-hormigón de junta plana, sin cohesión, con diferentes valores de esfuerzo normal en condición CNL: (a) arenisca(S1)-hormigón(C1), (b) arenisca(S2)-hormigón(C1), (c) arenisca(S3)-hormigón(C1). (datos experimentales tomados de Gu et al. 2003) (modificado de Gutiérrez-Ch et al. 2018, 2019d). 


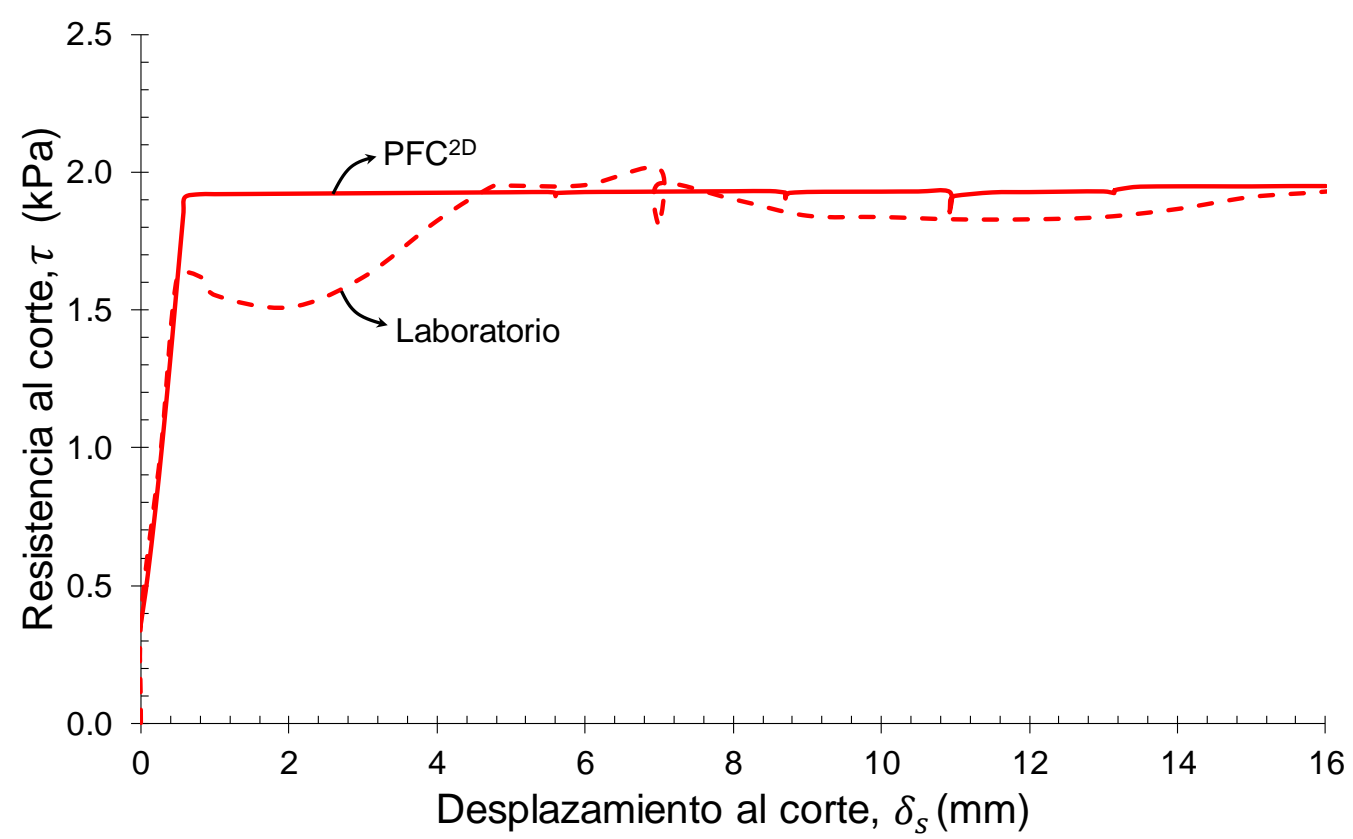

Figura 3.19. Comparación de los resultados de ensayo de corte directo con PFC2D en probeta de gneis-hormigón(C2) con junta plana, sin cohesión, en condición CNL $\left(\sigma_{n}=3.2 \mathrm{MPa}\right)$ (datos experimentales tomados de Gutiérrez 2013) (Gutiérrez-Ch et al. 2017).

Tabla 3.4. (a) Micropropiedades del modelo de contacto smooth-joint calibradas con PFC $2 \mathrm{D}$ para las diferentes juntas roca-hormigón empleadas en la validación; (b) comparación de los macroparámetros de la junta roca-hormigón obtenidos en los ensayos de corte directo experimentales y con PFC $2 \mathrm{D}$.

\begin{tabular}{|c|c|c|c|c|c|c|c|c|}
\hline \multirow{2}{*}{ a) } & \multicolumn{8}{|c|}{ Numérico $\left(\mathrm{PFC}^{2 \mathrm{D}}\right)$} \\
\hline & \multicolumn{2}{|c|}{ (I) } & \multicolumn{2}{|c|}{ (II) } & \multicolumn{2}{|c|}{ (III) } & \multicolumn{2}{|c|}{$(\mathrm{IV})$} \\
\hline $\begin{array}{l}\text { Rigidez normal, } k_{n} S J \\
(\mathrm{MPa} / \mathrm{mm})\end{array}$ & \multicolumn{2}{|c|}{10} & \multicolumn{2}{|c|}{10} & \multicolumn{2}{|c|}{10} & \multicolumn{2}{|c|}{10} \\
\hline $\begin{array}{l}\text { Rigidez al corte, } k_{s} S J \\
(\mathrm{MPa} / \mathrm{mm})\end{array}$ & \multicolumn{2}{|c|}{100} & \multicolumn{2}{|c|}{5} & \multicolumn{2}{|c|}{2} & \multicolumn{2}{|c|}{2} \\
\hline 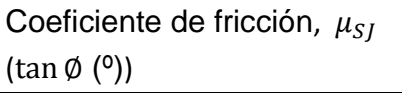 & \multicolumn{2}{|c|}{0.63} & \multicolumn{2}{|c|}{0.70} & \multicolumn{2}{|c|}{0.70} & \multicolumn{2}{|c|}{0.60} \\
\hline b) & $\left({ }^{*}\right)$ & $\left({ }^{* * *}\right)$ & $\left({ }^{\star}\right)$ & $\left({ }^{* * *}\right)$ & $\left({ }^{*}\right)$ & $\left({ }^{* \star *}\right)$ & $\left({ }^{* *}\right)$ & $\left({ }^{* \star \star}\right)$ \\
\hline $\begin{array}{l}\text { System normal stiffness, } K_{n} \\
(\mathrm{MPa} / \mathrm{mm})\end{array}$ & - & 9.91 & - & 9.50 & - & 10.30 & - & 16.49 \\
\hline $\begin{array}{l}\text { System shear stiffness, } K_{S} \\
(\mathrm{MPa} / \mathrm{mm})\end{array}$ & 3.37 & 3.40 & 1.84 & 1.81 & 1.37 & 1.36 & 2.51 & 2.53 \\
\hline Ángulo de fricción, $\tan \varnothing(\stackrel{\circ}{)})$ & 32.29 & 32.17 & 35.37 & 34.99 & 34.96 & 34.99 & 31.22 & 31.10 \\
\hline
\end{tabular}

(I) Arenisca(S1)-hormigón(C1), (II) Arenisca(S2)-hormigón(C1), (III) Arenisca(S3)-hormigón(C1), (IV) Gneishormigón(C2), $\left({ }^{\star}\right)$ Experimental por Gu et al. (2003), $\left({ }^{* *}\right)$ Experimental por Gutiérrez (2013), $\left(^{* * *}\right)$ Numérico $\left(\mathrm{PFC}^{2 \mathrm{D}}\right)$

\subsection{Resultados}

Una vez se ha completado el proceso de calibración de los microparámetros de los materiales (FJCM, ver la Sección 3.3.2) y de la junta (SJCM, ver la Sección 3.3.4) del ensayo de corte directo en probetas de roca-hormigón, es posible realizar múltiples ensayos de corte directo empleando $\mathrm{PFC}^{2 \mathrm{D}}$ con diferentes geometrías en la junta o con 
diversas condiciones (según el problema analizado). En las secciones siguientes, se presentan los resultados obtenidos en ensayos de corte directo realizados con $\mathrm{PFC}{ }^{2 \mathrm{D}}$ bajo condición CNL y CNS, y su comparación con los datos experimentales de Gutiérrez (2013) y Gu et al. (2003).

\subsubsection{Ensayos de corte directo en condición CNL}

A continuación, se muestran los resultados de siete (7) ensayos de corte directo realizados con $\mathrm{PFC}^{2 \mathrm{D}}$ en probetas de roca-hormigón, con juntas de morfología triangular simétricas, sin cohesión y análogas a los perfiles de rugosidad empleados en los ensayos experimentales de Gutiérrez (2013) y Gu et al. (2003). Estos modelos numéricos del ensayo corte directo se realizaron bajo condiciones de borde CNL, utilizando los microparámetros calibrados previamente (para más detalles consultar la Sección 3.3.4). Es importante resaltar, por tanto, que se está utilizando un modelo con $\mathrm{MED}^{2 \mathrm{D}}$ calibrado con datos experimentales de ensayos de corte directo realizados sobre junta plana, y que la calibración realizada con dichos ensayos (más sencillos) se emplea para predecir el comportamiento al corte en probetas roca-hormigón con interfaz rugosa. En este sentido, la comparación de los resultados obtenidos en los modelos con PFC ${ }^{2 D}$ y los experimentales sirve como ejercicio de validación de las capacidades que tienen los modelos numéricos realizados en esta investigación para evaluar el efecto de la rugosidad en el comportamiento al corte en juntas roca-hormigón.

En particular, la Figura 3.20 presenta los resultados de cuatro ensayos de corte directo realizados con $\mathrm{PFC}^{2 \mathrm{D}}$ empleando la metodología SBG descrita en la Sección 3.3.3, sobre probetas de arenisca(S3)-hormigón(C1), con junta sin cohesión y con diferentes perfiles de rugosidad de geometría triangular simétrica con diferente ángulo base respecto al plano horizontal $\left(\alpha=5^{\circ}, 20^{\circ}, 30^{\circ}\right.$ y $40^{\circ}$, ver la Figura 3.20), y bajo condición CNL (con un esfuerzo normal $\sigma_{n}=400 \mathrm{kPa}$ ). De manera similar, la Figura 3.21 presenta los resultados de tres ensayos de corte directo sobre probetas de gneis-hormigón(C2) 
con junta sin cohesión, con perfiles de rugosidad triangular simétrica con ángulo base de $\alpha=10^{\circ}, 20^{\circ}$ y $40^{\circ}$, y con condición CNL con $\sigma_{n}=3.2 \mathrm{MPa}$. A modo de comparación, y para evaluar la calidad de los resultados de los modelos PFC $2 \mathrm{D}$, se incluyen en ambas figuras los resultados de ensayos experimentales obtenidos para estas probetas por Gutiérrez (2013) y Gu et al. (2003).

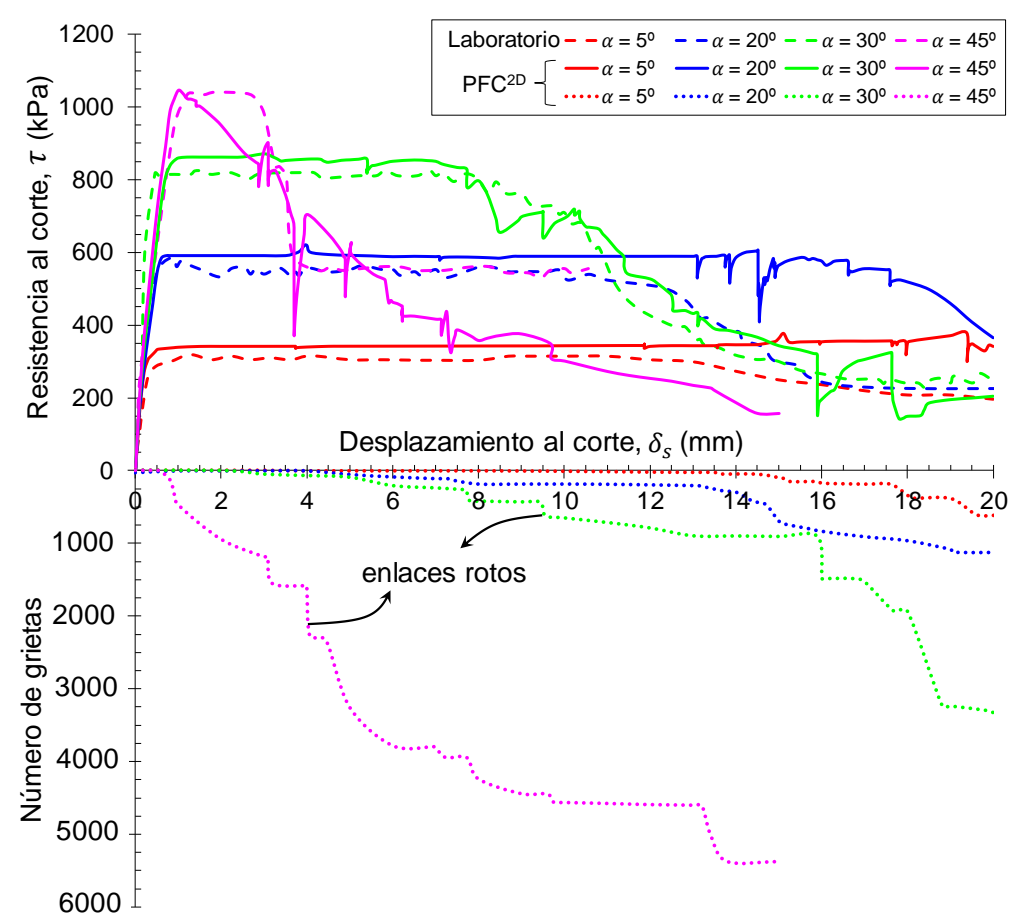

Figura 3.20. Comparación de resultados de ensayos de corte directo numéricos con $\mathrm{PFC}^{2 \mathrm{D}}$ y experimentales de Gu et al. (2003) realizados en probetas de arenisca(S3)-hormigón(C1) con junta sin cohesión y de diferentes perfiles de rugosidad bajo condición CNL con $\sigma_{n}=400 \mathrm{kPa}$ (modificado de Gutiérrez-Ch et al. 2018).

Los resultados presentados en la Figuras 3.20 y 3.21 muestran que los modelos con $\mathrm{PFC}^{2 \mathrm{D}}$ reproducen en forma adecuada el comportamiento experimental de las mismas probetas; es decir, los valores alcanzados para la resistencia al corte pico $\left(\tau_{p}\right)$ y su correspondiente desplazamiento al corte $\left(\delta_{p}\right)$, y por tanto, la rigidez al corte obtenidas con los modelos numéricos, es similar a la calculada en los ensayos experimentales. De forma similar, y en concordancia con investigaciones previas (Krounis et al. 2016; Gutiérrez 2013; Gu et al. 2003), se observa que los modelos numéricos predicen que la resistencia al corte pico aumenta con el aumento de la rugosidad (i.e., con el aumento del ángulo base) en la junta. 
Adicionalmente, se observa que la principal diferencia entre los resultados de los modelos numéricos y experimentales, para juntas rugosas, se produce en la etapa postpico. Puede notarse que las curvas experimentales muestran una disminución gradual de la resistencia al corte pico hasta su valor residual; lo cual puede deberse a una degradación progresiva de las asperidades. Sin embargo, los resultados de los modelos numéricos con $\mathrm{PFC}^{2 \mathrm{D}}$ muestran un comportamiento post-pico más fluctuante; esto podría atribuirse a que se produzca (durante el ensayo) una disminución repentina de las fuerzas que actúan en las asperidades cuando los enlaces entre las partículas se rompen (ver, e.g, Bahaaddini et al. 2016 y Asadi et al. 2013). De la Figuras 3.20 y 3.21 , puede inferirse que estas fluctuaciones parecen estar asociadas al incremento del número de enlaces que rompen durante la realización del ensayo. Además, en la Figuras 3.20 y 3.21 se nota que, solo una pequeña cantidad de micro-grietas se producen antes de alcanzar la resistencia al corte pico y que, una vez alcanzado dicho valor, el número de micro-grietas aumenta rápidamente. Esta tendencia del modelo está en concordancia con los resultados obtenidos por Bahaddinni et al. (2014), quienes sugieren que el comportamiento al corte en juntas está controlado por el inicio y propagación de las micro-grietas que se producen en las asperidades, cuyo número se incrementa rápidamente después de haber alcanzado la resistencia al corte pico del ensayo y que, por tanto, la aparición de estas micro-grietas traen consigo una disminución de la resistencia al corte pico hasta alcanzar su valor residual.

Otra explicación a las fluctuaciones observadas en la resistencia al corte en los modelos numéricos de las probetas más rugosas podría deberse a que las partículas no se rompen en PFC, mientras que en realidad estás partículas pueden degradarse progresivamente (recordar que las partículas con $\mathrm{PFC} 2 \mathrm{D}$ son discos rígidos que nunca se rompen durante la simulación). Para minimizar tales fluctuaciones, algunos autores sugieren disminuir el tamaño de las partículas en la zona adyacente a las juntas (ver e.g., Bahaddinni et al. 2016 y Asadi et al. 2013), sin embargo, debe recordarse que esto 
traería como consecuencia un incremento en el costo computacional de los modelos numéricos.

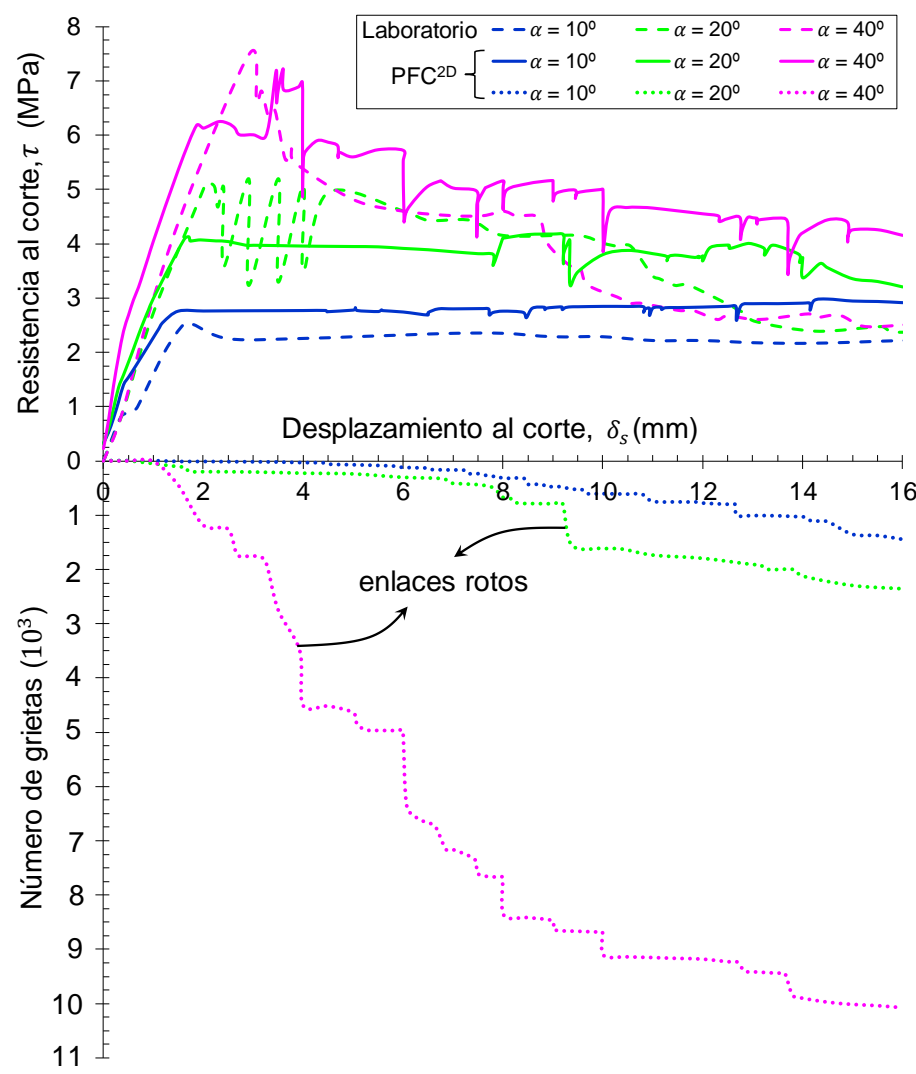

Figura 3.21. Comparación de resultados de ensayos de corte directo con $\mathrm{PFC}^{2 \mathrm{D}}$ y experimentales de Gutiérrez. (2013), realizados en probetas gneis-hormigón(C1) con junta sin cohesión y con diferentes perfiles de rugosidad bajo condición CNL con $\sigma_{n}=3.2 \mathrm{MPa}$ (modificado de Gutiérrez-Ch et al. 2018).

Por otra parte, en la Figuras 3.20 y 3.21 se observa que los modelos numéricos con $\mathrm{PFC}^{2 \mathrm{D}}$ son capaces de capturar en forma adecuada el mecanismo principal que controla el comportamiento post-pico. En particular, para juntas rugosas con $\alpha \leq 30^{\circ}$ se observa que reproducen el modo de fallo por deslizamiento en la interfaz roca-hormigón, mientras que para valores mayores de $\alpha$ reproducen la ocurrencia de una mayor degradación y rotura de las asperidades (ver la Figura 3.22; resultados análogos fueron reportados por Gu et al. 2003 y Gutiérrez 2013).

En la Figura 3.23 se presenta la degradación de las asperidades del modelo numérico con $\mathrm{PFC}^{2 \mathrm{D}}$ así como del experimental para la probeta de gneis-hormigón con junta triangular de $\alpha=40^{\circ}$ y condición CNL de $\sigma_{n}=3.2 \mathrm{MPa}$. Como puede observarse, los 
modelos numéricos reflejan correctamente el comportamiento experimental en este ensayo, es decir, las asperidades en el hormigón han sido completamente rotas y degradadas (notar que el esfuerzo normal aplicado es elevado; y que la resistencia del gneis es mucho mayor a la del hormigón, ver la Tabla 3.2). Este patrón también fue observado en las probetas arenisca(S3)-hormigón(C1), aunque en este caso la arenisca era más débil que el hormigón (ver la Tabla 3.2) y, en consecuencia, es la parte de la interfaz que sufre la mayor degradación (ver la Figura 3.22(a)).
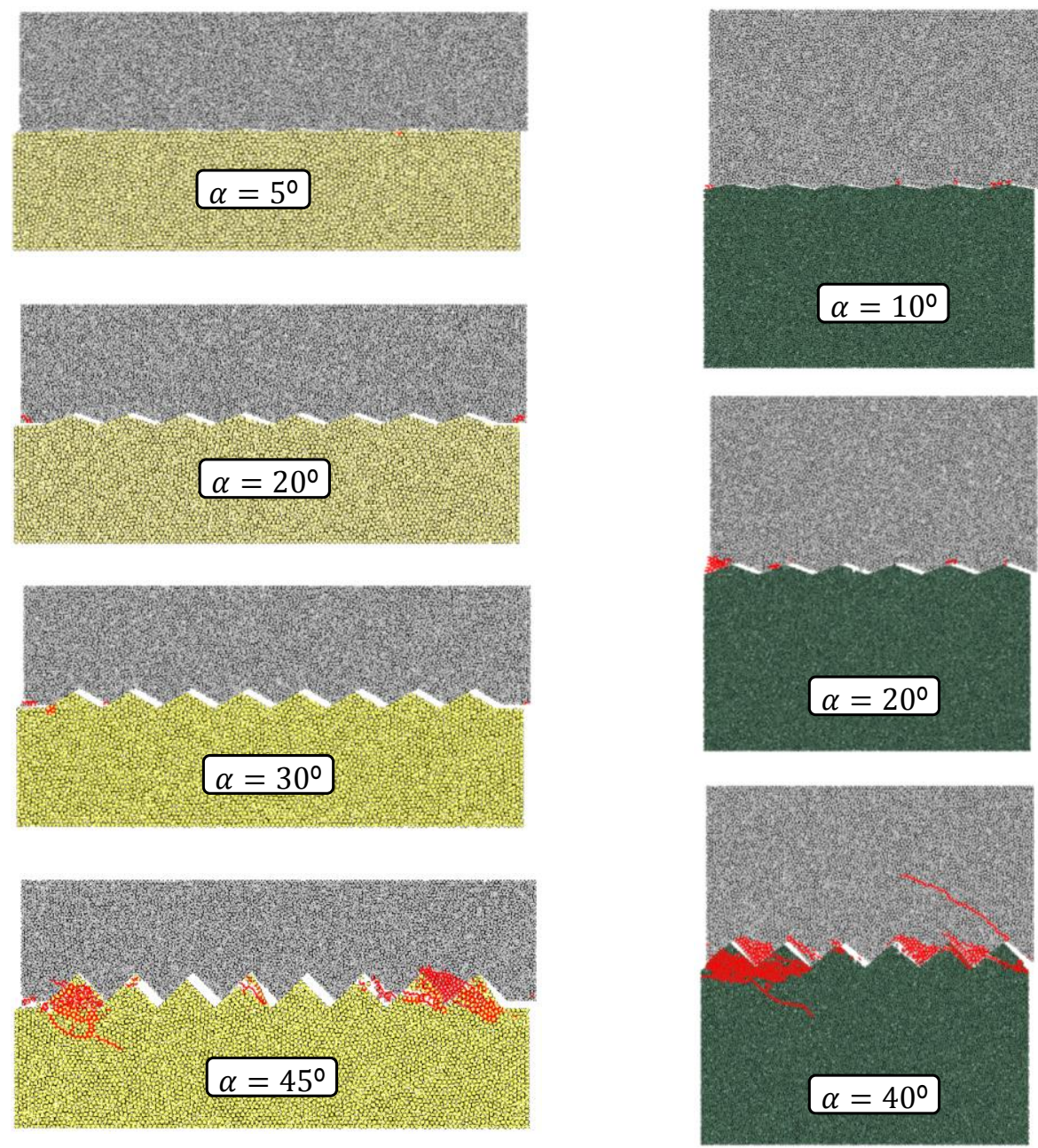

(a)

(b)

Figura 3.22. Daño observado en las asperidades de los modelos con PFC ${ }^{2 D}$ en juntas de morfología triangular sin cohesión, bajo condición CNL y diferentes valores de ángulo base $\alpha$ : (a) probeta de arenisca(S3)-hormigón(C1) para un desplazamiento al corte de $4 \mathrm{~mm}$ y $\sigma_{n}=400$ $\mathrm{kPa}$, (b) probeta de gneis-hormigón(C2) para un desplazamiento al corte de $4 \mathrm{~mm}$ y $\sigma_{n}=3.2$ MPa (las partículas de color gris, amarillo y verde representan al hormigón, arenisca y gneis, respectivamente; las líneas color rojo representan las micro-grietas) (Gutiérrez-Ch et al. 2018). 


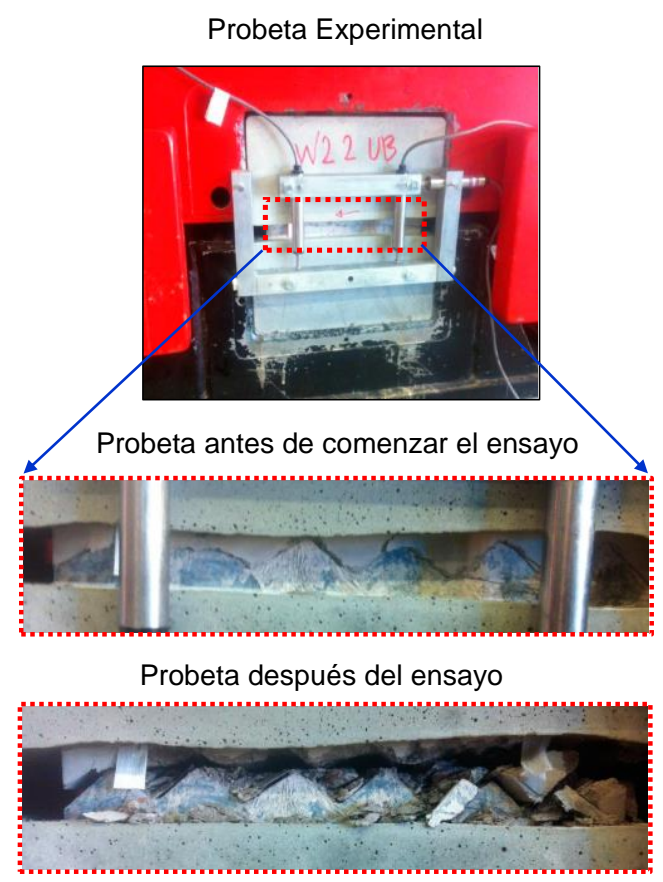

(a)

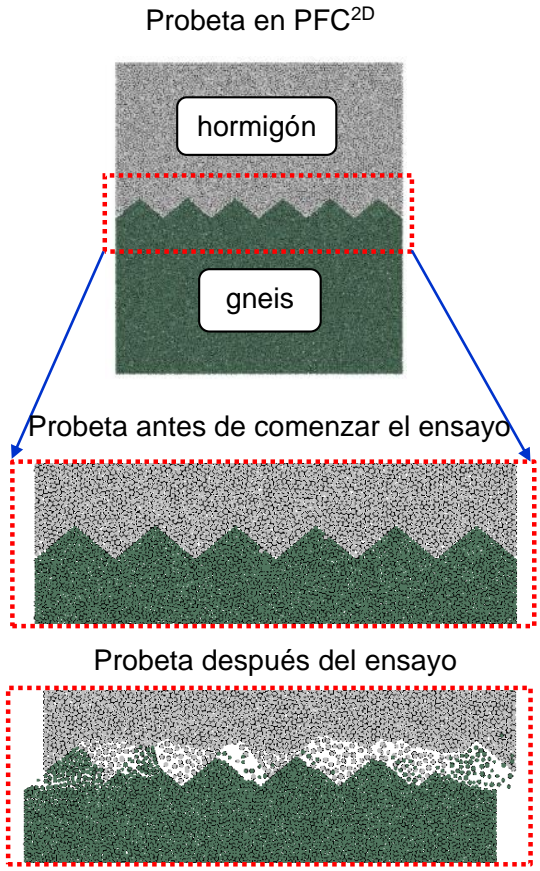

(b)

Figura 3.23. Rotura de las asperidades en el ensayo de corte directo de una probeta de gneishormigón(C2), con junta triangular simétrica $\left(\alpha=40^{\circ}\right)$ sin cohesión, y condición CNL con $\sigma_{n}=$ 3.2 MPa: (a) resultados experimentales de Gutiérrez (2013), (b) resultado del modelo numérico con PFC ${ }^{2 D}$ (Gutiérrez-Ch et al. 2018).

\subsubsection{Ensayos de corte directo en condición CNS}

A continuación, se presentan los resultados obtenidos en los modelos numéricos con $\mathrm{PFC}^{2 \mathrm{D}}$ para ensayos de corte directo en probetas arenisca(S1,S3)-hormigón(C1), sin cohesión, en juntas de morfología triangular simétrica y con diferentes valores del ángulo base $(\alpha)$, esfuerzo normal inicial $\left(\sigma_{n o}\right)$ y rigidez $(K)$. Para ello se ha empleado el modelo conceptual de condición de borde CNS ilustrado en la Figura 3.5(b), empleando las micropropiedades calibradas para los modelos FJCM (ver la Sección 3.3.2) y SJCM (ver la Sección 3.3.4).

En la Figura 3.24 se presenta la comparación de la respuesta al corte (resistencia al corte vs desplazamiento al corte) obtenidas en tres ensayos de corte directo realizados con $\mathrm{PFC}^{2 \mathrm{D}}$ en probetas de arenisca(S1)-hormigón(C1), con junta triangular simétrica $\left(\alpha=5^{\circ}, 10^{\circ}\right.$ y $\left.20^{\circ}\right)$, bajo condición CNS de $\sigma_{n o}=400 \mathrm{kPa}$ y con $K=800 \mathrm{kPa} / \mathrm{mm}$; mientras que en la Figura 3.25, se muestra los resultados obtenidos en tres ensayos de 
corte directo en probetas de arenisca(S1)-hormigón con junta triangular simétrica de $\alpha=$ $5^{\circ}$, bajo condición CNS de $\sigma_{n o}=400 \mathrm{kPa}$, y con $K$ variado desde 800 a $3200 \mathrm{kPa} / \mathrm{mm}$. Los resultados muestran que la rugosidad de la interfaz afecta en gran medida al comportamiento de resistencia al corte, con valores de resistencia al corte pico con PFC ${ }^{2 D}$ ligeramente superiores a los resultados experimentales de Gu et al. (2003).

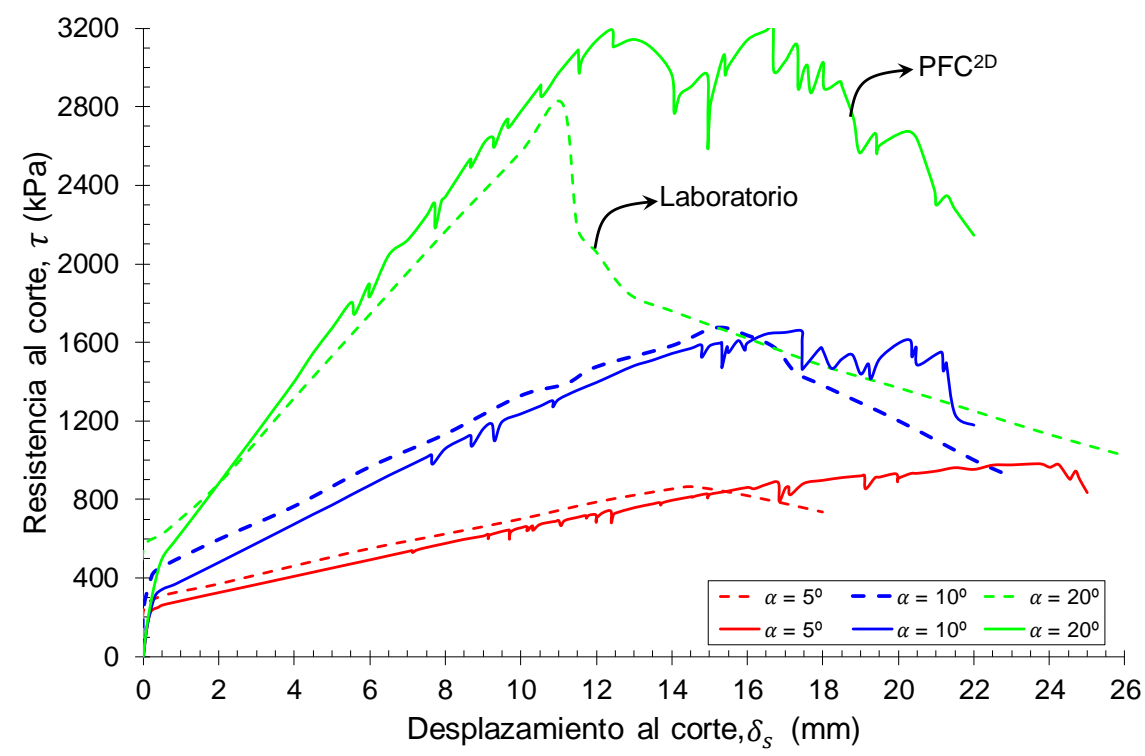

Figura 3.24. Comparación de resultados de ensayos de corte directo numéricos de esta investigación y experimentales de Gu et al. (2003), realizados en probetas de arenisca(S1)hormigón(C1), con junta triangular simétrica sin cohesión, bajo condición CNS de $\sigma_{n o}=400$ $\mathrm{kPa}$ y con $K=800 \mathrm{kPa} / \mathrm{mm}$ ) (Gutiérrez-Ch et al. 2018).

De manera similar, la Figura 3.25 muestra los resultados de tres pruebas en probetas de arenisca(S3)-hormigón(C1) con junta triangular simétrica, sin cohesión, con $\alpha=20^{\circ}$, con diferentes esfuerzos normales iniciales y con $K=800 \mathrm{kPa} / \mathrm{mm}$; los resultados numéricos muestran la misma tendencia que las pruebas experimentales de Gu et al. (2003), de modo que al aumentar el esfuerzo normal inicial se incrementa ligeramente la resistencia al corte pico. Sin embargo, puede observarse que las simulaciones con $\mathrm{PFC}^{2 \mathrm{D}}$ parecen sobreestimar ligeramente el valor de resistencia al corte pico para estas probetas (ver la Figura 3.26). 


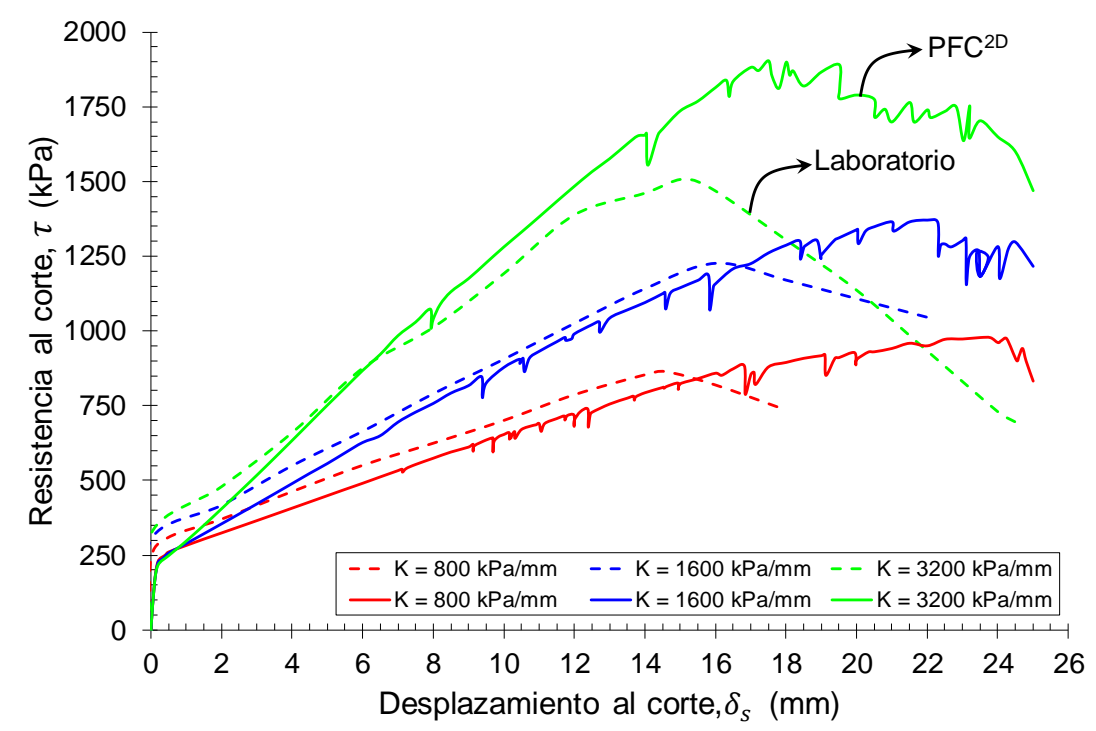

Figura 3.25. Comparación de resultados de ensayos de corte directo numéricos con $\mathrm{PFC}^{2 \mathrm{D}}$ y experimentales de Gu et al. (2003), realizados en probetas de arenisca(S1)-hormigón(C1), con junta triangular simétrica de $\alpha=5^{\circ}$ sin cohesión, bajo condición CNS de $\sigma_{n o}=400 \mathrm{kPa}$, y $K$ variado desde 800 a $3200 \mathrm{kPa} / \mathrm{mm}$ (Gutiérrez-Ch et al. 2018).

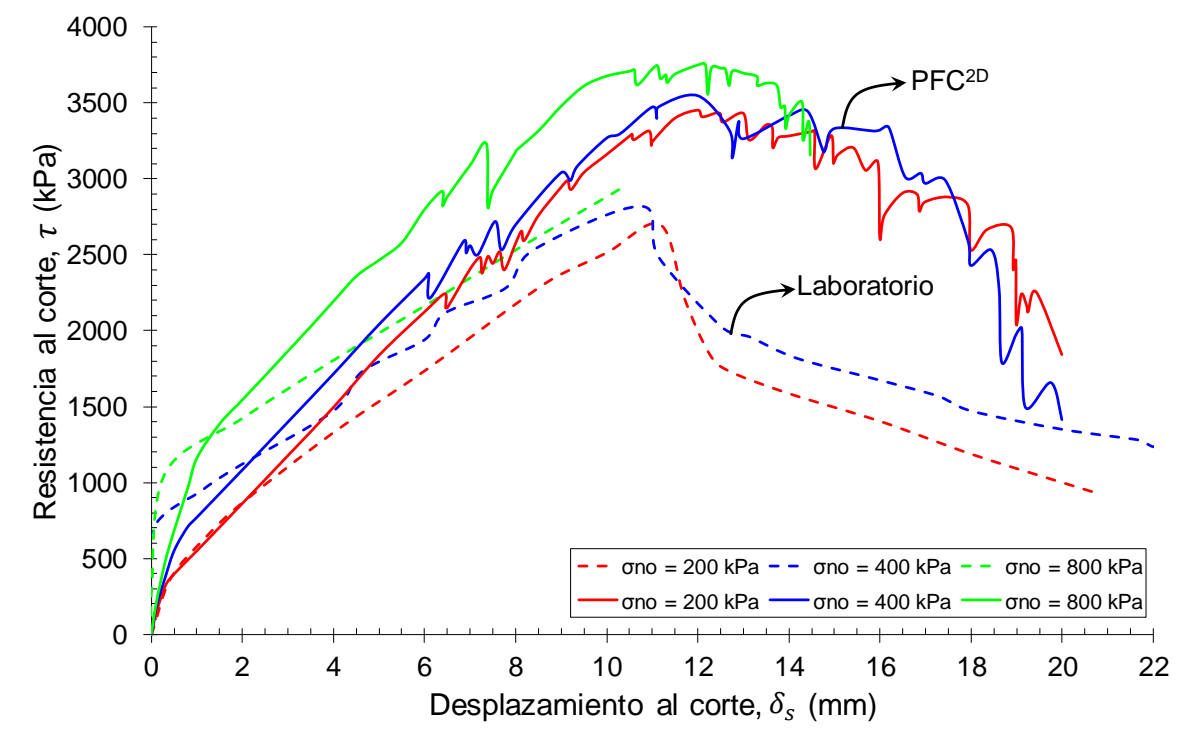

Figura 3.26. Comparación de resultados de ensayos de corte directo numéricos con $\mathrm{PFC}^{2 \mathrm{D}}$ y experimentales de Gu et al. (2003), realizados en probetas de arenisca(S3)-hormigón(C1), con junta triangular simétrica de $\alpha=20^{\circ}$ sin cohesión, bajo condición CNS con diferentes valores de esfuerzo normal inicial $\left(\sigma_{n o}\right)$, y $K=800 \mathrm{kPa} / \mathrm{mm}$ (Gutiérrez-Ch et al. 2018).

Finalmente, la Figura 3.27 muestra los resultados obtenidos en otra probeta de arenisca(S3)-hormigón(C1) con junta triangular simétrica, sin cohesión, para $\alpha=5^{\circ}$, con $\sigma_{n o}=400 \mathrm{kPa}$ y $K=800 \mathrm{kPa} / \mathrm{mm}$; en esta misma figura, se comparan los resultados obtenidos para esta misma probeta, con las mismas condiciones de $\sigma_{n o}$ y $K$, pero con interfaz más rugosa (de $\alpha=20^{\circ}$ ). Como puede observarse, los modelos numéricos con PFC $2 \mathrm{D}$ están de acuerdo con los resultados experimentales de Gu et al. (2003); sin 
embargo, se nota que la resistencia al corte pico y su correspondiente desplazamiento al corte son ligeramente sobreestimados por los modelos numéricos.

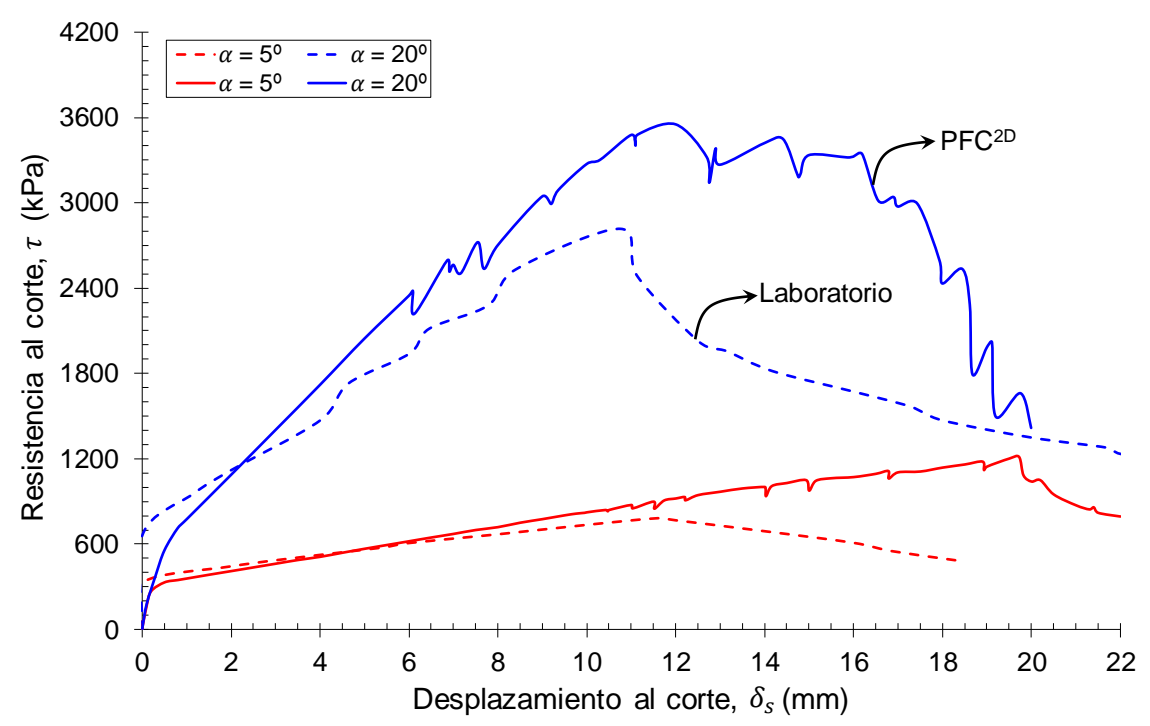

Figura 3.27. Comparación de resultados de ensayos de corte directo numéricos con $\mathrm{PFC}^{2 \mathrm{D}}$ y experimentales de Gu et al. (2003), realizados en probetas de arenisca(S3)-hormigón(C1), con junta triangular simétrica de $\alpha=5^{\circ}$ y $20^{\circ}$ sin cohesión, bajo condición CNS con $\sigma_{n o}=400 \mathrm{kPa}$, y $K=800 \mathrm{kPa} / \mathrm{mm}$ (Gutiérrez-Ch et al. 2018).

\subsubsection{Comparación entre la condición CNL y CNS}

En la Figura 3.28 se presenta una comparación entre los resultados numéricos y experimentales de ensayos de corte directo realizados en probetas de arenisca(S3)hormigón(C1), con junta triangular simétrica de $\alpha=20^{\circ}$ sin cohesión, bajo condición CNL y CNS. Como puede observarse, la condición de borde CNS tiene gran efecto sobre la resistencia al corte pico alcanzada, generándose mayor valor de resistencia al corte pico en las probetas CNS que en las CNL, para la misma condición de esfuerzo normal y morfología de la junta. Adicionalmente, se nota que los resultados numéricos concuerdan con este comportamiento obtenido en los ensayos experimentales de Gu et al. (2003), y con resultados de comportamiento similar reportados por otros investigadores (Shrivastava y Rao 2015; Seidel y Haberfield 2002).

Finalmente, los resultados presentados en la Figura 3.28 demuestran que la elección inadecuada de la condición de borde CNL o CNS puede sobreestimar o subestimar la 
resistencia al corte de juntas roca-hormigón, siendo esto de relevancia significativa en aplicaciones prácticas: por ejemplo, se ha demostrado que la condición de borde CNL no es adecuada en el estudio de pilotes empotrados en roca, debido a que se subestimaría considerablemente la resistencia al corte a lo largo de la interfaz piloteroca, y por tanto, su diseño no sería óptimo desde el punto de vista económico (Shrivastava y Rao, 2015). Es por ello que, en las simulaciones numéricas que se desarrollan más adelante (ver Capítulo 4) se emplea la condición CNS en las interfaces roca-pilote.

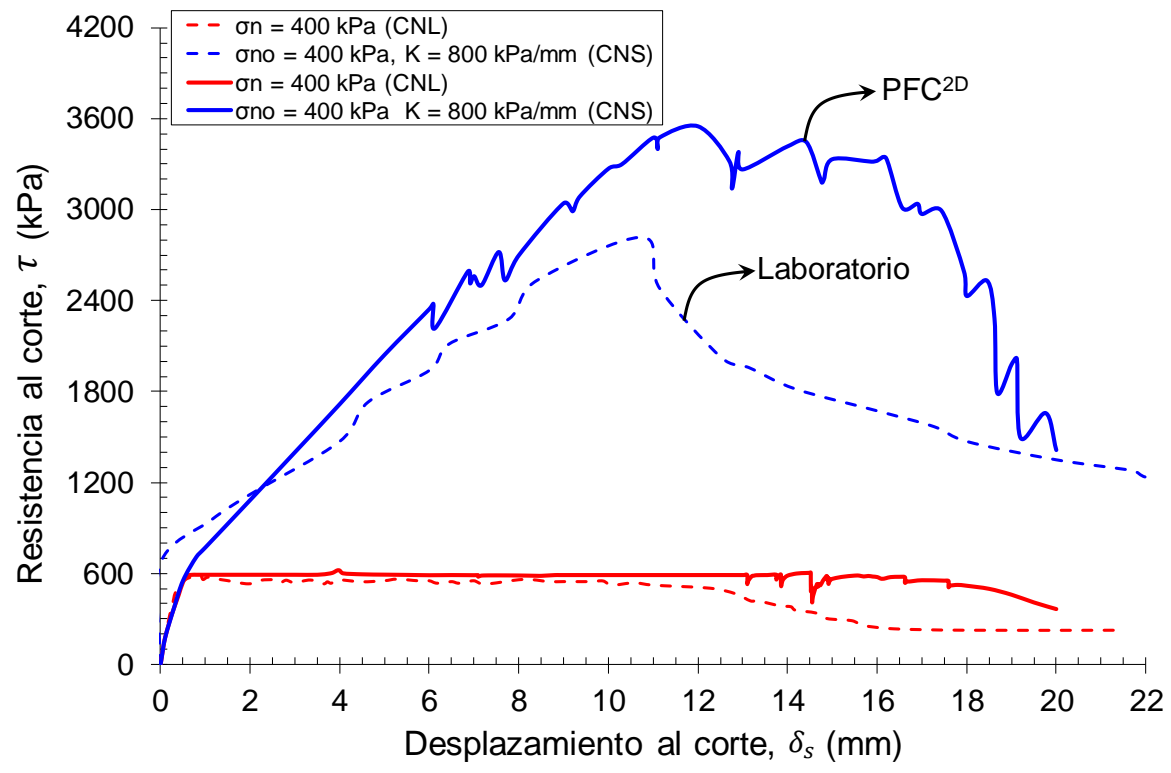

Figura 3.28. Comparación de resultados de ensayos de corte directo numéricos con $\mathrm{PFC}^{2 \mathrm{D}}$ y experimentales de Gu et al. (2003), realizados en probetas de arenisca(S3)-hormigón, con junta triangular simétrica de $\alpha=20^{\circ}$ sin cohesión, bajo condición CNL (línea roja) y CNS (línea azul) (Gutiérrez-Ch et al. 2018). 


\section{Modelos numéricos a gran escala de pilotes empotrados en roca}

\subsection{Introducción}

El objetivo principal de esta tesis doctoral es analizar la influencia de la rugosidad en la interfaz roca-pilote sobre el comportamiento por fuste de pilotes empotrados en roca. La motivación proviene de las evidencias obtenidas de (i) ensayos de carga sobre pilotes empotrados en roca, en los que se observó que la rugosidad tiene gran impacto sobre la resistencia por fuste (ver en el Capítulo 2, e.g., Pells et al. 1980; Horvath et al. 1983; Seol y Jeong 2007; Nam y Vipulanandan 2008; Dai et al. 2017) y, de (ii) que la rugosidad es, sin embargo, un factor que generalmente no se considera en los actuales diseños de pilotes empotrados en roca.

Para investigar la influencia de la rugosidad sobre el comportamiento de pilotes empotrados en roca, se ha empleado como medida de la rugosidad el factor de rugosidad $(R F)$ propuesto por Horvath et al. (1983) - que, para una cavidad dada, define $R F$ como una función del promedio de la altura de las asperidades y de la distancia total de un perfil trazado a lo largo de la cavidad- el cual ha servido para el desarrollo de modelos numéricos de pilotes empotrados en roca mediante el Método de Elementos Discretos (MED) y su comparación con el Método de Elementos Finitos (MEF) con los programas comerciales $\mathrm{PFC}^{3 \mathrm{D}}$-herramienta principal- y Abaqus -herramienta secundaria-, respectivamente. En particular, en los apartados siguientes se presenta (i) la aplicación del MED para simular pilotes empotrados en roca (ver la Sección 4.2), (ii) se analiza el efecto de la rugosidad de la pared de la cavidad sobre la resistencia por fuste de pilotes empotrados en roca (ver la Sección 4.3), y (iii) se analiza el mecanismo de transferencia de carga hacia la roca circundante, producto de la rugosidad en la interfaz roca-pilote (ver la Sección 4.3). Finalmente, en las Secciones 4.4 y 4.5 se presenta la aplicación y resultados obtenidos con la aplicación del MEF. Los modelos 
con $\mathrm{PFC}^{3 \mathrm{D}}$ y MEF desarrollados en esta investigación sólo consideran la porción del pilote empotrado en la roca; por tanto, se desprecia la contribución de la resistencia movilizada y el acortamiento elástico de la porción del pilote embebida en el estrato de suelo suprayacente.

\subsection{Configuración del modelo numérico con MED para pilotes cargados axialmente y empotrados en roca}

\subsubsection{Esquema del modelo MED ${ }^{3 D}$}

Uno de los propósitos de este capítulo es realizar la simulación numérica en $\mathrm{PFC}^{3 \mathrm{D}}$, de pilotes empotrados en rocas de diferente resistencia, y que han sido analizadas en el capítulo anterior -arenisca-S3, arenisca-S2 y gneis-, considerando un grado de rugosidad variable en la interfaz roca-pilote, mediante el uso de diversos valores del factor de rugosidad $(R F)$.

En la Figura 4.1 se presenta el esquema del modelo geotécnico empleado para el análisis, conformado por (i) el perfil idealizado de un pilote empotrado en roca (ver la Figura 4.1(a)) y (ii) su correspondiente dominio a modelar con PFC ${ }^{3 \mathrm{D}}$ (ver la Figura 4.1(b)). Se han realizado un total de 16 modelos de pilotes perforados en roca (6 en la arenisca-S3, 5 en la arenisca-S2, y 5 en el gneis) con rugosidad variable (ver la Sección 4.2.2). La metodología y detalles de la generación, así como los resultados obtenidos, se explican con detalle en las secciones siguientes.

\subsubsection{Rugosidad de la interfaz roca-pilote}

Para los modelos con PFC ${ }^{3 \mathrm{D}}$ considerados en esta investigación, la rugosidad en la interfaz roca-pilote se caracterizó por medio del factor de rugosidad $(R F)$ propuesto Horvath et al. (1983), definido como:

$$
R F=\frac{h_{m} L_{t}}{R L}
$$


Donde $h_{m}$ es la altura promedio de las asperidades (calculadas a partir de la línea segmentada roja de la Figura 4.2), $R$ es el radio nominal de la cavidad (o pilote), $L_{t}$ es la distancia total a lo largo de un perfil trazado en la cavidad, y $L$ es la longitud nominal de la cavidad (o pilote) (ver la Figura 4.2).

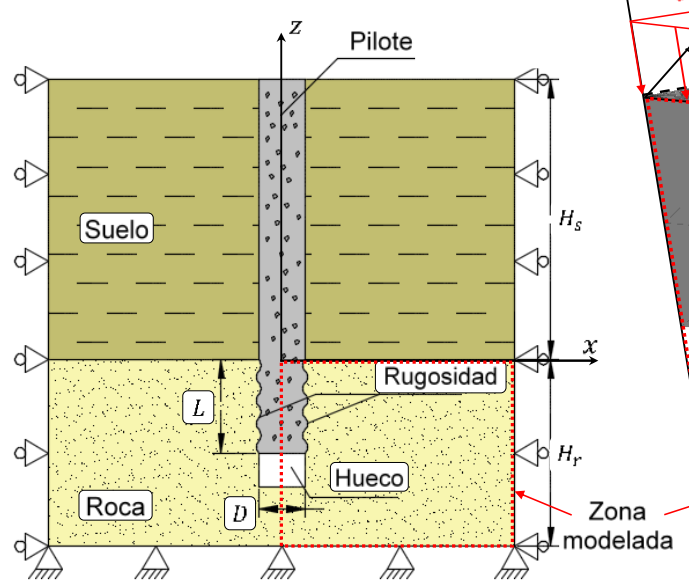

(a)

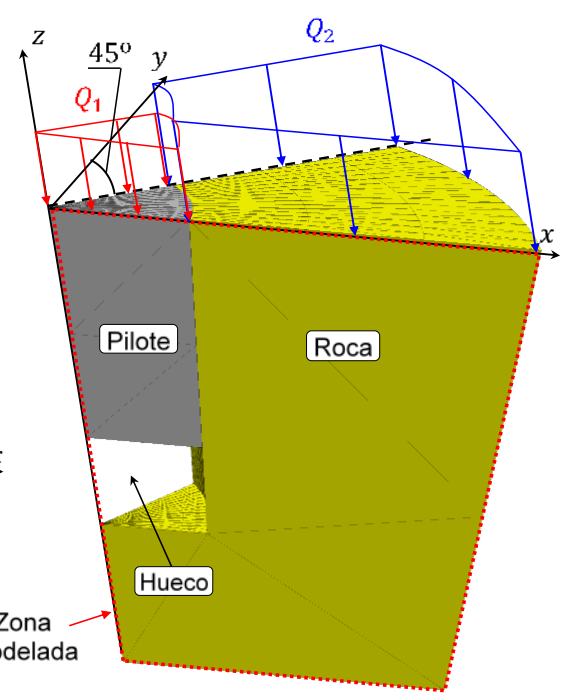

(b)

Figura 4.1. (a) modelo idealizado de un pilote empotrado en roca y su nomenclatura, (b) modelo con PFC ${ }^{3 D}$ de pilote empotrado en roca (Gutiérrez-Ch et al. 2020b).

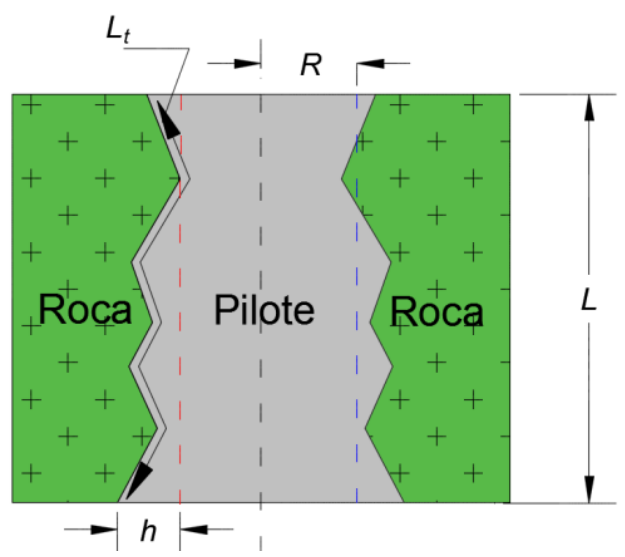

Figura 4.2. Parámetros para definir el factor de rugosidad $R F$ (modificado de Zhang 2004).

De acuerdo a O'Neill et al. (1996) y Hassan et al. (1997), la rugosidad de la pared de la cavidad ha sido simulada usando superficies sinusoidales con alturas de amplitudes de $h=\{0 ; 4 ; 10 ; 20 ; 32.5 ; 40\} \mathrm{mm}$ y con longitudes de onda de $500 \mathrm{~mm}$ en todos los modelos. Estos valores corresponden a factores de rugosidad de $R F=$ $\{0.000 ; 0.010 ; 0.025 ; 0.050 ; 0.085 ; 0.106\}$, respectivamente. En la Figura $4.3(a)$ se 
presenta la geometría resultante como consecuencia de emplear estos parámetros geométricos para modelizar la rugosidad en la interfaz roca-pilote, mientras que en la Figura 4.3(b) se muestran los parámetros geométricos antes mencionados, por ejemplo, para el modelo con $R F=0.106$.
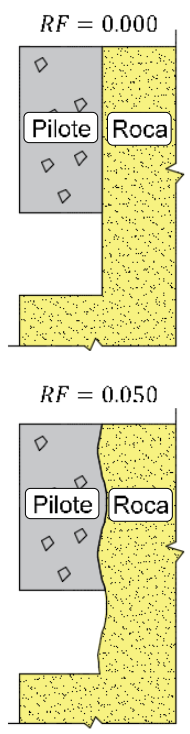
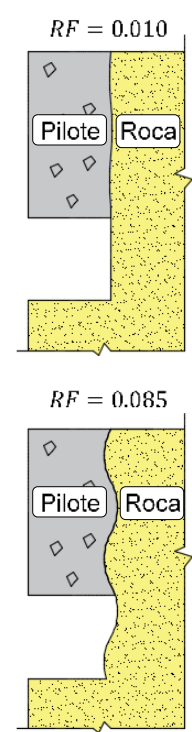
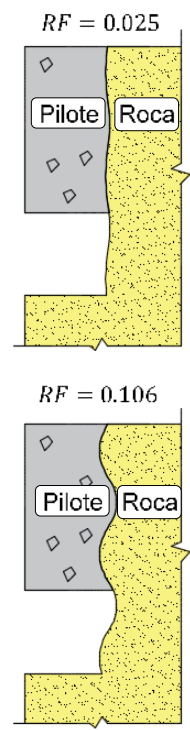

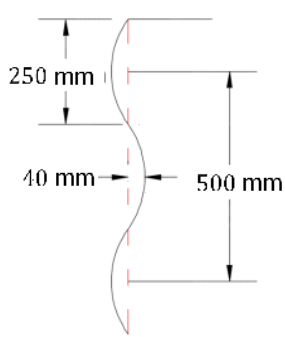

(b)

(a)

Figura 4.3. Esquema idealizado: (a) vista frontal 2D de los perfiles de rugosidad empleados en la interfaz roca-pilote, (b) ejemplo del detalle de los parámetros de la geometría sinusoidal para el modelo con $R F=0.106$ (modificado de Gutiérrez-Ch et al. 2019a).

\subsubsection{Calibración de los microparámetros de los materiales}

La simulación con el MED requiere de la selección previa del modelo de contacto que reproduzca el comportamiento del material o materiales -i.e., roca y hormigónpresentes en la modelización numérica, así como del modelo de contacto que reproduzca apropiadamente el comportamiento en la interfaz entre materiales diferentes. En este sentido, para los modelos numéricos de esta sección se ha utilizado el modelo de contacto FCJM (ver Capítulo 3, Sección 3.1.1) para simular la roca y el hormigón, mientras que para la interfaz de ambos materiales se ha empleado el modelo de contacto SJCM (ver Capítulo 3, Sección 3.1.2).

La calibración de los microparámetros del modelo de contacto FJCM se suele realizar por medio de ensayos de compresión uniaxial, a través de la comparación de 
macropropiedades -i.e., módulo de Young $(E)$, coeficiente de Poisson $(v)$ y la resistencia a compresión uniaxial pico $\left(\sigma_{c}\right)$ - obtenidas con el MED y mediante ensayos experimentales; mientras que para calibrar el modelo de contacto SJCM, se suele utilizar la comparación de los resultados de ensayos de corte directo (numéricos y experimentales) -i.e., la resistencia al corte pico $\left(\tau_{p}\right)$ y su correspondiente desplazamiento asociado $\left(\delta_{p}\right)$-. El detalle del proceso de calibración de los microparámetros de los modelos FJCM y SJCM, así como de los valores obtenidos para la roca (arenisca $(\mathrm{S} 2, \mathrm{~S} 3)$ y gneis), y para el hormigón $(\mathrm{C} 1, \mathrm{C} 2)$, de igual manera que para la interfaz de sus juntas, se presentaron en el Capítulo 3 (Secciones 3.3.2 y 3.3.4). Debe recordarse que, a pesar de que los parámetros usados en los modelos con PFC ${ }^{3 D}$ de esta sección provienen de la calibración de modelos con PFC ${ }^{2 D}$, en el Capítulo 3 (Sección 3.2.2) se demostró que la influencia de este aspecto (efecto escala de pasar del modelo 2D al modelo 3D) en los resultados es poco significativa.

\subsubsection{Ensamblaje inicial del modelo}

El pilote empotrado en roca considerado en el modelo de PFC ${ }^{3 D}$ (ver la Figura 4.4(b)) se compone de paredes sin fricción (para evitar el desarrollo de fuerzas de corte entre las partículas y las paredes, así como para reducir los efectos de la condición de borde), cuyo volumen interior es llenado con partículas distribuidas de forma aleatoria, simultánea e independiente dentro del cuerpo de la roca y del pilote (ver la Figura 4.4(a)). Adicionalmente, se asigna un valor de rigidez normal mucho mayor a las paredes del modelo que a las partículas; de esta manera, el solape entre el contacto partícula-pared es mínimo. El diámetro de las partículas que conforman la roca y el pilote siguen una distribución uniforme definida por sus radios mínimo $\left(R_{\min }\right)$ y máximo $\left(R_{\max }\right)$ (ver la Tabla 4.1), los cuales han sido definidos a partir de un análisis de sensibilidad, en el cual se ha verificado que el comportamiento del pilote no es afectado en gran medida por el tamaño de partícula (ver la Figura 4.5). 

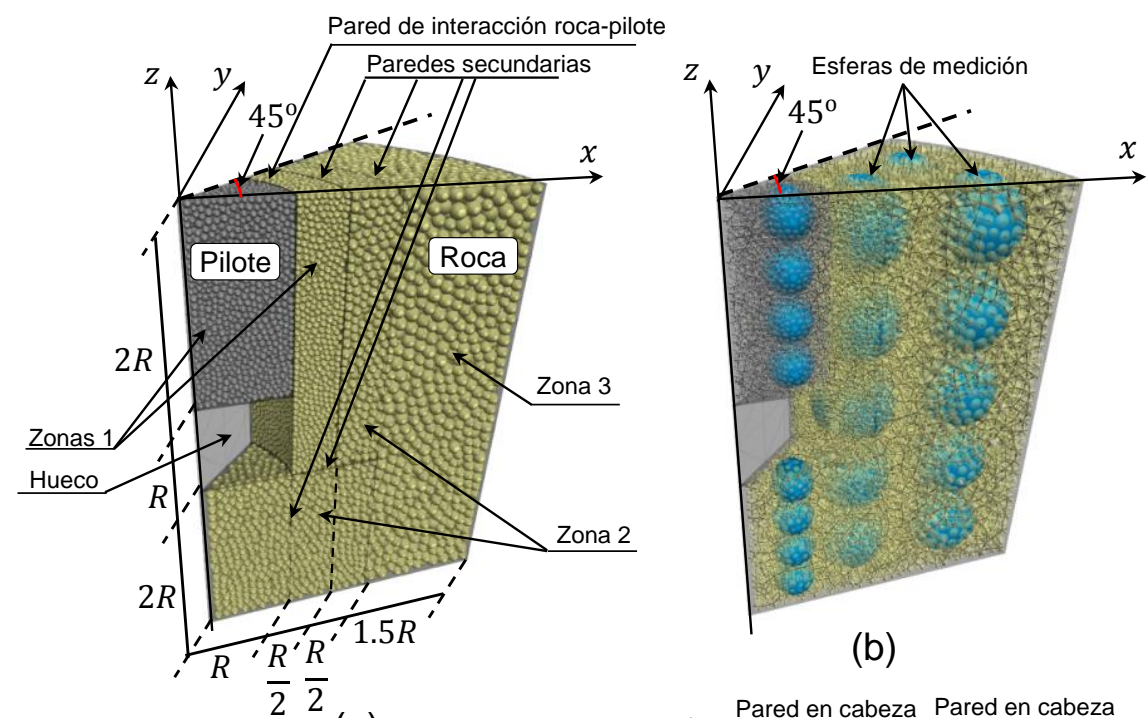

(b)

(a)

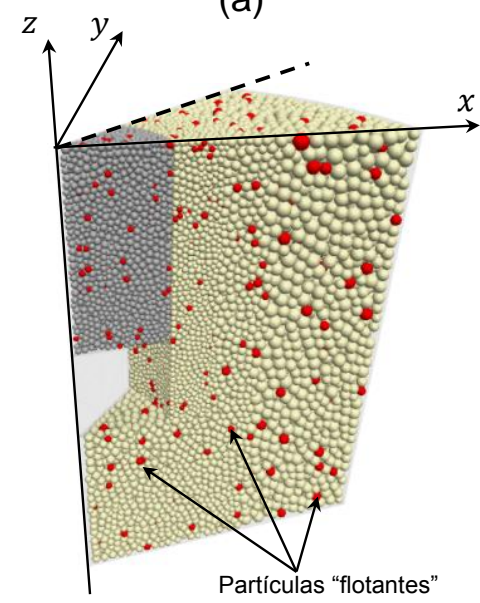

(c)

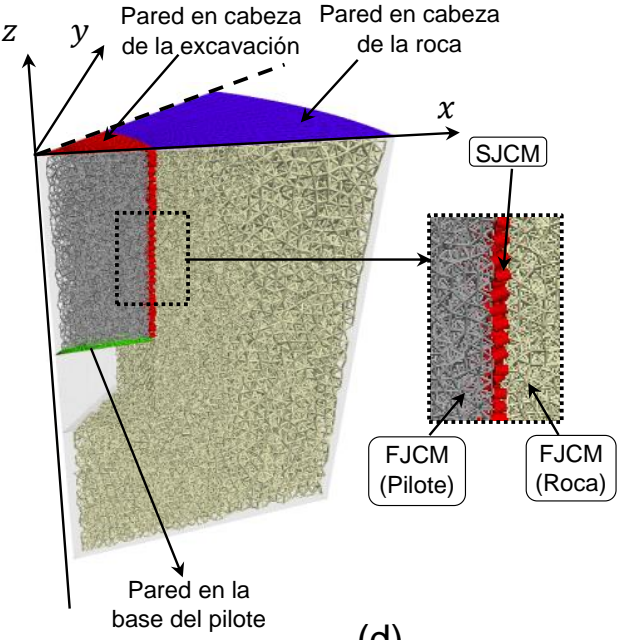

(d)

Figura 4.4. Generación del Modelo con PFC $3 \mathrm{D}$ : (a) geometría del ensamblaje inicial del modelo, (b) distribución de las fuerzas después de la instalación del esfuerzo isotrópico (las esferas azules representan las zonas de medición), (c) identificación de las partículas "flotantes" (esferas rojas), (d) asignación de los modelos de contacto FJCM y SJCM.

Por otra parte, para optimizar el tiempo de cálculo computacional, se ha realizado un refinamiento del tamaño de partículas en tres zonas del modelo, cuya distancia ha sido establecida en función del radio nominal del pilote $(R)$, de modo que se emplean las partículas más pequeñas en la zona de contacto roca-pilote (zona 1), (ver la Figura 4.4(a) y la Tabla 4.1); además, se ha considerado solo una porción de 1/8 de la sección transversal del pilote (i.e., de un ángulo de $45^{\circ}$ ), en lugar de la sección completa del pilote (ver la Figura 4.4(a)). Adicionalmente, para evitar movilizar la resistencia por punta, se ha dejado un hueco en la base del pilote con una altura equivalente a un radio 
del pilote. El radio $(R)$ y la longitud nominal empotrada en roca $(L)$ del pilote es de $0.4 \mathrm{~m}$ y $0.8 \mathrm{~m}$, respectivamente, para todos los modelos analizados.

Tabla 4.1. Distribución del tamaño de partícula empleado en la modelización con PFC ${ }^{3 D}$

\begin{tabular}{cccc}
\hline & & Radio mínimo, $R_{\min }(\mathrm{cm})$ & $R_{\max } / R_{\min }$ \\
\hline Roca & Zona 1 & 1.00 & 1.5 \\
$($ arenisca (S2,S3) y & Zona 2 & 1.50 & 1.5 \\
gneis) & Zona 3 & 2.25 & 1.5 \\
\hline Pilote & Zona 1 & 1.00 & \multirow{2}{*}{1.5} \\
\hline
\end{tabular}

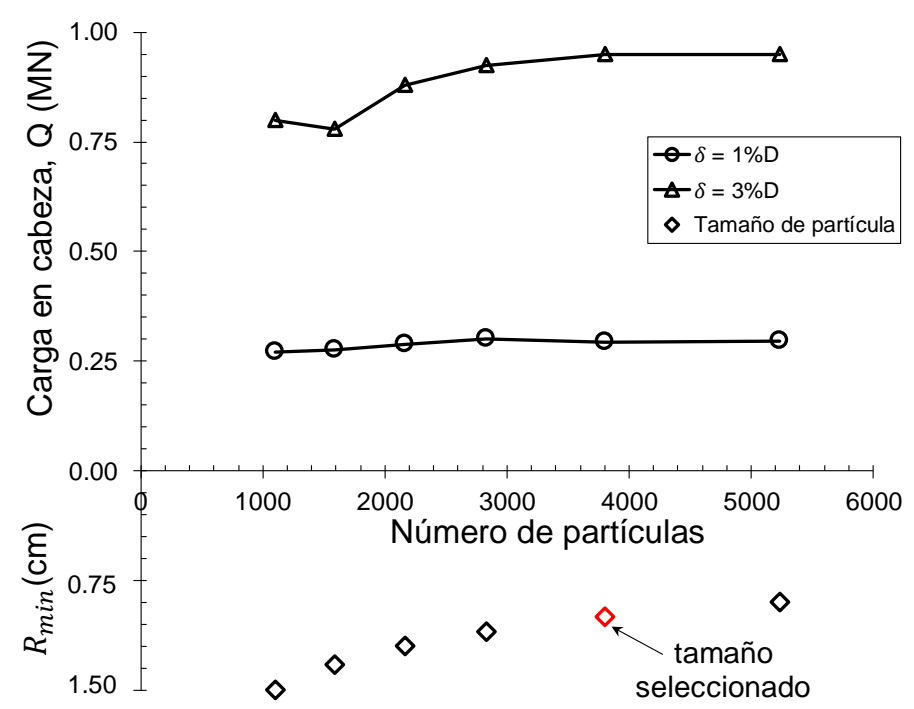

Figura 4.5. Selección del radio mínimo $\left(R_{\min }\right)$ de las partículas de los modelos con PFC ${ }^{3 \mathrm{D}}$ : análisis de sensibilidad realizado sobre el pilote empotrado en arenisca-S3 con $R F=0.025$ (para facilitar la ilustración solo se representaron el número de partículas correspondientes al cuerpo del pilote).

\subsubsection{Aplicación del esfuerzo isotrópico inicial}

Al inicio de esta fase, se han eliminado las paredes secundarias empleadas (temporalmente) para refinar del tamaño de partículas en el ensamblaje inicial. Luego, se han instalado regiones de medición (esferas azules en la Figura 4.4(b)) dentro de la roca y del pilote y se ha calculado, dentro de cada región, el esfuerzo normalizado $\sigma_{\text {step }}=\left(\sigma_{o}^{c}-\sigma_{o}\right) / \sigma_{o}^{c}$, donde $\sigma_{o}$ es el esfuerzo isotrópico $\left(\sigma_{o}=\left(\sigma_{11}+\sigma_{22}+\sigma_{33}\right) / 3\right) \mathrm{y}$ $\sigma_{o}^{c} \cong 1 \%$ de la resistencia a compresión uniaxial de cada material. Si $\sigma_{\text {step }} \geq \sigma_{\text {tol }}$, donde $\sigma_{t o l}$ es la tolerancia definida por el usuario, el radio de todas las partículas es cambiado 
de forma iterativa, en caso contrario, la iteración culmina (de acuerdo a Bahaaddini et al. 2013b y Gutiérrez-Ch et al. 2018 se ha empleado $\left.\sigma_{t o l}=0.5\right)$. De esta manera, se obtiene una mejor distribución de contactos entre partículas y se reduce la magnitud de las fuerzas localizadas dentro de cada material.

\subsubsection{Eliminación de partículas "flotantes"}

Es posible que durante el proceso de generación del modelo con PFC ${ }^{3 \mathrm{D}}$ resulten partículas con menos de tres contactos con sus partículas adyacentes (esferas rojas en la Figura 4.4(c)); esto no es un comportamiento "real" que puede esperarse en medios discretos de este tipo. Para corregir esto, se ha incrementado el radio de todas las partículas "flotantes" hasta que dichas partículas tengan, al menos, tres contactos con otras partículas de su entorno.

\subsubsection{Aplicación de los modelos de contacto FJCM y SJCM}

En esta fase, en primer lugar se ha aplicado el modelo de contacto FJCM (ver la Figura 4.4(d)), con sus correspondientes propiedades, al contacto entre partículas cuya separación es igual o menor que el gap inicial $\left(g_{o}\right)$ definido por el usuario. (Las propiedades calibradas para el modelo FJCM fueron presentadas en el Capítulo 3, Tabla 3.1). Posteriormente, se ha eliminado la pared que está en el plano de la interfaz rocapilote (nótese que, debido a que el valor de la cohesión entre el contacto partículapartícula del cuerpo de roca es elevado, no se producen caída de partículas hacia el hueco dejado en la punta). De esta manera, las partículas en la interfaz roca-pilote son fácilmente identificadas, y se les asigna el modelo de contacto SJCM (líneas rojas en la Figura 4.4(d)). Las propiedades del modelo SJCM, calibradas para cada interfaz rocapilote, fueron presentadas en el Capítulo 3, Tabla 3.4.

En la Figura 4.6(a) se muestran los vectores normales unitarios $\left(\hat{n}_{j}\right)$ de cada contacto del modelo SJCM. (Se ha implementado una función FISH -FISH es un lenguaje de 
programación que permite al usuario interactuar y realizar operaciones en PFC (Itasca Consulting Group Inc 2014)- para seguir el cambio de orientación de cada vector $\hat{n}_{j}$, desde la aplicación del modelo de contacto SJCM hasta la culminación del ensayo). Como puede observarse, su orientación -i.e., su ángulo de dirección $\left(\theta_{d}\right)$ y buzamiento $\left(\theta_{p}\right)$, para más detalles ver Capítulo 3 (Sección 3.2.2)- depende de la morfología de la superficie rugosa que conforma la interfaz roca-pilote.. En la Figura 4.6(b) se muestra cómo varía la orientación de los vectores $\hat{n}_{j}$ para dos modelos, con factores de rugosidad de $R F=0.025$ y $R F=0.106$. Para facilitar su visualización e interpretación, estos vectores se han representado en proyecciones estereográficas (Ragan 2009).
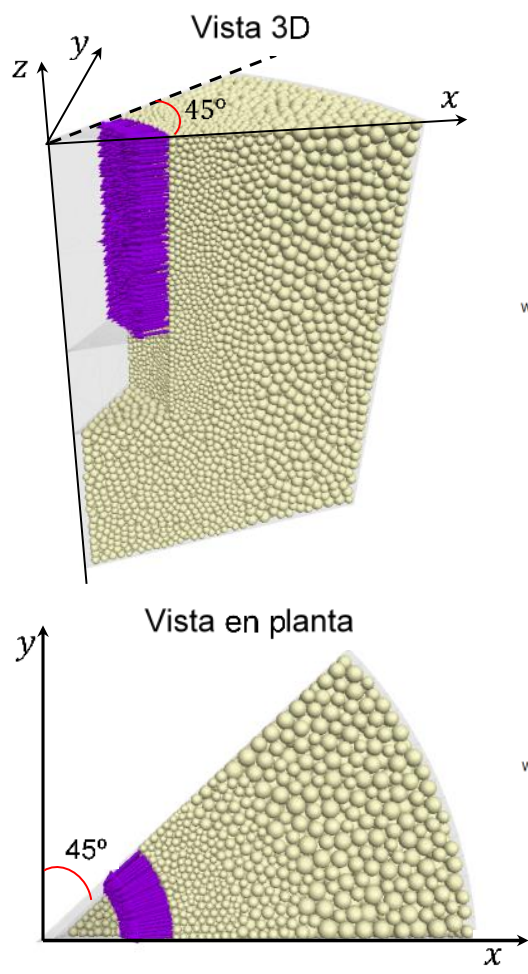

(a)
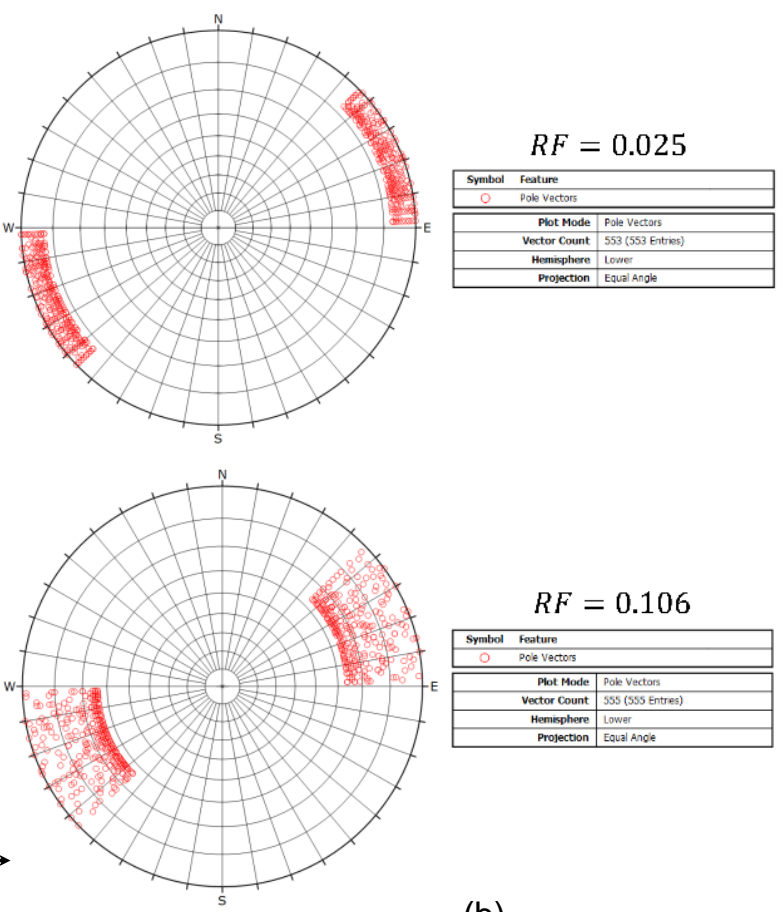

(b)

Figura 4.6. Representación de los vectores unitarios normales del modelo de contacto SJCM: (a) vista $3 \mathrm{D}$ y vista en planta de los vectores $\hat{n}_{j}$ (las flechas color purpura representan los vectores $\hat{n}_{j}$ en cada contacto partícula-partícula en el plano de la interfaz roca-pilote), (b) representación estereográfica de los vectores $\hat{n}_{j}$ para los modelos con $\mathrm{PFC}^{3 \mathrm{D}}$ con $R F=0.025$ y $R F=0.106$ (los puntos rojos representan los polos de los vectores $\hat{n}_{j}$ ). (Notar que para facilitar la ilustración se ha suprimido las partículas del cuerpo del pilote) (Gutiérrez-Ch et al. 2020b). 


\subsubsection{Aplicación de las cargas actuantes y condiciones de borde}

Los siguientes pasos son la aplicación de la fuerza de gravedad (para inducir los esfuerzos geoestáticos debidos al peso propio), y la aplicación de las cargas externas que representan el peso del espesor del estrato de suelo por encima del estrato de roca $\left(Q_{2}\right)$, y el peso propio de la porción de pilote embebido en el estrato de suelo $\left(Q_{1}\right)$ (ver la Figura 4.2(b)). Para ello, se ha empleado el mecanismo de servo-control de PFC (Itasca Consulting Group Inc 2014), el cual permite controlar la velocidad de traslación de la pared seleccionada -e.g., paredes de color rojo $\left(Q_{1}\right)$ y púrpura $\left(Q_{2}\right)$ de la Figura 4.7(a) - y así aplicar o mantener una fuerza dada sobre dicha pared. De esta forma, se ha modelado solo la porción de pilote empotrado en la roca, reduciendo significativamente el tamaño del modelo numérico y su correspondiente tiempo de cálculo.

En cuanto a las condiciones de borde, se han restringido los desplazamientos de todas las paredes de los bordes del modelo y se ha eliminado también la pared de color verde existente en la punta del pilote (ver la Figura 4.4(d)); de nuevo, nótese que la elevada cohesión entre partículas del cuerpo del pilote hace que no se produzcan caídas de estas partículas hacia el hueco dejado en la punta, ver la Figura 4.7(a). Para comenzar el ensayo de carga sobre el pilote del modelo con MED ${ }^{3 D}$, se ha aplicado una velocidad axial $\left(V_{z}\right)$ de $0.05 \mathrm{~m} / \mathrm{s}$-o lo suficientemente baja para garantizar que el modelo del pilote empotrado en roca permanezca en equilibrio cuasiestático- sobre la pared en la cabeza del pilote (pared de color rojo de la Figura 4.7(a)). Durante el ensayo la carga en cabeza (P) y el asiento en cabeza del pilote $(\delta)$ se han obtenido, respectivamente, mediante el registro de la fuerza de reacción axial $\left(F_{z}\right)$ sobre dicha pared y su respectivo desplazamiento $\left(u_{z}\right)$.

Finalmente, en la Figura 4.7(b) se muestra un ejemplo de la apariencia que tienen los modelos con $\mathrm{MED}^{3 \mathrm{D}}$ de los pilotes excavados en arenisca(S3) tras aplicar la 
metodología de preparación del modelo explicada en los apartados anteriores. Para facilitar la ilustración de la distribución de partículas, y la rugosidad en la interfaz rocapilote, se ha omitido la representación de las paredes en todos los modelos de la Figura 4.7(b). Adicionalmente, debe hacerse notar que pilotes "perfectamente lisos" con $R F=$ 0.000 (ver la Figura 4.7(b)) podrían no ser del todo "reales", al no ser posible de conseguir con las herramientas habituales de perforación de pilotes en roca; no obstante, este modelo numérico se ha utilizado como referencia del menor valor de la resistencia por fuste que podría obtenerse.

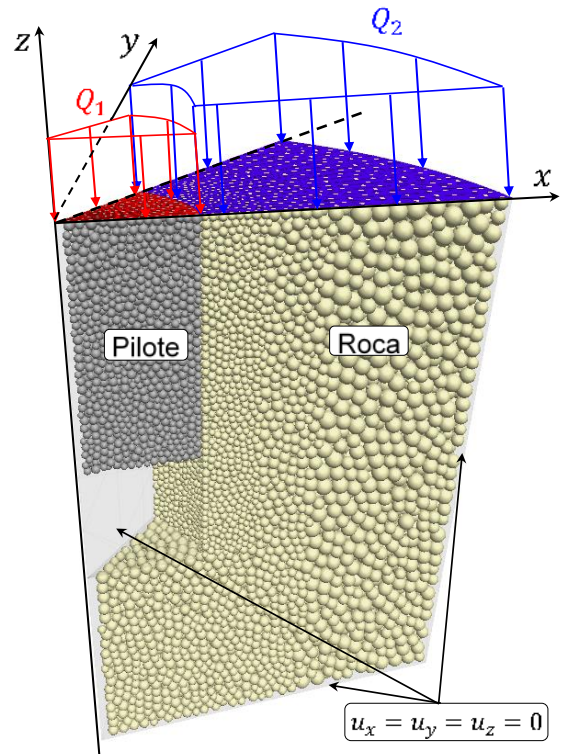

(a)

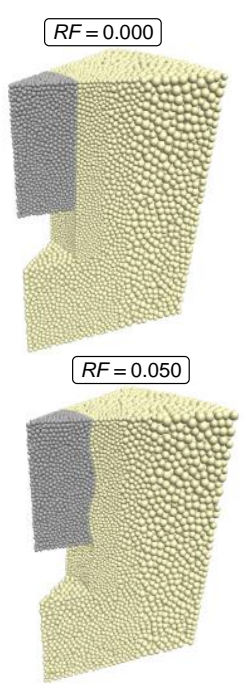

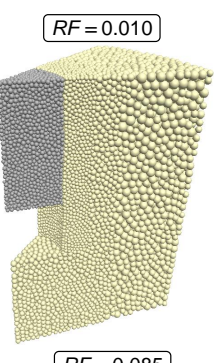

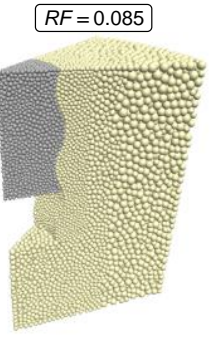

(b)
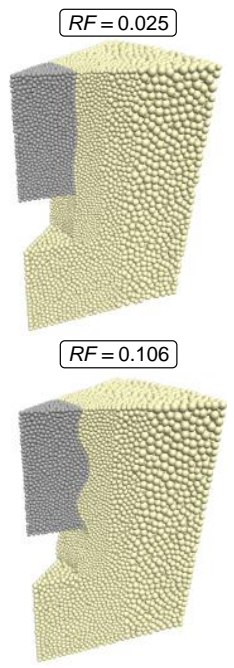

Figura 4.7. (a) condiciones de borde y cargas actuantes, (b) vista de los modelos con PFC ${ }^{3 D}$ de los pilotes empotrados en roca con diferente nivel de rugosidad en función del factor $R F$.

\subsection{Resultados de los modelos con MED}

En esta sección se presentan los resultados obtenidos, tras aplicar la metodología desarrollada en la Sección 4.2 para la simulación numérica de pilotes empotrados en roca mediante el uso de modelos con $\mathrm{PFC}^{3 \mathrm{D}}$.

\subsubsection{Comportamiento de carga axial-asiento}

En la Figura 4.8 se muestran las curvas de carga en cabeza vs asiento en cabeza (y vs el asiento normalizado por el diámetro del pilote, $\delta / D)$ obtenidas en los modelos con 
$\mathrm{PFC}^{3 \mathrm{D}}$ para los 6 pilotes empotrados en la arenisca-S3, usando diferentes rugosidades en la interfaz roca-pilote. Por otra parte, en la discusión que sigue, los términos "asiento en cabeza" y "carga en cabeza", están referidos al asiento y carga medidos en la pared ubicada en la cabeza del pilote (pared de color rojo de la Figura 4.7(a)). Todos los ensayos de carga se han realizado hasta alcanzar un asiento en cabeza $(\delta)$ del pilote de valor igual a $10 \%$ el diámetro del pilote $(\delta / D=10 \%)$.

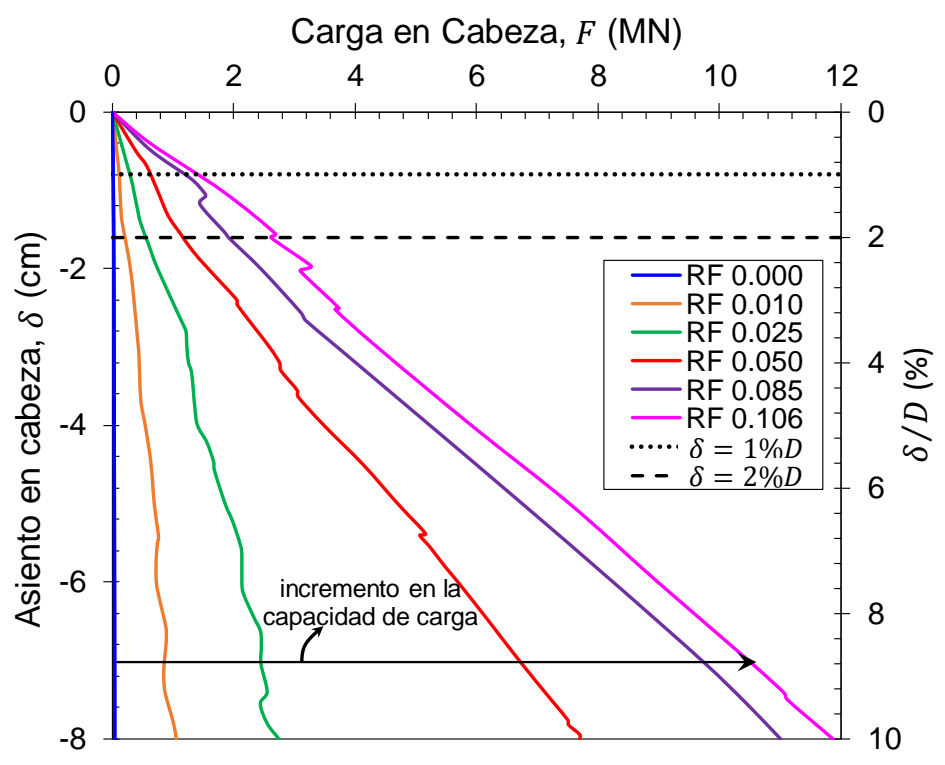

Figura 4.8. Comparación de las curvas carga en cabeza vs asiento cabeza obtenidas en los modelos con PFC ${ }^{3 \mathrm{D}}$ para los pilotes (hormigón-C1) empotrados en arenisca-S3 con diferente grado de rugosidad (modificado de Gutiérrez-Ch et al. 2020b).

Los resultados presentados en la Figura 4.8 muestran que la rugosidad en la interfaz roca-pilote aumenta significativamente la capacidad de carga y la rigidez de los pilotes empotrados en roca. Por ejemplo, como se muestra en la Tabla 4.2 y Figura 4.8, se observa que para un asiento en cabeza equivalente al $2 \% D$, el modelo de pilote con $R F=0.050$ resiste 2.1 veces más carga axial que el pilote con $R F=0.025$ (este comportamiento también fue observado para los modelos de pilotes excavados en la arenisca-S2 y en el gneis). Esto cualitativamente está en concordancia con los ensayos de carga realizados por Pells et al. (1980), en los que los pilotes rugosos soportaron una carga axial mayor -alrededor de 1.2 a 1.7 veces más dependiendo de la resistencia a compresión simple de la roca (areniscas con $\sigma_{c}=6-40 \mathrm{MPa}$ ), ver Capítulo 2 (Sección 
2.3.1.1) - que la soportada por los pilotes lisos. Una respuesta similar se observó en los ensayos de carga realizados por Horvath et al. (1983) (ver la Tabla 4.2), quienes indicaron que el pilote con $R F=0.076$ resistió 1.3 veces la carga axial soportada por el pilote con $R F=0.036$ para $\delta=2 \% D$ (ver Capítulo 2 (Sección 2.3.1.3)).

Tabla 4.2 Carga axial soportada en cabeza de pilotes excavados en roca con diferente nivel de rugosidad y para un asiento en cabeza equivalente a $2 \%$ el diámetro del pilote.

\begin{tabular}{ccccccc}
\hline a) Modelos con PFC & DD \\
\hline Modelo & 1 & 2 & 3 & 4 & 5 & 6 \\
Factor de rugosidad & 0.000 & 0.010 & 0.025 & 0.050 & 0.085 & 0.106 \\
Carga en cabeza, $P(\mathrm{kN})$ & 20.2 & 148.7 & 451.7 & 941.1 & 1675.4 & 2438.9 \\
\hline b) Ensayos de carga realizados sobre pilotes excavados en lutita por Horvath et al. (1983) \\
\hline Modelo & $\mathrm{P} 1$ & $\mathrm{P} 3$ & $\mathrm{P} 6$ & & & \\
Factor de rugosidad & 0.036 & 0.076 & 0.100 & & & \\
Carga en cabeza, $P(\mathrm{kN})$ & 3996.5 & 5189.3 & 7273.9 & & & \\
\hline
\end{tabular}

\subsubsection{Comportamiento de resistencia promedio por fuste-asiento}

Debido a que los modelos con PFC $^{3 D}$ desarrollados en esta tesis se componen de un ensamblaje de partículas y paredes, en lugar de un medio continuo, y debido que no se moviliza la resistencia por punta (a causa del hueco dejado en la base del pilote), la resistencia promedio por fuste movilizada a lo largo de la interfaz roca-pilote ha sido calculada a partir de las fuerzas de interacción entre las partículas de la interfaz rocapilote, mediante la siguiente ecuación:

$$
f_{\text {ave }}=\frac{\sum F_{z}}{A}
$$

donde $f_{\text {ave }}$ es la resistencia promedio por fuste (para un determinado asiento en cabeza), $\sum F_{z}$ es la sumatoria de la componente axial de las fuerzas de contacto actuantes sobre todas las partículas de la interfaz roca-pilote, y $A$ es el área nominal de la superficie cilíndrica que conforma la interfaz roca-pilote.

La Figura 4.9 muestran la evolución, para los 16 modelos de pilotes desarrollados en $\mathrm{PFC}^{3 \mathrm{D}}$ en función del asiento en cabeza del pilote $(\delta)$, de la resistencia promedio por 
fuste del pilote $\left(f_{\text {ave }}\right.$; calculado usando la Ecuación 4.2) y del número de grietas formadas entre partículas, por rotura de sus enlaces cohesivos. Los resultados muestran que $f_{\text {ave }}$ aumenta al incrementar la rugosidad en la interfaz roca-pilote; por ejemplo, en la Figura 4.9(a) se observa que la $f_{\text {ave }}$ movilizada para el modelo con $R F=0.025$ es aproximadamente 2 veces mayor que la $f_{\text {ave }}$ movilizada para el modelo con $R F=0.010$. Este comportamiento posiblemente se debe a que la junta más rugosa (i.e., con mayor $R F$ ), tiende a dilatar más y, debido a la restricción de movimiento en la interfaz -la cual es probablemente más cercana a la condición de borde de rigidez normal constante (CNS); ver el Capítulo 3 (Sección 3.4.2) para más detalles-, se produce un incremento significativo del esfuerzo normal actuante sobre la interfaz roca-pilote, lo cual a su vez genera un mayor aumento en la resistencia promedio por fuste de los modelos con mayor factor de rugosidad $R F$ (ver e.g., Seol y Jong 2007; Gutierrez-Ch et al. 2018).

Por otra parte, en la Figura 4.9 se presenta también el número de micro-grietas generadas (por corte o por tracción) en los contactos entre partículas en la roca y el pilote. Estas curvas aportan una información interesante, al observarse que el número de estás micro-grietas es pequeño para asientos en cabeza menores a $1 \% D$ (e.g., ver las micro-grietas generadas para los modelos de pilotes empotrados en la arenisca-S3 con $R F=0.010$ y $R F=0.050)$, y que este número de micro-grietas aumenta notablemente en todos los modelos para asientos en cabeza mayores a $1 \% D$. Estos resultados están de acuerdo con trabajos previos (ver e.g., Bahaaddini et al. 2013b; Gutierrez-Ch et al. 2018), que sugieren que en modelos con poca rugosidad (i.e., con bajo valor de $R F$ ), la rotura en el plano de contacto se produce por un mecanismo de deslizamiento, mientras que para superficies más rugosas (i.e., con mayor $R F$ ), se produce una mayor degradación y daño en las asperidades (ver la Figura 4.10). 


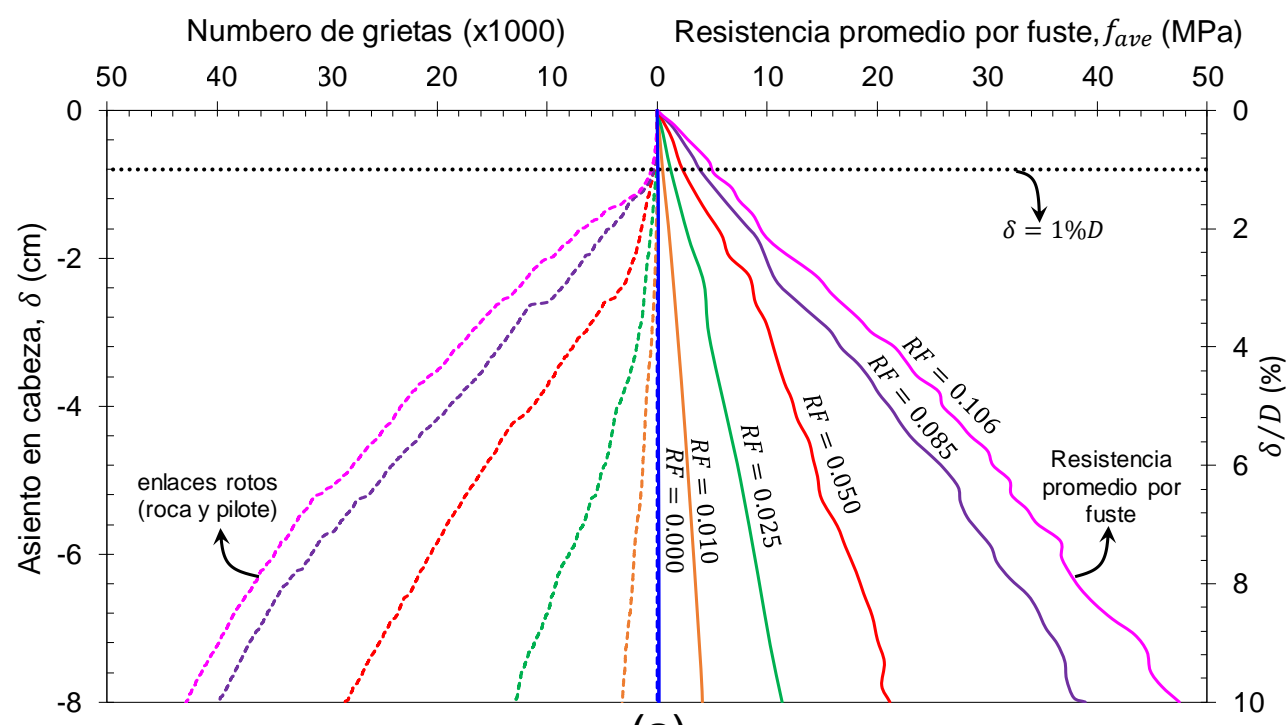

(a)

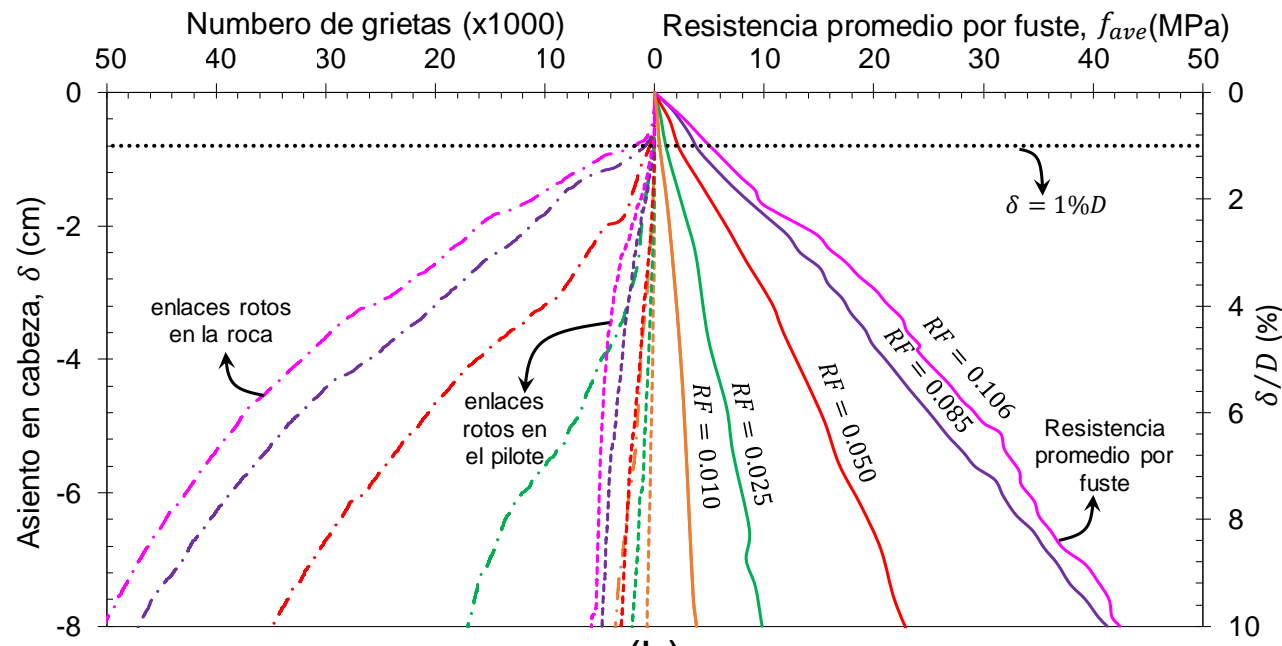

(b)

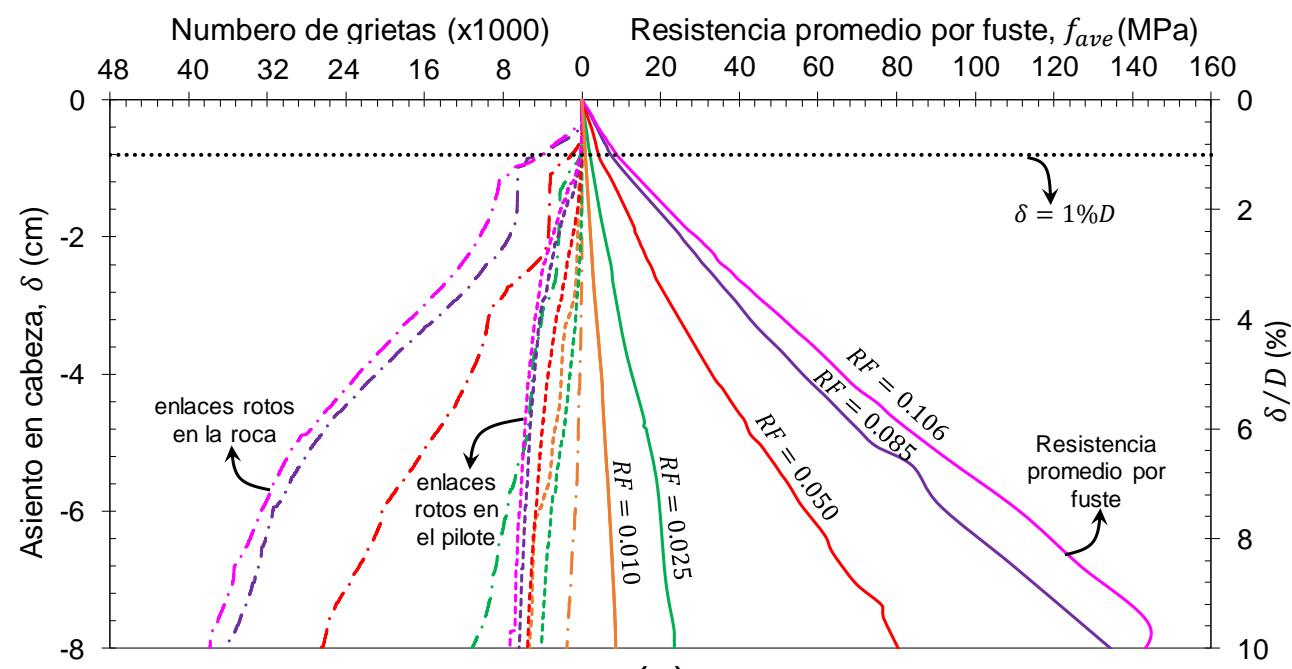

(c)

Figura 4.9. Resistencia promedio por fuste y numero de grietas vs asiento cabeza obtenidos en los modelos con PFC $3 \mathrm{D}$ en: (a) pilotes (hormigón-C1) empotrados en arenisca-S3 (modificado de Gutiérrez-Ch et al. 2020b), (b) pilotes (hormigón-C1) empotrados en arenisca-S2 (modificado de Gutiérrez-Ch et al. 2019c), (b) pilotes (hormigón-C2) empotrados en gneis, con diferente grado de rugosidad (modificado de Gutiérrez-Ch et al. 2020b). 

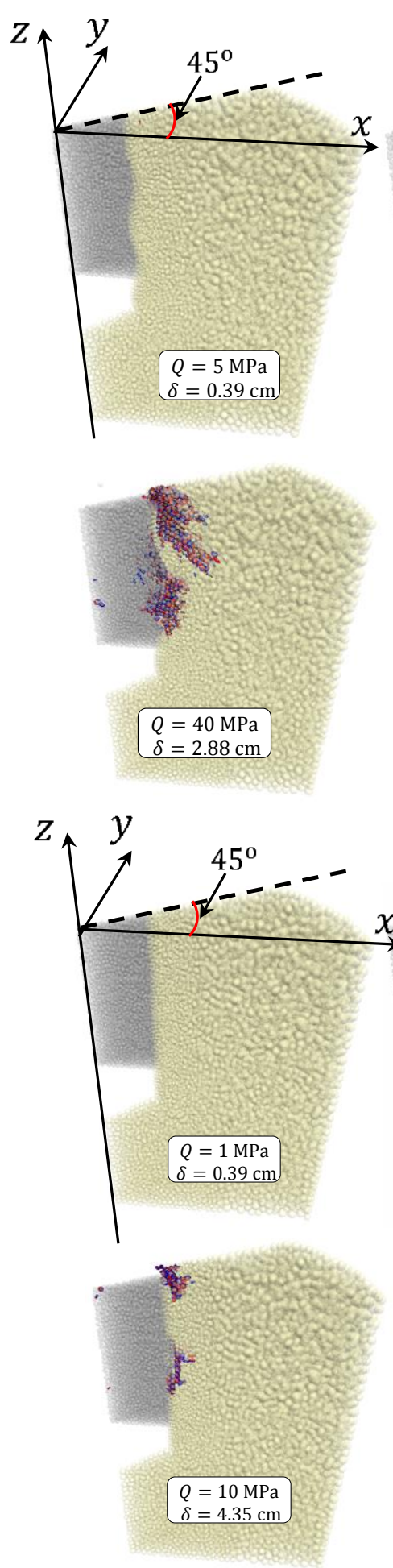
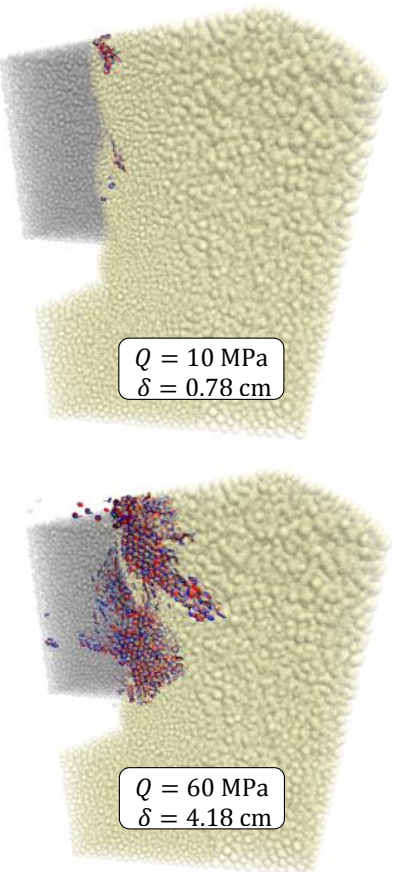

(a)
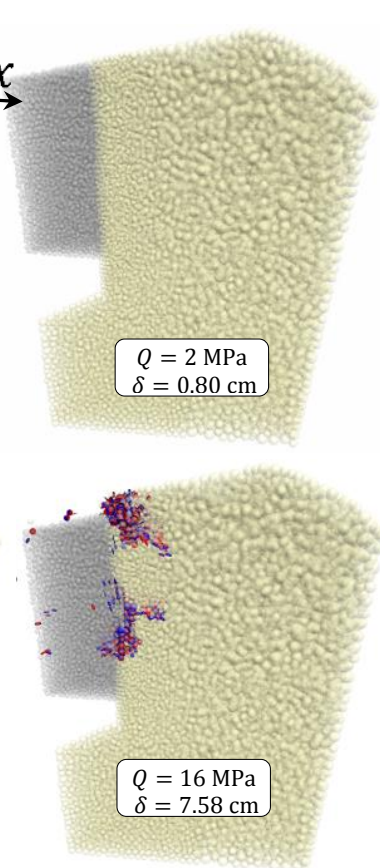

(b)
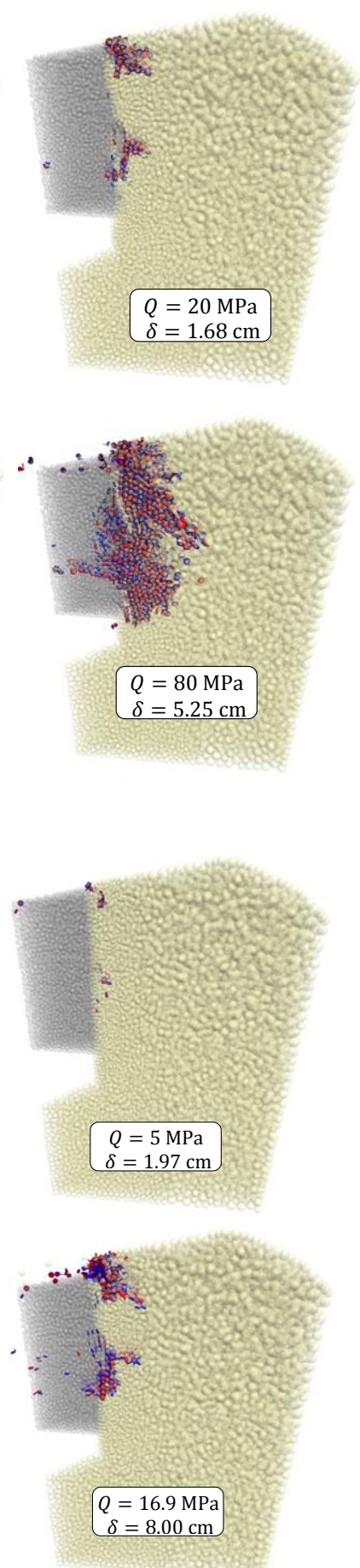

Figura 4.10. Propagación de micro-grietas bajo diferentes pasos de carga axial para los pilotes empotrados en arenisca-S3: (a) modelo con $R F=0.050$, (b) modelo con $R F=0.010$. (los colores azul y rojo representan las grietas por cortante y por tracción, respectivamente) (modificado de Gutiérrez-Ch et al. 2019b).

Adicionalmente, la Figura 4.9 muestran que el número de micro-grietas producidos en la roca es mayor que en el pilote. Este comportamiento quizás sería el esperado en el 
caso de las Figura 4.9(a)-(b), debido a que el hormigón-C1 es más resistente que la arenisca-(S2,S3) (ver sus micropropiedades en el Capítulo 3, Tabla 3.2); no obstante, en la Figura 4.9 (c) ocurre el mismo comportamiento -aunque con menos grietas en el homigón-C2 en comparación al hormigón-C1, por ser el homigón-C2 más resistente (ver Capítulo 3, Tabla 3.2)- a pesar de que el gneis tiene mayor resistencia a compresión simple que el hormigón-C2. (Esto puede atribuirse a la forma en que se produce la transferencia de carga, según principalmente se analiza en detalle en la Sección 4.3.4). En todo caso, el comportamiento reflejado en las Figuras 4.9 y 4.10 sugiere que el criterio de diseño de considerar como asiento máximo del pilote un valor de $1 \% D$ (o $0.01 D$ ) -ampliamente utilizado para la estimación de la resistencia por fuste de pilotes bajo carga axial empotrados en roca (Whitaker y Cooke 1966; Horvath y Kenney 1979)es apropiado para evitar un daño excesivo en la interfaz roca-pilote.

Por otra parte, la Figura 4.11 muestra la relación entre la rugosidad de la interfaz rocapilote (definida en función del factor de rugosidad $R F$ ) y las resistencias promedio por fuste calculadas en los modelos con PFC ${ }^{3 \mathrm{D}}$, para valores de asiento en cabeza del pilote de $1 \%$ al $3 \%$ del diámetro del pilote. Como se puede observar, las curvas muestran un comportamiento aproximadamente lineal para pequeños asientos en cabeza del pilote, cuando todavía no se ha producido un gran daño en la interfaz roca-pilote; sin embargo, esta relación parece aumentar su no-linealidad para asientos en cabeza mayores, en los cuales se ha producido un aumento significativo en el número de micro-grietas (ver las Figuras 4.9 a 4.11$)$.

De acuerdo con trabajos previos (e.g., Dai et al. 2017), los resultados presentados en la Figura 4.9 sugieren que podría existir un límite superior de la rugosidad, por encima del cual no aumenta significativamente la resistencia promedio por fuste a pesar de aumentar la rugosidad en la interfaz roca-pilote. En cualquier caso, los resultados 
presentados confirman que la rugosidad es un factor de gran importancia que debería ser incorporado en el diseño de pilotes empotrados en roca.

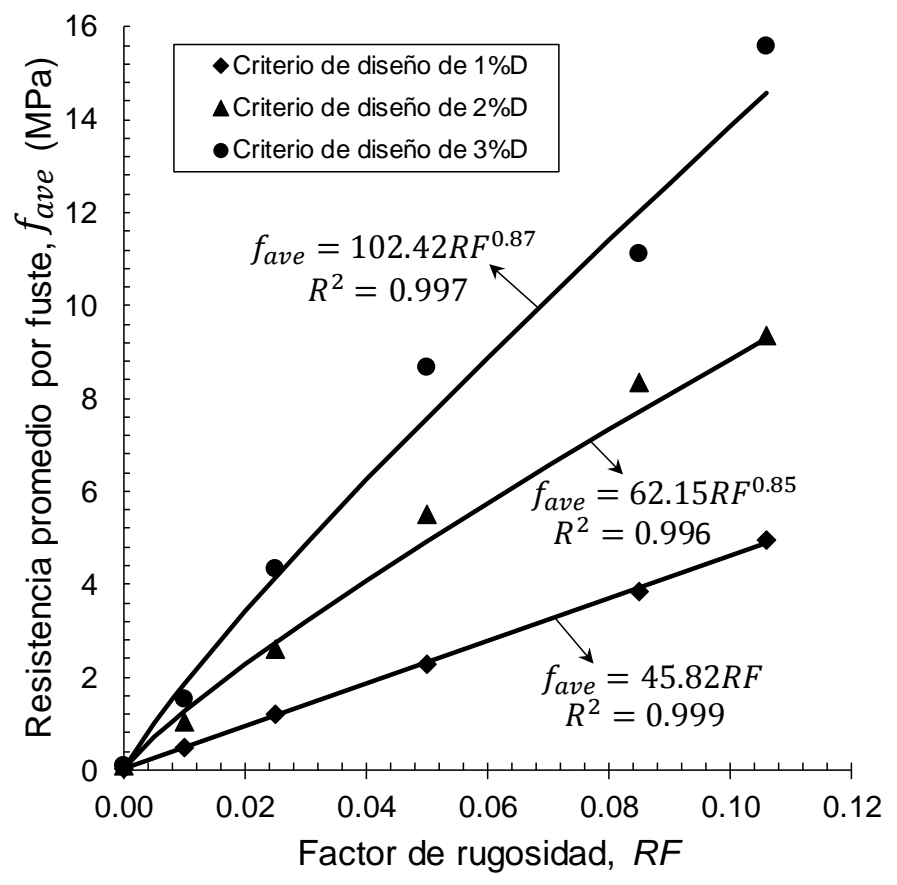

Figura 4.11. Resistencia promedio por fuste vs factor de rugosidad para valores de asiento en cabeza de $1 \%$ al $3 \%$, obtenidos con modelos $\mathrm{PFC}^{3 \mathrm{D}}$ en pilotes (hormigón-C1) empotrados en arenisca-S3 y con diferente grado de rugosidad (modificado de Gutiérrez-Ch et al. 2020b).

\subsubsection{Esfuerzo axial y resistencia por fuste movilizada a lo largo del pilote}

En la Figura 4.12 se muestra la distribución del esfuerzo axial $\left(\sigma_{z}\right)$ obtenida en $\mathrm{PFC}^{3 \mathrm{D}}$ a lo largo de los pilotes empotrados en la arenisca-S3, considerando diferente nivel de rugosidad (en función del factor de rugosidad $R F$ ) y diferentes valores de esfuerzo axial (Q) actuante en cabeza de dichos pilotes; en la Figura 4.13 se muestra también, la distribución de resistencia por fuste $(f)$ movilizada en la interfaz roca-pilote de estos mismos modelos. La distribución de esfuerzo axial ha sido estimada mediante esferas de medición distribuidas y espaciadas (cada $10 \mathrm{~cm}$ ) a lo largo de un eje axial que atraviesa todo el cuerpo del pilote (ver la Figura 4.12(a)), mientras que la distribución de resistencia por fuste se ha calculado mediante la Ecuación 4.2 (ver la Sección 4.3.2), a partir de las fuerzas axiales registradas a lo largo del pilote (ver la Figura 4.13(a)). 
En general, se observa que gran parte del esfuerzo axial es soportado en la parte superior del pilote -i.e., hasta una distancia de $0.5 \mathrm{D}$ medido desde la cabeza del pilotepara $R F$ desde 0.010 hasta 0.106 , mientras que la distribución de la resistencia movilizada por fuste parece depender de la geometría de las asperidades, de modo que es mayor en la zona de la interfaz en que se encuentra el frente de las asperidades (ver las Figuras 4.12(b) y 4.13(b)). Sin embargo, para el modelo perfectamente liso (con $R F=$ 0.000) se observa una disminución progresiva de $\sigma_{z}$ hacia la punta del pilote. Este comportamiento en el modelo con $R F=0.000$ puede deberse a que no hay efecto de la rugosidad en la interfaz, presentando así una distribución de $f$ casi uniforme a lo largo de todo el pilote (ver Figura 4.13(b))- y, en el que además, se ha movilizado totalmente su capacidad resistente; notar como no hay gran diferencia en la distribución de $f$ para valores de $\mathrm{Q}$ de $0.65 \mathrm{MPa}(\delta=6 \mathrm{~cm})$ y $0.64 \mathrm{MPa}(\delta=8 \mathrm{~cm})$, ver la Figura $4.13(\mathrm{~b})$.

Por otra parte, en las Figuras 4.13(b) y 4.14(b) se presenta el efecto de la rugosidad sobre la resistencia por fuste de pilotes empotrados en la arenisca-S3. La Figura 4.13(b) muestra la $f$ movilizada a lo largo de toda la interfaz roca-pilote para diversos valores de carga aplicada en cabeza (y sus correspondientes asientos asociados), mientras que la Figura 4.14(b) muestra la $f$ movilizada, en diferentes puntos de la interfaz roca-pilote, y en función del asiento en cabeza que sufre el mismo. Para facilitar la ilustración del efecto de la rugosidad sobre la resistencia por fuste, en la Figura 4.14(b) se ha empleado la misma escala en todos los modelos; notar, además, que para la sección de medición en la posición $-4 \mathrm{~cm}$ (línea roja), no hay registro de $f$ para asientos en cabeza aproximadamente mayores a $4.5 \mathrm{~cm}$ puesto que la cabeza del pilote ya se encuentra por debajo del lugar de medición-. Como puede apreciarse, para los primeros pasos de carga axial actuante en cabeza del pilote $(\mathrm{Q})$, la resistencia por fuste movilizada para los modelos con rugosidad (e.g., $R F \geq 0.010$ ) alcanza valores máximos por delante de las asperidades de la interfaz roca-pilote -ver e.g., desde 0 hasta $-15 \mathrm{~cm}$ y desde -40 hasta $-60 \mathrm{~cm}$ en la Figura 4.13(b), y la $f$ movilizada para $-4,-10$ y $-50 \mathrm{~cm}$ de la Figura 
4.14(b) - mientras que los tramos de fuste por detrás de una asperidad prácticamente no se cargan, tal y como se observa en el registro de la resistencia por fuste movilizada a diferentes profundidades -e.g., ver el tramo $-20 \mathrm{~cm}$ a $-40 \mathrm{~cm}$ en la Figura 4.13(b) y la referencia $\mathrm{a}-30 \mathrm{~cm}$ en la Figura 4.14(b).

Conforme sigue aumentando $Q$ y el correspondiente asiento en cabeza $(\delta)$ del pilote, se va agotando el fuste en la parte superior -e.g., ver en la Figura 4.14(b) el registro de $f$ en la posición - 4 cm (línea roja) - y se van movilizando hacia la parte inferior del pilote; obsérvese en la Figura 4.14(b) como en la posición - $15 \mathrm{~cm}$ la resistencia por fuste se comienza a movilizar para $\delta>-2 \mathrm{~cm}$ en los modelos con $R F \geq 0.050$. Para mayores asientos de los modelos con rugosidad $(R F \geq 0.010)$, los valores máximos de $f$ sólo se producen al frente de la siguiente asperidad ubicada cerca de la punta del pilote -i.e., desde -40 hasta $-60 \mathrm{~cm}$. Es importante resaltar, también, que este patrón de transferencia de carga (desde la parte superior a la parte inferior del pilote) quedó evidenciado en la forma de propagación de las grietas con el aumento de la carga mostrada en la Figura 4.10. Estos resultados son cualitativamente similares a las curvas de resistencia por fuste movilizada a lo largo del pilote obtenidas a partir de ensayos de carga ejecutados por Pells et al. (1980), sobre pilotes rugosos empotrados en las areniscas de Sídney (Australia) (ver Capítulo 2, Sección 2.3.1.1).

Finalmente, el efecto de la rugosidad en la interfaz roca-pilote observado en la Figura 4.13(b), podría ser la causa de que casi toda de la transferencia de $\sigma_{z}$ se produzca en la parte superior del pilote -i.e., desde 0 hasta $-40 \mathrm{~cm}$, ver la Figura $4.12(\mathrm{~b})-$, de modo que llegue muy poco $\sigma_{z}$ hasta la parte inferior del pilote (este mecanismo de transferencia se explica en detalle más adelante en la Sección 4.3.4). 


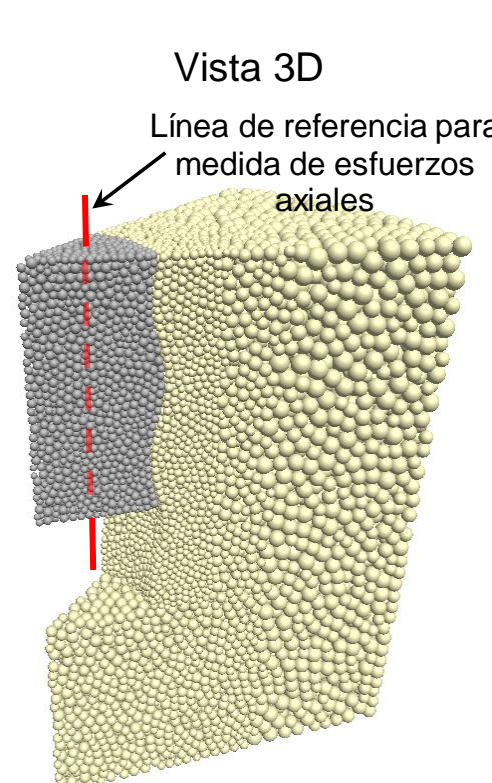

Vista en planta

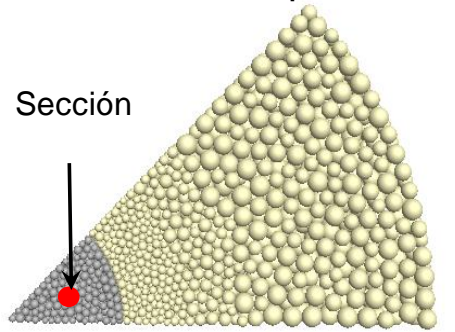

(a)

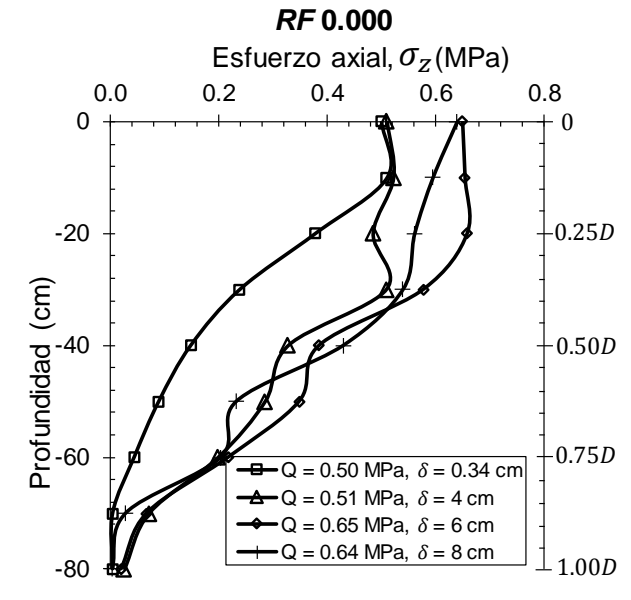

RF 0.050

Esfuerzo axial, $\sigma_{z}(\mathrm{MPa})$

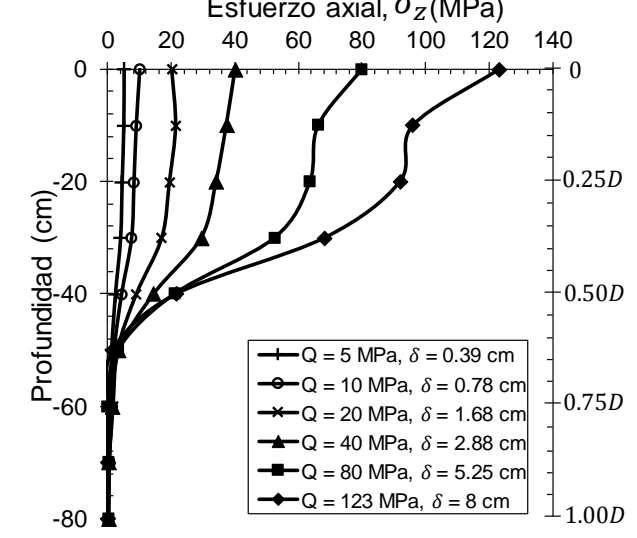

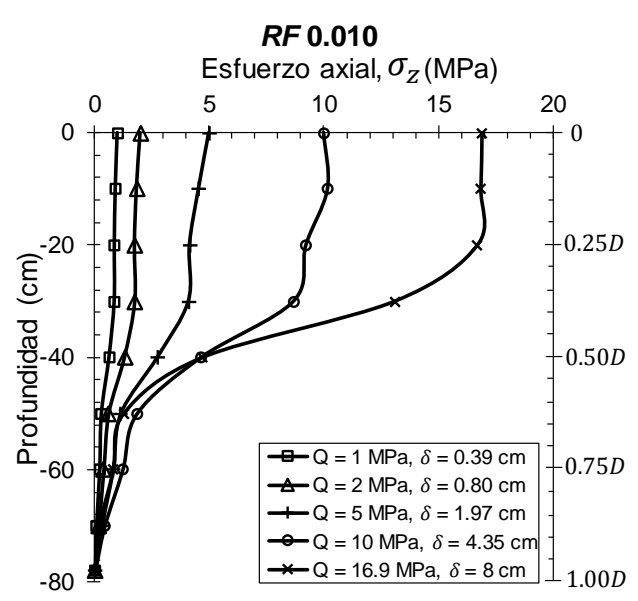

RF 0.085

Esfuerzo axial, $\sigma_{z}(\mathrm{MPa})$

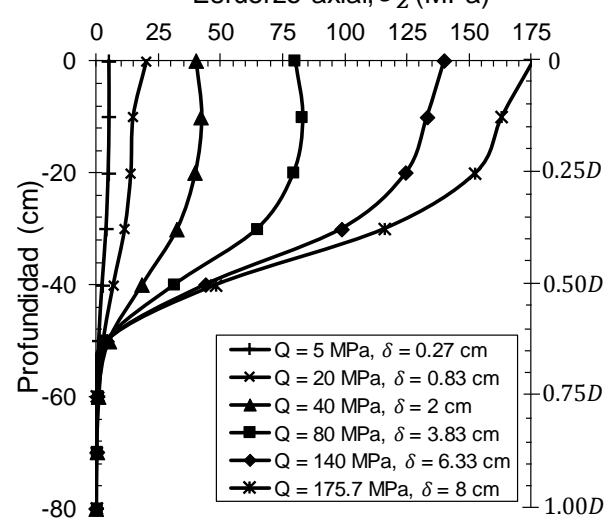

(b)

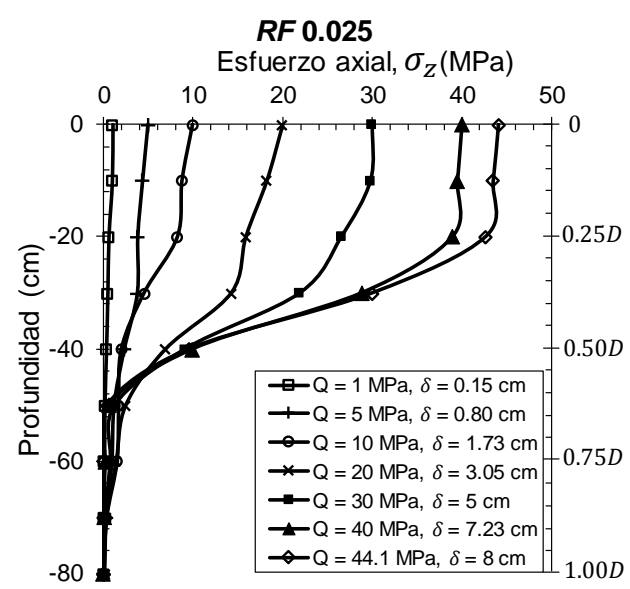

\section{$R F 0.106$}

Esfuerzo axial, $\sigma_{z}(\mathrm{MPa})$

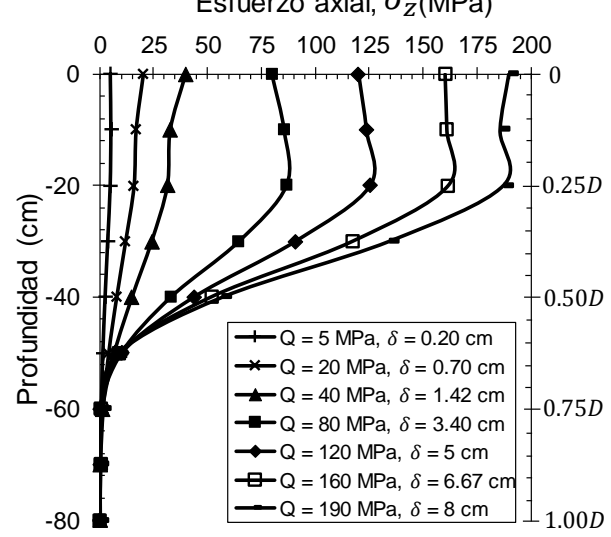

Figura 4.12. Esfuerzo axial movilizado para los modelos con PFC $3 \mathrm{D}$ de pilotes empotrados en la arenisca-S3 con diferente nivel de rugosidad: (a) ubicación de la sección axial trazada, (b) distribución de esfuerzo axial vs profundidad. 

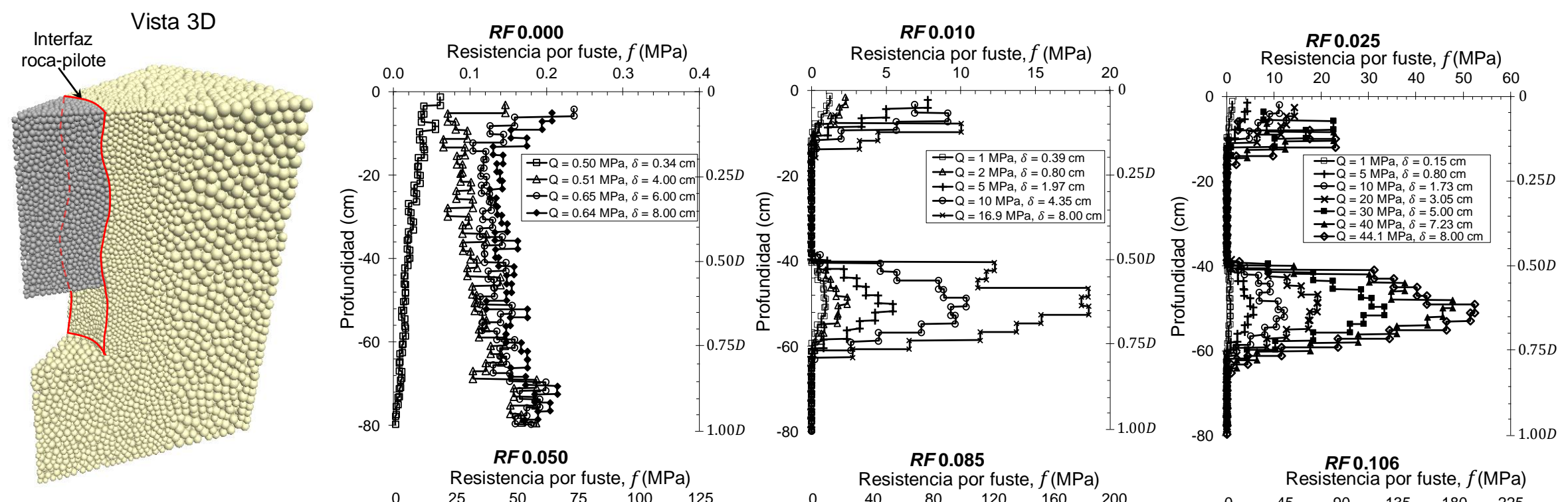

Vista en planta

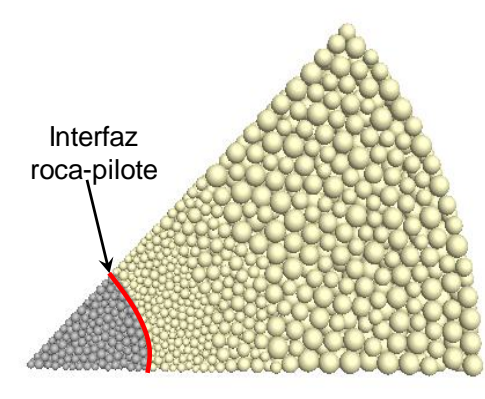

(a)

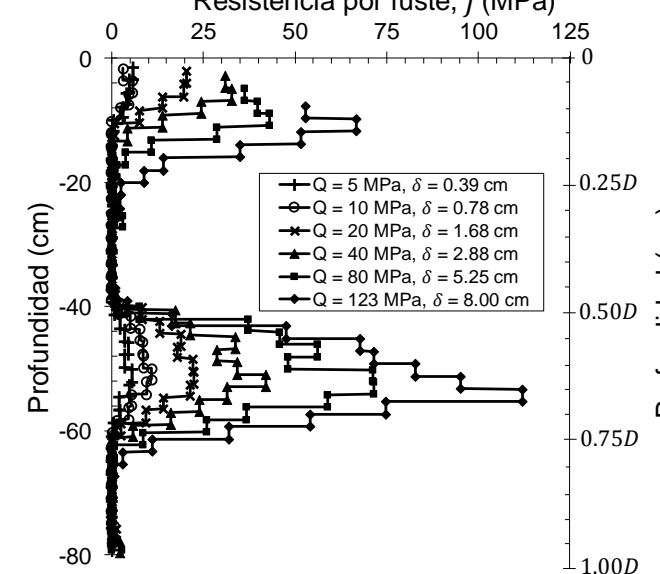

1.000

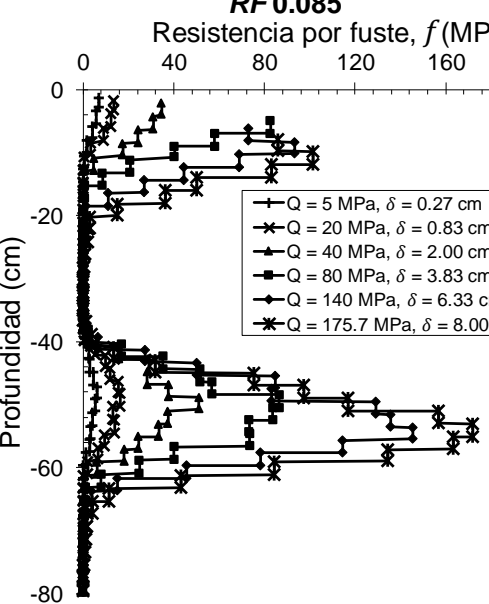

(b)

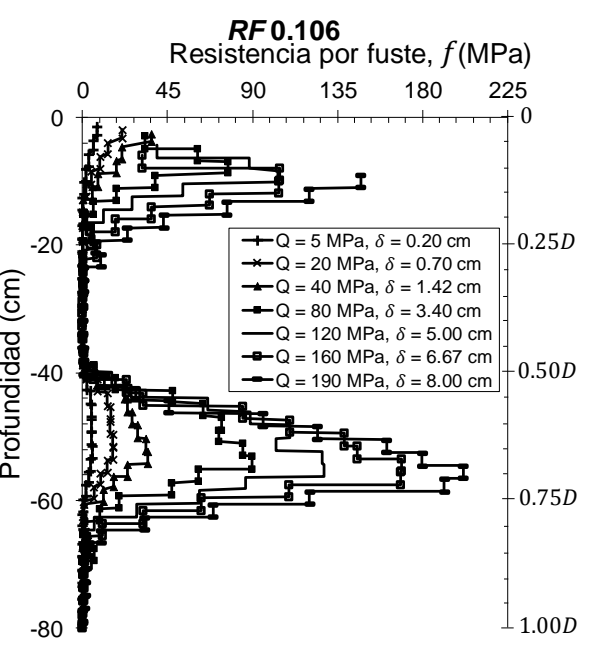

Figura 4.13. Resistencia por fuste movilizada para los modelos con PFC ${ }^{3 D}$ de pilotes empotrados en la arenisca-S3 con diferente nivel de rugosidad: (a) ubicación de la sección en la interfaz roca-pilote, (b) distribución de resistencia por fuste vs profundidad. 


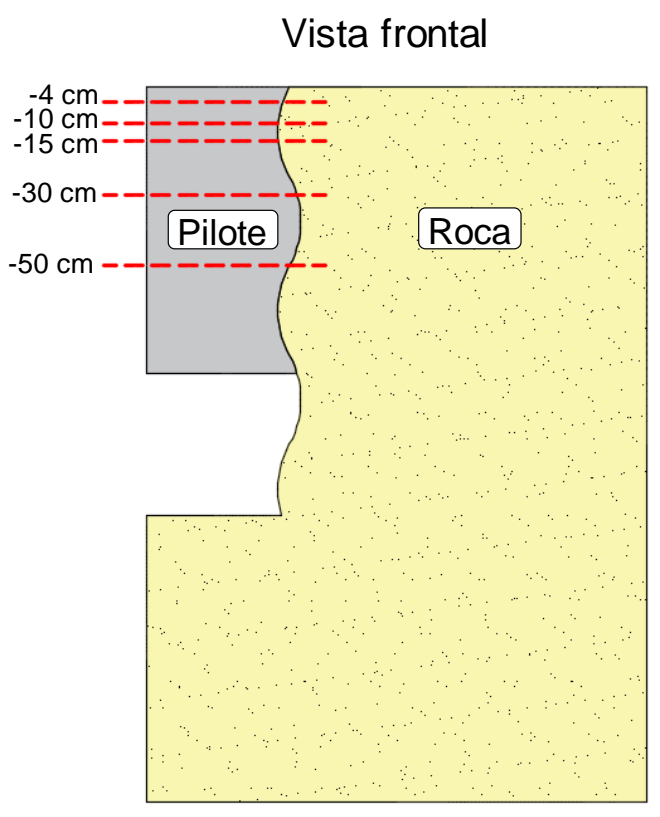

(a)

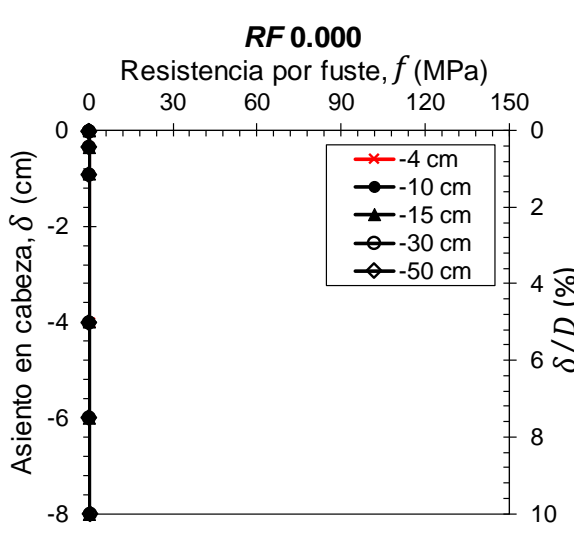

RF 0.050
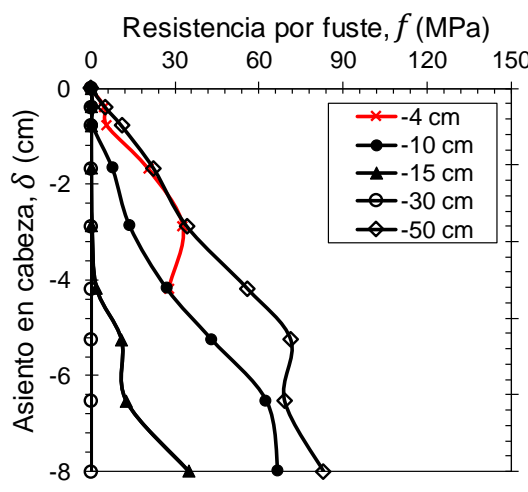

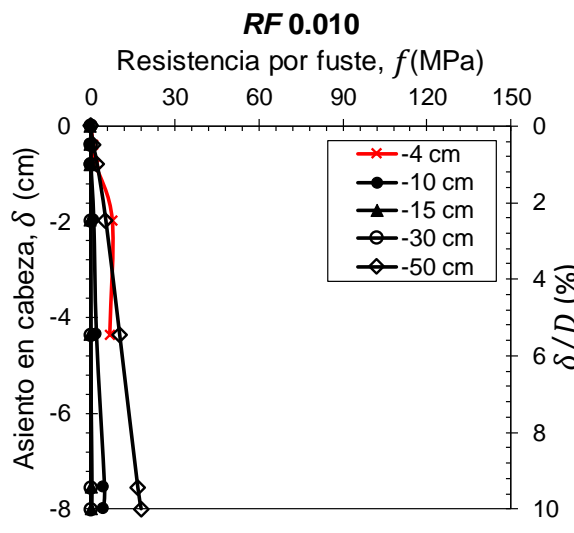

RF 0.085

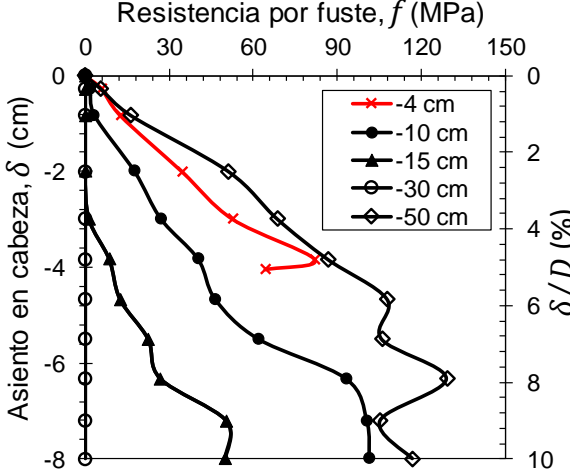

(b)

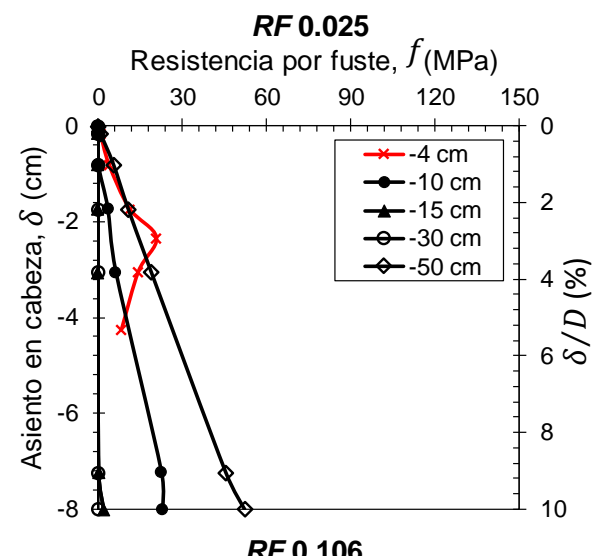

RF 0.106

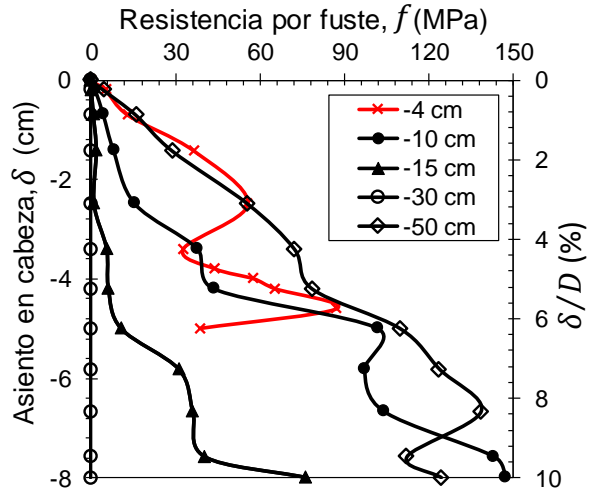

Figura 4.14. Resultados registrados a varios niveles en profundidad para los modelos con PFC ${ }^{3 \mathrm{D}}$ de pilotes empotrados en la arenisca-S3 con diferente nivel de rugosidad: (a) ubicación en profundidad de los puntos de medición en la interfaz roca-pilote, (b) resistencia por fuste movilizada vs asiento en cabeza. 


\subsubsection{Mecanismo de transferencia de carga}

En la Figura 4.15 se presenta un mecanismo idealizado para explicar el proceso de transferencia de carga de pilotes rugosos excavados en roca. La discusión está basada en el registro del estado tensional en tres puntos de observación, ubicados en tres zonas de la asperidad (frente, parte posterior y base, ver la Figura 4.15(a)-(b)). Durante los primeros estados de carga (en los que se obtienen asientos elásticos), el estado tensional se compone de esfuerzos compresivos y se mantiene totalmente el contacto a lo largo de toda la interfaz roca-pilote. Al incrementar la carga actuante en cabeza del pilote (aún en fase elástica), se incrementan en los esfuerzos de compresión en el frente de la asperidad, y se relajan la parte posterior y la base de la asperidad (ver la Figura 4.15(b)). Posteriormente, al seguir aumentando la carga actuante se alcanzan asientos elasto-plásticos en cabeza del pilote, manteniéndose los esfuerzos compresivos en el frente de la asperidad, pero iniciando la formación de un gap o separación en la interfaz roca-pilote (la formación de dicho indica de hecho el fin de la fase elástica). La formación de esta separación o gap en la interfaz, origina una relajación de los esfuerzos axial $\left(\sigma_{z}\right)$ y radial $\left(\sigma_{r}\right)$ en la parte posterior de la asperidad, y produce también esfuerzos de tracción axial y relajación radial en la base de la asperidad (ver la Figura 4.15(c)) (Hassan y O’Neill, 1997).

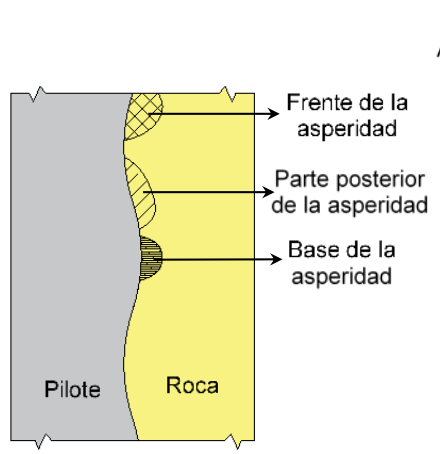

(a)

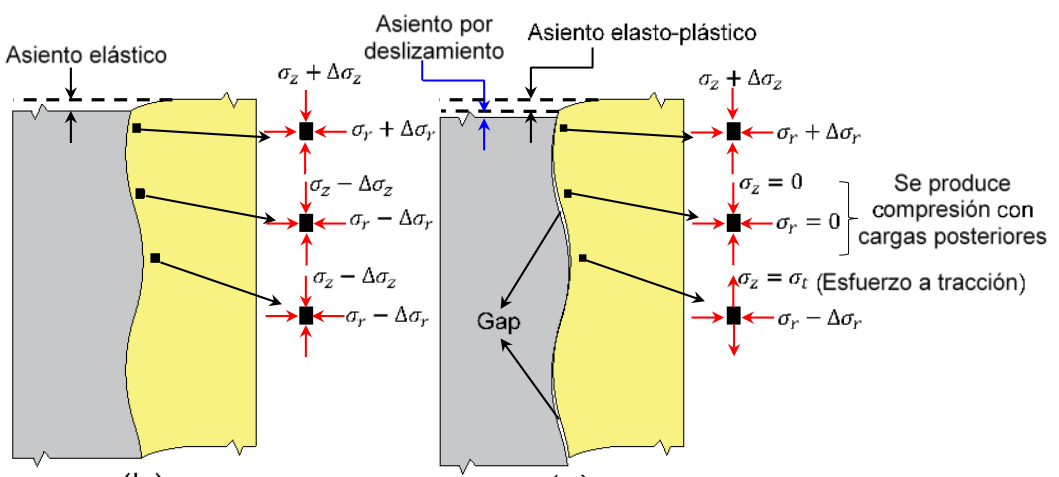

(b)

(c)

Figura 4.15. Mecanismo de transferencia de carga por fuste para pilotes rugosos empotrados en roca: (a) estado inicial y nomenclatura de la asperidad, (b) estado para deformación elástica, (c) estado para deformación inelástica (modificado de Hassan y O’Neill 1997). 
A continuación, se emplean los modelos con $\mathrm{PFC}^{3 \mathrm{D}}$ de pilotes empotrados en la arenisca-S3 con $R F=0.050$ y $R F=0.010$ para ilustrar el mecanismo de transferencia de carga mencionado previamente. Para ello, se instalaron esferas de medición en el cuerpo de roca, cuya localización estuvo sujeta a los mismos puntos de observación representados en el mecanismo de transferencia de la Figura 4.15 -i.e. en el frente, en la parte posterior y en la base de la asperidad-. Posteriormente, se calcularon en cada uno de estos puntos, las componentes de esfuerzo axial $\left(\sigma_{z}\right)$, radial $\left(\sigma_{r}\right)$ y transversal $\left(\sigma_{\theta}\right)$ para cada paso de carga actuante en cabeza del pilote. La evolución de dichos esfuerzos $-\sigma_{z}, \sigma_{r}$ y $\sigma_{\theta}$ - en función del asiento en cabeza, para cada punto de observación y para los dos modelos mencionados previamente, se presentan en las Figuras 4.16 y 4.17.

En el frente de la asperidad de ambos modelos, se observa que al aumentar el asiento en cabeza se producen esfuerzos de compresión -denotados con signo negativo- en la dirección axial $\left(\sigma_{z}\right)$ y radial $\left(\sigma_{r}\right)$ en el frente de la asperidad (ver las Figuras 4.16(b) y 4.17(b)). Por otra parte, en la dirección transversal $\left(\sigma_{\theta}\right)$ se aprecia la alternancia de pequeños esfuerzos de tracción (para $\delta<1 \% D$; y para $\delta>2 \% D$ ) y de compresión (para $1 \% D<\delta<2 \% D$ ) en el modelo con $R F=0.010$, mientras que para el modelo con $R F=$ 0.050 , se registraron esfuerzos de tracción (para $\delta<3 \% D$ ) y de compresión (para $\delta>$ $3 \% D)$. Este comportamiento evidencia que la dilatación en el modelo con $R F=0.050$ es mayor que en el modelo con $R F=0.010$, lo cual, además, está en concordancia con el número de micro-grietas desarrolladas en el frente de la asperidad en ambos modelos (ver la Figura 4.10).

En la parte posterior de la asperidad, el modelo con $R F=0.050$, se produce inicialmente una ligera relajación tensional para asientos muy pequeños -en rango elástico- (ver la Figura 4.16(b)) como consecuencia de los esfuerzos compresivos desarrollados en el frente de la asperidad. Luego, al incrementar la carga actuante en cabeza, se 
desarrollan esfuerzos de compresión en las componentes de $\sigma_{z}$ y de $\sigma_{r}$, y de tracción en $\sigma_{\theta}$ (para $\delta \geq 0.05 \% D$ ). Sin embargo, al aumentar la carga (y su correspondiente asiento), se observa una disminución en las componentes de $\sigma_{r}$ y de $\sigma_{\theta}$ hasta valores cercanos a cero (para $\delta \cong 1 \% D$ ). Esta tendencia sugiere el contacto se mantuvo en esta parte de la asperidad hasta alcanzar este valor de asiento en cabeza del pilote, de modo que al seguir aumentando la carga en cabeza se genera un gap en esta parte de la interfaz.

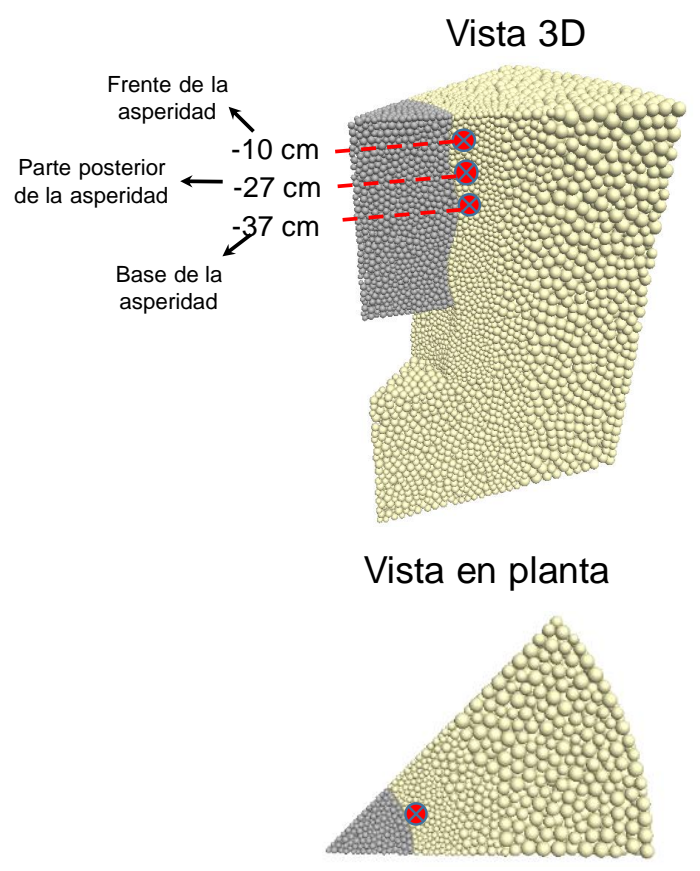

(a)

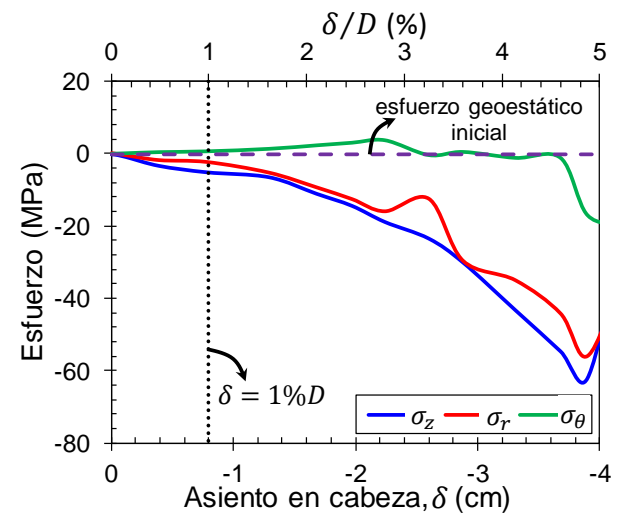

(b)

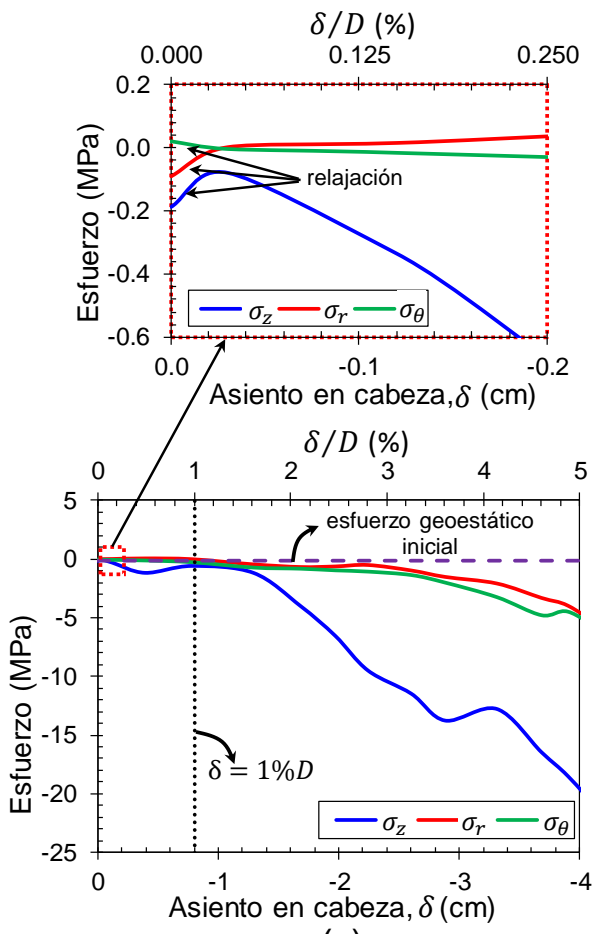

(c)

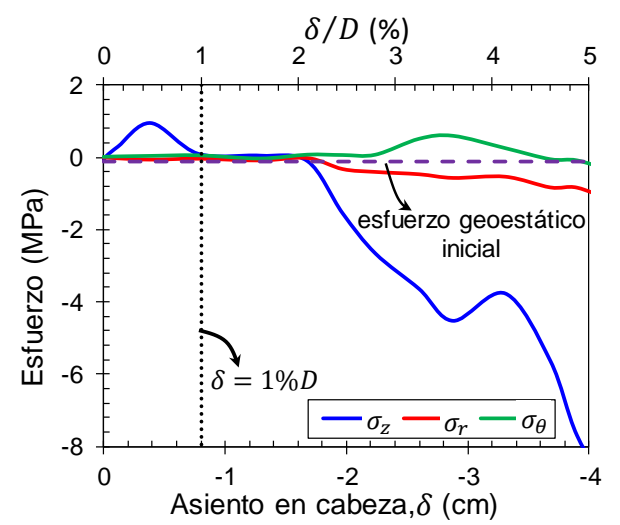

(d)

Figura 4.16. Curvas de las componentes de esfuerzos vs asiento en cabeza para el modelo con PFC $^{3 \mathrm{D}}$ del pilote con $R F=0.050$ empotrado en la arenisca-S3: (a) localización de los puntos de medición, (b) frente de la asperidad, (c) parte posterior de la asperidad, (d) base de la asperidad. 
Para asientos que producen una plastificación más relevante -i.e., por encima de $1 \% D$ en este caso-, se desarrollan nuevamente compresión en todas las componentes de esfuerzos de la parte posterior de la asperidad (ver la Figura 4.17(c)) debido al asiento del pilote y a las fuerzas de empujes que son transferidas desde el frente de la asperidad. Este patrón de transferencia de carga (en la parte posterior de la asperidad) descrito anteriormente para el modelo con $R F=0.050$, fue similar para el modelo con $R F=0.010$ (ver la Figura 4.18(c)) pero para mayores valores de asiento en cabeza del pilote y, con menor valor de esfuerzos respecto a los valores movilizados en el modelo con $R F=0.050$, puesto que, este último tiene mayor rugosidad, mayor dilatación, y en consecuencia, mayor daño en la asperidad (notar como el número de micro-grietas mostrado en la Figura 4.10 así lo confirma).

Por otra parte, los esfuerzos medidos en la base de la asperidad de ambos modelos i.e., $\operatorname{con} R F=0.050$ y $R F=0.010-$ se presenta en las Figuras $4.16(d)$ y $4.17(d)$. En el modelo con $R F=0.050$ se producen esfuerzos de tracción en la componente axial y transversal para asientos en rango elástico -i.e., $\delta<0.01 D$ en este caso-. Luego, se produce una relajación en el estado tensional (para $1 \% D<\delta<2 \% D$ ) y, para cargas superiores, se desarrollan nuevamente esfuerzos de compresión en la componentes de $\sigma_{z}$ y $\sigma_{r}$, mientras que en la componente de $\sigma_{\theta}$ se desarrollan esfuerzos de tracción. Este comportamiento fue similar para el modelo con $R F=0.010$ (ver la Figura 4.18(d)). No obstante, en el modelo con $R F=0.010$ no se observan grandes cambios en las componentes de esfuerzos medidos en la base de la asperidad para $\delta>2 \% D$, lo cual sugiere que no se produce más dilatación en la interfaz de este modelo. Notar, además, que el comportamiento en la parte posterior y base de la asperidad del modelo con $R F=$ 0.010 es ligeramente similar, lo que puede atribuirse a que esta interfaz presenta menor rugosidad que en el modelo con $R F=0.050$. 

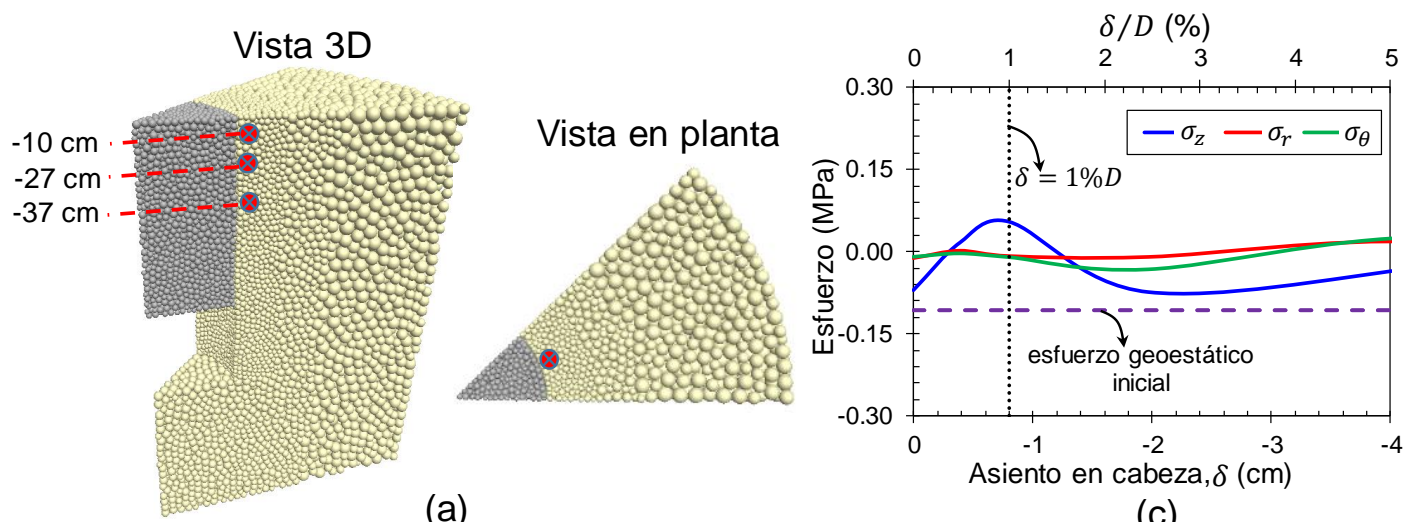

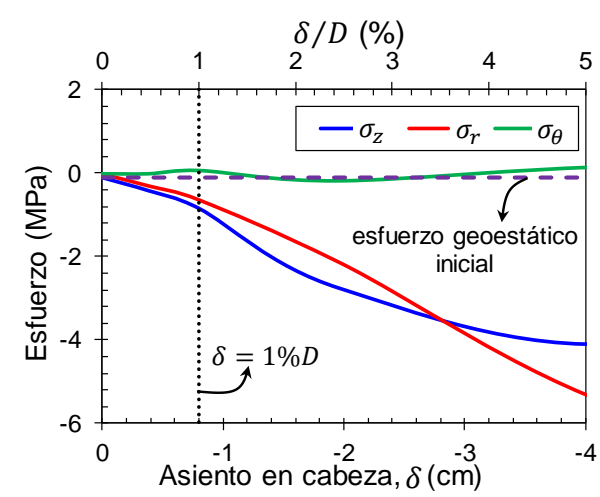

(b)

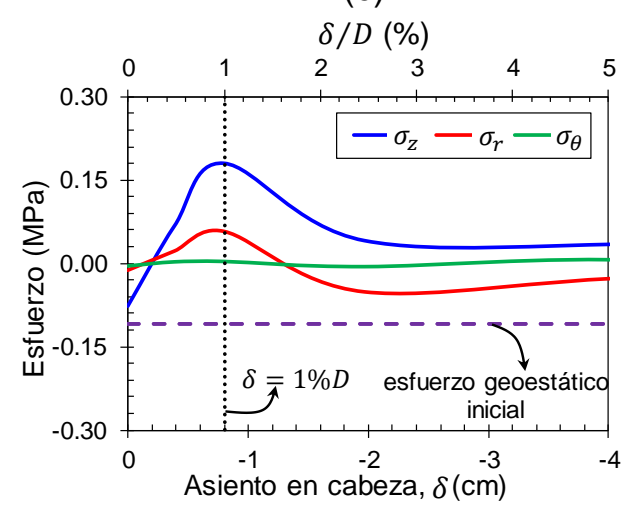

(d)

Figura 4.18. Curvas de las componentes de esfuerzos vs asiento en cabeza para el modelo con $\mathrm{PFC}^{3 \mathrm{D}}$ del pilote con $R F=0.010$ empotrado en la arenisca-S3: (a) localización de los puntos de medición, (b) frente de la asperidad, (c) parte posterior de la asperidad, (d) base de la asperidad.

Adicionalmente, con el objeto de determinar la zona de influencia durante el mecanismo de trasferencia de carga y analizar la distribución de esfuerzos alrededor de la roca circundante, se trazaron tres secciones de muestreo perpendiculares a la interfaz rocapilote (en la dirección radial), para los modelos con $\mathrm{PFC}^{3 \mathrm{D}}$ de pilotes empotrados en la arenisca-S3 con $R F=0.050$ y $R F=0.010$. Las secciones fueron trazadas en los mismos puntos de observación de la asperidad -i.e. en el frente, en la parte posterior y en la base de la asperidad- mostrados en la Figura 4.18. En cada sección se colocaron seis esferas de medición y se calcularon las componentes de esfuerzos $-\sigma_{z}, \sigma_{r}$ y $\sigma_{\theta}-$ para los distintos escalones de esfuerzo axial actuante en cabeza del pilote (ver las Figuras $4.18(a)$ y $4.19(a))$.

En las Figuras 4.18(b)-(d) y 4.19(b)-(d) se muestra la distribución de esfuerzos obtenida en cada una de las secciones de interés. Puede notarse que las curvas de esfuerzos 
muestran una tendencia similar a los incrementos de esfuerzos asociados a la expansión de cavidades cilíndricas. Al inicio, la roca está sometida a esfuerzos de compresión debido al esfuerzo geoestático inicial y, durante la aplicación de los primeros pasos de carga en los que se producen asientos elásticos, no se registran grandes variaciones en las componentes de esfuerzos en las tres partes de la asperidad de ambos modelos (e.g., ver líneas rojas y azules en las Figuras 4.18(b)-(d) y 4.19(b)-(d)). Luego, al aumentar el esfuerzo actuante en cabeza del pilote (Q), se produce una expansión alrededor de la roca circundante al pilote -debido a la dilatación en la interfaz roca-pilote, siendo ésta mayor en el modelo con $R F=0.050-$; en consecuencia, se incrementan los valores de esfuerzos de compresión en la dirección de $\sigma_{z}$ y $\sigma_{r}$ en la roca, mientras que disminuye $\sigma_{\theta}$, pasando de compresión a tracción (ver las Figuras 4.18(b)-(d) y 4.19(b)-(d)). Al seguir aumentando $Q$, se producen deformaciones permanentes (plásticas) en el cuerpo de la roca. Además, en las Figuras 4.18(b)-(d) y 4.19(b)-(d) se observa que la distribución de esfuerzos está en concordancia con las zonas en las que se propagan las micro-grietas del modelo (ver la Figura 4.10). Esta distribución de esfuerzos en la roca circundante es coherente con los resultados obtenidos por Xing et al. (2017), quienes reportaron que la zona con deformaciones plásticas alrededor del pilote disminuye con su profundidad.

Por otra parte, los resultados mostrados en las Figuras 4.18(b)-(d) y 4.19(b)-(d), sugieren que la transferencia de carga desde el pilote hacia la roca circundante ocurre principalmente en una zona de influencia de hasta un radio del pilote, siendo estos resultados congruentes con los reportados por Hassan y O'Neill (1997) a partir de modelos numéricos realizados con el MEF, sobre pilotes rugosos empotrados en roca blanda. 


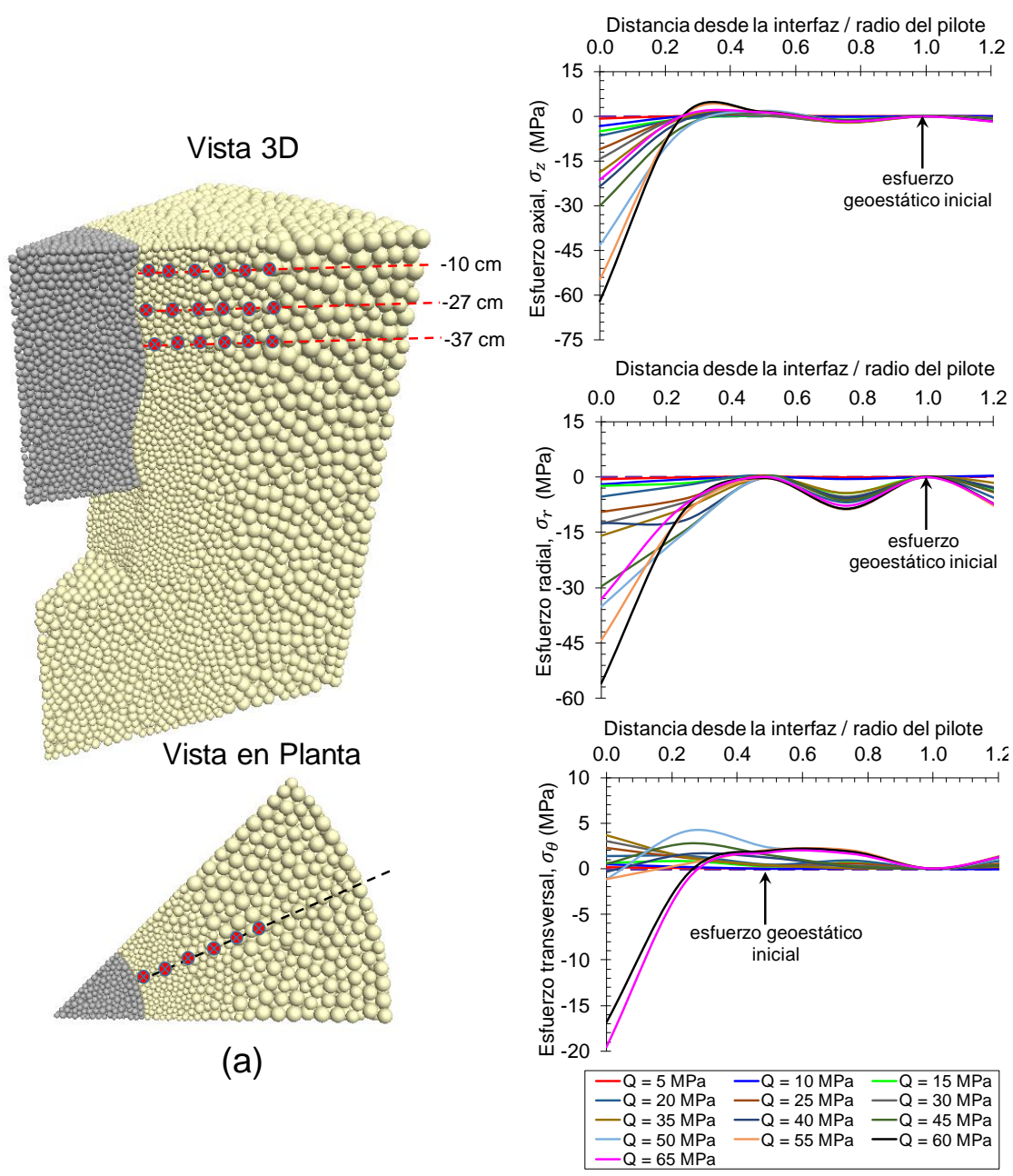

(b)

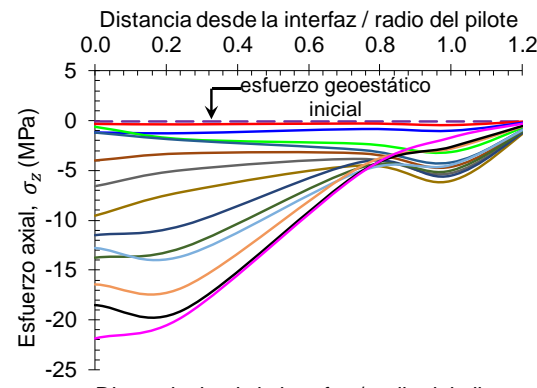

Distancia desde la interfaz / radio del pilote

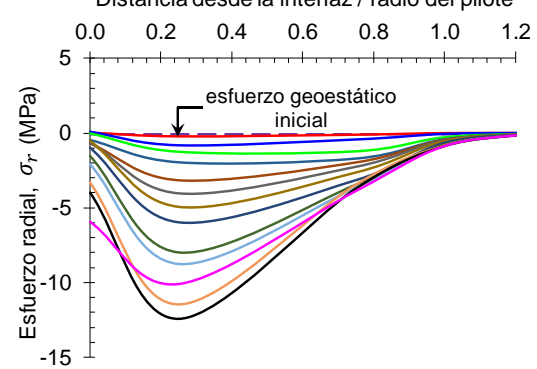

Distancia desde la interfaz / radio del pilote

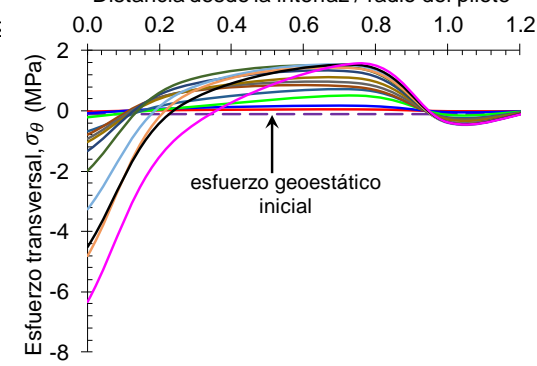

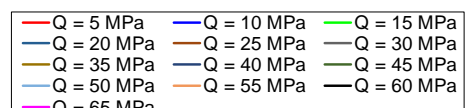

(c)

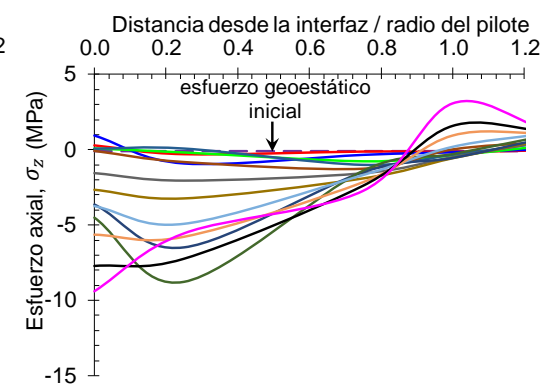

Distancia desde la interfaz / radio del pilote

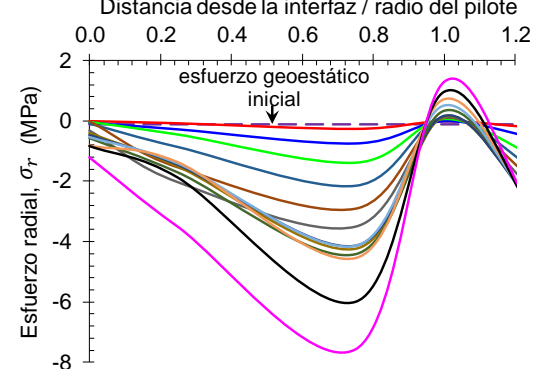

Distancia desde la interfaz / radio del pilote

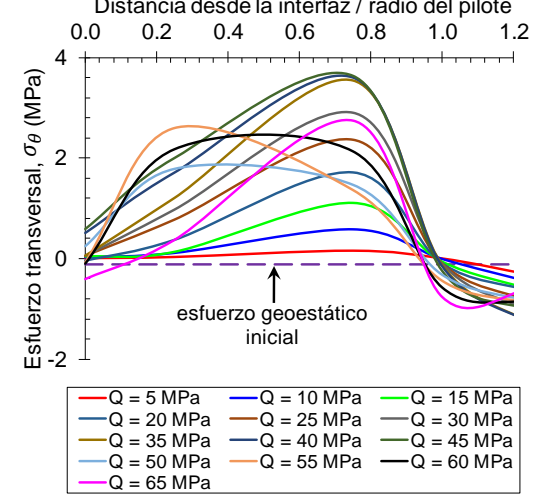

(d)

Figura 4.19. Distribución de esfuerzos alrededor de la roca circundante (arenisca-S3) del modelo con PFC 3D y $R F=0.050$ : (a) ubicación de las secciones, (b) esfuerzos en el frente de la asperidad $(-10 \mathrm{~cm})$, (c) esfuerzos en la parte posterior de la asperidad $(-27 \mathrm{~cm})$, (d) esfuerzos en la base de la asperidad $(-37 \mathrm{~cm})$. 

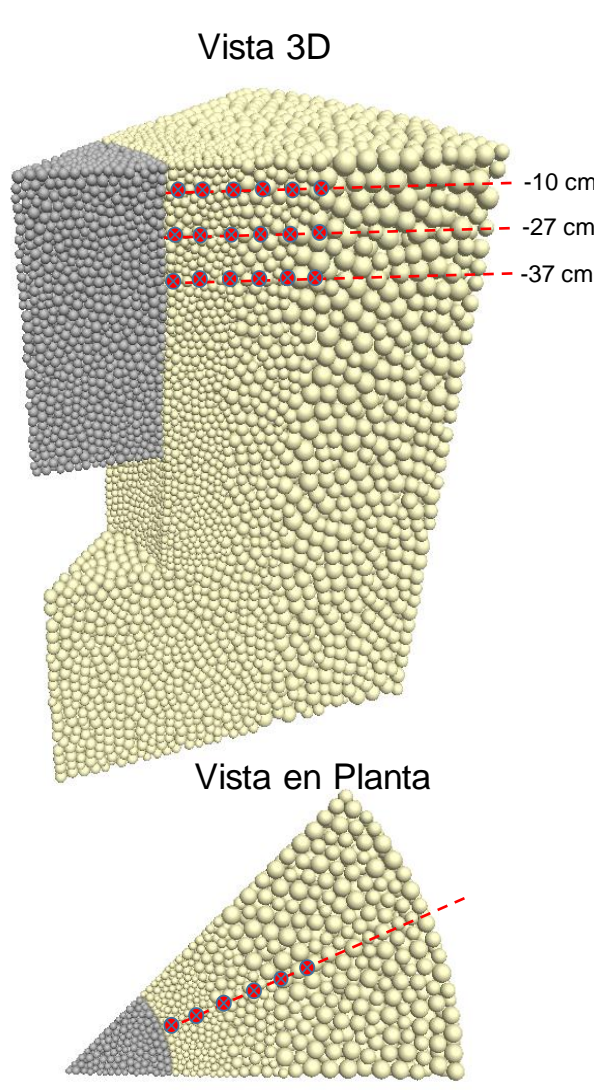

(a)
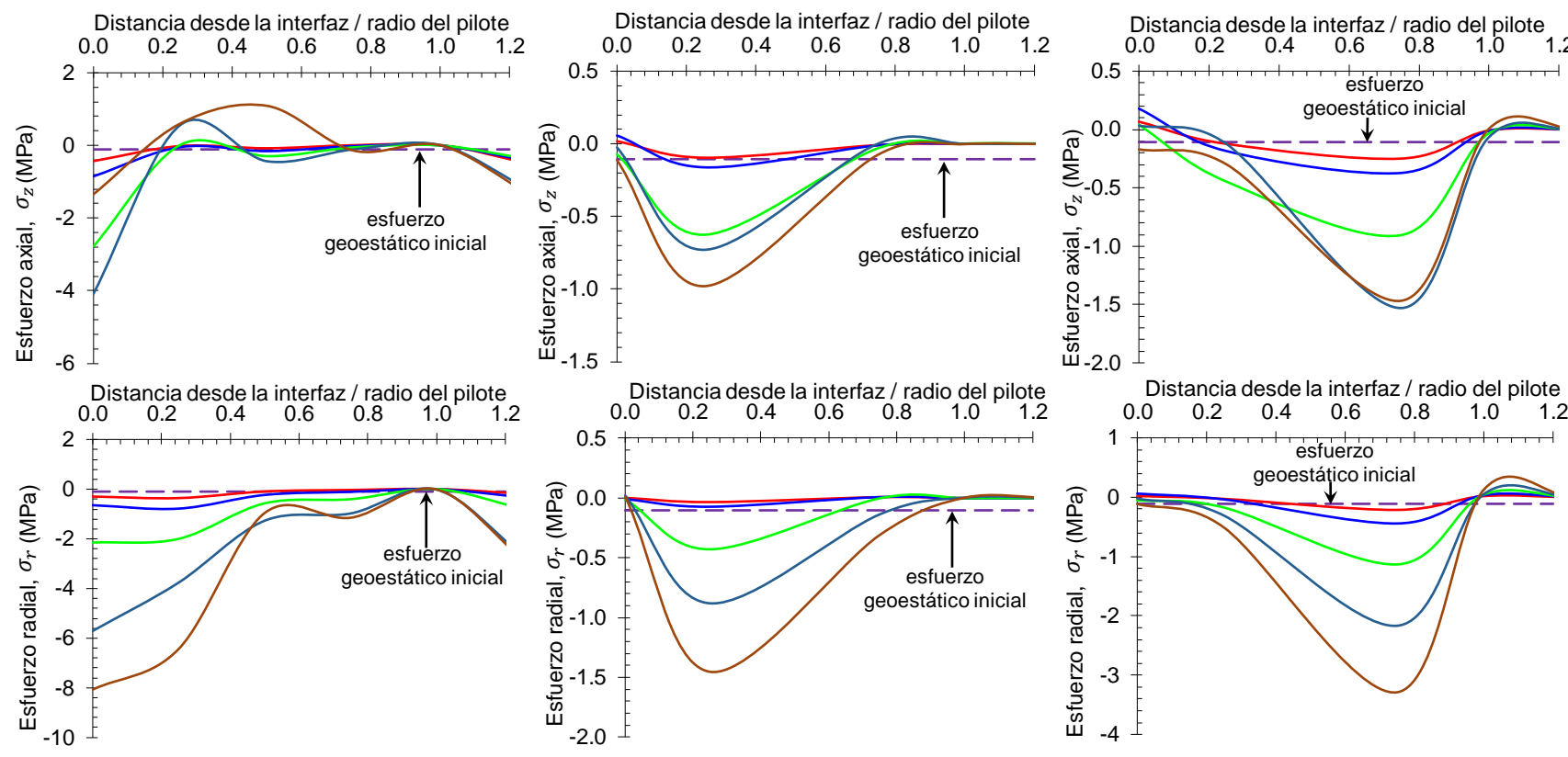

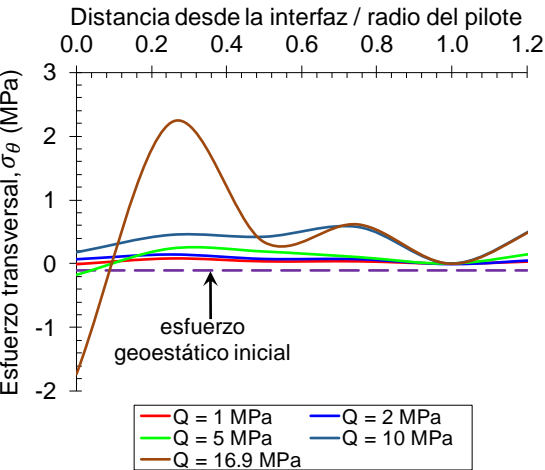

(b)

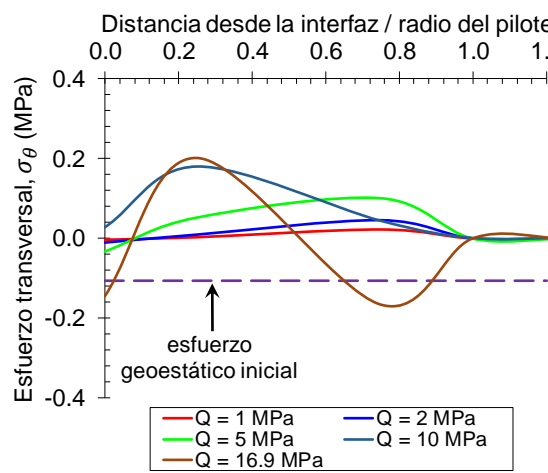

(c)

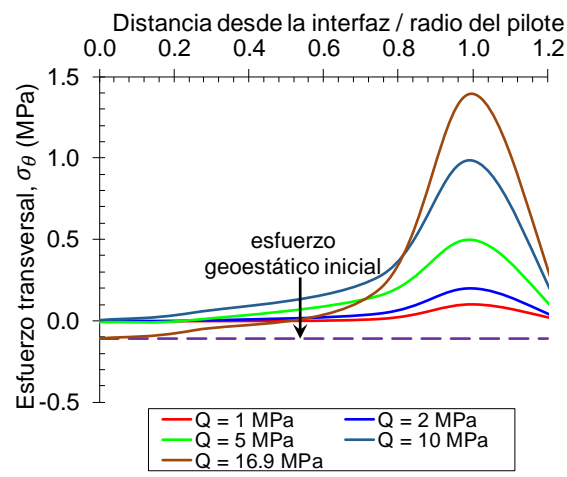

(d)

Figura 4.20. Distribución de esfuerzos alrededor de la roca circundante (arenisca-S3) del modelo con PFC $3 \mathrm{D}$ y $R F=0.010$ : (a) ubicación de las secciones, (b) esfuerzos en el frente de la asperidad $(-10 \mathrm{~cm})$, (c) esfuerzos en la parte posterior de la asperidad $(-27 \mathrm{~cm})$, (d) esfuerzos en la base de la asperidad $(-37 \mathrm{~cm})$. 
Otro aspecto de que puede ser observado en las Figuras 4.18(b)-(d) y 4.19(b)-(d) es que los máximos valores de esfuerzos (compresión y tracción) de las tres secciones trazadas en las zonas de la asperidad -i.e. en el frente, en la parte posterior y en la base de la asperidad-, corresponden con aquellos sitios donde se producen el mayor número de micro-grietas durante la carga del pilote (ver la Figura 4.10). Así, en el modelo con $R F=0.050$ para $\mathrm{Q}=60 \mathrm{MPa}$, los máximos en todas las componentes de esfuerzos se producen en la zona cercana a la asperidad, coincidiendo por tanto con la zona de mayor concentración de grietas en el frente y parte posterior de la asperidad (ver las Figuras 4.10(a) y 4.18(b)-(c)), mientras que en la base de la asperidad, los máximos valores de esfuerzos y las micro-grietas se producen a una distancia más alejada de la interfaz mayor a 0.5 veces el radio del pilote (ver las Figuras 4.10 (a) y $4.18(d)$ ).

Finalmente, la distribución de esfuerzos mostrada en la Figuras 4.18 y 4.19 arroja información interesante referente sobre los tipos esfuerzo que originaron las microgrietas en la roca. Así, las grietas producidas en el frente y parte posterior de la asperidad son producto, en gran medida, de esfuerzos de compresión en la dirección axial y radial, así como de la combinación de esfuerzos de compresión (zona próxima a la interfaz) y tracción (zona más alejada de la interfaz) en la dirección transversal. En la base de la asperidad el comportamiento es similar en la dirección axial, si bien se observa que la componente de tracción es más dominante. Al respecto, Hassan y O’Neill (1997) sugieren que este comportamiento dependerá de la morfología y grado de rugosidad en la interfaz roca-pilote.

\subsection{Configuración del modelo numérico con MEF para pilotes cargados axialmente y empotrados en roca}

El propósito de esta sección es realizar (i) una comparación de la resistencia promedio por fuste obtenida mediante modelos con MED (ver la Sección 4.2) y con MEF para los pilotes empotrados en la arenisca-S3 considerando un asiento de $1 \% D$ (ver la Sección 
4.4), y (ii) evaluar a priori el comportamiento y cargas máximas que se esperarían obtener durante la realización de los ensayos de centrífuga que se presentan en el Capítulo 5.

\subsubsection{Esquema del modelo MEF}

En la Figura 4.21 se presenta el proceso secuencial empleado en la simulación con MEF de todos los modelos de pilotes empotrados en roca considerados en esta sección. Estos modelos se han dividido en dos grupos:

a) Modelos de pilotes perforados en la arenisca-S3, Grupo 1: en estos modelos se considera solo la resistencia movilizada por fuste, con la misma geometría, carga y condiciones de borde empleadas en dichos modelos con PFC ${ }^{3 \mathrm{D}}$. (ver la Sección 4.2.1 y 4.2.2); en particular, para los modelos con $R F=$ $\{0.025 ; 0.050 ; 0.106\}$.

b) Modelos de pilotes perforados en pseudo-roca $\left(\sigma_{c}=1.51 \mathrm{MPa}\right.$, Dykeman y Valsangkar 1996), Grupo 2: en estos modelos se considera la resistencia simultánea por punta y fuste. La selección de este de roca se fundamenta en el hecho de que el efecto de la rugosidad tiene mayor relevancia en pilotes perforados en rocas blandas con $\sigma_{c}=1-12 \mathrm{MPa}$ (Seidel y Collingwood 2001). La geometría empleada en estos modelos se muestra en la Figura 4.22. En este grupo, la interfaz roca-pilote fue modelizada empleando las mismas amplitudes y longitudes de onda de los modelos de $\mathrm{PFC}^{3 \mathrm{D}}$, cuyos valores de factores de rugosidad resultan $R F=\{0.010 ; 0.025 ; 0.050 ; 0.106\}$ (ver Figura 4.3(b)). Como fue mencionado previamente, estos modelos se realizaron con fines de prediseño para los ensayos de centrífuga que se presentan en el Capítulo 5.

En todos los modelos, el pilote fue modelado como elástico, mientras que la roca fue modelada empleando el criterio elasto-plástico perfecto de Mohr-Coulomb. Las 
propiedades geomecánicas empleadas en cada caso se presentan en la Tabla 4.3. Adicionalmente, se empleó el modelo de fricción básico de Coulomb para asignar un coeficiente de fricción en la interfaz roca-pilote de 0.7 y 0.4 para los modelos empotrados en la arenisca-S3 (solo fuste) y en la pseudo-roca (punta y fuste), respectivamente. El estado tensional se aplicó como condición inicial, seguido de la aplicación del paso geostático de Abaqus para que el código equilibre las cargas gravitatorias con el estado tensional in-situ (Smith 2009). Finalmente, el pilote fue cargado axialmente mediante un procedimiento de desplazamiento controlado.

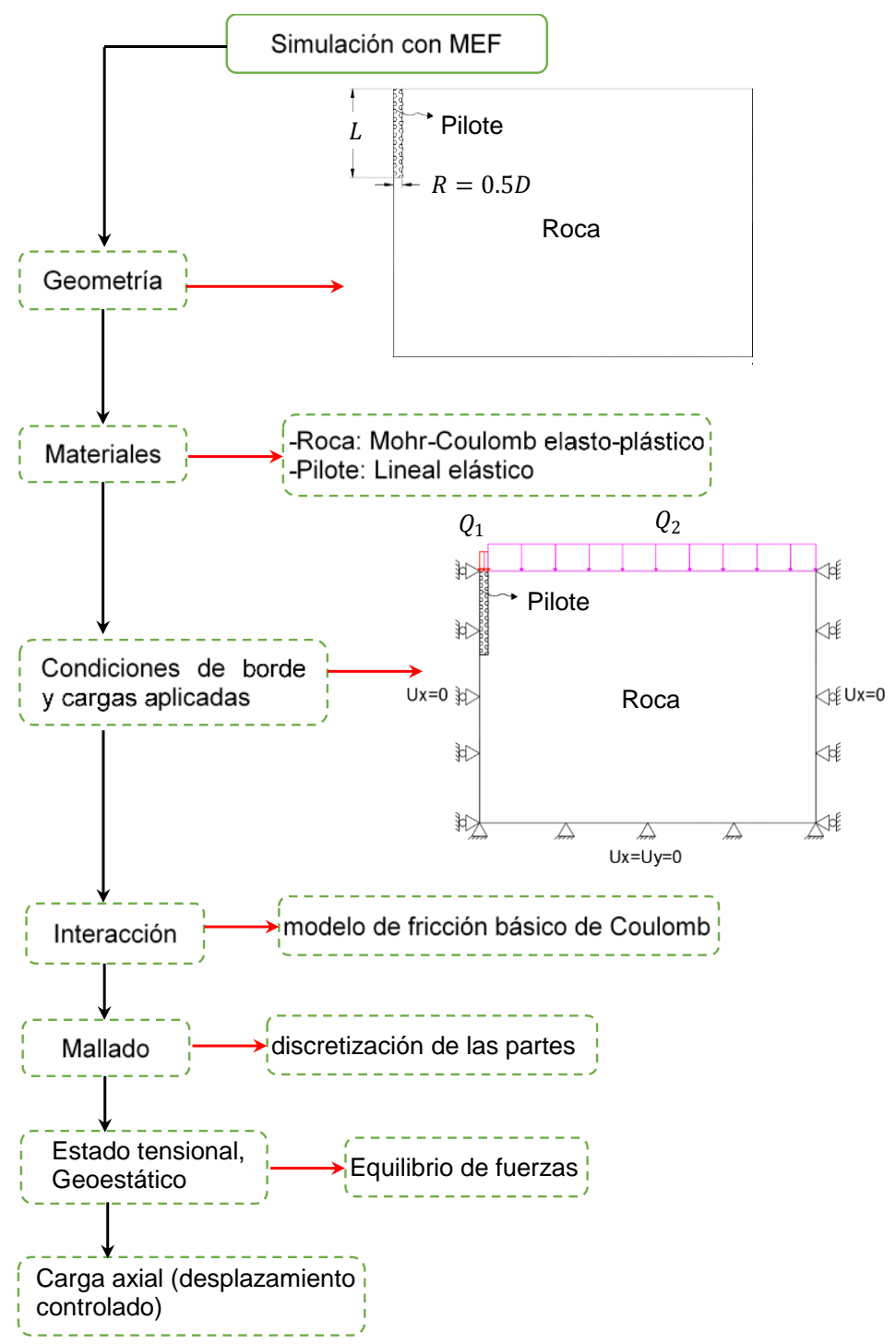

Figura 4.21. Esquema del proceso de simulación numérica con MEF en Abaqus (modificado de Gutiérrez-Ch y Melentijevic, 2016) 


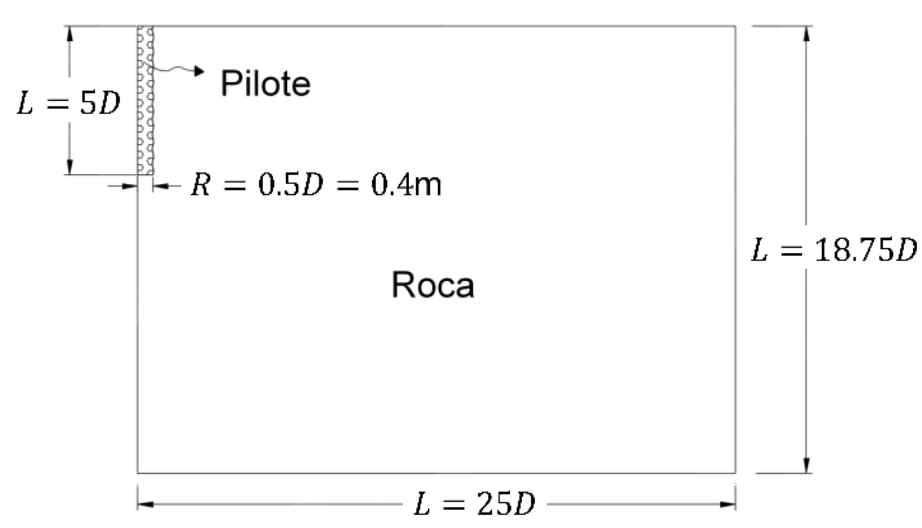

Figura 4.22. Geometría de los modelos con MEF considerando la resistencia por punta y fuste, Grupo 2.

Tabla 4.3. Propiedades de los materiales empleados en los modelos con MEF.

\begin{tabular}{lccc}
\hline Parámetro & $\begin{array}{c}\text { Arenisca-S3 } \\
\text { (solo fuste) }\end{array}$ & $\begin{array}{c}\text { Pseudo-roca }^{\mathrm{b}} \\
\text { (punta y fuste) }\end{array}$ & Pilote \\
\hline Densidad, $\rho\left(\mathrm{kg} / \mathrm{m}^{3}\right)$ & 2550 & 2242 & 2500 \\
Módulo de Young, $E(\mathrm{MPa})$ & 3250 & 365 & 30000 \\
Coeficiente de Poisson $v$ & 0.1 & 0.26 & 0.2 \\
Cohesión, $c(\mathrm{MPa})$ & 2.2 & 0.39 & - \\
Ángulo de rozamiento $\left(^{\circ}\right)$ & 35 & 31 & - \\
Resistencia a la compresión simple, $\sigma_{c}(\mathrm{MPa})$ & 21.77 & 1.51 & 40 \\
\hline
\end{tabular}

${ }^{a}$ tomados de Gu et al. (2003), ${ }^{b}$ tomados de Dykeman y Valsangkar (1996)

\subsection{Resultados de los modelos con MEF}

\subsubsection{Comportamiento de resistencia promedio por fuste-asiento para los modelos de pilotes empotrados en la arenisca-S3}

Debido a que estos modelos se desarrollaron con el MEF (medio continuo), la estimación de la resistencia por fuste se realizó como un promedio a partir esfuerzos tangenciales movilizados en los nodos de la interfaz roca-pilote. En la Figura 4.23(a) se presenta la evolución de la resistencia por fuste promedio $\left(f_{\text {ave }}\right)$ de los 3 modelos de pilotes empotrados en la arenisca-S3, en función del asiento en cabeza del pilote $(\delta)$, así como su comparación con los resultados obtenidos con el MED para los mismos modelos. Adicionalmente, la Figura 4.23(b) muestra la comparación de resultados de la resistencia promedio por fuste movilizada para un asiento en cabeza de $1 \% D\left(f_{\text {ave,1\%D }}\right)$ obtenida a partir de los modelos con el MED y con el MEF. 


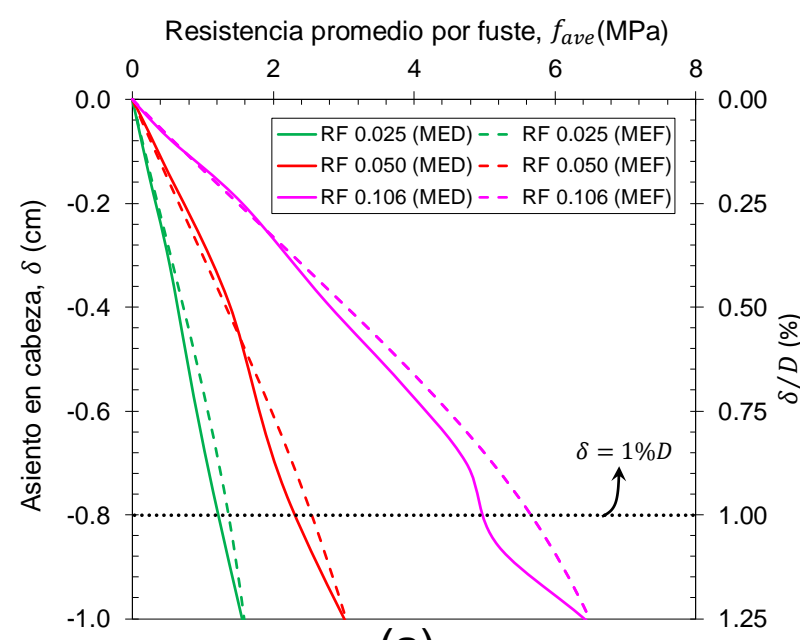

(a)

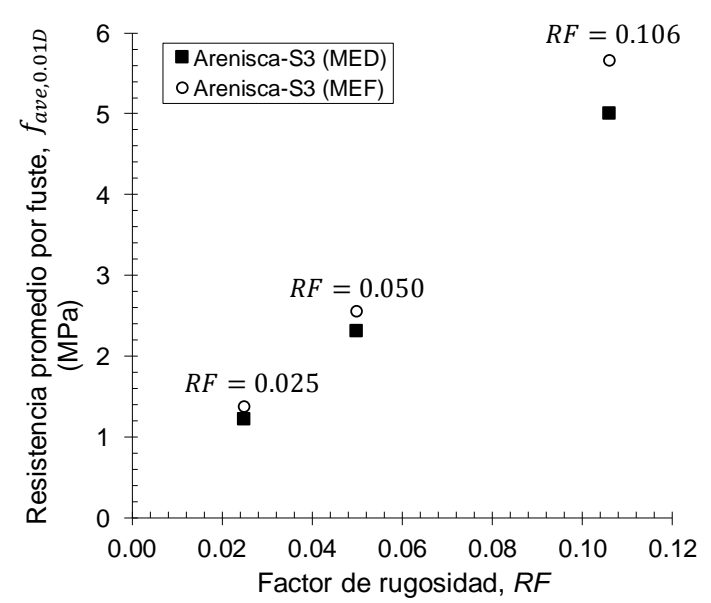

(b)

Figura 4.23. Comparación entre modelos con MED y con MEF: (a) resistencia promedio por fuste vs asiento en cabeza (b) resistencia promedio por fuste para un asiento de $1 \% D$ $\left(f_{\text {ave }, 0.01 D}\right)$ (modificado de Gutiérrez-Ch et al. 2019a).

Como puede observarse, los resultados obtenidos con el MEF son muy similares a aquellos hallados con el MED para un asiento en cabeza del pilote de $1 \% D$ (i.e., hay un incremento de la resistencia promedio por fuste con el aumento del nivel de rugosidad de la interfaz roca-pilote). Adicionalmente, en la Figura 4.24 se presenta un ejemplo de la distribución de los esfuerzos tangenciales movilizados por fuste para un asiento de $1 \% D$ en los modelos considerados en esta sección. De nuevo, los resultados obtenidos con el MEF concuerdan con lo reflejado por el MED para estos mismos modelos; en particular, a que la concentración de grietas (halladas con el MED) y la distribución de esfuerzos máximos (hallados con el MEF) se producen en el frente de las asperidades 
(ver la Figura 4.24(a)-(c)), así como también al gap generado en la parte posterior de la asperidad (ver la Figura 4.24(d)).

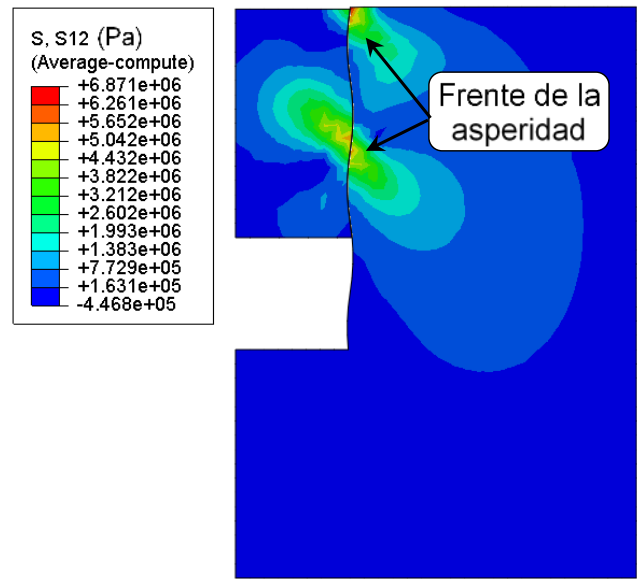

(a)
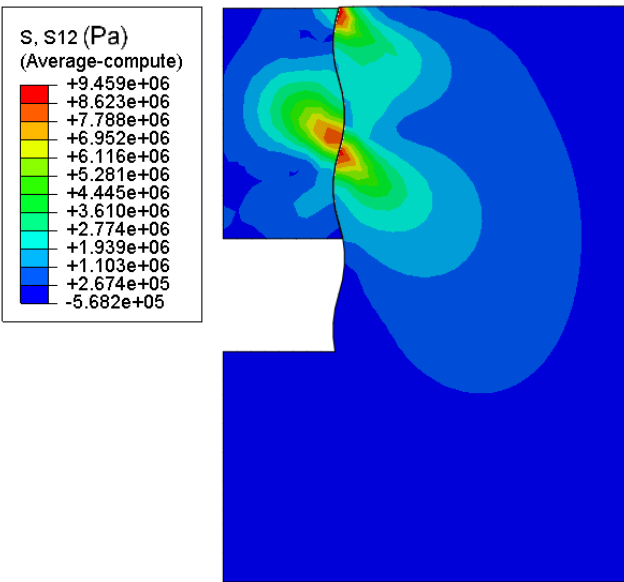

(b)

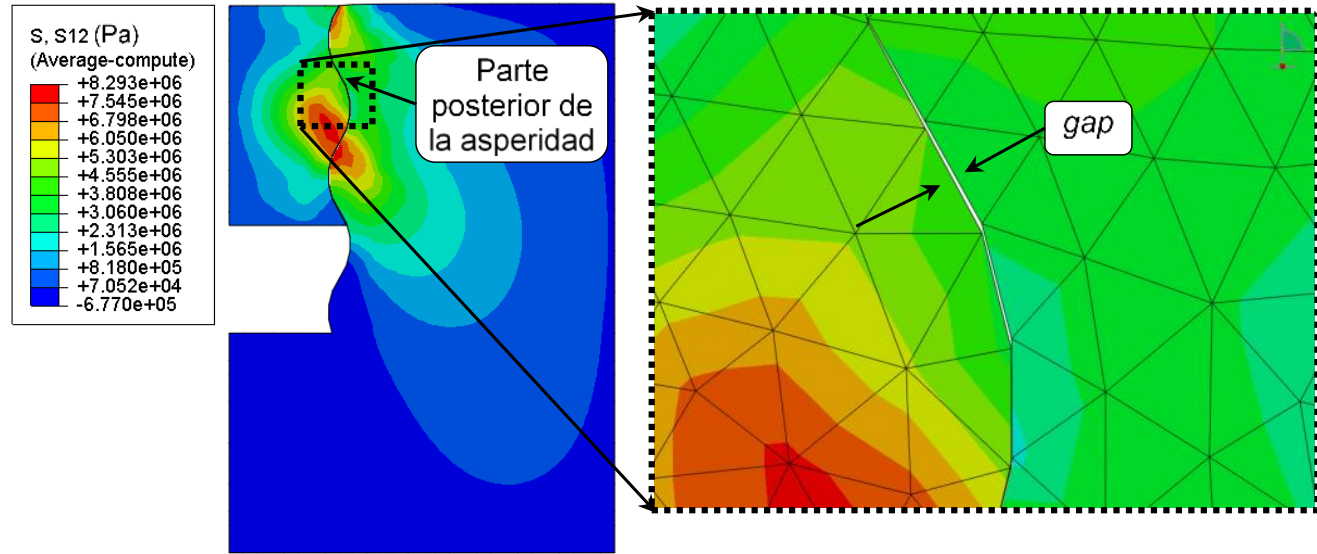

(c)

Figura 4.24. Ejemplo de los esfuerzos tangenciales movilizados por fuste para los modelos con Abaqus de pilotes empotrados en la arenisca-S3 para un asiento en cabeza de $1 \% D$ : (a) $R F=$ 0.025 , (b) $R F=0.050$, (c) $R F=0.106$.

\subsubsection{Comportamiento para los modelos de pilotes empotrados en la pseudo- roca}

A continuación, se presentan los resultados obtenidos para los pilotes empotrados en la pseudo-roca $\left(\sigma_{c}=1.51 \mathrm{MPa}\right.$, Dykeman y Valsangkar 1996), y en los que además se considera la resistencia simultánea por punta y fuste del pilote. 


\subsubsection{Respuesta carga axial-asiento}

En la Figura 4.25 se muestran las curvas de carga en cabeza vs asiento en cabeza obtenidas en la simulación numérica con Abaqus de los 5 pilotes empotrados en la pseudo-roca, usando diferentes rugosidades en la interfaz roca-pilote. Al igual que en los modelos realizados con MED, estos ensayos de carga se han realizado hasta alcanzar un asiento en cabeza $(\delta)$ del pilote de valor igual a $10 \%$ el diámetro del pilote $(10 \% D)$.

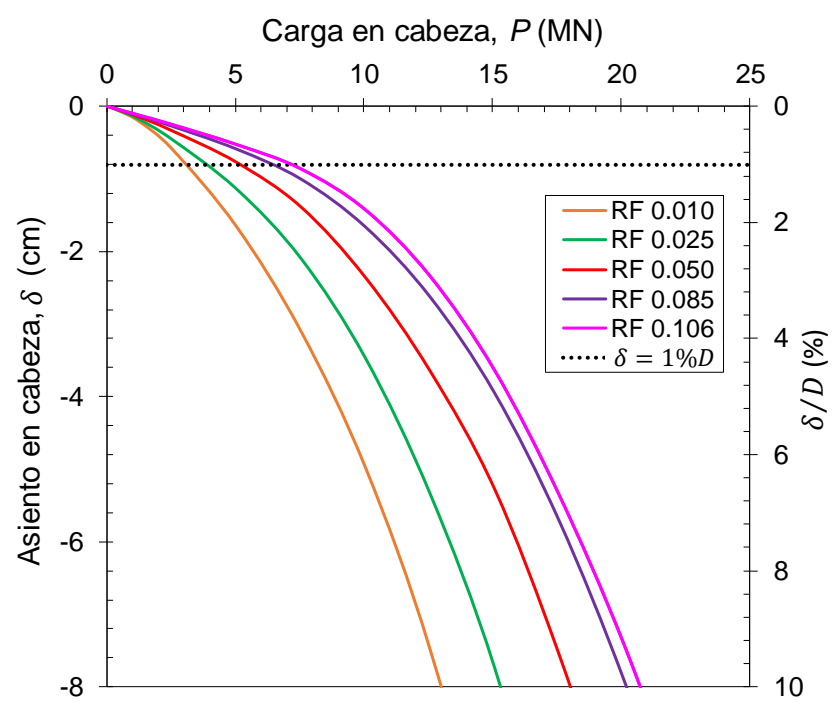

Figura 4.25. Comparación de las curvas carga en cabeza vs asiento cabeza obtenidas en los modelos con Abaqus para los pilotes empotrados en la pseudo-roca con diferente grado de rugosidad.

De nuevo, nótese como hay un incremento de la capacidad de carga y rigidez del pilote con el aumento de la rugosidad en la interfaz roca-pilote a pesar de movilizar de manera simultánea a la componente de resistencia punta y fuste del pilote; sin embargo, como ya ha sido evidenciado en los modelos con MED (ver la Sección 4.3) y en trabajos previos (e.g., Dai et al. 2017), los resultados de la Figura 4.25 indican que podría existir un límite superior de la rugosidad, por encima del cual no se produce un incremento importante en la capacidad de los pilotes.

Adicionalmente, las curvas mostradas en la Figura 4.25 aportan información relevante a las cargas obtenidas para un asiento en cabeza de $10 \% D$. Resulta oportuno recordar 
que, estos modelos se realizaron previo a la realización de los ensayos de centrifuga (ver el Capítulo 5) ejecutados en esta tesis doctoral. En este sentido, se observa que la carga actuante para el modelo con $R F=0.106$ es de alrededor de $20 \mathrm{MN}$. Luego, este valor de carga máxima en conjunto con la geometría de los perfiles de rugosidad, han sido empleados como punto de partida para el diseño de los ensayos de centrífuga (i.e., actuadores líneas de carga a emplear, factor de escala $N$, entre otros) que se presentan en el Capítulo 5.

\subsubsection{Respuesta de resistencia promedio por fuste y por punta-asiento}

Al ser modelos continuos, la resistencia promedio por fuste se calculó mediante el promedio de la tensión tangencial movilizada en los nodos de la interfaz roca-pilote, mientras que la resistencia por punta $\left(q_{p \text { ave }}\right)$ se obtuvo como el promedio de los esfuerzos axiales movilizados en los nodos de la punta del pilote.

En la Figura 4.26 se presenta el comportamiento de resistencia promedio por fuste y por punta para los modelos de pilotes empotrados en la pseudo-roca en función del asiento en cabeza del pilote $(\delta)$. Los resultados muestran nuevamente que hay un incremento de la resistencia promedio por fuste $\left(f_{\text {ave }}\right)$ debido al aumento del nivel de rugosidad en la interfaz roca-pilote; por ejemplo, nótese como el modelo con $R F=0.050$ moviliza 2.6 veces más $f_{\text {ave }}$ que el pilote con $R F=0.010$ para un asiento de $1 \% D$ (ver la Figura 4.26(a)). Además, nuevamente se evidencia la posible existencia de una cota superior en la cual la rugosidad en la interfaz no produce un incremento significativo de la resistencia por fuste de pilotes empotrados en roca, incluso en este caso en que se ha considerado la resistencia simultánea por punta y fuste del pilote.

Por otra parte, los resultados presentados en la Figura 4.26(b) sugieren que un aumento en el nivel de rugosidad en la interfaz roca-pilote produce una ligera disminución de la resistencia promedio movilizada en la punta del pilote ; por ejemplo, nótese como en el modelo con $R F=0.050$ la $q_{p \text { ave }}$ movilizada es un $15 \%$ menor a la del modelo con $R F=$ 
0.010 para un asiento de $5 \% D$ (ver la Figura 4.26(b)). Además, también se evidencia que este efecto de la rugosidad podría tener una cota superior en la cual no se registre un cambio importante en el porcentaje de la $q_{p}$ ave movilizada en el pilote.

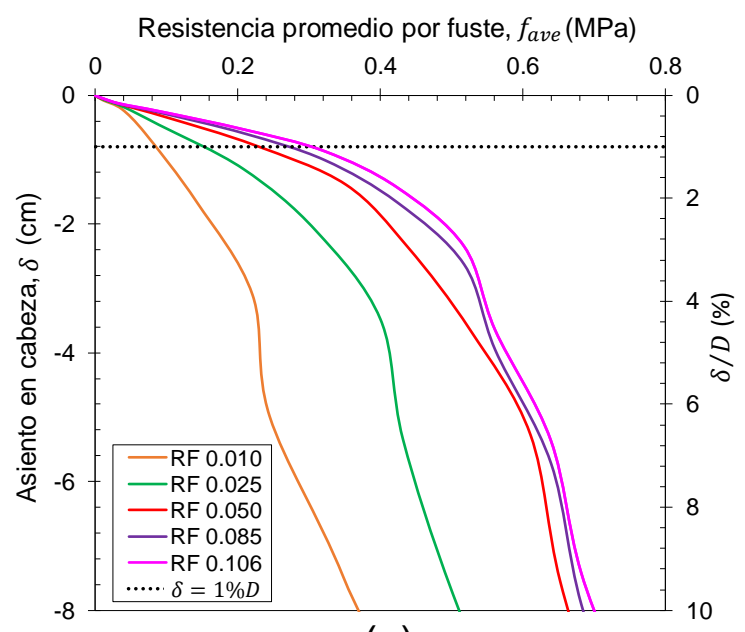

(a)

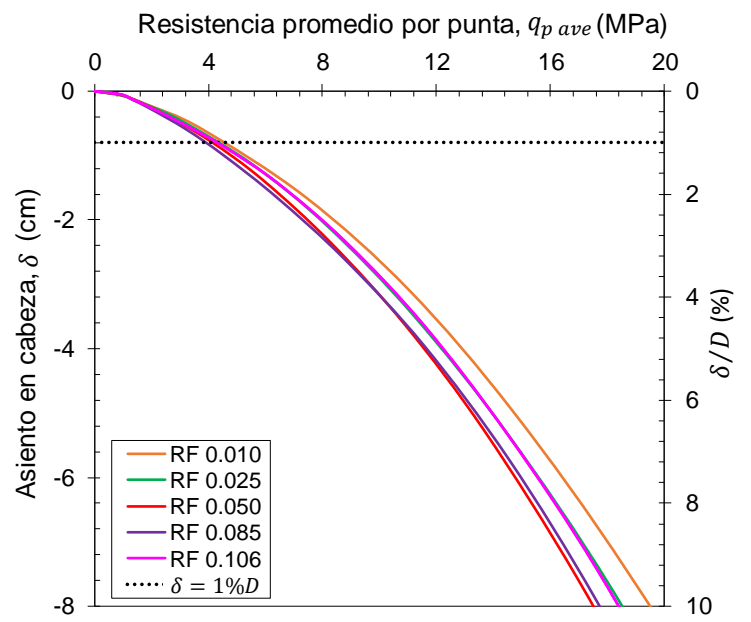

(b)

Figura 4.26. Resistencia promedio por fuste y por punta vs asiento cabeza obtenidos en los modelos con Abaqus para los pilotes empotrados en la pseudo-roca con diferente grado de rugosidad.

El efecto de la rugosidad sobre la resistencia promedio por fuste y por punta de los pilotes empotrados en la pseudo-roca también puede notarse cuando se analiza la evolución del porcentaje de resistencia movilizada por fuste y punta vs el asiento en cabeza. Por ejemplo, en la Figura 4.27 se presenta el porcentaje de resistencia movilizada por cada una de las componentes para todos los modelos de los pilotes y se observa que inicialmente alrededor del $80 \%$ es movilizado por la punta del pilote, y el $20 \%$ restante por el fuste; luego, para los siguientes incrementos de carga (o su asiento asociado), esta proporción va cambiando alcanzando los valores máximos del porcentaje de cada componente para asientos de menores o iguales a $1 \% D$. Posteriormente, se observa que los valores movilizados en cada componente disminuyen hacia un posible porcentaje de movilización residual. (Nótese como a pesar de seguir aumentando la rugosidad en la interfaz roca-pilote, el porcentaje de resistencia movilizado por fuste y por punta no superan el valor máximo de $60 \%$ y $40 \%$, respectivamente). 


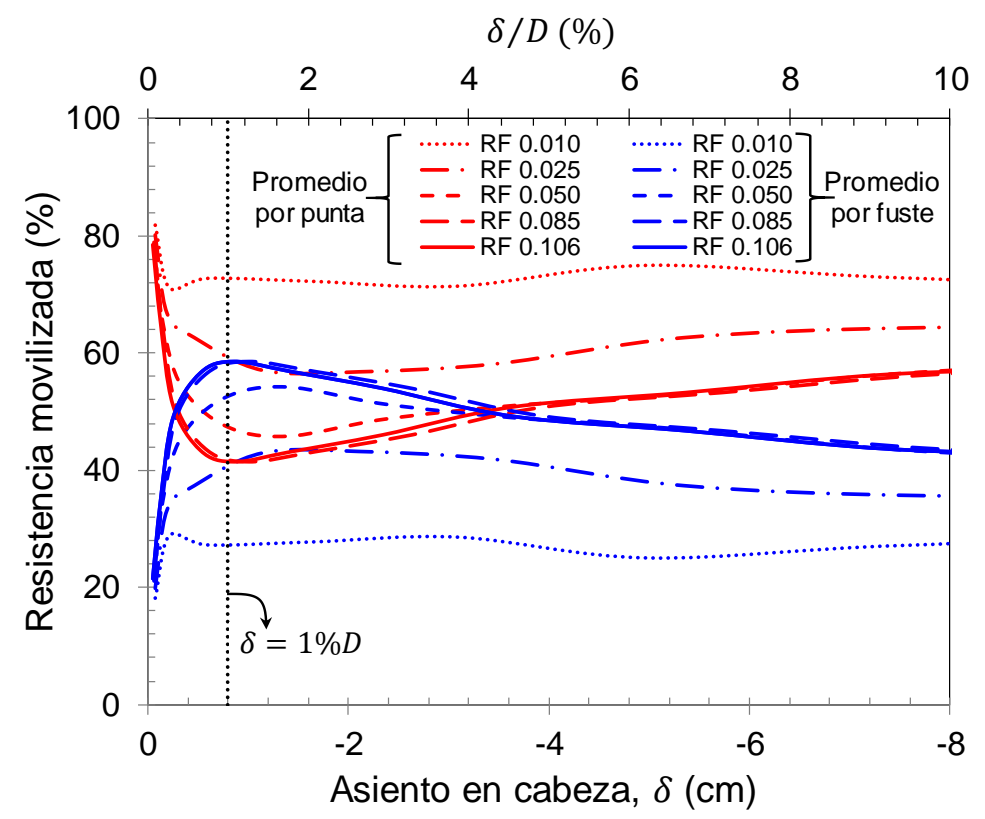

Figura 4.27. Contribución del porcentaje de resistencia promedio movilizada por fuste y por punta vs asiento cabeza obtenidos en los modelos con Abaqus para los pilotes empotrados en la pseudo-roca con diferente grado de rugosidad. 


\section{Ensayos de centrífuga de pilotes empotrados en roca}

\subsection{Introducción}

A pesar de los esfuerzos previos realizados para tratar de incorporar la rugosidad de la interfaz roca-pilote en la estimación de la resistencia por fuste de pilotes (ver e.g., Horvath et al. 1983; Seidel y Collingwood 2001; Dai et al. 2017; Gutiérrez-Ch et al. 2019a), aún es necesario realizar un análisis más profundo, mediante ensayos de carga in-situ, para mejorar las estimaciones de resistencia por fuste publicadas en la literatura. En este sentido, los ensayos de centrífuga (Leung y Ko 1993) proporcionan una interesante alternativa a los ensayos de carga ejecutados a escala real, debido a (i) las dificultades y costos asociados en la realización de nuevos ensayos de carga en campo; y a (ii) la ausencia de ensayos de carga publicados en la literatura en los que se reporte la información de la rugosidad medida en la cavidad de los pilotes empotrados en roca. Los ensayos de centrífuga permiten estudiar problemas geotécnicos por medio de modelos a pequeña escala, los cuales son sometidos a campos de aceleración de varias veces el valor de la gravedad $(g)$ de la Tierra. De este modo, los esfuerzos y procesos dependientes de la gravedad reproducen correctamente el problema en estudio a escala del prototipo (i.e. a escala real) durante la realización del ensayo, siempre y cuando se empleen de forma apropiada las leyes de escalado (Taylor 1995).

Como se indica en la Sección 2.4.1, los ensayos de centrífuga realizados por Leung y Ko (1993) y por Dykeman y Valsangkar (1996) demostraron un incremento en la capacidad axial de carga de pilotes al aumentar la rugosidad en la interfaz roca-pilote. Adicionalmente, los ensayos de centrífuga realizados por Xing et al. (2014), reflejaron la viabilidad de este tipo de ensayo para grupos de pilotes excavados en roca. Sin embargo, los ensayos antes mencionados proporcionan poca información sobre efecto de la rugosidad en el comportamiento de pilotes empotrados en roca. Ante lo anteriormente expuesto, el objetivo de este capítulo es evaluar el efecto de la rugosidad 
sobre la resistencia por fuste de pilotes empotrados en roca, mediante la realización de ensayos de centrífuga, considerando diferentes niveles de rugosidad en la interfaz rocapilote y siguiendo el esquema idealizado a escala del prototipo $(1 g)$ y del modelo $(N g)$ presentado en la Figura 5.1. Para ello, se emplean además las últimas tecnologías para medir deformaciones en los pilotes durante los ensayos a través del uso de fibra óptica.
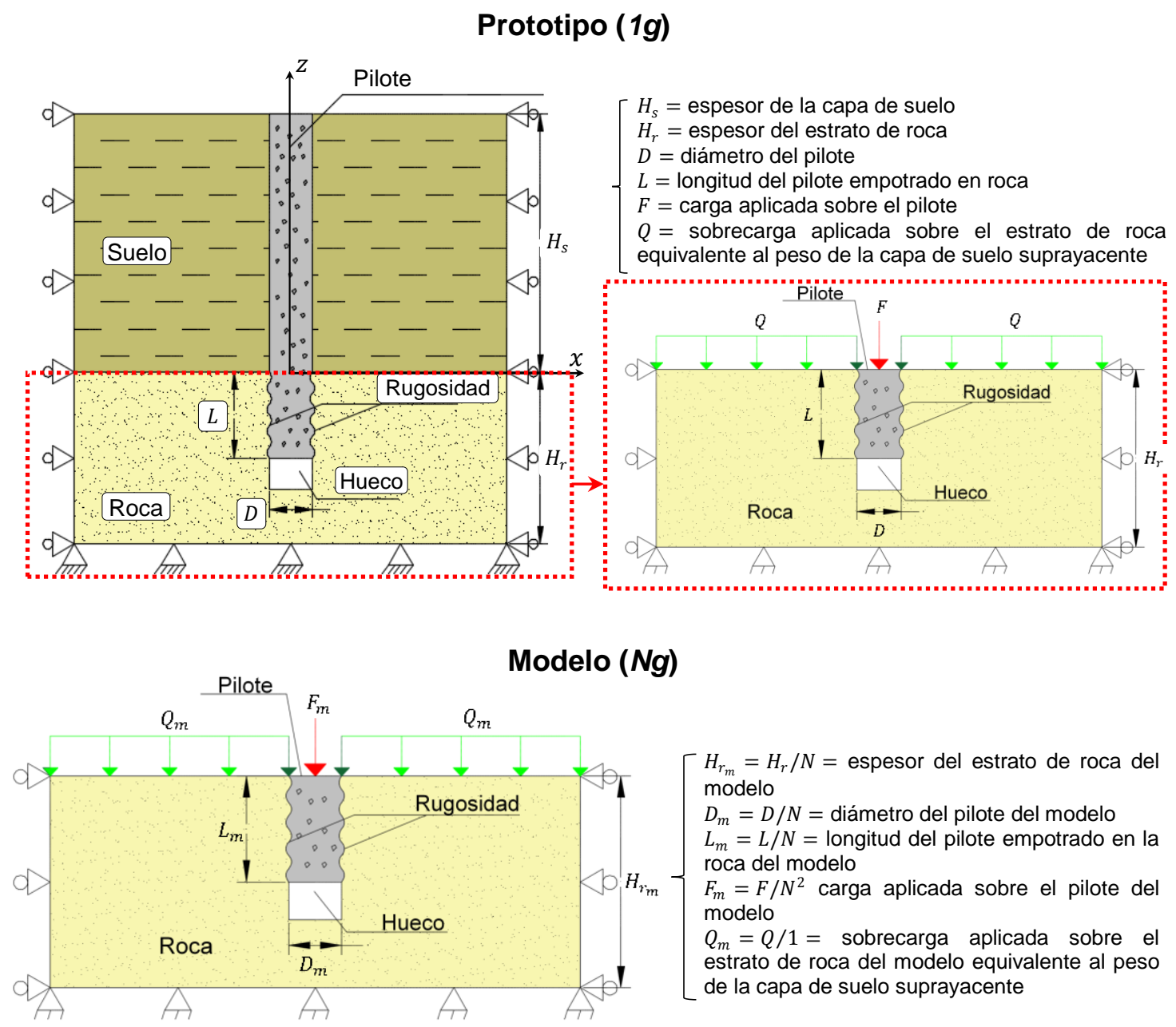

Figura 5.1. Esquema idealizado a escala del prototipo $(1 g)$ y del modelo $(N g)$.

\subsection{Modelación centrífuga}

Los ensayos de centrífuga que se presentan en este capítulo fueron realizados bajo una aceleración de $50 \mathrm{~g}$ (i.e., 50 veces la gravedad de la tierra) empleando la máquina de centrífuga del Centro de Geomecánica de la Universidad de Nottingham (ver la Figura 5.2), cuyo brazo giratorio asimétrico es de $2 \mathrm{~m}$ de radio, capaz de alcanzar una aceleración de hasta 150-g. De acuerdo a las leyes de escalado de los ensayos de 
centrífuga (ver la Sección 2.3), la longitud en un modelo de centrífuga se reduce en comparación con el prototipo a escala real por el factor de escala de gravedad $N\left(l_{m}=\right.$ $l / N$, donde $l$ y $l_{m}$ es la longitud a escala del prototipo y del modelo, respectivamente; el subíndice $m$ representa la propiedad a escala del modelo, ver la Figura 5.1), y la fuerza es escalada por $N^{2}\left(F_{m}=F_{p} / N^{2}\right.$, donde $F$ es la fuerza, ver la Figura 5.1). El adoptar $N=$ 50 en estos ensayos de centrífuga permitió reproducir, a escala del modelo, el rango de perfiles de rugosidades que se esperarían obtener en excavaciones reales de pilotes empotrados en roca, así como solicitaciones razonables para la capacidad de carga axial del pilote (menor que el límite de $10 \mathrm{kN}$ del actuador usado durante el ensayo de centrífuga). A continuación, se describen las características de la pseudo-roca (empleada para simular una roca de baja resistencia), de los pilotes del modelo y de su instrumentación, así como la configuración usada en los ensayos.

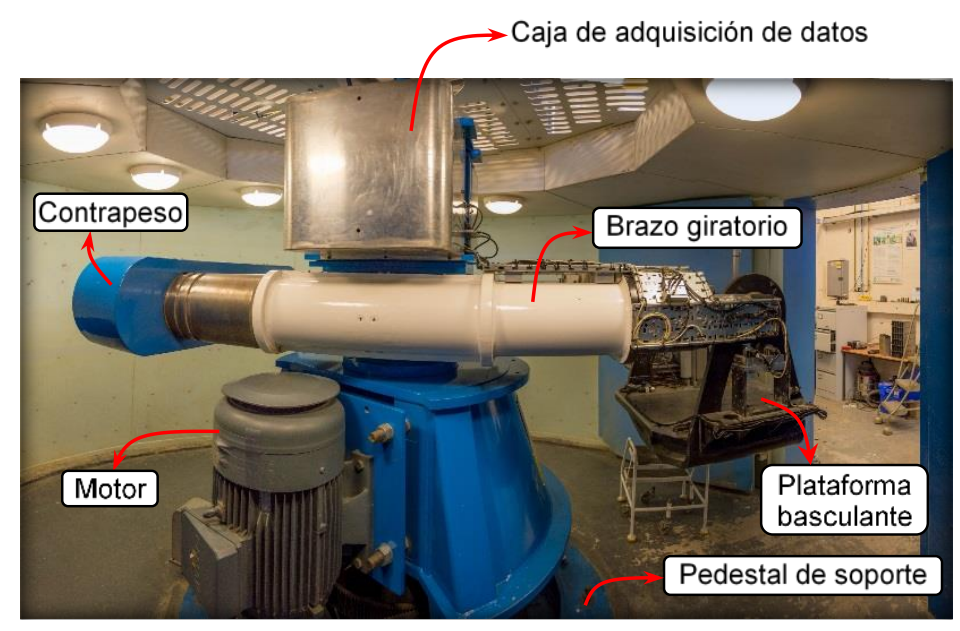

(a)

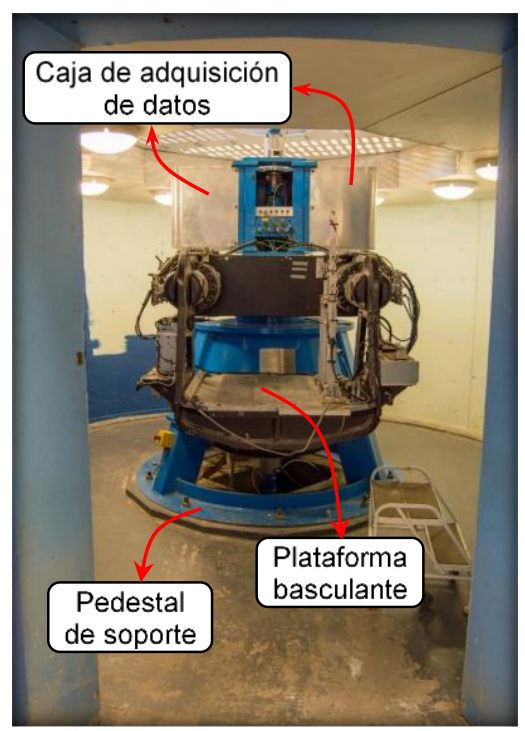

(b)

Figura 5.2. Máquina de centrífuga del Centro de Geomecánica de la Universidad de Nottingham y sus componentes: (a) vista panorámica, (b) vista frontal.

\subsubsection{Pseudo-roca}

El efecto de la rugosidad sobre la resistencia por fuste es de gran relevancia en pilotes excavados en rocas blandas con $\sigma_{c}=1-12 \mathrm{MPa}$ (Seidel y Collingwood 2001). En este sentido, se diseñaron diversas mezclas de arena, cemento, bentonita y agua (ver Figura 
5.3(a)-(c)) para obtener una roca sintética con $\sigma_{c}$ cercano a $1 \mathrm{MPa}$; tras varios ensayos, las proporciones adoptadas se presentan en la Tabla 5.1. Una vez preparada la mezcla de la pseudo-roca, la misma fue vertida y vibrada en tres capas iguales dentro de tres moldes cúbicos de dimensiones de $10.2 \times 10.2 \times 10.2 \mathrm{~cm}^{3}$ (ver Figura 5.3(d)), que fueron colocados en un lugar libre de vibración dentro de la cámara húmeda para su curado. Transcurrido un período de 44 días, las probetas fueron desmoldadas y sometidas a ensayos de compresión uniaxial (ver Figura 5.3(e)), resultando un valor promedio de $\sigma_{c}=1.14 \mathrm{MPa}(\operatorname{ver}$ Tabla 5.1).

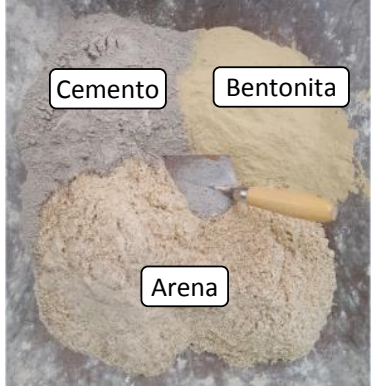

(a)

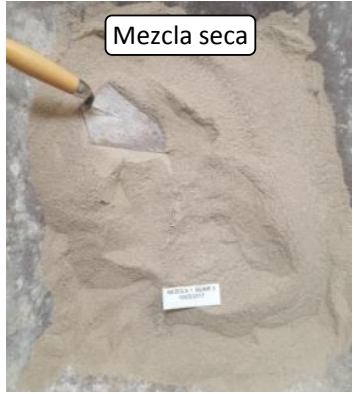

(b)

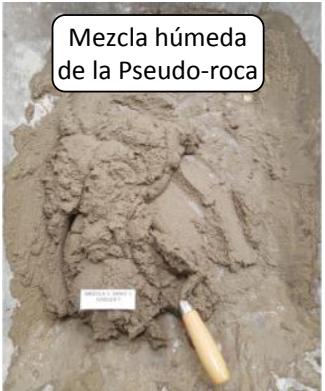

(c)

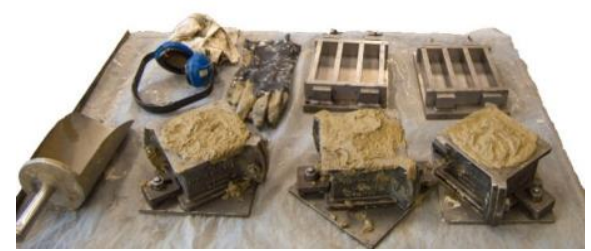

(d)

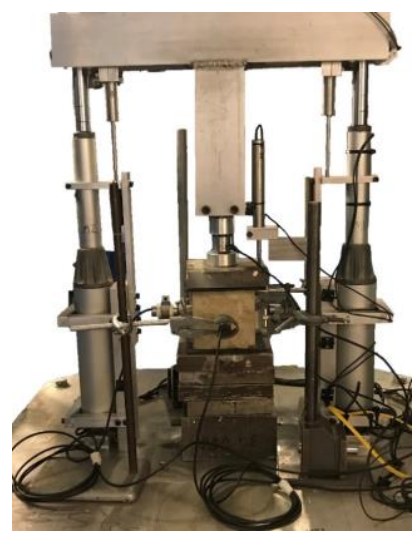

(e)

Figura 5.3. Proceso de preparación de la pseudo-roca: (a) arena, cemento y bentonita antes de mezclarlos, (b) arena, cemento y bentonita después de mezclarlos, (c) mezcla de arena, cemento y bentonita después de agregar la proporción de agua, (d) probetas cúbicas tras verter y vibrar la mezcla de la pseudo-roca en 3 capas iguales, (e) probeta de la pseudo-roca desmoldada a los 44 días para ensayo de compresión uniaxial.

Tabla 5.1. Proporciones de la mezcla expresadas como porcentaje en masa y resultados de ensayos de compresión uniaxial realizados en probetas a los 44 días.

\begin{tabular}{|c|c|c|c|}
\hline \multicolumn{4}{|c|}{ (a) Proporciones de la Mezcla (\%) } \\
\hline Arena & Cemento & Bentonita & Agua \\
\hline 52.3 & 12.2 & 6.5 & 29 \\
\hline \multicolumn{4}{|c|}{ (b) Ensayos realizados } \\
\hline & Probeta 1 & Probeta 2 & Probeta 3 \\
\hline UCS (MPa) & 1.14 & 1.15 & 1.12 \\
\hline
\end{tabular}




\subsubsection{Modelo de los Pilotes}

\subsubsection{Fabricación}

Los pilotes fueron fabricados de aluminio con un módulo de Young $E=69 \mathrm{GPa}$, con sección longitudinal hueca de diámetro nominal interno y externo de $11.81 \mathrm{~mm}$ y 15.87 $\mathrm{mm}$, respectivamente (ver la Figura 5.4). De esta manera y siguiendo las leyes de escalado (ver la Sección 2.3), cada pilote tiene una rigidez axial ( $E A$ donde $A$ es el área de la sección transversal) equivalente a la de un pilote a escala del prototipo de $0.8 \mathrm{~m}$ de diámetro, fabricado de hormigón y de sección sólida con un módulo de Young $E=$ $30 \mathrm{GPa}$. La longitud nominal del pilote ensayado en centrífuga es de $80 \mathrm{~mm}$ (4 m a escala de prototipo). El resume de las propiedades del pilote en ambas escalas se presenta en la Tabla 5.1.

Tabla 5.2. Propiedades del pilote a escala del prototipo y del modelo

\begin{tabular}{lccc}
\hline \multicolumn{1}{c}{ Propiedad } & Prototipo $(1 \mathrm{~g})$ & Modelo $(50 \mathrm{~g})$ & Modelo $(1 \mathrm{~g})$ \\
\hline Material & Hormigón & Aluminio & Aluminio \\
Módulo de Young (GPa) & 30 & 69 & 69 \\
Diámetro exterior $(\mathrm{mm})$ & 804 & 15.875 & 793.75 \\
Diámetro interior $(\mathrm{mm})$ & - & 11.811 & 590.55 \\
Longitud de empotramiento $(\mathrm{mm})$ & 4000 & 80 & 4000 \\
Rigidez axial $(\mathrm{GN})$ & 15.23 & $6.098 \times 10^{-3}$ & 15.24 \\
\hline
\end{tabular}

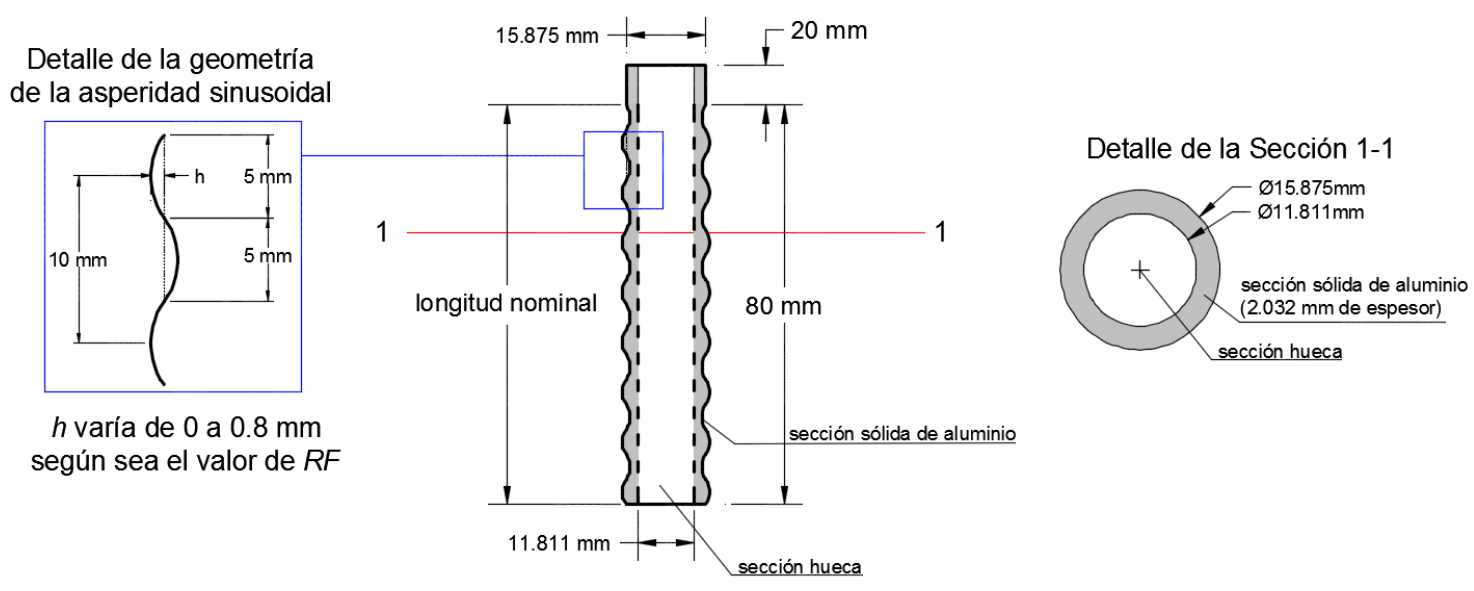

Figura 5.4. Detalle de la geometría idealizada del modelo de pilote.

Para poder estudiar el efecto de la rugosidad sobre el comportamiento bajo carga axial de pilotes empotrado en roca, los pilotes fueron fabricados con diferente grado de 
rugosidad. Para ello, y de acuerdo con O'Neill et al. (1996) y Hassan et al. (1997), se emplearon superficies sinusoidales con asperidades de amplitudes de $0,0.2,0.4$, y 0.8 mm, y con longitud de onda de $10 \mathrm{~mm}$ para todos los pilotes (ver la Figura 5.4) -notar que estos valores están escalados a $50 \mathrm{~g}$-. Usando el factor de rugosidad $(R F)$ propuesto Horvath et al. (1983, ver la Sección 4.2.2), estos modelos corresponden a valores de $R F=0.000, R F=0.025, R F=0.050$, y $R F=0.106$, respectivamente. La Figura 5.5 muestra fotografías de cada pilote ensayado.
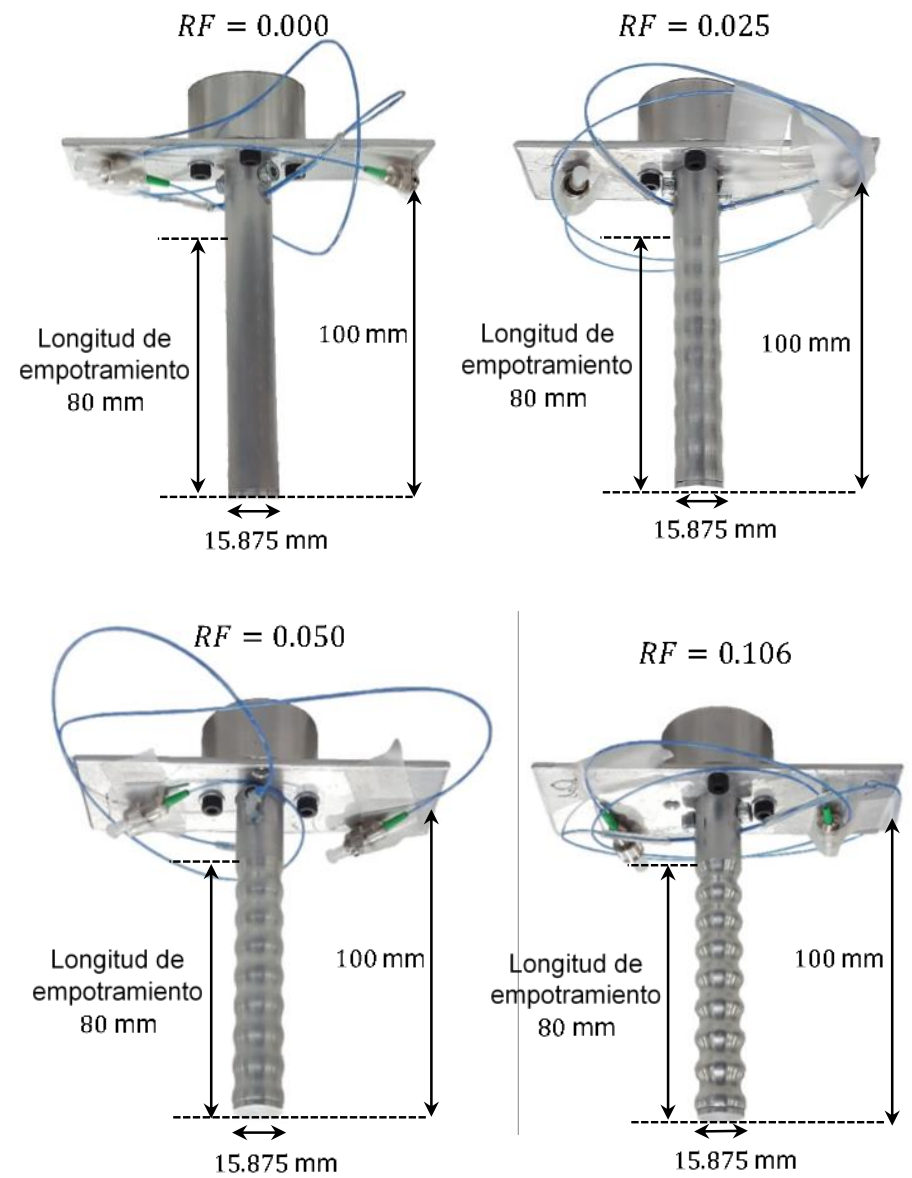

Figura 5.5. Pilotes de aluminio con diferente factor de rugosidad $(R F)$ a escala del modelo.

\subsubsection{Instrumentación}

Con el fin de registrar la fuerza axial movilizada a lo largo de cada pilote del modelo, se instalaron sensores de medición de fibra de óptica (Fiber Bragg Gratings, FBGs). Un sensor FBG es un filtro/reflector dependiente de la longitud de onda formada al introducir una perturbación periódica de la modulación del índice de refracción a lo largo de la 
longitud de la fibra (ver Figura 5.6) (Hill y Meltz 1997; Kashyap 2010; Alvárez-Botero et al. 2017). Las principales ventajas de los sensores FBG en relación a otras técnicas convencionales -e.g., bandas extensométricas, etc.- son (Kreuzer 2006): inmunidad al ruido electromagnético, fabricación ligera, dimensiones y peso reducido, y gran precisión en medir deformaciones (>10000 $\mu \mathrm{m} / \mathrm{m}$ ). Además, debido a su potencial de multiplexado, se puede insertar más de un sensor FBG a lo largo de una misma fibra óptica, siempre y cuando las longitudes de onda de cada sensor sean diferentes y no se solapen dentro de su intervalo de medición; e.g., si las longitudes de onda de los sensores FBG son $1510 \mathrm{~nm}, 1515 \mathrm{~nm}$ y $1520 \mathrm{~nm}$ y las deformaciones producidas son del orden de $\pm 2 \mathrm{~nm}$, no se produciría solape durante la medición (Kister et al. 2007). Figura 5.7(a)-(b) presenta un ejemplo de la estructura de una fibra óptica con varios sensores FBG.

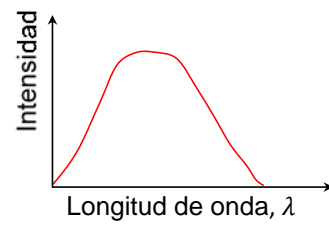

Señal de entrada

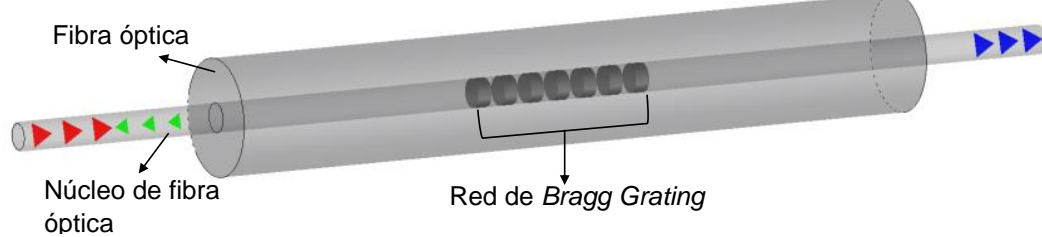

Figura 5.6. Esquema de la estructura y repuesta espectral de un sensor FBG (modificado de Kreuzer 2006).

La fibra óptica y sus respectivos sensores FBG fueron adheridos a la superficie interna de la sección hueca del modelo del pilote de aluminio. Para ello, se empleó el siguiente procedimiento de instalación (ver la Figura 5.7(c)): (1) se introdujo gran parte del cable FBG dentro de la sección hueca del pilote mediante un mini tubo plástico, para evitar así posibles daños sobre la estructura de la fibra; (2) una pequeña porción cable FBG (alrededor de $5 \mathrm{~mm}$ ) fue adherido con la ayuda de una barra de aluminio a la superficie interna del pilote mediante pegamento Super Glue; (3) la porción restante del cable FBG 
que no tiene tubo de protección -i.e., la porción de la fibra donde se encuentran los sensores FBG- fue extraída y "templada" mediante micrómetro modificado; y finalmente, (4) la fibra es fácilmente adherida a la superficie interna del pilote mediante pegamento Super Glue (con la ayuda de una barra de aluminio), y cubierto por adhesivo de curado UV. De esta forma, se garantiza que los sensores FBG fueran protegidos cuidadosamente y puestos en total contacto con la superficie interna del pilote de aluminio. Una vez adherida la fibra óptica con el procedimiento previamente descrito, es posible registrar el cambio de longitud de onda producto de la deformación del pilote.

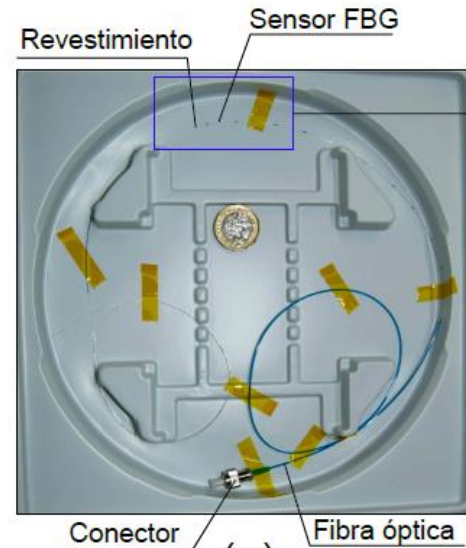

(a)

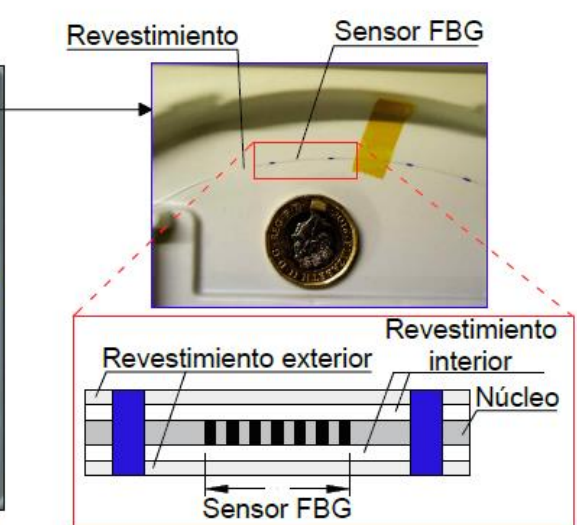

(b)

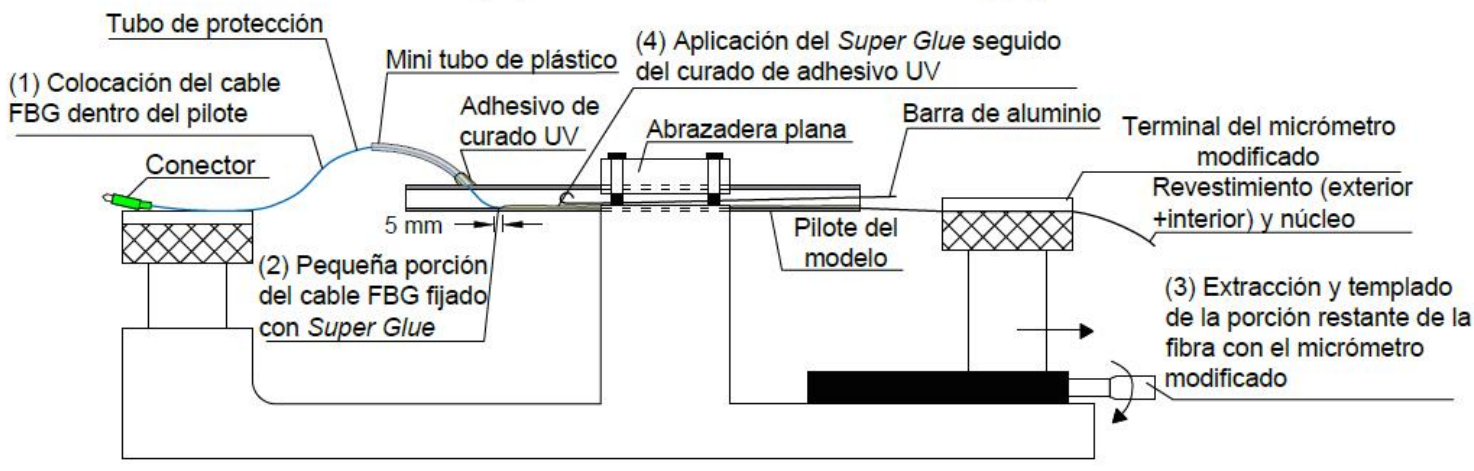

(c)

Figura 5.7. (a) ejemplo de la estructura de una fibra óptica con varios sensores FBG, (b) detalle de la fibra óptica y sensores FBG, (c) esquema del proceso de instalación de la instrumentación del modelo del pilote.

Cada modelo de pilote tiene dos líneas de fibra óptica (con sus correspondientes sensores FBG) localizados en los lados opuestos de la superficie interna de la sección hueca del pilote. Cada línea de fibra óptica contiene 3 sensores FBG fabricados con una longitud de onda de 1530 nm, 1535 nm, y 1540 nm ó de 1545 nm, 1550 nm, y 1555 nm 
(ver la Figura 5.8). Estos valores de longitud de onda fueron establecidos durante la fabricación de cada fibra óptica en función del rango de medición del interrogador FBG -e.g, rango de medición [1520; 1560] nm-. (Un interrogador FBG es el dispositivo que se encarga de registrar las señales recibidas por cada sensor FBG).

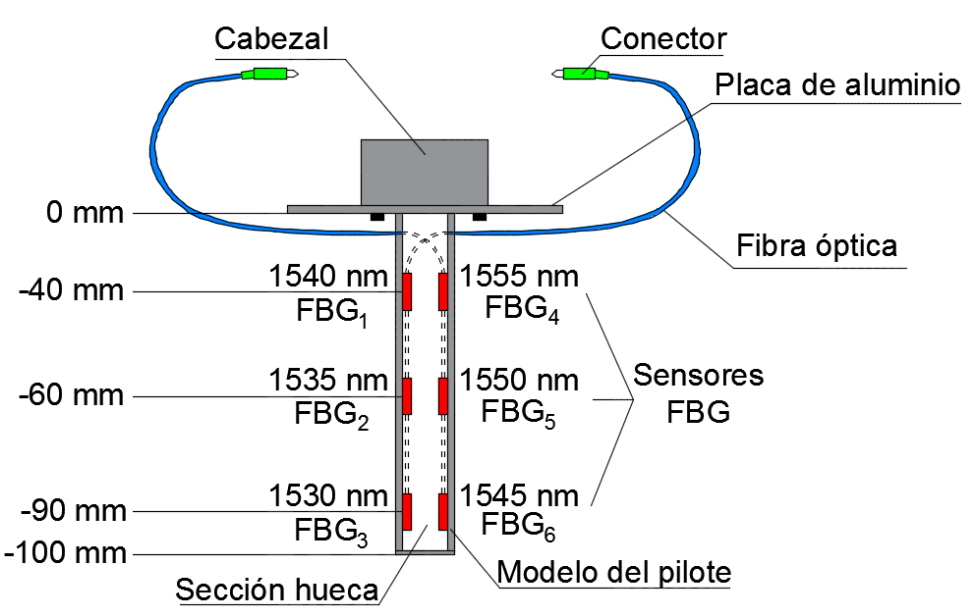

(a)

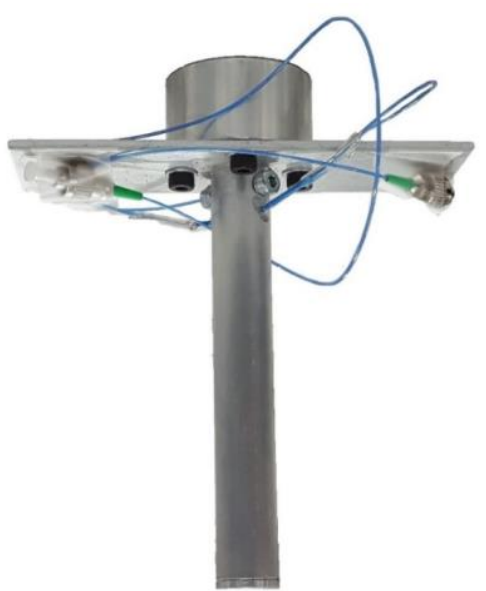

(b)

Figura 5.8. Instrumentación de cada modelo de pilote: (a) esquema ilustrativo de la localización de líneas de fibra óptica y localización de los sensores FBG $\left(F B G_{n}\right)$, (modificado de GutiérrezCh et al. 2020a), (b) modelo de pilote con $R F=0.000$ después de instalar su instrumentación.

\subsubsection{Calibración de los sensores FBG}

Para realizar el ensayo de centrífuga sobre cada uno de los modelos de pilotes empotrados en roca sometidos a carga axial, es necesario realizar una calibración previa de los sensores FBG, para caracterizar así la relación fuerza-cambio de longitud de onda de cada sensor. Dicha calibración se realiza 3 veces en cada modelo de pilote; y cada vez se aplican varios ciclos de carga y posterior descarga usando (i) un marco de carga de dos columnas -para aplicar la fuerza axial en compresión en cabeza del pilote-, (ii) una célula de carga calibrada -para registrar la fuerza axial movilizada en el extremo opuesto del pilote-, y (iii) un interrogador FBG -para medir el cambio de longitud de onda registrado en cada sensor FBG $\left(F B G_{i}\right)$ durante la aplicación de la carga- (ver la Figura 5.9). 


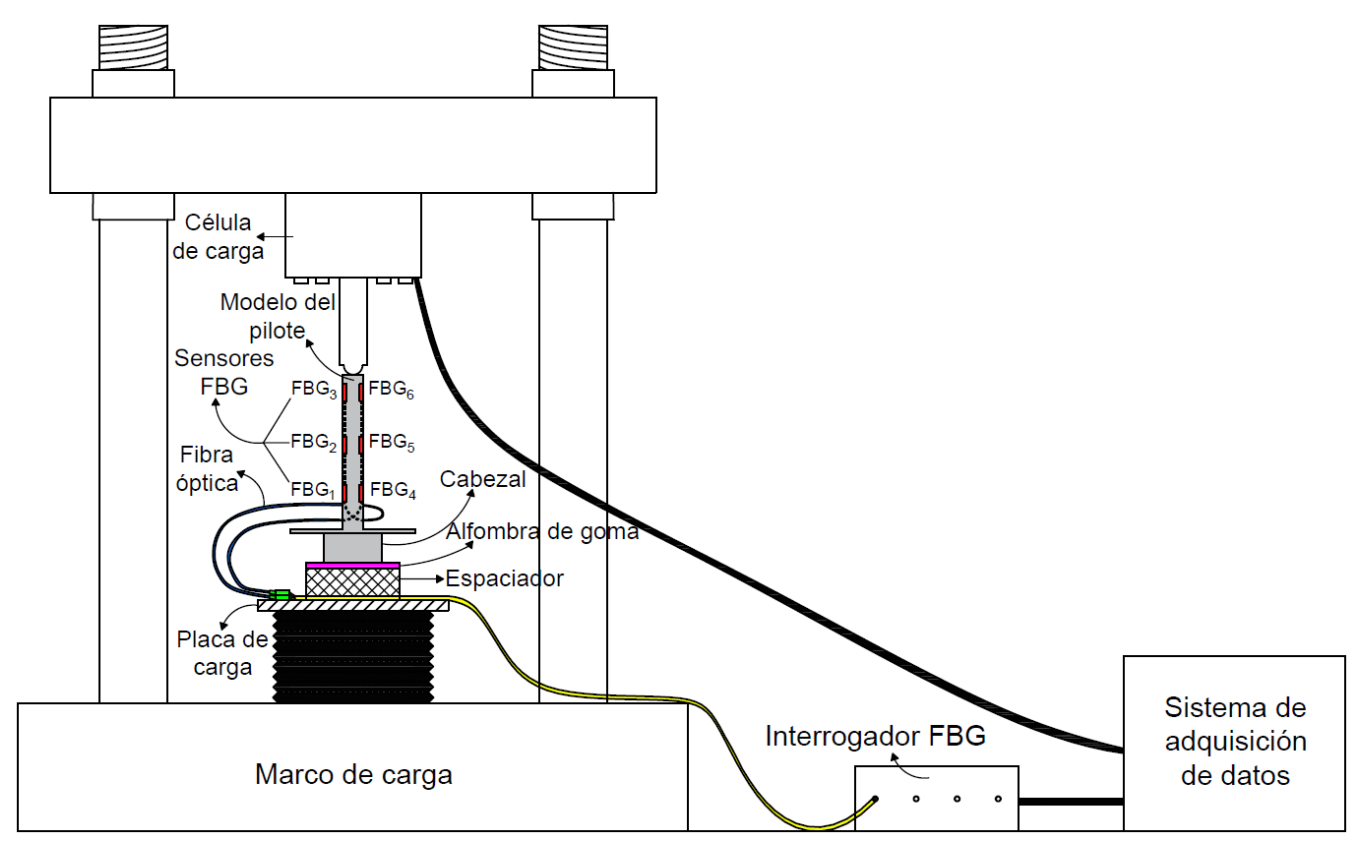

Figura 5.9. Esquema ilustrativo de la configuración adoptada durante el proceso de calibración de los sensores FBG.

En la Figura 5.10 se presenta un ejemplo de los resultados obtenidos en uno de los 3 ensayos realizados durante la calibración del modelo de pilote con $R F=0.025$. La Figura 5.10(a) muestra la fuerza axial (carga y descarga) registrada con la célula de carga en este ensayo como función del tiempo. Como puede observarse, una vez alcanzado el valor de cada escalón carga y descarga establecido, el mismo se mantuvo constante durante 15 segundos mediante el mecanismo de control del que dispone el marco de carga. En cada ensayo, el valor máximo de la carga aplicada fue de alrededor de $4000 \mathrm{~N}$, el cual es cercano al máximo valor de resistencia esperado durante la realización de los ensayos de centrífuga (i.e., alrededor de 3900 N). Adicionalmente, en la Figura 5.10(b) se muestra el cambio de longitud de onda registrado con el interrogador FBG para el sensor $F B G_{5}$, para los mismos intervalos de carga y descarga aplicados en dicho ensayo de calibración. Dado que el cambio de longitud de onda que se produce se debe a la fuerza axial aplicada, y dado que ambos registros (fuerza y cambio de longitud de onda) están sincronizados con el tiempo de realización del ensayo, es posible establecer la relación de fuerza-cambio de longitud de onda de la forma (ver Figura 5.10(c)-(d)): 


$$
F_{i}=K_{F B G_{i}} \Delta \lambda_{F B G_{i}}+F_{O S_{i}}
$$

donde $i$ es el número del sensor FBG, $F_{i}$ es la fuerza axial obtenida a partir del sensor $F B G_{i}, K_{F B G_{i}}$ es la pendiente de la línea de mejor ajuste de la Figura 5.10(c), $\Delta \lambda_{F B G_{i}}$ es el cambio de longitud de onda registrado en el sensor $F B G_{i}$, y $F_{O S_{i}}$ es la ordenada en el origen de la Figura 5.10(c). El valor de $K_{F B G_{i}}$ se considera único para cada sensor FBG $\left(F B G_{i}\right)$ (aunque pueden presentarse ligeros cambios en su valor entre los ensayos realizados durante la calibración), de modo que el valor final de $K_{F B G_{i}}$ (del sensor $F B G_{i}$ ) empleado para dicho sensor será, el promedio de los valores obtenidos en los 3 ensayos de calibración de cada sensor FBG (e.g., $\bar{K}_{F B G_{5}}=$ $\left(\left(K_{F B G_{5, \text { ensayo } 1}}+K_{F B G_{5, \text { ensayo } 2}}+K_{F B G_{5, \text { ensayo } 3}}\right) / 3\right)$.

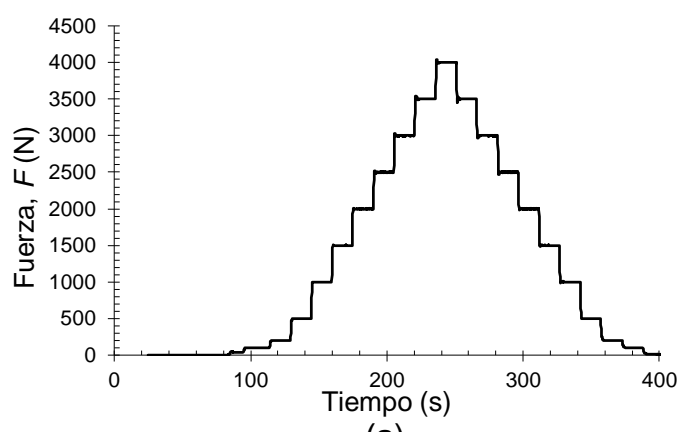

(a)

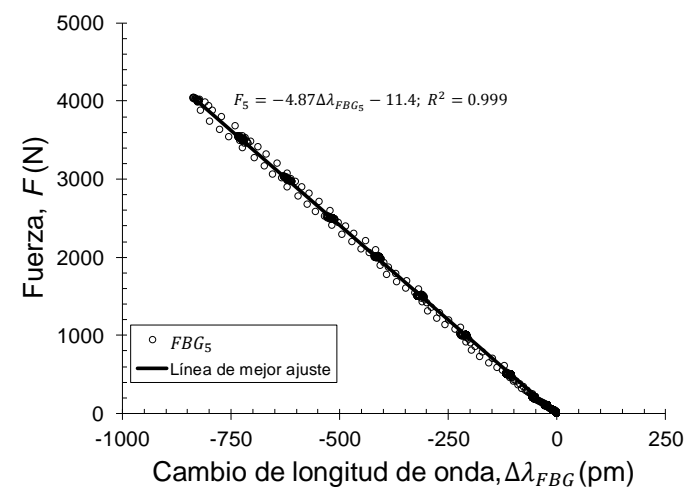

(c)

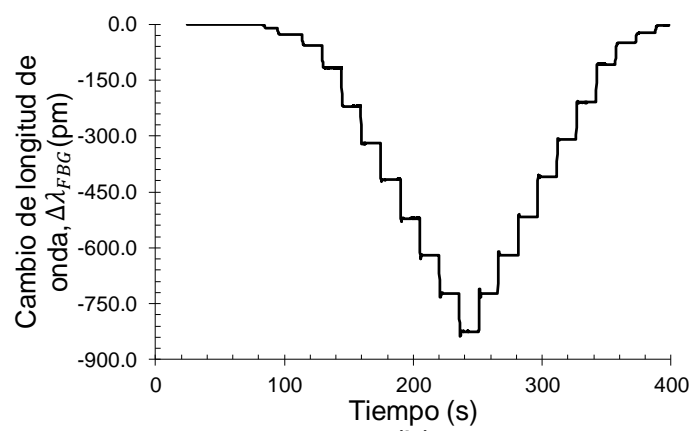

(b)

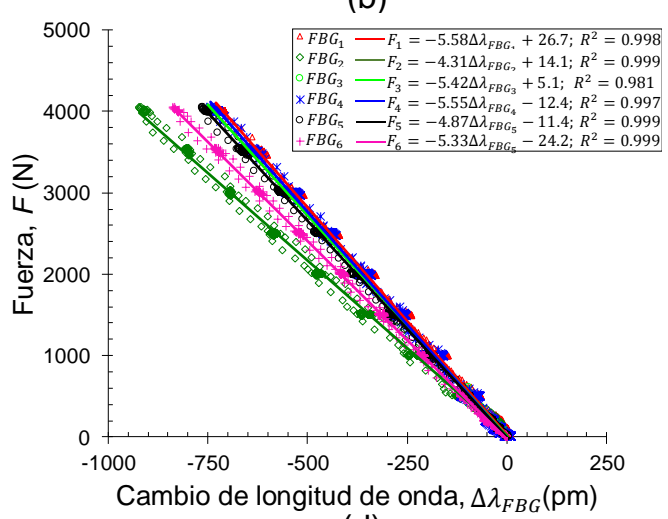

(d)

Figura 5.10. Ejemplo de los resultados obtenidos durante la calibración de los sensores para el modelo de pilote con $R F=0.025$ (para el sensor $F B G_{5}$ ): (a) ciclos de carga y descarga vs tiempo, (b) cambio en la longitud de onda vs tiempo, (c) relación fuerza-cambio de longitud de onda (modificado de Gutiérrez-Ch et al. 2020a); (d) relación fuerza-cambio de longitud de onda para todos los sensores. 


\subsubsection{Preparación del modelo de centrífuga}

Cada modelo para el ensayo de centrífuga se preparó de la siguiente manera:

a) Debido a que esta investigación analiza solo el comportamiento por fuste (i.e., sin considerar la punta), primero se adhirió un trozo de espuma de poliestireno blando (de altura y diámetro igual al diámetro del modelo del pilote de aluminio) a la punta de cada uno de los modelos de pilotes a ensayar (ver la Figura $5.11(a))$

b) Luego, se preparó la mezcla de la pseudo-roca usando las proporciones de arena, cemento, bentonita y agua presentadas en la Tabla 5.1. Una vez alcanzada una consistencia adecuada en la mezcla, la misma fue vertida (en 3 capas iguales) dentro de un molde metálico de forma cilíndrica, con altura y diámetro iguales a $20 \mathrm{~cm}$; cada una de estas capas fueron vibradas después de su colocación (ver la Figura 5.11(b)). Además, debido a la poca influencia de la condición de borde para distancias entre el borde del molde y el centro del pilote mayores a 4 veces su diámetro $(D)$ (Dykeman y Valsangkar 1996; Xing et al. 2014), se dejó una distancia mayor a $5 D$ entre el borde del molde y el eje del pilote (ver la Figura 5.11(c)).

c) Posteriormente, se introdujo el modelo del pilote de aluminio dentro de la mezcla (i.e., justo después de su vertido dentro del molde), tras lo que se realizó el vibrado del ensamblaje pilote-mezcla. De esta forma, y de acuerdo al procedimiento empleado en investigaciones previas (e.g., ver Dykeman y Valsangkar 1996; Dai et al. 2016), se logra una adhesión adecuada entre la mezcla de la pseudo-roca y el modelo del pilote (ver la Figura 5.11(c)).

d) Finalmente, cada uno de los modelos de centrífuga (ver la Figura 5.11(c)-(d)), fueron almacenados y curados normalmente dentro de una cámara húmeda 
(libre de vibraciones) hasta la realización del ensayo de centrífuga (en este caso, a los 44 días).

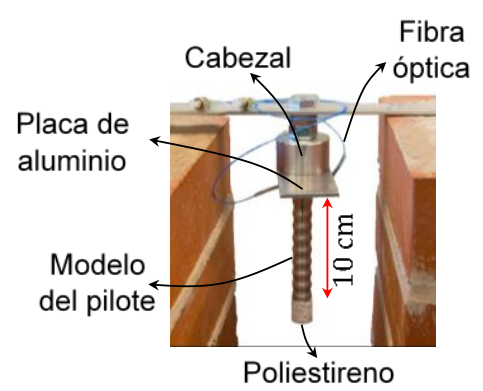

(a)

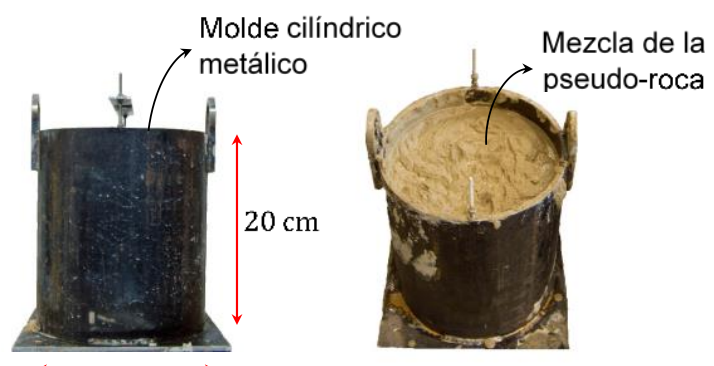

(b)

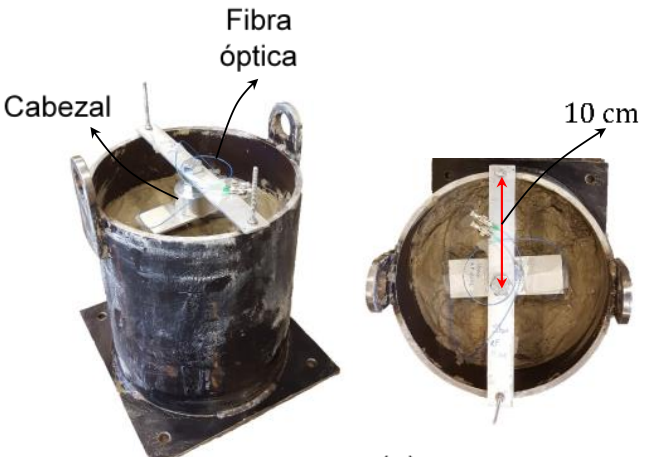

(c)

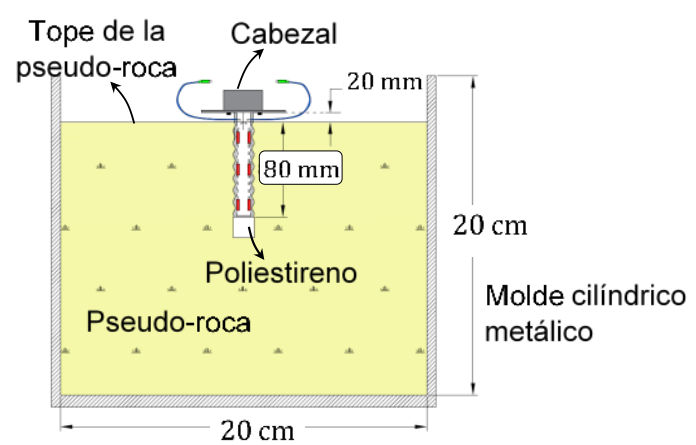

(d)

Figura 5.11. Preparación del modelo de centrífuga: (a) modelo del pilote de aluminio con $R F=$ 0.106 tras adherirle la pieza de poliestireno en la punta, (b) molde cilíndrico metálico tras verter la mezcla húmeda de la pseudo-roca, (c) ensamblaje pseudo-roca-pilote del modelo de centrífuga, (d) modelo idealizado de un ensamblaje típico pseudo-roca-pilote.

\subsubsection{Ensayos de centrífuga}

Transcurridos 44 días de curado, cada modelo de pilote fue colocado en su posición sobre la plataforma diseñada para su ensayo en la máquina de centrífuga, y se dispuso sobre la pseudo-roca del modelo una serie de placas metálicas (de $30 \mathrm{~mm}$ de espesor y de peso unitario de $80 \mathrm{kN} / \mathrm{m}^{3}$ ). Esto representa aplicar un esfuerzo, equivalente al esfuerzo in-situ (e.g., para simular un capa de suelo de $6 \mathrm{~m}$ suprayacente al estrato de roca con un peso unitario de $20 \mathrm{kN} / \mathrm{m}^{3}$ ) de alrededor de $120 \mathrm{kPa}$ a escala del prototipo (ver la Figura 5.12(a)-(c)). Luego, para registrar la fuerza axial aplicada en las cabezas de los pilotes durante el ensayo, se adhirieron células de carga con conectores a los mismos, y se posicionó y conectó el sistema de carga (i.e., el marco de carga de dos actuadores lineales de carga) al conector del modelo del pilote (ver la Figura 5.12(a)). 
Adicionalmente, para registrar el asiento producido en cabeza del pilote durante el ensayo, se posicionó un transductor diferencial de variación lineal (Linear Variable Displacement Transducer, LVDT) sobre la placa de aluminio. Notar que, por facilidad del ensayo, dicha placa se movió y se ubicó entre el cabezal del pilote y el espaciador, ver la Figura 5.12(c).

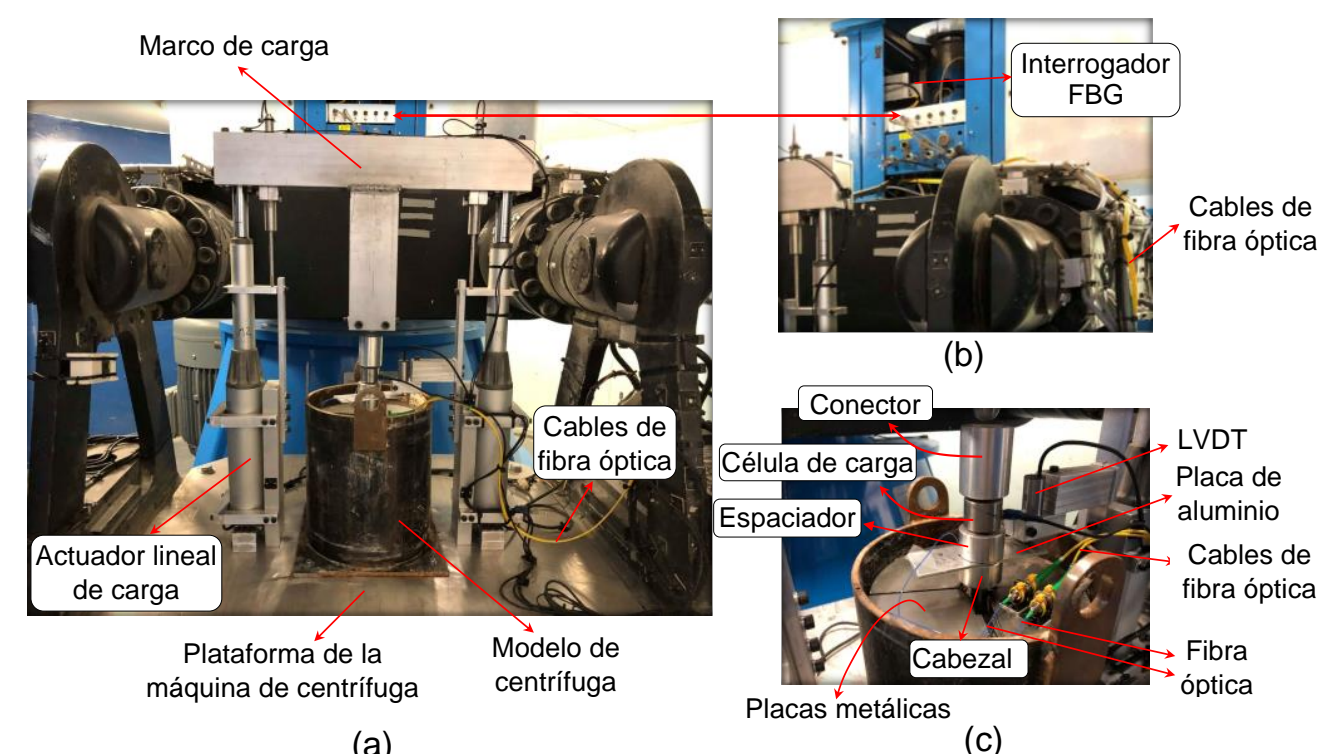

(a)

(c)

Figura 5.12. Configuración del ensayo de centrífuga: (a) sistema de carga, (b) localización del interrogador FBG, (c) detalle de la instrumentación de medición

Por otra parte, la fuerza axial movilizada a lo largo del modelo del pilote en cada sensor $F B G_{i}$ se calculó a través de la Ecuación 5.1 empleando: (i) el cambio de longitud de onda registrado durante la realización del ensayo en cada sensor $F B G_{i}$ (conectado mediante los cables de fibra óptica amarillos al interrogador FBG, ver la Figura 5.12(b)), y (ii) los valores de $K_{F B G_{i}}$ obtenidos durante la calibración de los sensores FBG. En cada ensayo de centrífuga, la aceleración de la máquina fue incrementada gradualmente hasta $50 \mathrm{~g}$. Una vez se alcanzó dicho valor, éste se mantuvo constante, y el modelo de pilote fue cargado axialmente mediante el uso del sistema de carga con una tasa de desplazamiento controlado de $0.1 \mathrm{~mm} / \mathrm{s}$. La fuerza axial aplicada y el cambio de longitud de onda en cada sensor $F B G_{i}$ fueron registrados cada $10 \mathrm{~Hz}$ por el sistema de adquisición de datos. Los resultados obtenidos en cada uno de los ensayos se describen a continuación. 


\subsection{Resultados}

\subsubsection{Respuesta carga-asiento}

En la Figura 5.13, se presentan las curvas de carga axial y rigidez global versus el asiento (normalizado por el diámetro del pilote) obtenidas durante los ensayos de centrífuga realizados sobre los 4 pilotes empotrados en roca considerando solo la resistencia por fuste (i.e., con poliestireno blando en la punta), y con diferente nivel de rugosidad en la interfaz roca-pilote. Estos resultados se presentan a escala del modelo -i.e., a un nivel de 50g-. La carga axial movilizada durante el proceso de aceleración de $1 g$ a $50 \mathrm{~g}$ fue despreciada. Notar, además, que los 4 ensayos se realizaron hasta alcanzar un asiento medido en cabeza del pilote $(\delta)$ mayor o igual al $20 \%$ de su diámetro $(\delta / D>20 \%)$

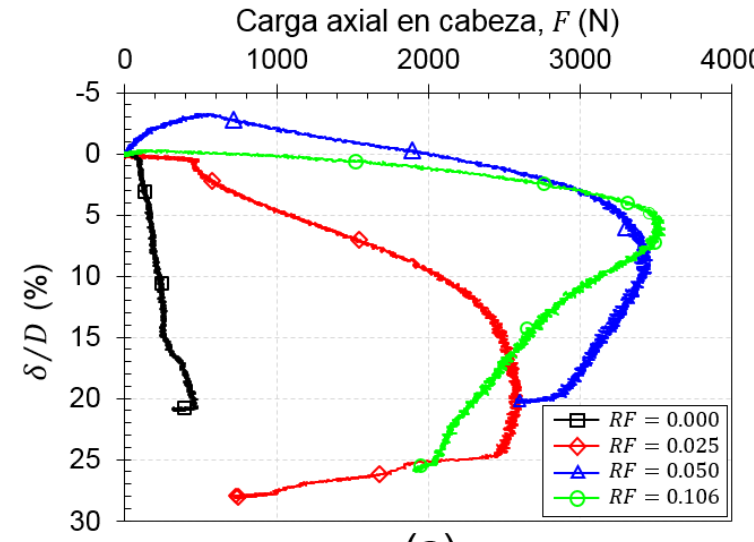

(a)

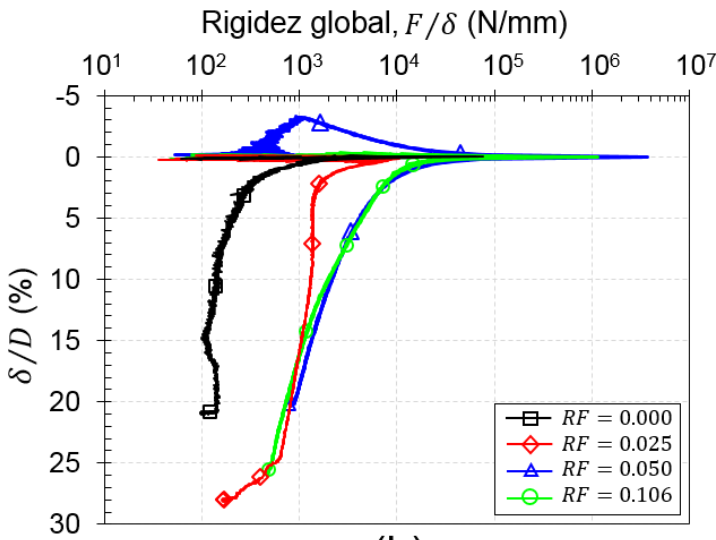

(b)

Figura 5.13. (a) curvas carga axial vs asiento normalizado por el diámetro, (b) curvas rigidez global vs asiento normalizado por el diámetro.

Los resultados experimentales presentados en la Figura 5.13 demuestran que la rugosidad en la interfaz roca-pilote es un factor con gran influencia sobre la capacidad de carga y rigidez global (i.e., la relación entre la carga axial aplicada y el asiento alcanzado, $F / \delta$ ) de pilotes empotrados en roca sometidos a carga axial. Por ejemplo, para un asiento en cabeza de $1 \% D$, la carga resistida fue de $100 \mathrm{~N}, 470 \mathrm{~N}, 2380 \mathrm{~N}$ y $1880 \mathrm{~N}$, y la rigidez global fue de $6.3 \times 10^{2} \mathrm{~N} / \mathrm{mm}, 3 \times 10^{3} \mathrm{~N} / \mathrm{mm}, 1.5 \times 10^{4} \mathrm{~N} / \mathrm{mm}$ y $1.2 \times 10^{4}$ 
$\mathrm{N} / \mathrm{mm}$, para los modelos de pilotes con $R F=0.000, R F=0.025, R F=0.050$ y $R F=$ 0.106 , respectivamente.

Además, en la Figura 5.13(a) se nota que las curvas carga-asiento de los modelos con $R F=0.000$ y $R F=0.025$ incrementan linealmente hasta alcanzar un primer valor de carga axial máximo (para $\delta=0.5 \% D$ ) seguido de una ligera disminución de su carga axial; esto podría atribuirse a la perdida de la cohesión existente en la interfaz rocapilote; luego, su capacidad resistente nuevamente aumenta con el incremento de la carga axial actuante hasta alcanzar un segundo valor de carga máximo para asientos de $\delta=20.5 \% D$ y $\delta=19.8 \% D$, respectivamente. Para el caso de los pilotes más rugosos $(R F=0.050$ y $R F=0.106)$, la disminución de la carga axial por la pérdida de cohesión en la interfaz roca-pilote no se evidencia; en estos modelos su capacidad de carga incrementó hasta alcanzar su correspondiente valor máximo, y posterior a dicho umbral se registró una disminución de su capacidad resistente. Adicionalmente, los resultados mostrados en la Figura 5.13(a) indican que el comportamiento resistente de los pilotes tras alcanzar su valor de carga máxima -i.e., su capacidad de mantener la resistencia alcanzada tras sobrepasar al asiento asociado a la carga axial máxima $\left(\delta_{\text {pico }}\right)-$ tiende a ser más dúctil en los pilotes rugosos. Al mismo tiempo, la Figura 5.13(b) muestra como la rigidez global de los pilotes ensayados incrementa rápidamente para asientos menores a $\delta \leq 0.5 \% D$; luego, se produce una rápida degradación de dicha rigidez de manera similar en los 4 pilotes hasta alcanzar asientos de $\delta=3 \% D$; tras superar dicho asiento la disminución de la rigidez tiende a estabilizarse en el caso de los pilotes con $R F=0.000$ y $R F=0.025$ o a disminuir de forma más gradual en el caso de los pilotes rugosos $(R F=0.050$ y $R F=0.106)$.

Por otra parte, de la curva carga-asiento del modelo con $R F=0.106$ se observa que el valor de carga axial máxima soportada es de alrededor de 3500 N (ver la Figura 5.13(a)), siendo este valor inferior al máximo -i.e., inferior a 8000 N (20 MN a escala del prototipo, 
ver el Capítulo 4, Sección 4.5.2.2)- que se esperaría movilizar según la simulación con el MEF de este modelo de pilote empotrado en una pseudo-roca con propiedades ligeramente superiores; si bien es cierto que el valor de carga axial obtenido en el ensayo de centrifuga para el modelo con $R F=0.106$ es casi la mitad del valor hallado en el MEF, resulta oportuno recordar que en el modelo numérico se consideró la movilización simultánea de la componente de resistencia por punta y fuste y por tanto era esperable que considerando sólo la resistencia por fuste el valor máximo de carga axial soportado resultara menor; en consecuencia, puede considerarse que las simulaciones numéricas realizadas con el MEF resultaron ser una herramienta alternativa adecuada para realizar el pre-diseño y analizar el comportamiento resistente de los pilotes empotrados en roca considerados en esta tesis doctoral.

Debe notarse, además, que la curva carga-asiento para el modelo de pilote con $R F=$ 0.050 sugiere que el mismo pudo tener un desplazamiento hacia arriba al inicio del ensayo. Esto, no representa un comportamiento "real" o esperado de un pilote bajo carga axial concéntrica y, probablemente puede deberse al diseño del sistema de aplicación de la carga, el cual puede generar la rotación del modelo del pilote cuando si se producen excentricidades durante la aplicación de la carga (i.e., si la carga no es aplicada en el centro del cabezal del pilote). En consecuencia, debido a la rotación del pilote, se produce un movimiento hacia arriba de la placa de aluminio sobre la cual está posicionado el transductor LVDT, siendo este movimiento registrado como un desplazamiento vertical del pilote. Este error podría haberse evitado si se hubiesen empleado múltiples transductores LVDT. La magnitud del error introducido sobre el asiento para este este ensayo el del orden de $\delta / D=3 \%$. Este error también podría haber afectado las mediciones de otras variables del modelo, sin embargo, basado en la tendencia de las curvas carga-asiento de los otros modelos no parece ser muy significativo. A pesar de este error, las cuatro curvas carga-asiento (ver la Figura 5.13) sugieren que podría existir un límite a partir del cual la rugosidad no produce más 
incremento sobre la capacidad de carga y rigidez global de pilotes empotrados en roca. Esta tendencia está en concordancia con los resultados reportados por Dai et al. (2017), quienes realizaron ensayos de carga a pequeña escala -i.e., a un nivel de $1-g-$ sobre pilotes empotrados en roca considerando diferentes niveles de rugosidad en la interfaz roca-pilote (ver la Sección 2.2.3.4).

\subsubsection{Fuerza axial}

La distribución de la fuerza axial en función de la profundidad para cada uno de los modelos de los pilotes fue obtenida a partir del cambio de longitud de onda (con el tiempo) registrada durante el ensayo en cada sensor. La localización de los sensores FBG fue presentada previamente en la Figura 5.8, y el procedimiento para calcular la fuerza axial en cada sensor FBG fue explicado en la Sección 5.2.2.3. Luego, la fuerza axial movilizada resultante en los tres niveles de ubicación de los sensores FBG se calculó como el promedio de las fuerzas registradas por los dos sensores ubicados en lados opuestos a la misma profundidad -e.g., la fuerza axial movilizada a $-40 \mathrm{~mm}$

$\left(F_{F B G_{1 / 4}}\right)$ es el promedio de la fuerza registrada por los sensores $F B G_{1}$ y $F B G_{4}-$. Además, debido a que en la punta de cada uno de los modelos de pilote de aluminio se adhirió una pieza de poliestireno blando, puede asumirse que sus puntas no movilizan resistencia.

La Figura 5.14 presenta la distribución de la fuerza axial movilizada a lo largo del pilote para diferentes valores de asientos medidos en cabeza del pilote (incluyendo el asiento pico, $\delta_{\text {pico }}$, asociado a la carga máxima registrada en la Figura 5.13). Notar, además, que debido a que el empotramiento del modelo del pilote en la pseudo-roca inicia a -20 mm de profundidad para todos los ensayos, la representación de la carga axial se realizó a partir de dicha profundidad (i.e., no hay transferencia de carga del pilote hacia la roca desde $0 \mathrm{~mm} \mathrm{a}-20 \mathrm{~mm}$ de profundidad por la no existencia de la misma, ver la Figura 5.11(d)). Como era de esperarse, se observa que: (i) la fuerza axial a lo largo del pilote, 
para cualquier asiento, disminuye con la profundidad, (ii) la carga axial a lo largo del pilote aumenta al aumentar la carga aplicada en cabeza, hasta que la carga axial pico es alcanzada y, (iii) el comportamiento tras alcanzar dicha carga (i.e., para $\delta>\delta_{\text {peak }}$ ) tiende a ser más dúctil en los pilotes más rugosos; esto es, los pilotes más rugosos mantienen un mayor porcentaje de su resistencia pico después de que se ha sobrepasado el asiento en cabeza asociado a su carga pico-.

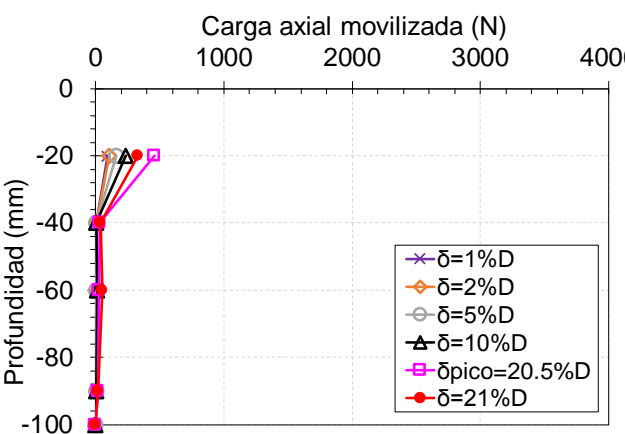

(a)

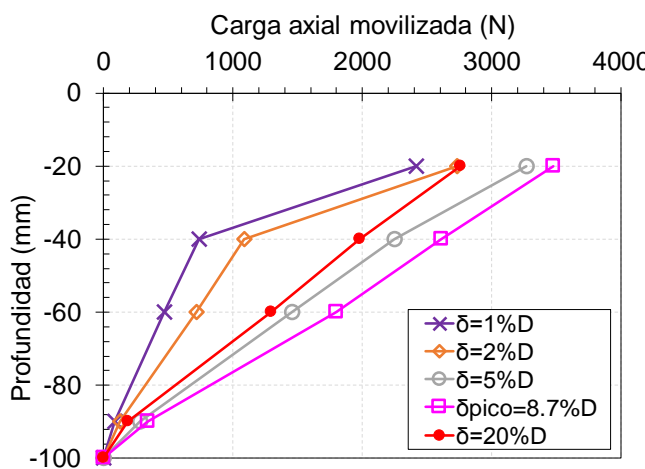

(c)

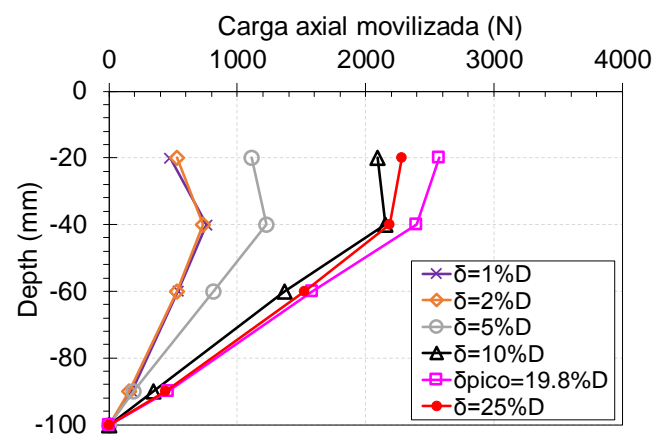

(b)

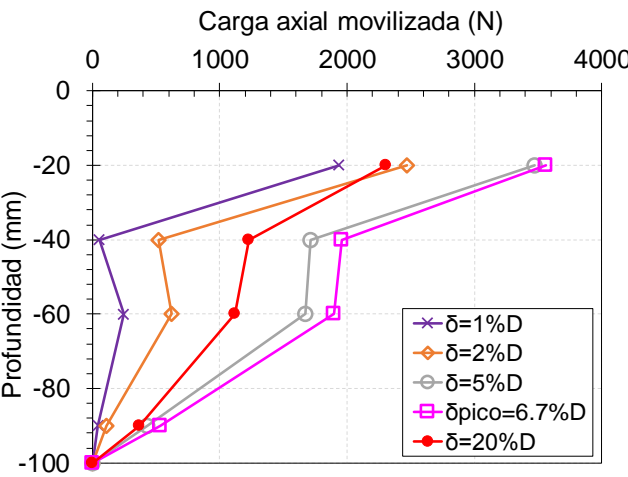

(d)

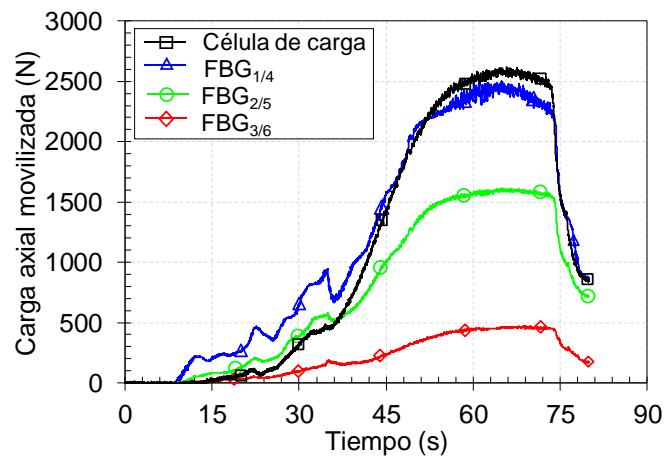

(e)

Figura 5.14. Distribución de la carga axial movilizada (en profundidad) para un asiento dado para los ensayos de centrifuga realizados sobre pilotes con diferente nivel de rugosidad: (a) $R F=0.000$, (b) $R F=0.025$, (c) $R F=0.050$, (d) $R F=0.106$; (d) carga axial movilizada vs tiempo, registrada por la célula de carga y por los sensores FBG para modelo de pilote con

$$
R F=0.025 \text {. }
$$


Además, en la Figura 5.14(b) se observa que la carga axial movilizada a una profundidad de $-40 \mathrm{~mm}$ es mayor que la carga axial aplicada en cabeza del modelo de pilote con $R F=0.025$. Este comportamiento no es real y se observa claramente cuando se analiza la evolución de la carga axial con el tiempo registrada por la célula de carga y por los sensores FBG (ver la Figura 5.14(d)). Como puede notarse, el promedio de la fuerza axial movilizada en los sensores FBG localizados a una profundidad de $-40 \mathrm{~mm}$ y de $-70 \mathrm{~mm}\left(F B G_{1 / 4}\right.$ y $F B G_{2 / 5}$, respectivamente, ver Figura 5.8(a)) es mayor a la carga registrada por la célula de carga para un tiempo menor a 33 segundos; esto puede atribuirse al hecho de que los factores de calibración $\left(K_{F B G_{i}}\right)$ de los sensores FBG (empleados para el computo de las fuerzas movilizadas durante el ensayo) se vieran afectados durante el proceso de 44 días de curado del modelo de centrífuga (ver la Figura 5.15)..

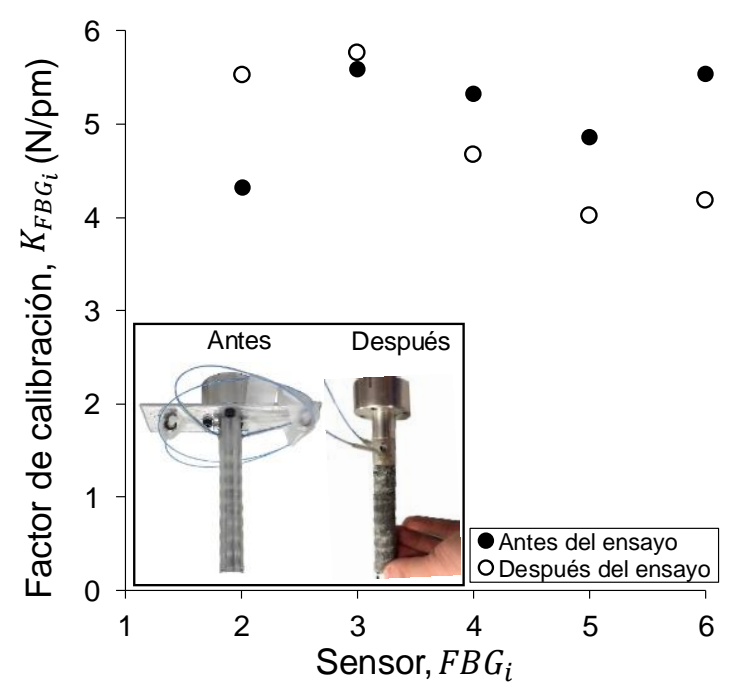

Figura 5.15. Variación del factor $K_{F B G_{i}}$ para el modelo de pilote con $R F=0.025$.

La Figura 5.15 muestra los factores $K_{F B G_{i}}$ obtenidos tras realizar la calibración (ver la Sección 5.2.2.3) de los sensores FBG, antes y después del ensayo de centrífuga sobre el modelo de pilote con $R F=0.025$. Estos resultados evidencian una variación de los factores $K_{F B G_{i}}$; en particular, se nota una disminución del factor $K_{F B G_{i}}$ en la mayoría de los sensores, y se cree que dicho comportamiento pueda deberse a una reacción química ocurrida entre el aluminio del pilote y la pseudo-roca circundante, alterando así 
la rigidez axial del pilote. En consecuencia, al emplear los factores $K_{F B G_{i}}$ hallados previo a la realización del ensayo de centrífuga produce la sobre-estimación del promedio de la fuerza axial movilizada presentada en la Figura 5.14(d).

\subsubsection{Resistencia por fuste}

La distribución de la resistencia promedio por fuste movilizada a lo largo del pilote (para un asiento determinado y entre puntos de referencia o medida) se determinó a partir de la diferencia de la fuerza axial entre dos puntos de referencia consecutivos, en los cuales se registró la fuerza axial durante la realización del ensayo de centrífuga. Para ello, se empleó la siguiente ecuación:

$$
f_{\text {ave }, \delta}=\frac{F_{i, \delta}-F_{i+1, \delta}}{\pi D L_{i \rightarrow i+1}}
$$

donde $f_{a v e, \delta}$ es la resistencia promedio por fuste (para un asiento determinado $\delta$, entre dos puntos de referencia consecutivos; $i$ e $i+1), F_{i, \delta}$ y $F_{i+1, \delta}$ son las fuerzas axiales registradas en dichos puntos consecutivos -e.g., en cabeza del pilote y a $40 \mathrm{~mm}$ por debajo de éste o donde se ubican los sensores $F B G_{1}$ y $F B G_{4}-$ para un asiento determinado; $D$ es el diámetro del pilote, y $L_{i \rightarrow i+1}$ es la longitud nominal entre los dos puntos consecutivos de referencia considerados.

En la Figura 5.16 se presenta la distribución de la resistencia promedio por fuste en profundidad, para un mismo porcentaje de asiento medido en cabeza del pilote (excepto para el valor pico) en todos los modelos ensayados. El valor pico representa la $f_{\text {ave }}$ calculada en el momento que se produce la máxima capacidad resistente del pilote, o para un asiento en cabeza $\left(\delta_{\text {peak }}\right)$ asociado al instante en que se alcanza la carga axial máxima de la Figura 5.13. (Notar que, debido a que el empotramiento del modelo del pilote en la pseudo-roca se inicia a $-20 \mathrm{~mm}$ de profundidad para todos los ensayos, no se muestra resistencia por fuste movilizada desde $0 \mathrm{~mm}$ hasta $-20 \mathrm{~mm}$ de profundidad). 


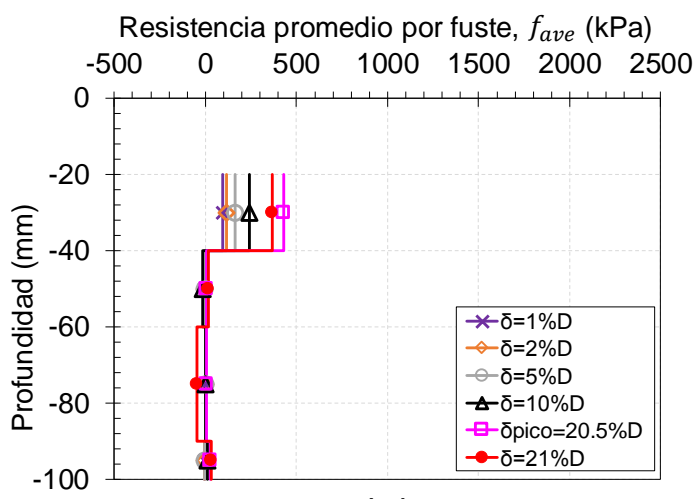

(a)

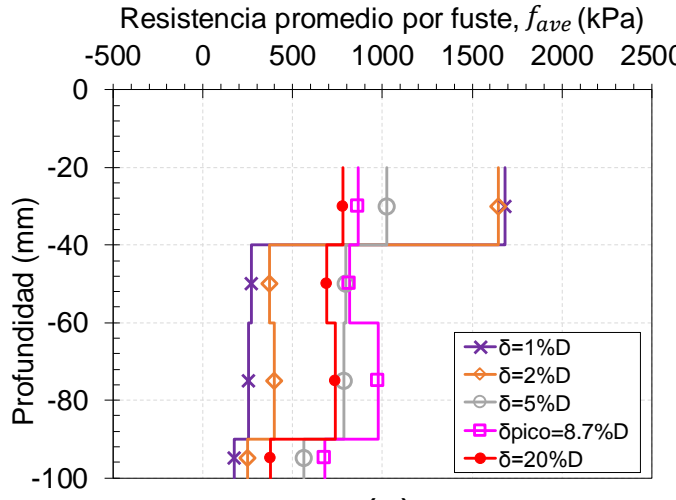

(c)

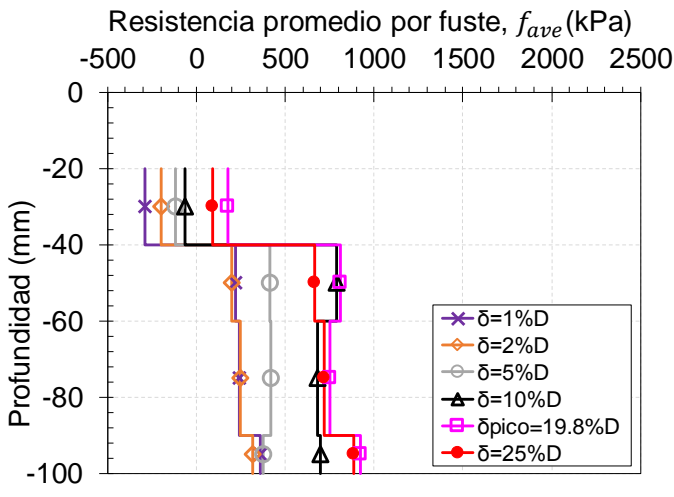

(b)

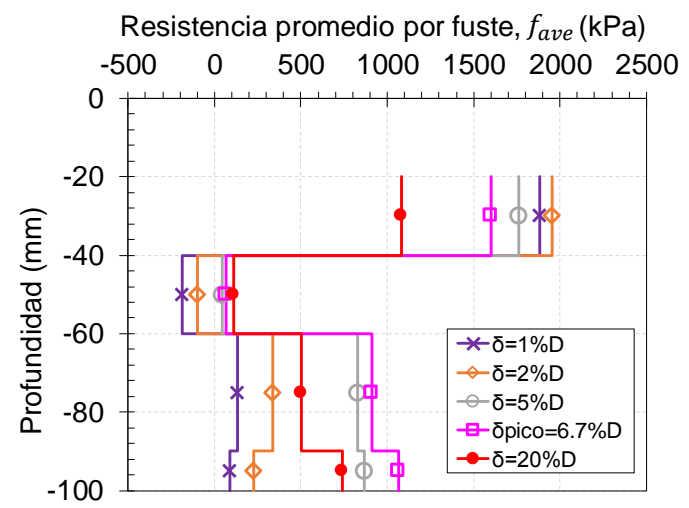

(d)

Figura 5.16. Resistencia promedio por fuste (vs profundidad) para todos los ensayos de centrífuga realizados sobre modelos de pilotes con diferente nivel de rugosidad en la interfaz roca-pilote: (a) $R F=0.000$, (b) $R F=0.025$, (c) $R F=0.050$, (d) $R F=0.106$.

Como puede observarse, la resistencia promedio por fuste en profundidad, para asientos en cabeza menores a $1 \% D$, es similar para todos los modelos (excepto para el modelo del pilote con $R F=0.025$ ): i.e., la resistencia promedio por fuste movilizada es mayor en el tramo cercano a la cabeza del pilote (desde $-20 \mathrm{~mm}$ hasta $-40 \mathrm{~mm}$ de profundidad) que hacia la punta del mismo. (Notar, además, que debido a la sobreestimación de la fuerza axial registradas por los sensores FBG a las profundidades de $-40 \mathrm{~mm}$ y $-70 \mathrm{~mm}$ (ver la sección 5.3.2), se produce resistencia por fuste negativa en el tramo de $0 \mathrm{~mm}$ a $-20 \mathrm{~mm}$ ). Sin embargo, a medida que se incrementa la carga axial durante el ensayo (i.e., para asientos en cabeza superiores a $1 \% D$ ), se produce una diminución de $f_{\text {ave }}$ en la parte superior del pilote (desde $-20 \mathrm{~mm}$ hasta $-40 \mathrm{~mm}$ en profundidad), así como un incremento de $f_{\text {ave }}$ en la porción restante del pilote (desde $-40 \mathrm{~mm}$ hasta $-100 \mathrm{~mm}$ en profundidad). Esta tendencia, que se aprecia mejor en los pilotes más rugosos $(R F=0.050$, y $R F=0.106$, ver la Figura 5.16(c)-(d)) que en los 
ligeramente lisos $(R F=0.000$, y $R F=0.025$, ver la Figura 5.16(c)-(d)), puede atribuirse al hecho que la $f_{a v e}$ es movilizada primero en las asperidades cercanas a la cabeza del pilote.

Este comportamiento también puede notarse cuando se analiza la evolución con el asiento de la resistencia promedio por fuste desarrollada a lo largo de diferentes segmentos del pilote; dicha evolución está referenciada al punto medio de cada segmento (e.g., en la mitad del segmento que separa los sensores $F B G_{1}$ y $F B G_{2}$ ) empleando para ello la relación normalizada $H / R$ (donde $H$ es la distancia media desde la placa de aluminio y $R$ es el radio del pilote, ver la Figura 5.18(a)). Por ejemplo, para los modelos con $R F=0.050$ y 0.106 de la Figura $5.18(\mathrm{c})$-(d), se observa que inicialmente (i.e., para asientos menores, aproximadamente, $3 \% D$ ), la $f_{\text {ave }}$ es movilizada por completo en el tramo superior del pilote (para $H / R=3.8$ ); luego, para los siguientes incrementos de carga (o para $\delta>3 \% D$ ), la $f_{\text {ave }}$ disminuye en este tramo del pilote y los valores máximos se desplazan hacia la parte inferior del pilote (i.e., para $H / R \geq 6$.3). Se cree que este comportamiento se debe a que durante los primeros pasos de carga, gran parte de la fuerza actuante sobre el pilote es transmitida al frente de las asperidades ubicadas en el tramo superior del pilote; luego, con el subsiguiente aumento de la carga actuante se produce rotura de las asperidades y un cambio de la rugosidad en la interfaz roca-pilote, en consecuencia, los valores de $f_{\text {ave }}$ se incrementan hacia el segmento inferior del pilote. Notar que estos valores de asientos son mucho mayores a los asientos asociados a un diseño apropiado para cargas en servicio (e.g., $\delta=1 \% D$, Whitaker y Cooke 1966). Estos resultados están en concordancia con la tendencia observada en las curvas de resistencia por fuste en profundidad reportadas por Pells et al. (1980) y Xing et al. (2017) y, además, son consistentes con el comportamiento de transferencia de carga de pilotes empotrados en roca reportado por Hassan y O’Neill (1997). 


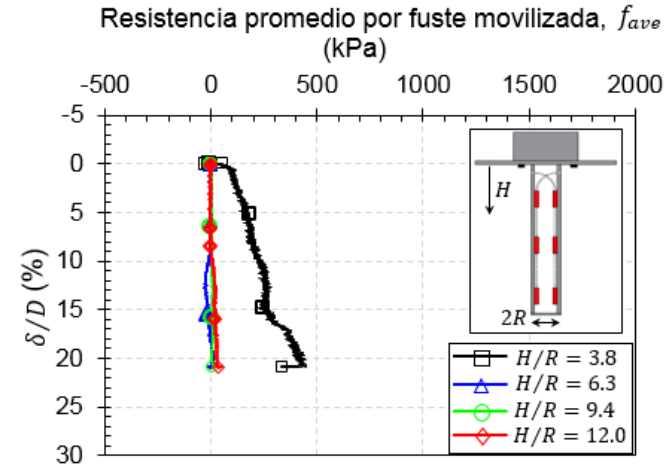

(a)

Resistencia promedio por fuste movilizada, $f_{\text {ave }}$

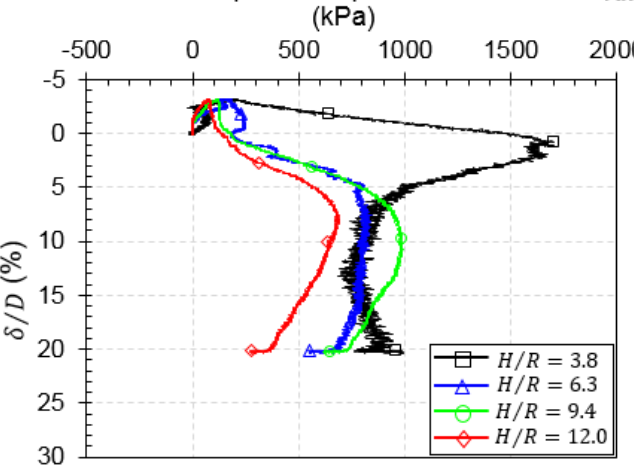

(c)
Resistencia promedio por fuste movilizada, $f_{\text {ave }}$ $(\mathrm{kPa})$

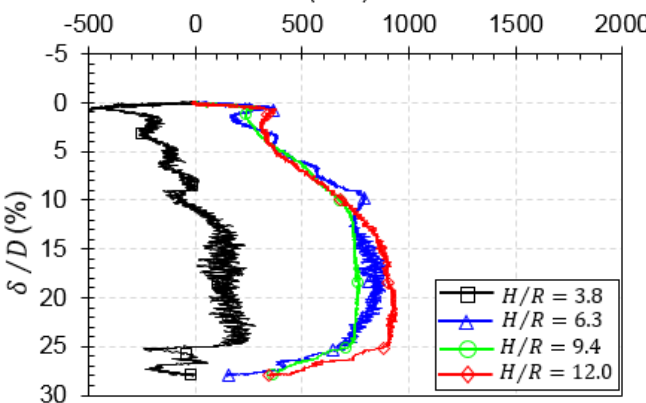

(b)

Resistencia promedio por fuste movilizada, $f_{\text {ave }}$ $(\mathrm{kPa})$

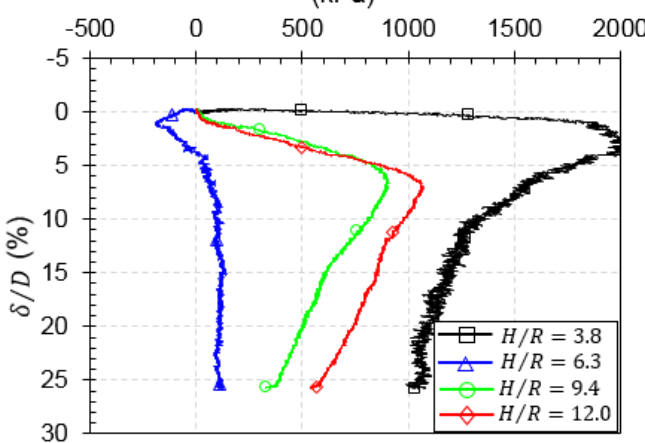

(d)

Figura 5.17. Resistencia promedio por fuste (vs asiento en cabeza del pilote) registrada durante todos los ensayos de centrífuga a diferentes profundidades: (a) $R F=0.000$, (b) $R F=0.025$, (c) $R F=0.050,(\mathrm{~d}) R F=0.106$.

Adicionalmente, en la Figura 5.18 se presenta la resistencia promedio por fuste (a lo largo de todo pilote) en función del asiento medido en la cabeza de los pilotes para todos los ensayos de centrífuga. Nuevamente, como ya fue comentado en la Sección 5.3.1, se evidencia la influencia de la rugosidad en la interfaz roca-pilote de modo que, por ejemplo, la $f_{\text {ave }}$ para el modelo con $R F=0.106$ es alrededor de tres veces mayor que la obtenida para el modelo con $R F=0.025$ para un asiento en cabeza de $\delta=5 \% D$, (ver la Figura 5.18). Estos resultados experimentales son cualitativamente consistentes con aquellos reportados por Seol y Jong (2007) y Gutiérrez-Ch et al. (2019b), quienes indicaron que la $f_{\text {ave }}$ movilizada en los pilotes más rugosos (i.e., los que tienen mayor $R F$ ) fue mucho mayor a la movilizada en los pilotes lisos debido que en los rugosos se produce una mayor dilatación y rigidez normal debido al esfuerzo actuante en la interfaz roca-pilote. 
Por otra parte, los resultados de los ensayos muestran un comportamiento elástico para asientos en cabeza menores a $1 \% D$. Una vez alcanzado y superado dicho valor, se observa un comportamiento plástico en las curvas (ver la Figura 5.18); este comportamiento es de gran relevancia y congruente con los resultados de Gutiérrez-Ch et al. $(2019 a, c)$ quienes, a partir de la propagación de micro-grietas en modelos numéricos simulados con el Método de Elementos Discretos, sugieren que el criterio de diseño de pilotes de considerar un asiento en cabeza de $1 \% D$ para cargas en servicio (e.g., Whitaker y Cooke 1966), es apropiado para evitar daños excesivos en la interfaz roca-hormigón de pilotes empotrados en roca.

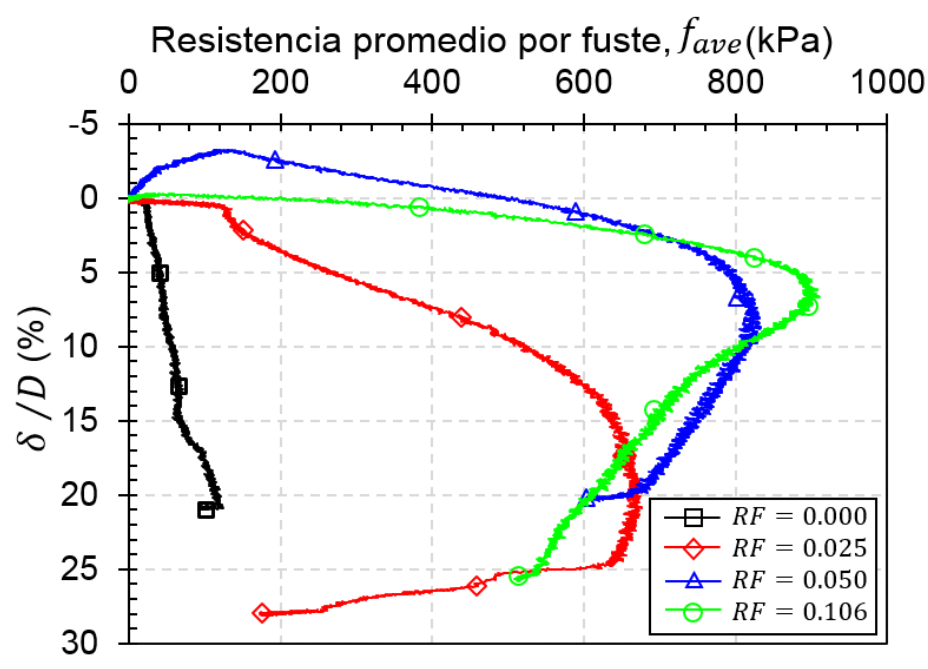

Figura 5.18. Resistencia promedio por fuste $\left(f_{\text {ave }}\right)$ versus el asiento en cabeza $(\delta)$ para los ensayos de centrífuga realizados sobre pilotes con diferente nivel de rugosidad.

\subsubsection{Comparación con modelos numéricos mediante el MEF}

Para comparar los resultados obtenidos en los ensayos de centrífuga, se realizó una simulación numérica mediante el Método de Elementos Finitos (MEF) con el código Abaqus (Smith 2009). En particular, se elaboró un modelo axisimétrico, con la misma geometría y condiciones de borde del modelo de pilote con $R F=0.106$, pero a escala del prototipo (usando las leyes de escalado, ver la Sección 2.4). El pilote fue modelado como elástico, con un módulo de Young de $E=30$ GPa y con un coeficiente de Poisson de $v=0.2$; de esta manera, el pilote tendría la misma rigidez axial que el pilote de 
aluminio a escala del modelo (i.e., a escala del ensayo de centrífuga). La pseudo-roca fue modelada empleando el criterio elasto-plástico perfecto de Mohr-Coulomb, con $E=$ $102 \mathrm{MPa}, v=0.27$, cohesión de $0.42 \mathrm{MPa}$ y ángulo de rozamiento de $\phi=31^{\circ}$ (estos parámetros fueron determinados, durante la fase experimental, para la pseudo-roca empleada para los ensayos de centrífuga, ver Anexo B). Además, se empleó el modelo de fricción básico de Coulomb para asignar un coeficiente de fricción en la interfaz rocapilote de 0.6, o similar al observado en los ensayos de corte directo roca-hormigón presentados en el Capítulo 4. El estado tensional in-situ se aplicó como condición inicial, seguido de la aplicación del paso geostático de Abaqus para que el código equilibre las cargas gravitatorias con el estado tensional in-situ (Smith 2009). Finalmente, el pilote fue cargado axialmente mediante un procedimiento de desplazamiento controlado, de tal forma que la carga aplicada y las condiciones de borde fueran equivalentes a las empleadas durante el ensayo de centrífuga.

En la Figura 5.19 se presenta la comparación de la resistencia promedio por fuste calculada con (i) el modelo del MEF descrito previamente, y (ii) los resultados del ensayo de centrífuga para el modelo de pilote con $R F=0.106$, para un asiento en cabeza de $0.01 D, 0.02 D$ y $0.05 D$. Como puede observarse, los resultados obtenidos con el MEF reproducen en forma adecuada el comportamiento observado en los resultados del ensayo de centrífuga: i.e., para asientos menores, el mayor valor de $f_{a v e}$ se moviliza cerca de la cabeza del pilote y, al aumentar la carga axial actuante en cabeza, el valor mayor de $f_{\text {ave }}$ comienza a desplazarse hacia la punta del pilote (ver la Figura 5.19(a)(d)). 


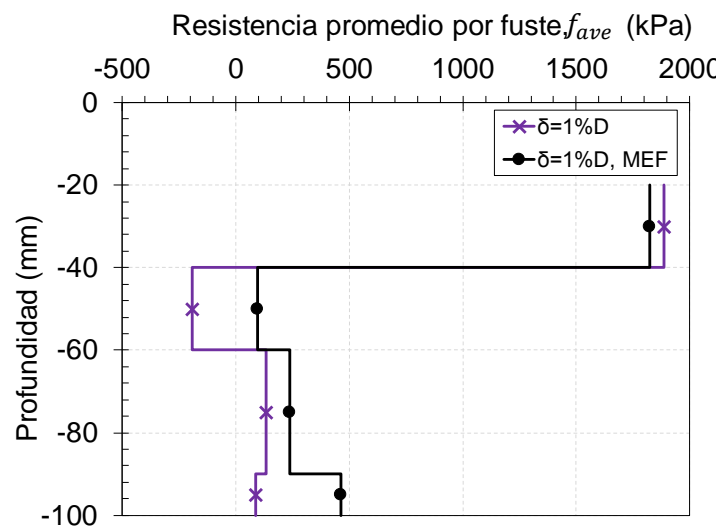

(a)

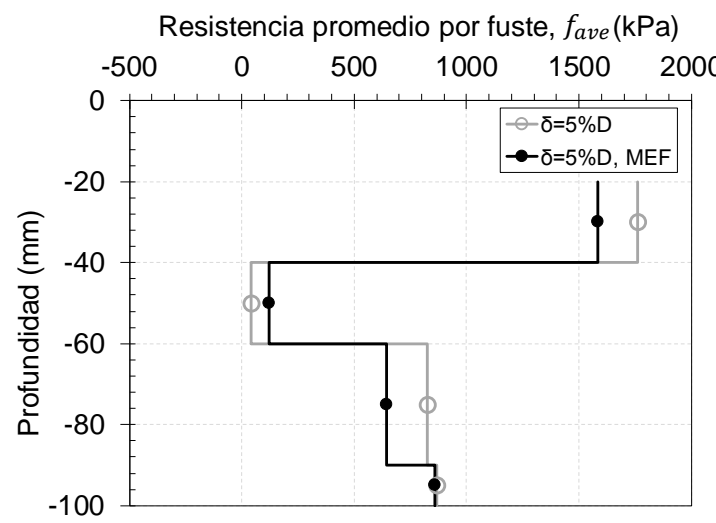

(c)

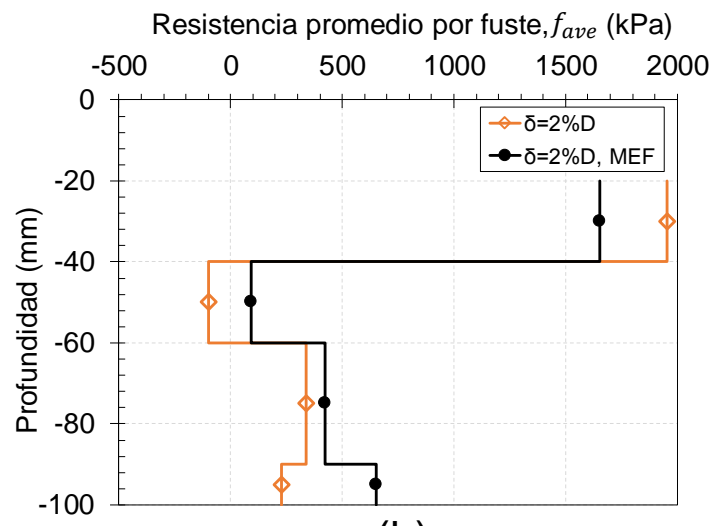

(b)

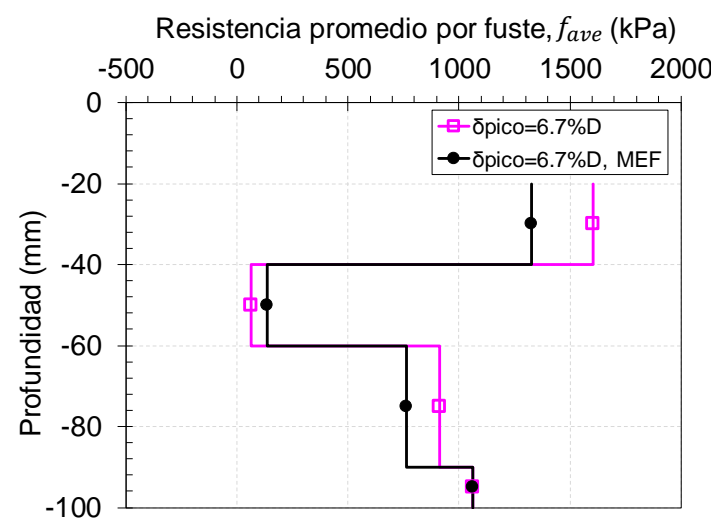

(d)

Figura 5.19. Comparación entre la distribución en profundidad de la resistencia promedio por fuste obtenida mediante el ensayo de centrífuga y el MEF para el modelo de pilote con $R F=$ $0.106: \delta=0.01 D$, (b) $\delta=0.02 D$, (c) $\delta=0.05 D$, (d) $\delta_{\text {pico }}=0.067 D$. 


\section{Formulación para la estimación de la resistencia por fuste de pilotes empotrados en roca considerando la rugosidad de la excavación}

\subsection{Introducción}

En este capítulo, se presenta un compendio de las diversas formulaciones publicadas en la literatura para la estimación, considerando la rugosidad del fuste, de la resistencia por fuste de pilotes empotrados en roca (ver la Sección 6.2), así como una comparación de las predicciones de dichas formulaciones con los resultados obtenidos mediante los modelos numéricos con PFC ${ }^{3 \mathrm{D}}$ (ver el Capítulo 4) y los ensayos de centrífuga (ver el Capítulo 5) con pilotes rugosos desarrollados en esta investigación (ver las Secciones 6.3 y 6.4). Seguidamente, se proponen curvas de diseño para estimar la resistencia por fuste de pilotes empotrados en roca, teniendo en cuenta su rugosidad. Luego, se realiza una validación de dichas curvas con ensayos de carga publicados en la literatura (ver Sección 6.5). Finalmente, se efectúa la aplicación de las curvas de diseño propuestas a nuevos casos de estudio (ver Sección 6.7).

\subsection{Formulaciones para el cálculo de la resistencia por fuste de pilotes empotrados en roca}

Usualmente, la estimación de la resistencia por fuste de pilotes empotrados en roca es realizada a través de (i) las normativas de cada país -e.g., en España se suele emplear el Código Técnico de la Edificación, etc.-, (ii) el conocimiento local obtenido a partir de pruebas de carga realizadas en rocas similares (Seidel y Collingwood 2001) y, (iii) correlaciones empíricas con la resistencia a compresión simple $\left(\sigma_{c}\right)$ del material intacto más débil (roca o pilote), cuya ecuación normalmente es de la forma (Rezazadeh y Eslani 2017):

$$
f_{\text {ave,peak }}[\mathrm{MPa}]=\alpha \sigma_{c}[\mathrm{MPa}]^{\beta}
$$


donde $f_{\text {ave,peak }}$ es la resistencia promedio pico por fuste (i.e., la mayor resistencia media desarrollada a lo largo de todo el fuste), $\alpha$ y $\beta$ son coeficientes empíricos que dependen del tipo de roca, y en algunos casos de la rugosidad (Seo et al. 2013). En la Tabla 6.1 se muestra el rango de valores que pueden tomar $\alpha$ y $\beta$ para la Ecuación 6.1. Adicionalmente, para aquellos casos en los que se disponía de dicha información en la referencia original, se incorporó a la Tabla 6.1 información sobre la altura promedio de las rugosidades de la excavación $\left(h_{m}\right)$ reportada en cada caso o inferida en función de la herramienta de perforación empleada y del tipo de roca (ver Tabla 6.2). Además, la Tabla 6.1 presenta también aquellas formulaciones que consideran la rugosidad de la excavación, según el sistema de clasificación de rugosidades propuesto por Pells et al (1980) (ver Capítulo 2, Tabla 2.1).

En la literatura existen también otro tipo de métodos para la estimación de la resistencia por fuste de pilotes empotrados en roca. Tal es el caso de la formulación analítica de Serrano et al. (2015), en la cual $f_{\text {ave,peak }}$ es estimada de la forma:

$$
\begin{gathered}
f_{\text {ave }, \text { peak }}=\beta_{a} \cdot \tau_{f m}{ }^{*} \\
\beta_{a}=\frac{m \cdot \sigma_{c, \text { roca }}}{s}
\end{gathered}
$$

donde $\beta_{a}$ es el módulo de resistencia de la forma paramétrica del criterio de rotura de Hoek y Brown expresado adimensionalmente según se indica en Serrano y Olalla (1994), $m$ y s son constantes que dependen del macizo rocoso y de su grado de fracturación (Hoek et al. 2002), $\sigma_{c, \text { roca }}$ es la resistencia a compresión simple de la roca intacta y $\tau_{f m}{ }^{*}$ es la resistencia promedio pico por fuste adimensional. Esta formulación es válida para pilotes “lisos” y asume que (i) sólo hay resistencia por fuste en la superficie del pilote empotrado en roca (no considera la contribución de $f_{\text {ave,peak }}$ de las capas de suelos que pudieran encontrarse por encima de la roca ni la posible contribución de la 
resistencia de la punta), (ii) la resistencia del material con que es fabricado el pilote es mayor a la de la roca.

Tabla 6.1. Coeficientes empíricos $\alpha$ y $\beta$ para la estimación de la resistencia por fuste promedio pico por fuste (ver Ecuación 6.1) (tomado principalmente de O’Neil et al. 1996 y Serrano et al. 2008, más algunos casos adicionales incorporados en esta investigación).

\begin{tabular}{|c|c|c|c|}
\hline Métodos de diseño & $\alpha$ & $\beta$ & $h_{m}{ }^{\mathrm{b}}(\mathrm{mm})$ \\
\hline Rosenberg y Journeaux (1976) & 1.11 & 0.51 & $\geq 35$ \\
\hline \multicolumn{4}{|l|}{ Horvath y Kenney (1979) } \\
\hline \multicolumn{4}{|l|}{ - Para pilotes de gran diámetro, $D \geq 410 \mathrm{~mm}$} \\
\hline a) Límite inferior & 0.20 & 0.50 & \\
\hline b) Límite superior & 0.25 & 0.50 & $\leq 10$ \\
\hline \multicolumn{4}{|l|}{ - Para pilotes de pequeño diámetro, $D<410 \mathrm{~mm}$} \\
\hline a) Límite inferior & 0.25 & 0.50 & \\
\hline b) Límite superior & 0.33 & 0.50 & \\
\hline Meigh y Wolski (1979) & 0.22 & 0.60 & $\leq 10$ \\
\hline Kaderbeck y Reynolds (1981) & 0.30 & 1.00 & - \\
\hline Williams et al. (1980) & 0.44 & 0.36 & $\leq 10$ \\
\hline Código de Pilotes Australiano (1980) & 0.15 & 1.00 & - \\
\hline \multicolumn{4}{|l|}{ Horvath et al. (1983) } \\
\hline a) Excavación lisa (valor inferior conservativo) & 0.20 & 0.50 & \multirow{2}{*}{$\leq 10$} \\
\hline b) Excavación rugosa (valor inferior conservativo) & 0.30 & 0.50 & \\
\hline Gupton y Logan (1984) & 0.20 & 1.00 & - \\
\hline \multicolumn{4}{|l|}{ Rowe y Armitage (1987) } \\
\hline a) Para R1, R2, $\mathrm{R}^{\mathrm{c}}, b=0.45$ & $0.70 b$ & 0.50 & \multirow{3}{*}{$\leq 70$} \\
\hline b) Para $\mathrm{R} 4^{\mathrm{c}}, b=0.60$ & $0.70 b$ & 0.50 & \\
\hline Toh et al. (1989) & 0.25 & 1.00 & \\
\hline \multicolumn{4}{|l|}{ Hooley y Lefroy (1993) } \\
\hline a) Para $0.25<\sigma_{c}$ & 0.30 & 1.00 & \multirow{3}{*}{$\leq 10$} \\
\hline b) Para $0.25 \leq \sigma_{c} \leq 3 \mathrm{MPa}$ & 0.15 & 0.50 & \\
\hline c) Para $\sigma_{c}>3 \mathrm{MPa}$ tomar $f_{\text {ave,peak }}=0.4 \mathrm{MPa}$ & - & - & \\
\hline \multicolumn{3}{|l|}{ Kulhawy y Phoon (1993) } & - \\
\hline a) Límite inferior & 1.34 & 0.50 & \multirow{2}{*}{ - } \\
\hline b) Límite superior & 0.22 & 0.50 & \\
\hline \multicolumn{4}{|l|}{ Carrubba (1997) } \\
\hline a) Límite inferior & 0.13 & 0.50 & \multirow[b]{2}{*}{$\leq 6$} \\
\hline b) Límite superior & 0.25 & 0.50 & \\
\hline \multicolumn{4}{|l|}{ Zhang y Einstein (1998) } \\
\hline a) Para $R 1, R 2, R 3^{c}$ & 0.40 & 0.50 & \\
\hline b) Para R4c & 0.80 & 0.50 & \\
\hline \multicolumn{4}{|c|}{$\begin{array}{l}\text { O'Neill y Reese }(1999)^{\mathrm{a}} \text {, Manual de Ingeniería de Cimentaciones } \\
\text { de Canada }(2006)^{\mathrm{a}} \text { y American Association of State Highway } \\
\text { and Transportation Officials AASHTO }(2008)^{\mathrm{a}}\end{array}$} \\
\hline a) Excavación lisa & 0.20 & 0.50 & \multirow{3}{*}{$\leq 20$} \\
\hline b) Excavación rugosa & 0.30 & 0.50 & \\
\hline c) Excavación muy rugosas (i.e., $h_{m} \geq 10 \mathrm{~mm}$ ) & 0.60 & 0.50 & \\
\hline Código Técnico de la Edificación, CTE (2006) & 0.20 & 0.50 & - \\
\hline Basarkar y Dewaikar (2006)a & 0.40 & 0.50 & - \\
\hline Sagong et al. (2007) $)^{a}$ & 0.38 & 0.51 & $\leq 6$ \\
\hline Rezazadeh y Eslami (2017)a & 0.36 & 0.36 & $\leq 10$ \\
\hline Arioglu et al. (2018)a & 0.36 & 0.40 & $\leq 10$ \\
\hline
\end{tabular}

a Esta correlación ha sido incorporada en esta investigación.

${ }^{\mathrm{b}}$ Esta información fue inferida desde la referencia original.

c Sistema de clasificación de rugosidades propuesto por Pells et al. (1980), ver Capítulo 2, Tabla 2.1. 
Más recientemente, Kulkarni y Dewaikar (2016) propusieron una formulación empírica como función de la resistencia a compresión simple promedio del macizo rocoso $\left(\sigma_{c m}\right)$ en lugar de la roca intacta $\left(\sigma_{c, \text { roca }}\right)$, e incorporando el factor $R Q D$ (Rock Quality Designation) del macizo de la forma:

$$
\begin{gathered}
f_{\text {ave }, \text { peak }}[\mathrm{MPa}]=0.2 \sigma_{c m}[\mathrm{MPa}]^{\beta} \\
\frac{\sigma_{c m}}{\sigma_{c, \text { roca }}}=10^{0.013 R Q D-1.34}
\end{gathered}
$$

Como ya se ha discutido esta tesis doctoral pretende incorporar la influencia de la rugosidad en la estimación de la resistencia por fuste de pilotes empotrados en roca. En este sentido, ante la evidencia registrada sobre dicha influencia, se han realizado algunos esfuerzos para tratar de incorporar este aspecto al diseño de este tipo de cimentación profunda. Tal es el caso de las curvas de diseño propuestas por Pells et al. (1980) y Horvath et al. (1983) (ver Figura 6.1) a partir de ensayos de laboratorio e in-situ (ver Capítulo 2, Secciones 2.2.3.1, 2.3.1.1, 2.3.1.3).

Posteriormente, partiendo de los ensayos realizados por Williams (1980) (ver Capítulo 2, Sección 2.2.1.2), Kodikara et al. (1992) propusieron una serie de curvas de diseño para la estimación de la resistencia por fuste de pilotes empotrados en roca (ver Figura 6.2) incorporando no sólo la rugosidad de la excavación -cuantificada según tres categorías: liso $\left(h_{m}=1-4 \mathrm{~mm}\right)$, intermedio $\left(h_{m}=4-20 \mathrm{~mm}\right)$ y rugoso $\left(h_{m}=20-80\right.$ $\mathrm{mm})$-, sino también el módulo de Young del macizo rocoso $\left(E_{m}\right)$ y el esfuerzo normal actuante en la interfaz roca-pilote $\left(\sigma_{n}\right)$.

De igual forma, Seidel y Collingwood (2001) propusieron un factor adimensional, el llamado "factor de resistencia por fuste" $(S R C)$, para la estimación de la resistencia por fuste de pilotes empotrados en roca. El $S R C$ fue formulado a partir de un estudio paramétrico realizado con el programa Rocket -desarrollado por Seidel (1993)- y empleando datos de ensayos de carga publicados previamente en la literatura. El $S R C$ 
considera el método constructivo empleado durante la perforación $\left(\eta_{c}\right)$, la relación entre el módulo de Young del macizo rocoso y la resistencia a compresión simple de la roca intacta $\left(n=E_{m} / \sigma_{c, \text { roca }}\right)$, el coeficiente de Poisson $(v)$, la altura promedio de las asperidades $\left(h_{m}\right)$ y el diámetro del pilote o excavación $(D)$, y se calcula según la siguiente formulación (para más detalles, véase Seidel y Collingwood (2001)):

$$
S R C=\eta_{c} \frac{n h_{m}}{(1+v) D}
$$

Una vez hallado el factor $S R C$, la resistencia por fuste es calculada a partir de la Figura 6.3.

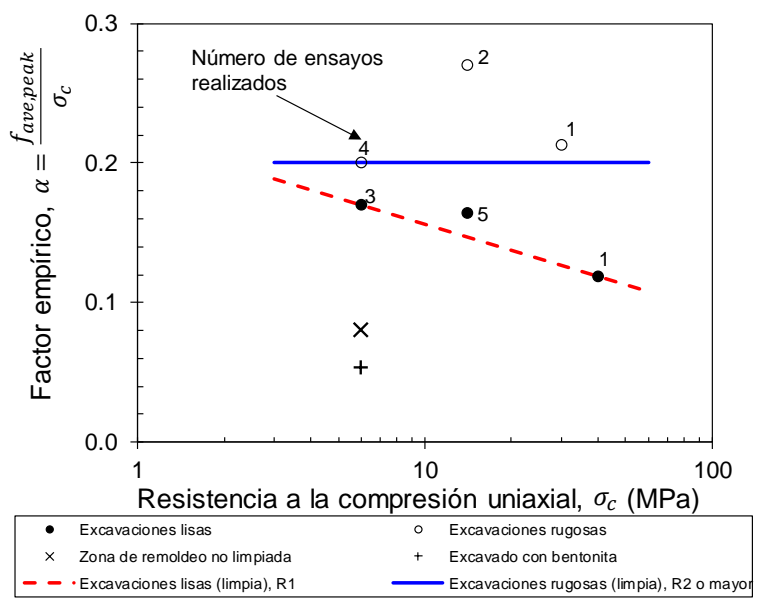

(a)

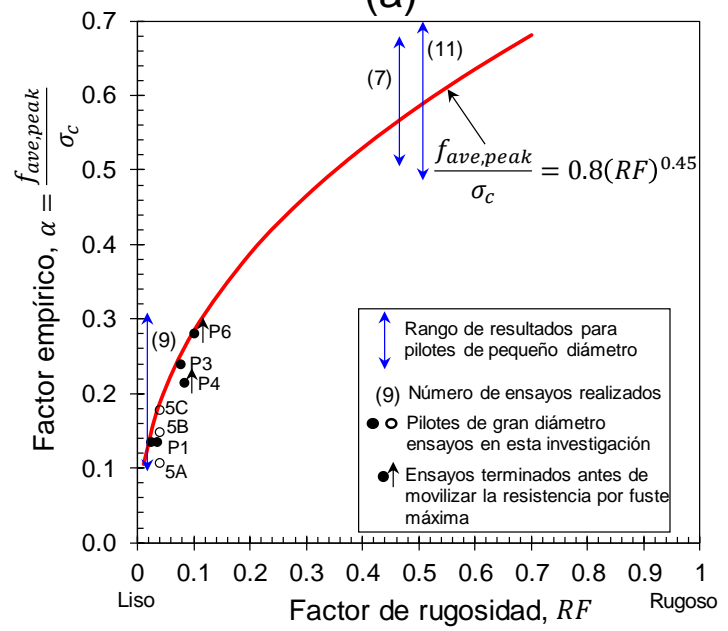

(b)

Figura 6.1. Curvas para la estimación de la resistencia media por fuste de pilotes empotrados en roca, considerando la rugosidad de la excavación: (a) modificado de Pells et al. (1980), (b) modificado de Horvath et al (1983). 

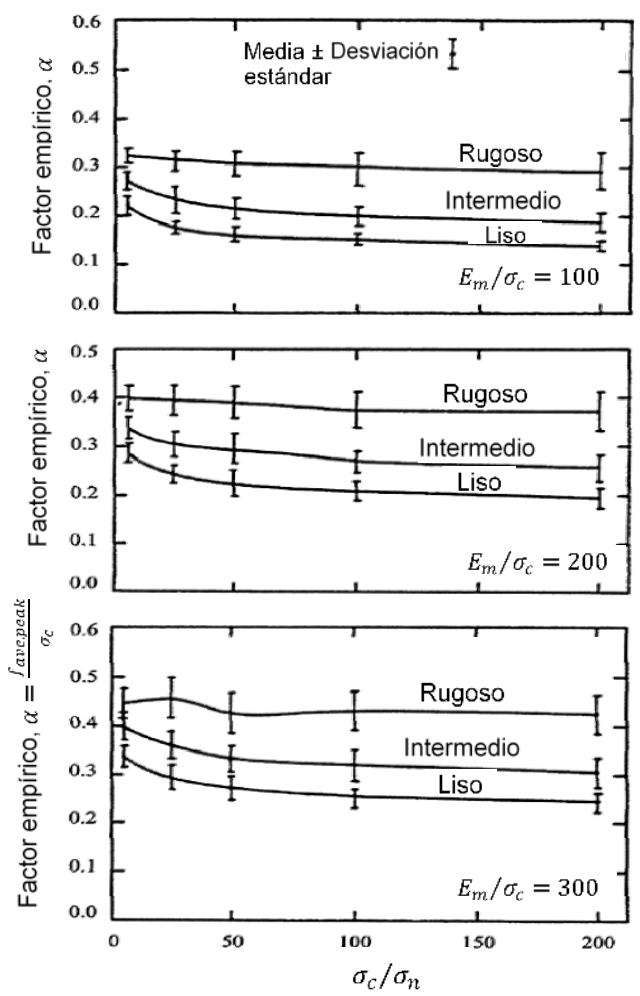

Figura 6.2. Curvas para la estimación de la resistencia por fuste de pilotes empotrados en roca propuesta por Kodikara et al. (1992).

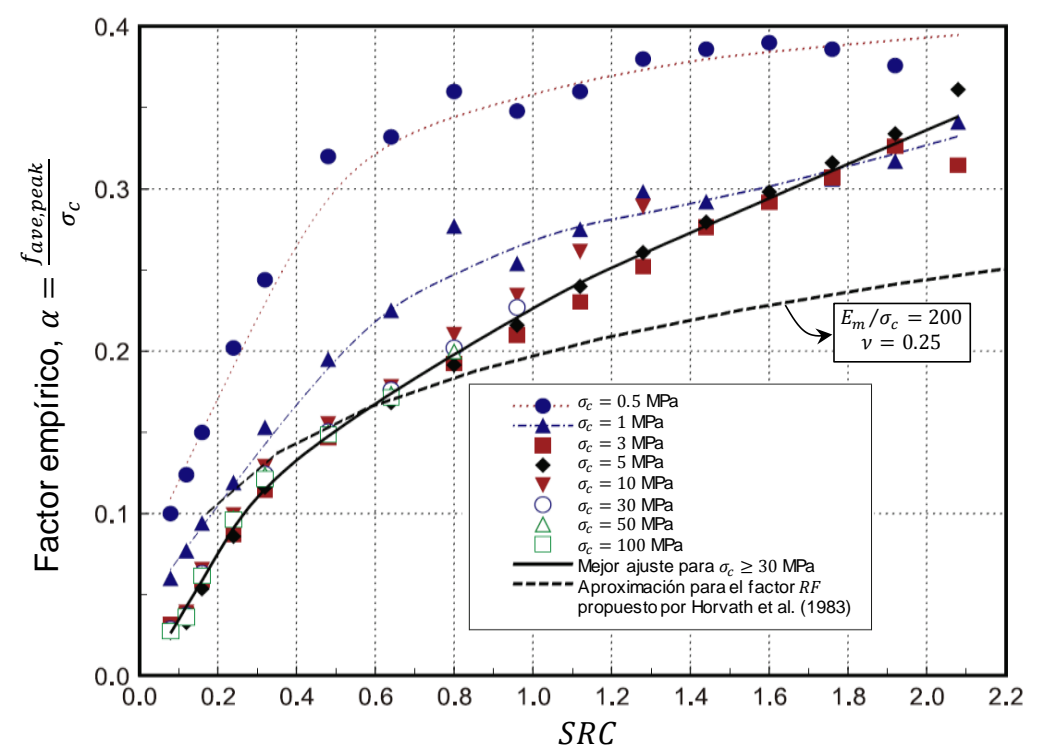

Figura 6.3. Curvas para la estimación de la resistencia por fuste de pilotes empotrados en roca propuesta por Seidel y Collingwood (2001).

\subsection{Comparación con formulaciones de diseño}

Esta sección compara los resultados calculados mediante algunas de las formulaciones presentadas en la Sección 6.2 con los resultados obtenidos mediante modelos numéricos con $\mathrm{PFC}^{3 \mathrm{D}}$ (ver Capítulo 4) y con ensayos de centrífuga (ver Capítulo 5). En 
particular, se comparan las estimaciones de resistencia por fuste calculadas para un asiento de $1 \% D$, a las que se denota como $f_{a v e, 0.01 D}$; el motivo es que, en la mayoría de los métodos empíricos discutidos anteriormente, las estimaciones de resistencia por fuste $\left(f_{\text {ave,peak }}\right)$ también se desarrollaron teniendo como referencia un asiento en cabeza del pilote de $1 \% D$.

Para ello, en primer lugar, se ha realizado un análisis del rango de altura de rugosidades esperadas en la ejecución de pilotes reales empotrados en roca. Los resultados de este análisis se presentan de forma resumida en la Tabla 6.2, evidenciando tal y como fue reportado en trabajos previos (ver e.g., Seidel y Collingwood 2001), que la rugosidad de la pared de la perforación depende principalmente de la herramienta de perforación empleada, así como del tipo y resistencia de la roca. En este sentido, se observa que las excavaciones realizadas con herramientas de perforación estándar, en rocas de resistencia baja a mediana, tienden a presentar excavaciones con paredes relativamente lisas, salvo que la roca se encuentre altamente fracturada o se empleen herramientas de perforación especiales para incrementar su rugosidad.

Las Figuras 6.4 y 6.5 comparan (i) los resultados de algunas de las formulaciones empíricas y analíticas discutidas con (ii) los resultados obtenidos en los modelos con $\mathrm{PFC}^{3 \mathrm{D}}$ de pilotes empotrados en la arenisca-S3. Resulta oportuno señalar que los métodos en los que los autores especifican una condición limitante en el uso de la formulación empleada -e.g., uso para pilote liso o pilote rugoso únicamente o, como en el caso de la formulación de Seidel y Collingwood, la condición de $S R C \leq 2.1$ - quedaron fuera de la comparación. Además, se señalaron en las Figuras 6.4 a la 6.9 con color rojo los resultados de los modelos en los que los valores de las alturas promedios de rugosidades (o su valor $R F$ ) son similares a las alturas de rugosidades que tenían los pilotes cuyos ensayos de carga fueron empleados para el desarrollo de cada método (ver la Tabla 6.1). 

considerando la rugosidad de la excavación

Tabla 6.2. Altura de rugosidades obtenidas en las excavaciones de pilotes empotrados en roca con diferentes herramientas de perforación (ver el Anexo A) (Gutiérrez-Ch et al. 2020b)

\begin{tabular}{|c|c|c|c|c|}
\hline Herramienta de perforación & $h_{m},(\mathrm{~mm})$ & Tipo de roca & $\sigma_{c, \text { roca }}(\mathrm{MPa})$ & $\begin{array}{c}\text { Referencia del ensayo de } \\
\text { carga }\end{array}$ \\
\hline Barrenab $^{b}$ & $75^{c}$ & Lutita & 0.48 & Matich y Kozicki 1967 \\
\hline Diamond tube & $<4^{\mathrm{e}}$ & Arenisca & 14 & Pells et al. 1980 \\
\hline Diamond tube & $<1^{\mathrm{e}}$ & Arenisca & 6 & Pells et al. 1980 \\
\hline Rock roller & $<4^{\mathrm{e}}$ & Arenisca & 6 & Pells et al. 1980 \\
\hline Rock roller & $<1^{\mathrm{e}}$ & Arenisca & 30 & Pells et al. 1980 \\
\hline Barrena & $<10^{\mathrm{e}}$ & Arenisca & 6 & Pells et al. 1980 \\
\hline Barrena 3 flight & $5.7^{d}$ & Lutita & 0.83 & Williams 1980 \\
\hline Barrena 3 flight $^{b}$ & $12.2^{d}$ & Lutita & 0.55 & Williams 1980 \\
\hline Barrena 3 flight & $4.8^{\mathrm{d}}$ & Lutita & 0.59 & Williams 1980 \\
\hline Casing barre/ ${ }^{b}$ & $10^{d}$ & Lutita & 0.58 & Williams 1980 \\
\hline Barrena Bucket & $7.3^{d}$ & Lutita & 2.46 & Williams 1980 \\
\hline Barrena Bucket ${ }^{b}$ & $17.3^{d}$ & Lutita & 2.3 & Williams 1980 \\
\hline Barrena Bucket & $5.9^{d}$ & Lutita & 2.3 & Williams 1980 \\
\hline Barrena Bucket & $5.2^{d}$ & Lutita & 3.06 & Williams 1980 \\
\hline Barrena & $12.7^{c}$ & Lutita & $5.4,5.6,11.1$ & Horvath et al. 1983 \\
\hline 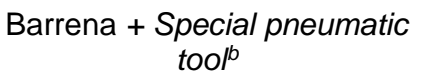 & $24.1^{c}$ & Lutita & 5.5 & Horvath et al. 1983 \\
\hline $\begin{array}{c}\text { Barrena }+ \text { Special pneumatic } \\
\text { tool }\end{array}$ & $24.1^{c}$ & Lutita & 10.4 & Horvath et al. 1983 \\
\hline Barrena reaming toola & $31.5^{c}$ & Lutita & 6.75 & Horvath et al. 1983 \\
\hline Barrenab & $76^{c}$ & Arenisca & $8.36-9.26$ & Gloss y Briggs 1983 \\
\hline Barrena & $<12.5^{\mathrm{c}}$ & Lutita & $0.5-5$ & O'Neil et al. 1996 \\
\hline $\begin{array}{l}\text { Barrena + "diente" de corte } \\
\text { ranurado }\end{array}$ & $11.1^{\mathrm{d}}$ & Limolita & $3.3-7.2$ & Baycan 1996 \\
\hline $\begin{array}{l}\text { Barrena + "diente" de corte } \\
\text { ranuradoa }\end{array}$ & $8.2^{d}$ & Limolita & $3.3-7.2$ & Baycan 1996 \\
\hline $\begin{array}{c}\text { Downhole jacket }+ \\
\text { pneumatic grooving toola }\end{array}$ & $100^{c}$ & Lodolita & 3.2 & Walter et al. 1997 \\
\hline $\begin{array}{c}\text { Downhole jacket }+ \\
\text { pneumatic grooving tool }\end{array}$ & $100^{\mathrm{c}}$ & Limolita & 8.9 & Walter et al. 1997 \\
\hline $\begin{array}{c}\text { Downhole jacket }+ \\
\text { pneumatic grooving toola }\end{array}$ & $100^{c}$ & Arenisca & 11.6 & Walter et al. 1997 \\
\hline Barrena & $10.2^{d}$ & Arenisca & - & Collingwood 2000 \\
\hline $\begin{array}{c}\text { Barrena flight }+ \text { herramienta } \\
\text { de corte circunferencial } \\
(\text { WRT1) }\end{array}$ & $7.1^{d}$ & Lutita & $7.5-20$ & Collingwood 2000 \\
\hline $\begin{array}{c}\text { Barrena flight + herramienta } \\
\text { de corte circunferencial } \\
(\text { WRT1) }\end{array}$ & $9^{d}$ & Lutita & $1.5-7.5$ & Collingwood 2000 \\
\hline $\begin{array}{c}\text { Barrena flight + herramienta } \\
\text { de corte circunferencial } \\
(\text { WRT1) }\end{array}$ & $16.1^{d}$ & Limolita & $0.8-1.35$ & Collingwood 2000 \\
\hline Barrena & $6^{d}$ & Arenisca & 6 & Collingwood 2000 \\
\hline Barrena & $7^{d}$ & Conglomerado & 6 & Collingwood 2000 \\
\hline Core barrel & $2.8^{\mathrm{d}}$ & Basalto & $50-75$ & Collingwood 2000 \\
\hline Core barrel & $4.1^{d}$ & Basalto & $75-100$ & Collingwood 2000 \\
\hline Barrena & $2.5^{\mathrm{c}}$ & Lutita & 1.2 & Nam y Vipulanandan 2008 \\
\hline Core barrel & $3.7^{\mathrm{c}}$ & Lutita & 1.2 & Nam y Vipulanandan 2008 \\
\hline Barrena & $1.6-2.6^{\mathrm{c}}$ & Lutita & 6 & Nam y Vipulanandan 2008 \\
\hline Core barrel & $4.2^{\mathrm{c}}$ & Lutita & 2.1 & Nam y Vipulanandan 2008 \\
\hline Barrena & $2.2^{\mathrm{c}}$ & Caliza & 10 & Nam y Vipulanandan 2008 \\
\hline Core barrel & $3.5^{c}$ & Caliza & 10 & Nam y Vipulanandan 2008 \\
\hline$R C D$ & $1-5.1^{\mathrm{c}}$ & Granito & $100-150$ & Jeong et al. 2010 \\
\hline$R C D$ & $1-4^{c}$ & Gneis & $30-130$ & Jeong et al. 2010 \\
\hline Core Bit & $1-7^{c}$ & Gneis & $5-50$ & Jeong et al. 2010 \\
\hline
\end{tabular}

${ }^{a}$ Método para generar mayor rugosidad en forma artificial.

${ }^{b}$ Posteriormente se incrementó la rugosidad en la excavación (no se indica la herramienta empleada para ello).

c Proporcionada en la referencia original del ensayo de carga.

${ }^{d}$ Calculada a partir de los perfiles de rugosidad presentados en la referencia original del ensayo de carga.

e Estimada usando el sistema de clasificación de rugosidades de Pells et al. (1980), ver Capítulo 2, Tabla 2.1. 
Más en concreto, la Figura 6.4 compara (i) la resistencia promedio por fuste para un asiento en cabeza del pilote de $1 \% D$ calculada en los modelos con $\mathrm{PFC}^{3 \mathrm{D}}$ y (ii) su valor estimado con las formulaciones empíricas y analíticas en las que no se considera la rugosidad de interfaz roca-pilote. Por tanto, se observa que la $f_{a v e, 0.01 D}$ alcanza el mismo valor (según la formulación empleada) y por supuesto sin importar el valor de $R F$ para todos los modelos. Por otra parte, los resultados en rojo sugieren que, a pesar del cierto grado de incertidumbre que pueda existir, los modelos con $\mathrm{PFC}^{3 \mathrm{D}}$ son generalmente capaces de reproducir las predicciones de cada método de cálculo, una vez que se considera el rango de altura de rugosidades (o su valor $R F$ ) con las que cada método fue formulado. Por ejemplo, el resultado con la formulación de Serrano et al. (2015) coincide con el valor de $f_{a v e, 0.01 D}$ para el modelo con $\mathrm{PFC}^{3 \mathrm{D}}$ y $R F=0.010$, el cual tiene un valor de $h_{m}=4 \mathrm{~mm}$ que, a su vez, estaría dentro la gama de pilotes reales "lisos" (ver Tabla 6.2).

De igual forma, las estimaciones de $f_{a v e, 0.01 D}$ con el método de Rosenberg y Journeaux (1976), arrojaron valores cercanos a los valores calculados a partir de los modelos con $\mathrm{PFC}^{3 \mathrm{D}}$ y $R F=0.106$. Esto, probablemente, se atribuye al hecho de que (i) el método de Rosenberg y Journeaux (1976) se formuló empleando resultados de ensayos de carga sobre pilotes excavados en roca, a cuyas paredes de excavación se les incrementó artificialmente su grado de rugosidad, según lo reportado por Matich y Kozicki (1967), así como también, de que (ii) la roca donde se realizaron estos ensayos presentaba un alto grado de fracturación, y en consecuencia, es posible que resultaran paredes muy rugosas tras la perforación del pilote (ver la Figura 6.4).

Las estimaciones realizadas con la formulación de Williams et al. (1980) son muy similares a las calculadas para los modelos con $\mathrm{PFC}^{3 \mathrm{D}}$ e interfaz relativamente lisa (ver la Figura 6.4); este comportamiento está en concordancia con lo presentado en la Tabla 6.2, según la cual Williams et al. (1980) se basaron en resultados de pilotes empotrados 
principalmente en lutitas blandas, cuyas alturas de rugosidad son pequeñas (a menos que se incremente artificialmente). De manera similar, la concordancia con la formulación de Arioglu et al. (2018) también es buena; aunque estos autores no proporcionan detalles sobre las herramientas de perforación empleadas, la información del tipo y resistencia de la roca en la que se ejecutaron los pilotes ensayados, sugiere que probablemente las paredes de excavación presentaron $h_{m} \leq 10 \mathrm{~mm}$ para las herramientas de perforación estándar empleadas (ver la Tabla 6.2).
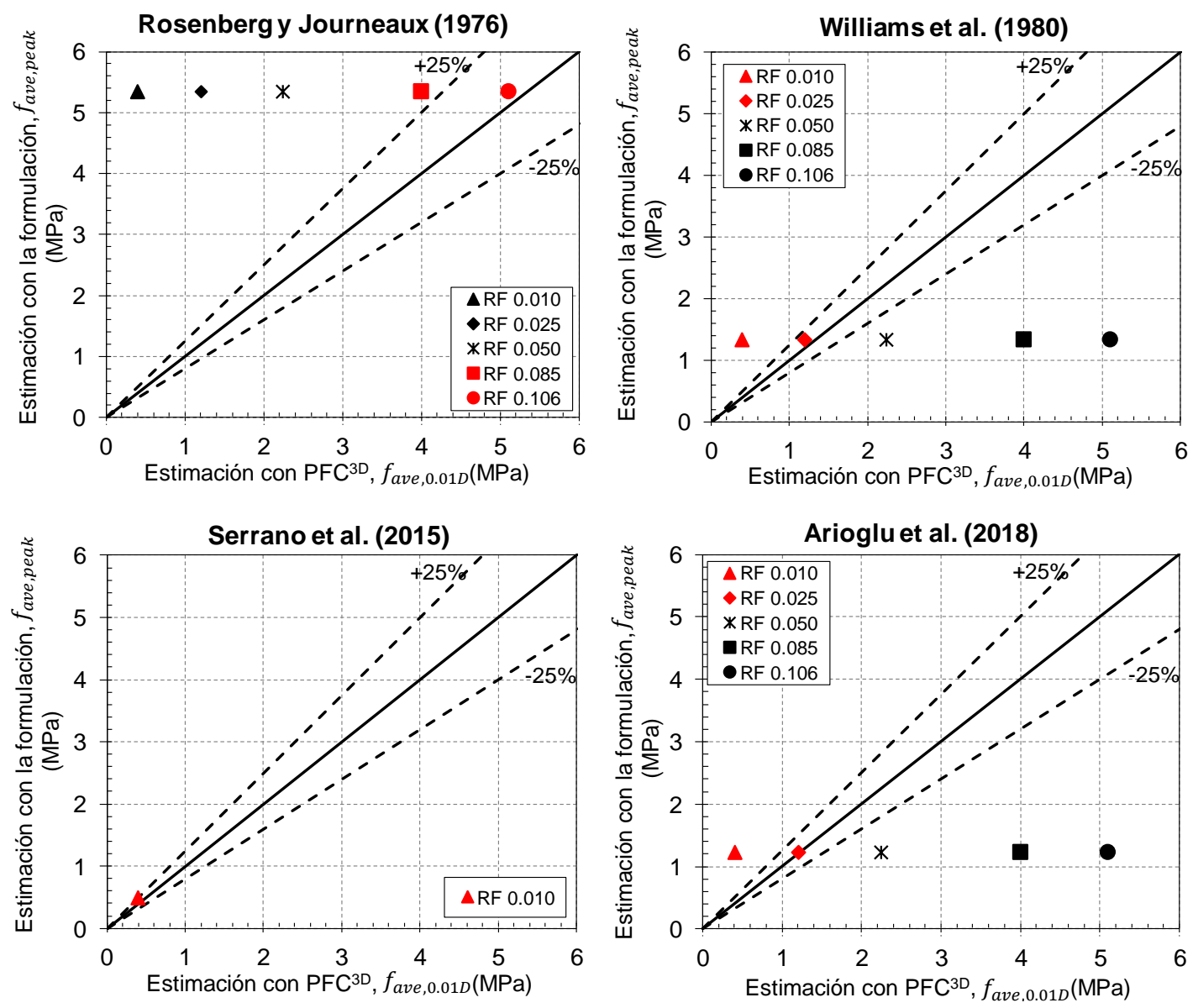

Figura 6.4. Estimación de la resistencia por fuste para los pilotes empotrados en la areniscaS3: comparación entre los resultados numéricos con $\mathrm{PFC}^{3 \mathrm{D}}$ vs las formulaciones analíticas y empíricas que no consideran la rugosidad de la interfaz roca-pilote.

Por otra parte, dentro de las formulaciones que consideran específicamente la rugosidad de la excavación, la formulación de Horvath y Kenny (1979) arrojó valores muy cercanos a los obtenidos para el modelo con $\mathrm{MED}^{3 \mathrm{D}}$ y $R F=0.025$ (ver la Figura 6.5). Esto puede deberse a que Horvath y Kenny (1979) indicaron en su investigación que "los ensayos 
de carga se realizaron sobre pilotes perforados en roca mediante herramientas convencionales", lo cual permite inferir que las alturas promedio de rugosidades en la interfaz roca-pilote probablemente resultaron menores a $10 \mathrm{~mm}$. En el caso de la formulación de Rowe y Armitage (1987), los resultados arrojaron valores similares a los obtenidos para las excavaciones relativamente lisas de los modelos con $\mathrm{MED}^{3 \mathrm{D}}$ y $R F \leq$ 0.050 (ver la Figura 6.5); mientras que para las excavaciones muy rugosas son significativamente más altos que los hallados con la formulación empírica de Rowe y Armitage (1987). Sin embargo, este comportamiento era de esperar, debido a que estos autores indicaron que "[su formulación] podría arrojar valores muy conservadores para excavaciones cuya rugosidad sea incrementada artificialmente".
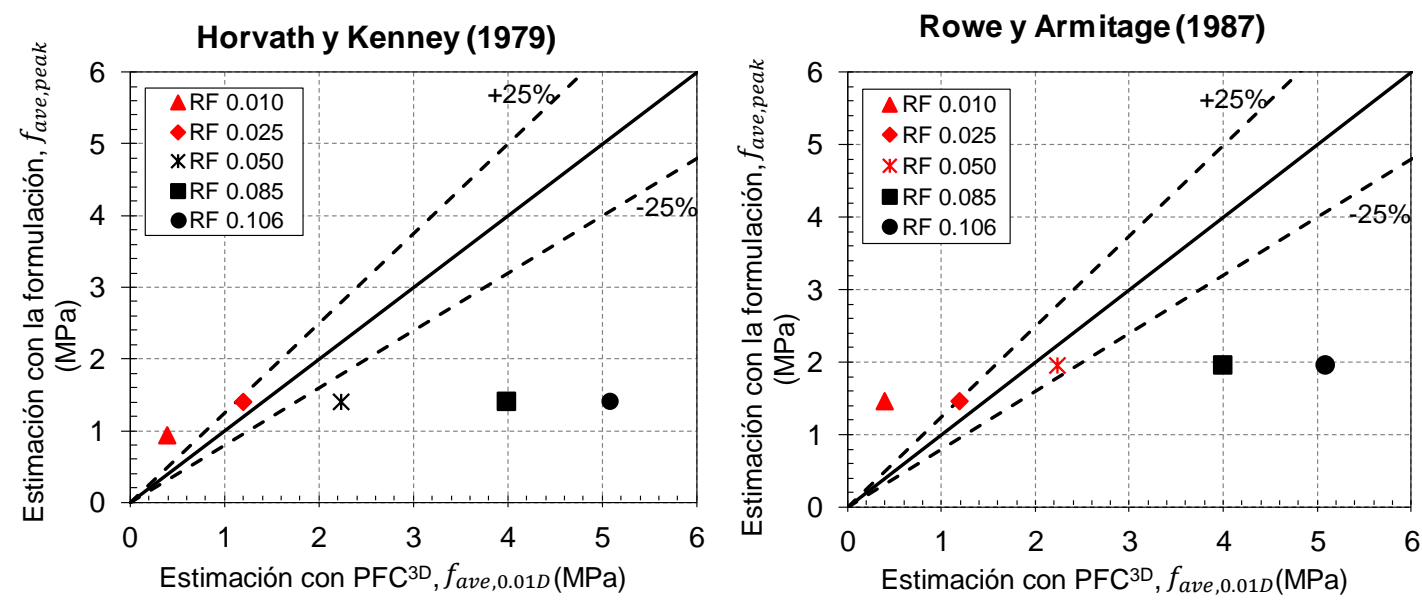

O’Neill y Reese (1999), Canadian
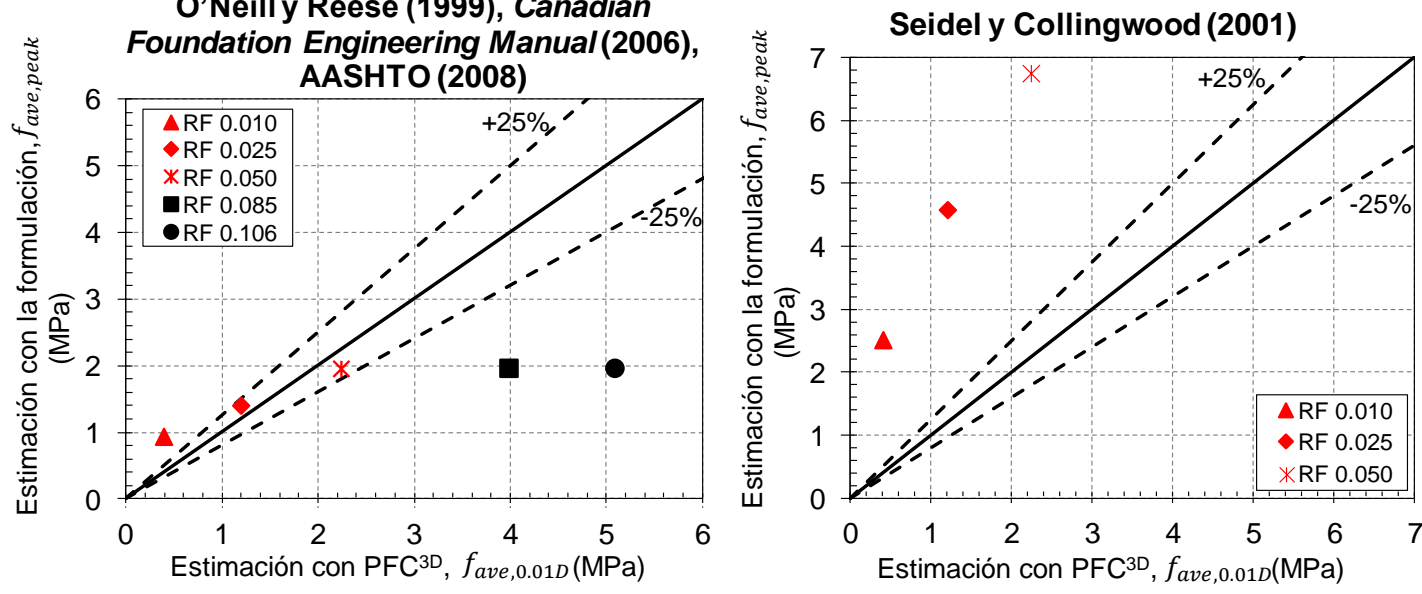

Figura 6.5. Estimación de la resistencia por fuste para los pilotes empotrados en la areniscaS3: comparación entre los resultados numéricos con $\mathrm{PFC}^{3 \mathrm{D}}$ y las formulaciones empíricas que consideran la rugosidad de la interfaz roca-pilote. 
Los métodos de O’Neill y Reese (1999), del Canadian Foundation Engineering Manual (2006) y de la Normativa AASHTO (2008), también proporcionan valores de resistencia por fuste muy similares a los calculados con los modelos de $\mathrm{PFC}^{3 \mathrm{D}}$ y $R F \leq 0.050$, mientras que para mayores valores de $R F$, las estimaciones con estas formulaciones producen resultados conservadores (ver la Figura 6.5). Nuevamente, esta tendencia era de esperar, dado que estas formulaciones se basaron en la combinación de las recomendaciones de Horvath y Kenny (1979) y de Rowe y Armitage (1987). Finalmente, los resultados numéricos de los modelos con $\mathrm{PFC}^{3 \mathrm{D}}$ arrojaron valores de $f_{\text {ave, } 0.01 D}$ significativamente menores a los obtenidos con la formulación de Seidel y Collingwood (2001). Nótese que los modelos con PFC ${ }^{3 \mathrm{D}}$ de los pilotes empotrados en la arenisca-S3 con $R F=0.085$ y $R F=0.106$ no se encuentran en la comparación; esto es debido a que, por sus características geométricas, arrojaron valores de $S R C>2.1$, con lo que la formulación de Seidel y Collingwood (2001) no puede ser aplicada.

En la Figuras 6.6 y 6.7 se muestra que el comportamiento para los pilotes empotrados en el gneis y la arenisca-S2, respectivamente, es similar al observado para los pilotes empotrados en la arenisca-S3. En cualquier caso, y aunque la discusión presentada a lo largo de esta sección es algo especulativa debido a las dificultades para reproducir el comportamiento real de campo, así como interpretar los ensayos de campo realizadas en otras investigaciones publicadas en la literatura, los resultados presentados anteriormente sugieren que, con una calibración adecuada, los modelos con PFC ${ }^{3 D}$ desarrollados en esta investigación pueden servir para cuantificar la influencia de la rugosidad del pilote empotrado en roca sobre la resistencia por fuste promedio. 

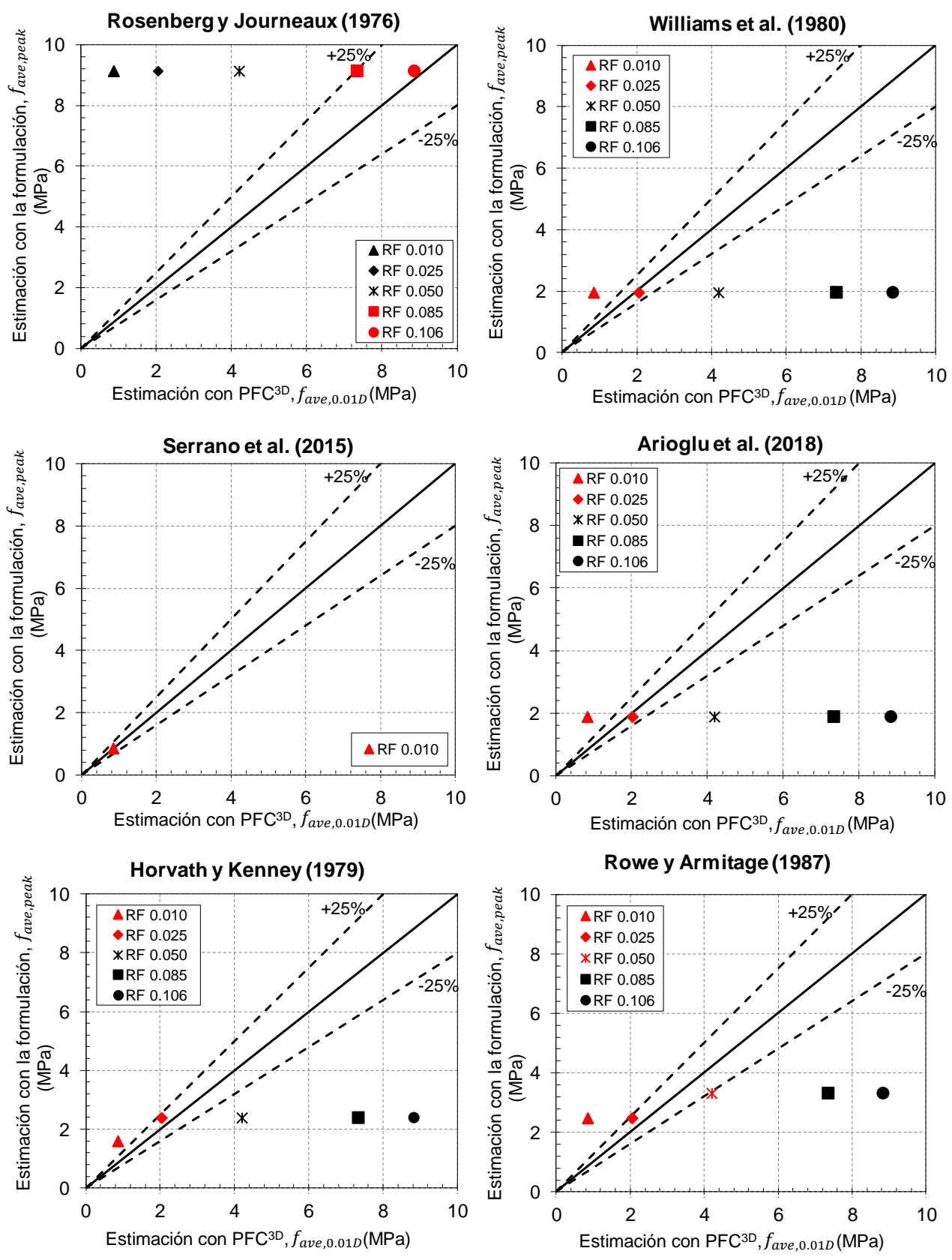

O'Neill y Reese (1999), Canadian
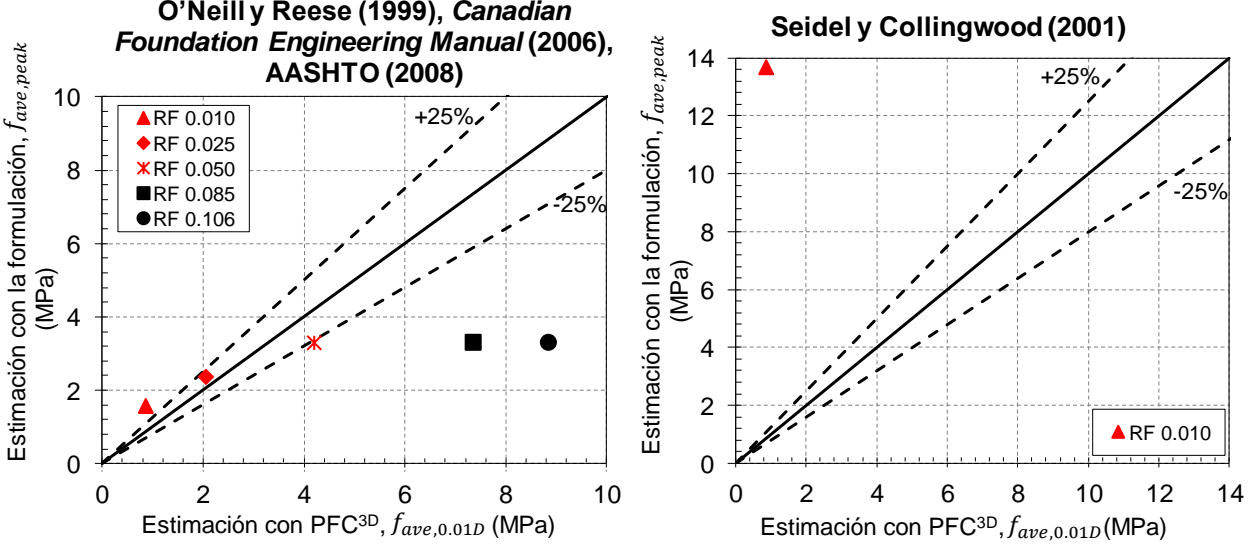

Figura 6.6. Estimación de la resistencia por fuste para los pilotes empotrados en el gneis: comparación entre los resultados numéricos con $\mathrm{PFC}^{3 \mathrm{D}}$ y las formulaciones empíricas y analíticas que consideran o no la rugosidad de la interfaz roca-pilote. 

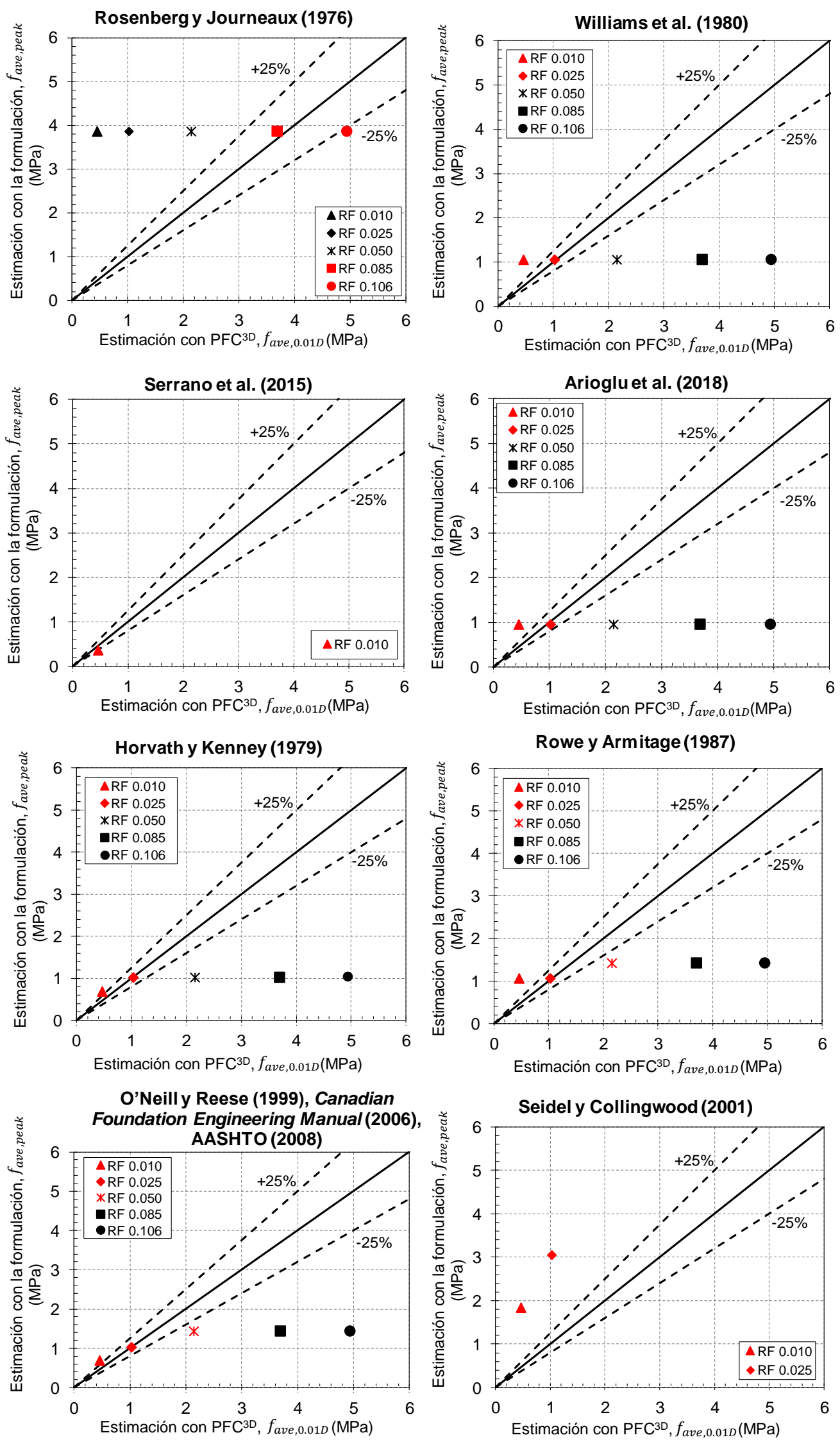

Figura 6.7. Estimación de la resistencia por fuste para los pilotes empotrados en la areniscaS2: comparación entre los resultados numéricos con MED ${ }^{3 \mathrm{D}}$ y las formulaciones empíricas y analíticas que consideran o no la rugosidad de la interfaz roca-pilote. 
Al comparar las estimaciones de las formulaciones (analíticas y empíricas) presentadas anteriormente con los ensayos de centrífuga realizados en esta investigación (ver Capítulo 5), se observa que todas las formulaciones predicen resultados muy superiores al valor de $f_{a v e, 0.01 D}$ obtenido en el ensayo de centrífuga para el modelo con $R F=0.000$ (ver la Figuras 6.8 y 6.9); este comportamiento era esperable puesto que el pilote de este ensayo se fabricó "perfectamente liso".
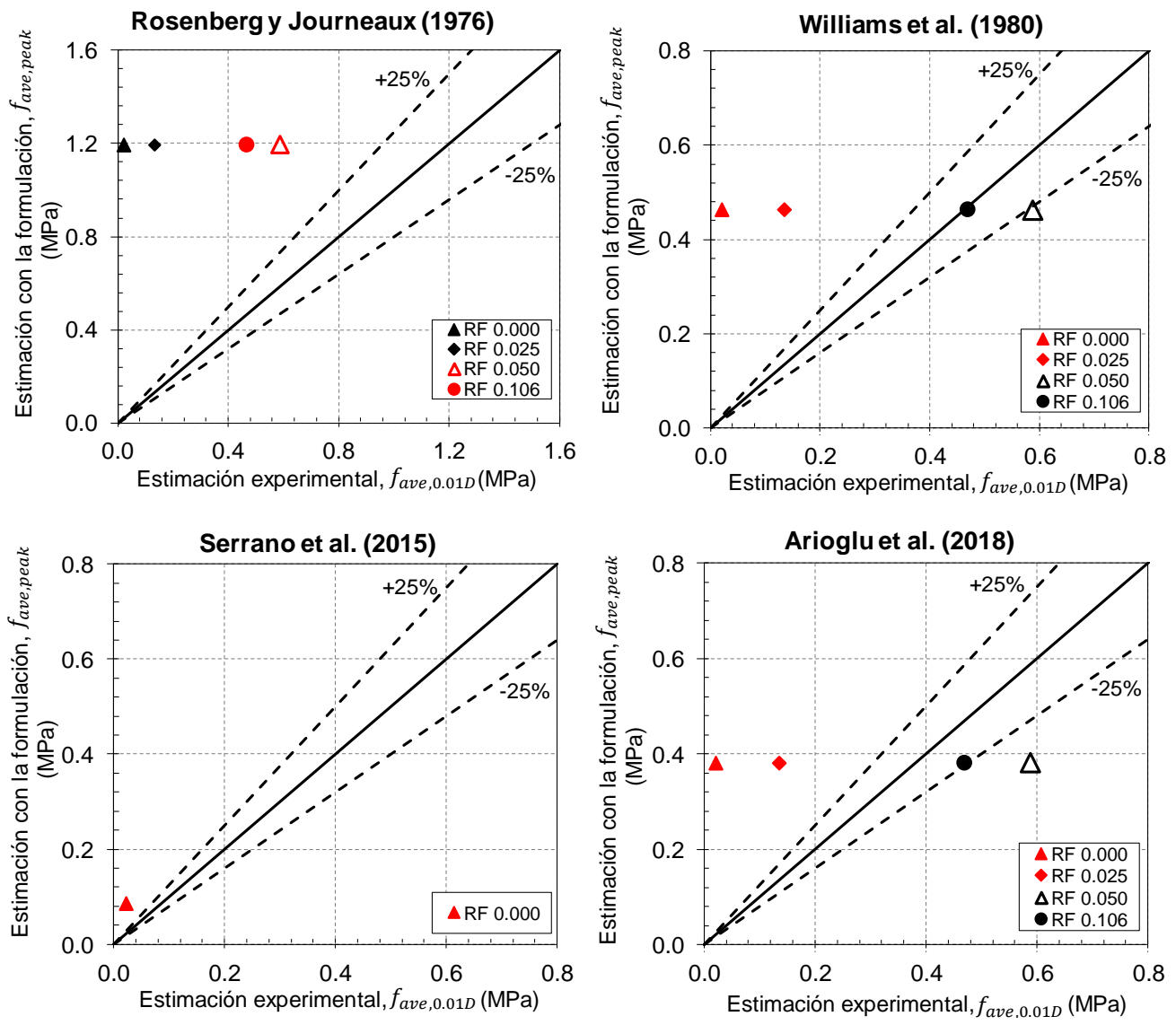

Figura 6.8. Estimación de la resistencia por fuste para los pilotes empotrados en la pseudoroca: comparación entre los resultados de los ensayos de centrífuga y las formulaciones analíticas y empíricas que no consideran la rugosidad de la interfaz roca-pilote.

Por otra parte, para los modelos con $R F=0.025, R F=0.050$ y $R F=0.106$, se observa una gran variabilidad de resultados, con una sobre-estimación o sub-estimación de la resistencia por fuste (según la formulación empleada) en comparación al valor hallado en el ensayo de centrífuga. Esta tendencia es congruente con los resultados obtenidos por Melentijevic et al. (2012), quienes reportaron un comportamiento similar entre los valores de resistencia por fuste obtenidos a partir de un ensayo de carga mediante célula 
Osterberg y los valores estimados mediante las diversas formulaciones existentes en la literatura. Notar, además, que el valor de resistencia por fuste obtenido en el modelo con $R F=0.050$ es mayor al del modelo con $R F=0.106$, la razón de este comportamiento se debe a un error de medición del asiento durante la realización de este ensayo (ver Capítulo 5), y a que su vez, induce a un valor de resistencia por fuste movilizado mayor al esperado para un asiento de $1 \%$ del diámetro del pilote.
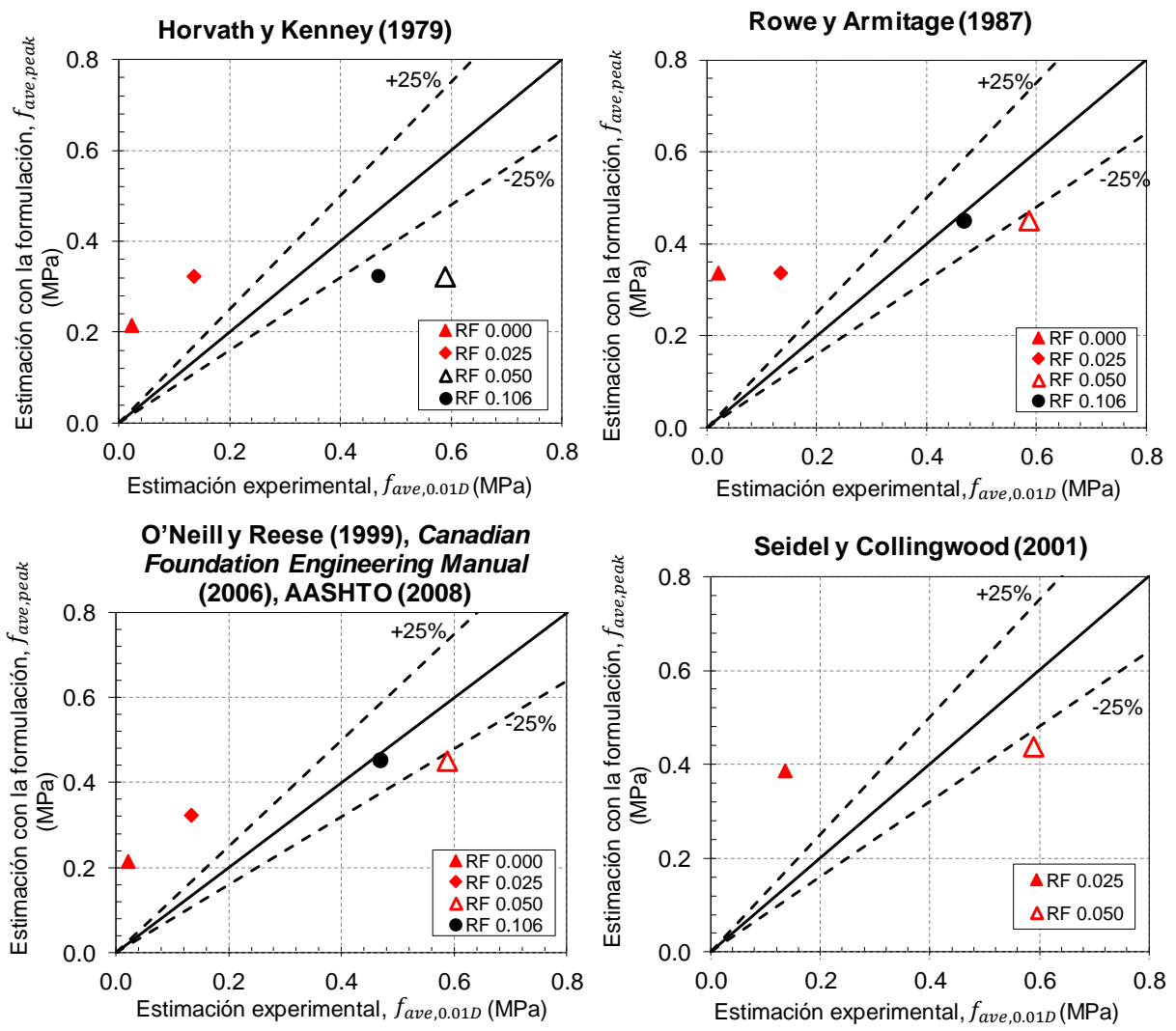

Figura 6.9. Estimación de la resistencia por fuste para los pilotes empotrados en la pseudoroca: comparación entre los resultados de los ensayos de centrífuga y las formulaciones empíricas que consideran la rugosidad de la interfaz roca-pilote.

\subsection{Comparación con el factor empírico $\alpha$}

A partir de los resultados de los modelos numéricos desarrollados en esta investigación, así como de los resultados de los de ensayos de centrífuga ejecutados, se calculó el factor empírico $\alpha=f_{\text {ave,peak }} / \sigma_{c}$ propuesto por algunos investigadores y se incorporó dentro de sus curvas de diseño. (Resulta oportuno recordar que, debido a que la mayoría de estas formulaciones proponen un valor de $f_{\text {ave,peak }}$ para un asiento en 
cabeza del pilote de $0.01 D$, es posible comparar con el valor de $f_{a v e, 0.01 D}$ hallado a partir de los modelos con $\mathrm{PFC}^{3 \mathrm{D}}$ y ensayos de centrífuga del presente trabajo).

En la Figura 6.10 se comparan los valores de $\alpha$ obtenidos para los modelos con PFC ${ }^{3 D}$ con los datos de ensayos de carga y curvas de diseño propuestas por Pells et al.(1980). Los datos de Pells et al. (1980) provienen de ensayos realizados sobre pilotes con diámetros menores a $300 \mathrm{~mm}$, con diferente rugosidad, y en los que la resistencia al fuste máxima se alcanzó para asientos en cabeza en un rango de $5-15 \mathrm{~mm}$. Nótese que los resultados de los ensayos de centrífuga (pseudo-roca, $\sigma_{c}=1.15 \mathrm{MPa}$ ) no han sido incorporados a la Figura 6.10, puesto que las curvas de diseño de Pells et al. (1980) son válidas para aproximadamente un rango de $3 \leq \sigma_{c} \leq 65 \mathrm{MPa}$.

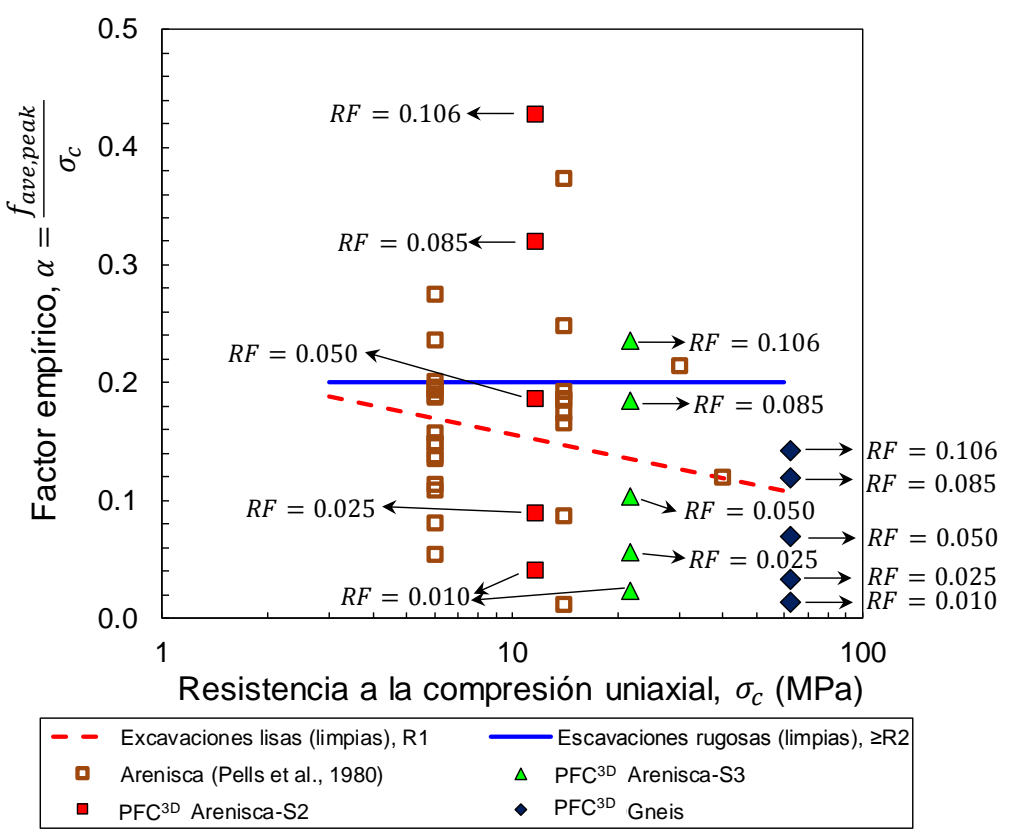

Figura 6.10. Comparación entre los resultados numéricos con PFC ${ }^{3 D}$ vs las curvas de diseño propuestas por Pells et al. (1980).

Como puede observarse en la Figura 6.10, los resultados numéricos obtenidos con $\mathrm{PFC}^{3 \mathrm{D}}$ para modelos con un rango amplio de rugosidades están dentro del rango de valores de $\alpha$ reportados por Pells et al.(1980); sin embargo, las curvas de diseño de Pells et al.(1980) no capturan la variabilidad de los resultados obtenidos en sus propios ensayos. Adicionalmente, Pells et al. (1998) indicaron que las discrepancias entre sus 
curvas de diseño y otras recomendaciones reportadas en la literatura (e.g., Williams et al. 1980) podrían deberse a factores como, por ejemplo, el proceso geológico de formación de la roca o el método de excavación, entre otros.

Por otra parte, en la Figura 6.11 se comparan los datos de ensayos de carga y curvas de diseño propuestas por Williams y Pells (1981) con los valores de $\alpha$ obtenidos con los modelos de PFC ${ }^{3 D}$ y con los ensayos de centrífuga. Williams y Pells (1981) emplearon para su formulación ensayos de carga realizados sobre lutitas y areniscas, en las cuales se aplicó un factor de 2.5 sobre la línea de mejor ajuste para obtener un límite inferior y superior de diseño. De la Figura 6.11 se observa que los modelos numéricos y de centrífuga reproducen bien los valores de $\alpha$ de los ensayos reales realizados por Williams y Pells (1981), incluso para rocas con un amplio rango de resistencias.

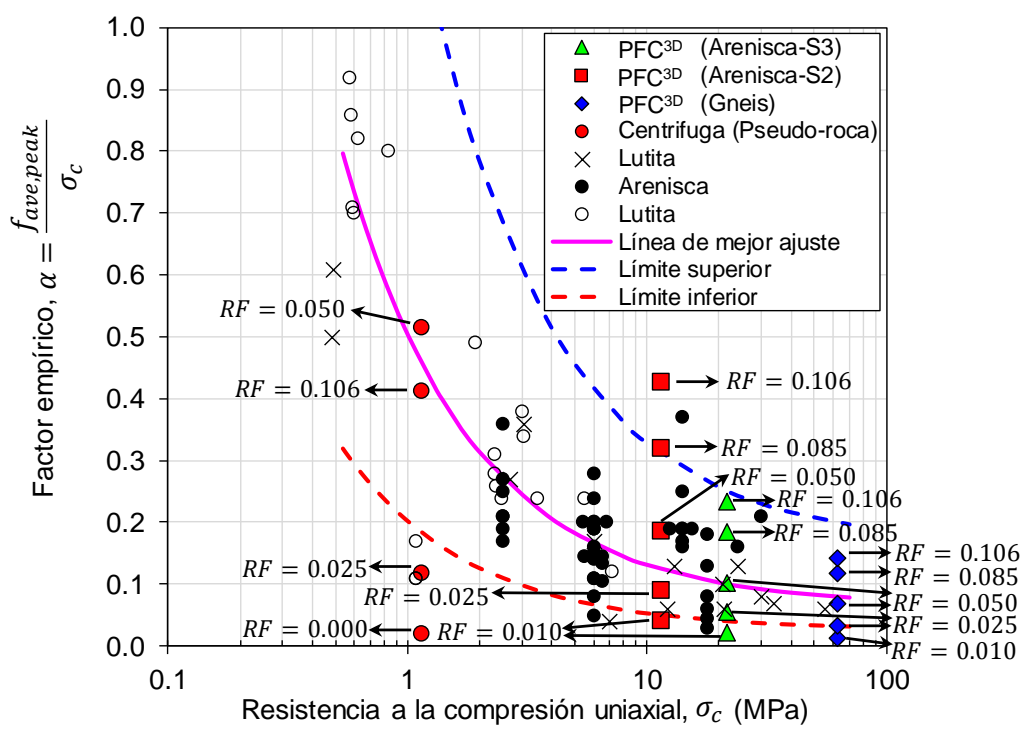

Figura 6.11. Comparación entre los resultados numéricos con $\mathrm{PFC}^{3 \mathrm{D}}$ y de centrífuga vs las curvas de diseño propuestas por Williams y Pells (1981).

Finalmente, en la Figura 6.12 se compara el factor $\alpha$ obtenido para los modelos con $\mathrm{PFC}^{3 \mathrm{D}}$ y para los ensayos de centrífuga con las curvas de diseño propuestas por Seidel y Collingwood (2001), después de incorporar los resultados de ensayos de carga de Kulhawy y Phoon (1993). Los límites de $S R C$ mostrados en la Figura 6.12 fueron definidos por Seidel y Collingwood (2001) a partir de pilotes con $D=450-1500 \mathrm{~mm}$, 
empotrados en roca con una relación de $E_{m} / \sigma_{c, \text { roca }}=50-200$ y $v=0.25$, y empleando una reducción por método constructivo de $\eta=0.75-1$ (ver Ecuación 6.6). Los límites superior e inferior efectivos fueron establecidos por Seidel y Collingwood (2001) a partir de los parámetros mencionados anteriormente, mientras que los límites superior e inferior extremo representan la mejor y peor combinación de estos mismos parámetros (ver Seidel y Collingwood 2001 para más detalles).

Debido a que el factor SRC (ver Ecuación 6.6) propuesto por Seidel y Collingwood (2001) parte del supuesto que el pilote es más resistente que la roca, para las estimaciones de resistencia al fuste de pilotes empotrados en el gneis se empleó el $\sigma_{c}$ del hormigón, debido a que era el más débil entre los dos materiales. En la Figura 6.12 se observa que los valores del factor $\alpha$ obtenidos a partir de los resultados de los modelos numéricos con $\mathrm{PFC}^{3 \mathrm{D}}$ y de los ensayos de centrífuga, para un asiento en cabeza de $1 \% D$, son consistentes con los datos de ensayos recopilados por Kulhawy y Phoon (1993) para pilotes construidos tanto en suelo como en roca, así como también con las curvas límites de diseño propuestas por Seidel y Collingwood (2001).

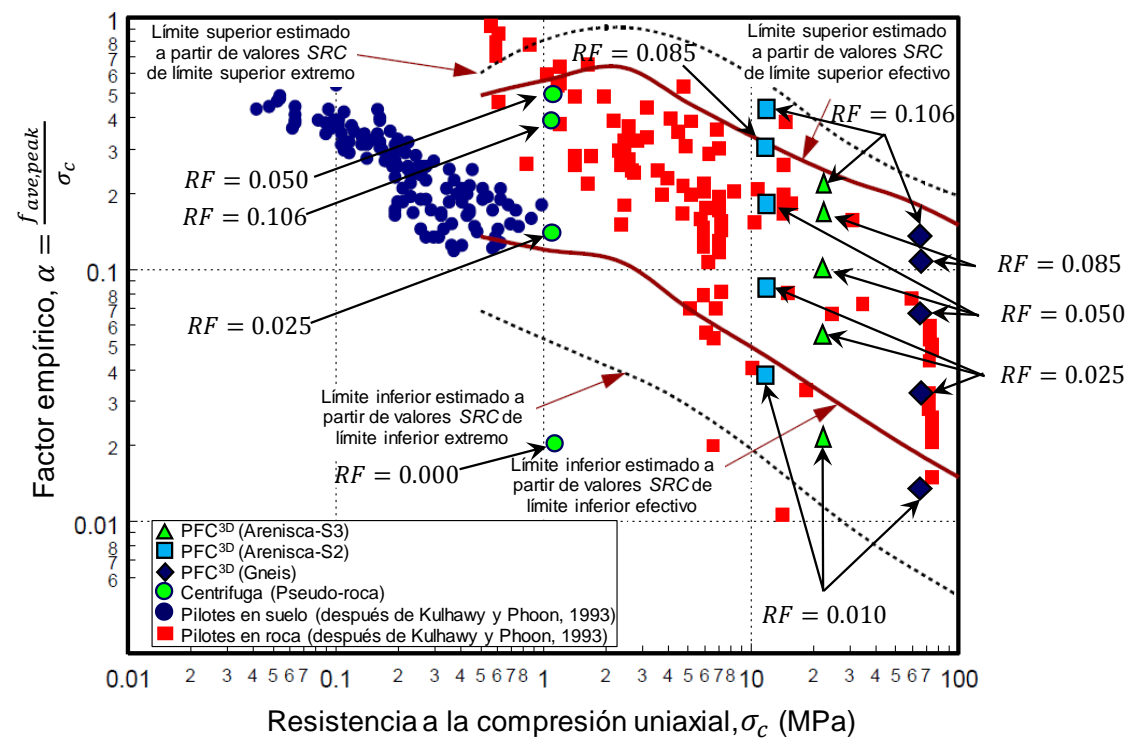

Figura 6.12. Comparación entre los resultados numéricos con $\mathrm{PFC}^{3 \mathrm{D}}$ y de centrífuga vs las curvas de diseño propuestas por Seidel y Collingwood (2001) después de incorporar los resultados de ensayos de carga de Kulhawy and Phoon (1993). 


\subsection{Factor $\alpha_{R F, 0.01 D}$ para la estimación de la resistencia por fuste}

Los resultados obtenidos mediante modelos numéricos con MED ${ }^{3 \mathrm{D}}$ (ver Capítulo 4) y con los ensayos de centrífuga (ver Capítulo 5), se han empleado para desarrollar el factor $\alpha_{R F, 0.01 D}$. Este factor permite la estimación de la resistencia promedio de pilotes empotrados en roca para un asiento en cabeza de $1 \% D$. Con base a lo mostrado en la Figura 6.13 -los casos en que el material más débil (roca o pilote) tienen $\sigma_{c} \geq 10 \mathrm{MPa}$ y $\sigma_{c}<10 \mathrm{MPa}$ fueron resaltados con color azul y rojo, respectivamente-, se proponen dos curvas de diseño de la forma:

$$
\begin{array}{ll}
\frac{f_{\text {ave }, 0.01 D}(\mathrm{MPa})}{\frac{L}{D} \sqrt{\sigma_{c}(\mathrm{MPa})}}=10 R F & \text { para } \sigma_{c} \geq 10 \mathrm{MPa} \text { y } L / D \leq 3 \\
\frac{f_{\text {ave }, 0.01 D}(\mathrm{MPa})}{\frac{L}{D} \sqrt{\sigma_{c}(\mathrm{MPa})}}=2.5 R F & \text { para } \sigma_{c}<10 \mathrm{MPa} \text { y } L / D \leq 3
\end{array}
$$

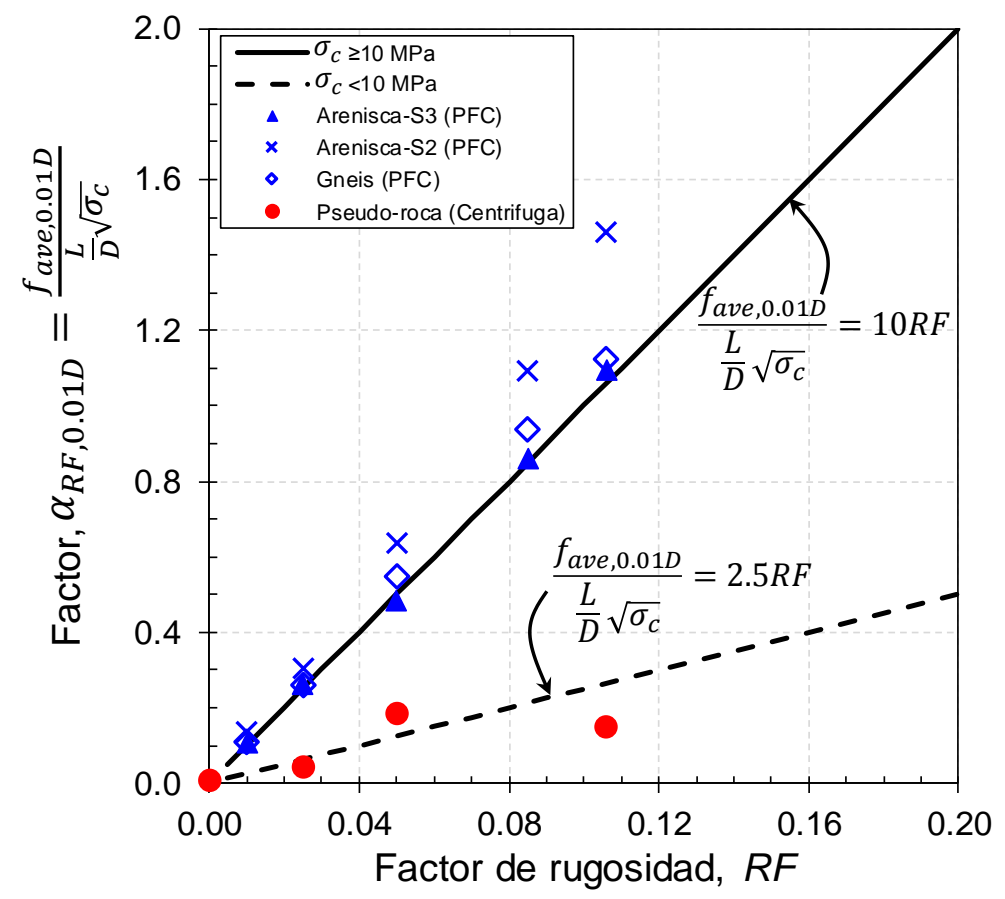

Figura 6.13. Factor $\alpha_{R F, 0.01 D}$ propuesto para la estimación de la resistencia por fuste de pilotes empotrados en roca considerando un asiento en cabeza de $1 \% D, f_{a v e, 0.01 D}$ (los puntos de color azul y rojo representan los casos en que el material el más débil -roca o pilote- con $\sigma_{c} \geq 10$ $\mathrm{MPa}$ y $\sigma_{c}<10 \mathrm{MPa}$, respectivamente).

Esta propuesta se basa en las siguientes hipótesis: (i) se considera que la rugosidad de la interfaz roca-pilote viene caracterizada por medio del factor de rugosidad $R F$, (ii) se 
usa la resistencia a compresión uniaxial del material intacto más débil (roca o pilote) para un amplio rango de resistencia de $0.5 \leq \sigma_{c} \leq 60 \mathrm{MPa}$, (iii) se parte del supuesto de la no existencia de cohesión $(c=0)$ en la interfaz roca-pilote, y por tanto, no se considera su posible contribución en la estimación de $f_{\text {ave, } 0.01 D} \mathrm{y}$, (iv) se incluye la relación geométrica de longitud de empotramiento-diámetro del pilote $(L / D)$. En el caso que la relación geométrica resulte $L / D>3$, se deberá tomar $L / D=3$ en las ecuaciones 6.7 y 6.8 .

La incorporación del factor geométrico $L / D$ en el factor $\alpha_{R F, 0.01 D}$, responde a la evidencia publicada la literatura, según el cual se observa un efecto de la relación $L / D$ sobre la contribución por el fuste de pilotes empotrados en roca (e.g., ver Osterberg y Gill 1973). Nótese, además, que este nuevo enfoque es equivalente a considerar $\beta=0.5$ en el criterio empírico general de la Ecuación 6.1; la selección de $\beta=0.5$ se debe a la simplicidad de la fórmula que resulta, y porque es el valor más comúnmente empleado en la práctica (ver la Tabla 6.1). Sin embargo, la principal diferencia de los métodos de la Tabla 6.1 con la propuesta realizada aquí es que, en contra de la mayoría de los enfoques previos, el método propuesto en esta tesis considera la rugosidad en la estimación del factor empírico $\alpha$.

\subsection{Validación del factor $\alpha_{R F, 0.01 D}$ con datos experimentales}

Con el objetivo de evaluar el comportamiento del factor $\alpha_{R F, 0.01 D}$ propuesto en la sección anterior, a continuación se presenta una validación del mismo realizada a partir ensayos de carga -in-situ y de laboratorio (1g)- publicados en la literatura. Para ello, (i) se estimó el factor de rugosidad $R F$ a partir de los datos geométricos de la pared del pilote, (ii) se estimó la $f_{a v e, 0.01 D}$ a partir de la curva carga-asiento reportada en el ensayo de carga de cada autor -i.e., la carga registrada para un asiento en cabeza de $0.01 D$ dividida por el área nominal por fuste del pilote- $\mathrm{y}$, (iii) se calculó el factor $\alpha_{R F, 0.01 D}=$ $f_{\text {ave }, 0.01 D} /\left((L / D) \sqrt{\sigma_{c}}\right)$. Los datos de geometría, factor de rugosidad, etc., empleados en 
cada uno de los ensayos se muestran en la Tabla 6.3. Los resultados obtenidos con la validación -i.e., $R F$ versus $\alpha_{R F, 0.01 D^{-}}$se muestran en la Figura 6.14. Nuevamente, se han resaltado con color azul y rojo, aquellos puntos cuyo material más débil (roca o pilote) tiene $\sigma_{c} \geq 10 \mathrm{MPa}$ y $\sigma_{c}<10 \mathrm{MPa}$, respectivamente.

Tabla 6.3. Datos empleados para la validación del factor $\alpha_{R F, 0.01 D}$.

\begin{tabular}{|c|c|c|c|c|c|c|c|c|c|c|}
\hline $\begin{array}{l}\text { Tipo de } \\
\text { Roca }\end{array}$ & Denominación & $R F$ & $\begin{array}{l}f_{\text {ave } 0,01 D} \\
(\mathrm{MPa})\end{array}$ & $\begin{array}{c}\sigma_{c} \text {, Pilote } \\
(\mathrm{MPa})\end{array}$ & $\begin{array}{c}\sigma_{c}, \text { Roca } \\
(\mathrm{MPa})\end{array}$ & $\begin{array}{l}\text { Longitud, } L \\
(\mathrm{~m})\end{array}$ & $\begin{array}{l}\text { Diámetro, } D \\
(\mathrm{~m})\end{array}$ & $\begin{array}{c}/ D \\
-\end{array}$ & Tipo de Ensayo & Referencia \\
\hline \multirow{11}{*}{ Lutita } & S1 & 0.029 & 0.566 & \multirow{11}{*}{41.5} & 0.83 & 1.52 & 0.66 & 2.30 & \multirow{11}{*}{$\begin{array}{l}\text { Ensayo de carga } \\
\text { (gato hidráulico } \\
\text { en superficie) }\end{array}$} & \multirow{11}{*}{ Williams (1980) } \\
\hline & S3 & 0.037 & 0.430 & & 0.55 & 2.59 & 1.12 & 2.31 & & \\
\hline & S5 & 0.011 & 0.470 & & 0.59 & 2.51 & 1.17 & 2.15 & & \\
\hline & S14 & 0.058 & 0.320 & & 0.58 & 0.87 & 0.40 & 2.20 & & \\
\hline & S15 & 0.052 & 0.235 & & 0.58 & 0.87 & 0.40 & 2.20 & & \\
\hline & S16 & 0.055 & 0.280 & & 0.58 & 0.87 & 0.40 & 2.20 & & \\
\hline & M1 & 0.027 & 0.520 & & 2.46 & 2.00 & 1.22 & 1.64 & & \\
\hline & M2 & 0.060 & 0.575 & & 2.30 & 2.00 & 1.30 & 1.54 & & \\
\hline & M3 & 0.014 & 0.680 & & 2.30 & 2.00 & 1.23 & 1.63 & & \\
\hline & M4 & 0.060 & 0.617 & & 2.34 & 2.00 & 1.35 & 1.48 & & \\
\hline & WG303/2 & 0.110 & 0.840 & & 3.49 & 2.00 & 1.58 & 1.27 & & \\
\hline \multirow{3}{*}{ Lutita } & P1 & 0.036 & 0.730 & \multirow{3}{*}{49} & 5.40 & & & & \multirow{3}{*}{$\begin{array}{l}\text { Ensayo de carga } \\
\text { (gato hidráulico } \\
\text { en superficie) }\end{array}$} & \multirow{3}{*}{ Horvath et al. (1983) } \\
\hline & P3 & 0.076 & 0.755 & & 5.60 & 1.37 & 0.71 & 1.93 & & \\
\hline & P6 & 0.100 & 1.115 & & 5.60 & & & & & \\
\hline \multirow{3}{*}{ Pseudo-roca } & & & 1.171 & \multirow{3}{*}{27.5} & 3.60 & & & & \multirow{3}{*}{$\begin{array}{c}\text { Ensayo de carga } \\
\text { en laboratorio } \\
(1 \mathrm{~g})\end{array}$} & \multirow{3}{*}{ Khan y Amadei (1993) } \\
\hline & MTS36 & 0.173 & 1.174 & & 6.54 & 0.25 & 0.06 & $4.00^{*}$ & & \\
\hline & & & 1.756 & & 9.53 & & & & & \\
\hline \multirow{3}{*}{ Pseudo-roca } & $\mathrm{P} 1$ con $\mathrm{P} 2$ & 0.157 & 1.250 & \multirow{3}{*}{22} & 4.70 & & & & \multirow{3}{*}{$\begin{array}{c}\text { Ensayo de carga } \\
\text { en laboratorio } \\
(1 \mathrm{~g})\end{array}$} & \multirow{3}{*}{ Benmokrane et al. (1994) } \\
\hline & $\mathrm{P} 1$ con $\mathrm{P} 1$ & 0.157 & 5.250 & & 22.00 & 0.10 & 0.05 & 2.00 & & \\
\hline & $\mathrm{P} 1 \operatorname{con} \mathrm{C}$ & 0.157 & 4.250 & & 42.00 & & & & & \\
\hline Lutita & HT & 0.020 & 0.094 & - & 1.34 & 3.00 & 0.76 & $3.95^{\star}$ & $\begin{array}{c}\text { Ensayo de carga } \\
\text { (O-cell) }\end{array}$ & $\begin{array}{l}\text { Nam y Vipulanandan } \\
(2008)\end{array}$ \\
\hline
\end{tabular}

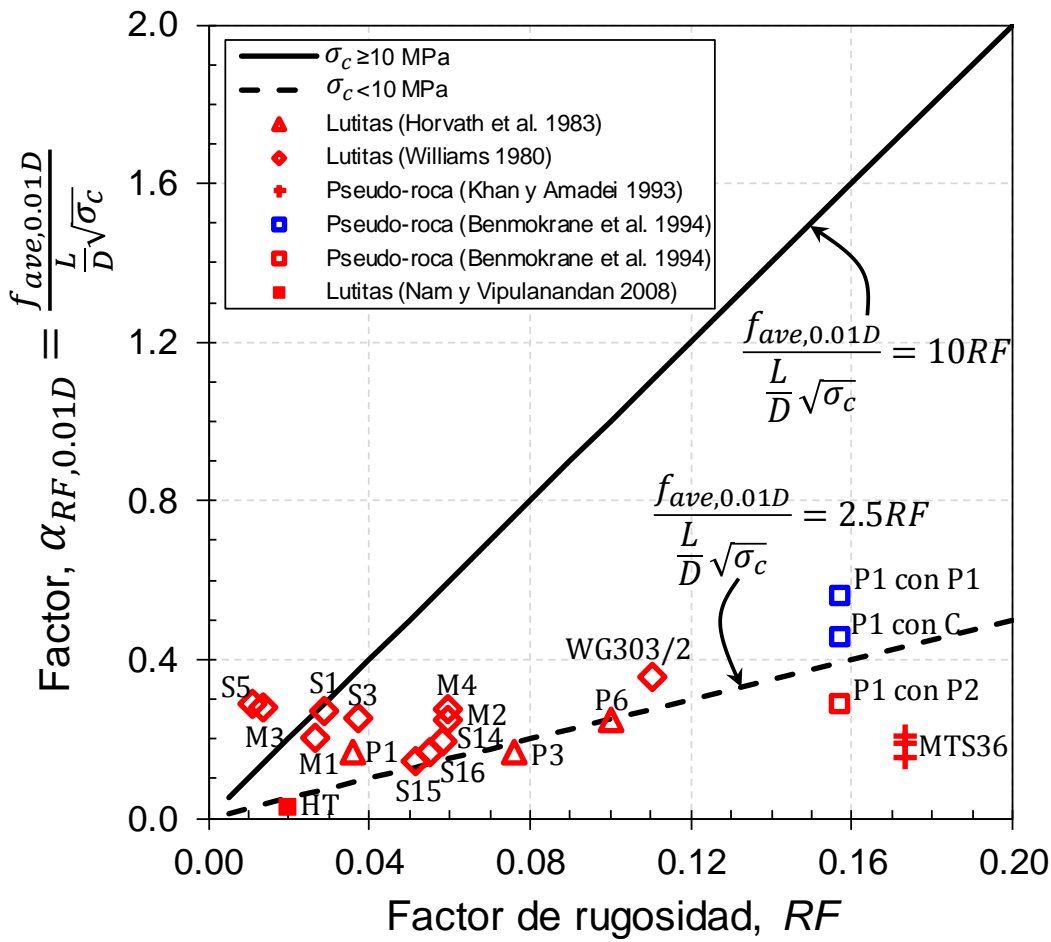

Figura 6.14. Validación del factor $\alpha_{R F, 0.01 D}$ a partir de ensayos sobre pilotes empotrados en roca publicados en la literatura (los puntos de color azul y rojo representan los casos en que el material el más débil -roca o pilote- presenta $\sigma_{c} \geq 10 \mathrm{MPa} \circ \sigma_{c}<10 \mathrm{MPa}$, respectivamente). 
De la Figura 6.14, se evidencia que la curva de diseño para materiales con $\sigma_{c}<10 \mathrm{MPa}$, presenta un buen comportamiento respecto a los valores registrados en los ensayos de carga realizado por Williams (1980), Horvath et al. (1983) y Nam y Vipulanandan (2008). En particular, se observa que, para las excavaciones con $R F \geq 0.020$, los resultados de los ensayos muestran una adecuada tendencia respecto la curva de diseño propuesta (línea negra segmentada). Notar, además, que para algunos de los ensayos realizados por Williams (1980) -e.g., ver pilotes S1 y S3-, los valores de $\alpha_{R F, 0.01 D}$ están muy por encima de la curva de diseño para $\sigma_{c}<10 \mathrm{MPa}$. Sin embargo, Williams (1980) indica que en el ensayo del pilote $\mathrm{S} 1$ no se llegó hasta la rotura y, además, observó la existencia de cohesión en gran parte de la interfaz roca-pilote. En consecuencia, su comportamiento por encima de la curva de diseño puede atribuirse a la posible contribución de la cohesión sobre la resistencia por fuste de este ensayo.

Para los pilotes S3 (al cual le fue incrementada artificialmente la rugosidad de la excavación) y S5, puede verse un comportamiento análogo al observado en el pilote S1, con valores similares de $\alpha_{R F, 0.01 D}$. A tales efectos, Williams (1980) indica que tras realizar ambos ensayos, se llevó a cabo la excavación de ambos pilotes y se evidenció la presencia de una pequeña capa de roca adherida firmemente a la superficie de ambos pilotes, seguida de una zona de falla por corte hacia la roca circundante. Además, en el parte constructivo de ambos ensayos, Williams (1980) manifiesta que, las paredes de la excavación fueron limpiadas antes de hormigonar el pilote; por tanto, esa pequeña capa de roca adherida en ambos pilotes sugiere la posible existencia de cohesión en el contacto roca-pilote tras su proceso de fraguado. Por tal razón, nuevamente, los altos valores de $\alpha_{R F, 0.01 D}$ de los pilotes S3 y S5 pueden estar asociados a la contribución de dicha cohesión en la interfaz-roca-pilote. En este sentido, resulta oportuno mencionar que las curvas de diseño para el cálculo de $f_{a v e, 0.01 D}$ (ver Figura 6.14) han sido propuestas considerando un comportamiento friccional $(c=0)$ en la interfaz roca-pilote, y que por tanto, la estimación de $f_{a v e, 0.01 D}$ es conservadora y no incluye la posible 
contribución de la cohesión en la interfaz roca-pilote. Sin embargo, en caso de tener seguridad de la existencia de cohesión en la interfaz roca-pilote, se deja a libre criterio del ingeniero su incorporación en la estimación de $f_{\text {ave, } 0.01 D}$.

Por otra parte, en el proceso de validación presentado en la Figura 6.14, también se han incluido los resultados de los ensayos de laboratorio $(1 \mathrm{~g})$, realizados por Kahn y Amedei (1993) y Benmokrane et al. (1994). Como puede observarse, estos ensayos dan resultados muy por debajo de lo esperado para pilotes tan rugosos -i.e., con valores altos de $R F-$. Esta tendencia de subestimación observada en ambos ensayos o de sobreestimación como lo reportado en los ensayos de Leung y Ko (1993, ver el Capítulo 2 (Sección 2.4.1.1)), puede atribuirse a un posible efecto escala, según el cual los modelos estudiados a pequeña escala $(1 g)$ no son representativos de la realidad, y por tanto pueden considerarse como un método menos fiable que otros métodos que reproducen mejor el estado tensional real en el entorno del pilote, como los ensayos insitu a escala "real" o en un ensayo de centrífuga $(\mathrm{Ng})$.

\subsection{Aplicación del factor $\alpha_{R F, 0.01 D}$ a nuevos casos de estudio}

La propuesta y validación del factor $\alpha_{R F, 0.01 D}$ presentada previamente (ver Secciones 6.5 y 6.6, respectivamente), se ha realizado considerando datos -numéricos y experimentales- de pilotes empotrados en solo una capa de roca; sin embargo, es posible que los pilotes a diseñar atraviesen terrenos multicapas. Por tal razón, esta sección presenta la aplicación del factor $\alpha_{R F, 0.01 D}$ a nuevos casos de estudio con estas características. A tales efectos, se han empleado los datos experimentales publicados por Miller (2003); a continuación, se presenta una descripción breve de cada uno de los ensayos de Miller (2003), así como también su comparación con la curva de diseño propuesta en esta tesis doctoral. 


\subsubsection{Ensayos de carga realizados en Lexington}

Como parte del proyecto para la construcción de un viaducto de $1244.5 \mathrm{~m}$ de longitud sobre el río Missouri (en la ciudad de Lexington del estado de Missouri (EE.UU.), el Departamento de Transporte de Missouri (DTM) realizó 2 ensayos de carga (TS-1A y TS-2) de pilotes mediante célula Osterberg (O-cell), en los cuales la selección de las ubicaciones de los ensayos fue realizada en función de la ubicación prevista para las pilas del viaducto (ver la Figura 6.15). Adicionalmente, debido a que las pilas proyectadas se cimentarían mediante pilotes que atraviesan varios estratos de rocas blandas (Formación Mulky, Formación Bevier, etc.), no fue considerada en el diseño de los pilotes, la posible contribución de la resistencia por punta (Miller, 2003). En este sentido, el objetivo principal de las pruebas de carga fue la estimación de la resistencia por fuste de los estratos rocosos sobre los que se cimentarían las pilas del viaducto.

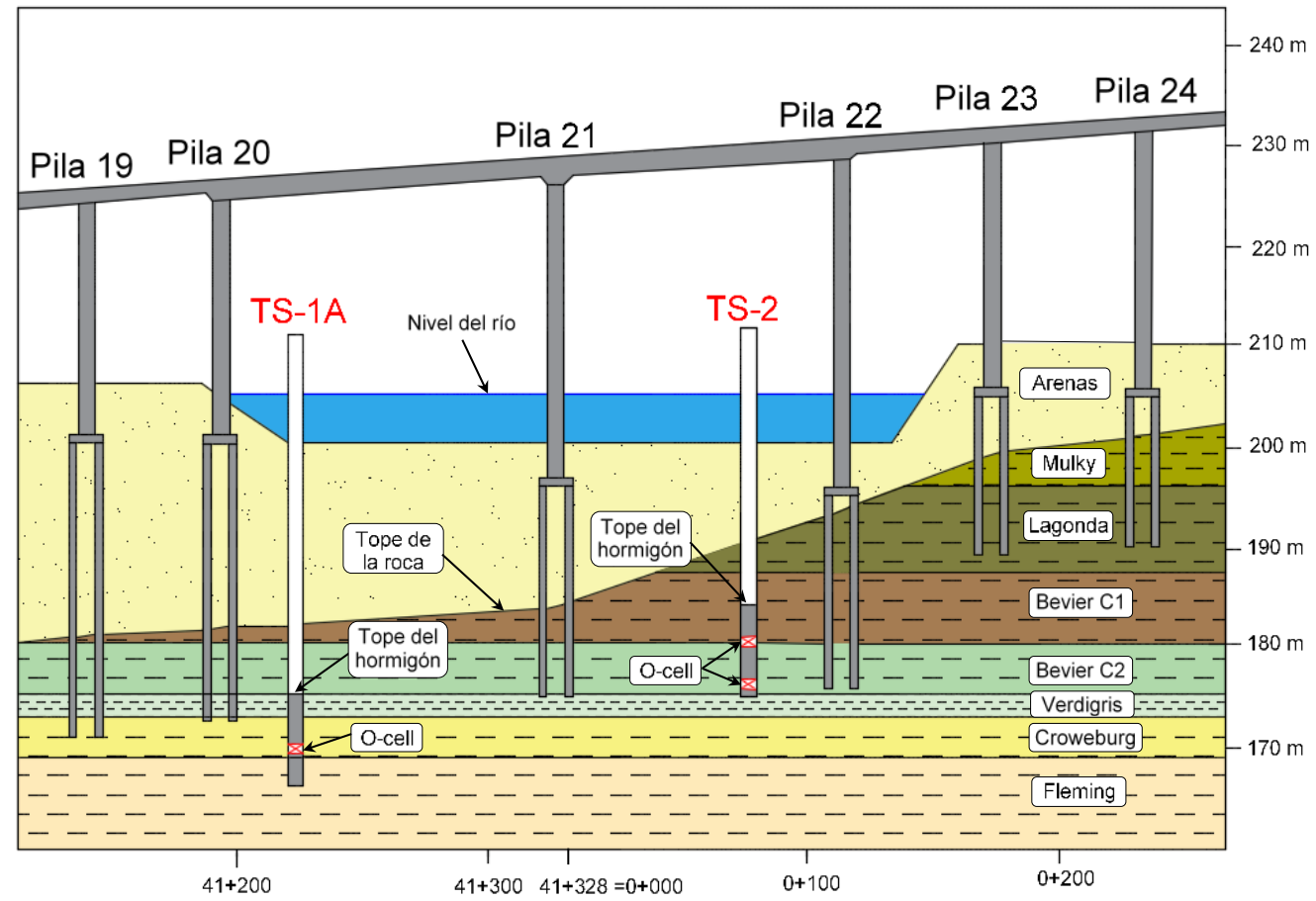

Figura 6.15. Vista longitudinal del viaducto: perfil geológico, ubicación de las pilas (19-24) de fundación y localización de las pruebas de carga (TS-1A y TS-2) (modificado de Miller 2003).

En cuanto al proceso de ejecución de estos ensayos, Miller (2003) reportó que se realizó

(i) una entibación temporal en las adyacencias de la zona del ensayo para evitar la 
entrada del agua del río Missouri durante el proceso de preparación y ejecución de los ensayos, y (ii) una entibación permanente a lo largo de todo el estrato de suelo suprayacente para evitar su contribución a la resistencia por fuste durante el ensayo. Adicionalmente, Miller (2003) indicó que la perforación de los estratos rocosos de ambos ensayos se hizo con barrenas de alta capacidad para rocas (Barrena Bullet Tooth Rock, ver el Anexo A), empleando agua como fluido de perforación. Posteriormente, una vez terminada la perforación, se midió la geometría de las paredes mediante el uso de un registro tipo Caliper. Finalmente, llevaron a cabo la colocación del conjunto armaduracélula Osterberg y el hormigonado del orifico (Miller, 2003). La tipología y geometría de cada ensayo se describe a continuación.

\subsubsection{Ensayo de carga TS-1A}

Este ensayo fue realizado cerca de la Pila 20, en las inmediaciones de la margen izquierda del río Missouri (ver la Figura 6.15). Para ello, se empleó una célula Osterberg de $660 \mathrm{~mm}$ de diámetro, localizada hacia la parte inferior del pilote, y presurizada de tal forma que su carga y posterior descarga permitieron la estimación de la resistencia simultánea por punta y fuste del pilote. La Figura 6.16(a) muestra el perfil geológico, las características y la geometría de la entibación (temporal y permanente) de la perforación, así como la localización de las bandas de medición (SG, rectángulos rojos en el pilote), etc. La morfología de la pared de la perforación (medida a partir de un registro Caliper) y los factores de rugosidad $(R F)$ de cada estrato, calculados para cada perfil (Oeste y Este), se presentan en la Figura 6.16(b) (para más detalles ver Miller, 2003).

\subsubsection{Ensayo de carga TS-2}

Esta prueba fue llevada a cabo entre las Pilas 21 y 22, cerca de la margen derecha del río Missouri (ver las Figuras 6.15 y 6.17). En esta prueba, a diferencia del ensayo TS1A, se emplearon dos células Osterberg (iguales a las del ensayo TS-1A) localizadas 
de tal manera que una se ubicó cerca de la punta del pilote, y la otra, en la parte media del mismo.

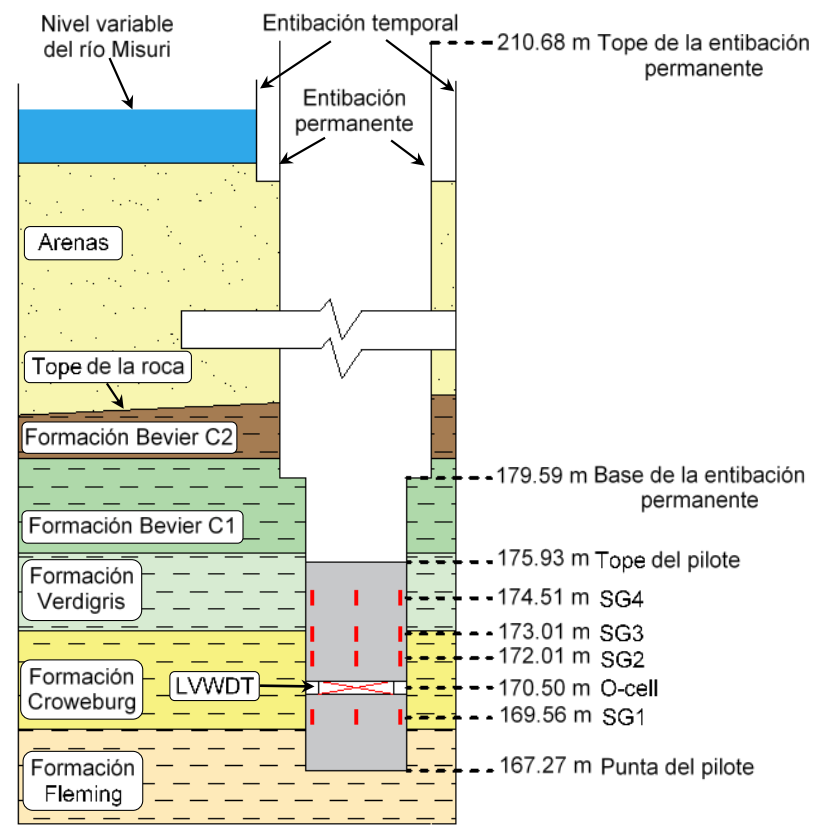

(a)

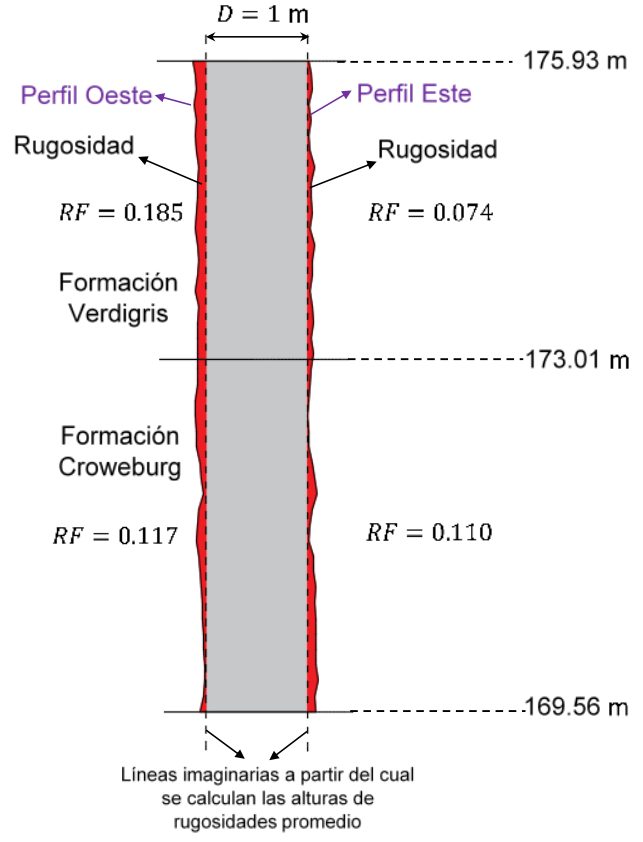

(b)

Figura 6.16. Esquema del ensayo de carga TS-1A: (a) perfil geológico y geometría del ensayo (modificado de Miller 2003), (b) perfil de rugosidad y factor de rugosidad calculado a partir de los datos de Miller (2003).

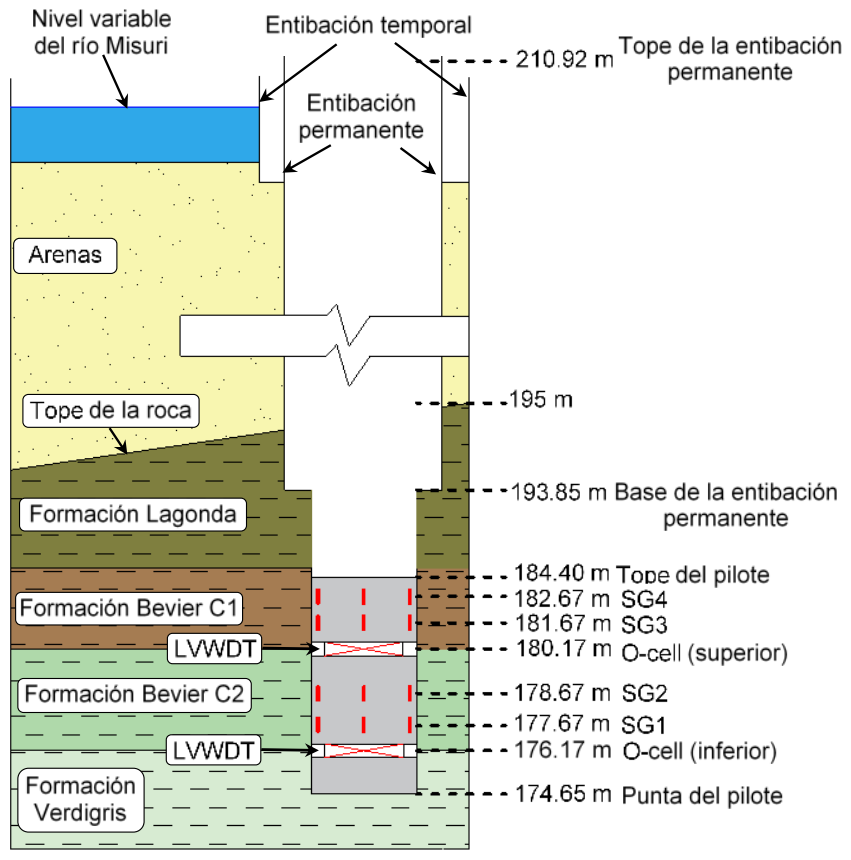

(a)

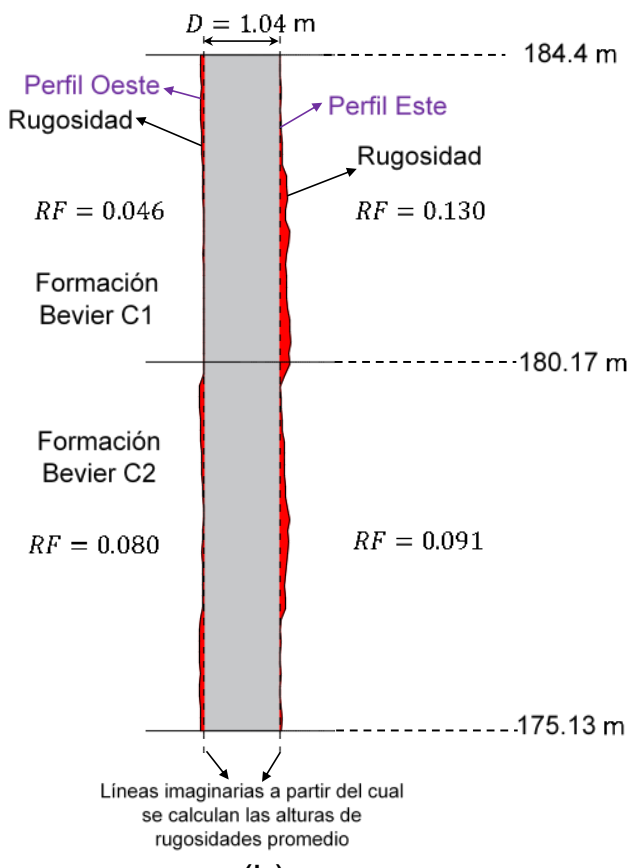

(b)

Figura 6.17. Esquema del ensayo de carga TS-2: (a) perfil geológico y geometría del ensayo (modificado de Miller 2003), (b) perfil de rugosidad y factor de rugosidad calculado a partir de los datos de Miller (2003). 
La prueba de carga fue realizada en tres etapas. En la etapa 1, la célula inferior fue presurizada para cuantificar la resistencia simultánea por punta y por fuste del tramo del pilote situado por debajo de la célula de carga inferior, y utilizando para ello como reacción la resistencia por fuste movilizada en el tramo de pilote ubicado entre las dos células de carga. Luego, en la etapa 2, se despresurizó la célula de carga inferior (simulando un hueco) y se presurizó la célula de carga superior; de esta forma, se midió la resistencia por fuste del tramo de pilote ubicado entre las dos células de carga utilizando como reacción la resistencia por fuste movilizada por encima de la célula de carga superior. Finalmente, en la etapa 3 , fue presurizada nuevamente la célula de carga inferior (movilizándose la resistencia por punta del pilote) antes de la aplicación del siguiente escalón de carga de la célula de carga superior; de este modo, se cuantificó la resistencia por fuste de la parte del pilote ubicada sobre la célula de carga superior, empleando como reacción la resistencia movilizada por punta y fuste del tramo de pilote ubicado debajo de la célula de carga superior (para más detalles, ver Miller 2003).

\subsubsection{Resultados}

En la Figura 6.18 se presentan las curvas de resistencia promedio por fuste $\left(f_{\text {ave }}\right)$ movilizada en cada uno de los estratos de roca de los ensayos de carga TS-1A y TS-2. El cálculo de $f_{\text {ave }}$ fue realizado a partir de los datos experimentales de Miller (2003), usando para ello la diferencia de fuerzas axiales en el pilote entre dos puntos sucesivos de medición, dividida por el área nominal de fuste entre ambos puntos. Además, en los estratos de roca en los que se tiene más de un punto de medición, la resistencia promedio por fuste se computó mediante el promedio de los valores registrados por dichos puntos: e.g., para el ensayo TS-1A, la resistencia promedio por fuste de la Formación Croweburg se calculó como el promedio de los valores de (i) la $f_{\text {ave }}$ movilizada entre la célula de carga y la banda SG2, y (ii) de la $f_{\text {ave }}$ movilizada en el tramo entre las bandas SG2 y SG3. Adicionalmente, debido a que el factor $\alpha_{R F, 0.01 D}$ va asociado a la resistencia promedio para un asiento de $1 \%$ del diámetro del pilote -i.e., 
$f_{a v e, 0.01 D^{-}}$, se extrapolaron las curvas para los casos en que el desplazamiento registrado en la célula Osterberg resultó menor a $0.01 D$ (e.g., ver en la Figura 6.18(a) las líneas segmentadas para la $f_{\text {ave }}$ movilizada en los estratos de la Formación Croweburg y Verdigris).

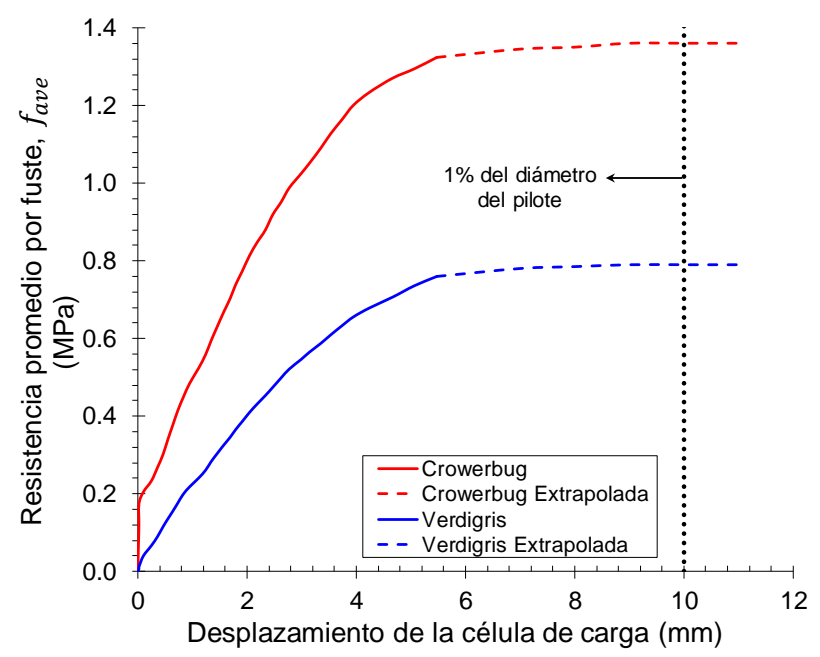

(a)

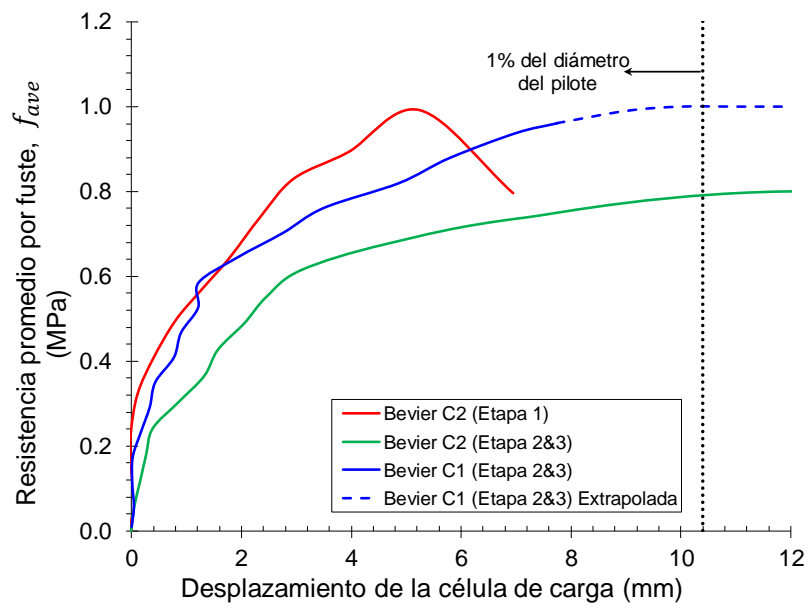

(b)

Figura 6.18. Resistencia promedio por fuste vs desplazamiento de la célula de carga obtenido en los ensayos de carga: (a) ensayo TS-1A, (b) ensayo TS-2 (datos experimentales tomados de Miller 2003).

En la Figura 6.19, se muestran los resultados del factor $\alpha_{R F, 0.01 D}$ calculado para cada uno de los estratos de roca presentes en los ensayos de carga TS-1A y TS-2. Para ello, (i) se calculó la resistencia por fuste movilizada para $0.01 D$-i.e., $f_{a v e, 0.01 D}-$ en cada estrato de roca, (ii) se calculó el factor $\alpha_{R F, 0.01 D}=f_{\text {ave, } 0.01 D} /\left((L / D) \sqrt{\sigma_{c}}\right)$ a partir de los datos de cada ensayo (ver la Tabla 6.4) y (iii) se representaron los valores del $R F$ (en 
particular, el menor de los dos valores correspondientes a los perfiles Este y Oeste) para los tramos de perforación ejecutados en cada estrato) en función de los $\alpha_{R F, 0.01 D}$ calculados para cada uno de los estratos de roca, dentro de las curvas de diseño propuestas en la Figura 6.13.

Tabla 6.4. Datos empleados para la aplicación del factor $\alpha_{R F, 0.01 D}$ a los ensayos de carga TS-1A y TS-2 (datos experimentales tomados de Miller 2003).

\begin{tabular}{|c|c|c|c|c|c|c|c|c|c|}
\hline Tipo de Roca & Ensayo & $R F$ & $\begin{array}{c}f_{\text {ave } 0,01 D} \\
(\mathrm{MPa})\end{array}$ & $\begin{array}{c}\sigma_{c}, \text { Pilote } \\
(\mathrm{MPa})\end{array}$ & $\begin{array}{c}\sigma_{c}, \text { Roca } \\
(\mathrm{MPa})\end{array}$ & $\begin{array}{l}\text { Longitud, } L \\
\quad(\mathrm{~m})\end{array}$ & $\begin{array}{l}\text { Diámetro, } D \\
(\mathrm{~m})\end{array}$ & $\begin{array}{c}L / D \\
- \\
\end{array}$ & Tipo de Ensayo \\
\hline $\begin{array}{c}\text { Caliza } \\
\text { (Formación Verdigris) }\end{array}$ & TS-1A & 0.074 & 0.790 & 28.1 & 1.21 & 2.92 & 1.00 & 2.92 & \multirow{4}{*}{$\begin{array}{l}\text { Ensayo de carga } \\
\text { (O-cell) }\end{array}$} \\
\hline $\begin{array}{c}\text { Caliza } \\
\text { (Formación Croweburg) }\end{array}$ & TS-1A & 0.110 & 1.360 & 28.1 & 1.72 & 2.51 & 1.00 & 2.51 & \\
\hline $\begin{array}{c}\text { Lutita } \\
\text { (Formación Bevier C1) }\end{array}$ & TS-2 & 0.046 & 0.970 & 28.1 & 3.81 & 4.23 & 1.04 & $4.07^{\star}$ & \\
\hline $\begin{array}{c}\text { Lutita } \\
\text { (Formación Bevier C2) }\end{array}$ & TS-2 & 0.080 & $0.900^{* *}$ & 28.1 & 3.00 & 4.00 & 1.04 & $3.85^{\star}$ & \\
\hline
\end{tabular}

Los resultados se muestra en la Figura 6.19, donde puede observarse que los valores obtenidos para el factor $\alpha_{R F, 0.01 D}$ reproducen adecuadamente las predicciones de la curva sugerida para el material más débil (roca o pilote) con $\sigma_{c}<10 \mathrm{MPa}$. Se observa también que dichos valores están ligeramente por encima de la curva propuesta, excepto (quizás) para el estrato de lutita de la Formación Bevier C2; esto puede deberse a que se empleó el promedio de la resistencia por fuste movilizada después de la realización de las tres etapas del ensayo, y a que además se cambió la dirección de aplicación de la carga. (Recuérdese que en la Etapa 1 del ensayo TS-2, la carga sobre el estrato de la Formación Bevier C2 se aplicó hacia arriba con la O-cell inferior, mientras que en las Etapas 2 y 3, la carga se aplicó sobre este mismo estrato, pero hacia abajo con la O-cell superior). Además, esto se correspondería con lo reportado por Asadi et al. (2013), quienes indicaron que, por ejemplo, la resistencia al corte movilizada de juntas roca-roca depende de la dirección de la carga aplicada (ver Capítulo 2, Sección 2.2.1.6). En cualquier caso, los resultados presentados en la Figura 6.19 indican que la curva propuesta para la estimación de la resistencia promedio por fuste para un asiento 
de $0.01 D$ para materiales con $\sigma_{c}<10 \mathrm{MPa}$ es apropiada y posiblemente conservadora en muchos casos.

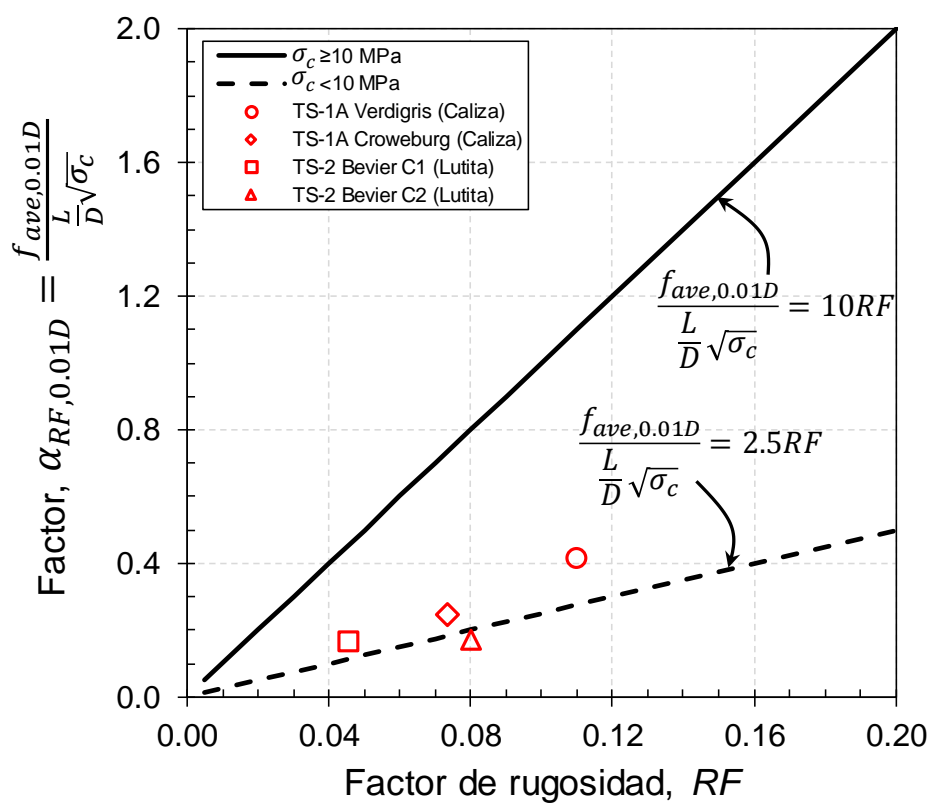

Figura 6.19. Resultados de la aplicación del factor $\alpha_{R F, 0.01 D}$ en los ensayos de carga TS-1A y TS-2 (los puntos de color rojo representan el material el más débil -roca o pilote- presenta $\sigma_{c}<$ $10 \mathrm{MPa})$.

\subsubsection{Implicación práctica}

Según se presentó en la Sección 6.3, las diversas formulaciones disponibles proporcionan una gran dispersión de resultados en la estimación de la resistencia por fuste de pilotes empotrados en roca. En consecuencia, el diseño de este tipo de cimentación suele ser muy conservador (con sus correspondientes incrementos del coste asociado en obra). La Figura 6.20 muestra la longitud de perforación prevista para cada pilote que soporta las pilas del viaducto, antes y después de realizar los ensayos de carga TS-1A y TS-2, y la Tabla 6.5 presenta una estimación del ahorro en los trabajos de perforación de dichos pilotes según lo reportado por Miller (2003) (estos costos no incluyen gastos de hormigonado, acero de refuerzo, etc.). Los resultados presentados reflejan una notable incidencia económica de realizar un análisis más detallado de los pilotes empotrados en roca, al que habría que añadir una posible disminución en los tiempos de ejecución de la obra, volumen de hormigón, acero de refuerzo, etc. 


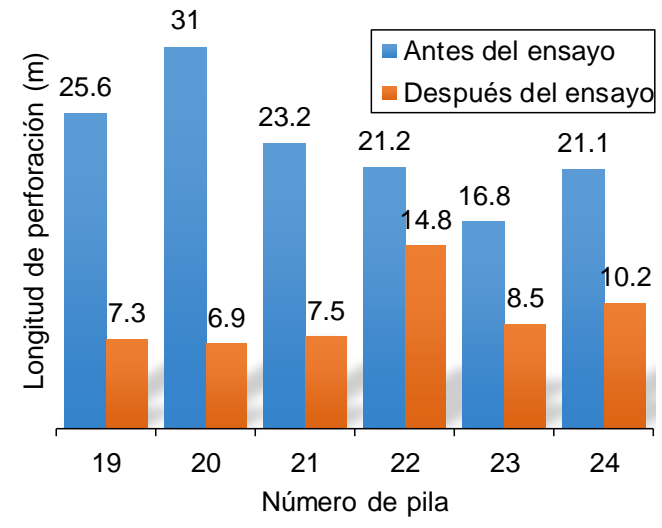

Figura 6.20. Longitud de perforación en roca por cada pilote de soporte de las pilas del viaducto (datos del proyecto tomados de Miller 2003).

Tabla 6.5. Costo total ahorrado en la perforación de los pilotes de soporte de las pilas del viaducto realizado en Lexington (datos del proyecto tomados de Miller 2003).

\begin{tabular}{|c|c|c|c|c|c|c|}
\hline \multirow[t]{2}{*}{ Pila } & \multicolumn{2}{|c|}{$\begin{array}{c}\text { Longitud de excavación } \\
\text { en roca }(\mathrm{m})\end{array}$} & \multirow{2}{*}{$\begin{array}{l}\text { Diferencia de } \\
\text { longitud } \\
\text { por pilote }(\mathrm{m})\end{array}$} & \multirow{2}{*}{$\begin{array}{l}\text { Número } \\
\text { de pilotes } \\
\text { por pila }\end{array}$} & \multirow{2}{*}{$\begin{array}{c}\text { Diferencia de } \\
\text { longitud } \\
\text { total }(\mathrm{m})\end{array}$} & \multirow{2}{*}{$\begin{array}{c}\text { Costo Total } \\
\text { ahorrado* } \\
(\$)\end{array}$} \\
\hline & Antes & Después & & & & \\
\hline 19 & 25.6 & 7.3 & 18.3 & 6 & 109.8 & \\
\hline 20 & 31 & 6.9 & 24.1 & 8 & 192.8 & \\
\hline 21 & 23.2 & 7.5 & 15.7 & 15 & 235.5 & 18194700 \\
\hline 22 & 21.2 & 14.8 & 6.4 & 8 & 51.2 & $10194 / 0.00$ \\
\hline 23 & 16.8 & 8.5 & 8.3 & 6 & 49.8 & \\
\hline 24 & 21.1 & 10.2 & 10.9 & 6 & 65.4 & \\
\hline
\end{tabular}

\subsubsection{Ensayo de carga realizado en Kansas}

Para mejorar el flujo vehicular en la intersección Grandview Triangle -una de las intersecciones con mayor intensidad de tráfico en la ciudad de Kansas del estado de Missouri (EE.UU)-, el DTM proyectó la construcción de varios viaductos elevados. Uno de estos viaductos es el A6252 (ver la Figura 6.21), en el cual se planteó el uso de pilotes empotrados en roca como cimentación de sus diferentes pilas. Se realizó un ensayo de carga (GT) pra determinar la resistencia por fuste de cada uno de los estrados rocosos (Formación Chanute, Formación Cement City, etc.) en los que penetran los pilotes de la cimentación (Miller 2003).

El pilote se perforó inicialmente con la ayuda de entibación y mediante la perforación con barrenas de alta capacidad para rocas (Barrena Bullet Tooth Rock, ver el Anexo A). 
Posteriormente, la perforación en los estratos de roca se realizó empleando de manera alternada las herramientas de perforación Barrena y Core Barrel (Miller 2003).

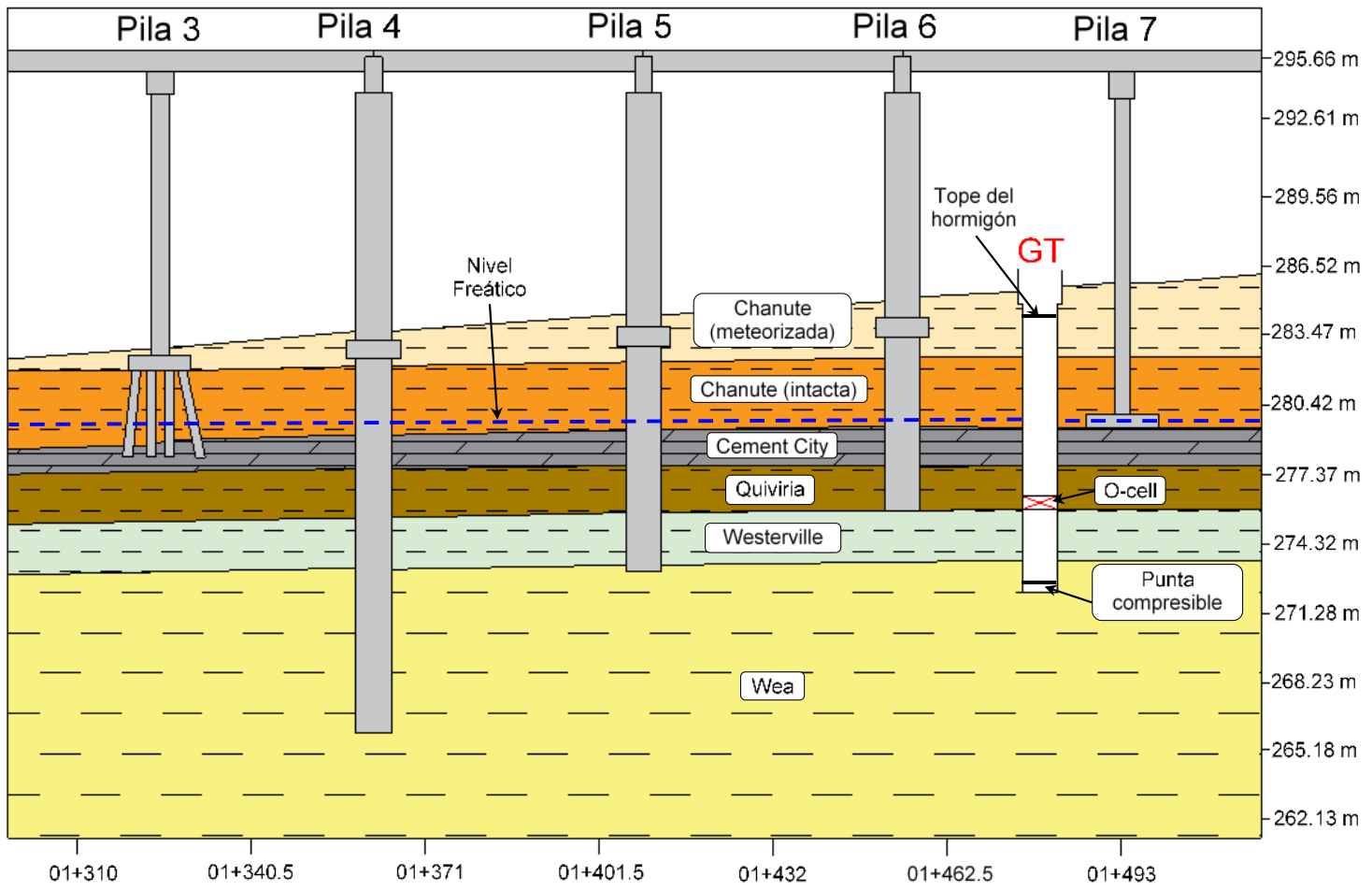

Figura 6.21. Vista longitudinal del viaducto A6252: perfil geológico, ubicación de las pilas (3-7) de dimentación y localización de la prueba de carga GT (modificado de Miller 2003).

\subsubsection{Ensayo de carga GT}

Este ensayo de carga se llevó a cabo entre las pilas 6 y 7 del viaducto A6252 (ver la Figura 6.21), y su profundidad se seleccionó con objeto de determinar la resistencia por fuste de la mayoría de los estratos de roca que serían atravesados para los pilotes proyectados. A tales efectos, se usó una célula Osterberg de $870 \mathrm{~mm}$ de diámetro, localizada hacia el tercio inferior del pilote (ver la Figura 6.22), y se empleó una inclusión compresible en la punta compuesta de espuma de poliestireno de $76 \mathrm{~mm}$ de espesor y localizada en medio de dos placas metálicas. La prueba de carga se realizó mediante la presurización de la célula Osterberg, en diferentes escalones de carga y descarga en ambas direcciones. En este caso, debido a la presencia de la inclusión compresible en la punta y la no existencia de material sobre la cabeza del pilote, es posible asumir que 
la carga aplicada es soportada solo por la componente movilizada de resistencia por fuste a lo largo del pilote del ensayo (Miller 2003).

El perfil geológico, las dimensiones y otros aspectos del ensayo, así como la instrumentación empleada, entre otros, se muestran en la Figura 6.22(a). Los perfiles de rugosidad de la pared de la perforación, obtenidos a partir de un registro Caliper, así como los factores de rugosidad $(R F)$ de cada estrato calculados para cada perfil (Oeste y Este) se presentan en la Figura 6.22(b) (para detalles adicionales ver Miller 2003).

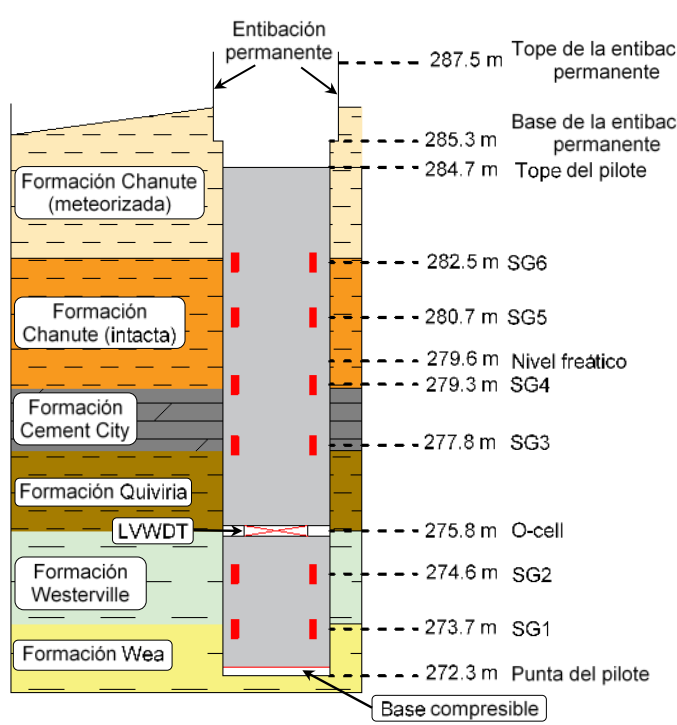

(a)

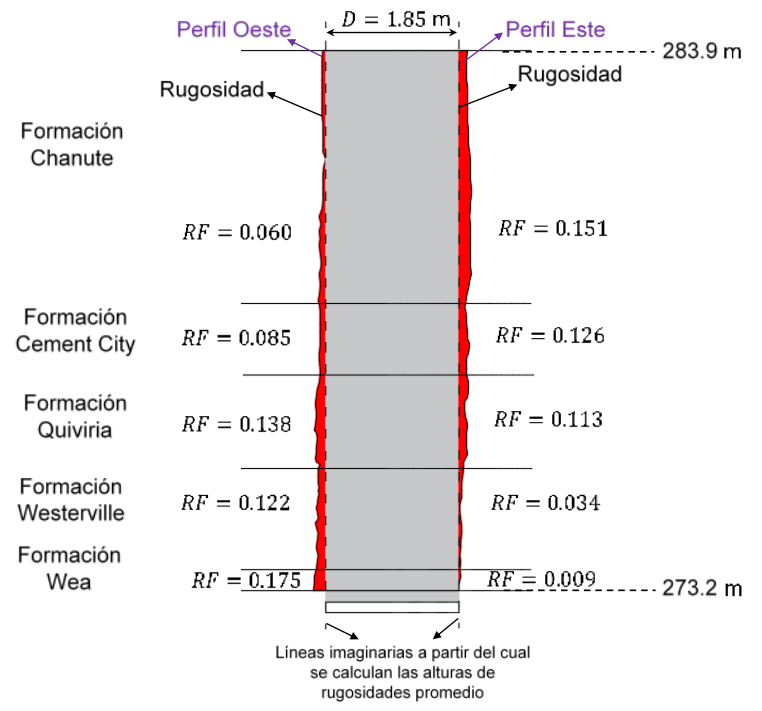

(b)

Figura 6.22. Esquema del ensayo de carga GT: (a) perfil geológico y geometría del ensayo (modificado de Miller 2003), (b) detalle de rugosidad del hoyo y factor de rugosidad calculado a partir de los datos de Miller (2003).

\subsubsection{Resultados}

En la Figura 6.23 se muestra la resistencia promedio por fuste movilizada para cada uno de los estratos de roca presentes en el ensayo de carga GT en función del desplazamiento registrado en la célula de carga. La estimación de $f_{\text {ave }}$ fue similar a la empleada en los ensayos de carga TS-1A y TS-2, y de nuevo se extrapolaron las curvas hasta alcanzar un desplazamiento de la célula Osterberg de $0.01 D$. Además, Miller (2003) reportó que, debido al inadecuado registro de la banda SG3 durante el ensayo, no fue posible estimar de forma independiente la resistencia movilizada en los estratos de la Formaciónes Cement City y Quiviria. A tales efectos, Miller (2003) sugirió un valor 
de resistencia por fuste movilizado para la Formación Quiviria de modo que, a partir de éste, puede inferirse las curvas extrapoladas para dichas Formaciones Quiviria y Cement City (ver líneas rojas continua y segmentadas en la Figura 6.23).

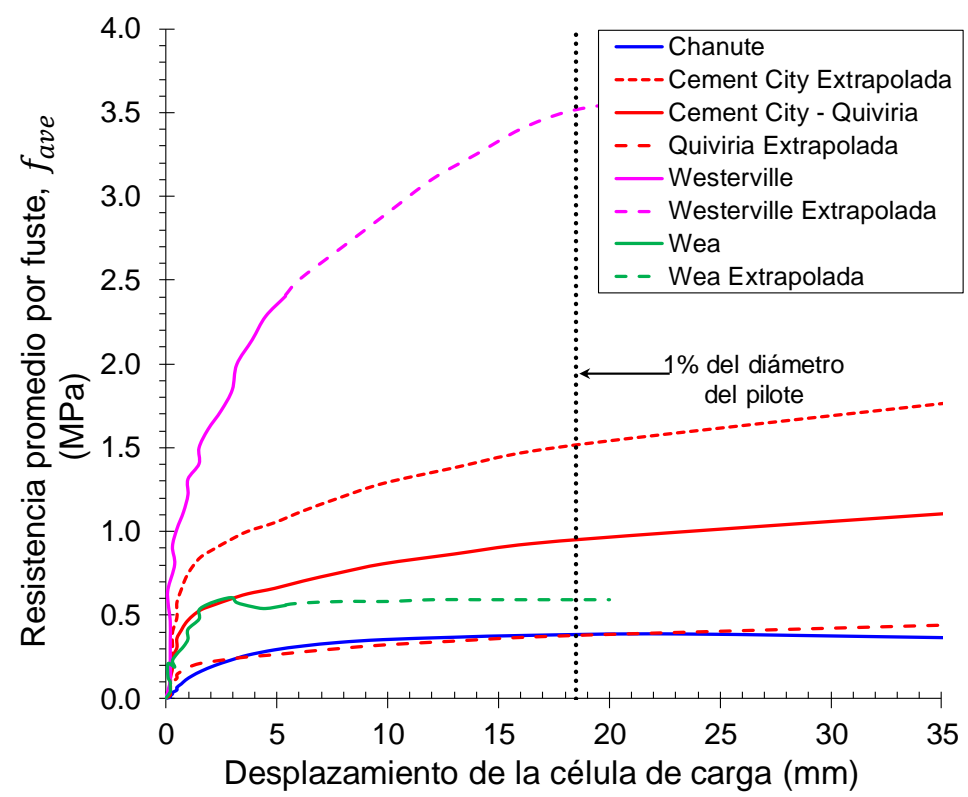

Figura 6.23. Resistencia promedio por fuste vs desplazamiento de la célula de carga obtenido en el ensayo de carga GT (datos experimentales tomados de Miller 2003).

En la Tabla 6.6 y en la Figura 6.24 se muestran los datos empleados para la estimación del factor $\alpha_{R F, 0.01 D}$ hallado para cada uno de los estratos de roca del ensayo de carga GT, así como los resultados obtenidos. El procedimiento de cálculo fue similar al empleado en los ensayos TS-1A y TS-2, empleando las curvas de $f_{\text {ave }}$ mostradas en la Figura 6.23 y el menor valor de $R F$ obtenido para estrato de roca.

Tabla 6.6. Datos empleados para la aplicación del factor $\alpha_{R F, 0.01 D}$ al ensayo de carga GT (datos experimentales tomados de Miller 2003).

\begin{tabular}{|c|c|c|c|c|c|c|c|c|c|}
\hline Tipo de Roca & Ensayo & $R F$ & $\begin{array}{c}f_{\text {ave } 0,01 D} \\
\text { (MPa) }\end{array}$ & $\begin{array}{c}\sigma_{c}, \text { Pilote } \\
(\mathrm{MPa})\end{array}$ & $\begin{array}{c}\sigma_{c}, \text { Roca } \\
(\mathrm{MPa})\end{array}$ & $\begin{array}{l}\text { Longitud, } L \\
\quad(\mathrm{~m})\end{array}$ & $\begin{array}{l}\text { Diámetro, } D \\
(\mathrm{~m})\end{array}$ & $\begin{array}{c}L / D \\
-\end{array}$ & Tipo de Ensayo \\
\hline $\begin{array}{c}\text { Lutita } \\
\text { (Formación Chanute) }\end{array}$ & GT & 0.060 & 0.30 & 41.4 & $0.81^{*}$ & 5.33 & 1.85 & 2.88 & \multirow{5}{*}{$\begin{array}{c}\text { Ensayo de carga } \\
\text { (O-cell) }\end{array}$} \\
\hline $\begin{array}{c}\text { Caliza } \\
\text { (Formación Cement City) }\end{array}$ & GT & 0.085 & 1.53 & 41.4 & 37.00 & 1.52 & 1.85 & 0.82 & \\
\hline $\begin{array}{c}\text { Lutita } \\
\text { (Formación Quiviria) }\end{array}$ & GT & 0.113 & $0.39^{* *}$ & 41.4 & 1.43 & 1.98 & 1.85 & 1.07 & \\
\hline $\begin{array}{c}\text { Lutita } \\
\text { (Formación Westerville) }\end{array}$ & GT & 0.034 & $3.50^{\star *}$ & 41.4 & 63.08 & 2.13 & 1.85 & 1.15 & \\
\hline $\begin{array}{c}\text { Lutita } \\
\text { (Formación Wea) }\end{array}$ & GT & 0.009 & 0.59 & 41.4 & 2.31 & 1.40 & 1.85 & 0.76 & \\
\hline
\end{tabular}




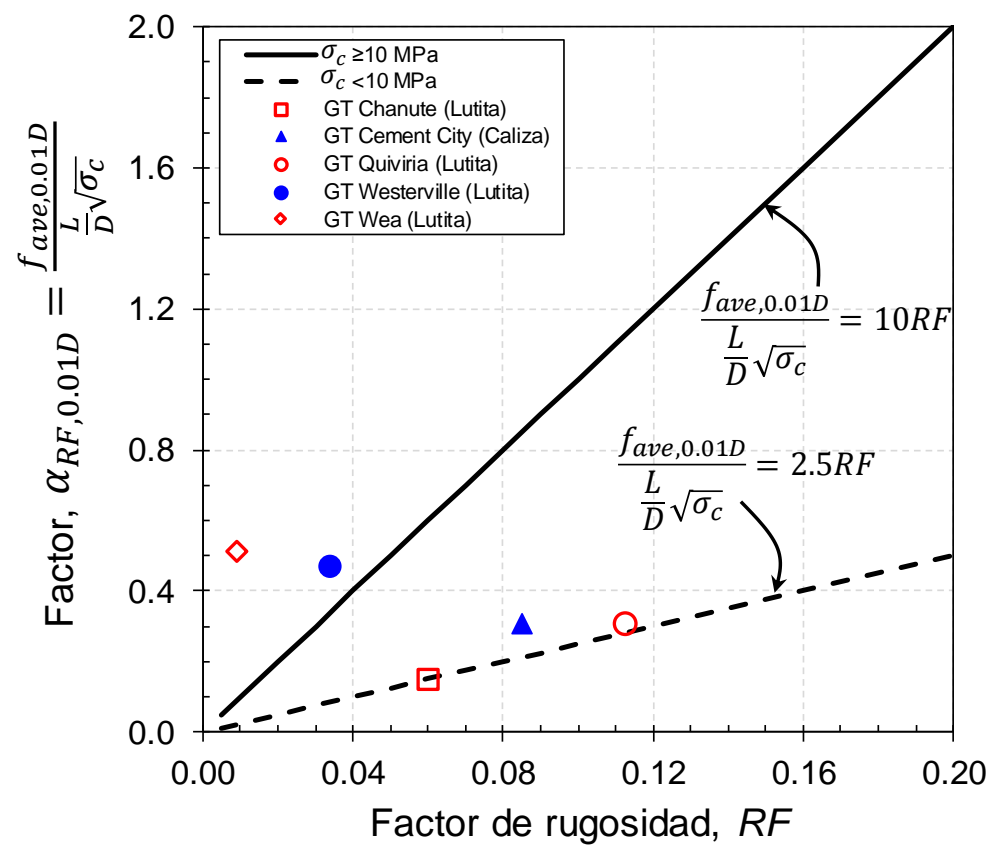

Figura 6.24. Resultados de la aplicación del factor $\alpha_{R F, 0.01 D}$ en el ensayo de carga GT (los puntos de color azul y rojo representan los casos en que el material el más débil -roca o pilotepresenta $\sigma_{c} \geq 10 \mathrm{MPa} 0 \sigma_{c}<10 \mathrm{MPa}$, respectivamente).

Como puede observarse en la Figura 6.24, los resultados obtenidos para las lutitas de la Formación Quiviria y Chanute se aproximan bien a la curva de diseño propuesta para la estimación de la resistencia promedio por fuste en materiales (el más débil entre la roca o pilote) con $\sigma_{c}<10 \mathrm{MPa}$. De la misma forma, el valor obtenido para la lutita de la Formación Westerville es acorde para la curva propuesta en la estimación de $f_{\text {ave }}$ en materiales con $\sigma_{c} \geq 10 \mathrm{MPa}$. Por otra parte, se observa que el valor de $\alpha_{R F, 0.01 D}$ obtenido para la lutita de la Formación Wea es muy superior al que se esperaría para un coeficiente de rugosidad tan bajo; sin embargo, esto hecho podría atribuirse a (i) un posible aporte de la cohesión en la interfaz roca-pilote, o (ii) a que su valor movilizado responda más a un valor de $R F$ mayor al considerado (recuérdese que se emplea el menos rugoso de los dos valores hallados; ver la Figura 6.22(b)).

Finalmente, se observa que el valor de $\alpha_{R F, 0.01 D}$ medido para la caliza de la Formación Cement City es muy inferior a las predicciones con la curva de diseño propuesta para materiales con $\sigma_{c} \geq 10 \mathrm{MPa}$. La razón de este resultado no está clara, si bien éste es uno de los estratos en los que Miller (2003) reportó problemas en la instrumentación y 
en los que la $f_{\text {ave }}$ movilizada se obtuvo por extrapolación. Quizás, el bajo valor de $\alpha_{R F, 0.01 D}$ de la Formación Cement City pueda atribuirse a la anisotropía y grietas observadas durante su excavación, y a través de las cuales se evidenció la entrada de agua hacia la excavación durante la construcción del pilote, lo que podría haber afectado la calidad de la interfaz.

\subsubsection{Implicación práctica}

En lo referente a la influencia de costos asociados a esta obra, la investigación de Miller (2003) no detalla las longitudes proyectadas para los pilotes del viaducto A6252, antes y después de la realización del ensayo de carga; no obstante, sí presenta información sobre una diferencia de longitud de perforación total de $65.2 \mathrm{~m}$ para los pilotes del proyecto, con un costo total ahorrado de $214000.00 \$$. 
Capítulo 6: Formulación para la estimación de la resistencia por fuste de pilotes empotrados en roca considerando la rugosidad de la excavación 


\section{Conclusiones y líneas de investigación futuras}

\subsection{Conclusiones}

El efecto de la rugosidad en el contacto pilote-roca sobre la resistencia por fuste de pilotes se analizó a través de cuatro componentes principales:

- Modelos a pequeña escala, Capítulo 3.

- Modelos a gran escala, Capítulo 4.

- Ensayos de centrífuga, Capítulo 5.

- Contraste, comparación y propuesta de diseño, Capítulo 6.

\subsubsection{Modelos a pequeña escala}

En el Capítulo 3 se presentaron los modelos numéricos desarrollados, mediante el Método de Elementos Discretos (MED) y su implementación en PFC ${ }^{2 D}$, para analizar el comportamiento al corte mediante ensayos de corte directo en juntas roca-hormigón con condiciones de borde de (i) carga normal constante (CNL) y (ii) rigidez normal constante (CNS). Dichos modelos fueron calibrados y empleados para reproducir el comportamiento experimental de ensayos de corte directo en juntas gneis-hormigón y arenisca-hormigón realizados por Gutiérrez (2013) y Gu et al. (2003), respectivamente.

Los resultados obtenidos indican que, cuando las micropropiedades empleadas en los modelos con MED son calibradas apropiadamente -a través de ensayos de compresión uniaxial y de corte directo (en juntas planas)-, el método Shear Box Genesis (SBG) propuesto por Bahaaddini et al. (2013b) permite reproducir satisfactoriamente el comportamiento al corte en juntas roca-hormigón sin cohesión, (i) con condición de borde CNL y CNS, (ii) en rocas de diferente resistencia (e.g., gneis y arenisca), y (iii) con diferente nivel de rugosidad en la interfaz roca-hormigón (e.g., juntas lisas o juntas con diferentes perfiles de rugosidad de geometría triangular simétrica con diferente 
ángulo base). En particular, los modelos numéricos con PFC ${ }^{2 \mathrm{D}}$, y sus modelos de contacto FJCM -para simular el comportamiento macroscópico de la roca y del hormigón- y SJCM -para la interfaz de ambos materiales-, pueden reproducir adecuadamente el comportamiento al corte en juntas roca-hormigón sin cohesión -i.e., resistencia al corte pico, rigidez al corte, comportamiento post-pico, etc.-, así como el modo de rotura (deslizamiento a lo largo de la junta o rotura de las asperidades) observado en ensayos de corte directo en juntas gneis-hormigón y arenisca-hormigón realizados por Gutiérrez (2013) y Gu et al. (2003), respectivamente; especialmente bajo condición de borde CNL.

Adicionalmente, los resultados de (i) los ensayos experimentales de Gutiérrez (2013) y Gu et al. (2003) y (ii) de los ensayos numéricos de esta investigación, demuestran que (además de la rugosidad de la interfaz roca-hormigón) el comportamiento al corte viene también afectado por la condición de borde (CNL o CNS) empleada durante la realización del ensayo. En consecuencia, la selección apropiada del tipo de ensayo trae implicaciones prácticas de gran relevancia, por ejemplo, para el estudio del efecto de la rugosidad sobre la resistencia de pilotes empotrados en roca, que se ha demostrado que debe ser estudiado mediante ensayos de corte directo bajo condición de borde CNS. De esta manera se consigue (i) evitar subestimar la resistencia al corte en la interfaz roca-hormigón y (ii) realizar diseños óptimos, económicos y más ajustados a las solicitaciones de las cargas actuantes.

\subsubsection{Modelos a gran escala}

En el Capítulo 4 se presentaron los modelos numéricos desarrollados, mediante el Método de Elementos Discretos (MED) y el Método de Elementos Finitos (MEF) y su implementación en $\mathrm{PFC}^{3 \mathrm{D}}$ y Abaqus, respectivamente, para analizar efecto de la rugosidad en la interfaz roca-pilote sobre el comportamiento por fuste de pilotes empotrados en roca. Los resultados numéricos demuestran que el comportamiento por 
fuste de pilotes empotrados en roca depende, en gran medida, de la rugosidad de la interfaz roca-pilote. $Y$, además, demuestran que los modelos numéricos elaborados con PFC $^{3 D}$ pueden ser empleados para (i) reproducir el comportamiento carga-asiento de pilotes empotrados en roca considerando diferentes niveles de rugosidad en la interfaz roca-pilote, (ii) para estimar su resistencia por fuste, y (iii) para analizar el mecanismo de transferencia de carga hacia la roca circundante (i.e., la distribución de esfuerzo axial, radial y trasversal, modo de fallo y propagación de grietas, zona de influencia, etc.).

Los resultados obtenidos con $\mathrm{PFC}^{3 \mathrm{D}}$ también sugieren que, para la transferencia de carga de pilotes empotrados en roca, la distribución de esfuerzos alrededor de la roca circundante es similar a aquella producida en la teoría de expansión de cavidades, con una tendencia que depende, a su vez, del nivel de rugosidad en la interfaz roca-pilote, y de la localización en la que se estiman los esfuerzos movilizados. Además, a partir de los resultados numéricos se evidenció que la zona de influencia para la transferencia de carga del pilote hacia la roca circundante tiene un alcance de, aproximadamente, un radio del pilote medido desde la interfaz roca-pilote.

Por otra parte, el análisis (a partir de los modelos con $\mathrm{PFC}^{3 \mathrm{D}}$ ) de la propagación del número de grietas, y de su localización, permite inferir que el criterio comúnmente empleado para la estimación de la resistencia por fuste de pilotes empotrados en roca considerando un asiento en cabeza equivalente al 1\% del diámetro del pilote, representa un criterio apropiado. El motivo es que, para asientos en cabeza menores a dicho valor, se puede evitar una degradación significativa de las asperidades en la interfaz rocapilote, especialmente para excavaciones muy rugosas.

Las principales ventajas de los modelos numéricos de pilotes empotrados en roca desarrollados en PFC en esta tesis doctoral es que (i) son más rápidos y económicos que los ensayos in-situ, y (ii) que son de fácil calibración mediante la realización de ensayos de laboratorio (compresión uniaxial y corte directo). Adicionalmente, otra 
ventaja es que estos modelos numéricos con MED permiten cuantificar la influencia de la rugosidad sobre la resistencia por fuste de pilotes empotrados en roca, de una forma más detallada que la que se podría realizar mediante muchas de las formulaciones empíricas existentes en la literatura.

Por otra parte, la comparación de resultados entre los modelos con el MEF y con el MED demostraron que los modelos numéricos con el MEF en Abaqus también puede ser una herramienta alternativa que puede ser empleada para analizar el comportamiento resistente de pilotes empotrados en roca, incluso para realizar diseños preliminares como los realizados previo a la ejecución de los ensayos de centrífuga de esta tesis doctoral. Además, los resultados numéricos con el MEF también evidenciaron el efecto de la rugosidad sobre el comportamiento resistentes de pilotes empotrados en roca no sólo cuando se considera sólo la componente de fuste del pilote, sino también cuando se moviliza de forma simultánea de resistencia por punta y por fuste del pilote.

\subsubsection{Ensayos de centrífuga}

En el Capítulo 5 se presentó el desarrollo, realización y resultados de los ensayos de centrífuga ejecutados en esta tesis doctoral para evaluar el efecto de la rugosidad sobre el comportamiento de pilotes empotrados en roca. Para ello: (i) se preparó, a partir de la mezcla de arena, cemento, bentonita y agua, una pseudo-roca de resistencia a la compresión uniaxial promedio de $1.1 \mathrm{MPa}$, y (ii) se fabricaron pilotes de aluminio con diferente grado de rugosidad, cuya instrumentación se realizó mediante el uso de fibra óptica, lo que permitió cuantificar la distribución de la fuerza axial y de la resistencia promedio por fuste movilizada a lo largo de los pilotes ensayados.

Los resultados obtenidos en estos ensayos de centrífuga demuestran que los mismos son una herramienta apropiada y de bajo costo para reproducir el comportamiento bajo carga axial de pilotes empotrados en roca. En particular, y según fue validado por los modelos numéricos con MEF en Abaqus, los resultados demuestran que los ensayos 
de centrífuga ejecutados en pilotes instrumentados con fibra óptica son adecuados para analizar (i) la respuesta carga-asiento de pilotes empotrados en roca, y (ii) el rango de valores promedio de resistencia por fuste típicos obtenidos a escala del prototipo, en pilotes de geometría y rugosidad análoga, y que fueron perforados en roca de resistencia similar; y, a partir de ello, es posible evaluar el efecto de la rugosidad sobre el comportamiento al fuste de pilotes empotrados en roca.

\subsubsection{Contraste, comparación y propuesta de diseño}

En el Capítulo 6, se presentaron las formulaciones existentes en la literatura para la estimación de la resistencia por fuste de pilotes empotrados en roca. Se observó que a pesar de la influencia que tiene la rugosidad sobre la resistencia por fuste de los pilotes en roca, la mayoría de formulaciones existentes no consideran la influencia de este factor, proporcionando métodos predictivos que sólo dependen de la resistencia a compresión simple del material intacto más débil (roca o pilote o en algunos casos del macizo rocoso). Además, a pesar de la existencia de algunas formulaciones en las cuales se considera la rugosidad de la excavación, se observa que el uso de las mismas arroja una gran dispersión en la estimación de la resistencia por fuste, especialmente para cavidades muy rugosas.

La comparación de los resultados de resistencia promedio por fuste obtenidos con los modelos numéricos y de ensayos de centrifuga de esta tesis doctoral, con aquellos obtenidos con algunas de las formulaciones publicadas en la literatura, muestran que $\mathrm{PFC}^{3 \mathrm{D}}$ y los ensayos de centrifuga son capaces de reproducir el rango de resultados obtenidos en ensayos de carga realizados in-situ (o mediante formulaciones analíticas cuando las mismas pueden ser empleadas) para pilotes de rugosidad y resistencia a la compresión uniaxial similar.

Por otra parte, la recopilación de los diversos trabajos publicados en la literatura (ver el Capítulo 6) sugiere que los pilotes perforados en rocas de baja a mediana resistencia, y 
con herramientas de perforación estándar, tienden a presentar paredes relativamente lisas, a menos que su rugosidad sea incrementada de manera artificial mediante el uso de herramientas especiales.

Basándose en los resultados obtenidos por medio de las cuatro componentes principales comentadas previamente -i.e., modelos a pequeña escala (ver el Capítulo 3), modelos a gran escala (ver el Capítulo 4), ensayos de centrífuga (ver el Capítulo 5), y el contraste, comparación y propuesta de diseño (ver el Capítulo 6)- se propone un nuevo factor $\alpha_{R F, 0.01 D}$ para la estimación de la resistencia promedio por fuste de pilotes empotrados en roca asociada a un asiento en cabeza de $1 \% D\left(f_{\text {ave, } 0.01 D}\right)$, considerando (i) la rugosidad en la interfaz roca-pilote caracterizada por medio del factor de rugosidad $R F$ propuesto por Horvath et al. (1983), y (ii) la resistencia a compresión simple del material intacto menos resistente (roca o pilote), y dentro de un rango de resistencia de $0.5-60 \mathrm{MPa}$.

\subsection{Líneas de investigación futuras}

Durante el desarrollo de esta tesis doctoral se investigó el efecto de la rugosidad sobre la resistencia por fuste de pilotes empotrados en roca. Sin embargo, hay algunos aspectos que resultaron interesantes o que requieren de mayor investigación o mejoras $y$, en consecuencia, que pueden ser desarrollados como futuros trabajos de investigación. A continuación, se presentan futuras líneas de investigación que se sugieren como continuación de esta tesis doctoral:

- Realizar un estudio integral que permita acoplar, para un mismo caso, ensayos in-situ (con medida de la rugosidad de la cavidad del pilote), ensayos de laboratorio y modelos numéricos. Se pretende poder evaluar así, y para pilotes empotrados en roca, el efecto de la rugosidad sobre la resistencia movilizada solo por fuste o de manera simultánea por punta y fuste. 
- Realizar ensayos de centrífuga y modelos numéricos de pilotes bajo carga axial empotrados en roca considerando: (i) diferentes niveles y tipos de rugosidad (sinusoidal, irregular, entre otras) en la interfaz roca-pilote, (ii) rocas de diferente resistencia, (iii) pilotes de diferente longitud y diámetro, etc. y considerando también tanto (iv) sólo la componente por fuste, y (v) la componente por fuste y punta de manera simultánea. Adicionalmente, para dichos ensayos de centrífuga, se sugiere cuidar el diseño del mecanismo de aplicación de carga axial para evitar la excentricidad durante la aplicación de la carga en cabeza del pilote.

- Realizar nuevos ensayos in-situ, ensayos de centrífuga y modelos numéricos que permitan evaluar el efecto de la rugosidad sobre el comportamiento resistente de grupos de pilotes empotrados en roca considerando los aspectos mencionados en el ítem anterior. 


\section{Referencias}

AASHTO (American Association of State Highway and Transportation Officials). (2008). LRFD bridge design specifications. Washington, DC.

Álvarez-Botero, G., Barón, F.E., Canom C.C., Sosa, O., Varón, M (2017). Optical sensing using Fiber Bragg gratings: Fundamentals and Applications. IEEE Instrum. Meas. Mag., 20(2): 33-38. https://doi.org/10.1139/t94-048.

Arioglu, E., Seker, P.T., Gokce, H.B. (2018). Discussion on empirical methods for determining shaft bearing capacity of semi-deep foundations socketed in rocks. J. Rock Mech. Geotech. Eng., 10(3): 603-606. https://doi.org/10.1016/j.jrmge.2018.01.003.

Asadi, M.S., Rasouli, V., Barla, G. (2012). A bonded particle model simulation of shear strength and asperity degradation for rough rock fractures. Rock Mech. Rock. Eng., 45: 649-675. https://doi.org/10.1007/s00603-012-0231-4.

Asadi, M.S., Rasouli, V., Barla, G. (2013). A laboratory shear cell used for simulation of shear strength and asperity degradation of rough rock fractures. Rock Mech. Rock. Eng., 46: 683-699. https://doi.org/10.1007/s00603-012-0322-2.

Bahaaddini, M., 2017. Effect of boundary condition on the shear behaviour of rock joints in the direct shear test. Rock Mech. Rock. Eng., 1-15. https://doi.org/10.1007/s00603016-1157-z.

Bahaaddini, M., Hagan, P.C., Mitra, R. Hebblewhite, B.K. (2013a). Numerical investigation of asperity degradation in the direct shear test of rock joints. ISRM International Symposium-EUROCK 2013. Wroclaw, Poland, 391-397.

Bahaaddini, M., Hagan, P.C., Mitra, R., Hebblewhite, B.K. (2014). Scale effect on the shear behaviour of rock joints based on a numerical study. Eng. Geol., 181: 212-223. https://doi.org/10.1016/i.enggeo.2014.07.018.

Bahaaddini, M., Hagan, P.C., Mitra, R., Khosravi, M.H. (2016). Experimental and numerical study of asperity degradation in the direct shear test. Eng. Geol., 204: 41-52. https://doi.org/10.1016/i.enggeo.2016.01.018.

Bahaaddini, M., Sharrock, G., Hebblewhite, B.K. (2013b). Numerical direct shear tests to model the shear behaviour of rock joints. Comput. Geotech., 51: 101-115. https://doi.org/10.1016/i.compgeo.2013.02.003.

Bandis, S.C., Lumsden, A.C., Barton, N.R. (1983). Fundamentals of rock joint deformation. Int. J. Rock Mech. Min. Sci. Geomech. Abstr., 20: 249-268. https://doi.org/10.1016/0148-9062(83)90595-8.

Barton, N.R. (1976). The shear strength of rock and rock joints. Int. J. Rock Mech. Min. Sci., 13, 255-279. https://doi.org/10.1016/0148-9062(76)90003-6.

Barton, N.R., Choubey, V. (1977). The shear strength of rock joints in theory and practice. Rock Mech. Rock. Eng., 10: 1-54. https://doi.org/10.1007/BF01261801. 
Basarkar, S.S., Dewaikar, D.M. (2006). Load transfer characteristics of socketed piles in Mumbai region. Soils Found., 46(2): 247-257. https://doi.org/10.3208/sandf.46.247.

Baycan, S. (1996). Field performance of expansive anchors and piles in rock. Ph.D. Thesis. Department of Civil Engineering, Monash University, Melbourne, Australia.

Benmokrane, B., Mouchaorab, K. S. Ballivy, G. (1994). Laboratory investigation of shaft resistance of rock-socketed piers sing the constant normal stiffness direct shear tests. Can. Geotech. J., 31(3): 407-419. https://doi.org/10.1139/t94-048.

Canadian Geotechnical Society. (2006). Canadian Foundation Engineering Manual. $4^{\text {th }}$ edition. Canadian Geotechnical Society. Calgary, Canada.

Carrubba, P. (1997). Skin friction of large-diameter piles socketed into rock. Can. Geotech. J., 34(2): 230-240. https://doi.org/10.1139/t96-104.

Castro-Filgueira, U., Alejano, L., Arzúa, J., Ivars, D.M. (2017). Sensitivity analysis of the micro-parameters used in a PFC analysis towards the mechanical properties of rocks. Procedia Eng., 191: 488-495. https://doi.org/10.1016/j.proeng.2017.05.208.

Chiu, H.K., Dight, P.M. (1983). Prediction of the performance of rock-socketed sideresistance-only piles using profiles. Int. J. Rock Mech. Min. Sci. Geomech. Abstr., 20(1): 21-32. https://doi.org/10.1016/0148-9062(83)91611-X.

Código Técnico de la Edificación CTE. (2006). Documento Básico de Ahorro de Energía CTE-DB-HE. Ministerio de Fomento. Madrid, España. http://www.codigotecnico.org.

Cundall, P.A., 2000. Numerical experiments on rough joints in shear using a bonded particle model. Aspects of tectonic faulting. Springer: Berlín; 1-9.

Dai, G., Salgado, R., Gong, W., Zhu, M. (2017). The effect of sidewall roughness on the shaft resistance of rock-socketed piles. Acta Geotechnica, 12(2): 429-440. https://doi.org/10.1007/s11440-016-0470-8.

Ding, X., Zhang, L., Zhu, H., Zhang, Q. (2014). Effect of model scale and particle size distribution on PFC ${ }^{3 D}$ simulation results. Rock Mech. Rock Eng., 47(6): 2139-2156. https://doi.org/10.1007/s00603-013-0533-1.

Dykeman, P., Valsangkar, A.L. (1996). Model studies of socketed caisson in soft rock. Can. Geotech. J., 33(5): 747-759. https://doi.org/10.1139/t96-100-321.

García-Luna, R., Senent, S., Jurado-Piña, R., Jimenez, R. Structure from Motion photogrammetry to characterize underground rock masses: Experiences from two real tunnels. Tunn. Undergr. Space Technol., 83: 262-273. https://doi.org/10.1016/j.tust.2018.09.026.

Gens, A., Carol, I., Alonso, E.E. (1990). A constitutive model for rock joints formulation and numerical implementation. Comput. Geotech. 9: 3-20. https://doi.org/10.1016/0266352X(90)90026-R.

Ghosh, A.K. (2010). Shear strength of dam-foundations rock interface-a case study. Indian Geotechnical Conference-2010, GEOtrendz: 1039-1042. Mumbai. 
Gloss, G.H., Briggs, O.H. (1983). Rock sockets in soft rock. J. Geotech. Eng., 109(4): 525-535. https://doi.org/10.1061/(ASCE)0733-9410(1983)109:4(525).

Grasselli, G., Egger, P. (2003). Constitutive law for the shear strength of rock joints based on three-dimensional surface parameters. Int. J. Rock Mech. Min. Sci., 40, 2540. https://doi.org/10.1016/S1365-1609(02)00101-6.

Gu, X.F., Seidel, J.P., Haberfield, C.M. (2003). Direct shear test of sandstone-concrete joints. Int. J. Geomech., 3: 21-33. https://doi.org/10.1061/(ASCE)15323641(2003)3:1(21).

Gupton, C., Logan, T. (1984). Design guidelines for drilled shafts in weak rocks of South Florida. Proc. South Florida Annual ASCE Meeting, ASCE, Miami, Florida.

Gutiérrez, M. (2013). Shear resistance for concrete dams: Laboratory tests. Master Thesis. Department of Hydraulic and Environmental Engineering, Norwegian University of Science and Technology. Trondheim.

Gutiérrez-Ch, J.G. (2015). Análisis numérico del efecto de la rugosidad en el contacto pilote-roca sobre la resistencia por fuste y punta. Tesina de Máster. CEDEX-UNED.

Gutierrez-Ch, J.G., Melentijevic, S. (2016). Análisis numérico del efecto de la rugosidad en el contacto pilote-roca sobre la resistencia por fuste y punta. Ingeniería Civil 182: 4153. http://ingenieriacivil.cedex.es/index.php/ingenieria-civil/article/view/446/419.

Gutiérrez-Ch, J.G., Melentijevic, S., Senent, S., Jimenez, R. (2017). Simulación numérica de juntas roca-hormigón a través de ensayos de corte directo en PFC ${ }^{2 D}$. Congress on Numerical Methods in Engineering, 3-5 July. Valencia, España.

Gutiérrez-Ch, J.G., Senent, S., Melentijevic, S., Jimenez, R. (2018). Distinct element method simulations of rock-concrete interfaces under different boundary conditions. Eng. Geol., 240: 123-139. https://doi.org/10.1016/..enggeo.2018.04.017.

Gutiérrez-Ch, J.G., Melentijevic, S., Senent, S., Jimenez, R. (2019a). DEM models to predict side shear resistance of rock-socketed piles considering socket roughness. 53rd U.S. Rock Mechanics/Geomechanics Symposium, 23-26 June: ARMA 19-1757. New York City, New York.

Gutiérrez-Ch, J.G., Melentijevic, S., Senent, S., Jimenez, R. (2019b). A new factor design considering socket roughness for side shear resistance prediction of rocksocketed piles. Congress on Numerical Methods in Engineering, 1-3 July. Guimaraes, Portugal.

Gutiérrez-Ch, J.G., Melentijevic, S., Senent, S., Jimenez, R. (2019c). Socket roughness effect on side shear resistance prediction of rock-socketed piles. 1st Mediterranean Young Geotechnical Engineers Conference, 23-24 September. Mugla, Turkey.

Gutiérrez-Ch, J.G., Song, G., Heron, C., Marschall, A., Jimenez, R., (2020a). Centrifuge modelling of shaft resistance of a rock-socketed pile considering socket roughness. 4th European Conference on Physical Modelling in Geotechnics-ECPMG, 15-17 March, Lulea, Sweden. Accepted 
Gutiérrez-Ch, J.G., Melentijevic, S., Senent, S., Jimenez, R. (2020b). Distinct element method simulations of rock-socketed piles: estimation of side shear resistance considering socket roughness. J. Geotech. Geoenviron. Eng. Under review.

Haberfield, C.M. (2013). Performance of footings in rock based on serviceability. Australi. Geomech. J., 48(1): 1-49.

Haberfield, C.M., Lochaden, A.L.E. (2019). Analysis and design of axially loaded piles in rock. J. Rock Mech. Geotech. Eng., 11(3): 535-548. https://doi.org/10.1016/i.jrmge.2018.10.001.

Haque, A. (1999). Shear behaviour of soft rock joints under constant normal stiffness. PhD Thesis. Deparment of Civil, Mining and Environmental Engineering, University of Wollonfong.

Haque, A., Kodikara, J. (2012). A simplified analytical model for predicting the shear behaviour of regular triangular rock/concrete joints under constant normal stiffness. Geotechnique, 62: 171-176. https://doi.org/10.1680/geot.8.T.018.

Hassan K.M., O'Neill, M.W., Sheikh, S.A., Ealy, C.D. (1997) Design method for drilled shafts in soft argillaceous rock. J. Geotech. Geoenviron. Eng., 123(3): 272-280. https://doi.org/10.1061/(ASCE)1090-0241(1997)123:3(272).

Hill, K.O., Meltz, G. (1997). Fiber bragg grating technology fundamentals and overview. J. Lightwave Technol., 15(8): 1263-1276. https://doi.org/10.1109/50.618320.

Hoek, E., Carranza-Torres, C., Corkum, B. (2002) Hoek-Brown failure criterion. Proc. of NARMS-TAC 2002, mining innovation and technology, Toronto: 267-273.

Hooley, P., Lefroy, S.R. (1993). The ultimate frictional resistance mobilized by bored piles in over-consolidated clays and socketed into weak and weathered rock. The Engineering Geology of Weak Rock. Rotterdam: Balkema, 447-455.

Horvath, R.G., Kenney, T.C. (1979). Shaft resistance of rock-socketed drilled piers. Proc. Symp. on Deep Found., ASCE, 182-214. Atlanta, USA.

Horvath, R.G., Kenney, T.C., Kozicki, P. (1983). Methods for improving the performance of drilled piers in weak rock. Can. Geotech. J., 20(4): 758-772. https://doi.org/10.1139/t83-081.

Indraratna, B., Thirukumaran, S., Brown, E.T., Zhu, S. (2015). Modelling the shear behaviour of rock joints with asperity damage under constant normal stiffness. Rock Mech. Rock. Eng., 48: 179-195. https://doi.org/10.1007/s00603-014-0556-2.

ISSMFE. (1985). Axial pile loading test-Part 1: Static loading. Geotech. Test. J., 8(2): 79-89.

Itasca Consulting Group Inc., 2014. PFC ${ }^{2 D}$ Manual, Version 5.0. (Minnneapolis, Minnesota).

Ivars, D.M., Pierce, M.E., Darcel, C., Reyes-Montes, J., Potyondy, D.O., Young, R.P., Cundall, P.A. (2011). The synthetic rock mass approach for jointed rock mass modelling. Int. J. Rock Mech. Min. Sci., 48: 219-244. https://doi.org/10.1016/j.ijrmms.2010.11.014. 
Ivars, D.M., Potyondy, D.O., Pierce, M., Cundall, P.A. (2008). The smooth-joint contact model. Proc. 8th. World Congress on Computational Mech. - 5th. European Congress on Computational Methods in Applied Sci. and Eng. Venice, Italy.

Jeong, S., Ahn, S., Seol, H. (2010). Shear load transfer characteristics of drilled shafts socketed in rocks. Rock Mech. Rock Eng., 43(1): 41-54. https://doi.org/10.1007/s00603009-0026-4.

Johnston, I.W., Lam, T.S.K., Williams, A.F. (1987). Constant normal stiffness direct shear testing for socketed pile design in weak rock. Geotechnique, 37: 83-89. https://doi.org/10.1680/geot.1987.37.1.83.

Kaderabek. T.J., Reynolds, R.T. (1981). Miami limestone foundation design and construction. J. Geotech. Eng., 107(7): 859-872.

Karandikar, D.V. (2018). Challenges to quality control in bored cast-in-situ piling in growing urban environment. Indian Geotech. J., 48(2): 360-376. https://doi.org/10.1007/s40098-017-0277-z.

Kashyap, R. (2010). Fiber bragg gratings. $2^{\text {nd }}$ Edition, Academic Press.

Khan, A.R., Amadei, B. (1993). Physical modelling of piers socketed into soft rocks. Int. J. Rock Mech. Min. Sci. Geomech. Abstr., 30(7): 747-750. https://doi.org/10.1016/01489062(93)90017-8.

Kister, G., Winter, D., Gebremichael, Y. M., Leighton, J., Badcock, R.A., Tester, P.D., Krishnamuthy, S., Boyle, W.J.O., Grattan, K.T.V., and Fernando G. F. (2007). Methodology and integrity monitoring of foundation concrete piles using Bragg grating optical fibre sensors. Eng. Structures., 29(9): 2048-2055. https://doi.org/10.1016/i.engstruct.2006.10.021.

Kodikara, J.K., Johnston, I.W. (1994). Shear behaviour of irregular triangular rockconcrete joints. Int. J. Rock Mech. Min. Sci. Geomech. Abstr., 31: 313-322. https://doi.org/10.1016/0148-9062(94)90900-8.

Kreuzer M. (2006). Strain measurement with Fiber Bragg grating sensors. HBM: Darmstadt, Germany.

Krounis, A., Johansson, F., Larsson, S. (2016). Shear strength of partially bonded concrete-rock interfaces for application in dam stability analyses. Rock Mech. Rock. Eng., 49: 2711-2722. https://doi.org/10.1007/s00603-016-0962-8.

Kulatilake, P.H.S.W., Shreedharan, S., Sherizadeh, T., Shu, B., Xing, Y., He, P. (2016). Laboratory estimation of rock joint stiffness and frictional parameters. Geotech. Geol. Eng., 34: 1723-1735. https://doi.org/10.1007/s10706-016-9984-y.

Kulhawy, F.H., Phoon, K.K. (1993). Drilled shaft resistance in clay soil to rock. Proc. Conference on Design and Performance of Deep Foundations: Piles and Piers in Soil and Soft Rock., ASCE. Geotech. Spec. Publ., 38: 172-183. New York, USA.

Kulkarni, R.U., Dewaikar D.M. (2016). An empirical approach to assess socket friction and point resistance of axially loaded rock socketed piles of Mumbai region. Int. J. Geotech. Eng., 11(5): 479-486. https://doi.org/10.1080/19386362.2016.1237607. 
Kusumi, H., Matsuoka, T., Ashida, Y., Tatumi, S. (2005). Simulation analysis of shear behavior of rock joint by distinct element method. EUROCK 2005-Impact of Human Activity on the Geological Environment: 281-286.

Ladanyi, B., Archambault, G. (1980). Direct and indirect determination of shear strength of rock mass. Preprint No. 80-25, A.I.M.E. Annual Meeting. Las Vegas, Nevada, 24-28.

Lambert, C., Coll, C. (2014). Discrete modeling of rock joints with a smooth-joint contact model. J. Rock Mech. Geotech. Eng., 6: 1-12. https://doi.org/10.1016/i.jrmge.2013.12.003.

Leichnitz, W. (1985). Mechanical properties of rock joints. Int. J. Rock Mech. Min. Sci., 22(5): 313-321. https://doi.org/10.1016/0148-9062(85)92063-7.

Leung, C.F., Ko, H-Y. (2001). Centrifuge model study of piles socketed in soft rock. Japanese Soc. Soil Mech. Found. Eng., 33(33): 88-91. https://doi.org/10.3208/sandf1972.33.3 80.

Liahagen, S.A. (2012). Stabilitet av betongdammer - Ruhetens påvirkning på skjærkapasiteten mellom betong og berg. Master Thesis. Department of Hydraulic and Environmental Engineering, Norwegian University of Science and Technology. Trondheim.

Lo, K.Y., Ogawa, T., Lukajic, B., Dupak, D.D. (1991). Measurements of strength parameters of concrete-rock contact at the dam-foundation interface. Geotechnical Test. J: 14, 383-394.

LOADTEST. (2012). Deep foundation quality assurance. Consultada el 15 de julio de 2017, en: http://www.loadtest.com/services_int/SoniCaliper.pdf.

Malkowski, P. (2015). Behaviour of joints in sandstones during the shear test. Acta Geodyn. Geomater., 12: 399-410. https://doi.org/10.13168/AGG.2015.0034.

Matich, M.A.J., Kozicki, P. (1967). Some load tests on drilled cast in place concrete caissons. Can. Geotech. J., 4(4): 367-375. https://doi.org/10.1139/t67-065.

Meigh, A.C., Wolshi, W. (1979). Design parameters for weak rocks. Proc. 7th European Conf. on Soil Mech. Found. Eng., 55-57. Brighton, British Geotechnical Society.

Melentijevic, S., Olalla, C. (2014). Different FEM models for simulation of the Osterberg load test in rock shafts. ISRM Regional Symp. EUROCK 2014. London, United Kingdom.

Melentijevic, S., Olalla, C., Prieto., I. (2012). Comportamiento del pilote por fuste en roca estudiado mediante célula Osterberg". $9^{\circ}$ Simposio sobre Cimentaciones y Excavaciones Profundas, Sevilla: 451-466.

Miller, A.D. (2003). Prediction of ultimate side shear for drilled shafts in Missouri shales. Master Thesis. Faculty of the Graduate School, University of Missouri-Columbia.

Mollon, G. (2015). A numerical framework for discrete modelling of friction and wear using Voronoi polyhedrons. Tribology Int., 90: 343-355. https://doi.org/10.1016/i.triboint.2015.04.011. 
Nam, M.S., Vipulanandan, C. (2008). Roughness and unit side resistances of drilled shafts socketed in clay shale and limestone. J. Geotech. Geoenviron. Eng., 134(9): 1272-1279. https://doi.org/10.1061/(ASCE)1090-0241(2008)134:9(1272).

O'Neill, M.W., Reese, L.C. (1999). Drilled shafts: construction procedures and design methods. FHWA-IF-99-025, Federal Highway Administration, U.S. Department of Transportation, Washington, DC.

O'Neill, M.W., Townsend, F.C., Hassan, K.M., Buller, A., Chan, P.S. (1996). Load transfer for drilled shafts in intermediate geomaterials. U.S. Department of Transportation, FHWA-RD- 95-172, Final Report.

Osterberg, J.O., Gill, S.A. (1973). Load transfer mechanism for piers socketed in hard soils or rock. Proc. 9th Canadian Symp. Rock Mech., Montreal, Canada: 235-262.

Park, J., Song, J. (2009). Numerical simulation of a direct shear test on a rock joint using a bonded-particle model. Int. J. Rock Mech. Min. Sci., 46: 1315-1328. https://doi.org/10.1016/j.ijrmms.2009.03.007.

Patton, F.D. (1966). Multiple modes of shear failure in rock. 1st ISRM Congress. Lisbon, Portugal, 509-513.

Pells, P. J. N., Douglas, D. J., Rodway, B., Thorne, C. P., McMahon, B. R. (1978). Design loadings for shales and sandstones in the Sydney region. Austral. Geomechs., G8: 3139.

Pells, P.J.N., Mostyn, G., Walker, B.F. (1998). Foundations on sandstone and shale in the Sydney region. Australi. Geomech., 33(3), 17-29.

Pells, P.J.N., Rowe, R.K., Turner, R.M. (1980). An experimental investigation into shear for socketed piles in sandstone. Proc. Int. Conf. on Struct. Found. on Rock, 1: 291-302. Sydney, Australia.

Poturovic, S., Schubert, W. Blümel, M. (2015). Comparison of constant normal load (CNL) and constant normal stiffness (CNS) direct shear tests. ISRM Regional Symposium-EUROCK 2015. Salzburg, Austria.

Potyondy, D.O. (2012). A flat-jointed bonded-particle material for hard rock. 46th US Rock Mechanics/Geomechanics Symposium. Chicago, Illinois.

Potyondy, D.O. (2015). The bonded-particle model as a tool for rock mechanics research and application: current trends and future directions. Geosyst. Eng., 18: 1-28. http://dx.doi.org/10.1080/12269328.2014.998346.

Potyondy, D.O., Cundall, P.A. (2004). A bonded-particle model for rock. Int. J. Rock Mech. Min. Sci., 41: 1329-1364. https://doi.org/10.1016/i.ijrmms.2004.09.011.

Ragan, D.M. (2009). Structural geology: an introduction to geometrical techniques. Cambridge (UK): Cambridge University Press. https://doi.org/10.1017/CBO9780511816109. 
Rezazadeh, S., Eslami, A. (2017). Empirical methods for determining shaft bearing capacity of semi-deep foundations socketed in rocks. Int. J. Rock Mech. Geotech. Eng., 9(6): 1140-1151. https://doi.org/10.1016/.j.jmge.2017.06.003.

Riquelme, A.J., Tomás, R., Abellán, A. (2016). Characterization of rock slopes through slope mass rating using 3D point clouds. Int. J. Rock Mech. Min. Sci., 84: 165-176. https://doi.org/10.1016/j.ijrmms.2015.12.008.

Rosenberg, P., Journeaux, N.L. (1976). Friction and end bearing tests on bedrock for high capacity socket design. Can. Geotech. J., 13(3): 324-333. https://doi.org/10.1139/t76-033.

Rowe, R.K., Armitage, H.H. (1987). A design method for drilled piers in soft rock. Can. Geotech. J., 24(1): 126-142. https://doi.org/10.1139/t87-011.

Royo, J.M., Melentijevic, S. (2015). Comparison of laboratory direct shear test results with the numerical analysis. Analytical Methods in Petroleum Upstream Applications: Numerical Methods in Geotechnical Engineering, 199-204.

Sagong, M., Paik, K., Kim, D. (2007). A new approach to estimate side resistance of rock socketed drilled shafts. Soils Found., 47(2): 415-421. https://doi.org/10.3208/sandf.47.415.

Seidel, J.P. (1993). The analysis and design of pile shafts into weak rock. Ph.D. Thesis. Department of Civil Engineering, Monash University, Melbourne, Australia.

Seidel, J.P., Collingwood, B. (2001). A new socket roughness factor for prediction of rock socket shaft resistance. Can. Geotech. J., 38(1): 138-153. https://doi.org/10.1139/t00$\underline{083}$.

Seidel, J.P., Haberfield, C.M. (1995). The axial capacity of pile sockets in rocks and hard soils. Ground Eng.: 28(2), 33-38.

Seidel, J.P., Haberfield, C.M. (2002). A theoretical model for rock joints subjected to constant normal stiffness direct shear. Int. J. Rock Mech. Min. Sci., 39: 539-553. https://doi.org/10.1016/S1365-1609(02)00056-4.

Seo, H., Prezzi, M., Salgado, R. (2013). Instrumented static load test on rock-socketed micropile. J. Geotech. Geoenviron. Eng., 139(12): 2037-2047. https://doi.org/10.1061/(ASCE)GT.1943-5606.0000946.

Seol, H.I., Jeong, S.S. (2007). Shaft resistance characteristics of rock-socketed drilled shafts based on pile load tests. J. Korean Geotech. Soc., 23(9): 51-63.

Seol, H.I., Jeong, S.S., Cho, S. (2009). Analytical method for load-transfer characteristics of rock-socketed drilled shafts. J. Geotech. Geoenviron. Eng., 135(6): 778-789. https://doi.org/10.1061/(ASCE)1090-0241(2009)135:6(778).

Serrano, A., Olalla, C. (1994). Ultimate bearing capacity of rock masses. Int. J. Rock Mech. Min. Sci., 31(2): 93-106. 
Serrano, A., Olalla, C., Aguilar, D. (2008). Resistencia por fuste de pilotes empotrados en roca: Estudio comparativo entre distintos métodos de cálculo y normativas. Ingeniería Civil 149: 1-14.

Serrano, A., Olalla, C., Galindo, R.A. (2015). Shaft resistance of a pile in rock based on the modified Hoek-Brown criterion. Int. J. Rock Mech. Min. Sci., 76: 138-145. https://doi.org/10.1016/j.ijrmms.2015.03.007.

Serrano, A., Olalla, C., Galindo, R.A. (2014). Micromechanical basis for shear strength of rock discontinuities. Int. J. Rock Mech. Min. Sci. 70: 33-46. https://doi.org/10.1016/j.ijrmms.2014.02.021.

Shang, J., Zhao, Z., Ma, S. (2018). On the shear failure of incipient rock discontinuities under CNL and CNS boundary conditions: Insights from DEM modelling. Eng. Geol., 234: 153-166. https://doi.org/10.1016/j.enggeo.2018.01.012.

Shrivastava, A.K., Rao, K.S. (2015). Shear behaviour of rock joints under CNL and CNS boundary conditions. Int. J. Geotech. Geol. Eng., 33: 1205-1220. https://doi.org/10.1007/s10706-015-9896-2.

Shrivastava, A.K., Rao, K.S. (2018). Physical modelling of shear behavior of infilled rock joints under CNL and CNS boundary conditios. Rock Mech. Rock Eng., 51: 101-118. https://doi.org/10.1007/s00603-017-1318-8.

Smith, M. (2009). ABAQUS/Standard User's Manual, Version 6.12. Providence, RI: Simulia.

Song, G., Marshall, A.M., Heron, C.M. (2019a). Load redistribution of pile affected by tunneling: hybrid centrifuge tests using fibre Bragg grating. Proc. XVII European Conference on Soil Mech. Geotech. Eng. Reykjavik, Iceland https://doi.org/10.32075/17ECSMGE-2019-0236.

Song, G. (2019b). The use of protective structures to reduce tunneling induced damage to buildings. Ph.D. thesis, Faculty of Engineering, University of Nottingham, UK.

Taylor, R.N. (1995). Geotechnical centrifuge technology. London: Blackie Academic \& Professional.

TEREX. (2015). Auger Tooling. https://www.terex.com/docs/.

Thirukumaran, S., Indraratna, B. (2016). A review of shear strength models for rock joints subjected to constant normal stiffness. J. Rock Mech. Geotech. Eng., 8: 405-414. https://doi.org/10.1016/i.jrmge.2015.10.006.

Tian, H.M., Chen, W.Z., Yang, D.S., Yang, J.P. (2015). Experimental and numerical analysis of the shear behaviour of cemented concrete-rock joints. Rock Mech. Rock. Eng., 48: 213-222. https://doi.org/10.1007/s00603-014-0560-6.

Tian, Y., Quansheng, L., Hao, M., Liu, Q., Deng, P. (2018). New peak shear strength model for cement filled rock joints. Eng. Geol., 233: 269-280. https://doi.org/10.1016/j.enggeo.2017.12.021. 
Toh, C.T., Ooi, T.A., Chiu, H.K., Chee, S.K., Ting, W.H. (1989). Design parameters for bored piles in a weathered sedimentary formation. Proc. 12th Int. Conf. on Soil Mech. Found. Eng., 2:1073-1078. Rio de Janeiro.

Walter, D.J., Burwash, W.J., Montgomery, R.A. (1997). Design of large-diameter drilled shafts for Northumberland Strait bridge project. Can. Geotech. J., 34(4): 580-587. https://doi.org/10.1139/t97-036.

Wang, C., Tannant, D.D., Lilly, P.A. (2003). Numerical analysis of the stability of heavily jointed rock slopes using PFC ${ }^{2 D}$. Int. J. Rock Mech. Min. Sci., 40: 415-424. https://doi.org/10.1016/S1365-1609(03)00004-2.

Whitaker, T., Cooke, R.W. (1966). An investigation of the shaft and base resistances of large bored piles in London Clay. Proc., Symp., of Large Bored Piles, 7-49, London, UK.

Williams, A.F. (1980). The side resistance of piles socketed into weak rock. Ph.D. Thesis. Department of Civil Engineering, Monash University, Melbourne, Australia.

Williams, A.F., Johnston, I.W., Donald, I.B. (1980). The design of socketed piles in weak rock. Proc. Int. Conf. Struct. Found. Rock, 1: 327-347. Sydney, Australia.

Williams, A.F., Pells, P.J.N. (1981). Side resistance in sandstone, mudstone, and shale. Can. Geotech. J., 18(4): 502-513. https://doi.org/10.1139/t81-061.

Xing, H., Zhang, Z., Meng, M., Luo, Y., Ye, G. (2014). Centrifuge tests of superlargediameter rock-socketed piles and their bearing characteristics. J. Bridge Eng., 19(6): 04014010. https://doi.org/10.1061/(ASCE)BE.1943-5592.0000582.

Xu, M., Ni, P., Mei, G., Zhao, Y. (2018). Load-settlement behaviour of bored piles with loose sediments at the pile tip: Experimental, numerical and analytical study. Comput. Geotech., 102: 92-101. https://doi.org/10.1016/j.compgeo.2018.06.010.

Zhang, L. (2004). Drilled shafts in rock: analysis and design. London (UK): Balkema.

Zhang, L.M., Wong, E. Y. (2007). Centrifuge modelling of large-diameter bored pile groups with defects. J. Geotech. Geoenviron. Eng., 133(9): 1091-1101. https://doi.org/10.1061/(ASCE)1090-0241(2007)133:9(1091).

Zhang, L., Einstein, H. H. (1998). End bearing capacity of drilled shafts in rock. J. Geotech. Geoenviron. Eng., 124(7): 574-584. https://doi.org/10.1061/(ASCE)10900241(1998)124:7(574). 


\section{Anexo A: Herramientas de perforación}

A continuación, se presentan una serie de imágenes de algunas de las herramientas de perforación empleadas durante la perforación de pilotes en roca, y que han sido mencionadas a lo largo de esta tesis doctoral.

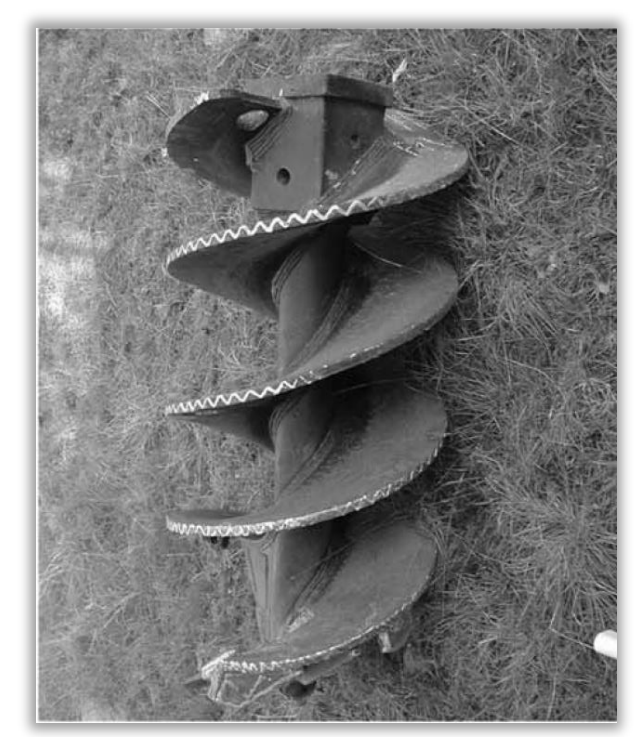

Figura A.1. Barrena (Auger) (tomado de Nam y Vipulanandan 2008).

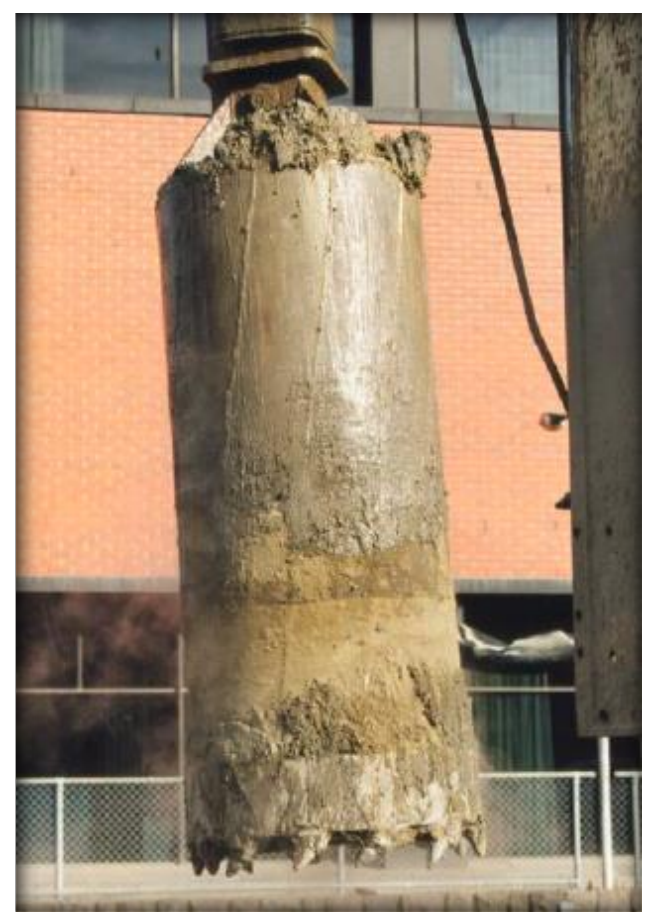

Figura A.2. Core Barrel (tomado de Collingwood 2000). 


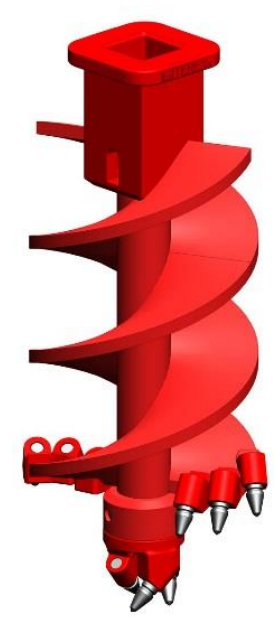

Figura A.3. Barrena Bullet Tooth Rock (tomado de TEREX 2015).

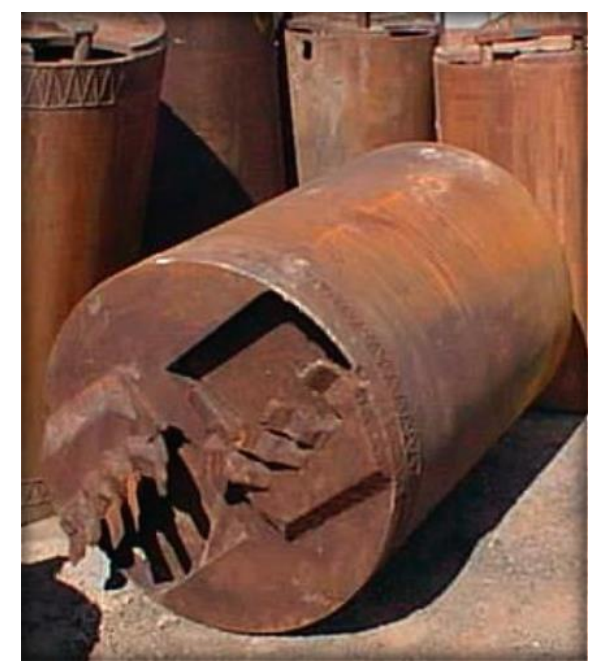

Figura A.4. Barrena Bucket. (tomado de Collingwood 2000).

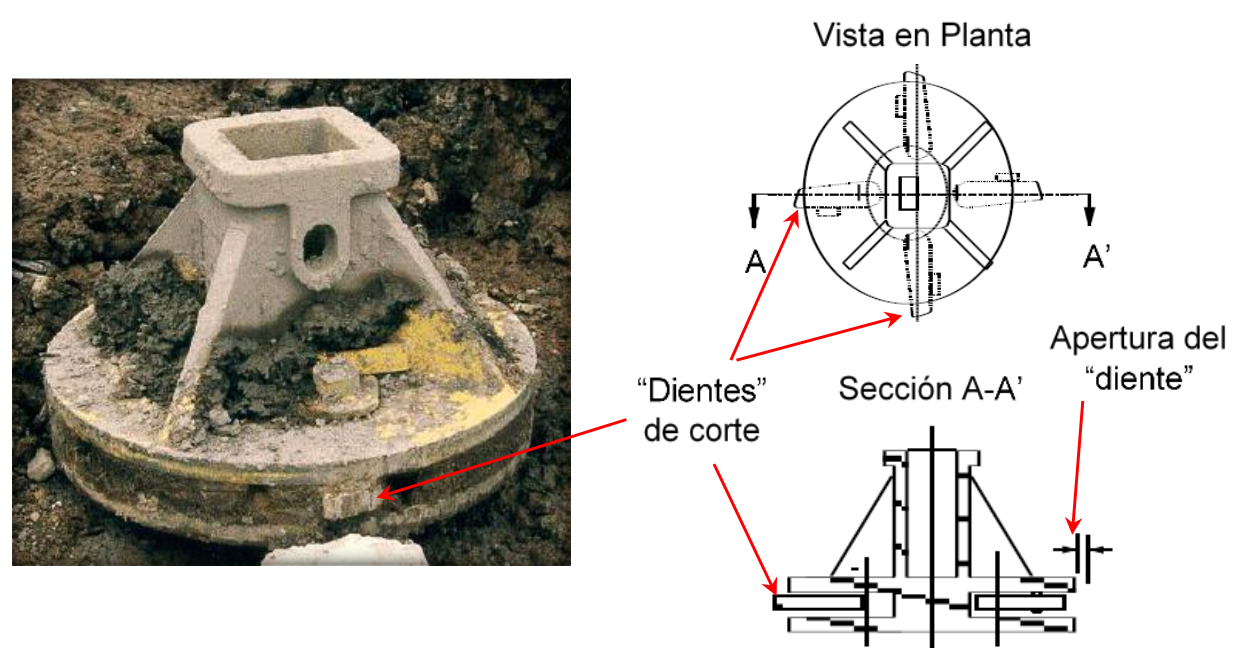

Figura A.5. Herramienta de corte circunferencial (WRT1) para incrementar la rugosidad de la excavación (modificado de Collingwood 2000). 


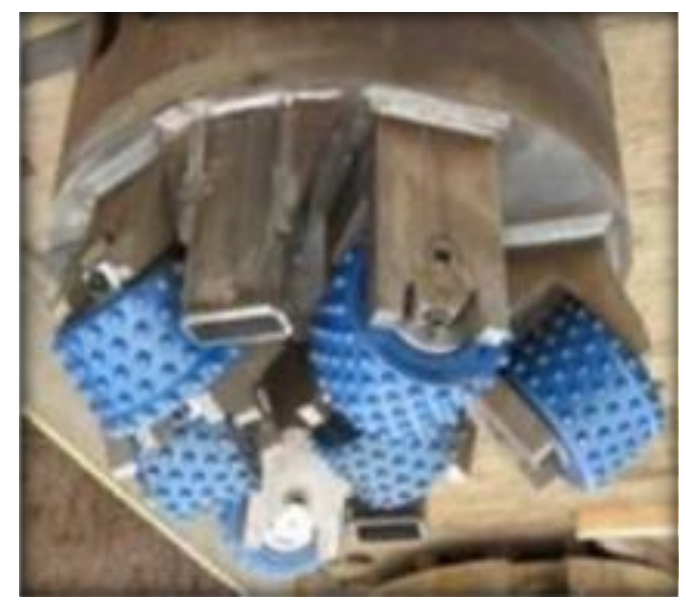

Figura A.6. Core Bit (tomado de Karandikar 2018).

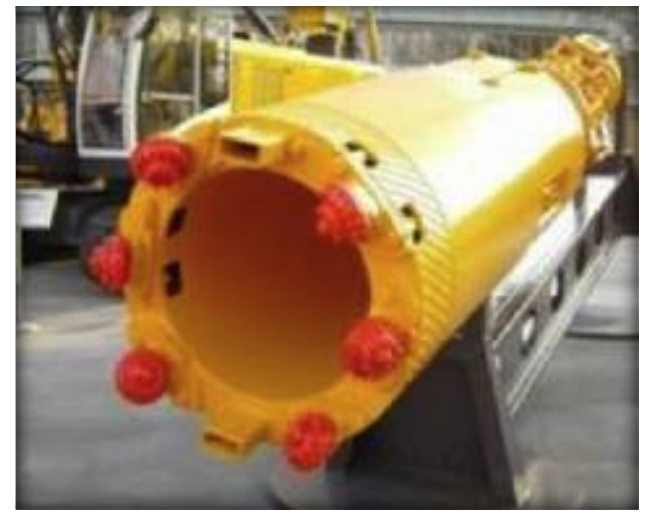

Figura A.7. Rock roller (tomado de Karandikar 2018).

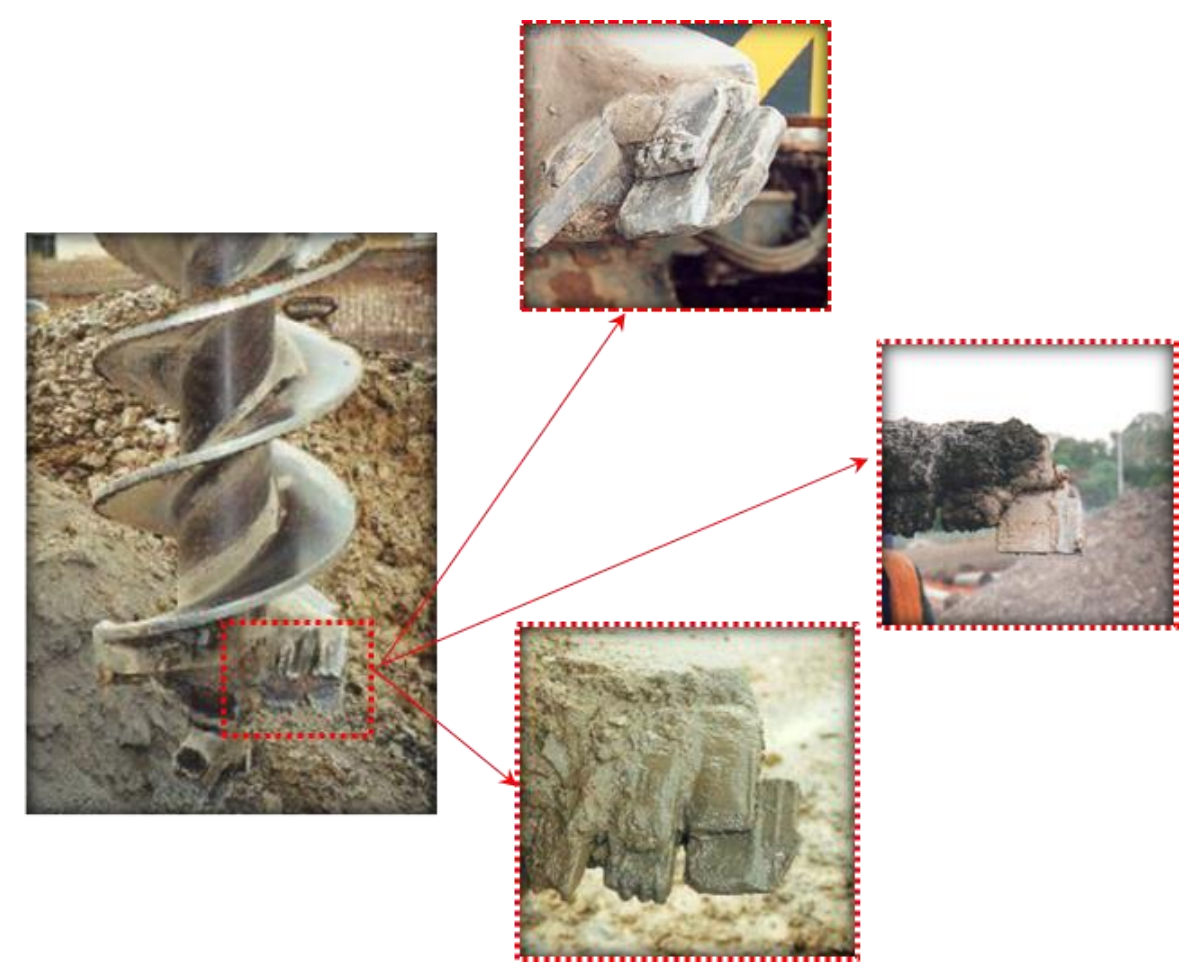

Figura A.8. Barrena con "dientes" de corte para incrementar la rugosidad de la excavación (modificado de Collingwood 2000). 


\section{Anexo B: Registro fotográfico de los ensayos de laboratorio}

A continuación, se presenta parte del registro fotográfico de los ensayos realizados durante la preparación de la pseudo-roca a usar en los ensayos de centrífuga realizados en esta tesis doctoral (ver el Capítulo 5).

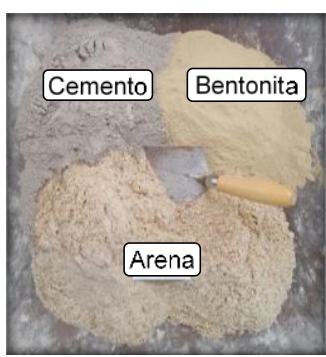

(a)

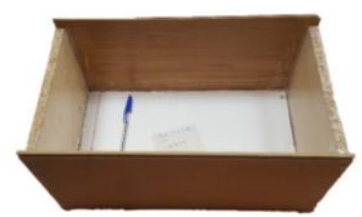

(d)

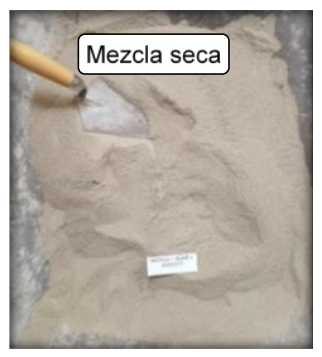

(b)

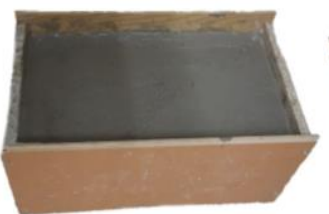

(e)

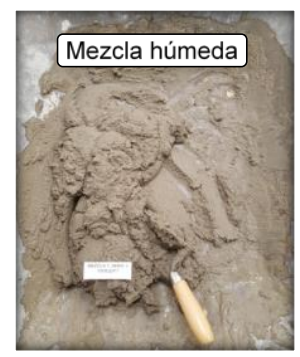

(c)

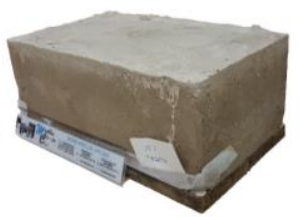

(f)

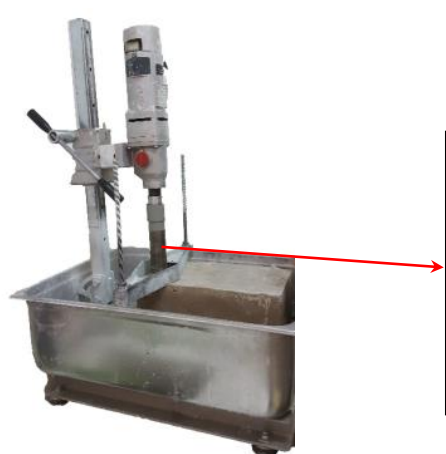

(g)

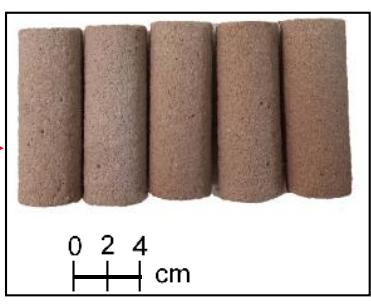

(h)

Figura B.1. Ejemplo del proceso de preparación de la pseudo-roca: (a) arena, cemento y bentonita antes de mezclarlos, (b) arena, cemento y bentonita después de mezclarlos, (c) mezcla de arena, cemento y bentonita después de agregar la proporción de agua, (d) molde de madera de $36 \times 21 \times 15 \mathrm{~cm}^{3}$ antes de verter la mezcla, (e) molde de madera después de verter y vibrar la mezcla de la pseudo-roca en 3 capas iguales, (f) probeta de la pseudo-roca desmoldada después del proceso de curado, (g) extracción de probetas cilíndricas de la pseudo-roca para realización de ensayos. 


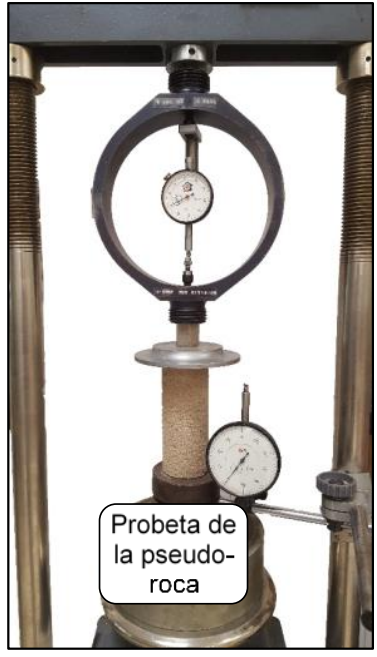

(a)

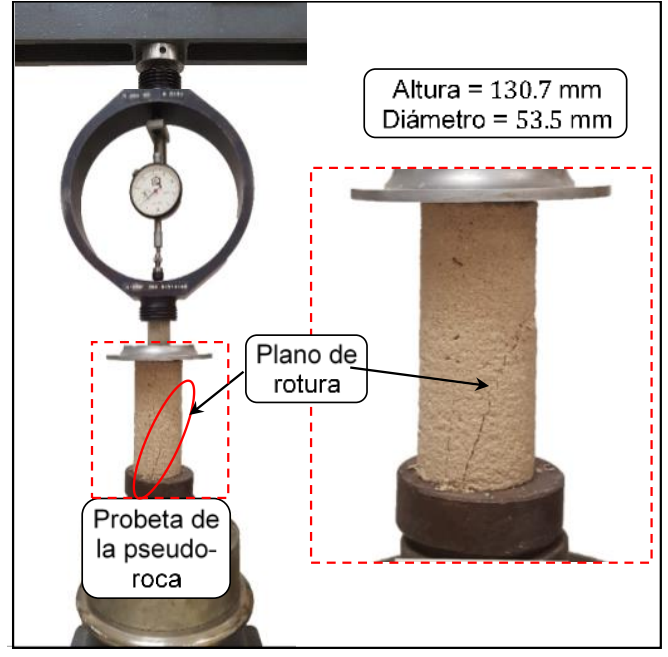

(b)

Figura B.2. Ejemplo de ensayo de compresión uniaxial: (a) probeta de la pseudo-roca antes de comenzar el ensayo, (b) modo de fallo de la probeta.

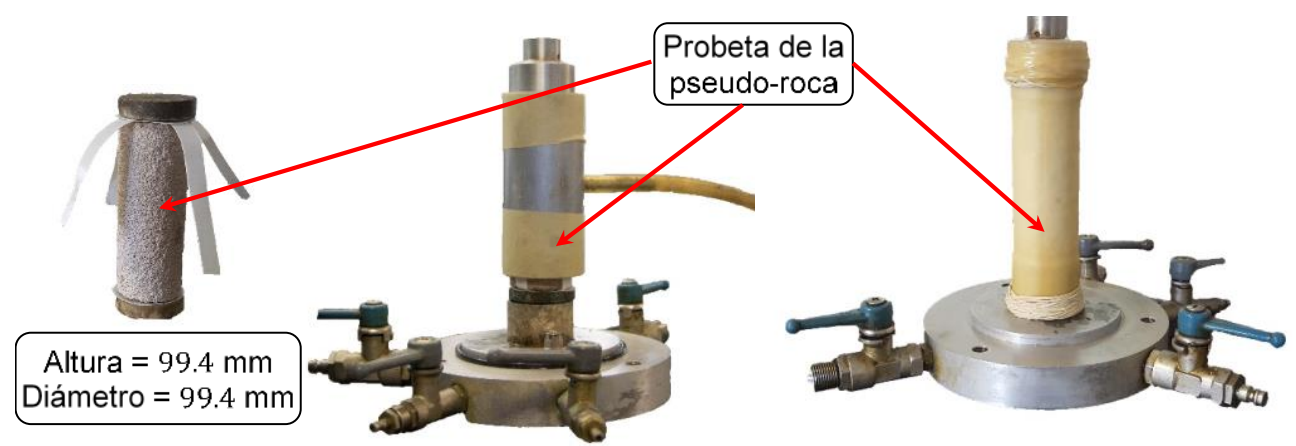

(a)

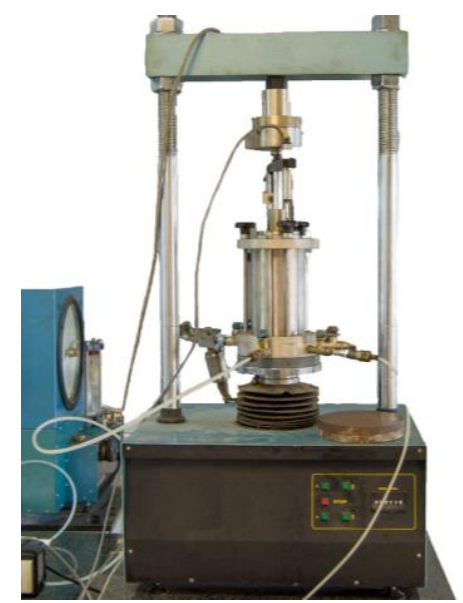

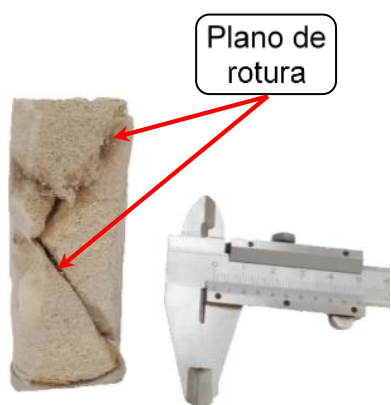

(c)

(b)

Figura B.3. Ejemplo de ensayo triaxial consolidado no drenado: (a) preparación de la probeta de la pseudo-roca, (b) probeta durante el ensayo, (c) modo de fallo de la probeta. 


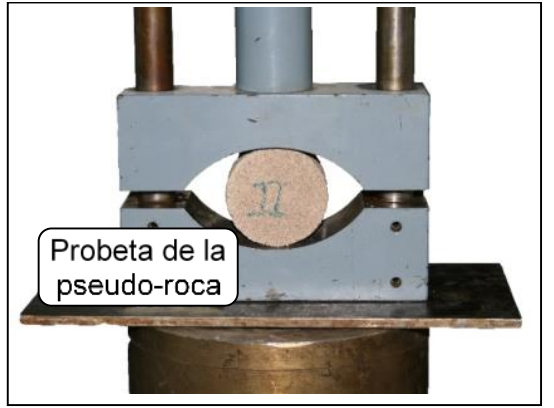

(a)

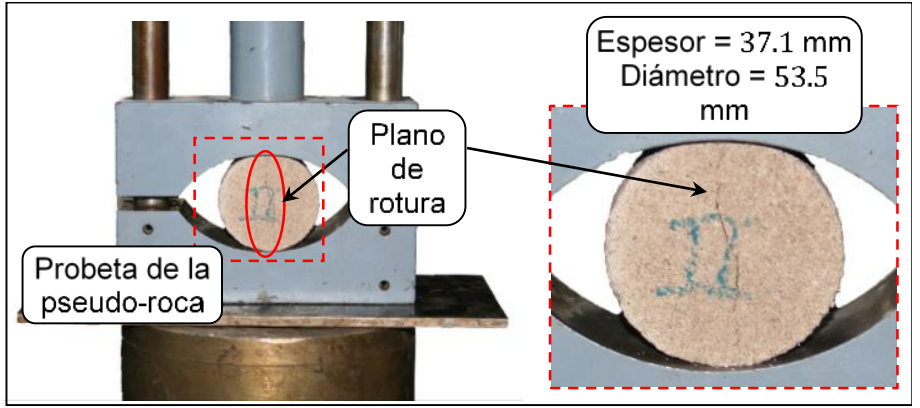

(b)

Figura B.4. Ejemplo de ensayo brasileño (tracción indirecta): probeta de la pseudo-roca antes de comenzar el ensayo, (b) modo de fallo de la probeta.
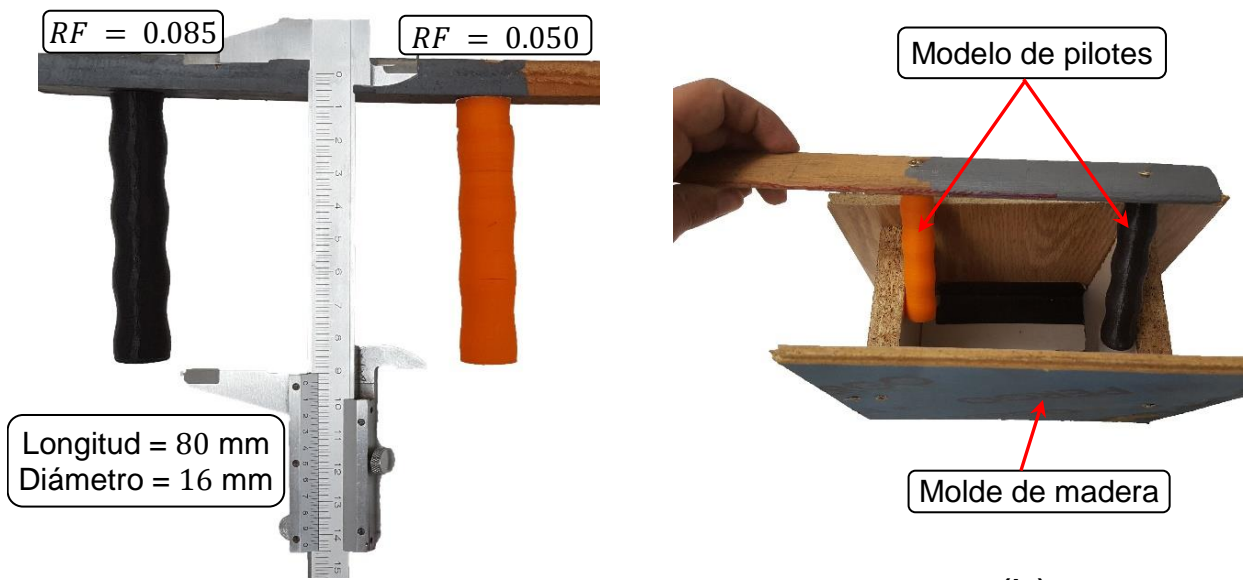

(b)

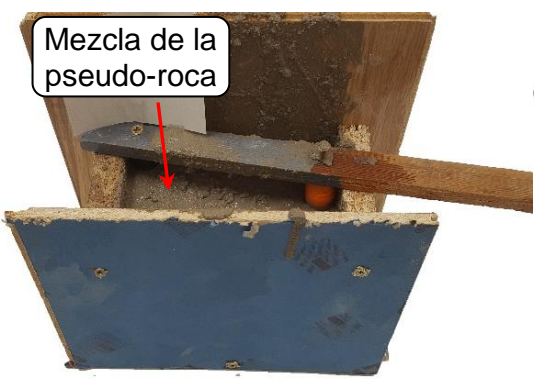

(C)

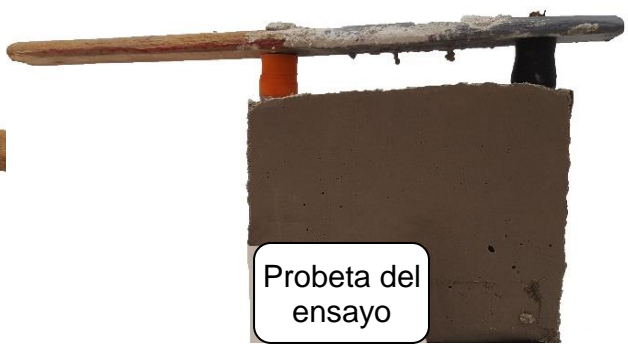

(d)

Figura B.5. Ejemplo de prueba realizada para evaluar la rugosidad resultante en la interfaz roca-pilote: (a) modelos de pilotes de plástico con diferente nivel de rugosidad, (b) colocación de los modelos de pilotes dentro del molde de madera, (c) probeta del ensayo después de verter y vibrar la mezcla de pseudo-roca, (d) probeta del ensayo después de ser desmoldada a las 24 horas. 


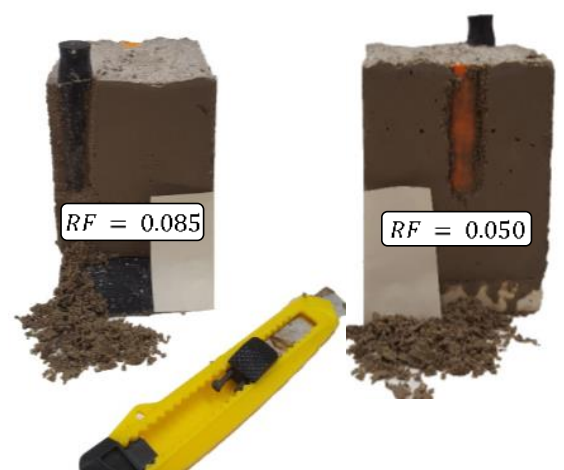

(a)

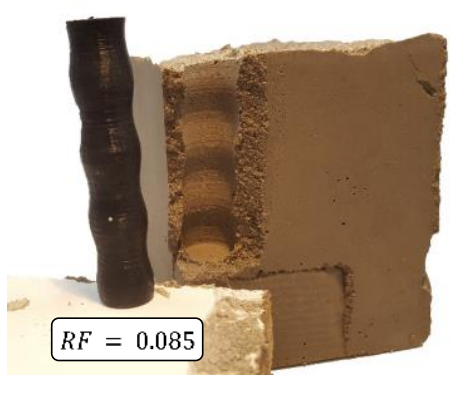

(c)

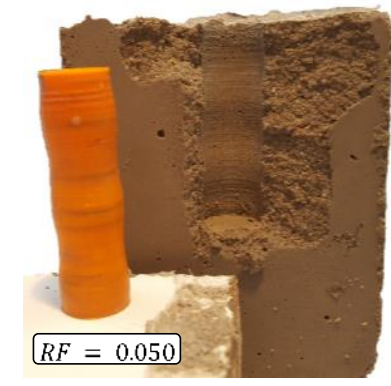

(b)

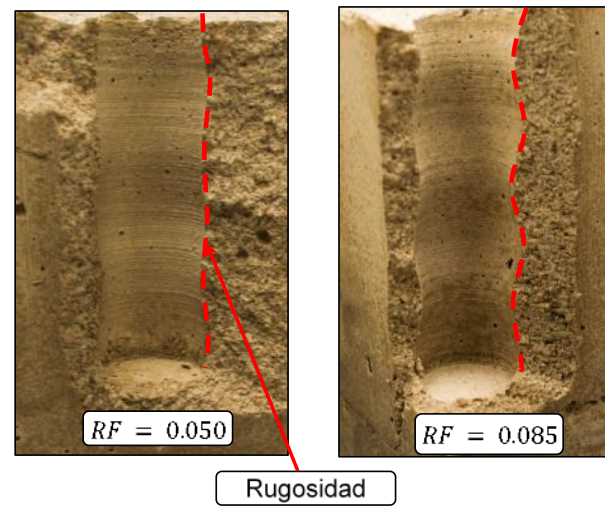

(d)

Figura B.6. Ejemplo de la rugosidad en la interfaz roca-pilote: (a) remoción de los modelos de pilotes a las 24 horas, (b) rugosidad resultante en la mezcla de la pseudo-roca luego de remover el modelo de pilote con $R F=0.050,(\mathrm{C})$ rugosidad resultante en la mezcla de la pseudo-roca luego de remover el modelo de pilote con $R F=0.085$, (d) detalle de la rugosidad resultante en la pseudo-roca del ensayo. 